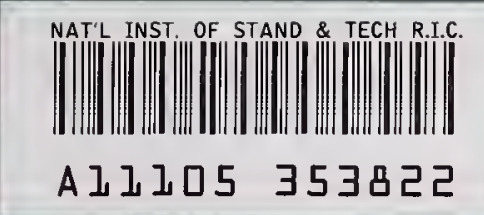

Mechanicall and Thermal Properties of Ceramics

$$
\begin{gathered}
\text { Precceding of a Symposium } \\
\text { Aprit 1m. } 1968
\end{gathered}
$$

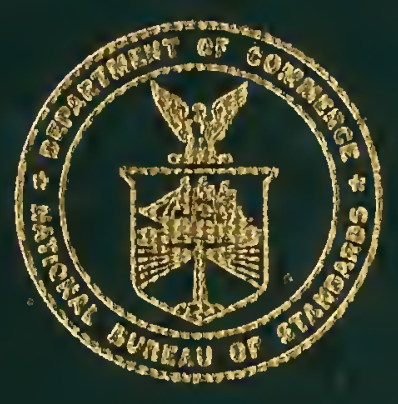

Uximed States Department of Conmarce

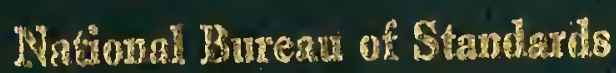

Syegenal Publication 303 



UNITED STATES DEPARTMENT OF COMMERCE

Maurice H. Stans, Secretary

NATIONAL BUREAU OF STANDARDS • A. V. Astin, Director

\title{
Mechanical and Thermal Properties of Ceramics
}

\author{
Proceedings of a Symposium \\ Held at Gaithersburg, Maryland \\ April 1-2, 1968
}

\author{
Edited by \\ J. B. Wachtman, Jr. \\ Institute for Materials Research \\ National Bureau of Standards \\ Washington, D.C. 20234
}

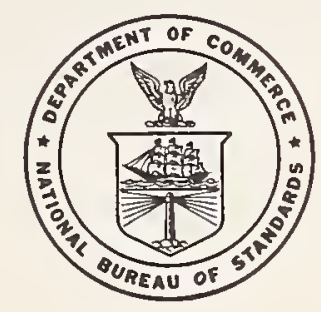

Sponsored by

The American Ceramic Society

The American Society for Testing and Materials

and

The National Bureau of Standards

U.S. National Bureau of Standards, Special Publication 303

Issued May 1969

For sale by the Superintendent of Documents, U.S. Government Printing Office, Washington, D.C. 20402

Price \$4. 25 
JUL 23969

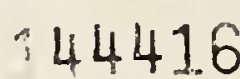

20100

U. 57

No. 303

1905

\begin{abstract}
The proceedings of the Symposium on Mechanical and Thermal Properties of Ceramics, held at the National Bureau of Standards in Gaithersburg, Maryland, on April 1-2, 1968, are presented. The symposium was cosponsored by the American Ceramic Society, the American Society for Testing and Materials, and the National Bureau of Standards. Attention was concentrated upon properties primarily of structural and/or high temperature use including melting points, thermal expansion, thermal conductivity, thermal radiation properties, elasticity, viscoelasticity, inelastic deformation, and fracture. The dependence of these properties upon composition and microstructure was surveyed and the importance of controlling these features of character to insure reliability of ceramics was emphasized. The procedures by which American ceramists formulate standards and specifications to assist in insuring reliability were summarized.
\end{abstract}

Key Words: Ceramies; elasticity; fracture; inelastic deformation; melting point; standards; thermal conductivity; thermal expansion; thermal radiation; viscoelasticity.

Library of Congress Catalog Card Number: 68-61960 


\section{Foreword}

A major responsibility of the NBS Institute for Materials Research is to insure the availability of those basic data on the properties of materials which are urgently needed to meet the requirements of the Nation's scientists and engineers. To obtain reliable data of this kind not only must the experimental procedures be tailored to permit the required measurement accuracies, and the results correctly analyzed, but also the sample materials must be sufficiently uniform and well characterized to permit measurements amenable to meaningful interpretation and practical use.

The reliability of data needed for structural or high-temperature use of ceramics is an important example. Sensitivity of strength data to test conditions and complications introduced by high measurement temperatures plague the measurements and make experimental procedures a prime concern. Also, several important properties, including thermal conductivity, inelastic deformation, and strength, are sensitive to small variations in microstructure and composition. To provide a basis for understanding the dependence of ceramic properties on various characterizing features, a Symposium on Mechanical and Thermal Properties of Ceramics was held at the National Bureau of Standards laboratories in Gaithersburg, Md., in April 1968. The meeting was jointly sponsored by the American Ceramic Society, the American Society for Testing and Materials, and NBS.

The National Bureau of Standards is pleased to have the opportunity of making the papers presented at the Symposuim available in this publication. In this way we hope to assist those attending the conference in optimizing the use of ceramics, to make the conference results available to a wider audience, and to show appreciation for the essential contributions of the participants and the cosponsor organizations.

\section{A. V. Astin, Director}




\section{Introduction}

This is a collection of the proceedings of the symposium, Mechanical and Thermal Properties of Ceramies, held at the National Bureau of Standards laboratories at Gaithersburg, Maryland, on April 1-2, 1968. Approximately 210 scientists and engineers participated.

The proper selection and effective use of materials is one of the fundamental factors upon which advances in technology depend. Ceramic materials have a long history of effective use in many applications for which their hardness, stiffness, strength, resistance to corrosion, thermal and electrical insulating qualities, high melting points, optical properties, semiconducting properties, dielectric properties, magnetic properties, piezoelectric properties, nuclear properties, or other special qualities are appropriate. The ceramic industry in the United States continues to grow and is approaching the ten billion dollar a year level. Every type of material, including ceramics, is challenged, however, both by competition from other materials in its traditional areas of use and by the requirements for improved properties needed in new applications.

The development of new materials and the improvement of properties depend partly upon an empirical approach in which the composition and manufacturing procedure are systematically varied and correlation with resulting properties is attempted. This empirical method will probably continue indefinitely to be an important part of materials development and need not conflict with the use of materials science. The latter is helpful in predicting areas where improvement appears possible, choosing the parameters to be varied, designing an efficient processing-property study, and interpreting the results.

Ceramics are frequently manufactured by a heating process which appears deceptively simple but which usually involves a number of competing reactions and transport processes occurring at different rates. Optimizing a particular property requires a starting composition and heat treatment which produces the final composition and microstructure (usually denoted by the word "character") needed for that property. In principle one should begin with the properties needed, determine the character required from an understanding of the propertycharacter relationship, and design the processing from an understanding of the chemistry and mass transport which are involved. In practice the understanding of both areas is limited so that empirical procedures, guided by some degree of fundamental understanding, are generally used.

The current status of ceramic processing was surveyed by the Materials Advisory Board ad hoc Committee on Ceramics Processing, chaired by Professor J. A. Pask, and their conclusions are available in Ceramic Processing, National Academy of Sciences Publication 1576 (1968). The importance of an improved understanding and use of the property-character relationship by users of ceramics as well as producers is emphasized. In particular, the user should understand that many properties depend on other aspects of character in addition to gross chemical composition. The engineer who specifies that a part be made of "alumina" is leaving the strength very poorly specified; commercial alumina ceramics range in strength from values below 2000 psi for certain refractories to values approaching 100,000 psi for certain tool bits and other special purpose ceramics. The present symposium is aimed primarily at the users of ceramics but it is hoped that producers will also find a systematic review of property-character relationships useful.

Properties of ceramics in general form too broad and diverse a subject to be effectively treated in this symposium; the properties selected for treatment are those related to mechanical and thermal behavior. Ceramics are frequently used to exploit their thermal properties and also as structural members in which case their deformation and ultimate fracture under load are of obvious importance. In many applications ceramics are used to exploit other properties, but mechanical and thermal properties are usually still important in determining the size, weight, and cost of the parts involved. Ceramics are, of course, particularly well suited to high temperature applications but the conditions of high temperature use frequently create thermal gradients with accompanying thermal stress. The symposium accordingly includes 
thermal factors not only because of their direct engineering importance, but also because of their role in limiting load-bearing capacity at high temperatures; i.e., the melting points, thermal expansion, thermal conductivity, and thermal radiation properties are surveyed.

The practical utilization of high-performance ceramics requires more than their successful production on a laboratory scale and favorable economic factors. Such ceramics must be reliable in production, that is, their properties must be uniform within each piece and reproducible from piece to piece. Small lots of special ceramics frequently show significant variation in structure-sensitive properties including strength. This variation has raised questions concerning the permissible stress levels in structural applications and is currently inspiring research aimed at determining to what extent this variability in strength results from variability in processing, variability in testing, or from the intrinsic nature of brittle materials. This research aims at the ultimate establishment of a degree of processing control, testing accuracy, and design understanding to permit ceramics to be used routinely and reliably at high stress levels. Such use depends on acceptance of testing standards by the producers and users of ceramics.

This symposium accordingly opens with a survey of the activities of the American Ceramic Society and the American Society for Testing and Materials, two of the principal institutions through which American ceramists communicate and formulate standards. The needs for high-performance ceramics are next discussed and the importance of reliability is stressed. The thermal properties are then treated, being taken first because of their role in determining stresses resulting from heat flow. Finally, the mechanical properties which determine response to stress, whether of thermal or mechanical origin, are discussed. Throughout the discussion of properties the emphasis is placed on the property-character relationship;i.e., on the features of composition and microstructure upon which the properties depend. Some property tabulations are given to illustrate property ranges, but exhaustive tabulation of numerical values has not been attempted. References are given to many existing tabulations which, excellent as some are, need to be used carefully with an understanding of how variation in character of the ceramic under consideration can cause properties to vary from the handbook values.

Each of the following papers was written as a separate entity and can be read without reference to the others. A few comments on some of the highlights of the papers and on the interrelation of the various properties should, however, give a helpful perspective on the general subject of the symposium. An important point concerning high-performance ceramics is made in the paper on the American Ceramic Society and Ceramic Materials Technology. The example of ceramic insulators for spark plugs is quoted to make the point that when large volume requirements exist ceramics can be and are routinely produced at modest cost to reliably meet high-performance specifications. This example raises the question of how well expertise developed in producing a specialized ceramic can be transferred to the production of other ceramics to meet somewhat different high-performance requirements. A purely empirical production process developed around a specific raw material and product may not be a good guide in developing a new product; understanding of the property-character relationship and of the process of character development during processing are needed. Standard test methods for both character determination and property measurement are therefore important to aid in developing this understanding in relation to commercial manufacturing of ceramics. The process by which United States Standards are developed and the role of the American Ceramic Society and the American Society for Testing and Materials in the development of standards for ceramics is accordingly described.

Commercial development of new ceramics will occur only if justified by the demand; the paper on "Properties of Ceramics for Structural and/or High Temperature Use; Need for Control, Measurement and Compilation" discusses new markets open to ceramics and emphasizes the requirement of reliability and the consequent need for understanding the characterproperty relationship and for good testing procedures.

The discussion of thermal properties begins with a paper on melting points. This might incorrectly be thought to be a simple subject but the engineer unfamiliar with phase equilibria who attempts to use ceramics at high temperatures may be in for a surprise. Thus, for example, $\mathrm{TiO}_{2}$, melting at about $1840{ }^{\circ} \mathrm{C}$, and $\mathrm{CaO}$, melting at about $2570{ }^{\circ} \mathrm{C}$, might appear to be safe for use at $1500{ }^{\circ} \mathrm{C}$. Anyone who has tried using $\mathrm{CaO}$ and $\mathrm{TiO}_{2}$ in contact with each other at $1500{ }^{\circ} \mathrm{C}$ or many similar combinations of ceramics above their eutectic temperature is referred to the paper on melting points for an explanation of what happened to his ceramic parts. 
One of the factors limiting use of ceramics at high temperature, as mentioned previously, is the development of stress accompanying thermal gradients. Thermal shock parameters involving elastic moduli and thermal expansion have been used as a rough guide to the "thermal shock resistance" of materials, but it has long been recognized that a complete analysis of the heat flow and temperature distribution for each particular device is needed. High-speed computers make this feasible so that modern designs are increasing based on an analysis which requires the thermal radiation properties (emissivity, etc.) and thermal conductivity to calculate the temperature distribution and then requires the thermal expansion and elastic moduli (plus equations giving plastic response if it is involved) in order to calculate the stress distribution.

Fracture of polycrystalline ceramics as a result of stress of thermal or mechanical origin usually occurs suddenly and completely, at least at temperatures below the range of appreciable plastic deformation. The need to understand the characteristics of brittle fracture and to design for the use of ceramics with this in mind is apparent. The fracture paper in this symposium deals with this subject from the point of view of fracture mechanics as a branch of continuum mechanics as well as from the point of view of the effects of defects and environment. Finally, the subject of mechanical testing is treated in a separate paper because of its difficulty and importance.

J. B. Wachtman, Jr.

\section{Acknowledgment}

The editor wishes to thank the following for their assistance in organizing and holding the symposium:

R. T. Cook, National Bureau of Standards

P. M. Corbett, Glidden-Durkee Division, SCM Corp.

J. J. Diamond, National Bureau of Standards

G. T. Leighty, National Bureau of Standards

J. L. Pentecost, W. R. Grace \& Co., Washington Research Center

D. W. Robertson, General Refractories Company 


\section{Contents}

Page

Foreword.$\ldots \ldots \ldots \ldots \ldots \ldots \ldots \ldots \ldots \ldots \ldots \ldots \ldots \ldots \ldots \ldots \ldots \ldots \ldots \ldots \ldots \ldots$ III

Introduction $\ldots \ldots \ldots \ldots \ldots \ldots \ldots \ldots \ldots \ldots \ldots \ldots \ldots \ldots \ldots \ldots \ldots \ldots \ldots \ldots \ldots \ldots$ IV

The American Ceramic Society and Ceramic Materials Technology............. 1 J. S. Owens

ASTM : Its Function and Philosophy $\ldots \ldots \ldots \ldots \ldots \ldots \ldots \ldots \ldots \ldots \ldots \ldots \ldots \ldots \ldots \ldots$

F. J. Mardulier

ASTM Activities in Ceramics................................. 9

J. C. Richmond

Properties of Ceramics for Structural and/or High Temperature Use: Need for Control, Measurement, and Compilation........................ 13 C. S. Bersch

Melting Points of Ceramics. . . . . . . . . . . . . . . . . . . . . . . . . . . . . . . . 19

S. J. Schneider

Thermal Expansion of Ceramics............................. 4.1 R. K. Kirby

Thermal Conductivity of Ceramics............................. 63 D. R. Flynn

Thermal Radiation Properties of Ceramic Materials.................... 125 J. C. Richmond

Elastic Deformation of Ceramics and Other Refractory Materials............. 139 J. B. Wachtman, Jr.

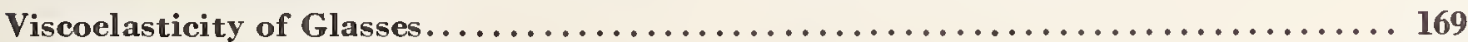

P. B. de Macedo

Inelastic Deformation of Oxide Ceramics . . . . . . . . . . . . . . . . . . . . . . . . . . 189

R. M. Spriggs

Plastic Deformation of Carbides..................................201

G. E. Hollox

Fracture of Ceramics. . . . . . . . . . . . . . . . . . . . . . . . . . . 217

S. M. Wiederhorn

Strength Testing of Ceramies $-A$ Survey $\ldots \ldots \ldots \ldots \ldots \ldots \ldots \ldots \ldots \ldots \ldots \ldots$

L. Mordfin and M. J. Kerper

Subject Index. . . . . . . . . . . . . . . . . . . . . . . . . . . . . . . . . 263 



\title{
The American Ceramic Society and Ceramic Materials Technology
}

\author{
James S. Owens \\ President, The American Ceramic Society
}

\author{
Champion Spark Plug Company, Ceramic Division, Detroit, Michigan 48211
}

\begin{abstract}
The ceramic industry in the United States, through the continued growth of the use of traditional ceramics and the development of many new products and applications, has a sales volume approaching ten billion dollars a year. The American Ceramic Society, which was founded 69 years ago and represents all segments of the industry, has grown into the world's largest ceramic technical organization and is still growing at the rate of 50 percent per decade. It has diversified into ten Divisions to serve the economic, technological and scientific interests associated with ceramics. High performance ceramic materials with unique properties inherently dependent upon microstructure have been developed for specific applications that require either limited quantities or high volume mass production. Careful production control to insure a high level of performance is receiving close attention.
\end{abstract}

Key Words: Ceramics; ceramic industry; American Ceramic Society; microstructure; properties; special ceramics.

It is an honor to be invited to take part with you in this significant Symposium on Mechanical and Thermal Properties of Ceramics, and it is a pleasure to do so. Since its founding, The American Ceramic Society has been extremely interested in encouraging and supporting the continuation of the technical education of the members of the ceramic industry and of those associated with it. This meeting is an excellent example of such continuing education. Our Society is happy and proud to sponsor this Symposium through its Baltimore-Washington Section in cooperation with the Middle Atlantic District of the American Society for Testing and Materials and the National Bureau of Standards.

It is particularly fitting that this program should be held here at the National Bureau of Standards since the Bureau has done outstanding research in ceramics and this Symposium was organized by J. B. Wachtman, Jr., who is Chief of the Physical Properties Section of the Bureau and is an officer of the Baltimore-Washington Section of The American Ceramic Society.

The ceramic industry is a large one with estimated sales this year of about ten billion dollars. Ceramics include the inorganic nonmetallic solid materials. They have great importance for a wide variety of industrial, commercial and consumer uses, and to our Government for both national defense and civilian purposes. Their broad range of uses includes, for example, refractory ceramics for application at very high temperatures, in nuclear and aerospace technology, and in industrial furnaces and kilns; electronic ceramics in miniaturized microelectronic circuitry that helps to make possible new generations of computers and other complex, yet compact, electronic equipment; spark plug and jet igniter insulators; ceramic protective coatings for metals; brick, glass, and tile structural and decorative ceramics; glass containers; and dinnerware.

Since the success of many applications of ceramic materials depends on their mechanical and thermal properties, this Symposium is an important and timely one.

The American Ceramic Society had its inception seventy years ago in February 1898 at the 12 th Annual Convention of the National Brick Manufacturers' Association in Pittsburgh where the first scientific paper of its kind was presented before that Association. As a result, there developed in the next two days the idea for a separate society for the presentation of technical papers on ceramics that culminated a year later on February 6, 1899 in the official chartering of 'The American Ceramic Society in Columbus, Ohio, with 15 charter members. These included General Edward Orton, Jr., who was Secretary of the Society until 1917, and A. V. Bleininger who was associated with the National Bureau of Standards for several years.

Our Society has grown from those very small beginnings into the largest ceramic technical organization in the world with just under 10,000 members and subscribers. It is dynamic and progressive and has developed to its present significant position because it was needed to aid in the development and the improvement of the ceramic profession and industry, and it has made a continuing, conscientious, successful effort to fill this 
need. As a result, the Society has grown tremendously and is still growing in membership at the rate of over 50 percent per decade. However, many of the activities of our members are increasing twice that rapidly. Thus, the number of technical papers published annually, the number of papers presented at the Annual Meeting and the fall Divisional Meetings, the attendance at the fall meetings, and the number of abstracts published yearly has doubled in the past decade.

The primary services of the Society are the holding of meetings for the sharing of technical ceramic information by the presentation and discussion of papers and the publication of these papers for the wider dissemination of the information. These are extremely important services, but, in addition, the Society provides other significant services for its members and for the whole ceramic industry and the public generally. These include authoritative, specialized publications on selected, important subjects such as Phase Diagrams for Ceramists, Engineering Properties of Selected Ceramic Materials, Large Scale Phase Diagrams of Oxide Systems, and Symposium on Nucleation and Crystallization in Glasses and Melts; the encouragement and sponsorship of continuing education programs such as this Symposium and the programs carried out under the auspices of other Sections of the Society; investigation of the needs of the ceramic industry for technical information services and its possible sequel for the determination of optimum methods to provide the services found to be needed; liaison through the National Institute of Ceramic Engineers with other engineering disciplines; cooperation with related scientific and technical societies in this country and with ceramic organizations abroad on matters of common interest; and cooperation with governmental agencies and public organizations such as the National Research Council through members of the Society serving on advisory panels, boards and committees of these organizations in the public interest. I might also mention that many members of our Society serve on appropriate committees of AS'TM to help advance the work of that distinguished organization for the public welfare.

The American Ceramic Society is international in scope with the staff housed in a functional headquarters building in Columbus, Ohio. 'The Society holds an annual meeting each spring at which technical programs are presented in the fields of interest of all of its Divisions and programs are also presented by its Classes. The Society is organized functionally into two Classes and ten Divisions. The Classes are the National Institute of Ceramic Engineers which provides coordination between our Society and other engineering fields and the Ceramic Educational Council which is concerned primarily with educational policies, college curricula, and other similar educational aspects of ceramics. The Divisions represent ten different categories of interest in ceramics of the members of the Society namely Basic Science, Ceramic-Metal Systems, Design, Electronics, Glass, Materials and Equipment, Nuclear, Refractories, Structural Clay Products, and White Wares. Each Division, with one exception, holds a fall meeting for the presentation of technical papers of interest to its members. The Society is also organized regionally by means of thirty Sections located throughout the United States which carry out its activities on a local or regional basis. The Sections hold evening meetings at regular intervals; some also hold meetings of one to three days duration, from once to three times a year, at which papers are presented on different technical ceramic subjects. Some Sections carry on continuing education programs, of which this Symposium is a good example.

We in The American Ceramic Society are proud to be able to contribute in a measurable way to the technical advancement of our country. We invite you who may be concerned with ceramic materials, but who are not members of the Society, to join with us in this progress.

I should like now to discuss briefly some general aspects of the microstructure of ceramic materials in view of its influence on their properties. Polycrystalline ceramic systems are quite complex, usually consisting of two or more phases, pores of varying size and size distribution and of varying shape and shape distribution, grain boundaries, grains of varying size and size distribution that may range from quite broad to very narrow, distribution of impurities within grains and in grain boundaries, partitioning of impurities between phases, and a crystallographic orientation that may range from a random one to an extremely well defined one. The arrangement of such internal features on a microscopic scale constitutes the microstructure. Sub-grain features, such as dislocations, complicate the microstructure even further. While shape, size, surface condition, and other physical factors of a polycrystalline ceramic article of a given composition may have definite effects, the microstructure will, to a considerable extent, influence its physical, mechanical, thermal, and electrical properties.

An understanding of the effect of specific microstructural characteristics on a property or properties of a ceramic is often an inducement to endeavor to change these characteristics. Thus Coble [1] achieved translucency and enhanced strength in a high alumina ceramic, later called Lucalox, by the elimination of porosity and the control of grain growth by a special sintering process. Spriggs [2] has reported that a transverse bend strength of up to 130,000 psi has been obtained for dense, submicron grain size alumina prepared by vacuum pressure sintering to maintain minimum grain size and porosity. These developments were made possible by the application of the knowledge that porosity and large grain size weaken brittle materials and that porosity increases the reflection and refraction of light. These 
microstructural characteristics could readily be observed, but there are other characteristics that are not so easily measurable. Insley and Barczak [3] demonstrated that polycrystalline alumina ceramics could be strengthened by heat treatment that causes a re-solution of phases present at grain boundaries that have precipitated from solution in the grains. This thermal conditioning illustrates the dependence of the strength of a ceramic on a rather subtle change of its microstructure caused by heat trea tment. Jorgensen and Westbrook [4] believe that solute segregation at grain boundaries is a significant mechanism for control of grain growth and porosity; they have used the results of such refined techniques as autoradiography and microhardness testing in support of their hypothesis.

The announcement of this Symposium pointed out that the control and reproducibility of many desirable physical properties of a given ceramic material depend upon the characterization and control of its microstructure and composition. It has been demonstrated, for example, that the mechanical strength of a particular type of ceramic will depend markedly on both grain size and porosity and that the thermal conductivity can be strongly affected by composition and also by compound formation. Riddle [5] reported experimental results which show that an intermediate compound in a binary system will have a lower conductivity than either end member.

Any type of ceramic with specifically required properties will require careful control of its production to assure its optimum behavior in the environment to which it is to be subjected. The specifications for a ceramic do not usually include a specification of the microstructure although such properties as nominal chemical composition, specific gravity, flexural strength, dielectric constant, and thermal conductivity, for example, may be specified. However, almost all of such properties are dependent to some extent on the microstructure, and the specification of microstructure might be helpful for the improvement of the quality and uniformity of ceramic materials. In this regard, some more effort might be directed toward more rapid and efficient methods of preparing sections for microstructural examination and determination. We are able to determine the composition of materials by instrumental techniques in a matter of minutes. However, the composition alone will not adequately characterize a material for all purposes and a rapid method of microstructural analysis would undoubtedly be of great value for process control.

Some of you may be concerned with ceramic materials that have rather unique properties for highly specialized applications and which may be required in only relatively small quantities. The development of such materials may be very time consuming and costly, and their subsequent small scale production may likewise be expensive. It should, however, be realized that there are other ceramic materials that have the excellent properties needed to meet equally stringent requirements but which are used in much larger volume and have much lower production costs. One example would be the ceramic insulators for spark plugs. These are necessarily made of highly refined, carefully developed and manufactured ceramics that must withstand extremely stringent conditions in use. These ceramic insulators must have excellent electrical resistance over a broad temperature range, high dielectric strength, excellent resistance to thermal shock, high mechanical strength, good thermal conductivity, great hardness and resistance to abrasion, and excellent resistance to chemical attack by combustion products. The development of a ceramic with a suitable balance of these electrical, thermal, mechanical, and chemical properties and the development of well controlled processes to manufacture insulators from it require a great amount of intensive research and engineering work. However, the development costs may be distributed over a large volume of product so that the special ceramic insulators with carefully specified and controlled properties can be produced at moderate cost in the large volume required.

The technical papers that will be presented in the program that has been arranged for this Symposium should be of great benefit to all of us who have the responsibility of fundamentally studying, developing, endeavoring to improve, producing, testing, specifying, or using ceramic materials.

\section{References}

[1] Coble, R. L., Sintering crystalline solids: I, Intermediate and final state diffusion models, J. Appl. Phys., 32, 787 (1961).

Coble, R. L., Sintering crystalline solids: II, Experimental test of diffusion models in powder compacts, ibid., 32, 793 (1961).

[2] Spriggs, R. M., Chapter 7, Microstructure and its influence on strength, in Strengthening Mechanisms, Metals and Ceramics (Syracuse University Press, Syracuse, New York, 1966).

[3] Insley, R. H., and Barczak, V. J., Thermal conditioning of polycrystalline alumina ceramics, J. Am. Ceram. Soc., 47, 1 (1964).

[4] Jorgensen, P. J., and Westbrook, J. H., Role of solute segregation at grain boundaries during the finalstage sintering of alumina, J. Am. Ceram. Soc. 47, 332 (1964).

[5] Riddle, F. H., Ceramic spark-plug insulators, J. Am. Ceram. Soc. 32, 333 (1949). 



\title{
ASTM-Its Function and Philosophy
}

\author{
Francis J. Mardulier, President, ASTM
}

\author{
Manager, Rock Products Chemicals, Dewey and Almy Chemical Division, W. R. Grace and Co., \\ Cambridge, Mass. 02140
}

\begin{abstract}
The American Society for Testing and Materials is a nonprofit privately operated interdisciplinary materials-oriented society which specializes in standards rather than a discipline-oriented society. ASTM provides the meeting ground for the technically competent engineers and scientists who comprise the technical committees which develop the realistic compromises, known as standards, which govern our economic life. Its membership comes from and is supported by industrial companies, government bureaus, educational institutions, testing laboratories, etc., all of whom are vitally interested in the voluntary standardization process.
\end{abstract}

Key Words: Compromises; consensus; materials; standards; technology; voluntary standardization process.

It is a privilege to be here to tell you about ASTM and the part it plays in voluntary standardization and its relation to the other organizations represented here in this forum. I welcome particularly this opportunity to discuss ASTM's principles of operation. We are grateful indeed to the National Bureau of Standards for providing this second opportunity to join with them and the American Ceramic Society in a technical conference.

Founded in 1898 as the American Section of the old International Association for Testing and Materials, ASTM's original charter, dated 1902, stated its purpose to be "the promotion of the knowledge of materials of engineering, and the standardization of specifications and the methods of testing." Inherent in this statement of purpose is ASTM's fundamental interest in research and education as well as its standardization work. ASTM's specialty, therefore, is materials research and standards, although it has formulated nonmaterials standards on request when no other organization was available to fill an existing need. As a result of its research and standards development activities, ASTM has achieved worldwide recognition in the field of standard methods of testing, whether applied to materials, products, or assemblies.

ASTM, like the American Ceramic Society, is not a discipline-oriented professional society, like ASCE, AIA, AIME, ASME, etc. Instead, because it is materials oriented and its specialization is standards, it must be considered an interdisciplinary society in which the standardization interests of its members predominate. Its membership comes from and is supported by industrial companies, governmental bureaus, educational institutions, testing laboratories, etc., all of whom are vitally interested in the voluntary standardization process. ASTM provides the meeting ground for the technically competent engineers and scientists who comprise the technical committees which develop the realistic compromises, known as standards, which govern our economic life.

Standards are necessarily compromises-compromises among the many conflicting producer and nonproducer interests involved. Each participant in the standardizing process wants a standard that helps him but the self-interests of these of these participating individuals or organizational representatives are necessarily diverse. The standardization process thus must involve concessions by all the interested parties to the extent needed to achieve an acceptable compromise which protects all and seriously injures no one.

Such compromises-not of the technical truths or principles involved but rather of the quantitative restrictions needed for the maintenance of reasonable quality levels-represents a realistic evaluation of the relationship between quality and cost. Highest quality and lowest cost to the consumer are not compatible. However, with an agreed upon quality level acceptable to the producer, user, and general interests involved, goods may be mass produced utilizing the most modern technologies and processes to provide the consumer with standard quality at minimum cost.

I can assure you, ladies and gentlemen, that the ASTM procedures involved in the development of standards by our technical committees do guarantee a consensus of the parties involved, and, at the same time, protect the interests of minorities. In addition, these procedures require that the representatives of the various interests involved be technically competent and support 
actively the interests they represent to the end that the resulting standard be an effective, realistic compromise of all the interests involved. The procedural details whereby this is accomplished will be given to you by Mr. Richmond, who follows me.

Reference to the word "consensus" brings to light one of the probably unique features of our technical committee procedures. Webster's Seventh New Collegiate Dictionary defines "consensus" as "general agreement-unanimity." "To me there is a gap of about 49 percentage points between these two definitions, since the first can be taken to mean majority agreement, while the second definitely calls for 100 percent agreement. In ASTM balloting on proposed or revised standards, a consensus of the magnitude of 95 percent or more is usually achieved, and even then it is required that negative votes be carefully considered and resolved if possible. A large majority of ASTM standards are, as a result, unanimously approved and accepted.

However, consensus of the committee membership would be meaningless if any one interestparticularly a producer interest-predominated. For this reason, each ASTM technical committee is required to maintain a balance of interest between the producer and nonproducer interests so that the producer voting membership never exceeds 50 percent. It may be less, but too much less is not desired either. Unfortunately, for a variety of reasons-mostly financial-it is much more difficult to get technically competent nonproducers to assist in the preparation of ASTM standards. We are particularly grateful, therefore, to the General Services Administration, the National Bureau of Standards, the U.S. Bureau of Reclamation, the U.S. Corps of Engineers and many other governmental agencies for their active participation in our standardization work as representatives of consumer interests. I might mention in passing that the National Buresu of Standards whose primary interest is the standardization of units of measurement has one or more representatives on a majority of ASTM's technical committees and contributes significantly to the technical soundness of ASTM standards.

We would similarly welcome the greater participation by consumer and general interest representatives from other areas of our free-enterprise economy so that its present and future needs will be made known and provided for in terms of new standards or revisions of old standards. Technological change is impossible without the development of appropriate standards. Consumers and industry use ASTM standards extensively by referring directly or indirectly to many ASTM standards in purchasing, design, and construction specifications. Yet consumers and even industry in some cases participate in ASTM standards work in far less proportion than established by their standards usage and needs. To maintain ASTM's balance of interest in its technical committees, we need the active participation of all interested parties, but more particularly that of consumers.

ASTM's scrupulous observance of its democratic principles in its standardizing procedure have caused ASTM standards not only to receive national and international acceptance, but has legal implications of tremendous importance to industry and our free enterprise system. It also guarantees the continuation of industry's direct participation in standards development and of government's participation as a representative of the consuming public. Strict adherence to ASTM operating procedures make it possible for technical committee members to articulate their organization's special interests without fear of antitrust violation, provided, of course, there is adequate representation of the parties at interest on the committee. This emphasizes the balance-of-interest requirement in the membership of ASTM committees.

As an interesting side effect of its requirement that there be balance of interest in committee membership, ASTM's standards when submitted through USASI for acceptance as an ISO standard are generally well received by the other members of the ISO committees having jurisdiction over the materials area in question. Thus, ASTM standards proposed for such acceptance are frequently converted into ISO standards with little or no revision of technical content and requirements. For example, the bulk of the ISO standards on plastics are essentially ASTM standards as originally formulated by ASTM Committee D-20. The significance of this fact to the U.S. plastics industry which is interested in export business is inescapable.

It seems appropriate at this point to digress in order to describe the relationship between ASTM and the USA Standards Institute (USASI). USASI is a private organization, of which the former American Standards Association was the prototype, whose function is that of a USA standards coordinator and clearing house of information on standards. It is the official USA member of those international organizations which establish international standards. Except for the unusual circumstances when existing standards organizations are unable or unwilling to provide needed standards, USASI does not itself actively participate in standards development and then only through the medium of a USA Standards Committee sponsored or administered by some outside organization or a USASI member body.

ASTM is such a member body. competent and willing to develop standards where needed. ASTM has formulated more standards than any other organization, private or public. It has in fact provided approximately 40 percent of the standards which to date have been processed through USASI and published as USA Standards. Its only requirement for such standards development activity is that there be a clearly defined standards need and to this end ASTM has issued an invita- 
tion to all organizations, be they companies, governmental bureaus, educational institutions, etc., to bring to our attention standards needs where they exist, and join with us in formulating the appropriate standards.

I hope that in this brief presentation I have given you a clear picture of the basic principles governing ASTM procedures, and the importance of meticulous observance of its democratic procedures to the continuation of voluntary standardization in the U.S.A. I hope I have made clear ASTM's relationship to USASI as one of the latter's most important member bodies and overseas representatives. I would be remiss indeed if I did not emphasize that USASI is also dedicated to the same principles in the development of standards and as U.S. coordinator of standards is in a position to see that all USA standards similarly conform.

In closing, I should like to call to your attention a final and most important requirement of a good standard-it should be realistic and legally defendable. A unilaterally developed standard may be technically correct but legally and politically unacceptable if it does not recognize the needs and rights and represent the participative effort of all the parties at interest. This is a fact of life which becomes increasingly clear as the needs and rights of the consumer are daily accorded growing recognition.

Two years ago at a similar symposium I told you of a decision which had been made in a restraint of trade case which ASTM had instigated to clear itself of any stigma which might exist as a result of its involvement in a prior criminal suit brought against three USA manufacturers of asbestos-cement pipe for alleged antitrust violation. One of Judge Van Deusen's findings of fact in the U. S. District Court for Eastern Pennsylvania sums up far better than I can the basis on which ASTM won its case. And so, in closing, I take the liberty of quoting these findings once more:

"The technically qualified balanced membership of ASTM committees, and the democratic procedure governing their operation, make it likely that the results reached by them will be scientifically sound and will represent the general interest".

"Because of the heavy reliance of federal, state and municipal governments on ASTM for specifications, the society may be regarded as an essential arm or branch of the government and its acts may be entitled to the immunity from antitrust laws accorded governmental acts". 



\title{
ASTM Activities in Ceramics
}

\author{
Joseph C. Richmond \\ Institute for Basic Standards, \\ National Bureau of Standards, Washington, D.C. 20234
}

\begin{abstract}
The organization of the American Society for Testing and Materials is described, the procedure followed in the promulgation of ASTM Standards is briefly outlined, and the scope of ASTM activities in the field of ceramics is indicated.
\end{abstract}

Key words: ASTM; committees; definitions; promulgation; standards; tentatives.

The American Society for Testing and Materials is the largest voluotary standardizing crganization in the world. Its membership is made up of over 11,000 individual engineers and scientists, 2700 companies, associations, and research institutes, 1300 educational institutions, governmental agencies (federal, state, and municipal) and professional societies, and 900 engineering and science students. It functions by the committee system, with some 106 main technical committees that are primarily responsible for the development of standards.

The large membership of highly qualified individuals with widely diverse technical backgrounds is particularly advantageous for the development of standards. A technically sound standard is likely to be approved by such technically qualified members and the standards that are approved will receive wide distribution and acceptance.

The membership of each technical committee is made up of individuals knowledgeable in the specific field covered by the committee, each of whom, it is assumed, represents the interests of his employer, or himself, if self-employed. Membership on a committee is attained by election to the committee after application by an individual, stating his qualifications for membership. In general only those considered capable of contributing to the work of the committee are elected to membership. Membership is lost automatically when a member changes employment, or ceases to participate in the work of the committee.

The primary objective of the society is to promulgate the standards prepared by the technical committees. The gen eric term "standard" includes documents that differ both in the degree of acceptance and in content. With respect to content, they generally fall into one of the following classes: a) definitions of terms, b) recommended practices, c) methods of test, d) classifications, and e) specifications. With respect to the degree of acceptance they are published as: a) Proposed
Standards, b) Tentatives, or c) Standards. As a matter of Society policy its Standards are submitted for recognition as USA Standards, and currently more than 40 percent of all USA Standards are ASTM Standards.

Many technical terms are not adequately defined in standard dictionaries or if they are defined, the technical meaning is different from that which is technically accepted. Adequate definitions of terms are thus the first prerequisite for meaningful communication, not only between individuals in the same area of technology, but particularly between individuals in different areas of technology.

Engineers and scientists in many fields have found that in many cases certain ways of doing things are likely to lead to trouble or yield erroneous results, while other procedures will gen erally avoid such trouble or errors. Information of this kind that is not properly a part of a test procedure or specification is termed a recommended practice.

The detailed description of test apparatus and techniques to be used in evaluating the properties of materials and equipment form the basis for a method of test. If the techniques are intended for verifying the calibration of testing machines or equipment, such a document is another form of an ASTM method of test. A specification may include one or more test procedures, but its primary purpose is to set limiting values for the parameters of a material or equipment for a specific application; it may classify a material into different grades on the basis of its physical or chemical properties.

Documents may be published for information only, particularly when in a new or rapidly expanding area of technology. This type of publication implies no acceptance or approval by the Society. In the case of new areas of technology, or controversial subjects, a document may be published as a proposed method or specification in order to bring it to the attention of interested members of the Society, so that if they wish they may express their opinion regarding the merits of 
the proposal before it is accepted as a Tentative. Publication as a Tentative implies that the document is new and requires more testing data. Such a document has the approval of the sponsoring committee and the Administrative Committee on Standards representing the Society. Publication as a Standard implies full approval by the sponsoring committee and the Society as a whole. Acceptance of an ASTM Standard by the U.S.A. Standards Institute transforms it into a U.S.A. Standard. Not all U.S.A. Standards originate in ASTM, but far more come from ASTM than from any other source.

The term "Tentative" has an unfortunate connotation, and the Society is actively searching for a better term to express the thought that such a document does indeed represent the best available technical knowledge on the subject, but cannot be granted the status of a Standard because of lack of knowledge of the accuracy and precision of the test methods involved, or the correlation of test and service data, or some similar lack.

The procedures followed in the promulgation of an ASTM Standard are quite involved. This involvement results in part from the elaborate safeguards that have been instituted (1) to ensure that every interested individual member of the Society will have an opportunity to express his opinion and be heard, and (2) to prevent domination of any group within ASTM by special interest representatives.

ASTM Standards are developed by the democratic process implied in the term "consensus." Each Standard is subjected to letter ballot at least three times before adoption, twice in the technical committee and once by the Society. A key provision in the adoption process is that any negative vote in a letter ballot must be accompanied by valid technical reasons, and must be considered and reconciled if possible before adoption of the Standard.

The first draft of a standard is generally prepared by a small task group appointed for that purpose, made up of technically qualified individuals who represent as many diverse interests as is practical. Development of a suitable test procedure, for instance, may involve research work in the laboratories of the members of the task group, followed by round-robin tests to prove that the method is precise and reliable. After the group has agreed upon the technical details, a draft is prepared and presented to the subcommittee, where it is discussed, revised if necessary in the light of the discussion, and after approval by letter ballot, is presented to the main committee, where it is again submitted to letter ballot. Although only a two-thirds favorable vote is required in subcommittee and committee letter ballots, the ballot is usually unanimous in the subcommittee and nearly unanimous in the main committee. When approved by the committee it is submitted to the Society as a whole, either at the Annual Meeting or through the Administrative
Committee on Standards, where it is accepted as a Tentative. After one year as a Tentat ve, the sponsoring committee may recommend that it be advanced to a Standard, with or without revision, which must again be confirmed by a two-thirds favorable letter ballot. After such a recommendation, it is submitted to letter ballot of the Society as a.whole. After approval by two-thirds of these voting, it is formally accepted and published as an ASTM Standard. If immediate adoption as a Standard is recommended by sponsoring committee, approval by nine-tenths of those voting is required in the Society letter ballot.

Any technically sound proposal that accurately represents the best knowledge available, that is capable of the desired precision and accuracy, and that is arrived at by the concensus process mentioned above, may and should be submitted for immediate adoption as a Standard, without prior adoption as a Tentative.

ASTM Standards are subject to periodic revision and reapproval by the sponsoring committee at least once in each six year period. They do not remain in force unless the committee makes such a recommendation, confirmed by letter ballot.

The Regulations Governing ASTM Technical Committees provide that the members of each Committee shall be classified as either producers, consumers, or general interest members, on the basis of their interest in the material or product involved in a document. The regulations also require that in no case shall the producers dominate any committee. No producer shall serve as chairman of a committee that prepares or recommends adoption of a specification. As a further check on domination by producer interests, the regulations require that each member indicate on each letter ballot his classification with respect to the material or product involved, and a two-third vote requires that two-thirds of the producers and two-thirds of the nonproducers separately approve the recommendation. This insistance on participation by consumers and general-interest members in the development and approval of ASTM documents has contributed greatly to their wide acceptance by industry, government, and consumers generally, and has kept the Society free from involvement in antitrust actions.

As a further restriction, while each member has an individual vote in the subcommittee, the representatives of each organization have only one vote collectively in the letter ballots of the committee and of the Society.

There are 106 technical committees. These are classified as follows: 7 "A" Committees on Ferrous Metals, 9 "B" Committees on Nonferrous Metals, 24 "C" Committees on Cementitious, Ceramic, Concrete, and Masonry Materials, 30 "D" Committees on Miscellaneous Materials, 27 " $\mathrm{E}$ " Committees on Miscellaneous Subjects, 6 " $\mathrm{F}$ " Committees on Materials for Specific Applica- 
tions, and 3 "G" Committees on Corrosion, Deterioration, and Degradation of Materials.

Most of the "C" Committees are concerned with ceramic materials. Time does not permit a detailed account of the activities of these Committees, but a list of the major " $\mathrm{C}$ " Committees will indicate something of the breadth of their scope: $\mathrm{C}-1$ on Cement, $\mathrm{C}-3$ on Chemical Resistant Nonmetallic Materials, C-4 on Clay Pipe, C-7 on Lime, C-8 on Refractories, C-11 on Gypsum, $\mathrm{C}-14$ on Glass and Glass Products, $\mathrm{C}-15$ on Manufactured Masonry Units, C-16 on Thermal Insulating Materials, $\mathrm{C}-17$ on Asbestos-cement Products, C-20 on Acoustical Materials, C-21 on Ceramic Whitewares, C-22 on Porcelain Enamel and Related Ceramic-Metal Systems, C-25 on Ceramics for Electronics. In addition, several other committees also deal with ceramic materials. D-9 on Electrical Insulating Materials has a subcommittee on Ceramic Products. E-12 on Appearance of Materials covers test methods for measuring reflectance, gloss, and color of ceramic as well as other materials. E-20 on Temperature Measurement covers methods of calibrating thermometers, thermocouples, and pyrometers. $\mathrm{F}-1$ on Materials for Electron Devices covers ceramic-metal seals.

The standardization of methods of testing and specifications is the primary objective of ASTM. In 1967, 2565 Standards and 1403 Tentatives were published in 32 volumes, with over 25,000 pages. The complete Book of Standards is republished annually, in order to incorporate all revisions as well as new Tentatives and Standards. Standards dealing with ceramic materials are included primarily in Part 13, and comprise 127 Standards (including 34 U.S.A. Standards) and 31 Tentatives.
A recent bulletin from ASTM Headquarters states that about 30 percent of the contents of the ASTM Book of Standards is obsolete after one year, and 65 percent after two years. This statement indicates the effort put forth by ASTM technical committees to keep their standards up to date.

In addition to its primary work of promulgating standards, ASTM sponsors and publishes the proceedings of a wide variety of symposia, generally in the fields of properties of materials and materials research. The fact that our meeting here is jointly sponsored by ASTM is an example of such sponsorship. ASTM also sponsors research, either directly or through the technical committees. The Cement Reference Laboratory and the project on $\mathrm{x}$-ray powder diffraction patterns here at NBS are good examples.

ASTM has a number of other publications. Materials Research and Standards is a monthly magazine which includes announcements of all ASTM sponsored meetings, general information on Society activities, and technical articles of general interest. The quarterly Journal of Materials contains technical papers on materials research. The annual Proceedings contains the annual reports of all technical committees. Many special publications containing technical papers on a single general topic are published each year.

This is an abbreviated account of some of the activities of the American Society for Testing and Materials, but indicates something of their scope, particularly in the field of ceramics. We expect that these activities will continue and even expand in years to come. 



\title{
Properties of Ceramics for Structural and/or High Temperature Use: Need for Control, Measurement, and Compilation
}

\author{
Charles F. Bersch \\ Naval Air Systems Command, \\ Code AIR-52032A, Washington, D.C. 20360
}

\begin{abstract}
Extensive new markets are available to ceramics as soon as weight-efficient applications involving primary and secondary structural requirements can be satisfied. The major obstacle and its causes are discussed and long and short range approaches for dealing with those causes are suggested.
\end{abstract}

Key Words: Applications of ceramics; brittleness; brittle design; ceramics; strength; structural ceramics; variability; weight-efficiency.

\section{New Markets Open to Ceramics}

The opportunities for new or improved materials with the properties to perform new functions, or to operate in more extreme environments, or to fulfill their functions more efficiently are commonplace in the aerospace industry and are increasingly evident in the hydrospace and automotive industries.

Ceramics, by virtue of the spectrum of functional and structural properties they offer, are an especially attractive source for such materials. Prominent among their functional properties are extreme resistance to heat, oxidation, corrosion and abrasion; outstanding dimensional stability (in thermal, corrosive, abrasive or mechanical environments) ; and unique nuclear, optical, magnetic, electronic and energy-conversion capabilities. Unparalleled stiffness and specific stiffness are available for structural purposes, and achievements of substantial portions of the predicted theoretical strength (and the associated specific strength) are increasingly frequent and sometimes commercially successful $[4,8,9]$.

Functional applications of ceramics are widespread and common. They often include lowlevel structural requirements (secondary structural requirements), as in sanitary ware, electrical insulators, windows, and corrosion-resistant processing equipment, but functional considerations are predominant. Ceramics have also been used routinely for many years in a wide variety of thermal and structural applications, oftentimes in systems incorporating both modes simultaneously, such as kiln construction and furnace linings.

However, virtually all current applications of ceramics in which there have been appreciable mechanical stresses due to thermal or mechanical loading have been successful, or at least adequate, because one or more of three conditions prevailed:

(1) The loads were accommodated by gross overdesign.

(2) All appreciable stresses were successfully confined to the compression mode.

(3) Failure, if it occurred, was not catastrophic either in mode or else in consequences.

In almost no instance could one say that the system or part was weight-efficient, that is, that it fulfilled its purpose at the lowest possible weight. Yet weight-efficiency is almost a sine qua non in industries where each pound saved in functional or structural weight during the conceptual stage can be cascaded on the drawing board, via savings in structural, powerplant, and fuel requirements, into savings of tens and hundreds of pounds, and, in the case of missiles, thousands of pounds in total systems weights.

\section{The Obstacle to Weight-Efficient Uses of Ceramics}

One persistent obstacle prevents a burgeoning use of ceramics in applications where primary or secondary structural properties are needed. That obstacle has many descriptors. Most commonly they are "brittleness" or an "inability to design with brittle materials." But one can live with the brittle failure mode when one is certain that failure will not occur or when one can predict and control when it will occur. And, though the techniques may be primitive because of insufficient usage and lack of accrued experience there is some ability to design using brittle materials. A more accurate descriptor is "lack of reliability" or, as some designers say, the "variability" of 
ceramics. But even controlled or bounded variability could be endured if its limits could be predicted. "Poor predictability" is probably the best descriptor for the major obstacle.

An even finer focus on the real obstacle to more widespread applications of ceramics is that "variability" and "unpredictability" apply primarily to the strength properties of ceramics. Though thermal, electrical, elastic, and other properties are influenced by compositional and microstructural variations, they are usually much less variable than the strength properties. That lesser variability is usually attributed to a lower sensitivity, but it may also suggest that the first two of the following suggested causes of variability in strength properties are the major ones, since they should have no effect on the nonstrength properties and they are also the most readily identifiable causes of variability in strength.

\section{The Causes of Variability}

Possibly the major contributor to variability in strength data is the testing situation. Just as special design techniques are needed for brittle materials, so also special test procedures performed by skilled and careful operators are needed to evaluate brittle materials. A ductile material will ignore or forgive parasitic stresses, due to misalignment and mistreatment, that are catastrophic to a ceramic material. The paper by Mordfin and Kerper in this proceedings discusses this subject in greater detail.

The surface of a ceramic specimen is also of major importance, especially when the part is loaded in tension or flexure. But the variability of a surface finish is likely to be a greater cause of variability in strength data than is the surface finish itself. A uniformly poor surface finish will cause low strength values but will not necessarily cause a scatter in data. However, as the average surface finish improves, the effects of surface finish variations will be more prominent. An added complication becoming increasingly evident is environmental sensitivity, which is also experienced via the surface and which is also more prominent as the surface finish improves. This is discussed further in the paper by S. M. Wiederhorn.

The character of the ceramic specimen, the resultant of the raw materials and the processing as evidenced by the composition and microstructure, has a prominent but mixed role in ceramic property variability. Pores, microcracks and cracks will have some effect on variability, but the size, shape, location, and distribution of defects are probably more critical than their frequency. Grain size variability, grain shape, and orientation can also be factors. But, except for cracks, it is probable that body character is important to variability only after test and surface deficiencies are under control.

\section{Steps to a Brighter Future for Ceramics}

\subsection{Design Techniques Based on Predictability}

From the foregoing it would appear that there are several steps that must be taken for ceramics to fulfill their potential in future hardware. One is a substantial advance in ability to design for brittle materials. This subject is beyond the scope of this discussion; however, it must be obvious that the availability of ceramic materials having predictable performance would provide both an impetus to advances in design techniques and the means or substance for physical substantiation of theoretical advances.

One contribution to predictability might be a total confrontation with brittle failure - an intensive study and analysis of the failure mechanism to determine where and how and why failures begin and how and why they proceed to completion. Modest efforts of this nature have been very fruitful. Griffith flaws have provided a useful concept for theoretical analysis. Their physical reality has been demonstrated in a variety of forms ranging from mechanical damage [1-3] through thermal shock damage [4] to stress corrosion sites [5] and possibly phase separation boundaries that occur at the surface. Once the flaw and its genesis have been identified, it is possible to conceive of prevention, elimination, or at least control. Current techniques for providing compressive stresses in the surface of a body $[6,7]$ are essentially efforts to prevent Griffith flaws from acting as stress concentrators and failure initiation sites. Efforts along this line are encouraging; bending strengths above 200,000 psi after abrasion have been achieved with glass ceramics [8] and the techniques have been shown to be feasible with conventionally processed ceramics [4, 7]. More recently a procedure for coating glass that appears to prevent Griffith flaws due to either mechanical or stress corrosion effects has allowed bending strengths of seven percent of the elastic modulus to be realized in glass rods of five different compositions [9].

Such studies, some aimed at understanding brittle failure and some aimed at enhancing useful strength, tread a common ground and are mutually supporting, suggesting that predictability and great strength may be achieved simultaneously. What an impetus to advances in design techniques that would be!

\subsection{More Useful Tests and Data}

A second essential step toward weight-efficient structural applications of ceramics is an improvement in data, primarily strength data. Several facets are involved: improvements of tests to minimize variability due to the tests; sufficient identification of any test specimen or part so 
it can be duplicated; and a concept of strength and measurements of strength that extends from properties to behavior.

An improvement in test procedures and techniques with a resultant reduction in variability of strength data and a concomitant increase in predictability is necessary but not sufficient to advance the use of ceramics. It is necessary to provide sufficiently sensitive comparative data to facilitate process or product improvement studies and it is necessary to provide useful numbers to evaluate probable material performance in various stress states. Part of the insufficiency in the case of development studies is obvious, since the development activity itself is also essential. And part of the insufficiency in design analysis is so fundamental that its neglect is possibly the greatest paradox in the ceramic industry: the failure to identify or characterize the test specimen or the piece of hardware sufficiently so it can be reproduced so that the test data or the service experience that have been acquired achieve meaning via future utility. This deficiency is by no means confined to strength data though it may be more critical for strength data. The distressing fact is that much of the data in the literature including some of the most carefully acquired data is useful only to suggest trends in behavior; it cannot be used with exactitude because the ceramic material from which it was taken can never intentionally be reproduced. The incredible truth is that there are a few instances where there was no further identification than the word "alumina."

The accumulation of carefully controlled and reliable data on reproducible ceramic materials is necessary but not yet quite sufficient; the data must be meaningful and useful. Strength is not an invariable condition; it is rather a numerical measure of a process that is influenced by many external features. It is sensitive to the rate of loading; to the duration over which the load is applied; to the temperature of the stressed material; to chemical influences such as those which cause corrosion or stress-corrosion; to the kind of stress, its origin and uniformity; to the simultaneous presence of other stresses, macro and micro; to surface energy effects which, in boundary cases, may include size and geometry effects. In fact, in many cases test data do not so much describe a property as a behavior in the presence of a variety of conditions. Thermal shock behavior is a good example. A less clearcut but equally important example is the ability to sustain long enduring loads during immersion in seawater or some other uncommon medium.

It should be evident that test data obtained at random, that is, without a specific use in mind for that data with that use determining the conditions under which the data are obtained, will be of value only to suggest trends in behavior. It will not suffice for any specific decisions.
A supplier of metals to the aerospace industry reports that tensile data on his material was all that the designer desired 35 years ago. Today he must obtain data from 63 different test specimen configurations to obtain the variety of data demanded by aerospace designers. The ceramic industry, with a product that is presently much less predictable, will need more sophistication than ar advance from three to four point loading.

\subsection{Improved Ceramic Processes and Parts}

Several volumes might be written about the general and specific actions related to processing that could be taken to improve the predictability of ceramic materials. Each individual composition and each separate process has its merits and shortcomings, its areas especially susceptible to improvement. But what is improvement? It may be higher or more uniform properties achieved via smaller average grain size, or smaller grain size range, or more or less orientation of anisotropic grains, or fewer, or rounder, or smaller pores. An almost endless list can be made and it would be rather fruitless to complete it or to attempt to act on it. What seems more appropriate is to apply to the materials presently in use, and to a few model materials, the best principles available at the time, such as uniform small grain size, minimum porosity, etc., as the material and process combination allow, in order that uniformity within a part, reproducibility from part to part, and reliability in service may be improved. Usable strength levels would undoubtedly rise as a consequence.

One area that merits special attention is the surface, and one process that requires special attention, since it is used on most test specimens and parts, for shaping, dimensioning, or application of a specified finish, is abrasive machining or grinding. Another paradox of the ceramic industry is the evident recognition of the contribution of the surface of a ceramic part to the strength and reliability of the part set off against the failure to study what grinding, which is a rather brutal treatment, does to that surface. A knowledge of the nature and the extent of the influence of grinding on a ceramic surface is a prerequisite to any efforts to prevent, control, or cure such influence.

\section{Characterization}

The character of a ceramic has been defined as the sum of the compositional and structural descriptions which identify a specimen uniquely, may be correlated with its properties, and suffice for the reproduction of the material [10]. It is apparent that the need for characterization has been a consistent thread throughout the previous discussion. In particular, characterization was emphasized as essential to provide meaning to test data by providing the opportunity to use that 
knowledge again in further production of the same material. Less emphasized, but equally important, is the need for characterization during process and product improvement. This is particularly so as technology becomes more sophisticated and subtle and the earlier direct relationships between processing and properties become insufficient. Character then becomes, in addition to all above, the link joining processing and properties.

Complete or total characterization $\left(C_{T}\right)$ consists of the sum of all character features on the atomic scale $\left(C_{A}\right)$, on the microstructural scale $(C \mu)$ and on the macrostructural scale $\left(C_{\Delta}\right)$ and is often expressed in the equation:

$$
C_{T}=C_{A}+C_{\mu}+C_{M} .
$$

Atomic character includes chemical composition and uniformity. Microstructural character includes the size, shape and orientation of grains; the nature of interfaces and grain boundaries; the presence and disposition of additional phases; the distribution of dislocation and intergranular stresses. Macrostructural character includes the size and shape of the part, its uniformity, density, gross chemical composition and surface topography, and the major stresses present.

Complete characterization is an ideal state; it may never be realized in entirety for any body and it is certainly too extensive, complex, and expensive to do routinely. Accordingly, a second concept is available to provide, simultaneously, a means to approach the ideal state and a more practical exercise, so benefits can be realized at once. The concept is labeled "description" (D) and it is the sum of the total characterization available $\left(C_{T}\right)$ plus data on the properties $(P)$ of the body plus the sample history $(H)$, including raw material background and processing data. This too has an equation:

$$
D=C_{T}+P+H \text {. }
$$

In the hope that the current partial reliance on properties and sample history may eventually be discarded, the equation is expressed as:

$$
D=X C_{T}+(1-X)(P+H)
$$

where as $X$ approaches 1 , description will equal total characterization.

The provision of the second and third equations recognizes the need for a practical approach to characterization but it $\mathrm{s}$ till does not suggest what a happy medium might be or should be. In trying to make specific recommendations one is torn between the desirability of having the maximum amount of information available versus the practicality of a minimum expenditure of time and effort. The most practical suggestion is not, of course, to do no characterization; that is shortsighted and self destructive; it is, rather, to do as much as is known to be important, to do as much as provides a useful immediate return in fostering growth. Wachtman's [11] recommendations concerning conventional, augmented conventional and more intensive characterization, as shown in table I, seem to be the most reasonable. Thus, in the most routine cases one might accept only chemical composition, average grain size, and density. 'This is not in any way adequate, but if confined to production materials it has the added benefits of accumulated experience in the typical properties and histories of the materials.

TABLE 1. Parameters needed to specify the mechanical properties of a ceramic

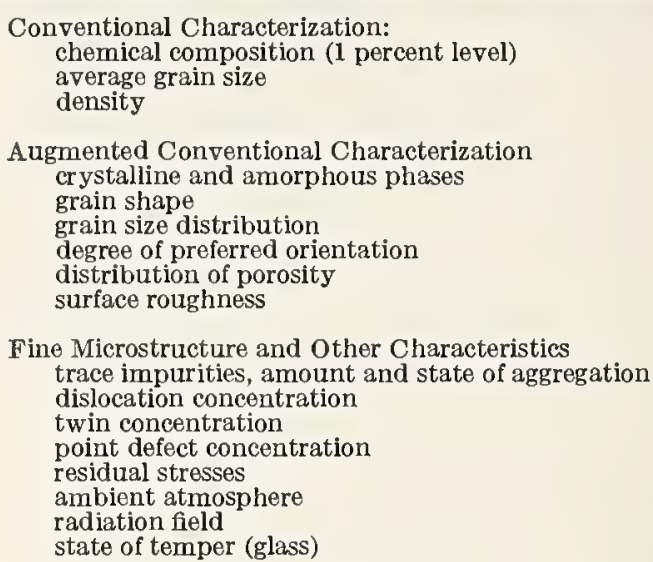

Conventional Characterization: chemical composition (1 percent level) average grain size density

Augmented Conventional Characterization crystalline and amorphous phases grain shape

grain size distribution

degree of preferred orientation

distribution of porosity

surface roughness

Fine Microstructure and Other Characteristics trace impurities, amount and state of aggregation dislocation concentration dislocation concentr
twin concentration

point defect concentration

residual stresses

ambient atmosphere

radiation field

state of temper (glass)

In any effort other than routine production, for example in product or process development, the augmented conventional characterization outline should be followed. This, again, is minimally adequate, but assumes prior knowledge about the process or product that helps complete the knowledge and understanding of the material.

Lastly, in any research study on ceramics, the last grouping of character features must be considered in addition to the ones already discussed. Not all of them will be needed in all circumstances. In fact, some may be quite difficult to determine. But the effort must be made to foster an optimum practical growth of the state of the art and the range of applications of ceramics.

\section{Summary}

Broad opportunities exist for much wider and more extensive applications of ceramics, in primary structural applications ranging to the hulls of deep submergence vehicles and beyond, and in secondary structural applications from rod end bearings in helicopter blade control rods to gas bearings, fuel cell components, and yet unconceived applications, if:

- poor predictability is overcome by confron ting brittle behavior and learning to prevent, overcome or control it;

- poor predictability is eliminated by the skillful use of well-conceived tesl procedures;

- poor predictability is overcome through achievement of understanding and control of 
ceramic processing, in all stages from the preparation of raw materials through surface finishing and assembly.

\section{References}

[1] Griffith, A. A., Phenomena of rupture and flow in solids, Phil. Trans. Roy. Soc. (London) R21A, 163-98 (1920)

[2] Ernsberger, F. M., Detection of strength-impairing surface flaws in glass, Proc. Roy. Soc. (London) A25\%, 213-23 (1960).

[3] — , A study of the origin and frequency of occurrence of Griffith microcracks on glass surfaces, pp 511-24 in Advances in Glass Technology (Plenum Press, New York, 1962).

[4] Kirchner, H. P., Gruver, R. M., Platts, D. R. Rishel, P. A., and Walker, R. E., Chemical Strengthening of Ceramic Materials, Summary Report for Naval Air Systems Command Contract N00019-67-C-0489 (6 April 1968).

[5] Schmitz, G. K., Gulden, M. E., and Metcalfe, A. G., A Study of Stress Corrosion of Glass Fibers, Final
Report for Naval Air Systems Command Contract N00019-67-C-0409 (June 1968).

[6] Stookey, S. D., Olcott, J. S., Garfinkel, H. M., and Rothermel, D. L., Ultra-high strength glasses by ion exchange and surface crystallization, Advances in Glass Technology (Plenum Press, New York, 1962).

[7] Kirchner, H. P., and Gruver, R. M., Chemical strengthening of polycrystalline ceramics, J. Am. Ceram. Soc. 49, 330-3 (1966).

[8] Duke, D. A., MacDowell, J. F., and Karstetter, B. R., The crystallization and chemical strengthening of nepheline glass-ceramics, J. Am. Ceram. Soc. 50, 67-74 (1967)

[9] Hallman, R. W., and Anderson, J. S., Ultra-High Strength Bulk Glass, Final Report for Naval Air Systems Command Contract N00019-67-C-0435 (15 May 1968).

[10] Ceramic Processing, Materials Advisory Board, National Academy of Sciences, Publication MAB-233, February 1968. Also National Academy of Sciences Publication 1576.

[11] Wachtman, J. B., Jr., Mechanical properties of ceramics: An introductory survey, Bull. Am. Ceram. Soc. 46, 756-72 (1967). 



\title{
Melting Points of Ceramics
}

\author{
S. J. Schneider \\ Institute for Materials Research, \\ National Bureau of Standards, Washington, D.C. 20234
}

\begin{abstract}
The melting behavior of a ceramic, as for any material, can be described by the phase rule and its graphical expression, the phase equilibrium diagram. The solid to liquid transition (melting point) is discussed in terms of a one-component system and extended to more complex systems utilizing unary principles as basic building blocks. Phase equilibrium diagrams of hypothetical and of real ceramic systems are presented to illustrate the relationship between melting point and the pertinent intensive variables-pressure, temperature and composition. The effect of metastable equilibrium upon the melting point is also emphasized. Experimental methods for melting point determinations are outlined and include the thermal analysis and quenching techniques. Relevant details of temperature measurement are given.
\end{abstract}

Key Words: Ceramics; fusion curve; melting point; melting point methods; metastability; phase rule.

\section{Melting Point and the Phase Rule}

Ceramics as a general class are materials with rather high melting points, characteristically having a somewhat viscous liquid phase. Although ceramics possess distinguishing features which impart experimental difficulties in their study, the general principles governing the fusion and crystallization process are the same as that inherent in all materials. Conceptually, the melting point of a ceramic cannot be viewed any differently from that of any other material and is the temperature at which solid and liquid of the same chemical composition are in equilibrium for a given confining pressure. Normally, the pressure is inferred to be equal to $1 \mathrm{~atm}$ but most certainly is not limited to that value. An equally valid but less widely used definition would be the pressure at which solid and liquid are in equilibrium for a given temperature.

The significant thoughts embodied by both definitions are related by the familiar Gibbs phase rule for heterogeneous systems:1

where

$$
P+F=C+2
$$

$P=$ the number of homogeneous phases present at equilibrium. Phases may consist of either solid, liquid, or vapor and must be mechanically separable one from another.

$F=$ the number of intensive variables of a system such as pressure, temperature, and composition which can be arbitrarily changed

1 Numerous publications are available describing the theory and utilization of the phase rule. Some of these are tabulated in references [1] through [14].
(Figures in brackets indicate the literature references at the end of this paper.) without disturbing the equilibrium. These variables are commonly termed degrees of freedom or variance of the system.

$C=$ the least number of independent components from which $P$ phases can be made.

Equation (1) applies to all noncondensed systems. Inasmuch as many ceramic materials have low vapor pressures, the vapor phase is often neglected, thus reducing eq (1) to

$$
\begin{gathered}
(P-1)+F=C+2-1 \\
\text { or } \\
P+F=C+1
\end{gathered}
$$

where $F$ and $C$ have the same meaning as previously stipulated and $P$ refers only to solid and liquid phases. The condensed phase rule, however, is not strictly applicable to true one-component systems.

The phase rule says nothing about rates of reactions between the components, nor does it infer the amount of each phase present at equilibrium. It does, however, stipulate the number of phases allowable for a given set of conditions. ${ }^{2}$ At times the utility of the phase rule may appear to be purely academic, but a real insight into the behavior of materials can be gained through its use. Of course, the entire field of phase equilibria is merely the experimental extension of the phase rule. When applied to the melting phenomenon the aforementioned definitions of melting point become more clear. For example, in the familiar water system, ice and water are in equilibrium at the melting point. Here the number of

\footnotetext{
2 For noncondensed and condensed systems the maximum number of phases equals $C+2$ and $C+1$, respectively.
} 
components is one $\left(\mathrm{H}_{2} \mathrm{O}\right)$, the number of phases, two (solid and liquid), and the variance, one, $(F=C+2-P=1+2-2=1)$. Thus, if one degree of freedom is utilized by holding the system at constant pressure, the temperature of melting is automatically determined. Conversely, when the temperature is held constant the pressure is then specified.

At the melting point the number of degrees of freedom $(F)$ always equals unity for a noncondensed single-component system. Moreover, expansion to a multicomponent system does not permit an increase in the variance of the solidliquid equilibrium at the melting point of a compound. Since the composition is fixed by specifying the compound, the only remaining variables are either pressure or temperature. Hence $F=1$, the same as for a one-component system. In essence, regardless of the number of elemental constituents involved in the reaction, proper application of the phase rule requires that only the smallest number of components necessary to describe the equilibria need be considered. By way of illustration, the compound $\mathrm{CaO} \cdot \mathrm{Al}_{2} \mathrm{O}_{3} \cdot 2 \mathrm{SiO}_{2}$ melts to all liquid at $1553{ }^{\circ} \mathrm{C}$ [15]. Treating the system as containing three components $\left(\mathrm{CaO}, \mathrm{Al}_{2} \mathrm{O}_{3}, \mathrm{SiO}_{2}\right)$ would yield at the melting point of the compound a variance of three, an obviously false conclusion. The degree of freedom, of course, equals unity as for a one-component system.

Furthermore, the number of components under consideration may be altered within a system depending upon the conditions of experiment. The compound $3 \mathrm{CaO} \cdot \mathrm{Al}_{2} \mathrm{O}_{3}$, for instance, melts at $1535{ }^{\circ} \mathrm{C}$ and atmospheric pressure to $\mathrm{CaO}$ and liquid [16]. At temperatures below melting and at fixed pressure, the compound can be thought of as one component possessing one degree of freedom for the condensed system. At $1535{ }^{\circ} \mathrm{C}$ three phases, $\mathrm{CaO}, 3 \mathrm{CaO} \cdot \mathrm{Al}_{2} \mathrm{O}_{3}$, and liquid are in equilibrium with a variance of zero. The number of components then must equal two. Basically, the system appears to change from one to two components $\left(3 \mathrm{CaO} \cdot \mathrm{Al}_{2} \mathrm{O}_{3} \rightarrow \mathrm{CaO}\right.$ and $\left.\mathrm{Al}_{2} \mathrm{O}_{3}\right)$ all within the framework of the $\mathrm{CaO}-\mathrm{Al}_{2} \mathrm{O}_{3}$ binary.

The phase rule is basically a statement of the conditions which exist when a system is at thermodynamic equilibrium. Theoretically, equilibrium conditions require that a system cannot change with time without a gain or loss in energy. Here, the Gibbs energy of the system is at a minimum and the entropy at a maximum. The attainment of equilibrium may be difficult and of ten presents a dilemma to the investigator. In certain systems, particularly those involving ceramics, reactions and transformation may proceed at such a slow rate that a quasi state of equilibrium will be obtained. Such a situation, termed metastable equilibrium [2, 3], is often consistent with the phase rule. A good case in point is supercooled water which is in metastable equilibrium with its vapor at temperatures below its freezing point. More pertinent to this discussion is the metastable equi- librium exhibited by fused silica when supercooled to room temperature [17]. The liquid is metastable with respect to quartz, the stable room temperature modification. The system strictly obeys the phase rule and yet is not at thermodynamic equilibrium. It is axiomatic that compliance with the phase rule is not a test for equilibrium but only a guide. However, disobedience of the phase rule furnishes positive evidence that equilibrium conditions are absent. The importance of metastable equilibrium cannot be stressed too strongly. Many significant processes depend upon it for the manufacture of common, everyday products such as glass.

\section{One-Component Systems}

\subsection{Stable Equilibria}

For practical use the phase rule must be combined with experimental information. The graphical representation of the data encompassing the principles set forth by the phase rule is the phase equilibrium diagram. Figure $1 \mathrm{~A}$ gives a hypothetical phase diagram for a single-component system using pressure and temperature as the independent variables. Real systems can be infinitely more complicated in detail but the figure illustrates the essential features inherent in all single-component systems. The diagram consists of three individual curves, intersecting at point $b$, which separate solid, liquid, and vapor phases. The variance of each area and curve is indicated by an $F$ value, the maximum being two (divariant) for singlephase areas and the minimum, zero (invariant) for point $b$. Univariant curves $a b$ and $b c$ are the vapor pressure curves for the solid and liquid phases, respectively. At point $b$, the triple point, three phases, solid, liquid, and vapor, are in equilibrium.

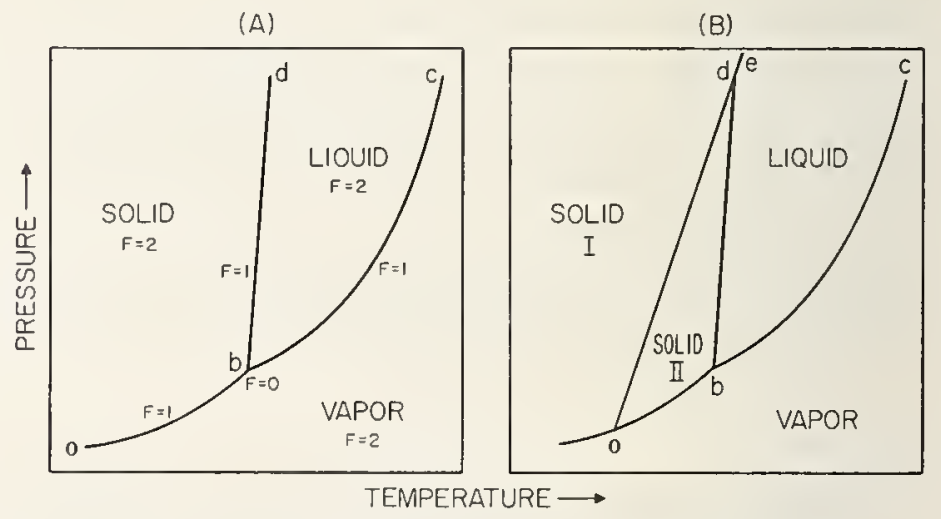

FIgURE 1. Schematic phase diagrams for one-component systems. F equals number of degrees of freedom.

Systems A and B:

$a b$-univariant sublimation curve,

bc-univariant liquid-vapor curve (boiling point curve),

bd-univariant melting point curve,

b-invariant triple point (solid, liquid, and vapor).

System B only:

ad-univariant solid 1-solid 11 transition curve

de-univariant melting point curve of solid 1 ,

d-invariant triple point (solid 1, solid II, and liquid), 
Curve bd represents the melting curve and indicates the change in the solid-liquid equilibria (melting points) with pressure and temperature. The curve indicates quite clearly that any definition of nielting point must include statements regarding both variables. It is insufficient to quote a melting temperature without specifying the pressure.

Theoretically, the melting curve is involved only with solid-liquid equilibria and only at the triple point does the vapor phase enter into the reaction. Take for instance, the compound $A_{x} O_{y}$ which vaporizes congruently to a number of gaseous species. For simplicity the reaction can be written as $A_{x} O_{y}(\mathrm{~s}, l) \rightarrow A_{x}(\mathrm{~g})+O_{y}(\mathrm{~g})$. The reaction equation describes the stoichiometry of vaporization and will be reflected in the onecomponent phase diagram by the location of curves $a b$ and bc, figure 1. Even here the twovapor species, $A_{x}(\mathrm{~g})$ and $O_{y}(\mathrm{~g})$, still only constitute a single-vapor phase and consequently obey the phase rule. In effect, the vaporization curves for $A_{x} O_{y}(\mathrm{~s}, l)$ are dissociation curves. The mechanism of vaporization will have no definitive effect upon the fusion curve in a one-component system, for in theory, there is no vapor phase to contend with at melting and the equilibrium of interest is: $A_{x} O_{y}(\mathrm{~s}) \rightarrow A_{x} O_{y}(l)$. In this hypothetical situation the pressure confining the system must be visualized as that exerted by a frictionless piston. In actuality, however, all real experiments must be carried out in some type of environment, say air or inert gas. Now the system becomes binary because a second component, the environment (air), has been added and the melting equilibrium is describable as:

$$
\begin{aligned}
A_{x} O_{y}(\mathrm{~s}) \text { +environment } & \rightarrow A_{x} O_{y}(l) \\
& + \text { environment }(\mathrm{a} \mid \mathrm{r}) .
\end{aligned}
$$

Both the solid and liquid then are also in equilibrium with a vapor phase and vapor pressures may be influential. In terms of the phase rule along the melting curve of $A_{x} O_{y}:[P+F=C+2]$ $=[3(\mathrm{~s}, l, \mathrm{v})+F=2+2]$ or $F=1$, the same as for a true one-component system. If the vapor pressure of the compound is very low, as is the case for many ceramics, the condensed phase rule can be applied: $[P+F=C+1]=[2(\mathrm{~s}, l)+F$ $=2+1]$ or $F=1$, again the same $F$ value as previously obtained when considering the vapor phase. It should be emphasized that neglecting the vapor as a possible phase does not remove it as one of the components $\left(A_{x} O_{y}+\right.$ environment). The condensed phase rule, eq 2 , only accounts for a vapor phase deletion and the system, in essence, is not truly a one-component system.

The assumption that the presence of an environment will not affect a melting curve is normally valid, provided the vapor pressures of the condensed components are low and provided that the environment is nonreactive. For example, if the component $A_{x} O_{y}$ dissociated to $A_{x} O_{y-z}(\mathrm{~s})$
$+O_{z}(\mathrm{~g})$ or $A_{x}(\mathrm{~s})+O_{y}(\mathrm{~g})$, the determined melting curve then would be representative of the new system, $A_{x} O_{y-z}(\mathrm{~s})$ or $A_{x}(\mathrm{~s})$, not the original system under investigation. In this context, the dissociation (or oxidation) pressures become extremely important to the melting point determination.

Figure $1 \mathrm{~B}$ indicates another hypothetical diagram for a one-component system in which a polymorphic change occurs in the solid state. Curve ad represents the transition curve for solid phases I and II. Point $a$ is another triple point where the two solids and vapor are in equilibrium. The transition curve, ad, is drawn to intersect the melting curve, bd, at the triple point, d, where solids I and II and liquid exist. With increased temperature and pressure solid I melts directly to liquid, thus exhibiting its own melting curve, de. This situation, where at high pressures the low form melts at a higher temperature than the maximum fusion temperature of the high form, is not at all uncommon. In ceramic systems there is a general scarcity of data showing such phenomena because of the extreme experimental conditions involved. Silica, the mainstay of all ceramics, does exhibit this type of behavior when subjected to high pressures. Figure 2 gives a composite phase diagram for $\mathrm{SiO}_{2}$ drawn from the data presented by several investigators $[6,18,19,20]$. High quartz is seen to melt at higher temperatures than cristobalite, the high

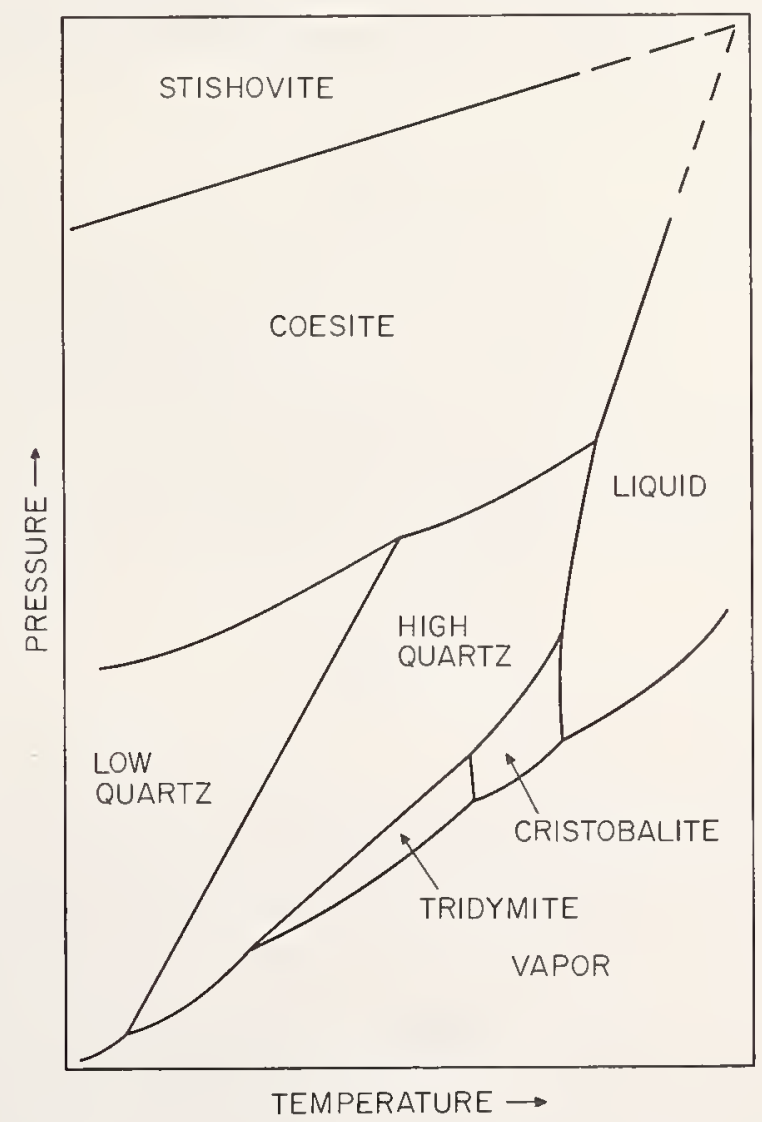

FIguRe 2. Schematic phase diagram for the onecomponent system, $\mathrm{SiO}_{2}$. Portions of diagram after Levin et al. [6], Ostrovsky [18], Boyd and England [19], and Fenner [20]. 
temperature-atmospheric pressure form. Coesite, the high pressure-low temperature phase, melts at still higher temperatures and although data are not available, stishovite conceivably would fuse at temperatures successively higher.

In the silica diagram the majority of the melting and transition curves all have positive slopes, that is, an increase in pressure will cause an increase in temperature for the transition. Negative slopes, although somewhat rare, do occur in a number of materials including water, bismuth, and gallium [2, 21]. Normally the overall change in melting point with pressure is rather small and generally is disregarded. However slight, these changes can be approximated from the Clapeyron equation provided values of the molar volumes of the solid and liquid phases as well as the heat of fusion are available:

$$
\frac{d T}{d P}=\frac{T_{m}\left(V_{l}-V_{s}\right)}{\Delta H_{f}}=\frac{T_{m} \Delta V}{\Delta H_{f}}
$$

where

$d T=$ change in melting temperature from $T_{m}$,

$d P=$ change in confining pressure,

$T_{m}=$ melting temperature at given pressure,

$V_{l}=$ molar volume of liquid,

$V_{\mathrm{s}}=$ molar volume of solid,

$\Delta H_{f}=$ molar heat of fusion.

A semi-empirical equation describing the melting curve has been derived by Simon [21, 22, 25] for the case where the coordinates of the triple point are known:

$$
\frac{P-P_{0}}{a}=\left(\frac{T}{T_{0}}\right)^{c}-1
$$

where

$P_{0}=$ pressure of the triple point,

$T_{0}=$ temperature of the triple point,

$P$ and $T=$ pressure and temperature of some point on melting curve,

$a$ and $c=$ constants characteristic of the material. At the triple point the Simon equation is related to the Clapeyron equation by [21]:

$$
\frac{d T}{d P}=\frac{T}{c(a+P)}
$$

where $P>>P_{0}$

The Simon equation is in essence a curve-fitting equation requiring extensive and accurate experimental data to be of any great utility. For ceramic systems the Clapeyron relationship will suffice for most purposes. As a practical example the slope of the melting curve for $\mathrm{Al}_{2} \mathrm{O}_{3}$ is 0.02 deg per atm assuming $\Delta H_{f}=28.3 \mathrm{kcal} / \mathrm{mol}$, $V_{\mathrm{l}}=0.338 \mathrm{~cm}^{3} / \mathrm{g}$ and $V_{\mathrm{s}}=0.251 \mathrm{~cm}^{3} / \mathrm{g}$. For a change in melting point of only $2 \mathrm{deg}$, a pressure of at least 100 atm would be required-indeed a very negligible pressure effect. Similar calculations applied to the cristobalite-liquid transformation yield a $0.07 \mathrm{deg} / \mathrm{atm}$ slope, still a slight effect.
These small pressure effects are characteristic of ceramic systems involving only solid-liquid equilibria.

The differential form of the Clapeyron equation is seldorn applicable over very wide pressure ranges, for both $\Delta H_{f}$ and $\Delta V$ are functions of pressure and temperature. The compressibility of the liquid phase generally is greater than that of the solid [3] so at very high pressures $V_{l}$ will become less than $V_{\mathrm{B}}$. At some point $V_{l}$ will equal $V_{\mathrm{s}}$ and $\frac{d T}{d P}$ will then be zero, resulting in a maximum or reversal point on the melting curve. Few ceramics have been studied at sufficiently high pressures and temperatures to determine whether or not they exhibit this type of behavior. However, some data are available and figures 3 and 4 illustrate such maxima found for the carbon [18, 24] and $\mathrm{Li}_{2} \mathrm{CrO}_{4}$ [25] systems. The reversal phenomenon, of course, is an experimental observation and a detailed explanation of why it occurs for some materials involves careful analysis of the structures of both the liquid and solid phases at fusion temperatures.

A maximum in the fusion curve dictates that $\Delta V$ is zero. If, coincident with $\Delta V=0$, the change in entropy were also zero, the system then would cease to be heterogeneous and the solid and liquid phases would be indistinguishable, analogous to the critical phenomena which exist for liquid-vapor transitions. The fusion curve at this

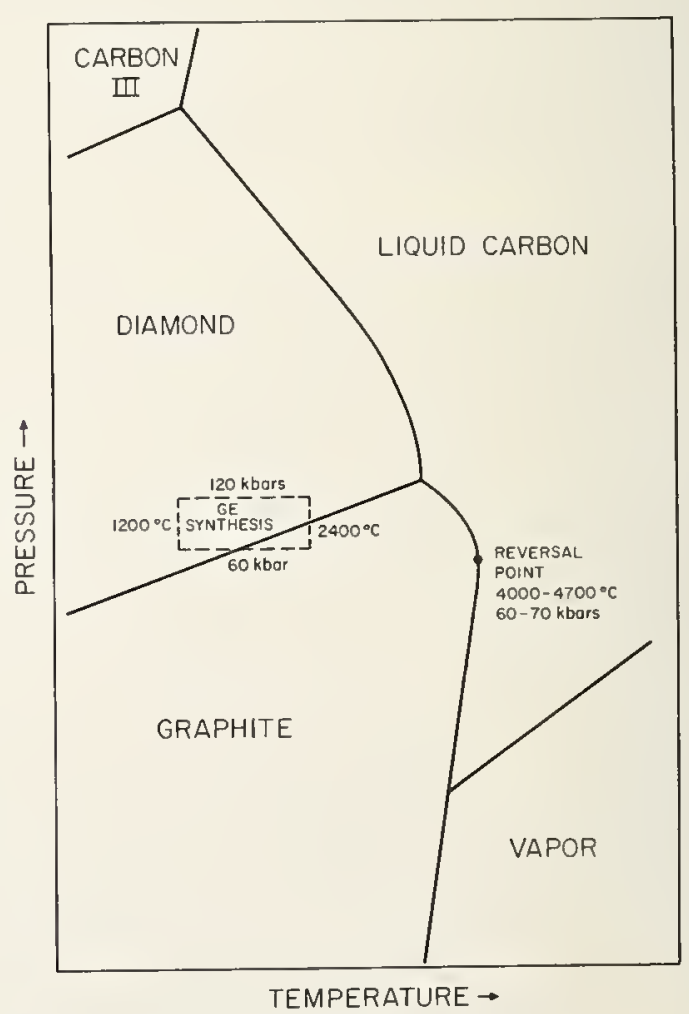

FIgURE 3. Schematic phase diagram for the one-component system carbon. Diagram redrawn from that given by Bundy [24]. 


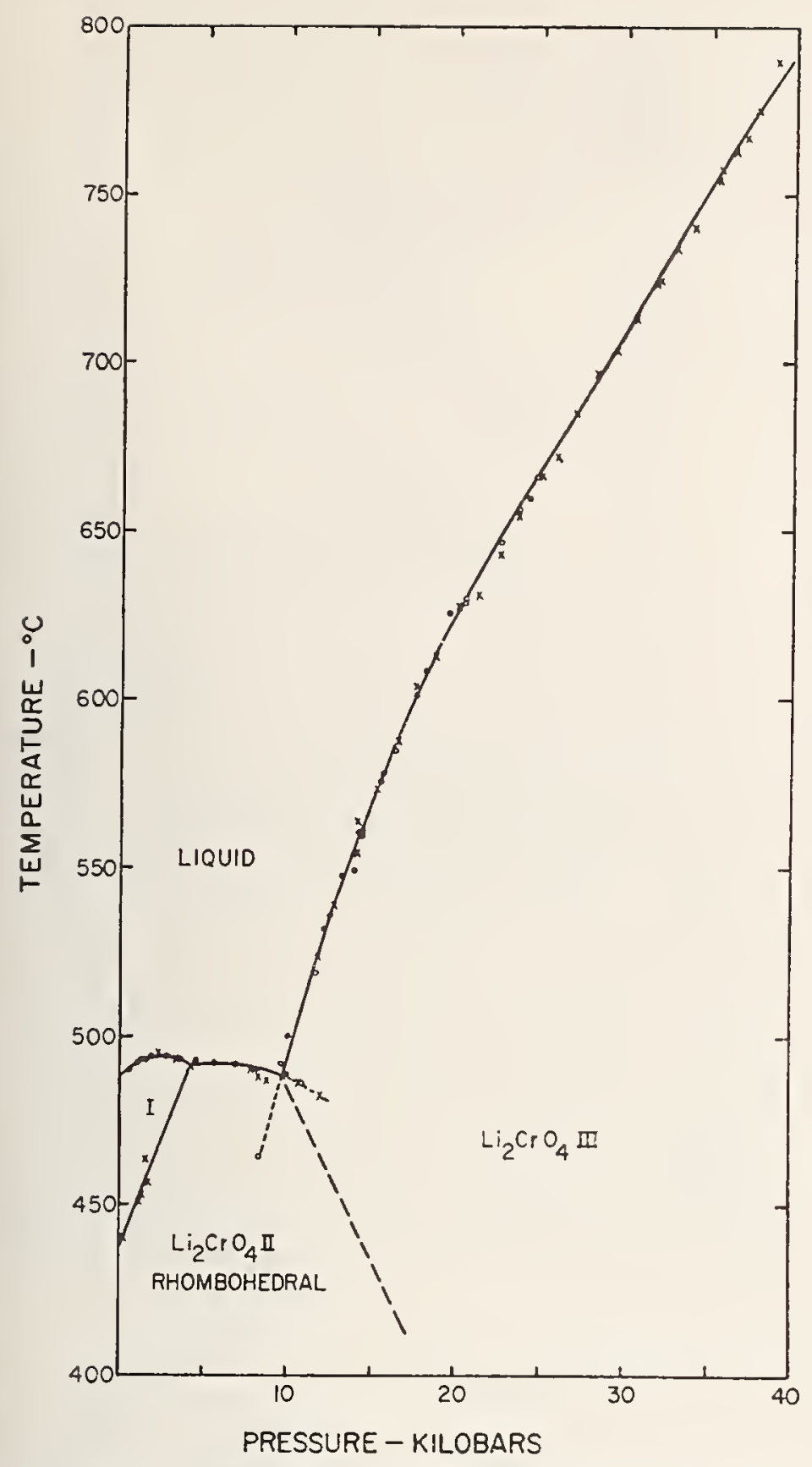

Figure 4. Phase diagram for the one component system, $\mathrm{Li}_{2} \mathrm{CrO}_{4}$. Afler Pislorius [25].

critical temperature and pressure point would terminate in a manner similar to the upper limit found for liquid vaporization curves. Critical melting has been postulated but not verified for several materials [21]. If such melting occurs in ceramics the pressures involved would be exceedingly high.

\subsection{Metastable Equilibria}

Metastable equilibria can be depicted graphically in a manner similar to that illustrated for stable systems. However, a certain definite relationship always exists between the two types of equilibria which dictates the behavior of metastable phases with pressure and temperature. Figure 5 gives diagrams for three one-component

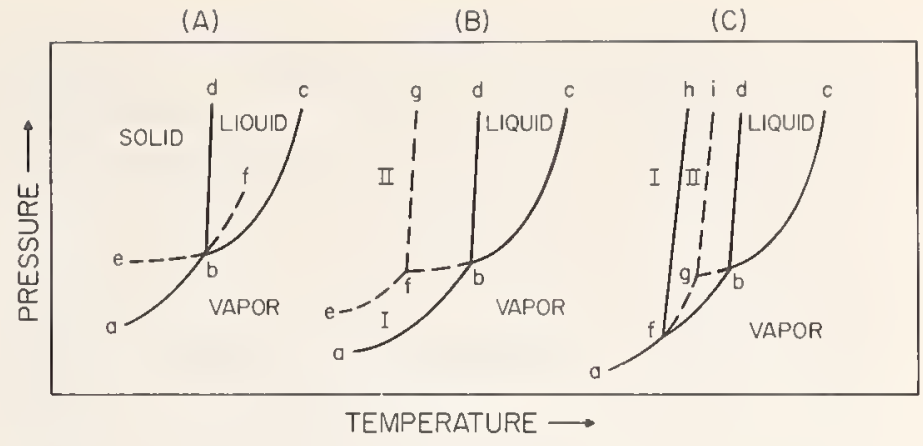

FiguRE 5. Schematic phase diagrams for one-component systems showing slable (solid lines) and melastable (dashed lines) univariant curves.

eb-supercooling,

bf-superheating (system A),

fg and gi-metastable melting point curves.

systems showing the effect of metastability upon the fusion curve. In the general case, $(\mathbb{A})$, the metastable univariant curves (dashed lines) are extensions through the triple point, b, of the stable curves (solid lines) with bf representing superfusion, and eb, supercooling. Superfusion, that is, the heating of a solid above the melting point without the appearance of the liquid phase, has seldom, if ever, been found experimentally [3]. Supercooling on the other hand, is quite common and as mentioned previously, ordinary glass offers a perfect example.

Both metastable extensions, curves bf and eb, must be drawn between the stable univariant curves so that the stable phases will be always illustrated as having the lowest vapor pressures. This requirement is necessary because a phase is always stable with respect to another if it possesses a lower Gibbs energy or, in practical terms, lower vapor pressure $[2,3]$.

In case (B), figure 5, the system is seen to contain a phase II which has no stability field whatsoever. The vapor pressure curve of II, ef, intersects the extension of the liquid-vapor curve at $f$, a metastable triple point. The fusion curve, $\mathrm{fg}$, indicates metastable melting at temperatures lower than those for stable melting, curve bd. Example (C) of the figure illustrates metastable melting, curve gi, as influenced by the presence of a stable polymorph, I. Here solid I melts rather than transforming to solid II as required by the stable equilibrium relationships. It is interesting to note that both situations, (B) and (C), show how it is possible for certain materials upon heating, to melt, then solidify, and finally remelt again.

The question sometimes arises whether or not a metastable phase can melt at temperatures higher than its stable counterpart. The metastability represented by (B) and (C), fig. 5, indicate lower melting. In these examples the metastable liquid phase is always unstable with respect to a true equilibrium solid. For metastable melting to occur at higher temperatures, a liquid phase would 
have to be formed which is unstable with respect to an equilibrium liquid. Figure 6 (A) and (B) gives hypothetical examples of higher melting in which liquid II would be unstable. In essence two immiscible liquids would have to be postulated for a one-component system. This, of course, represents an improbable situation and consequently higher melting cannot occur. It should be pointed out that example (A), figure 6, represents nothing more than superfusion previously illustrated in figure 5A. The unlikelihood of liquid immiscibility in a one-component system accounts for the fact that superfusion has never been demonstrated experimentally.

Instances of metastable equilibria occurring in ceramic materials are numerous. The $\mathrm{GeO}_{2}$ system [26], figure 7, perhaps offers a perfect example of the types of metastability given in figure 5. It is evident from the figure (curve jc) that liquid $\mathrm{GeO}_{2}$ can be supercooled from above $1116^{\circ} \mathrm{C}$, the true melting point, to room temperature. The rutile form of $\mathrm{GeO}_{2}$, the stable low temperature form, is seen also to melt metastably at $1086{ }^{\circ} \mathrm{C}$ instead of transorming to the stable $\beta$ quartz polymorph at $1049{ }^{\circ} \mathrm{C}$. Furthermore, the $\beta$ quartz form on cooling, can transform metastably at $1000{ }^{\circ} \mathrm{C}$ to $\alpha$ quartz, a form that presumably has no stability field whatsoever.

Other examples of the prominent role played by metastable equilibria in the behavior of ceramics are given by the $\mathrm{SiO}_{2}[20]$ and $\mathrm{Bi}_{2} \mathrm{O}_{3}$ [27] systems, figures 8 and 9. Here again metastable melting is a significant feature of both systems.

\section{Binary Systems}

Although few commercially important ceramics involve only single-component systems, their basic behavior can be developed rather nicely from one-component principles. Consider first a onecomponent system, solid $A$, to which small increments of an impurity, solid B, have been

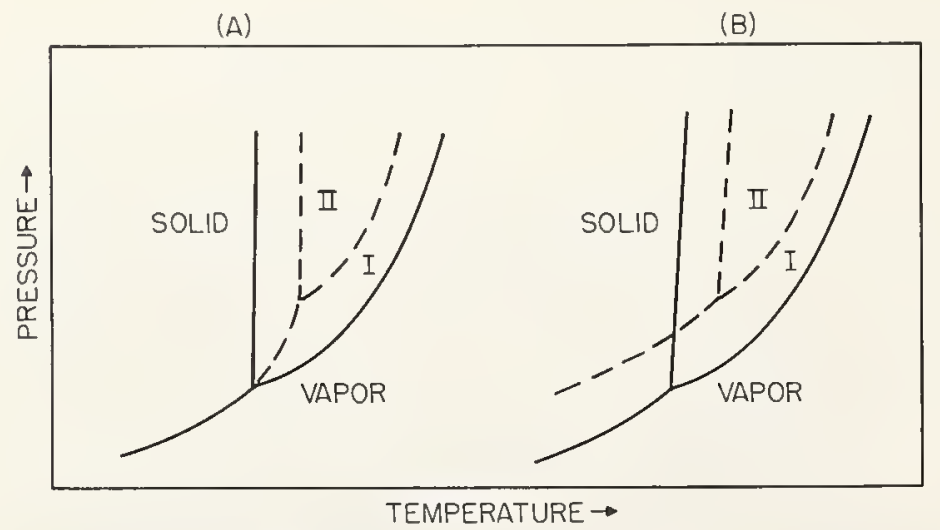

FIGURE 6. Phase diagrams for one-component systems showing two situations of superheating.

Solid lines-stable univariant curves,

Dashed lines-metastable univariant curves,

1-stable liquid,

II-metastable liquid.

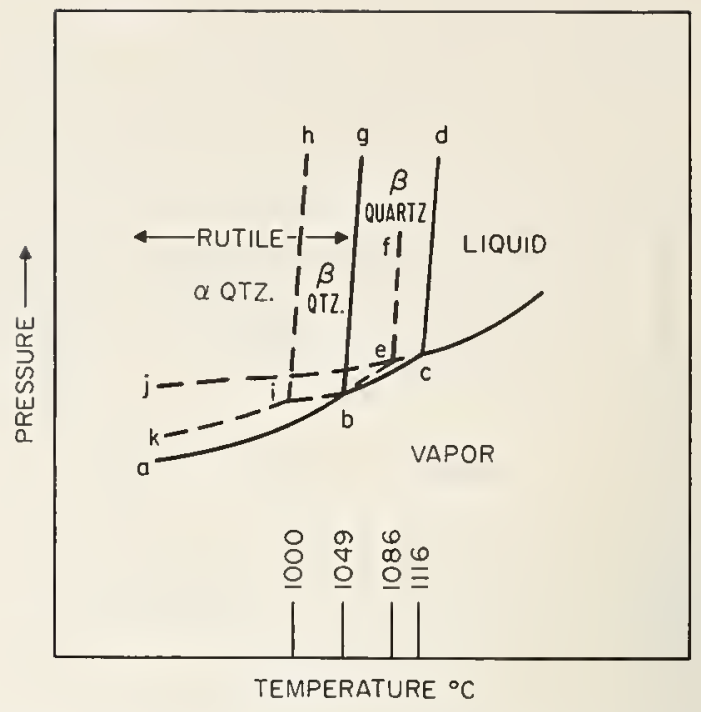

FiguRe 7. Phase diagram for the one-component system, $\mathrm{GeO}_{2}$. Essentially after Sarver and Hummel [26].

Dashed lines-metastable univariant curves, Solid lines-stable univariant curves,

fe-metastable melting point curve of rutile form, de-stable melting point curve of $\beta$-quartz form, hi-metastable transition curve of metastable form $\alpha$-quartz and stable form $\alpha$-quartz.

\section{$\mathrm{SiO}_{2}$}

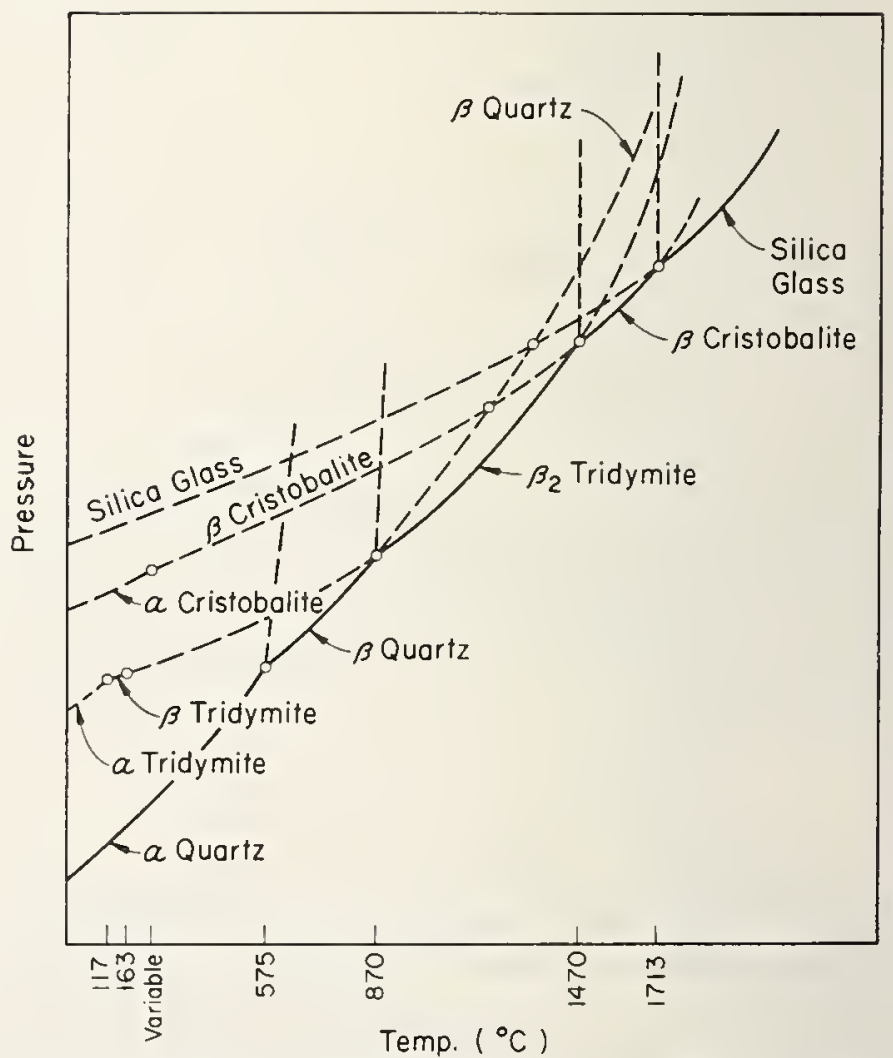

Figure 8. Stability relations of the silica minerals. After C. N. Fenner [20]. 


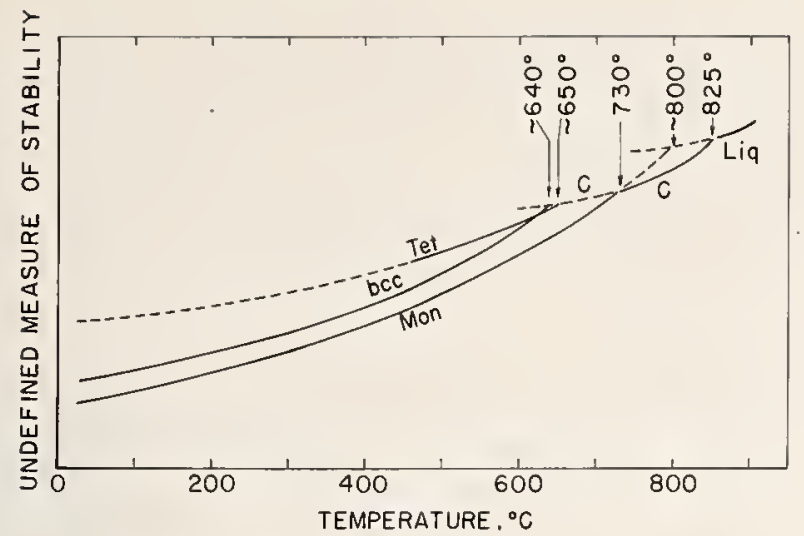

FIgURE 9. Postulated stability diagram of $\mathrm{Bi}_{2} \mathrm{O}_{3}$. After Levin and Roth [27].

Polymorphs: Mon-monoclinic, C-cubic, tet-tetragonal, b.c.c.-body-centered cubic, Liq-liquid. Metastable melting point $\sim 800^{\circ} \mathrm{C}$.

added. Further presuppose that no reaction occurs between solids, but that the impurity does react to form a liquid solution containing both constituents. If each binary combination is viewed as an individual one component, then a series of typical solid-liquid-vapor curves can be established for every compositional increment as given in figure 10A. An orthogonal projection of the pseudo one-component curves on the 100 percent composition plane of A produces a two-dimensional representation, figure $10 \mathrm{~B}$, which clearly indicates the effect caused by the addition of the second component. The impurity, B, reacting in the liquid only, causes a sequential lowering of the liquidvapor pressure curves as more and more of the second component goes into solution. Because of nonreaction between solids, the solid-vapor pres-

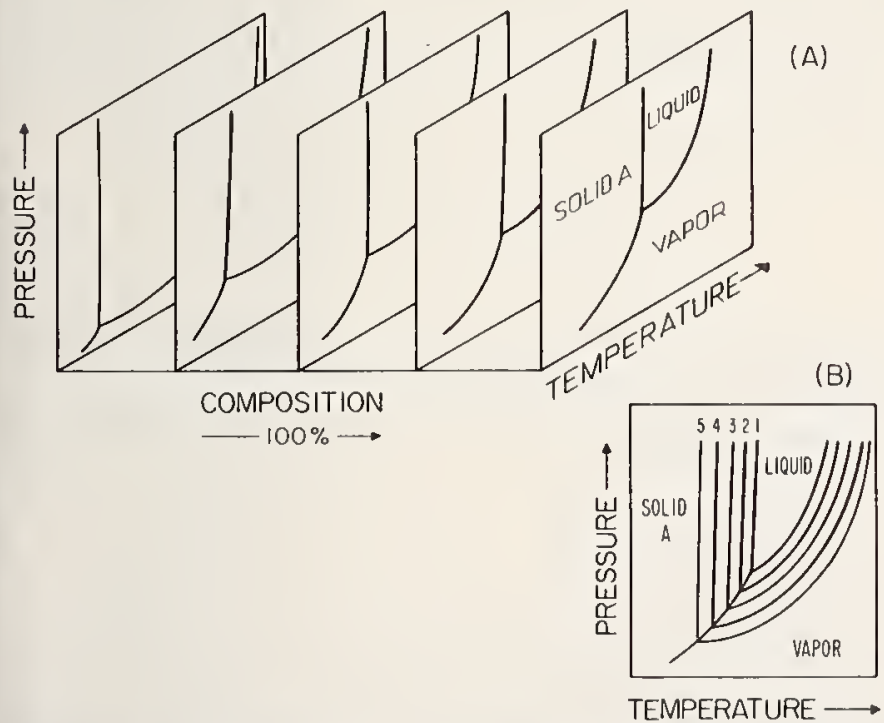

FIGURE 10. Schematic representation showing the effect on the univariant curves of a one-component system by the addition of a second component.

(A) Three-dimensional model illustrating progressive change of one-component univariant curves.

(B) Projection of binary univariant curves on pressure-temperature plane of pure component $A$. Curve $1-$ melting point curve of solid A; Curves $2,3,4$, and 5 -melting point curves of binary mixtures. sure curve remains unchanged. Intersection of the liquid-vapor curves with the unaltered solidvapor univariant forms a series of triple points and consequently a number of melting point curves (1 through 5, fig. 10B). The fusion curves are seen to occur at progressively lower temperatures, indicative of the amount of impurity added to the pure component. The area defined by curves 1 and 5 and the solid-vapor pressure curves represent, in three dimensions, a volume containing both solid, $\mathrm{A}$, and liquid phases.

For experimental convenience, it is customary, and sometimes necessary, to investigate the behavior of binary and other multicomponent systems at a fixed pressure. The constant pressure plane of importance to ceramics normally would be at pressures greater than those of the binary triple points. Graphically, figure 11 would represent an isobaric study of a binary system in which the pressure plane intersects only the melting and liquid-vapor curves. The melting point of the pure component, $A$, at the fixed pressure is indicated by $T_{1}$ while $T_{2}, T_{3}, T_{4}$, and $T_{5}$ are melting points of the binary increments. If the various melting points $\left(T_{1} \rightarrow T_{5}\right)$ are connected as shown in figure $12 \mathrm{~A}$, the resulting curve,

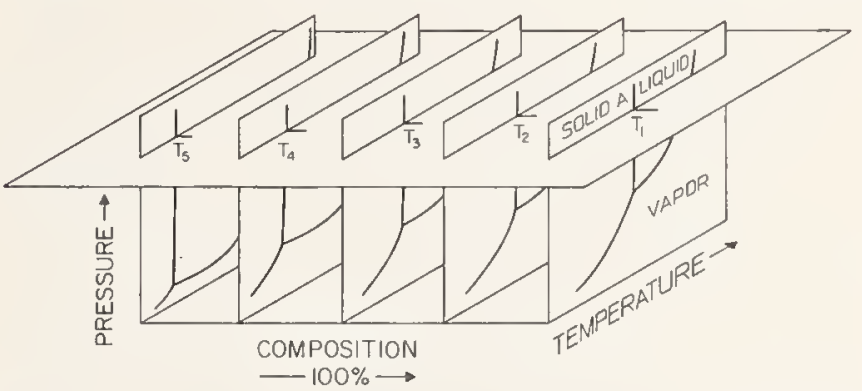

FIGURE 11. Isobaric cut through melting point and boiling point curves of binary mixtures and pure component, $A$.

$T_{1}, T_{2}, T_{3}, T_{4}$, and $T_{5}$ - - melting points at constant pressure for pure component $\mathbf{A}$ and binary mixtures.

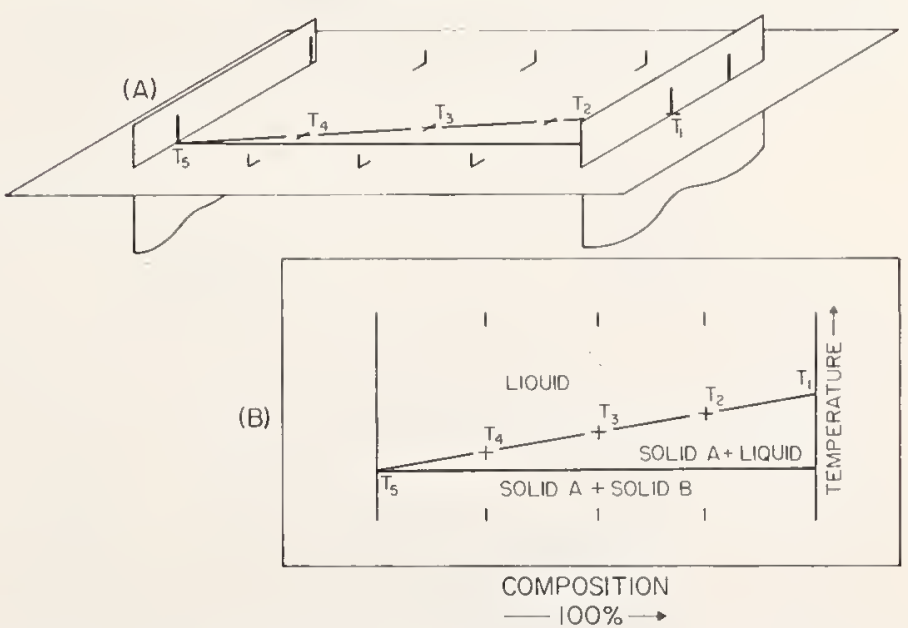

FiguRE 12. Schematic derivation of liquidus curve for portion of binary system at constant pressure.

(A) Portion of three-dimensional model given in figure 11.

(B) Conventional two-dimensional phase diagram for a portion of a two component system. $T_{1}, T_{2}, T_{3}, T_{4}$, and $T_{6}$-liquidus temperatures at constant pressure. 
given on the two dimensional temperature-composition plot, figure $12 \mathrm{~B}$, becomes the familiar liquidus curve found for so many phase diagrams of binary systems.

The same general treatment can be applied to the other end member, $\mathrm{B}$, with component A now acting as the impurity. Lower meltlng temperatures for B would occur with the end result being a simple eutectic system as shown in figure 13 . The intersection of the two depressed liquidus curves defines the eutectic temperature and composition for a given pressure.

The extent of the melting point depression of each end member can be approximated from the Raoult-Van't Hoff equation for dilute solutions $[2,3]$ :

$$
\Delta T=\frac{R T^{2}}{\Delta H_{f}}\left(N_{\mathrm{BL}}\right)
$$

where

$\Delta T=$ change in melting point,

$T=$ melting point of pure component A,

$N_{\mathrm{BL}}=$ mole fraction of impurity $\mathrm{B}$ in liquid solution,

$R=$ gas constant,

$\Delta H_{f}=$ molar heat of fusion.

Quite obviously eq (6) is based on ideal solutions and therefore is of limited use for real systems. General trends, however, can be anticipated from the relationship and when applied to $\mathrm{Al}_{2} \mathrm{O}_{3}$, for instance, it is found that the melting point would be depressed approximately $3^{\circ}$ for every mole perceni contaminant. For $\mathrm{SiO}_{2}$ a similar calculation indicales a change in melting point of about $80^{\circ}$ for each mole percent of added impurity. Thus, these data suggest that the slope of any liquidus curve involving $\mathrm{Al}_{2} \mathrm{O}_{3}$ as the pure component would be less than that for $\mathrm{SiO}_{2}$. This fact is borne out by experimental results on the $\mathrm{Al}_{2} \mathrm{O}_{3}$ $\mathrm{SiO}_{2}$ system [28], figure 14, which indicates a greater depression for the melting point of $\mathrm{SiO}_{2}$ than for $\mathrm{Al}_{2} \mathrm{O}_{3}$.

Systems exhibiting only liquid solution are the ultimate in simplicity and seldom are found in real situations. Most systems contain one or

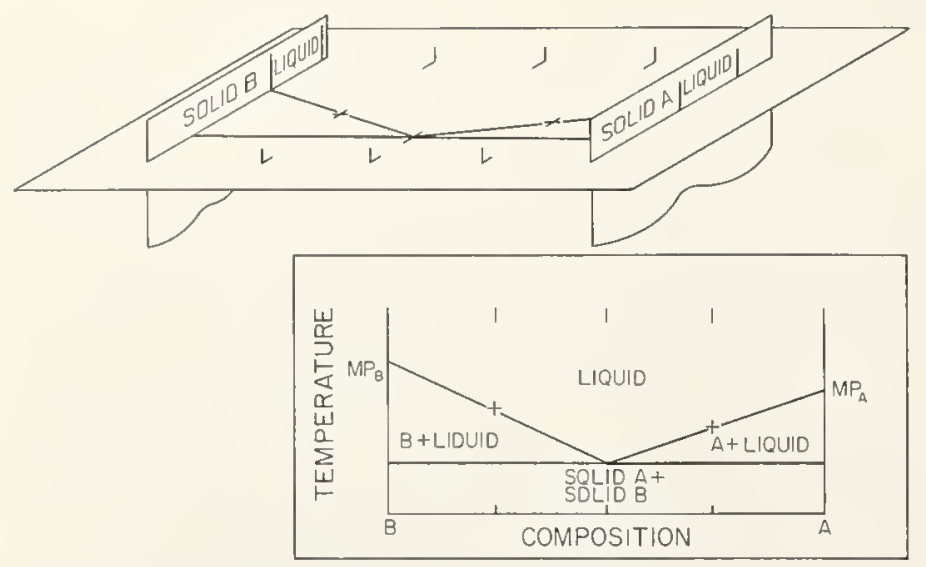

FIGURE 13. Schematic derivation of liquidus curves for binary system $A-B$ at constant pressure.

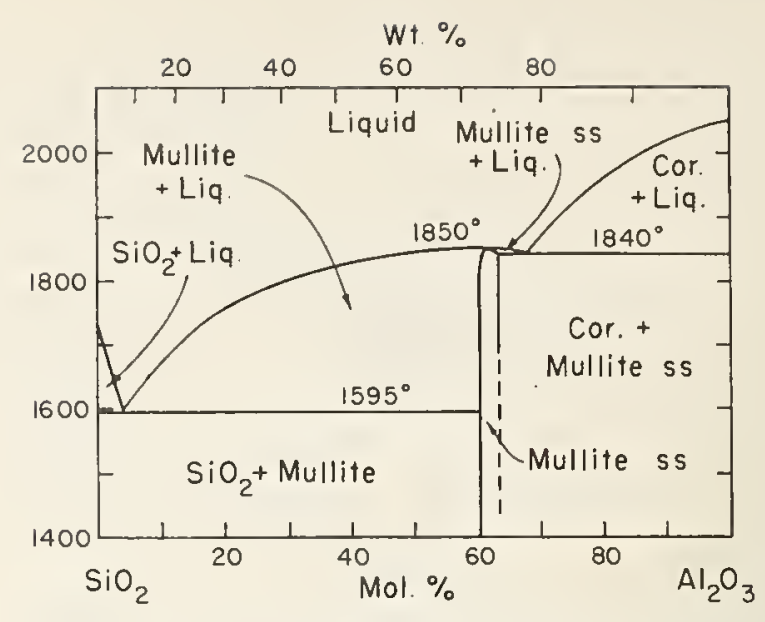

Figure 14. Phase diagram for the $\mathrm{SiO}_{2}-\mathrm{Al}_{2} \mathrm{O}_{3}$ system. After Aramaki and Roy [28]. Figure reproduced from Levin et al. [6].

more intermediate compounds, and more often than not, some solid solubility between end members. Compounds which melt to a liquid and solid of different composition are termed incongruent and their presence will be reflected by an inflection or break in the liquidus curves indicative of the melting temperature (peritectic point). Congruently melting compounds are those which fuse to a liquid of the same composition. They can be treated in entirety as a one-component system with the melting point always appearing as a maximum on the liquidus curve of the full binary.

Approximations are difficult to establish concerning the effect of solid solution upon the melting point of a compound or pure component. Solid solution can either raise or lower the melting point depending upon the solubility of the solute in both the liquid and solid phase. To show the influence of solid solution the Raoult-Van't Hoff relationship, eq (6), can be modified to [3]:

$$
\Delta T=\frac{R T^{2}}{\Delta H_{f}} N_{\mathrm{BL}}\left[1-\frac{N_{\mathrm{BS}}}{N_{\mathrm{BL}}}\right]
$$

where, $\Delta T, R, T$, and $\Delta H_{f}$ have the same meaning as described for eq (6) and

$N_{\mathrm{BL}}=$ mole fraction of impurity $\mathrm{B}$ in liquid solution,

$N_{\text {BS }}=$ mole fraction of impurity $\mathrm{B}$ in solid solution.

It is readily apparent that if $N_{\mathrm{BS}}$ is zero then eqs (6) and (7) become equivalent and a simple melting point depression will occur. On the other hand, if $N_{\mathrm{BS}}$ is greater than $N_{\mathrm{BL}}$, then the melting point will be elevated. Figure 15 graphically illustrates this principle by indicating an effect on the vapor pressure curves caused by both liquid and solid solutions. Example A shows greater vapor pressure change for liquid solution $\left(N_{\mathrm{BL}}>N_{\mathrm{BS}}\right)$ and thus a lower melting curve. For case $B$ the solid vapor pressure curve is 
(A)
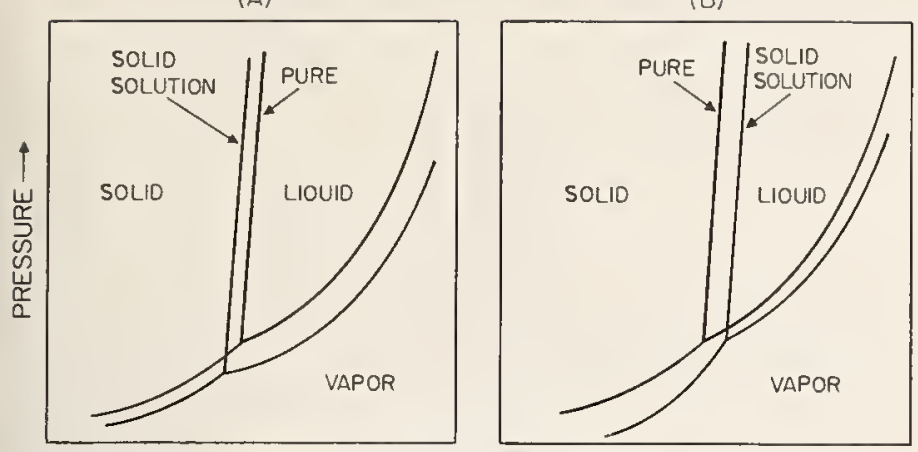

TEMPERATURE $\longrightarrow$

Frgure 15. Schematic phase diagrams for one-component systems showing effect of solid solution on the meling point curve.

(A) Melting point curve lowered by solid solution.

(B) Melting point curve raised by solid solution.

affected to a greater degree $\left(N_{\mathrm{BL}}<N_{\mathrm{BS}}\right)$ and a raised melting point results.

\section{Pseudo One-Component Systems}

Many ceramic materials seemingly behave as single components in that they exhibit all the overt characteristics of one-component systems, melting at defined pressures and temperatures. Some compounds, for example, will melt at a given temperature in one atmosphere oxygen and simply by changing the environment to one atmosphere of air, a different melting point will be observed. If the course of each different heat treatment is followed with, say, simultaneous x-ray examination of the samples, it will become evident that compositional changes have taken place. Because of the environment the resulting equilibrium cannot be described in terms of the starting material. Titanium dioxide for instance is known to lose oxygen upon heating in a supposedly oxidizing environment such as air. Consequently the determined melting point probably will not be representative of stoichiometric $\mathrm{TiO}_{2}$ and the composition of interest would lie in the $\mathrm{Ti}-\mathrm{O}_{2}$ binary system. Examination of the $\mathrm{Ti}^{-\mathrm{TiO}_{2}}$ diagram [29], figure 16, clearly indicates that an

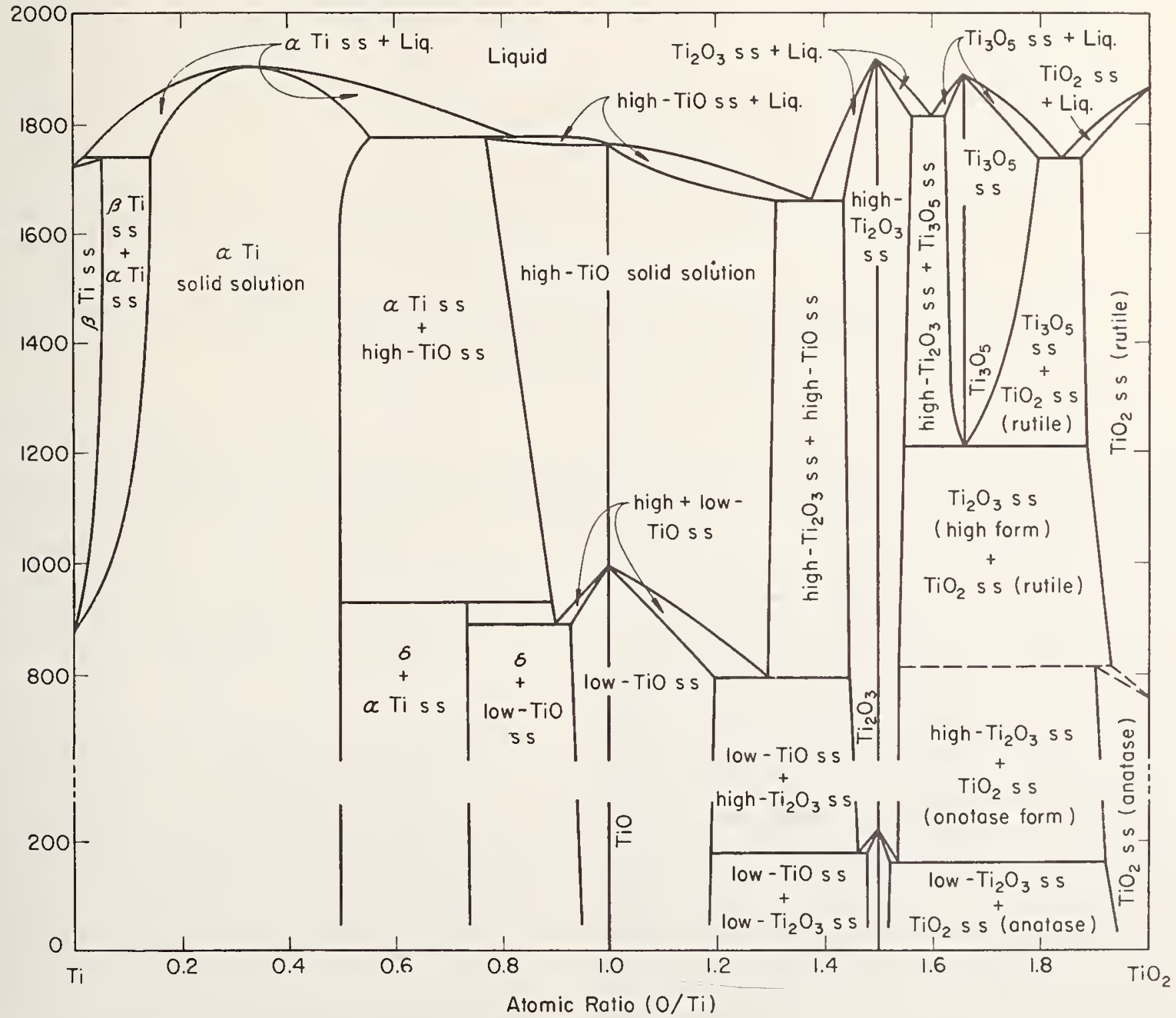

FIGURE 16. Phase diagram for the Ti-TiO ${ }_{2}$ system. After DeVries and Roy [29]. Figure reproduced from Levin et al. [6]. 


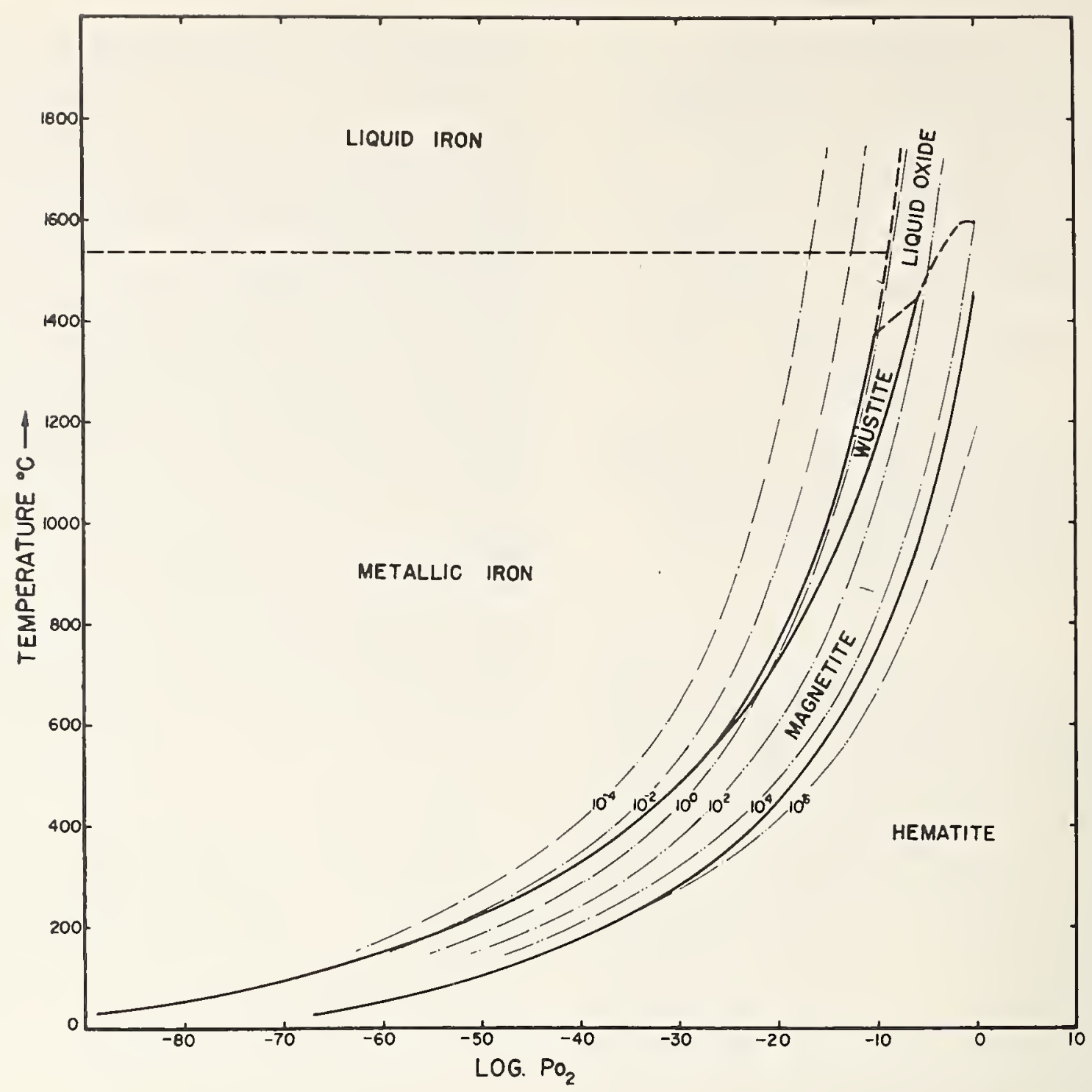

FIgURE 17. Phase diagram for the iron-iron oxide system as a function of oxygen partial pressure in atm. After Muan [30] and after Darken and Gurry [31, 32].

Dot-dash-lines of equal $\mathrm{PH}_{2} \mathrm{O} / \mathrm{PH}_{2}$ ratio of gas phase at 1 atm.

infinite number of "melting points" are possible, all dependent upon the extent of reduction and the final composition at fusion. Unfortunately, the type of diagram illustrated lacks one important feature; it gives no information concerning the amount of reduction or change in the condensed phases which could occur for each environment.

Knowledge of the phase relations as a function of both temper ature and oxygen partial pressures is therefore of great importance, particularly in those systems involving the transition metal oxides where oxidation and reduction are common occurrences. As an illustration, figure 17 gives information of this type for the $\mathrm{Fe}-\mathrm{O}$ binary system $[30,31,32]$. In this plot the stability fields of the condensed phases, iron and iron oxides in various oxidation states, are given as a function of temperature and oxygen partial pressure. Overall, the diagram has the appearance of the typical one-component system with each boundary curve representing a univariant situation. Although not shown, each phase is assumed to be in equilibrium with a gas phase. If the oxygen partial pressure were held constant say at log $\mathrm{P}_{\mathrm{O}_{2}} \sim-0.7$ (one atmosphere air) hematite would upon heating transform through reduction to magnetite before melting. On the other hand, if the hematite composition were fixed, the oxygen partial pressure would continually change during the course of the heat treatment. It is interesting to note that for stoichiometric $\mathrm{Fe}_{2} \mathrm{O}_{3}$ (hematite) to melt, the oxygen pressure would have to be much greater than 1 atm. As far as is known, " $\mathrm{Fe}_{2} \mathrm{O}_{3}$ " as such has never been melted.

The phases listed in fig. 17 have been designated as magnetite, hematite, etc. for good reason. The psuedo one-component diagram provides no information on the exact compositions ( $\mathrm{Fe} / \mathrm{O}$ ratios) of the condensed phases. Wüstite, for example, can vary in composition depending upon the temperature and oxygen partial pressure. The same is true for hematite and magnetite. To alleviate this problem the conventional binary plot can be 


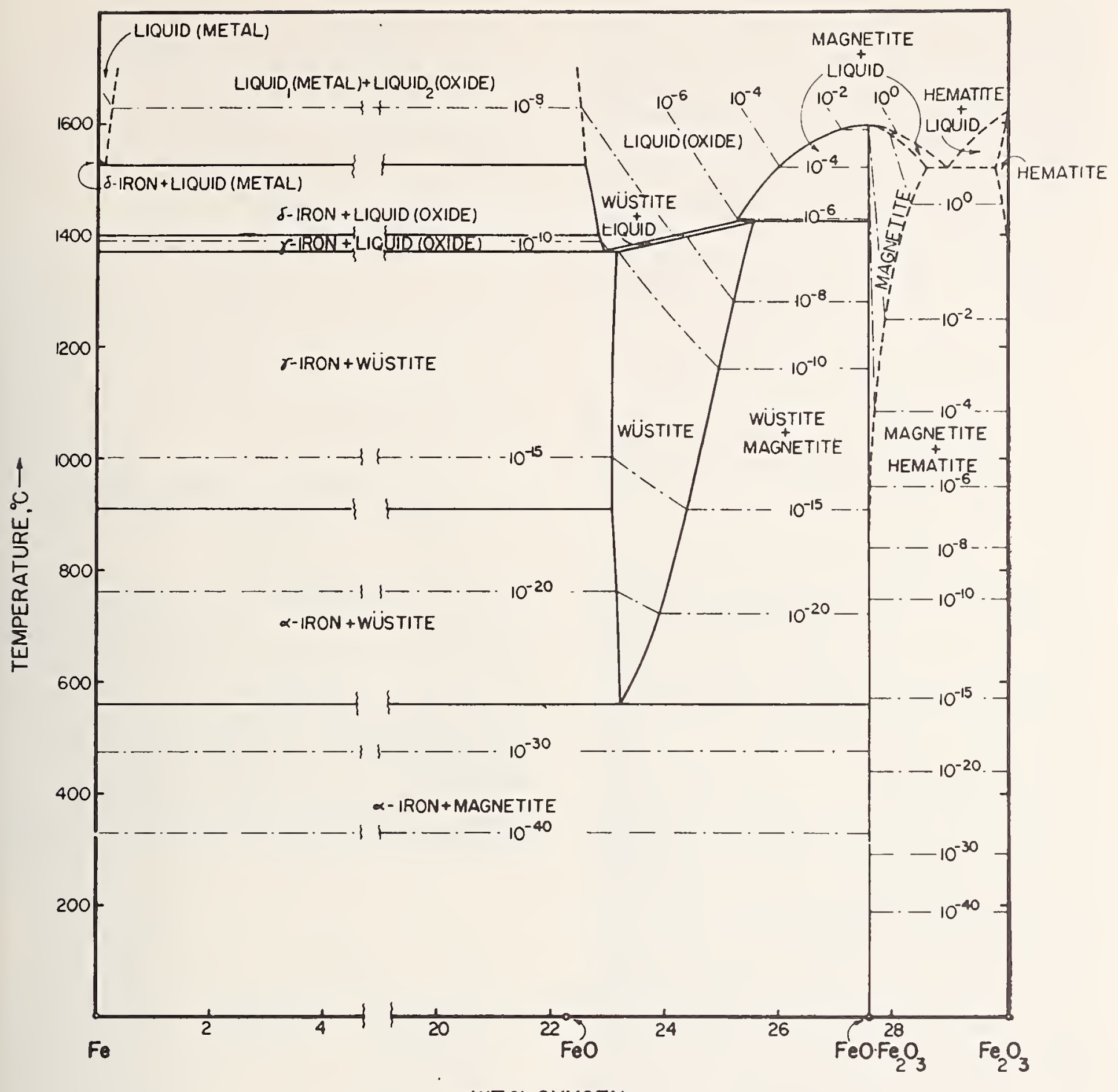

WT.\% OXYGEN

Figure 18. Phase diagram of the $\mathrm{Fe}_{-} \mathrm{Fe}_{2} \mathrm{O}_{3}$ system. After Darken and Gurry [31, 32] and after Muan [30]. Heavy solid lines are boundary curves separating labeled phase areas. Dash-dot lines are $\mathrm{O}_{2}$ isobars in atm.

combined with the one-component representation in a manner as shown in figure 18 [30, 31, 32]. Here, various oxygen isobars are plotted across compositional lines and indicate the pressure of the gas in equilibrium with the condensed phases. Each two-phase region is equivalent to the univariant boundary curves given in figure 17 . Single-phase areas in addition to being defined by temperature and oxygen pressure, are also located with respect to composition.

Some exceedingly useful information can be gained from proper utilization of this type of diagram. Certain rules, however, must be adhered to in its use. If the oxygen partial pressure is held constant, the path or course of events for a particular composition would follow that given isobar. For instance, $\mathrm{Fe}_{3} \mathrm{O}_{4}\left(\mathrm{FeO} \cdot \mathrm{Fe}_{2} \mathrm{O}_{3}\right)$, upon heating from room temperature in a gas of $10^{-4}$ atm oxygen partial pressure and one atmosphere total pressure, would oxidize at low temperatures until $\mathrm{Fe}_{2} \mathrm{O}_{3}$ is formed. This is necessary because the $10^{-4}$ atm isobar does not intersect the $\mathrm{Fe}_{3} \mathrm{O}_{4}$ composition at low temperatures, thus making the phase metastable with respect to $\mathrm{Fe}_{2} \mathrm{O}_{3}$. Further 
increases in temperature would cause a subsequent reformation of magnetite (" $\mathrm{Fe}_{3} \mathrm{O}_{4}$ ") but of a different composition than the starting material. The magnetite phase would continue to change in composition with temperature until melting occurred. At the melting point the solid (magnetite) would be in equilibrium with a liquid only at one temperature for the $10^{-4}$ atm isobar, thus giving the appearance of congruently melting. If the oxygen partial pressure were altered, a different "congruent" melting temperature would be observed. In these cases, congruent melting is a misnomer since the solid and liquid in equilibrium do not have the same composition.

\section{Multicomponent Systems}

To the novice, the phase diagram of a multicomponent system may appear as a quagmire of points, curves, areas, and polyvariant situations. Multicomponent systems present a problem not only in their investigation but also in the mere graphic representation of their equilibrium relations. As stated previously, the independent variables of any system are pressure, temperature, and composition (of $n$ components), each requiring an axis in space for pictorial representation. It is apparent that a major problem exists in representing all variables in three-dimensional space.

A plane, two-dimensional figure is suitable for a one-component system with an axis each for pressure and temperature. Likewise, a binary system can be depicted in two dimensions if one of the variables is fixed; or in three dimensions, if both the pressure and temperature variables are utilized. Ternary systems can be illustrated fully by a three-dimensional model only by fixing the pressure (or temperature) and setting up a coordinate system of temperature and the concentration of the three components. For systems containing four components graphic representation is impracticable because both pressure and temperature then must be held constant. Thus, for a system of $3+-n$ components a complete graphic model cannot be formulated since $3+n$ dimensional space would be required. Fortunately, phase equilibrium relationships between materials are such that the multicomponent system can be viewed as groups of interrelated limiting boundary systems consisting of a lesser number of components. The four-component system, A-B-C-D, for example, can be thought of as the four boundary ternary systems; $[\mathrm{A}-\mathrm{B}-\mathrm{C}],[\mathrm{A}-\mathrm{C}-\mathrm{D}],[\mathrm{A}-\mathrm{B}-\mathrm{D}]$, and $[\mathrm{B}-\mathrm{C}-\mathrm{D}]$. These ternary systems in turn can be broken down into other boundary subgroups which are more conducive to investigation.

For apparent reasons, the teruary system plays a prime role in the understanding of more complex systems. Similar to binary mixtures, the ternary system also can be developed using one-component systems as basic building blocks. Consider first, three one-component systems $\mathrm{A}, \mathrm{B}$, and $\mathrm{C}$, which react to form only liquid solution. The individual

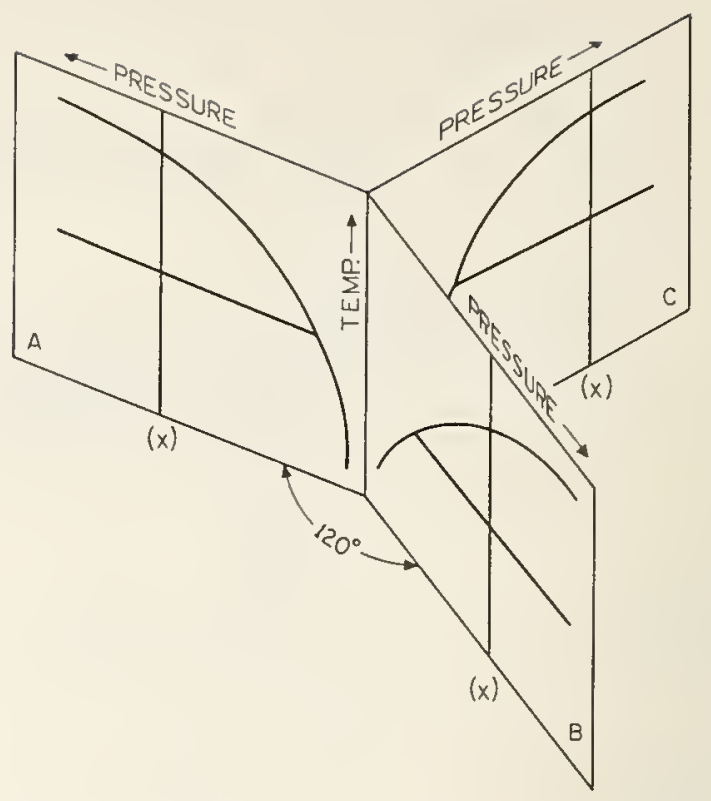

FIgURE 19. Schematic derivation of the threecomponent system, $A-B-C$, from individual one-component systems.

$$
\mathrm{X} \text {-isobaric lines. }
$$

systems can be arranged about a common temperature axis $120 \mathrm{deg}$ apart in the manner illustrated by figure 19. Lines of equal pressure (X) drawn to intersect the univariant curves, in essence fix the pressure $v$ ariable at the same value for each onecomponent system. By connecting the isobaric lines with planes of constant pressure a threedimensional model (figure 20) is obtained which relates the three components with temperature at constant pressure. Points $T_{\mathrm{A}}, T_{\mathrm{B}}$, and $T_{\mathrm{C}}$ indicate the melting temperatures of components $\mathrm{A}, \mathrm{B}$, and $\mathrm{C}$ for that given pressure. The resulting

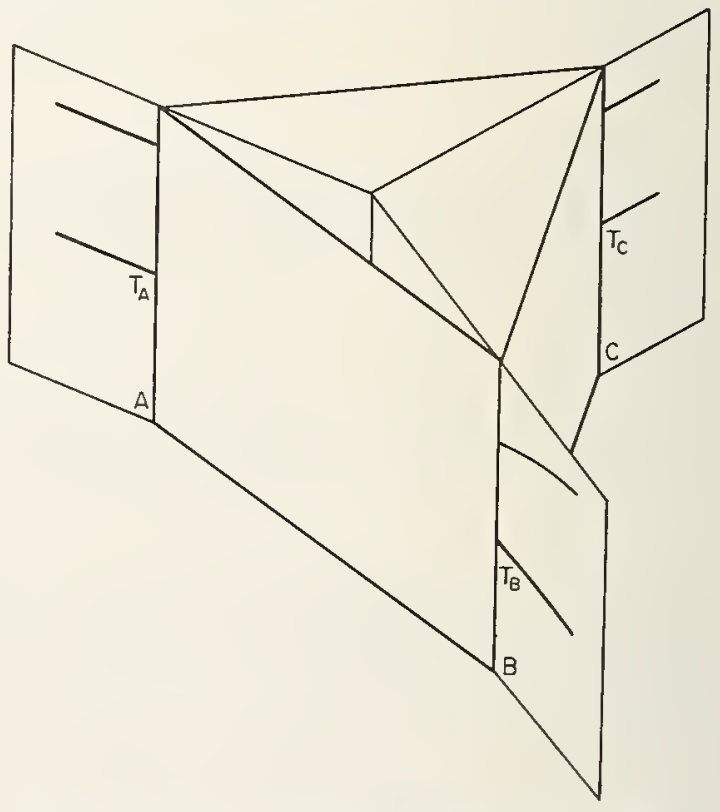

F1GURE 20. Schematic derivation of the threecomponent system, $A-B-C$, showing the three-dimensional relationships between components at constant pressure.

$T_{\mathrm{A}}, T_{\mathrm{B}}$, and $T_{\mathrm{C}}-$ melting points of components A, $B$, and $C$, respectively, at constant pressure. 
model, more clearly shown in figure $21 \mathrm{~A}$, consists of three planes, each representing a limiting boundary binary system at constant pressure, which intersect at $60 \mathrm{deg}$. Temperatures are indicated by the vertical axis. Concentration of each end member can be located by the coordinate system shown by the equilateral triangle projected below the solid figure. The apex and corresponding base of the triangle represent 100 percent and 0 percent, respectively, of the designated component. Point 4 , for example, would have an overall composition of 40 percent $A, 40$ percent $B$, and 20 percent $\mathrm{C}$.

As described before, the melting point of each component in binary combination will be depressed to form a eutectic. Figure 21B indicates the liquidus curves for the three binary systems with points 1, 2, and 3 locating the binary eutectic compositions and temperatures. The addition of the third component to a binary combination will cause further depression of melting points, thus forming a series of curved liquidus surfaces meeting at a common ternary eutectic point. The complete ternary model is given in figure $21 \mathrm{C}$. Projection of the various important points to the base of the solid model results in the two dimensional plane triangle given below the triangular prism. Point 4 is the ternary eutectic and as before points 1,2, and 3, the binary eutectics. Arrows indicate the direction of falling temperatures. Although not shown, temperatures on the liquidus surface can be indicated by contour lines similar to thase designating elevations on topographic maps. The entire liquidus surface can be seen as a group of individual surfaces, each indicating a primary phase field. Upon cooling from a melt the primary phase would be the first solid phase to

(A)
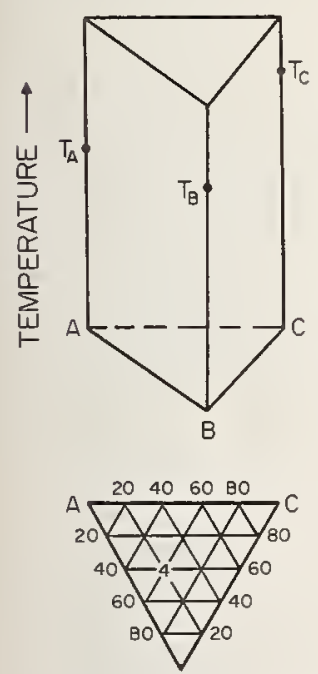

B
(B)
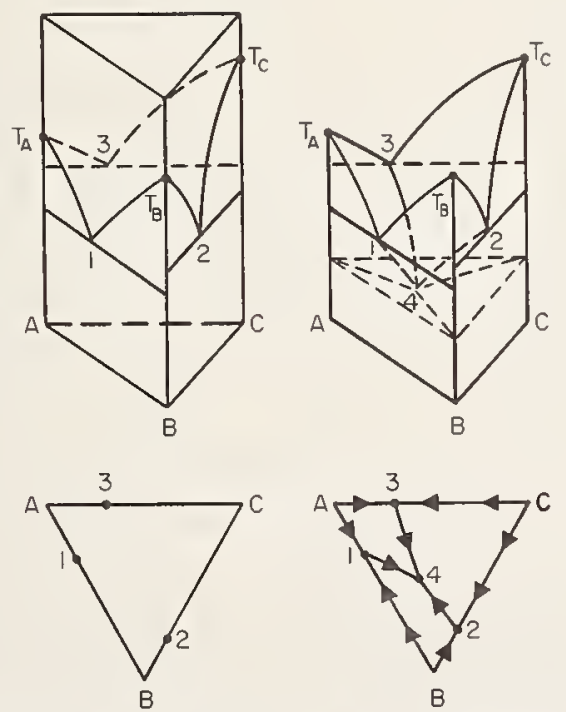

FIGURE 21. Schematic representation of the three-component system, $A-B-C$, in various stages of development. Upper figures indicate three-dimensional models; lower, the projection of important points on base.

$T_{\mathrm{A}}, T_{\mathrm{B}}$, and $T_{\mathrm{C}}-$ melting points of components $\mathrm{A}, \mathrm{B}$, and $\mathrm{C}$. Numerals $(1,2,3$, and 4$)$-eutectic temperatures and compositions. Curves $1-4,3-4$, and 2-4-boundary curves separating primary phase areas. Arrows-indicate direction of falling temperatures. crystallize. The intersection of the individual surfaces form a series of boundary curves 1-4, $2-4$, and $3-4$. The projection sbows clearly which primary phase is in equilibrium with the melt along the liquidus surface. Phase $\mathrm{A}$, for illustration, will be in equilibrium with liquid for those compositions in area A-1-4-3. Along univariant curve 1-4, phases A, B, and liquid will coexist. Likewise, curves $3-4$ and 2-4 separate primary phases $\mathrm{A}$ and $\mathrm{C}$, and $\mathrm{B}$ and $\mathrm{C}$, respectively. At point 4 (invariant) all three components plus liquid will comprise the equilibrium assemblage.

As a consequence of Raoult's law, congruently melting compounds will always melt at temperatures higher than those of adjacent compositions (excluding some solid solutions). Thus, the liquidus surface will appear as a dome or mountain with the peak occurring at the composition of the compound. On the other hand an incongruently melting compound will produce a terrace effect in the liquidus surface. A general guide to the behavior of ternary liquidus surfaces is given by the Alkemade theorem [6] which in essence states that the direction of falling temperatures on a boundary curve is always away from the straight line (Alkemade live) connecting the composition points of adjacent primary phase fields. The point of intersection of an Alkemade line and a boundary curve or its extension will be a maximum on that boundary curve. In figure $21 \mathrm{C}$ (lower triangle) line $A B$ (or $A C$ and $B C$ ) can be thought of as an Alkemade line. The intersection of $\mathrm{AB}$ with boundary curve 1-4 at point 1 is seen to be at a higher temperature than any cther point along 1-4. Similarly points 2 and 3 must represent the highest temperatures on boundary lines 2-4 and $3-4$, respectively.

Figure 22 (upper) gives six hypothetical systems which demonstrate typical situations encountered in ternary mixtures. The middle set of binary systems indicate the phase relations between the two base end members of the ternary system. The lower diagrams illustrate binary representations of segments or joins (dashed lines) through the ternary systems. With the exception of system $A$, the join (dashed line) is coincident with an Alkemade line. Temperatures are indicated on the tbree sets of diagrams by numerals.

The ternary system $A$, is a simple eutectic system similar to that developed for figure 21 . The binary system, A-B, exhibits elementary eutectic behavior while the join, $\mathrm{X}-\mathrm{C}$, does not. Line $\mathrm{X}-\mathrm{C}$ fails to meet the requirement of an Alkemade line in that composition $\mathrm{X}$ has no primary phase field. Consequently point 5 will not be a maximum along boundary curve 1-4 or along the extension of curve $2-4$.

The remainder of the ternary systems, B through $F$, are somewhat more complicated with each containing an intermediate binary or ternary compound. System B indicates a congruently melting compound $\mathrm{AB}$ and two ternary eutectics. In this case line $A B-C$ (Alkemade line) represents 

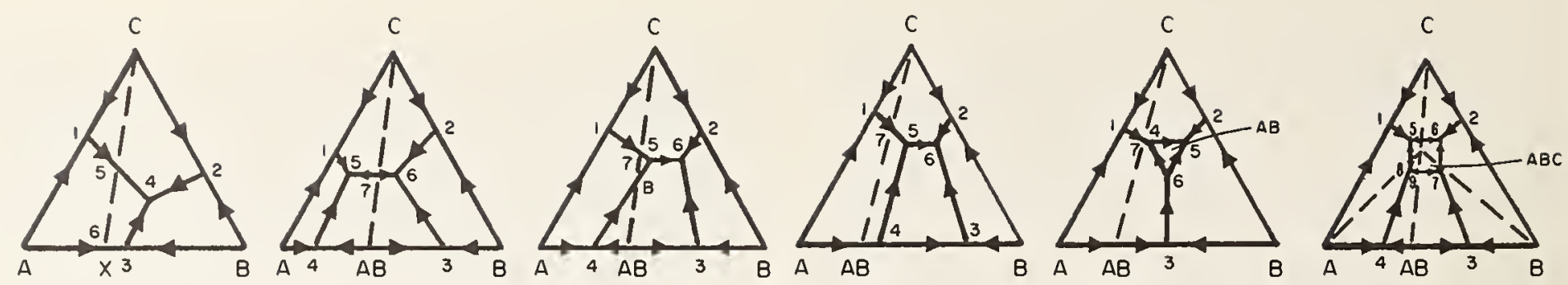

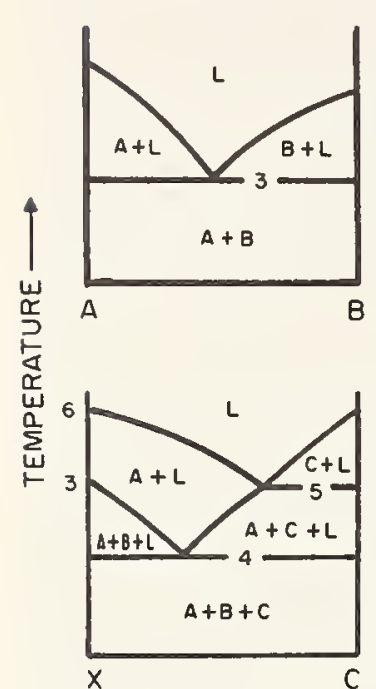

A
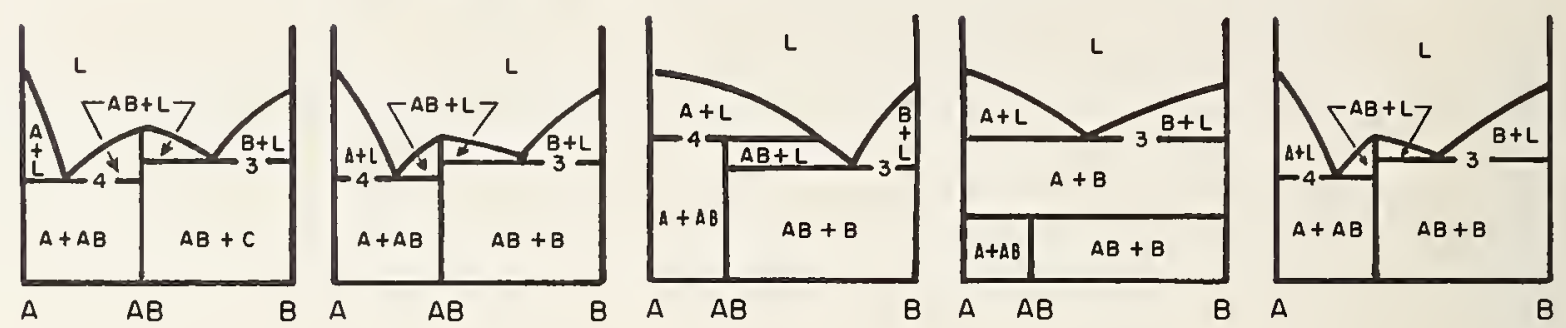

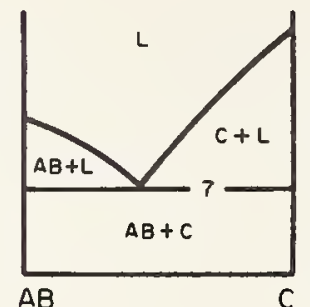

$B$

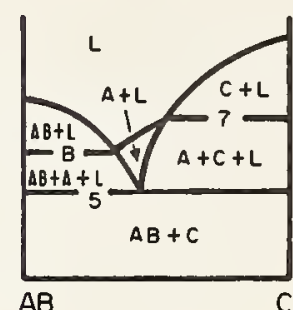

C

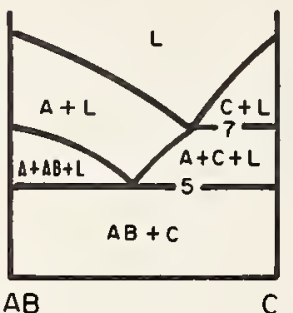

D

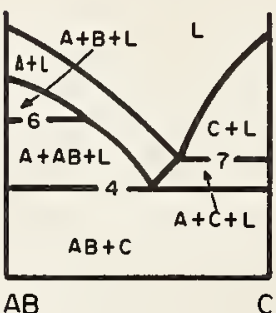

$E$

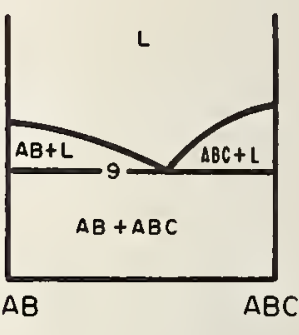

$\mathrm{F}$

Figure 22. Typical three-component systems of various complexity.

Upper-After Levin et al. [6]. Arrows indicate direction of falling temperatures.

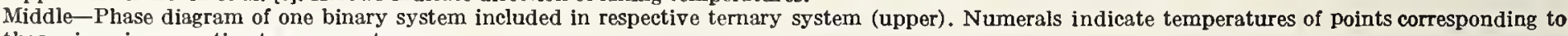
those given in respective ternary systems.

Lower-Binary representations of joins through ternary systems (dashed lines). Systems B and F (lower) are true binary systems.

a true binary subsystem and triangles $\mathrm{A}-\mathrm{AB}-\mathrm{C}$ and $\mathrm{AB}-\mathrm{C}-\mathrm{B}$ true subternaries. Each subsystem may be considered individually, independent of the overall larger system. The distinction here between true and pseudo system becomes important. A true system is one in which all primary phases formed in that system can be expressed in terms of the end member components. As illustrated by the binary diagram, $\mathrm{AB}-\mathrm{C}$ (figure $22 \mathrm{~B}$, lower), the primary phases are either $\mathrm{AB}$ or $\mathrm{C}$ and thus $\mathrm{AB}-\mathrm{C}$ is a true system.

Conversely, system C, figure 22, indicates a situation in which compound $\mathrm{AB}$ does not form a true subsystem with component $\mathrm{C}$. The diagram of the $\mathrm{AB}-\mathrm{C}$ join (Alkemade line) shows this clearly. Phase A which occurs throughout cannot be formed from any combination of $\mathrm{AB}$ and $\mathrm{C}$, thereby relegating system $\mathrm{AB}-\mathrm{C}$ to a pseudo binary status. Furthermore the $\mathrm{AB}-\mathrm{C}$ pseudo system yields three phase equilibrium at regions other than invariant points, a situation which is not allowed by the phase rule for two components. It should be noted that points 7 and 8 will not be temperature maxima of curves $1-5$ and $4-5$, respectively. This is due to the fact that the Alkemade theorem is applicable only to boundary curves of adjacent primary fields. The intersection of line $A B-C$ with the extension of the $C$ and $A B$ boundary curve, line 5-6 will be at a maximum. Thus, point 5 in the ternary system $\mathrm{C}$ represents a ternary peritectic and point 6 a ternary eutectic. System D, figure 22, illustrates an incongruently melting compound, $\mathrm{AB}$, with its primary phase field indicated by area 3-4-5-6. Again, point 5 is a peritectic and point 6 a eutectic. As before in system $C$, the subsystem $A B-C$ is not a true binary.

System E indicates a situation not at all uncommon for ceramic systems. The binary compound, $\mathrm{AB}$, decomposes before melting to solids $A$ and $B$. In the ternary system, however, $A B$ is seen to be the primary phase in equilibrium with liquid in field $4-5-6$. Both points 4 and 6 are peritectics while point 5 is a ternary eutectic. It is evident that the appearance of $\mathrm{AB}$ as primary phase in ternary mixtures offers interesting possibilities for single crystal growth not possible in the binary system, A-B, alone.

System F, figure 22, contains two intermediate congruently melting compounds, ABC (ternary) and $\mathrm{AB}$ (binary). The larger system, A-B-C, can be subdivided into four true ternary systems, $\mathrm{A}-\mathrm{ABC}-\mathrm{AB}, \mathrm{A}-\mathrm{ABC}-\mathrm{C}, \mathrm{ABC}-\mathrm{C}-\mathrm{B}$, and $\mathrm{ABC}-$ $\mathrm{B}-\mathrm{AB}$. Each sub-binary in turn is a true system. System AB-ABC (lower, figure 22), typical of each subsystem, illustrates only eutectic configuration.

Often it is difficult to visualize the distinct relationships which exist between various subregions of multicomponent systems. Levin et al. 
[6] have formulated a useful graph, reproduced in figure 23, which aids immensely in clarifying matters. For a complete discussion of the figure reference must be made to the original article. In essence the figure basically relates the variance and its geometrical representation to the number of solid phases (squares) in equilibrium with liquid for a given number of components in a condensed system. As a simple example, the figure indicates that at a eutectic point in a condensed ternary system, three solid phases and liquid will be in equilibrium. Similarly the liquidus surface of a ternary is a divariant situation in which one solid phase and liquid are in equilibrium. The vertical arrows and numbers in parentheses indicate the interrelationships between regions of any given system. For illustration, in a ternary system two divariant surfaces will intersect to form an univariant curve. Three univariant curves in turn will intersect to form an invariant point (eutectic) at which three solid phases are in equilibrium with liquid. Finally, the diagonal arrows relate the origin or terminus of regions at bourdary systems. For instance, a univariant curve in a ternary system will originate (or terminate) at an invariant point of a binary boundary system. In essence the region loses one degree of freedom because the boundary system always has a lesser number of components.

\begin{tabular}{|c|c|c|c|c|c|c|}
\hline \multirow{2}{*}{$\begin{array}{l}\text { Degrees } \\
\text { af } \\
\text { Freedom }\end{array}$} & \multirow{2}{*}{$\begin{array}{c}\text { Geometrical } \\
\text { Representa - } \\
\text { tion }\end{array}$} & \multicolumn{5}{|c|}{$\begin{array}{l}\text { NUMBER OF SOLID PHASES IN EQUILIBRIUM } \\
\text { WITH LIQUID IN SYSTEMS: }\end{array}$} \\
\hline & & Binory & Ternary & Quoter- & Quinary & $n$ \\
\hline $\begin{array}{c}0 \\
\text { Invariant }\end{array}$ & Point & 2 & 3 & 4 & 5 & $n$ \\
\hline $\begin{array}{c}1 \\
\text { Univoriont }\end{array}$ & Curve & 1 & 2 & 3 & 4 & $n-1$ \\
\hline $\begin{array}{c}2 \\
\text { Divariont }\end{array}$ & Surfoce & 0 & 1 & 2 & 3 & $n-2$ \\
\hline $\begin{array}{c}3 \\
\text { Trivoriont }\end{array}$ & Volume & & 0 & 1 & 2 & $n-3$ \\
\hline $\begin{array}{c}4 \\
\text { Tetrovoriont }\end{array}$ & $\begin{array}{c}\text { None } \\
\text { (4 dimen- } \\
\text { sional) }\end{array}$ & & & 0 & 1 & $n-4$ \\
\hline $\begin{array}{c}5 \\
\text { Pentavoriont }\end{array}$ & $\begin{array}{c}\text { None } \\
(5 \text { dirnen - } \\
\text { sionol) }\end{array}$ & & & & 0 & $n-5$ \\
\hline $\begin{array}{l}F+P= \\
C+1=\end{array}$ & & 3 & 4 & 5 & 6 & $n+1$ \\
\hline
\end{tabular}

FIGURE 23. The interrelationships of subregions of multicomponent condensed systems at constant pressure. After Levin et al. [6].

\section{Methods}

\subsection{Dynamic Methods}

Tradit: onally, the thermal analysis method has been widely accepted as one of the more desirable procedures in establishing the melting or freezing point of a material. All first order transitions such as the solid to liquid and solid I to solid II transformations, produce an energy change which is reflected by a corresponding change in the heat content of the system. Heat will be absorbed from the surrounding areas upon heating, and conversely, will be evolved during cooling. At the transformation point, simultaneously with the heat change, there will also be a discontinuous change in a number of physical properties, as for example, thermal expansion or electrical resistivity. Basically the thermal analysis method utilizes these changes to detect the occurrence of the transition. Once detected, the problem resolves itself to one of the accurate measurement of the temperature at which these overt changes indicative of melting take place.

The thermal analysis method most commonly employed makes use of the change in heat content inherent in the solid to liquid transition. Generally, the experimental arrangement simply consists of a crucible containing the sample in which a temperature measuring device has been centrally located. A continuous record during controlled heat treatment of the specimen will result in a time-temperature plot commonly referred to as a heating or cooling curve (direct method). If no transition takes place during cooling the curve will be smooth and continuous similar to that shown by $A$, figure 24 . At the freezing point heat will be evolved thus causing an arrest or halt in the cooling process. For ideal situations the temperature of the specimen will remain constant until the last trace of liquid has solidified, at which point further cooling takes place. The freezing point will be reflected by that portion of the cooling curve which is flat and parallel to the time axis (B, figure 24). Usually, the solidification rate of most materials is slow enough to cause some supercooling, the end result being a cooling curve illustrated by $\mathrm{C}$, figure 24 . If the rate of cooling exceeds the rate of evolution of heat the true freezing point will not be indicated by the

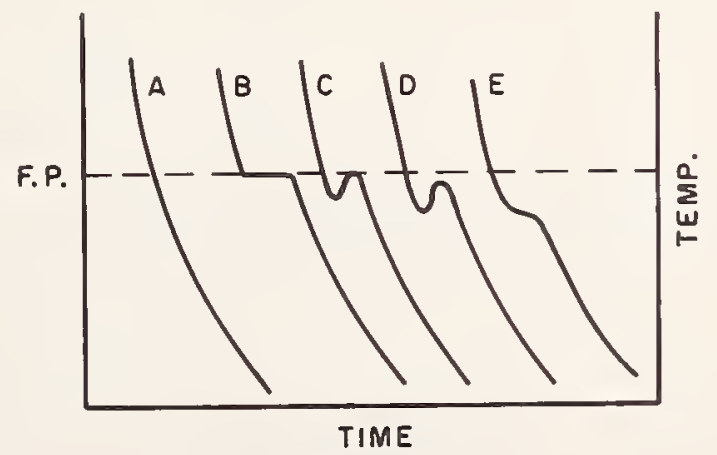

Figure 24. Typical thermal analyses cooling curves. Essentially after Weber [36].

A-no transition,

B-theoretical cooling curve with freezing arrest, C-cooling curve illustrating supercooling,

$\mathrm{D}$ and $\mathrm{E}-\mathrm{imperfect}$ cooling curves which give false indication of freezing point. 
cooling curve. Curves D and E, figure 24 exemplify this type of behavior with both curves yielding a freezing point less than the true value.

Freezing and melting points are numerically equal, the terminology being merely a reflection of the experimental approach to the solid-liquid transition. Heating curves generally are less well defined than the corresponding cooling curves. A cooling liquid is more likely to possess a uniform temperature because of greater thermal conductivity throughout the melt. Furthermore on heating, small variations in power supplied to the furnace will cause abnormalities in the heating curve not related to melting. Supercooling, especially for ceramics, can be troublesome in utilizing cooling curves. On the other hand, superheating does not occur, at least readily, thus making the heating curve method more desirable in this respect. The choice between a heating or cooling curve as a means of establishing the transition point is strictly material dependent. Figures 25 [33] and 26 [34] give examples of experimental determinations of the freezing points of gold and several ceramics. Figure 27 [35] shows both the heating and cooling curve obtained for alumina $\left(\mathrm{Al}_{2} \mathrm{O}_{3}\right)$. Here supercooling is so pronounced that the observed freezing point of $\mathrm{Al}_{2} \mathrm{O}_{3}$ is some 200 degrees less than the observed melting point.

The thermal analysis method, of course, is not limited to the study of pure materials. It is equally adaptable to binary and other multicomponent systems. Figure 28A, curves I and II, give illustrations of cooling curves for a pure component and a mixture. Line A-B, curve I, represents the freezing temperatures for the pure component while the inflections $\mathrm{C}$ and $\mathrm{D}$ indicate the liquidus and solidus temperatures, respectively, for the mixture. The temperature interval between $\mathrm{C}$ and $\mathrm{D}$ indicates the region in which both the solid pure component and liquid containing the mixture are in equilibrium. Obviously, it is possible to construct a phase diagram from thermal analysis data

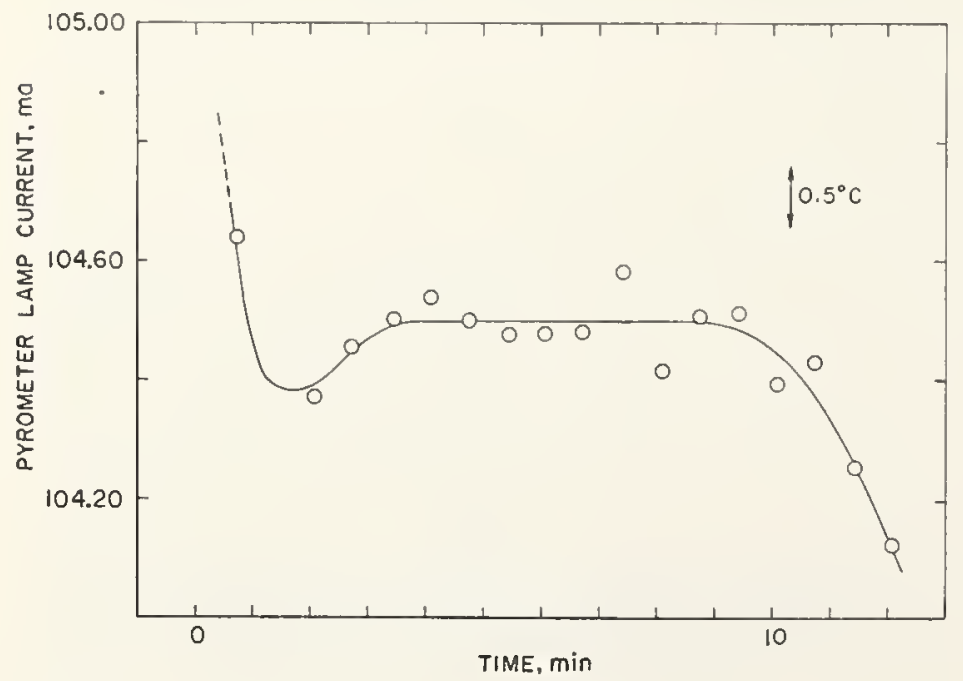

Figure 25. Cooling curve of gold. After Kostkowski and Lee [33].

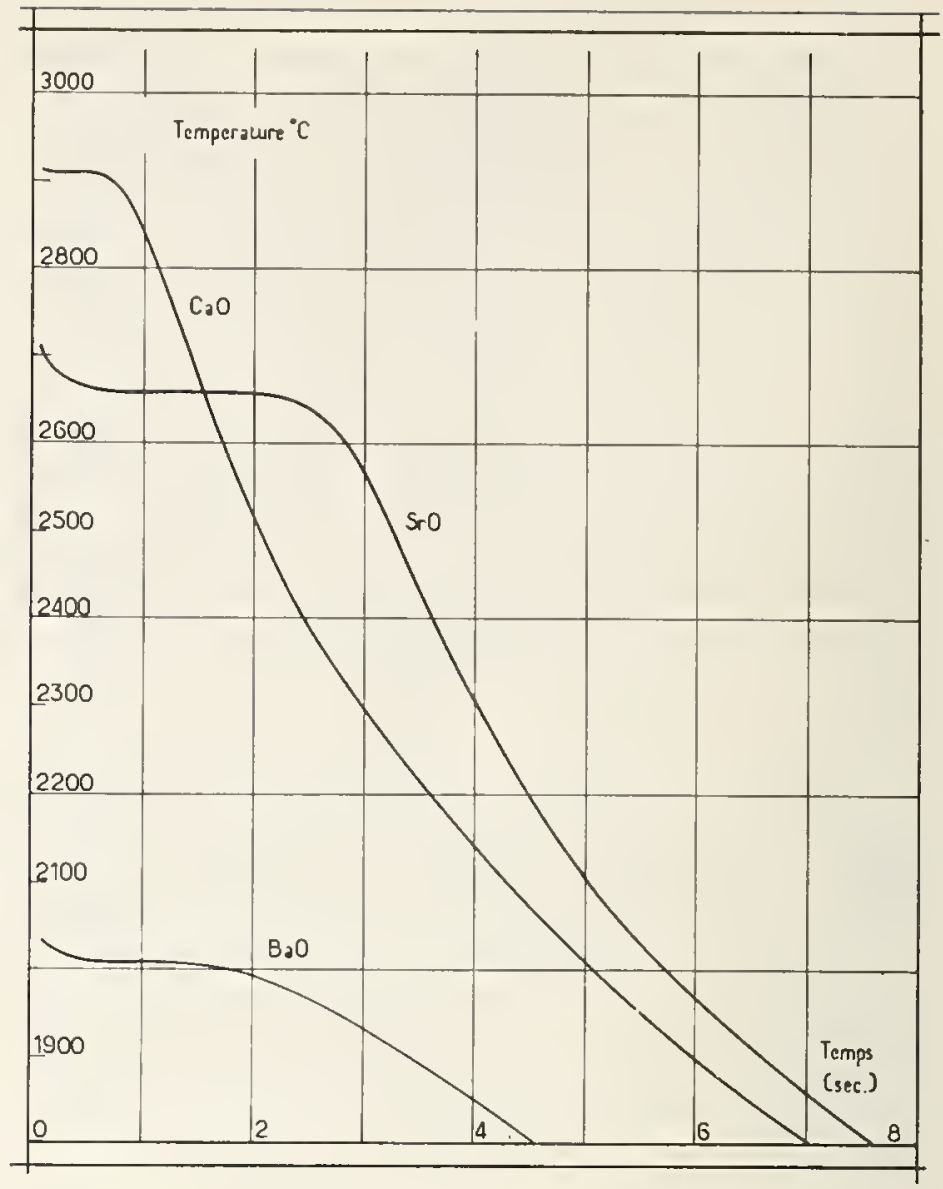

Figdre 26. Cooling curves of several ceramics. After Foex [34].

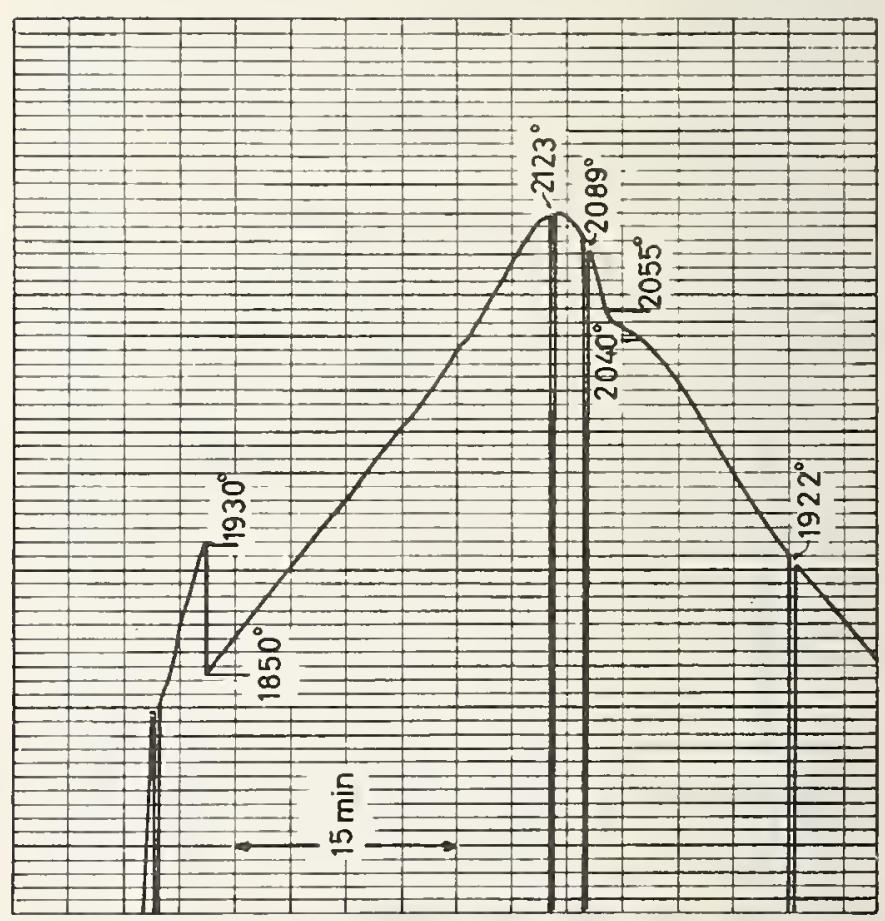

Frgure 27. Heating and cooling curves of $\mathrm{Al}_{2} \mathrm{O}_{3}$. After Gitlesen and Motzfeld [35].

Heating curve begins at right axis; cooling curve begins at maximum temperature. Sharp vertical breaks in curve are due to pyrometer measurements. 

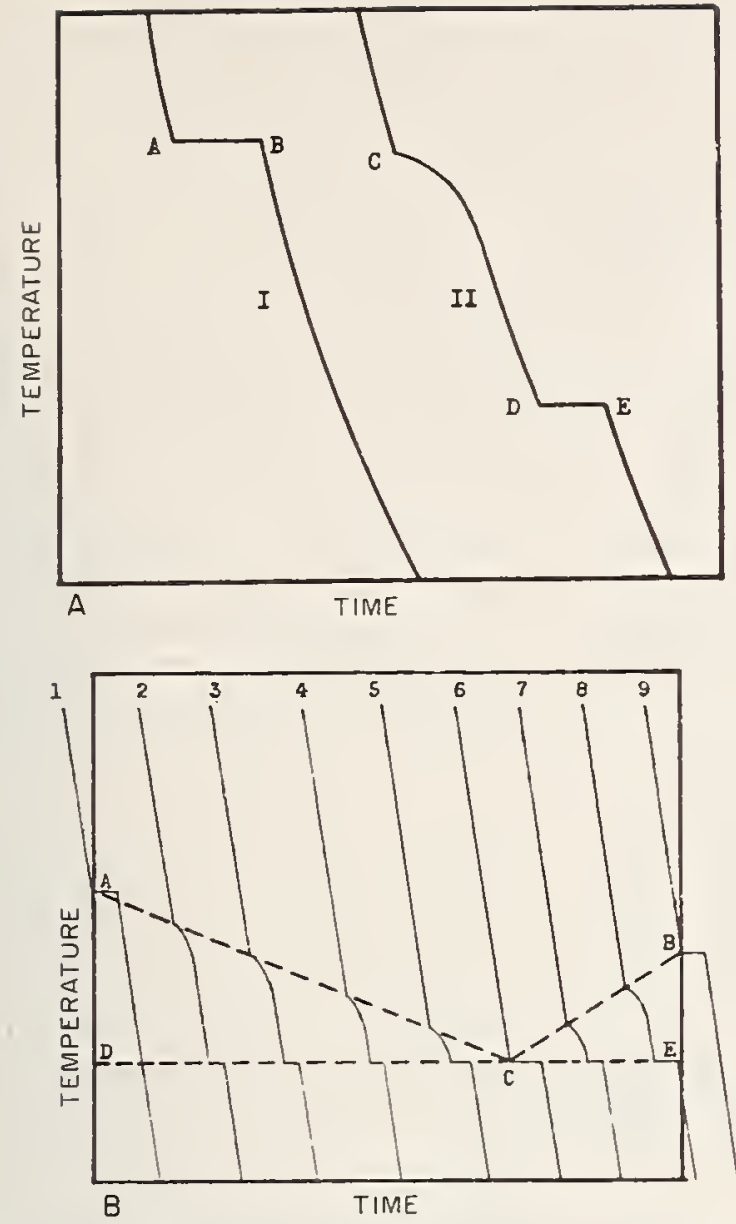

FIGURE 28. Typical cooling curves as applied to a binary mixture. After McNamara [42].

A-Curve I-pure component; Curve II-a mix ture, B-relationship between cooling curves and $\mathrm{a}$ binary phase diagram. Curves 1 and 9-pure components; Curves 2 through $8-$ mixtures. Time axis and composition axis are superimposed.

on various compositional increments. The relationship between these data and the phase diagram for a simple eutectic system is given by figure $28 \mathrm{~B}$. Curves 1 and 9 indicate the cooling behavior for the end members while curves 2 through 8 represent similar data for intermediate compositions.

Normally the heat of fusion (or crystallization) is not large in comparison with the total heat content of the system; consequently, the melting or freezing arrest usually is not very pronounced. To alleviate this problem, various alternate methods have been devised to enhance the inflection points. In all, there are four principal methods which are used to obtain thermal data $[36,37]$ :

1. Direct (heating and cooling curves),

2. Inverse rate,

3. Differential (D.T.A.),

4. Derived differential.

The direct and inverse rate procedures differ only in the method which is used to record the data. The same experimental apparatus is appropriate for both. The inverse rate method consists of determining the time required for the specimen to change a definite temperature interval, which in effect, amounts to differentiating the temperature-time curve (direct method) with respect to temperature. Methods 3 and 4 require the same experimental setup and, as before for methods 1 and 2, differ only in the way the data is collected. The differential method records the difference in temperature between the specimen and an inert body which undergoes no transformation. These differences are plotted against either the temperature of the specimen or that of the furnace cavity. The derived-differential method (4) consists of differentiating the differential curve with respect to temperature. Typical examples [36] of the four types of plots are given in figure 29. Each indicates the relative sensitivities in establishing the same transition point.

Various other dynamic methods have been devised for melting point determinations [37, 38, $39,40,41]$. Although these methods differ somewhat in detail, conceptually, they all involve visual observation of a specimen during heat treatment. The temperature at which a specimen is seen to fuse is taken as the melting point. Here, the measurement of temperature becomes the major obstacle in obtaining an accurate value. The direct thermal analysis method is generally more conducive to accurate temperature measurement in that a blackbody can be built into the experimental setup, thereby permitting a realistic assessment of true temperatures. The other dynamic methods depend on the ability of an observer to distinguish a specimen from surrounding areas to detect melting. This requirement is not compatible with the achievement of the

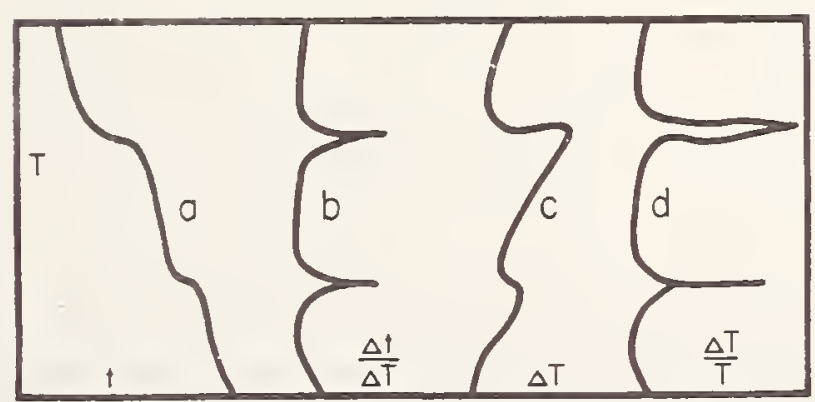

Figure 29. Four methods of thermal analysis. After Weber [36]. a-direct b-inverse rate c-differential (D.T.A.) d-derived differential $T=$ temperature $t=$ time 
blackbody conditions as a specimen located in a blackbody enclosure for all practical purposes becomes invisible. The obvious recourse has been to allow the necessary deviation from perfect blackness to see the specimen and to incorporate a temperature correction factor obtained from emissivity data or from in situ calibration. Published emissivity data as well as some calibration procedures, however, are not always reliable and are generally not applicable to specific experiments.

\subsection{Static Methods}

The quenching method $[6,42,43,44]$ is the one procedure specifically developed for study of ceramic cystems. The method relies upon the fact that many reactions in ceramics are sluggish and may be arrested or "frozen in" by rapid cooling or quenching. In general, a small homogeneous charge is placed in a container which in turn is heated at a constant temperature until equilibrium is attained. The specimen is then quenched by dropping the container into a cooled liquid or alternatively, by rapidly cooling the furnace. Examination of the cooled specimen by various means reveals the degree of melting. If the specimen shows no signs of melting, additional heat treatments are performed at successively higher temperatures. After a series of heatings the melting point can be established as that temperature midway between the maximum temperature at which no melting occurred and the minimum temperature where fusion was complete. Quite obviously the success of the method depends upon the ability of the examiner to establish the degree of melting the specimen had undergone. For glass-forming materials evidence of fusion can be detected by microscopic techniques which will identify the presence of liquid by the appearance of isotropic material (glass). For nonglass formers the problem of liquid detection becomes more formidable. Physical appearance of the cooled specimen is a useful guide. A sample which had lost its original shape and flowed to surrounding areas can, of course, be considered completely melted. Unfortunately, many ceramics when melted tend to form viscous liquids and consequently little slumping or flow occurs. In these cases the problem is minimized if single crystals are used as test specimens [45]. Unless melted, the single crystal will retain its identity, that is, remain single. If fusion occurs the cooled specimen will appear as a polycrystalline mass which is readily identifiable.

\section{Temperature Measurement}

\subsection{Temperature Scales}

Perhaps the greatest difficulty inherent in the determination of a melting point is the accurate measurement of temperature. The thermodynamic temperature scale [46] is the fundamental scale, to which all temperatures should be referred. The scale is based on the second law of thermodynamics which allows complete definition by fixing one thermodynamic temperature. At present this temperature is designated as exactly 273.16 $K$, the triple point of water [46]. Because of experimental problems associated with the measurement of temperatures on the thermodynamic scale (gas thermometry), a practical temperature scale was established in 1927 by international agreement [47]. A major revision of the 1927 scale was made in 1948 [48] with subsequent text revision in 1960 [46]. Further changes in this scale are anticipated to make it conform more closely to the thermodynamic scale. The International Practical Temperature Scale of 1948 (IPTS) [46], the scale now in use, ${ }^{3}$ in essence represents a realistic experimental approach to the ideal thermodynamic scale.

Currently six points define IPTS between the lowest assigned value, the oxygen point $(-182.97$ $\left.{ }^{\circ} \mathrm{C}\right)$ and the highest, the gold point $\left(1063{ }^{\circ} \mathrm{C}\right)$. Certain thermometric instruments and formulas are specified as a means to realize these fixed points and intermediate temperatures. Above the gold point, temperatures are based upon the Planck relationship:

$$
\frac{J_{\ell}}{J_{\mathrm{Au}}}=\frac{\exp \left[\frac{C_{2}}{\lambda\left(t_{\mathrm{Au}}+T_{0}\right)}\right]-1}{\exp \left[\frac{C_{2}}{\lambda\left(t+T_{0}\right)}\right]-1}
$$

where,

$J_{\mathrm{Au}}$ and $J_{t}=$ radiant energies per unit wavelength emitted by unit area of a blackbody at temperature $t$ and the gold point ${ }_{\mathrm{Au}}\left(1063^{\circ} \mathrm{C}\right)$,

$\lambda=$ wavelength,

$C_{2}=$ second radiation constant (1.438 cm deg),

$T_{0}=273.15 \mathrm{deg}$.

Although no instrument of measurement is designated by IPTS for temperatures above $1063^{\circ} \mathrm{C}$, the optical pyrometer has been traditionally utilized for this purpose. In addition to the six primary points IPTS recommends certain other points which may be used for calibration purposes. Table 1 summarizes important features of the practical scale.

\subsection{Instruments}

Since IPTS specifies certain instruments it is generally advisable to limit their use to the designated temperature ranges. This is not always possible, however, and frequently the investigator must extend the range of the instrument beyond that stated by IPTS. Of prime importance to the study of ceramics are the (1) thermoelectric, and (2) radiation pyrometers. The theory, use, and calibration of these temperature measuring de-

${ }^{3}$ Recently a new scale (IPTS 1968) was adopted, official text of which will
appear in Metrologia, April 1969. 
TABLE 1. Fixed and secondary points on the International Practical Temperature Scale of $1948^{\prime}$ [46].

\begin{tabular}{|c|c|c|c|}
\hline \multirow{2}{*}{ Material } & \multirow{2}{*}{ Property ${ }^{2}$} & \multicolumn{2}{|c|}{ Temperature, ${ }^{\circ} \mathrm{C}$. } \\
\hline & & $\begin{array}{l}\text { Primary } \\
\text { point }\end{array}$ & $\begin{array}{l}\text { Secondary } \\
\text { point }\end{array}$ \\
\hline 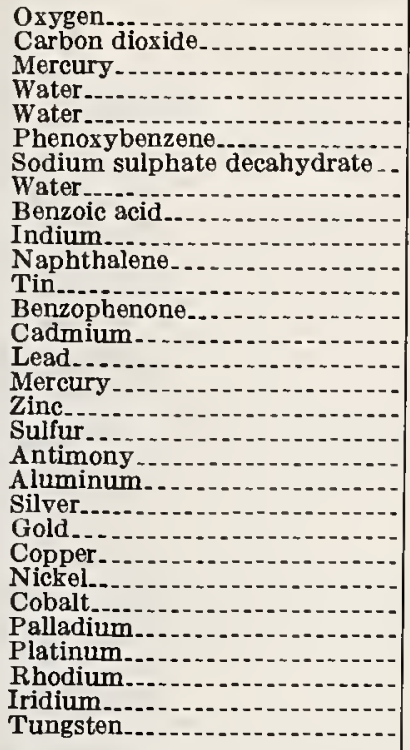 & $\begin{array}{l}\text { B.P. } \\
\text { S.P. } \\
\text { F.P. } \\
\text { F.P. } \\
\text { T.P. } \\
\text { T.P.P. } \\
\text { B.P. } \\
\text { T.P. } \\
\text { F.P. } \\
\text { B.P. } \\
\text { F.P. } \\
\text { B.P. } \\
\text { F.P. } \\
\text { F.P. } \\
\text { B.P. } \\
\text { F.P. } \\
\text { B.P. } \\
\text { F.P. } \\
\text { F.P. } \\
\text { F.P. } \\
\text { F.P. } \\
\text { F.P. } \\
\text { F.P. } \\
\text { F.P. } \\
\text { F.P. } \\
\text { F.P. } \\
\text { F.P. } \\
\text { F.P. } \\
\text { M.P. }\end{array}$ & 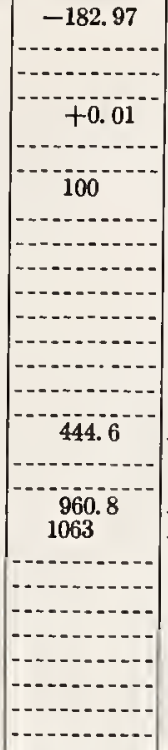 & \begin{tabular}{|c|}
-78.5 \\
-38.87 \\
0.000 \\
\hdashline 26.88 \\
32.38 \\
122.36 \\
156.61 \\
218.0 \\
231.91 \\
305.9 \\
321.03 \\
327.3 \\
356.58 \\
419.505 \\
--630.5 \\
660.1 \\
-1083 \\
-1453 \\
1492 \\
1552 \\
1769 \\
1960 \\
2443 \\
3380
\end{tabular} \\
\hline
\end{tabular}

1 Certain instruments of measurement are specified as a means to realize the scale between different fixed points. These are: -182.97 to $630.5^{\circ} \mathrm{C}$, platinum resistance thermometer; 630.5 to $1053^{\circ} \mathrm{C}$, platinum versus platinum$10 \%$ rhodium thermocouple; $1063{ }^{\circ} \mathrm{C}$ and above, no designated instrument although an optical pyrometer is generally utilized.

${ }_{2}$ The symbols B.P., S.P., T.P., F.P., M.P., and Tr.P. refer to boiling
. point, sublimation point, triple point, freezing point, melting point, and transition point, respectively. All points except the triple points applicable when determined under one standard atmosphere. The copper point requires a reducing environment.

vices have been well documented [33, 36, 49, $50,51,52,53,54]$.

Thermoelectric pyrometers, thermocouples, are, of course, the mainstay of the ceramic industry. Quite simply, thermocouples consist of two dissimilar electrical conductors joined together at their ends. A temperature difference between the two junctions will produce an emf which is characteristic of the type of material and of the magnitude of the temperature gradient. Calibration of a particular thermocouple at several fixed and secondary reference points will yield an emf versus temperature plot generally in the form of

where,

$$
E=a+b \Delta t+c(\Delta t)^{2}
$$

$$
\begin{aligned}
E & =\text { emf, } \\
\Delta t & =\text { temperature of hot junction minus } \\
& \text { temperature of cold junction, }
\end{aligned}
$$

$a, b$ and $c=$ constants.

The types of thermocouples available are many and varied [50, 52, 53]. Generally it is advisable for the thermocouple to have as large a thermoelectric power $\left(\frac{d E}{d t}\right)$ as possible. Resistance to deterioration by oxidation or reduction is also of great importance. The melting point of the lowest melting member will set the maximum temperature of use. However, other factors generally limit its utility to lower values.

Radiation pyrometry can be divided into two categories: (a) total, and (b) spectral. Total radiation pyrometry employs radiation from a large part of the spectrum according to the StefanBoltzmann relationship:

$$
J=e_{\mathrm{T}} \sigma T
$$

where,

$$
\begin{aligned}
& J=\text { total radiant flux per unit area per second, } \\
& T=\text { temperature }(\mathrm{K}), \\
& e_{\mathbf{T}}=\text { total emissivity, } \\
& \sigma=\text { Stefan-Boltzmann constant. }
\end{aligned}
$$

The radiation pyrometer in effect collects the energy emitted by a definite area of a hot body within a limited solid angle and converts that energy to some measurable quantity such as emf. The emf or other deflection, $D$, of the indicating instrument is related to temperature by:

$$
D=c\left(T^{4}-T_{0}^{4}\right)
$$

or

$$
D \approx c T^{n}\left(\text { when } T>>T_{0}\right)
$$

where,

$$
\begin{aligned}
D & =\text { deflection of indicating instrument, } \\
T & =\text { blackbody temperature }(\mathrm{K}), \\
T_{0} & =\text { pyrometer reference temperature }(\mathrm{K}), \\
c & =\text { empirical constant, } \\
n & =\text { exponent, approximately equal to } 4 .
\end{aligned}
$$

For a nonblackbody the total radiation pyrometer will indicate temperatures lower than the actual target temperatures. Observed temperatures are related to the true temperature by:

$$
T_{0}=T_{t} \sqrt[4]{e_{t}}
$$

where,

$$
\begin{aligned}
& T_{0}=\text { observed temperature }(\mathrm{K}) \\
& T_{t}=\text { true temperature }(\mathrm{K}), \\
& e_{t}=\text { total emissivity. }
\end{aligned}
$$

Spectral pyrometry uses only a narrow region of the spectrum, traditionally about $0.65 \mu \mathrm{m}$. The spectral pyrometer compares the intensity of filtered radiation emitted from a target with that of a comparison source of known but variable temperature. Since its inception, this type of pyrometer has utilized the human eye as the means to compare the two intensities and thus has become known as an optical pryrometer. Of late, however, a photoelectric tube has been used for this purpose $[45,51]$. The comparison source is generally a lamp filament contained within the pyrometer. By varying the current of the lamp filament its intensity can be adjusted until a match in brightness is obtained with the target. 
Calibration of the pyrometer against targets of known temperatures gives an equation:

$$
T=a+b I+c I^{2}+d I^{3}
$$

where,

$$
\begin{aligned}
T & =\text { blackbody temperature }\left({ }^{\circ} \mathrm{C}\right), \\
I & =\text { pyrometer lampcurrent, } \\
a, b, c \text { and } d & =\text { empirical constants. }
\end{aligned}
$$

In spectral radiation pyrometry the measuring instrument determines a brightness temperature, not the true temperature of an incandescent body. Using Wien's law (an approximation) the relationship between these two temperatures is given by:

$$
\frac{1}{T_{t}}-\frac{1}{T_{0}}=\frac{\lambda_{e}}{C_{2}} \ln e_{\lambda}
$$

where,

$T_{t}=$ true temperature $(\mathrm{K})$,

$T_{0}=$ observed (brightness) temperature $(\mathrm{K})$,

$\lambda_{6}=$ effective wavelength,

$\mathrm{C}_{2}=$ second radiation constant,

$e_{\lambda}=$ spectral emissivity.

\subsection{Blackbodies}

Radiation pyrometry plays a prominent role in the determination of the melting point of ceramics primarily because of the high temperatures involved. The theory behind this type of pyrometry is well established and easy to utilize. Equations 13 and 15 of the previous section relate the true temperature $\left(T_{t}\right)$ of a body with the observed temperature $\left(T_{0}\right)$ as monitored by a total and a spectral pyrometer, respectively. Quite obviously, for a given wavelength band or effective wavelength, the difference between $T_{t}$ and $T_{0}$ may be rather large, depending upon the emissivity. For example, if the true temperature of a material having a total or a spectral emissivity of 0.5 is $2000{ }^{\circ} \mathrm{C}$, an optical pyrometer would indicate about $1880^{\circ} \mathrm{C}$ or $120^{\circ} \mathrm{C}$ too low. Similarly, for the same situation, a total radiation pyrometer would read a temperature of about $1640^{\circ} \mathrm{C}$ or be in error about $360^{\circ} \mathrm{C}$.

Emissivity is known but for a few materials; thus, a real problem exists in the accurate measurement of temperatures by radiation methods. The only practical solution available is to incorporate a klackbody into the temperature measuring system. By definition, a blackbody is a substance or object which absorbs all incident radiation, and reflects or transmits none. A blackbody is also a perfect radiator, emitting the maximum possible energy as the result of temperature alone. Consequently, the emissivity of a perfect blackbody is equal to unity. It is evident, then that if a radiation pyrometer is sighted on a blackbody the observed temperature would necessarily be equal to the true temperature.

Fortunately, experimental blackbodies can be constructed so that their effective emittances are sufficiently close to unity as to make errors in temperature measurement small. An experimental blackbody generally consists of a uniformly heated opaque enclosure containing a small aperture. The enclosure may be any shape but usually is in the form of a sphere, cylinder, or closed end tube. Providing isothermal conditions exist, radiation within the enclosure, after numerous reflections, emerges through the hole as nearly black. All interior details of the enclosure are indistinguishable. The smaller the area of the aperture in comparison with the total internal area of the enclosure, the greater the emittance. The emittance of any enclosure supposedly built as a blackbody is extremely dependent upon the nature of the internal reflections. Even enclosures in which the geometric design is correct, can have low emittances if the internal reflections are specular rather than diffuse. A roughened internal surface aids in this respect.

A perfect blackbody, like a perfect gas, represents an ideal situation unobtainable in practice. Therefore, an estimate must be made of the quality of the experimental blackbody. Excellent papers by several authors $[55,56,57,58]$ give methods of evaluating the effective emittances of blackbodies having different configurations. It should be emphasized that these estimates can be valid only for enclosures which are uniformly heated. If thermal gradients exist along any enclosure there is little possibility that its effective emittance can approach the theoretical limit of unity.

\section{Melting Point Standards}

Numerous ceramics have been utilized throughout the years as high temperature melting point standards. Materials such as $\mathrm{Al}_{2} \mathrm{O}_{3}, \mathrm{Cr}_{2} \mathrm{O}_{3}, \mathrm{MgO}$, $\mathrm{NiO}, \mathrm{CaMgSi}_{2} \mathrm{O}_{3}$ (diopside), $15 \% \mathrm{CaO}: 85 \% \mathrm{SiO}_{2}$, and $\mathrm{CaSiO}_{3}$ [59] all, at one time or another, have been used, often as fixed points on practical temperature scales [37]. These materials, however, only can be regarded as pseudostandards for there is no common agreement as to their exact melting point. There is such a wide divergence between published melting points for the same material [59] that the user more often than not selects a value that best conforms with his own experimental data. In reality, the only materials acceptable for use as high temperature standards are those having internationally recognized melting points such as given by the International Practical Temperature Scale. Unfortunately, the materials specified by IPTS which melt above the gold point are too few in number and generally not adaptable to a wide variety of experimental conditions. Furthermore, many of the melting points were established many years ago $[60,61,62,63]$ and thus a reevaluation might be in order. The need for additional secondary reference points is all too apparent. The metal oxides as well as other ceramics, indeed, warrant consideration for this purpose. 
Many thanks are due to Dr. T. Negas and other members of the phase equilibria group, High Temperature Chemistry Section, National Bureau of Standards, for their helpful discussions and suggestions which aided greatly in the preparation of the text.

\section{References}

[1] Bowden, S. T., Phase Rule and Phase Reactions (Macmillan and Co., London, 1938).

[2] Findlay, A., Campbell, A. N., and Smith, N. D., The Phase Rule and Its Application, 9th ed., 494 pp. (Dover Publs., Inc., New York, 1951).

[3] Darken, L. S., and Gurry, R. W., Physical Chemistry of Metals, 535 pp. (McGraw-Hill Book Co., New York, 1953).

[4] Gibbs, J. W., Trans. Conn. Acad. Sci. 3, 108-248, 343-525 (1874-78); also Gibbs, J. W., The Collected Works of J. Willard Gibbs 1, 54-371 (Longmans, Green and Co., New York, 1928).

[5] Knapp, W. J., J. Am. Ceram. Soc. 36, 43-47 (1953).

[6] Levin, E. M., Robbins, C. R., and McMurdie, H. F., Phase Diagrams for Ceramists, 601 pp. (Am. Ceram. Soc., Columbus, Ohio, 1964).

[7] Marsh, J. S., Principles of Phase Diagrams, 1st ed., 193 pp. (McGraw-Hill Book Co., New York, 1935).

[8j Morey, G. W., The Glass Industry 12, No. 4, 69-80 (1931).

[9] Newton, R. F., Science 79, 321-322 (1934).

[10] Ricci, J. E., The Phase Rule and Heterogeneous Equilibrium, 505 pp. (D. Van Nostrand Co., Inc., New York (1951).

[11] Slater, J. C., Introduction to Chemical Physies, 3-32, 166-182, 256-307 (McGraw-Hill Book Co., New York, 1939).

[12] Turnbull, D., Phase Changes, Solid State Physics, Advances in Research and Application, 225-306 (Academic Press Inc., New York, 1955).

[13] Wetmore, F. E. W., and Leroy, D. J.. Principles of Phase Equilibria, 1st ed., 200 pp. (McGraw-Hill Book Co., Inc., New York, 1951).

[14] Zernike, J., Chemical Phase Theory, N. V. UitceversMaatschappij, 494 pp. (Ae. E. Kluwer DeventerAntwerp-Djakarta, 1955).

[15] Osborn, E. F., and Muan, A., Phase Equilibrium Diagrams of Oxide Systems, Plate 1, Large Scale diagrams (Am. Ceram. Soc. and Edward Orton Ceram. Foundation, 1960).

[16] Lea, F. M., and Desch, C. H., The Chemistry of Cement and Concrete, 2nd ed., 52 (Edward Arnold and Co., London, 1956).

[17] Sosman, R. B., The Phases of Silica, 388 pp. (Rutgers University Press, New Brunswick, New Jersey, 1965).

[18] Ostrovsky, I. A., Geol. J. 5, No. 2, 321-328 (1967).

[19] Boyd, F. R., and England, J. L., J. Geophys. Res. 65, No. 2, 752 (1960).

[20] Fenner, C. N., Am. J. Sci., 4th Ser. 36, 338 (1913); and Greig, J. W., Am. J. Sci., 5th Ser. 13, 1-44 (1927).

[21] Ubbelohde, A. R., Melting and Crystal Structure (Clarendon Press, Oxford, 1965).

[22] Simon, F. E., and Glatzel, G., Z. Anorg. u. Allgem. Chem. 178, 309 (1929).

[23] Babb, Jr., S. E., Rev. Mod. Phys. 35, 400-413 (1963).

[24] Bundy, F. P., Science 137, 1057-1058 (1962).

[25] Pistorius, C. W. F. T., J. Phys. Chem. Solids 28, Pergamon Press, Great Britain, 1811-1819 (1967).

[26] Sarver, J. F., and Hummel. F. A., J. Am. Ceram. Soc. 43, 366 (1960).
[27] Levin E. M., and Roth, R. S., J. Res. NBS 68A (Phys. and Chem.) No. 2, 189-195 (1964).

[28] Aramaki, S., and Roy, R., J. Am. Ceram. Soc. 42, 644 (1959).

[29] DeVries, R. C., and Roy, R., Am. Ceram. Soc. Bull. 33, 370-372 (1954).

[30] Muan, A., Am. J. Sci. 256, 171-207 (1958).

[31] Darken, L. S., and Gurry, R. W., J. Am. Chem. Soc. 68, 798-816 (1946).

[32] Darken, L. S., and Gurry, R. W., J. Am. Chem. Soc. 67, 1398-1412 (1945).

[33] Kostkowski, H. J., and Lee, R. D., NBS Mono. 41 (1962).

[34] Foex, M., Solar Energy 9, 61-67 (1965).

[35] Gitlesen, G., and Motzfeldt, K., Rev. Hautes Tempér. et Réfract. 3, 343-350 (1966).

[36] Weber, R. L., Heat and Temperature Measurement (Prentice-Hall, Inc., New York, 1950).

[37] Foote, P. D., Fairchild, C. O., and Harrison, T. R., Tech. Pap. BS, 14, 1-326 (1920-1921) T170.

[38] Gutt, W., J. Sci. Instrum. 41, 393-394 (1964).

[39] Hill, V. G., Roy, R., and Osborn, E. F., J. Am. Ceram. Soc. 35, 135-142 (1952).

[40] Riley, B., Rev. Hautes Tempér. et Réfract. 3, $327-336$ (1966).

[41] Statton, W. D., J. Chem. Phys. 19, 33-40 (1951).

[42] McNamara, E. P., Ceramics II, 350 pp. (Penn. State College, State College, Penn., 1949).

[43] Rankin, G. A., and Wright, F. E., Am. J. Sci. 39, 4th Ser., 1-79 (1915).

44] Schneider, S. J., and Waring, J. L., J. Res. NBS 67A, (Phys. and Chem.) No. 1, 19-25 (1963).

45] Schneider, S. J., and McDaniel, C. L., J. Res. NBS 71A, (Phys. and Chem.) No. 4, 317-333 (1967).

[46] Stimson, H. F., J. Res. NBS 65A, (Phys. and Chem.) No. 3, 139-145 (1961).

[47] Burgess, G. K., BS J. Research 1, 635-640 (1928) RP22.

[48] Stimson, H. F., J. Res. NBS 42, 209-217 (1949) RP1962.

[49] Olsen, L. O., and Halpern, C., Bibliography of Temperature Measurement, NBS Mono. 27, Supplement 2 (1967).

[50] Margrave, J. L., Temperature Measurement, Physicochemical Measurements at High Temperatures, Edited by Bockris, J. O'M., White, J. L., Mackenzie, J. D., 6-46 (Butterworth Scientific Publ., 1959).

[51] Lee, R. D., Metrologia 2, No. 4, 150-162 (1966).

[52] Roeser, W. R., and Lonberger, S. T., NBS Circ. 590 (1958).

[53] Caldwell, F. R., NBS Mono. 40 (1962).

[54] Temperature, Its Measurement and Control in Science and Industry 3, Parts 1 and 2, Edited by C. M. Herzfeld (Reinhold Publ. Corp., New York 1962).

[55] Gouffé, A., Rev. Opt. 24, 1-10 (1945).

[56] DeVoss, J. C., Physica 20, 669-689 (1954).

[57] Quinn, T. J., Brit. J. Appl. Phys. 18, 1105-113 (1967).

[58] Sparrow, E. M., Albers, L. U., and Eckert, E. R. G., J. Heat Transfer, Trans. Amer. Soc. Mech. Engs. Series C, 84, 73-81 (1962).

[59] Schneider, S. J., NBS Mono. 68, 31 pp. (1963).

[60] Wensel T., and Roeser, W. F., BS J. Research 5, 1309-1318 (1930) RP258.

[61] Roeser, W. F., and Wensel, H. T., BS J. Research 12, 519-526 (1934) RP676.

[62] Roeser, W. F., Caldwell, F. R., and Wensel, H. T., BS J. Research 6, 1119-1129 (1931) RP326.

[63] Henning, F., and Wensel, H. T., BS J. Research 10, 809-827 (1933) RP568. 



\title{
Thermal Expansion of Ceramics
}

\author{
Richard K. Kirby \\ Institute for Basic Standards, \\ National Bureau of Standards, Washington, D.G. 20234
}

\begin{abstract}
Various types of coefficients of thermal expansion are defined and their application described. The theory of thermal expansion is also reviewed and several applications to ceramics are given. Variations of four general test methods are described with special emphasis placed on techniques of obtaining high accuracy. Included are three variations of the vitreous silica dilatometer, two types of interferometers, an optical comparator, and two types of x-ray diffractometers. The effect of several characterization parameters on the expansion of ceramics is discussed. These include crystalline and amorphous phases, fictive temperature of glassy phase, quantity of phases, grain size, orientation of anisotropic grains, microcracks, and stress.
\end{abstract}

Key Words: Anisotropic grains; ceramics; composites; glass-crystal system; interferometer; microcracks; optical comparator; stress; thermal expansion; vitreous-silica dilatometer; x-ray diffractometer.

\section{Introduction}

During the last twenty years over 200 papers have been published in the Journal of the American Ceramic Society that have contained information on thermal expansion [1-216] ${ }^{1}$. All but a few of these papers have presented experimental data obtained by the author(s), two have been review papers on thermal expansion [17 and 35], and several have described equipment for measuring thermal expansion $[16,37,81,85,130,138,139$, $150,156,168,169$, and 180]. This represents about 12 percent of all papers published by the Journal during this period. Of these papers about 30 percent have given data on crystalline oxides, 20 percent on glasses, 15 percent on multiphase systems, 15 percent on porcelains, enamels, etc., and about 6 percent on carbides, nitrides, and borides. Very little of this body of information, however, has been obtained on materials sufficiently characterized to be of usefulness beyond knowing the expansion of some particular specimens.

In general, thermal expansion is not greatly dependent upon the history and character of materials, the variation of coefficient of linear thermal expansion being about the same order as the coefficients themselves. This is in contrast with the behavior of transport properties and some mechanical properties where the variations reach several orders of magnitude. History, however, is of relative importance in its effect on thermal expansion of ceramics.

1 Figures in brackets indicate the literature references at the end of this paper.
History includes the method of preparation and thermal treatment and to a great extent determines the character of the material. Of the many parameters of character which affect the properties of ceramics, the following will sometimes have a significant effect on thermal expansion:

Chemical composition (1 percent level)

Crystalline and amorphous phases [32, 141, $185,197]$

Quantity and size of phases $[92,125,184$, 201]

Orientation of anisotropic grains (87, 165, 183]

Residual stress [55, 93, 198]

Fictive temperature of glassy phase $[6,83$, $94,155]$

Formation of cracks [113]

Formation of point defects

Chemistry of surface [205, 206, 207]

Those parameters which usually do not have a significant effect on thermal expansion include:

Density [83]

Grain size [65, 75, 165]

Porosity [59, 82, 165]

Stoichiometry of grains [196, 212]

Impurities $(<1$ percent level)

Dislocations

Grain boundaries

Topography of surface [60]

There are always exceptions to the above rule, however. For instance, in graphitic materials the grain size and porosity have very significant 
effects on the thermal expansion through the orientation of the anisotropic grains [217].

The general range of values of the coefficient of linear thermal expansion of ceramics is indicated in table 1. Compilations of thermal expansion can be found in five readily available sources [218-222]. Literature values for the expansion of ceramic materials vary on the average by about 10 percent but variations as large as 40 percent also appear. It is difficult to judge how much of this variation is due to experimental procedures and how much is due to history and character differences. An interesting interlaboratory study has been made on magnesium oxide [223], however, in which it was found that an average variation of about 5 percent was due to experimental procedures.

TABLE 1. Coefficients of linear thermal expansion of some ceramic materials at $300 \mathrm{~K}$

\begin{tabular}{|c|c|}
\hline Material & Coefficient \\
\hline 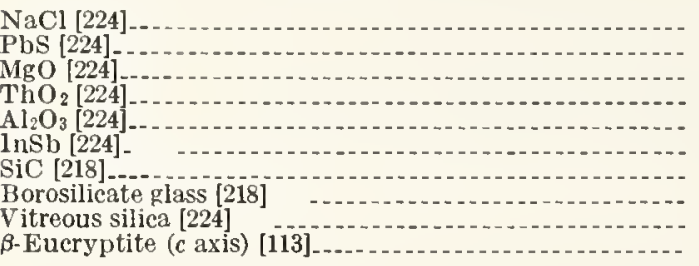 & $\begin{array}{c}40 \times 10^{-6 / K} \\
18 \\
11 \\
7 \\
5 \\
5 \\
4 \\
3 \\
0.4 \\
-17\end{array}$ \\
\hline
\end{tabular}

\section{Definitions}

The thermodynamic coefficient of thermal expansion is defined as

$$
\beta=\frac{1}{V}\left(\frac{\partial V}{\partial T}\right)_{P}
$$

where the volume, $V$, is a function of temperature, $T$, at constant pressure, $P$. The coefficient of linear thermal expansion is defined as

$$
\alpha=\frac{1}{L}\left(\frac{\partial L}{\partial T}\right)_{P}
$$

where the length, $L$, is a function of temperature at constant pressure. If the material is isotropic then

$$
\beta=3 \alpha \text {. }
$$

Single crystals having cubic symmetry, annealed polycrystalline solids having randomly oriented grains, and annealed glasses or amorphous materials are isotropic.

The coefficients of linear thermal expansion needed to describe the expansion of crystals of various symmetries are as follows [225]:

$\begin{array}{lllllll}\text { Cubic } & \alpha_{1} & \alpha_{1} & \alpha_{1} & 0 & 0 & 0 \\ \text { Hexagonal } & \alpha_{1} & \alpha_{1} & \alpha_{3} & 0 & 0 & 0 \\ \text { Tetragonal } & \alpha_{1} & \alpha_{1} & \alpha_{3} & 0 & 0 & 0 \\ \text { Trigonal } & \alpha_{1} & \alpha_{1} & \alpha_{3} & 0 & 0 & 0 \\ \text { Orthorhombic } & \alpha_{1} & \alpha_{2} & \alpha_{3} & 0 & 0 & 0 \\ \text { Monoclinic } & \alpha_{1} & \alpha_{2} & \alpha_{3} & 0 & \alpha_{5} & 0 \\ \text { Triclinic } & \alpha_{1} & \alpha_{2} & \alpha_{3} & \alpha_{\alpha_{1}} & \alpha_{5} & \alpha_{6} .\end{array}$

The principal coefficients $\left(\alpha_{1}, \alpha_{2}\right.$, and $\left.\alpha_{3}\right)$ describe the volume change of the crystal so that

$$
\beta=\alpha_{1}+\alpha_{2}+\alpha_{3} \text {. }
$$

These coefficients are not necessarily in the direction of the crystallographic translation vectors but are in the direction of the principal axes [225]. The remaining coefficients $\left(\alpha_{4}, \alpha_{5}\right.$, and $\left.\alpha_{6}\right)$ describe the change in shape (but not the symmetry) of the crystal. A monoclinic or triclinic crystal will change shape during a temperature change even if it is cut in the crystallographic reference system. For example, the lattice parameters of a monoclinic crystal are given as $a$, $b, c, 90^{\circ}, \beta 90^{\circ}$ (see fig. 1). The special relations $\alpha=\gamma=90^{\circ}$ cannot be changed by temperature change. While $\alpha_{2}$ and $\alpha_{3}$ are the coefficients along the crystallographic translation vectors $\bar{b}$ and $\bar{c}$, $\alpha_{1}$ is the coefficient in the direction of the principal axis $X_{1}$ which is orthogonal to the $X_{2}(\bar{b})$ and $X_{3}(\bar{c})$ axes. The remaining coefficient, $\alpha_{5}$, represents the change in the angle $\beta$ and is given by

$$
\alpha_{5}=\frac{1}{\pi / 2} \frac{d \beta}{d T}
$$

Except for monoclinic and triclinic symmetries, the expansivity of a single crystal in a direction different from the crystallographic axes is given by

$$
\alpha_{\omega}=\Sigma \alpha_{i} \cos ^{2} \omega_{i}
$$

where $\omega_{i}$ is the angle between the direction of $\alpha_{i}$ and the direction of interest. For hexagonal, tetragonal and trigonal symmetries this relationship reduces to

$$
\alpha_{\omega}=\alpha_{1}+\left(\alpha_{3}-\alpha_{1}\right) \cos ^{2} \omega_{3} .
$$

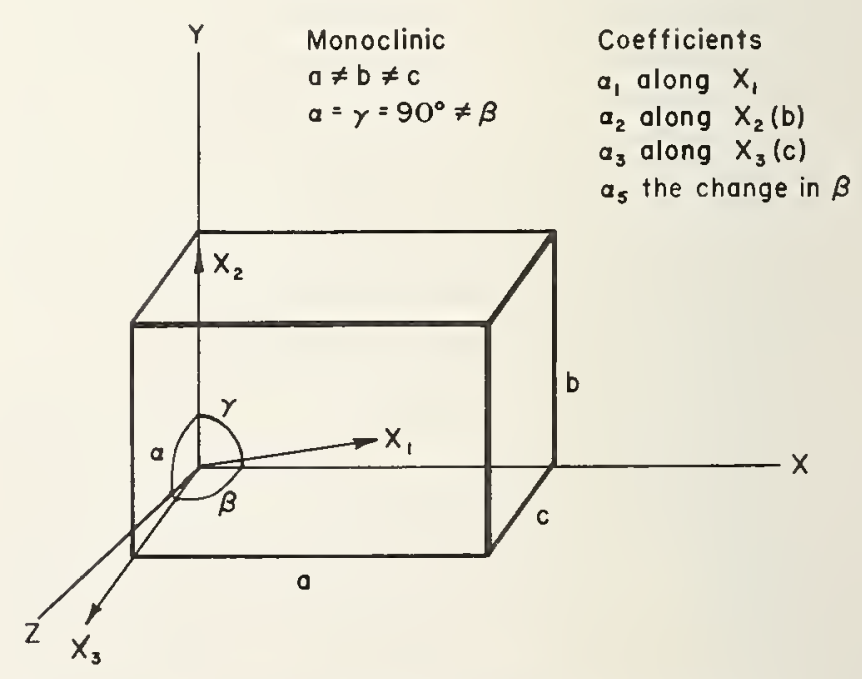

FIgure 1. Coefficients of thermal expansion for a monoclinic crystal.

The direction $X_{1}$ (orthogonal to $X_{2}$ and $X_{3}$ in the $x z$ plane) varies with temperature and $\alpha_{5}$ is a measure of the change in the angle $\beta$. 
If the values of $\alpha_{\omega}$ are plotted against the corresponding values of $\cos ^{2} \omega_{3}$ the result is very close to a straight line. Deviations from the straight line can result from residual stresses. Data obtained by the author on several crystals of $\mathrm{TiO}_{2}$ having different orientations are illustrated in figure 2.

The thermodynamic coefficient of thermal expansion can be calculated from an equation that represents the expansion of the material or by graphically determining the slope of the expansion data at a particular temperature and dividing by the volume at that temperature. The difficulty with the first procedure is that while equations (usually polynominals) can be obtained in limited temperature ranges that appear to fit the data, a closed equation will not in fact exactly represent the thermal expansion. This is especially true in the lower temperature range as can be seen in figures 3, 4, and 5. Equations derived from Grüneisen's theory of the solid state are probably the best to use for representing thermal expansion. Derivation of these equations will be given in the next section.

Under certain conditions the instantaneous coefficient of thermal expansion will satisfactorily

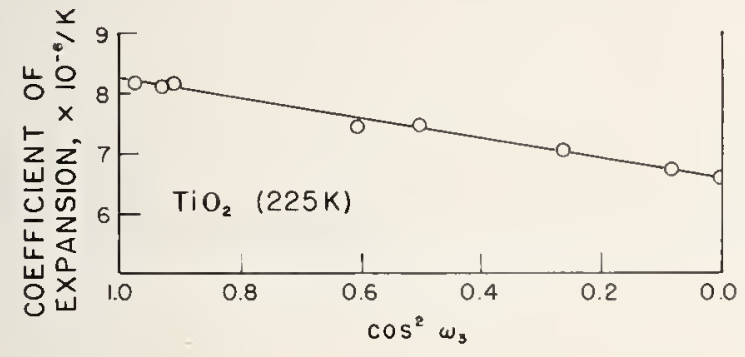

Figure 2. Coefficients of thermal expansion of single-crystal rutile in various directions from the c axis.

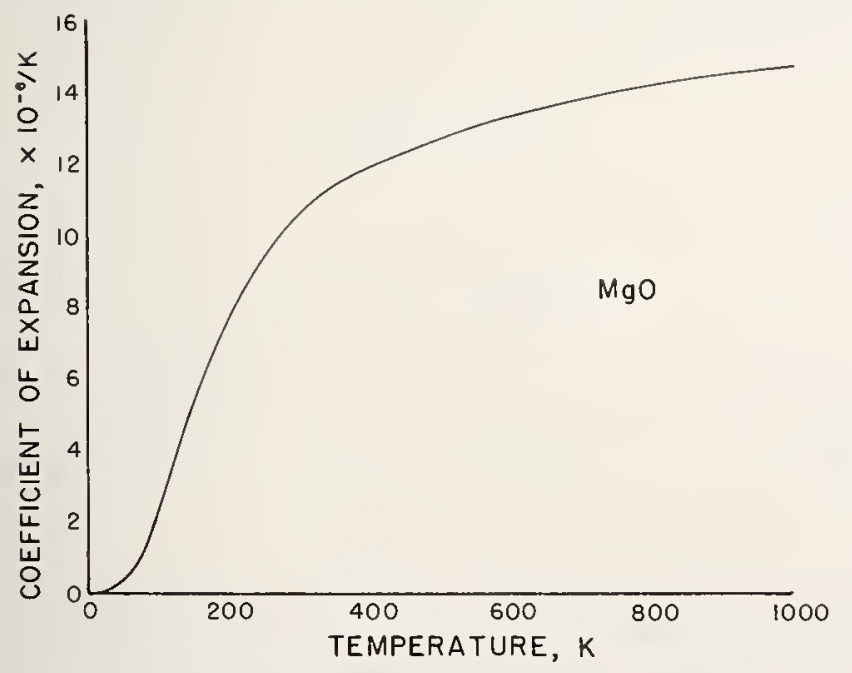

FiguRE 3. Expansivity of magnesium oxide as a function of temperature.

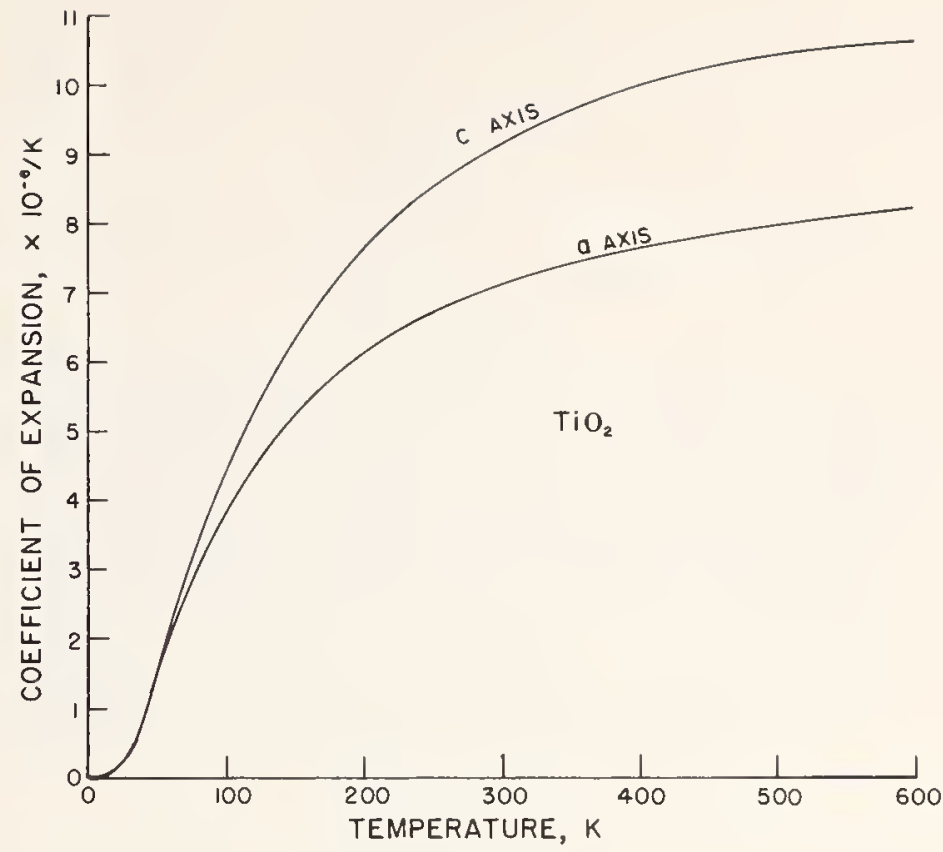

FIGURE 4. Expansivity of single-crystal rutile as function of temperature.

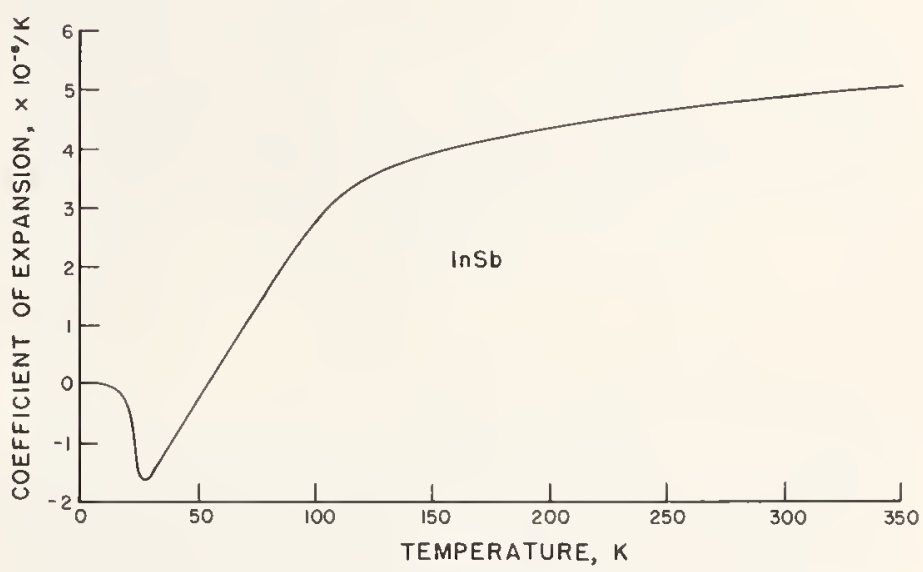

FIGURE 5. Expansivity of indium antimony as a function of temperature.

represent the thermodynamic coefficient. The instantaneous coefficient is defined as

$$
\beta_{i}=\frac{1}{V_{0}} \frac{d V}{d T}=\underset{T_{1} \rightarrow T_{2}}{\operatorname{limit}} \frac{V_{2}-V_{1}}{V_{0}\left(T_{2}-T_{i}\right)}
$$

at

$$
\bar{T}=\frac{T_{2}+T_{1}}{2}
$$

where $V_{0}$ is the volume at some reference temperature, usually room temperature. The instantaneous coefficient of linear thermal expansion is given by

$$
\alpha_{i}=\underset{T_{1} \rightarrow T_{2}}{\operatorname{limit}} \frac{L_{2}-L_{1}}{L_{o}\left(T_{2}-T_{1}\right)} .
$$

If the expansion over a limited temperature range can be approximated by a quadratic, 
then

$$
L=L_{o}+a T+b T^{2}
$$

and

$$
\alpha_{i}=\frac{a+2 b \bar{T}}{L_{o}}
$$

$$
\alpha=\frac{a+2 b \bar{T}}{L_{o}+a \bar{T}+b \bar{T}^{2}} .
$$

For the case of a quadratic, the limit of $T_{1} \rightarrow T_{2}$ does not need to be applied. For low-expansion materials such as vitreous silica, $\alpha_{i}$ equals $\alpha$ for temperatures as high as $1200 \mathrm{~K}$. If the expansion curve takes the shape of higher polynominals the limit of $T_{1} \rightarrow T_{2}$ must be applied if it is desired that $\alpha_{i} \rightarrow \alpha$.

For practical applications the mean coefficient of linear thermal expansion between temperatures $T_{1}$ and $T_{2}$,

$$
\alpha_{m}=\frac{L_{2}-L_{1}}{L_{0}\left(T_{2}-T_{1}\right)},
$$

is very useful. Values of the mean and instantaneous coefficients for quartz are shown in figure 6 . It is quite apparent that the mean coefficient is relatively insensitive to changes in expansion. The mean coefficient of volume thermal expansion is not equal to three times the mean coefficient of linear thermal expansion but can be approximated by

$$
\beta_{m} \simeq 3 \alpha_{m}+3\left(T_{2}-T_{1}\right) \alpha_{m}^{2} .
$$

For example, if $\alpha_{m}=10 \times 10^{-6} / \mathrm{K}$ and $T_{2}-T_{1}^{*}=1000$ $\mathrm{K}$ then $\beta_{m}=30.3 \times 10^{-6} / \mathrm{K}$.

\section{Theory of Thermal Expansion}

It has been known for years [226] that thermal expansion originates in the anharmonic vibrations of atoms in a solid. It is only recently, however, that real progress has been made in obtaining

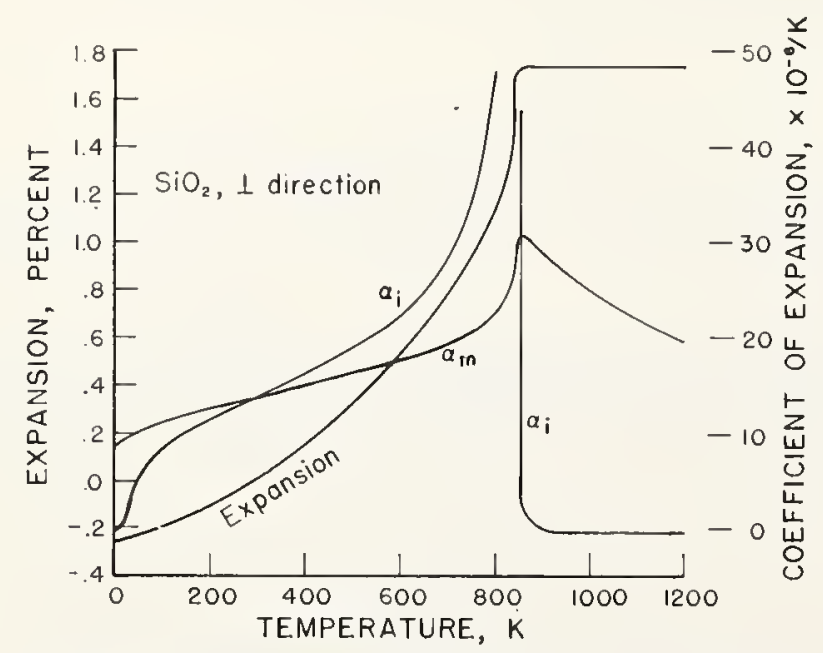

Figure 6. Comparison of the mean and instantaneous coefficients of quartz. reliable information about the anharmonic terms in the potential energy function. The inelastic scattering of neutrons by a lattice has been studied as a function of volume by application of external force [227]. The frequency shifts of the lattice vibrations that are thus produced reflect the deviation of the crystal potential from the harmonic approximation. These measurements have resulted in calculated values for thermal expansion and confirm what has been approximated by simple approaches.

From thermodynamics we have the following set of definitions and relationships:

and

$$
\kappa_{T}=-\frac{1}{V}\left(\frac{\partial V}{\partial P}\right)_{T}, \beta=\frac{1}{V}\left(\frac{\partial V}{\partial T}\right)_{P}
$$

$$
\frac{\beta}{\kappa_{T}}=\left(\frac{\partial P}{\partial T}\right)_{V}=\left(\frac{\partial S}{\partial V}\right)_{T}=-\frac{\partial^{2} F}{\partial V \partial T}
$$

where $\kappa_{T}$ is the isothermal compressibility, $S$ is the entropy, and $F$ is the free energy. Applying statistical mechanics to the quasi-harmonic approximation (the interaction constants of the harmonic theory are allowed to be volume dependent) for a set of harmonic oscillators it can be shown that

$$
\frac{\partial^{2} F}{\partial V \partial T}=-\frac{1}{V} \sum_{i=1}^{3 N} \gamma_{i} C_{i}
$$

where

$$
\gamma_{i}=-\frac{V}{\omega_{i}} \frac{d \omega_{i}}{d V}
$$

and

$$
C_{i}=k\left(\hbar \omega_{i} / k T\right)^{2} \exp \left(\hbar \omega_{i} / k T\right) /\left[\exp \left(\hbar \omega_{i} / k T\right)-1\right]^{2} .
$$

$C_{i}$ is the contribution of each vibrational mode to the heat capacity, $k$ is Boltzmann's constant, $\hbar=h_{/}^{\prime} 2 \pi$ where $h$ is Planck's constant, and $\omega_{i}$ is the frequency of the $i$ th mode. If $\gamma$ is the weighted average of the individual $\gamma_{i}$ 's,

$$
\gamma=\sum_{i=1}^{3 N} \gamma_{i} C_{i} / \sum_{i=1}^{3 N} C_{i}
$$

then

$$
\frac{\partial^{2} F}{\partial V \partial T}=-\frac{\gamma C_{V}}{V}
$$

Grüneisen's relation is obtained from eqs (1) and (2),

$$
\beta=\gamma \frac{\kappa_{T} C_{V}}{V},
$$

where $\gamma$, the Grüneisen parameter, is a measure of the average anharmonic interaction.

At temperatures higher than the Debye characteristic temperature, $\theta_{D}$, the value of $\gamma$ is nearly constant since the whole spectrum of vibrational 
frequencies is excited and $\gamma_{\infty}$ is merely the arithmetic average of the $\gamma_{i}$ 's,

$$
\gamma_{\infty}=\frac{1}{3 N} \sum_{i=1}^{3 N} \gamma_{i}
$$

In the low-temperature limit as $T \rightarrow O \mathrm{~K}$, the Debye continuum is a valid model and again $\gamma$ will approach a constant value,

$$
\gamma_{0}=-\frac{V}{\theta_{0}} \frac{d \theta_{0}}{d V}
$$

where $\theta_{0}$ is the limiting value of $\theta_{D}$ at low temperatures and is directly related to the elastic constants. At intermediate temperatures the behavior of $\gamma$ may be investigated through Grüneisen's relation,

$$
\gamma(T)=\frac{V \beta}{\kappa_{T} C_{V}}=\frac{V \beta}{\kappa_{S} C_{P}}
$$

The variation of $\gamma$ for $\mathrm{Al}_{2} \mathrm{O}_{3}$ is shown in figure 7 .

Since the free energy is an additive function of state, eq (3) can be expressed as a sum of the contributions from the lattice vibrations (both the acoustical and optical), the conduction electrons in metals, magnetic interactions, etc.,

$$
\beta=\frac{\kappa_{T}}{V}\left(\gamma_{\mathrm{aco}} C_{\mathrm{aco}}+\gamma_{\mathrm{opt}} C_{\mathrm{opt}}+\gamma_{e} C_{e}+\gamma_{m} C_{m}+\ldots\right) .
$$

In the case of nonmagnetic metals, the heat capacity of the conduction electrons and the lattice can be approximated by

$$
C_{e} \simeq a T \text { and } C_{l} \simeq b T^{3}
$$

at very low temperatures.

Equation (4) can therefore be written as

$$
\beta=\frac{\kappa_{T}}{V}\left(\gamma_{e} a T+\gamma_{l} b T^{3}\right)
$$

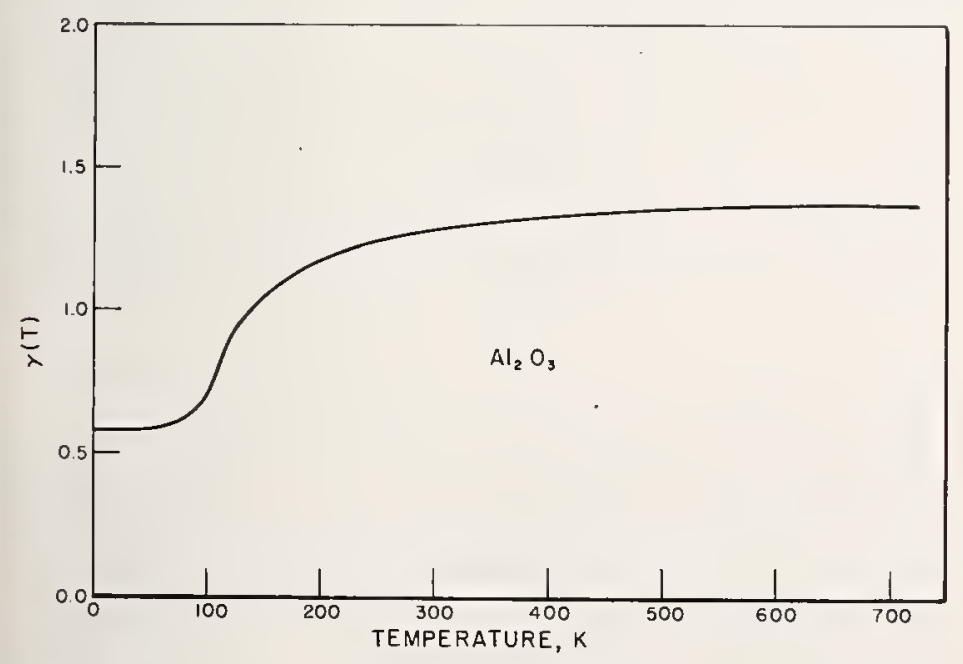

FIGURE 7. Grüneisen's parameter for alumina as a function of temperature.
If the terms are rearranged,

$$
\frac{\beta V}{\kappa_{T} T}=\gamma_{e} a+\gamma_{l} b T^{2},
$$

and the values of $\left(\frac{\beta V}{\kappa_{T} T}\right)$ are plotted against $\left(T^{2}\right)$

a straight line should result. Such a plot for copper is shown in figure 8.

The separation of the free energy terms for the acoustical and optical vibrations in a polyatomic crystal can also be obtained [228]. The heat capacity of the optical modes is determined from Einstein's function using the value of the infrared resonance frequency at the absorption maximum,

$$
C_{\mathrm{opt}}=f\left(\frac{\Theta_{E}}{T}\right) \text { where } \Theta_{E}=\frac{h \omega}{k} .
$$

The heat capacity of the acoustical modes is taken as the difference between observed values of $C_{\mathrm{V}}$ and calculated values of $C_{\text {opt }}$. If values of $\left(\beta V / C_{\text {aco }} \kappa_{\mathrm{T}}\right)$ are plotted against the values of $\left(C_{\text {opt }} / C_{\text {aco }}\right)$, a straight line should result. Such a plot for $\mathrm{TiO}_{2}$ is shown in figure 9 .

Equations can also be derived to represent the anisotropic expansion of any class of crystal [226]. Using matrix notation, eq (3) can be expressed as a sum of the expansion in the crystallographic directions,

$$
\alpha_{i}=\frac{C_{V}}{V} \sum_{j=1}^{6} s_{i j} \gamma_{j}, i=1,2,3,
$$

where $s_{i j}$ are the elastic compliances for the lattice. This equation gives a linear relation between components of the expansion tensor and the heat capacity. The volume coefficient is the trace of the expansion tensor so that

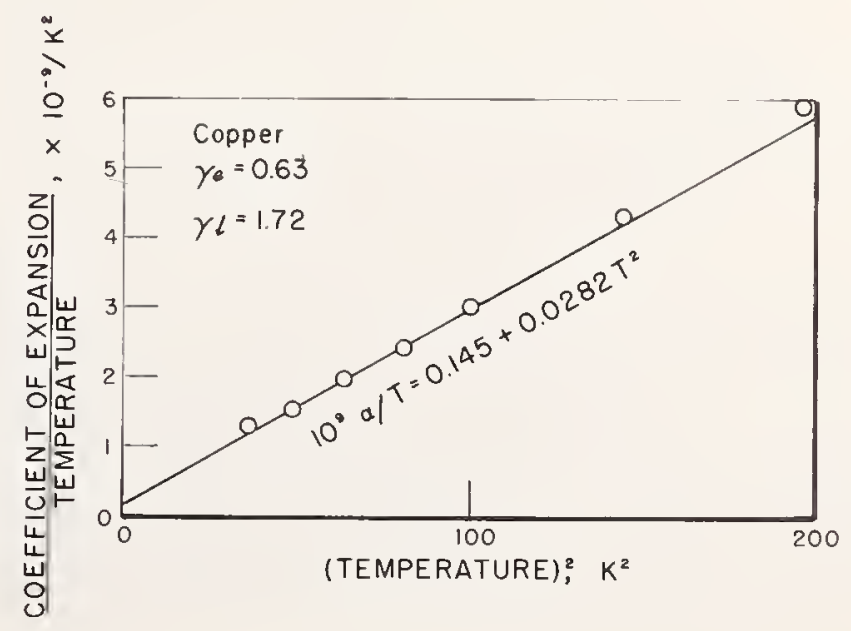

FIGURE 8. Contributions to the thermal expansion of copper of the conduction electrons and the lattice vibrations. 


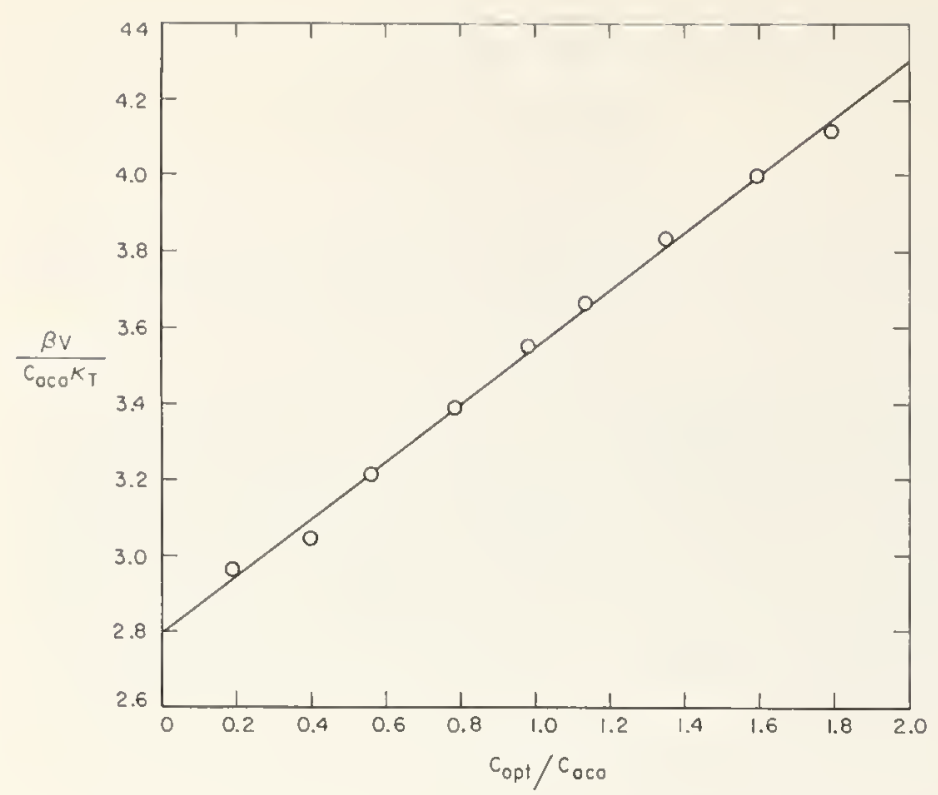

FIGURE 9. Contributions to the thermal expansion of rutile of the acoustical and optical vibrational modes.

$$
\beta=\frac{C_{V}}{V} \sum_{i=1}^{3} \sum_{j=1}^{6} s_{i j} \gamma_{j}
$$

When $s_{i j}(j=4,5,6)=0$, as in cubic, hexagonal, orthorhombic, and some tetragonal crystals, a single value of $\gamma$ can be determined by

$$
\gamma=\sum_{i, j=1}^{3} s_{i j} \gamma_{j} / \sum_{i, j=1}^{3} s_{i j}
$$

For the case of a hexagonal crystal,

$$
\begin{aligned}
\alpha_{1} & =\frac{C_{V}}{V}\left[\left(s_{11}+s_{12}\right) \gamma_{1}+s_{13} \gamma_{3}\right], \\
\alpha_{3} & =\frac{C_{V}}{V}\left[2 s_{13} \gamma_{1}+s_{33} \gamma_{3}\right], \\
\beta & =\frac{C_{V}}{V}\left[2\left(s_{11}+s_{12}+s_{13}\right) \gamma_{1}+\left(2 s_{13}+s_{33}\right) \gamma_{3}\right],
\end{aligned}
$$

and

$$
\gamma=\frac{2\left(s_{11}+s_{12}+s_{13}\right) \gamma_{1}+2\left(s_{13}+s_{33}\right) \gamma_{3}}{2 s_{11}+s_{33}+2 s_{12}+4 s_{13}}
$$

Observed and calculated values of $\alpha_{1}$ and $\alpha_{3}$ for $\mathrm{Al}_{2} \mathrm{O}_{3}$ are shown in figure 10 .

The use of the equations derived so far requires knowledge of the compressibility or the elastic compliances as a function of temperature. A useful variation of Grüneisen's relation can be obtained that circumvents a possible lack of this knowledge [226]. The Debye equation of state [229] is given by

$$
P V=-V\left(\frac{\partial U_{0}}{\partial V}\right)+\gamma U_{D}
$$

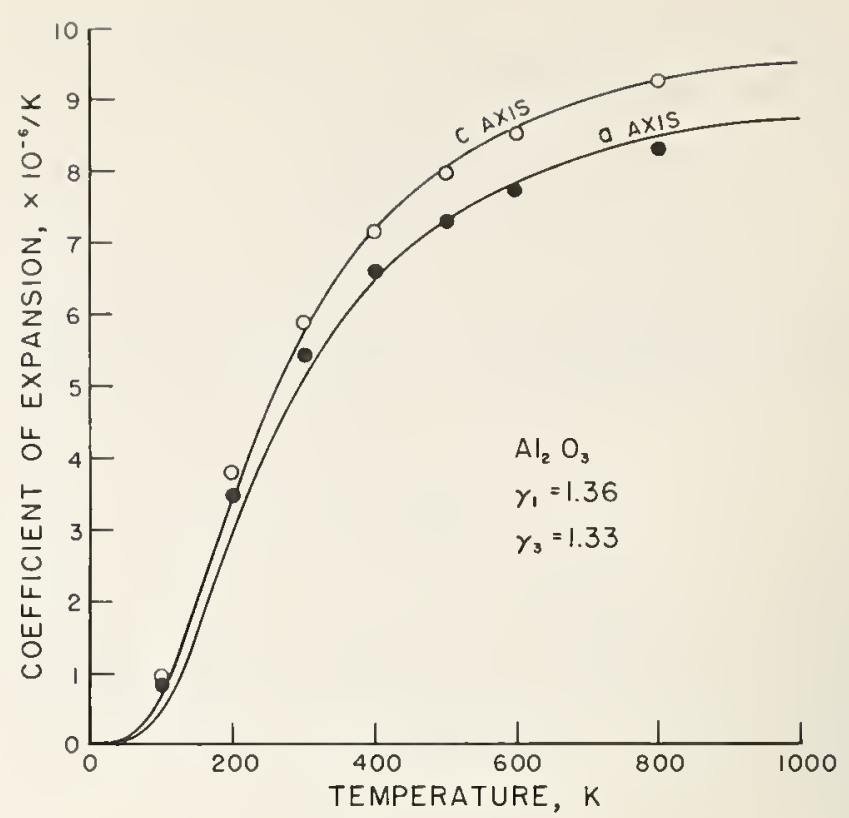

Figure 10. Comparison between observed (solid lines) and calculated values (points) of the expansivity of alumina in the crystallographic directions.

where $U_{0}$ is the internal energy at $0 \mathrm{~K}$ and $U_{D}$ is the contribution of the lattice vibrations to the internal energy. On setting $P$ equal to zero eq (5) becomes

$$
V \frac{\partial U_{0}}{\partial V}=G(V)=\gamma U_{D}
$$

On expanding $G(V)$ in a Taylor series, eq (6) be-

$$
\begin{aligned}
\text { comes } G\left(V_{0}\right)+\left(V-V_{0}\right) \frac{d G\left(V_{0}\right)}{d V_{0}}+\frac{\left(V-V_{0}\right)^{2}}{2} \\
\frac{d^{2} G\left(V_{0}\right)}{d V_{0}^{2}}+\ldots=\gamma U_{D}
\end{aligned}
$$

where $V_{0}$ is the volume at $0 \mathrm{~K}$. Assuming a reasonable model for the potential energy of the lattice, it can be shown that

$$
\begin{gathered}
G\left(V_{0}\right)=0 \\
\frac{d G\left(V_{0}\right)}{d V_{0}}=\frac{1}{\kappa_{0}} \\
\frac{d^{2} G\left(V_{0}\right)}{d V_{0}^{2}}=-\frac{2 A}{V_{0} \kappa_{0}}
\end{gathered}
$$

where $\kappa_{0}$ is the compressibility at $0 \mathrm{~K}$. Substituting these terms in eq (7) one obtains

$$
\left(\frac{V-V_{0}}{V_{0}}\right)-A\left(\frac{V-V_{0}}{V_{0}}\right)^{2}+\ldots=\frac{\kappa_{0}}{V_{0}} \gamma U_{D}
$$

If the approximation

$$
\frac{V-V_{0}}{V_{0}} \simeq \frac{U_{D}}{Q} \text { where } Q=\frac{V_{0}}{\kappa_{0} \gamma}
$$


is used in eq (8), a variation of Grüneisen's relation is obtained:

$$
\frac{V-V_{0}}{V_{0}}=\frac{U_{D}}{Q-A U_{D}}
$$

This equation is best used to represent expansion data by determining the values of $Q$ and $A$ through a least squares calculation. This has been done for $\mathrm{Al}_{2} \mathrm{O}_{3}$ and $\mathrm{ThO}_{2}$ with excellent results over a temperature range of 100 to $1100 \mathrm{~K}$ [139].

\section{Methods of Measuring Thermal Expansion}

While there are many different methods and variations of methods of measuring thermal expansion, for reasons of space, only four general types will be described: (1) The vitreous-silica dilatbmeter is very useful in the range 100 to $1200 \mathrm{~K}$. The measurements of thermal expansion $\left(\frac{\Delta L}{L}\right)$ with a dilatometer are usually accurate ${ }^{2}$ to $25 \mathrm{ppm}$ but the precision can be as good as $0.1 \mathrm{ppm}$. (2) The interferometric method is useful in the range 20 to $1200 \mathrm{~K}$. Smaller specimens are used, which increases the accuracy of the temperature measurements. The measurements of expansion with this method are usually accurate to $4 \mathrm{ppm}$ but can be accurate to $1 \mathrm{ppm}$. (3) The optical comparator method is most useful in the range 1000 to $3500 \mathrm{~K}$. The measurements of expansion can be accurate to $50 \mathrm{ppm}$. (4) The Debye-Scherrer $x$-ray diffractometer can be used in the range 20 to $2500 \mathrm{~K}$. Only crystalline materials can be measured but very small amounts are sufficient. The measurements of expansion can be accurate to $50 \mathrm{ppm}$.

\subsection{Vitreous Silica Dilatometers}

Three general types of dilatometers are shown in figure 11. Two of these types are described in an ASTM method of test [230]. The tube type, shown on the right, is the best known and the most widely used. The single-rod type shown on the left is the easiest to use and is more accurate than the tube type. The double-rod type shown in the center is in principle the most accurate but has been used very little. In all three types, the thermal expansion of the test material is given by

$$
\frac{\Delta L}{L_{0}}=A \frac{(\Delta L)_{a}}{L_{0}}+B
$$

where $(\Delta L)_{a}$ is the observed length change, $A$ is a calibration constant for the system, and $B$ is the net expansion of the dilatometer.

There are three ways of using and calibrating a dilatometer. These are, (1) Very careful calibra-

\footnotetext{
2 The following values for the accuracy of the thermal expansion measurements are based on a temperature interval of $100 \mathrm{~K}$ and a material having an expansivity of $10 \times 10^{-8 / K}$.
}

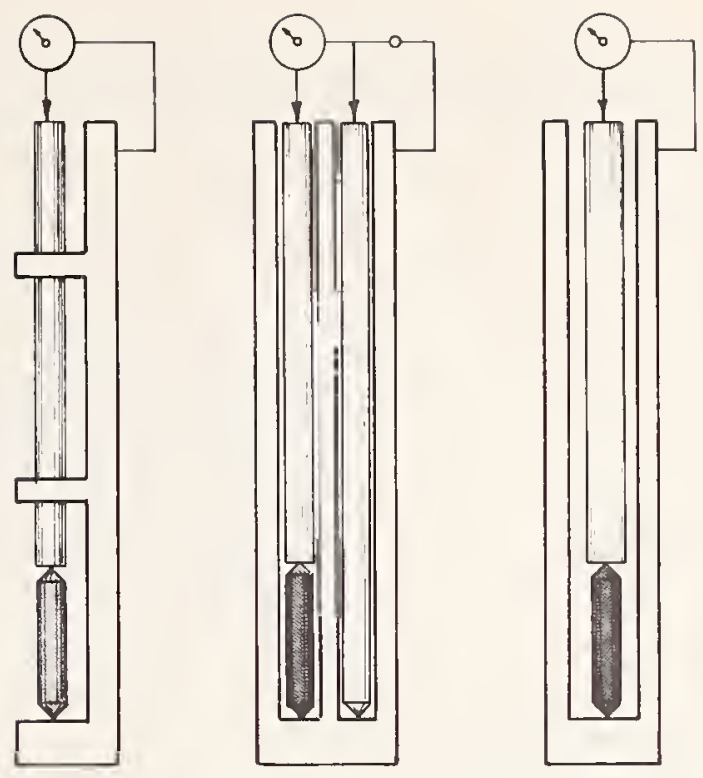

FIgURE 11. Variations of the vitreous-silica dilatometer.

tion of the extensometer and thermometer so that $A=1$ and assume a value for the expansion of the vitreous silica. (2) Same as (1) but a reference material of known expansion is used to determine the expansion of the vitreous silica. (3) Two reference materials are used to determine the values of $A$ and $B$ (this is not a substitute for the calibration of the extensometer and thermometer but provides additional information on the behavior of the system). It can be seen in table 2 that the method of using two reference materials is in general somewhat better than the other methods except that a more accurate calibration can be obtained with just one reference material if the expansivity is within 2 percent of the test material.

The vitreous silica parts of a dilatometer should be fine annealed ${ }^{3}$ after they have been fabricated and should thereafter not be heated above $1200 \mathrm{~K}$. Dial indicators and linear variable differential transformers (LVDT) [81] are used the most for measuring the length change but many other types of extensometers are also used. These include optical levers [231], interferometers [232], strain gages [16], and optical gratings [37].

One of the most common sources of error in using dilatometers is the measurement of temperature. All too often, the temperature that is measured is not the temperature of the specimen. This is especially true if measurements are made while the temperature is changing. If a thermocouple is used it is recommended that the junction and at least $3 \mathrm{~cm}$ of the leads should be at the same temperature (preferably by being in contact with the specimen). This procedure will prevent heat from being transported away from or to the junction.

3 Heat at $100 \mathrm{~K} / \mathrm{hr}$ to $1475 \mathrm{~K}$, hold at that temperature for $2 \mathrm{hr}$, cool at 60 $\mathrm{K} / \mathrm{hr}$ to $1275 \mathrm{~K}$ at $120 \mathrm{~K} / \mathrm{hr}$ to $900 \mathrm{~K}$ and at $200 \mathrm{~K} / \mathrm{hr}$ to room temperature. Care should be taken to minimize viscous distortion during the annealing. 
TABLE 2. Accuracy that can be obtained with a vitreous silica dilatometer on measurements of coefficients of thermal expansion (a one percent linear error is assumed in either the length or temperature measurements), $\times 10^{-6} / \mathrm{K}$.

\begin{tabular}{|ccccc|}
\hline \multicolumn{5}{|c}{ Method of using dilatometer } \\
\hline $1 *$ & 2 & 2 & 3 & 3 \\
\hline
\end{tabular}

\begin{tabular}{|c|c|c|c|c|c|}
\hline $\begin{array}{l}\text { 1st reference material } \\
\text { Accuracy }\end{array}$ & $\begin{array}{l}\text { None } \\
\text { used }\end{array}$ & $\begin{array}{r}0.50 \\
\pm 0.02\end{array}$ & $\begin{array}{r}10.00 \\
\pm 0.04\end{array}$ & $\begin{array}{r}0.50 \\
\pm 0.02\end{array}$ & $\begin{array}{r}0.50 \\
\pm 0.02\end{array}$ \\
\hline $\begin{array}{l}\text { 2nd reference material } \\
\text { Accuracy }\end{array}$ & $\begin{array}{r}\text { None } \\
\text { used }\end{array}$ & $\begin{array}{r}\text { None } \\
\text { used }\end{array}$ & $\begin{array}{r}\text { None } \\
\text { used }\end{array}$ & $\begin{array}{r}4.00 \\
\pm 0.03\end{array}$ & $\begin{array}{r}10.00 \\
\pm 0.04\end{array}$ \\
\hline
\end{tabular}

\begin{tabular}{|c|c|c|c|c|c|c|c|}
\hline $\begin{array}{l}\text { Nominal expansivity of } \\
\text { test material and pre- } \\
\text { cision of measurement. }\end{array}$ & $\begin{array}{r}0.0 \\
0.5 \\
4.0 \\
10.0\end{array}$ & $\begin{array}{l} \pm 0.10 \\
\pm 0.10 \\
\pm 0.10 \\
\pm 0.12\end{array}$ & $\begin{array}{l} \pm 0.17 \\
\pm 0.17 \\
\pm 0.17 \\
\pm 0.20\end{array}$ & $\begin{array}{l} \pm 0.15 \\
\pm 0.15 \\
\pm 0.15 \\
\pm 0.18\end{array}$ & $\begin{array}{l} \pm 0.19 \\
\pm 0.19 \\
\pm 0.18 \\
\pm 0.17\end{array}$ & $\begin{array}{l} \pm 0.16 \\
\pm 0.14 \\
\pm 0.15 \\
\pm 0.36\end{array}$ & $\begin{array}{l} \pm 0.15 \\
\pm 0.14 \\
\pm 0.13 \\
\pm 0.18\end{array}$ \\
\hline
\end{tabular}

* For these calculations it was assumed that the value used for the expansivity of the dilatometer was accurate to $\pm 0.12 \times 10^{-6} / \mathrm{K}$.

Dilatometers may be used in either the vertical or horizontal position. For use at temperatures above $1200 \mathrm{~K}$, a dilatometer can be made from sapphire [233], $\mathrm{SiC}[234]$, or other refractory materials [150].

\section{2. Interferometers}

Three general types of interferometers that produce fringes of equal thickness are also described in an ASTM method of test [235]. These are the Fizeau, Abbe-Pulfrich, and Priest interferometers. Because of its limited use, the Priest type will not be described here. 'The AbbePulfrich type (see fig. 12), like the dilatometer, measures the thermal expansion of a specimen relative to the expansion of the material from which it is constructed. The Fizeau type, on the
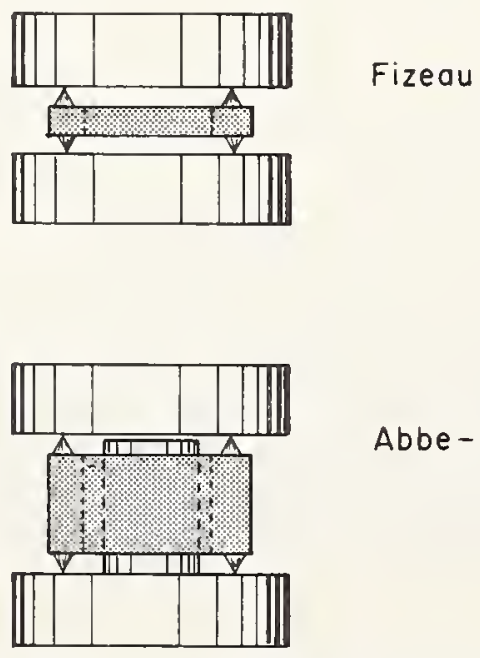

Abbe-Pulfich

Figure 12. The Fizeau and AbbePulfrich types of interferometer. other hand, measures the "absolute" expansion of the specimen.

When using a Fizeau interferometer the expansion of the specimen from $T_{1}$ to $T_{2}$ is given by

$$
\frac{\Delta L}{L_{0}}=\frac{N \lambda_{V}}{2 L_{0} n_{2}}+\frac{n_{1}-n_{2}}{n_{2}}
$$

where $N$ is the number of fringes, including fractional parts, that have moved past the reference point, $\lambda_{V}$ is the vacuum wavelength of the monochromatic light used to produce the fringes, and $n_{1}$ and $n_{2}$ are the indices of refraction of the gas between the optical flats at pressure $P$ and temperatures $T_{1}$ and $T_{2}$ respectively.

Experimental values of the index are not available over extended temperature ranges and therefore it is recommended that the Fizeau interferometer be placed in a reduced pressure atmosphere. When helium is used, a pressure of 5 torr or less will usually not produce a significant fringe shift over a range of 50 to $1200 \mathrm{~K}$. Air should not be used in any case at temperatures below $250 \mathrm{~K}$. Vacuum is not recommended, however, except in specially designed equipment because of the difficulty of temperature control and measurement. The index of refraction at a fixed wavelength can be estimated by

$$
n=1+\left(n_{r}-1\right) 288 P / 760 T
$$

where $n_{r}$ is the index at a reference temperature of $288 \mathrm{~K}$ and a pressure of 760 torr. For air, $n_{r}=$ 1.0002771 (yellow light) or $n_{r}=1.0002779$ (green light). For helium, $n_{r}=1.000036$.

In the case of the Abbe-Pulfrich interferometer, the effect of the index change is made insignificant by reducing the length of the optical path to a 
sufficiently small value. Under this condition the expansion of the specimen is given by

$$
\frac{\Delta L}{L_{o}}=\frac{N \lambda_{V}}{2 L_{o} n_{1}}+\frac{(\Delta L)_{\tau}}{L_{o}}
$$

where $(\Delta L)_{r}$ is the length change of the material from which the interferometer is made.

With the Fizeau interferometer reliable results can be obtained with pin specimens, see figure 13 , but special care must be taken. One technique is to place the three pins so that one of them carries
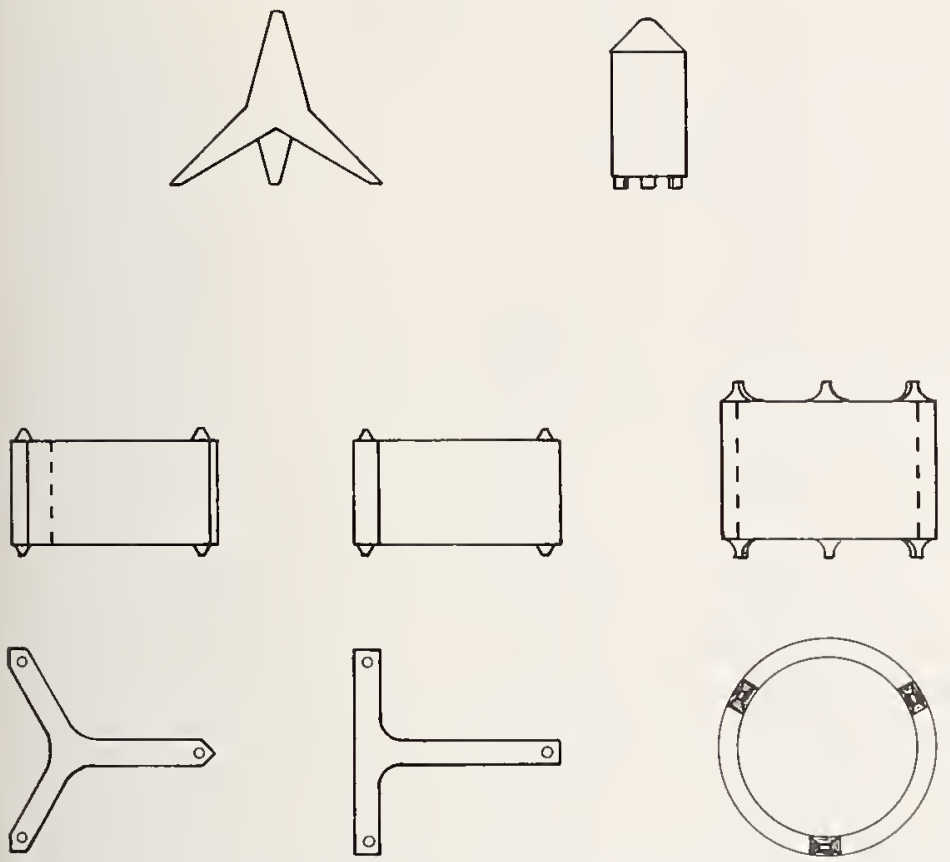

FIgURE 13. Variations of specimen shape for use in interferometers. most of the weight of the top optical flat [236]. That pin is taken as the reference point for the fringe system so that if one or both of the cther pins tip it will not effect the measurement of expansion. A "ring" or "T" shaped specimen, however, is the easiest to work with because it cannot tip. The specimen used in the AbbePulfrich interferometer may be the "ring" etalon or the center post. The ends of the post are polished optically flat and parallel and it is rung or contacted to the lower optical flat.

A Pulfrich viewer, shown in figure 14, is usually used to observe the fringe system. The position of the fringes with respect to a reference point, see figure 15, can be estimated with the unaided eye to 0.1 of a fringe separation $(\sim 0.03 \mu \mathrm{m})$ and easily measured with a micrometer eyepiece to 0.03 . The number of fringes that move past the reference point can be counted by eye or by photographic [237, 238] or photoelectric [239] techniques. It is also possible to avoid the counting problem by determining the correct order of the fringes at each temperature with two or more different wavelengths of light [240].

\subsection{Optical Comparators}

The optical comparator method is most useful for measuring thermal expansion at high temperatures. There are many variations $[85,156,168$, $169,241]$ of this type of apparatus but the best results are obtained when two general techniques are followed, see figure 16 . The first of these is that two microscopes should be used. They should be rigidly mounted and the length change measured with filar micrometer eyepieces. The second technique is that the fiducial marks observed with the

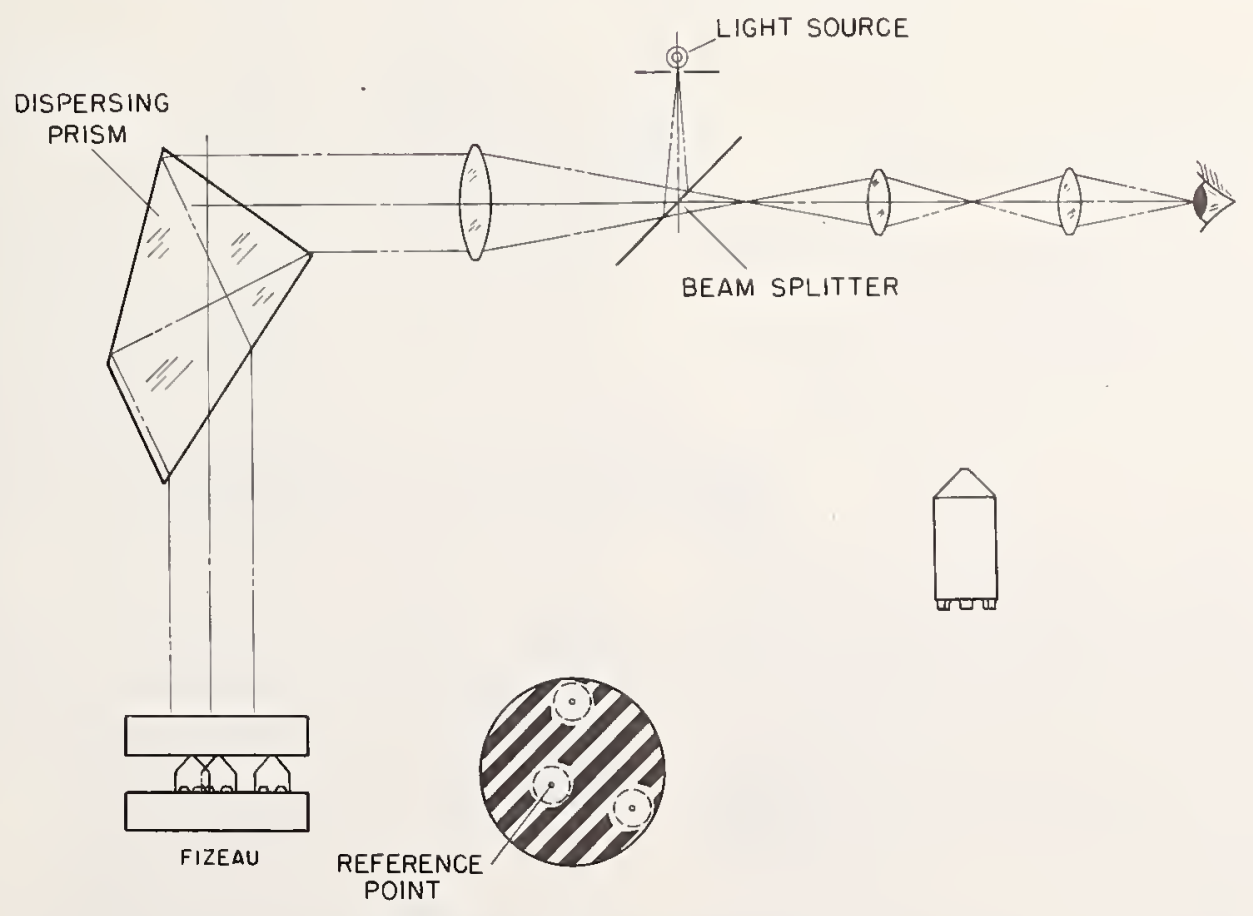

Figdre 14. Pulfrich viewer. 


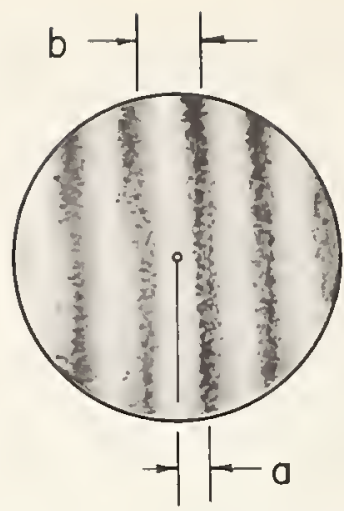

Fractional Part of Fringe Displacement is $\frac{a}{b}$

Figure 15. Measurement of fringe fraction.

(a)

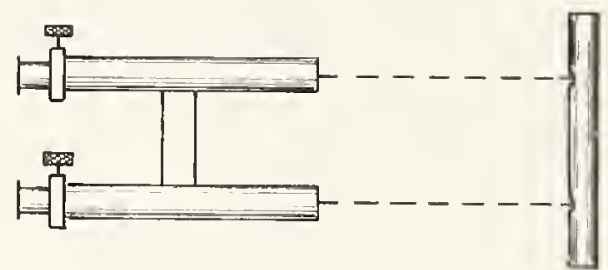

(b)
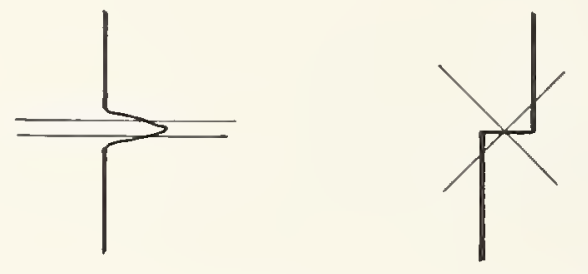

FIGURE 16. (a) Schematic drawing of optical comparator.

Microscopes mounted on invar bar can be either vertical or horizontal.

FIGURE 16. (b) Two types of fiducial marks that can be cut into the specimen.

Parallel $\Leftrightarrow$ hairs in the microscope ey epiece should be used to sight on the cut or nick type of fiducial mark and cross $(X)$ hairs should be used to sight on the step or knife edge type.

the microscopes should be machined into the specimen in such a way that they will be in sharp focus. If a cut or nick is used then it should be symmetrical and of a size that when viewed with the microscope it appears to be comparable to the separation between the parallel hairs in the eyepiece. If a knife edge is used then crossed hairs (X) should be used in the eyepiece. Small, clean holes drilled through the specimen are also satisfactory for fiducial marks.

In the optical comparator used at the National Bureau of Standards [242] the specimen is hung vertically in the furnace, see figure 17. The gage length of the specimen, between the fiducial marks, is $10 \mathrm{~cm}$. Since the windows may cause a significant error in the measurement of absolute length, a light source is built into the furnace to illuminate the specimen for a room-temperature measurement. The windows are flat to one wavelength and parallel to one second. Since the maximum temperature of the furnace is $1900 \mathrm{~K}$, the measurement of temperature can be made with thermocouples. Both W-Re and Pt-Rh 30-6 thermocouples are used. These thermocouples are calibrated in the furnace by a probe thermocouple inserted in place of the specimen.

This probe is also used to measure the temperature profile in the furnace. As seen in figure 18, the microscopes are mounted on an invar bar and as a unit can be rotated and moved vertically. The microscopes have a magnification of $50 \times$ and a working distance of $16 \mathrm{~cm}$. During each measurement, the length of the specimen is compared to the length of a reference scale kept at room temperature. This procedure compensates for any apparent change in distance between the microscopes that might be caused by the expansion of the invar bar or by the tilting of a microscope.

When using the optical comparator method, a vacuum can be used but an argon atmosphere is sometimes necessary to suppress vaporization. When an atmosphere is used, care must be taken in the measurement of length to avoid refraction effects in optical paths that travel through temperature gradients [110, 241]. An optical pyrometer should be used to measure temperature in the range above $2000 \mathrm{~K}$. When a pyrometer is used, care must be taken to provide blackbody conditions and to keep the furnace window clean. The pyrometer and the window should be calibrated together.

\subsection{X-Ray Techniques}

The thermal expansion of crystalline materials can be measured with x-ray cameras or with x-ray diffractometers. This method is important because it provides accurate data under conditions that preclude the use of any other method, for instance, when only a small amount of material is available. The method is unique in that it can provide data on expansion in different directions in anisotropic crystals and permits direct observation of phase changes, measurement of the expansion of each phase when two phases coexist [243], and measurement of the change in specific volume resulting from the phase change. There is a further advantage in that the expansion measured is that of the crystal lattice and does not include effects that are observed in the macroscopic expansion [244].

The use of the x-ray method depends upon the Bragg equation

$$
d=n \lambda / 2 \sin \theta
$$

where $d$ is the distance between crystal planes, $n$ is the order of reflection, $\lambda$ is the wavelength of 


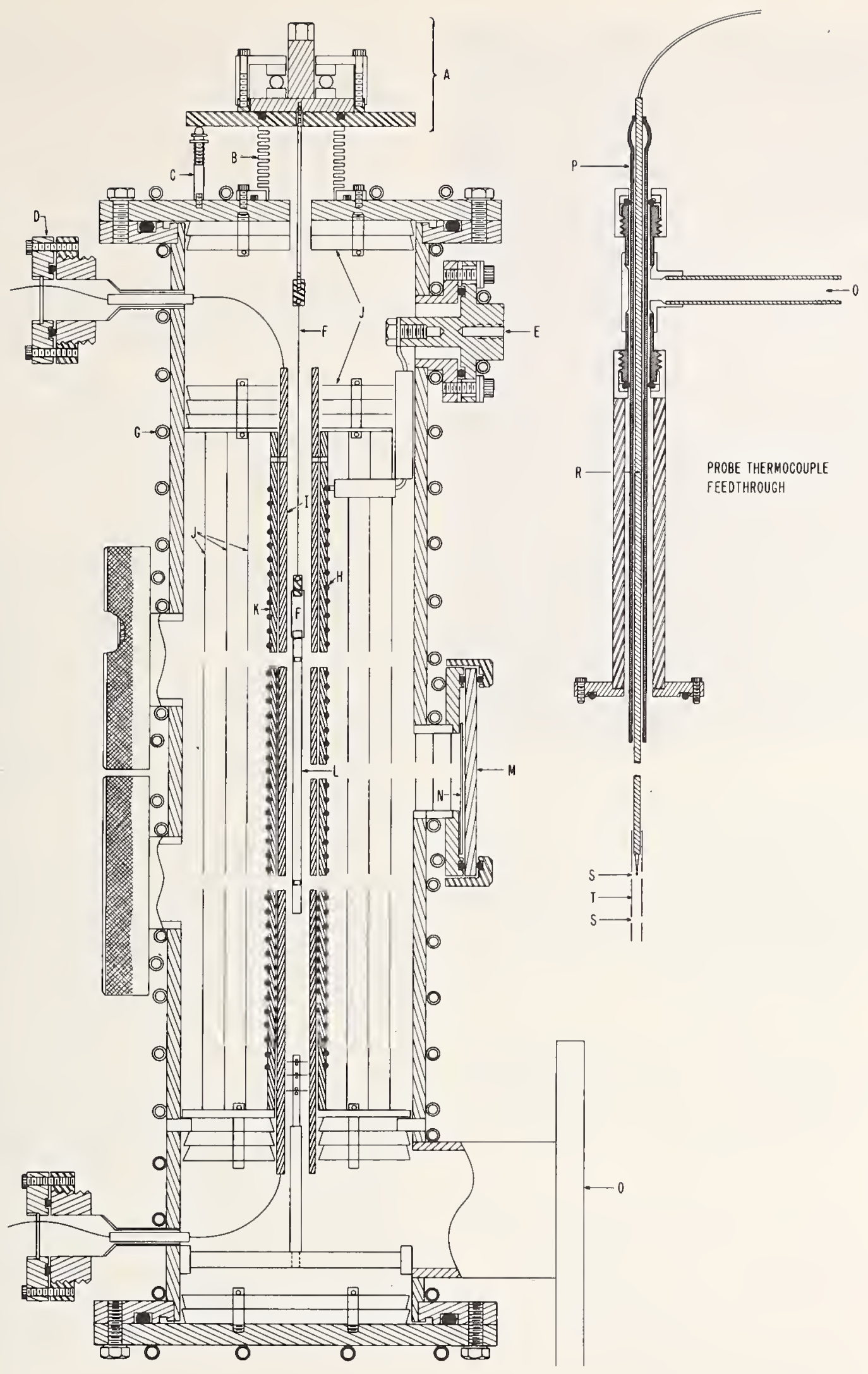

FIGURE 17. Schematic drawing of controlled-gradient vacuum furnace and probe thermocouple for measurement of expansion.
A. Specimen support
B. Bellows
C. Position adjusting screw, one of three
D. Thermocouple feedthrough
E. Electrical feedthrough, one of ten
F. Tungsten wire loop
G. Water cooling coil
H. Resistance beating wire
I. Thermocouple protection tube
J. Radiation heat shields
K. Alumina Core
L. Specimen
M. Window
N. Shutter
o. Vacuum port
P. V ycor tube
Q. Vacuum
R. Thermocouple protection tube
S. Pyrometer viewing hole
T. Tantalum tube 


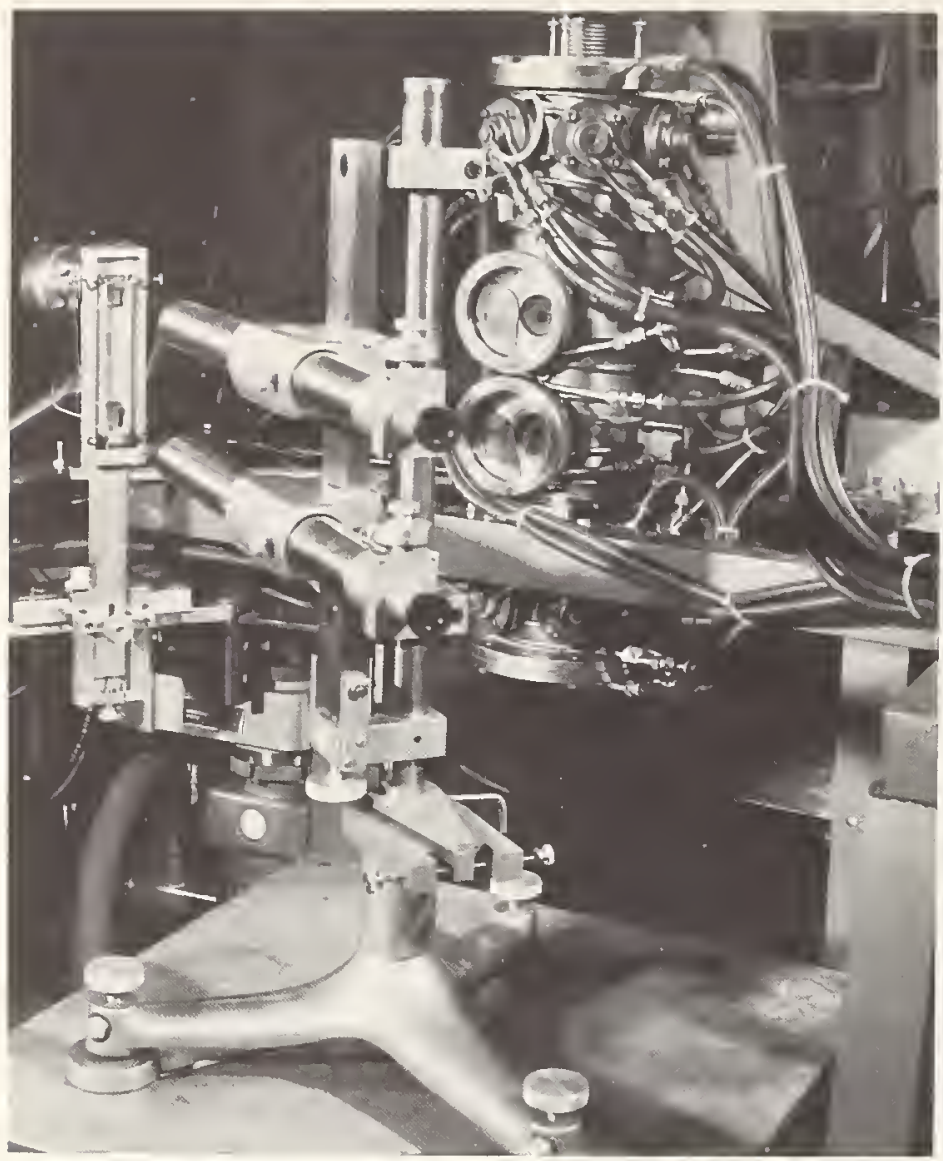

Figure 18. Photograph showing relative position of comparator, furnace, and reference scale.

the $\mathrm{x}$-ray beam, and $\theta$ is the angle of incidence of the beam with the crystal plane. From the Bragg equation it is easy to show that the change of the spacing between crystal planes is given by

$$
\frac{\Delta d}{d}=\frac{\sin \theta_{1}-\sin \theta_{2}}{\sin \theta_{2}}
$$

Except for a small correction due to refraction of the $\mathrm{x}$-rays [223] this measurement of expansion is independent of wavelength.

The distance between planes in crystals of various symmetries is given by

$$
\begin{aligned}
d= & \left\{\left[1-\cos ^{2} \alpha-\cos ^{2} \beta-\cos ^{2} \gamma+2 \cos \alpha \cos \beta \cos \gamma\right]\right. \\
& /\left[\left(\frac{h}{a}\right)^{2} \sin ^{2} \alpha+\left(\frac{k}{b}\right)^{2} \sin ^{2} \beta+\left(\frac{l}{c}\right)^{2} \sin ^{2} \gamma\right. \\
& -\frac{2 k l}{b c}(\cos \alpha-\cos \beta \cos \gamma)-\frac{2 h l}{a c}(\cos \beta \\
& \left.\left.-\cos \alpha \cos \gamma)-\frac{2 h k}{a b}(\cos \gamma-\cos \alpha \cos \beta)\right]\right\}^{1 / 2}
\end{aligned}
$$

where $h, k$, and $l$ are the Miller indices. Using this equation it can be shown that for cubic crystals $\left(a=b=c\right.$ and $\left.\alpha=\beta=\gamma=90^{\circ}\right)$ the expansion

$$
\frac{\Delta d}{d}=\frac{\Delta a}{a}
$$

can be measured by using the reflection from any crystal plane. In the case of hexagonal crystals $\left(a=b \neq c\right.$ and $\left.\alpha=\beta=\gamma=90^{\circ}\right)$

$$
\begin{aligned}
\frac{\Delta d}{d}=\left[\frac{4 c^{2}}{3 a^{2}}\left(h^{2}+h k+k^{2}\right) \frac{\Delta a}{a}+l^{2} \frac{\Delta c}{c}\right] & \\
& /\left[\frac{4 c^{2}}{3 a^{2}}\left(h^{2}+h k+k^{2}\right)+l^{2}\right]
\end{aligned}
$$

the expansion in the $a$ and $c$ directions must be determined from simultaneous equations except for special planes such as (100) and (010) where

$$
\frac{\Delta d}{d}=\frac{\Delta a}{a}
$$

and (001) where

$$
\frac{\Delta d}{d}=\frac{\Delta c}{c}
$$

To obtain accurate data with an x-ray camera it is necessary to measure the film shrinkage so that the angles can be determined. The position of the diffraction lines should be determined with a travelling microscope or a microdensitometer. To obtain accurate data with an x-ray diffractometer the furnace must be mounted on a precision wideangle goniometer. In both techniques it must be possible to aline and maintain the sample on the focusing circle, see figure 19. Any tilt of the sample surface about the axis of the goniometer or the plate holder results in broadened x-ray lines, as part of the surface will be above or below the focusing circle. A displacement of the sample surface in the $X$ direction from the focusing circle by an amount $S$ will cause an error in the measurement of $d$ by as much as

$$
\frac{\Delta d}{d}=\frac{S}{R} \frac{\cos ^{2} \theta}{\sin \theta}
$$

where $R$ is the radius of the circle. Determinations of $d$, therefore, are very sensitive to sample dis-

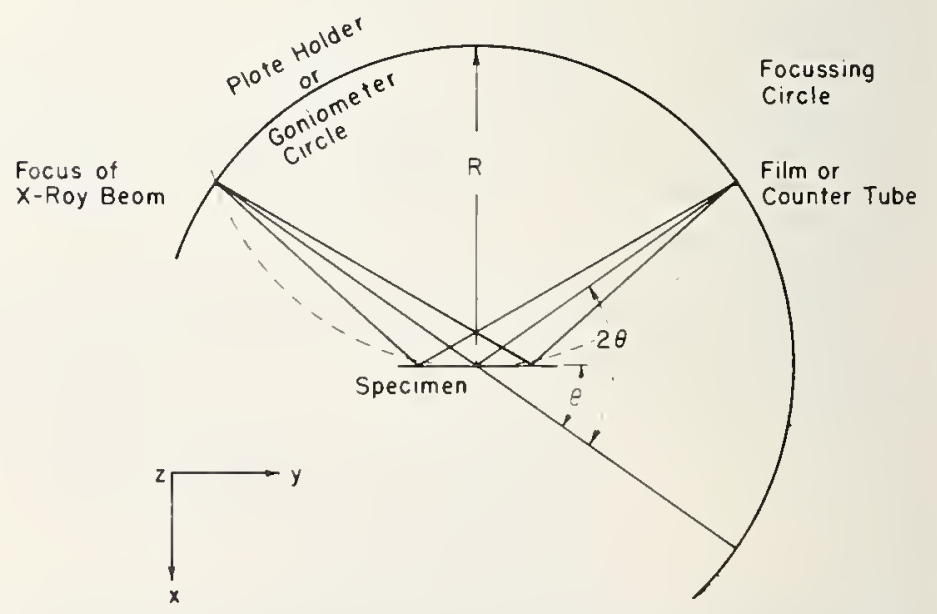

FIgURE 19. Focusing conditions for an $x$-ray diffractometer. 
placement in the front reflection region with sensitivity rapidly decreasing at higher angles.

Furnaces that have precision sample movements $[245,246]$ provide results that are equivalent in resolution and line profile to conventional $\mathrm{x}$-ray techniques. There is a 10 to 25 percent loss of intensity due to absorption in the furnace windows and a reduction of from 5 to $40^{\circ}, 2 \theta$, in useful angular range due to limiting windows, radiation shields, etc. Because of this reduction in angular range some back-reflection cameras and diffractometers are not able to measure the more complex crystal systems such as the orthorhombic, monoclinic, and triclinic.

Because of the small size of x-ray furnaces and the relatively large window area, thermal gradien ts exist in the sample which makes accurate temperature measurements difficult to obtain. Temperature gradients across the sample also results in broadened x-ray lines. At temperatures around $1300 \mathrm{~K}$ there can be thermal gradients of 20 to $30 \mathrm{~K} / \mathrm{cm}$ across the sample and 100 to $600 \mathrm{~K} / \mathrm{cm}$ through the sample holder. Since the penetration depth of the $x$-rays is only about $0.01 \mathrm{~cm}$, the temperature of the surface must be measured. If a thermocouple is used it should, therefore, be attached to the front surface of the sample with the junction in the x-ray beam and the leads also in contact with the surface. If possible, providing there is no alloying or reactions, an internal standard of known expansion should be used to measure the temperature.

\subsection{Reference Materials}

Two reference materials, chemically pure platinum and copper, are recommended by ASTM standards [230, 235] for calibrating thermal expansion apparatus in the range 100 to $1200 \mathrm{~K}$. Platinum, silver, gold, iron, tungsten, and $\mathrm{MgO}$ have also been used with success in high-temperature $\mathrm{x}$-ray measurements. In using these materials it is hoped that their thermal expansion, even when purchased from different suppliers, will be close to the tabulated literature values. Since there is some doubt that these materials will behave as hoped, the National Bureau of Standards, through its Office of Standard Reference Materials, will shortly offer a group of materials for sale that will have certified values of thermal expansion. These reference materials will include vitreous silica, borosilicate glass, single-crystal sapphire, copper, and tungsten and will be certified within the range 20 to $1900 \mathrm{~K}$.

\section{Effect of Some Characterization Parameters on Thermal Expansion}

As indicated in the introduction, the thermal expansion behavior of a ceramic is in general not greatly influenced by its history or character. A glass, for example, should have about the same expansivity as a crystal from the same melt even though it does not have the long-range structure of the crystal. Porosities of up to 50 percent do not alter the expansivity but do have a drastic effect on mechanical properties. Shifts from stoichiometry, a slight loss of oxygen in $\mathrm{TiO}_{2}$, for instance, can cause a striking change in electrical conductivity but little or no change in expansivity. Some parameters of character that do affect thermal exparsion will be discussed.

\subsection{Amorphous and Crystalline Phases}

Materials that form glasses usually have a crystalline form characterized by an open structure and because of the high viscosity of their melts a random structure characteristic of the melt can be frozen in by rapid cooling. Glasses are therefore said to be in metastable equilibrium relative to the crystalline form. An idealized diagram of a glass-crystal system is shown in figure 20. In the range between the melting temperature and the glass transition temperature, $T_{g}$, the atomic configuration possesses a degree of randomness which is temperature dependent. Because the viscosity is increasing very rapidly in this range, it is possible to freeze in different configurations by varying the rate of cooling or by quenching from different temperatures within the range. This results in glasses having different specific volumes at room temperature.

Figure 21 indicates the sort of results that are obtained with borosilicate glass [247]. On fast cooling from above $T_{g}$ a high volume structure is obtained and on very slow cooling a low volume structure is obtained. On slow heating of the quenched glass it will expand normally to a temperature near $T_{g}$ but then its volume will decrease toward that of the fine annealed glass before

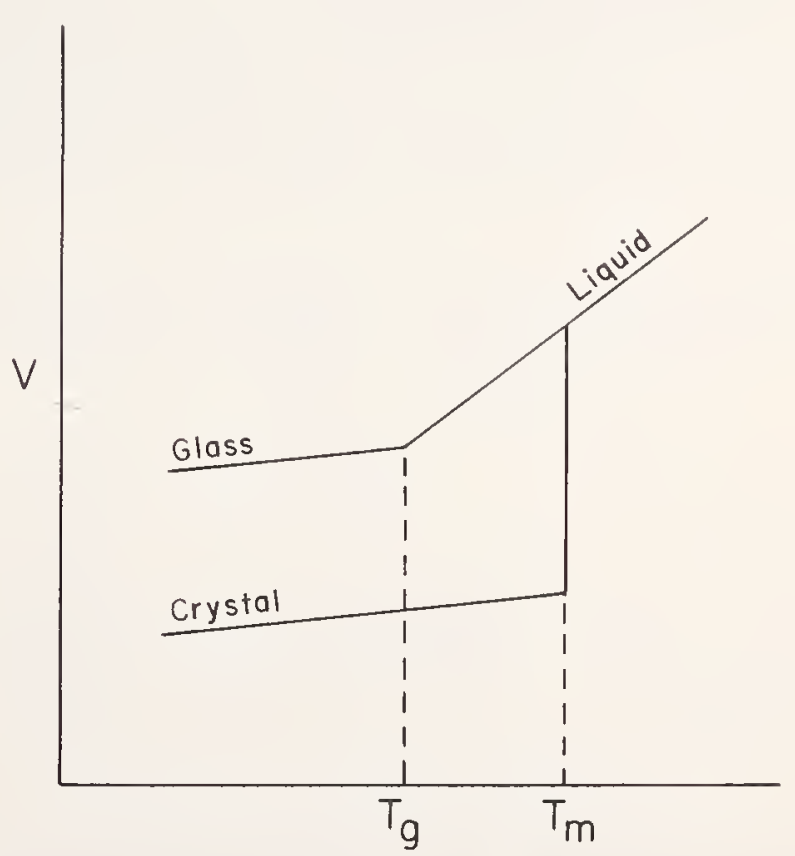

Figure 20. Volume-temperature diagram for an ideal glass former. 


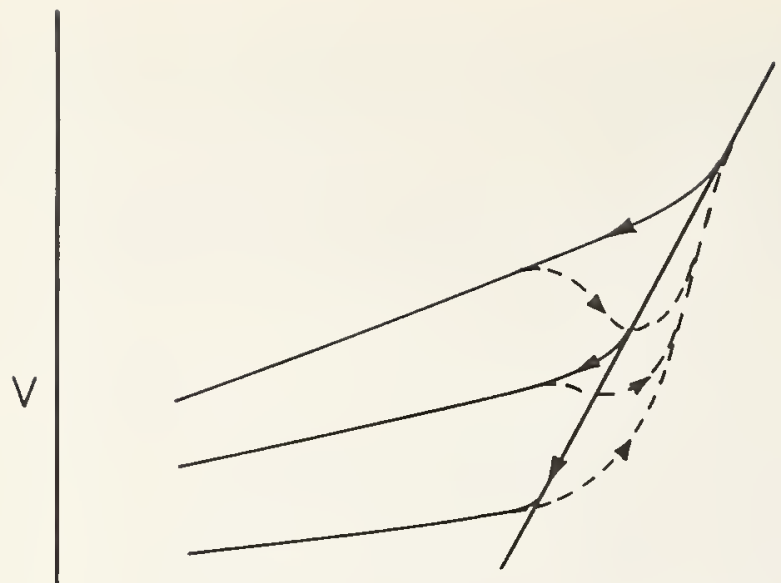

$T$

FIGURE 21. Volume-temperature diagram for a borosilicate glass.

again expanding. On the other hand, the rapid heating of the fine annealed glass will carry its low volume structure to temperatures above $T_{\mathrm{g}}$.

It is expected that a glass characterized by different densities would have different expansivities. Ritland [83] has found this to be the case for borosilicate glasses, as indicated in figure 21 , and Brueckner [248] has found the same behavior in vitreous silica. Ritland also pointed out that there is a difference between the expansivity of a glass that has been quenched and that of the same glass that has been slowly cooled, even though the same specific volume had been obtained.

The volume dependence of several glass-crystal systems is shown in figures 22,23 , and 24 . In the $\mathrm{B}_{2} \mathrm{O}_{3}$ system the relationship between the crystalline and glassy states is more or less normal with the glass having the largest expansivity. The situation for $\mathrm{SiO}_{2}$ is not so clear, however, because of the 22 phases that can form. It is of interest, however, that all of the stable high-temperature

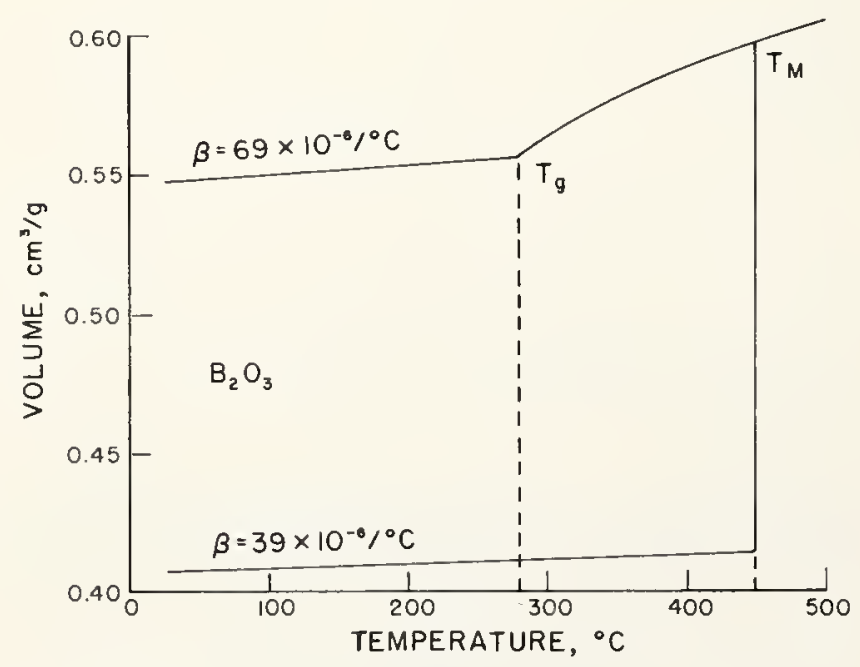

Figure 22. Volume-temperature diagram for $\mathrm{B}_{2} \mathrm{O}_{3}$.

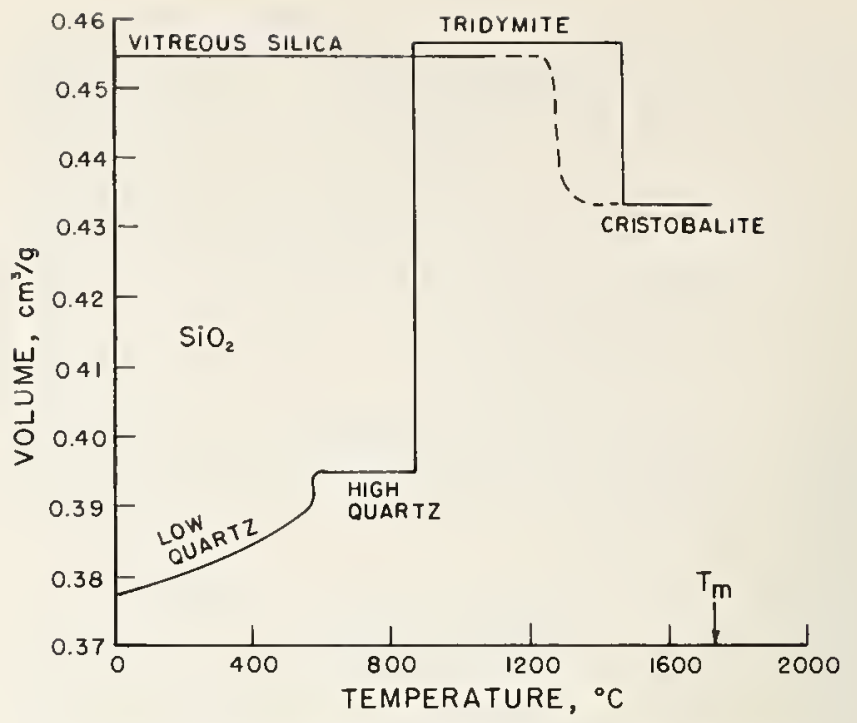

Figure 23. Volume-temperature diagram for $\mathrm{SiO}_{2}$.

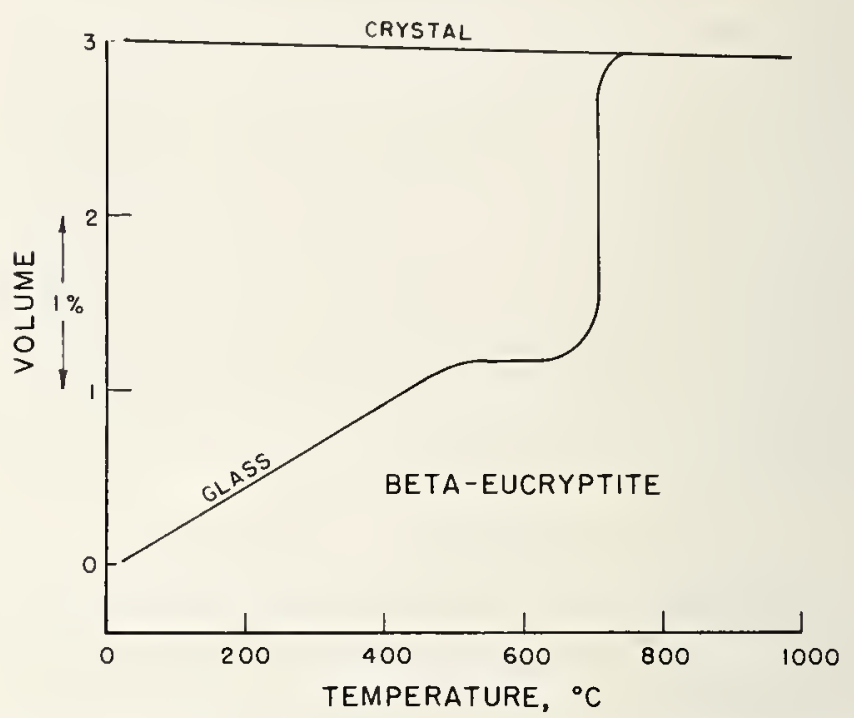

FIGURE 24. Volume-temperature diagram for eucryptite $\left(\mathrm{Li}_{2} \mathrm{O} \cdot \mathrm{Al}_{2} \mathrm{O}_{3} \cdot 2 \mathrm{SiO}_{2}\right)$.

crystalline phases have near-zero expansivity and that when silica glass is heated to a high enough temperature it will crystallize in the cristobalite phase. Silica is therefore normal in that the glassy and crystalline states of the same configuration have about the same expansivity. It is, of course, the near-zero expansivity of vitreous silica that makes it so useful.

A metastable crystalline phase of $\mathrm{Li}_{2} \mathrm{O} \cdot \mathrm{Al}_{2} \mathrm{O}_{3}$ .2 $\mathrm{SiO}_{2}, \quad \beta$-eucryptite, also has a near-zero expansivity at room temperature. This glass-crystal system does not seem to be normal in that the glassy phase has a higher density and expansivity than the crystalline phase. The structure of $\beta$-eucryptite is similar to that of silica, however, and is described as a stuffed derivative of the $\beta$-quartz structure where $\mathrm{Al}^{3+}$ ions are substituted for $\mathrm{Si}^{4+}$ ions in the network and $\mathrm{Li}^{+}$ions are positioned in the interstitial openings to preserve electrical balance [207]. In these materials the near-zero expansion is presumably associated 
with transverse optical modes, the transverse vibrations of the oxygen atoms in the open network structure.

Figure 25 indicates other compositions in the $\mathrm{Li}_{2} \mathrm{O}-\mathrm{Al}_{2} \mathrm{O}_{3}-\mathrm{SiO}_{2}$ system which also have the property of near-zero expansion. Compositions in this system are used in the glass ceramic materials that are finding wide application. The expansivities of compositions along the section between $\mathrm{SiO}_{2}$ and $\mathrm{Li}_{2} \mathrm{O} \cdot \mathrm{Al}_{2} \mathrm{O}_{3}$ are shown in figure 26. The expansivity of the crystalline phases stays near zero from high $\mathrm{SiO}_{2}$ content to eucryptite. The expansivity of the glassy phase, however, increases linearly from $\mathrm{SiO}_{2}$ to spodumene. This type of behavior is expected when two network formers are involved. The significantly lower expansivity of the glass corresponding to the eucryptite composition is probably caused by the interstitial $\mathrm{Li}^{+}$ions.

\subsection{Quantity of Phases}

The thermal expansion of two-phase composite bodies can be predicted with some but not

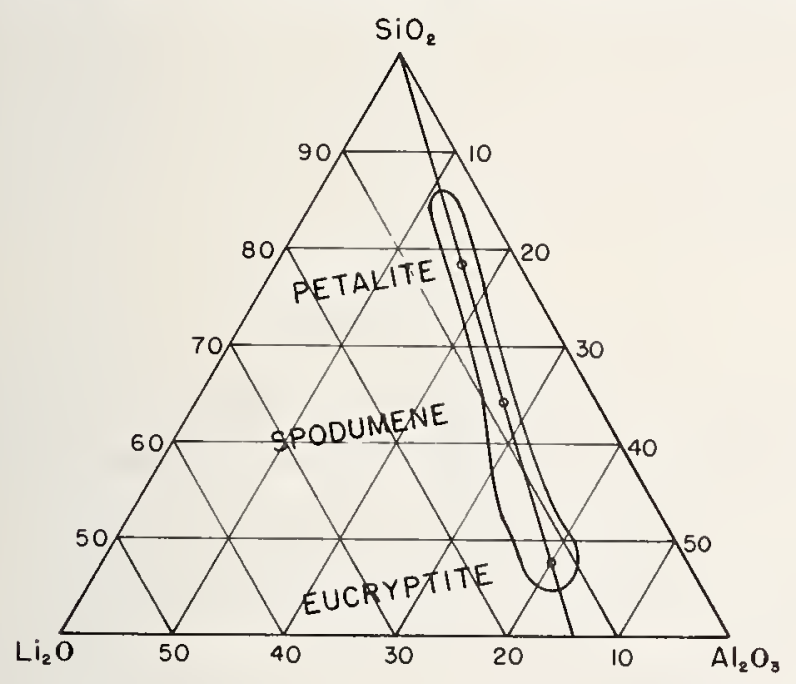

FIguRe 25. Ternary diagram of the $\mathrm{Li}_{2} \mathrm{O}-\mathrm{Al}_{2} \mathrm{O}_{3}-\mathrm{SiO}_{2}$ system showing the compositions that have nearzero expansivity.

$10-$

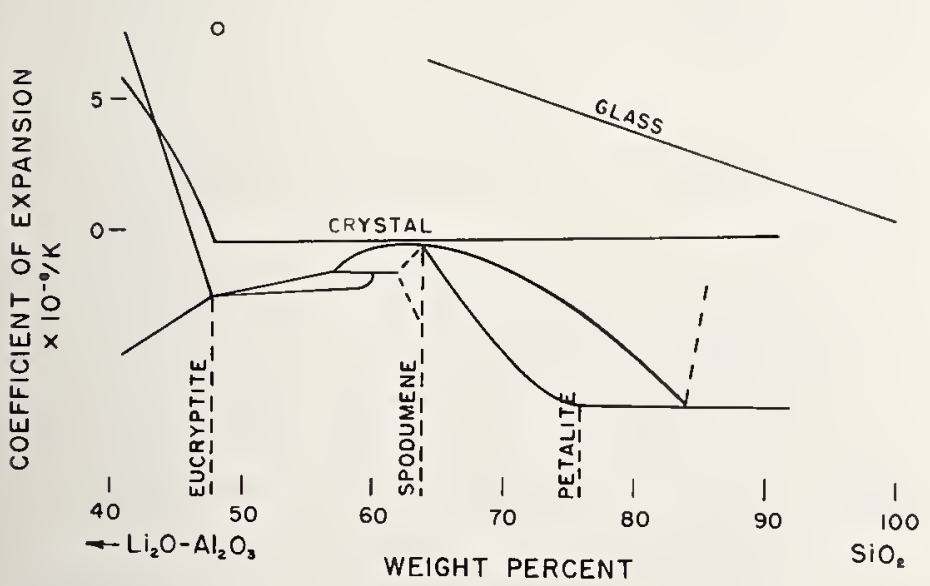

FIGURE 26. Expansivities of the crystalline and glassy phases of the $\mathrm{SiO}_{2}-\mathrm{Li}_{2} \mathrm{O} \cdot \mathrm{Al}_{2} \mathrm{O}_{3}$ system. complete success. An equation derived by Turner [249] seems to be very useful in spite of its simplicity. In his derivation the average coefficient of the composite is given by

$$
\alpha=\frac{\frac{\alpha_{1} E_{1} P_{1}}{d_{1}}+\frac{\alpha_{2} E_{2} P_{2}}{d_{2}}}{\frac{E_{1} P_{1}}{d_{1}}+\frac{E_{2} P_{2}}{d_{2}}}
$$

where $\alpha_{1}$ and $\alpha_{2}, E_{1}$ and $E_{2}, d_{1}$ and $d_{2}$, and $P_{1}$ and $P_{2}$ are the coefficients, Young's modulus, density, and weight fractions of the two phases, respectively. Kerner [250] has developed an equation based on a model that takes shear stresses into consideration. In his derivation the average coefficient is given by

$$
\alpha=\left(4 \frac{G}{K}+3\right)\left[\frac{\alpha_{1} P_{1}}{4 \frac{G}{K_{1}}+3}+\frac{\alpha_{2} P_{2}}{4 \frac{G}{K_{2}}+3}\right]
$$

where $G$ and $K$ are the shear and bulk moduli of the composite body and $K_{1}$ and $K_{2}$ are the bulk moduli of the two phases. Kerner has also developed equations for predicting the values of the shear and bulk moduli in composites but they are very difficult to use.

Fortunately, a paper by Hunter and Brownell [201] provides all of the data that is needed to compare Turner's and Kerner's equations. Figure 27 shows such a comparison for composites formed from spinel and alumina. It can easily be seen that the values of the coefficient calculated with Turner's equation are in good agreement with the experimentally determined values. Figure 28 shows the same comparison for composites formed from a borosilicate glass and alumina. In this case neither equation represents the experimental values.

\subsection{Grain Size and Formation of Cracks}

Grain size, as such, does not affect the thermal expansion of ceramics. In anisotropic crystals, however, two effects of grain size have been

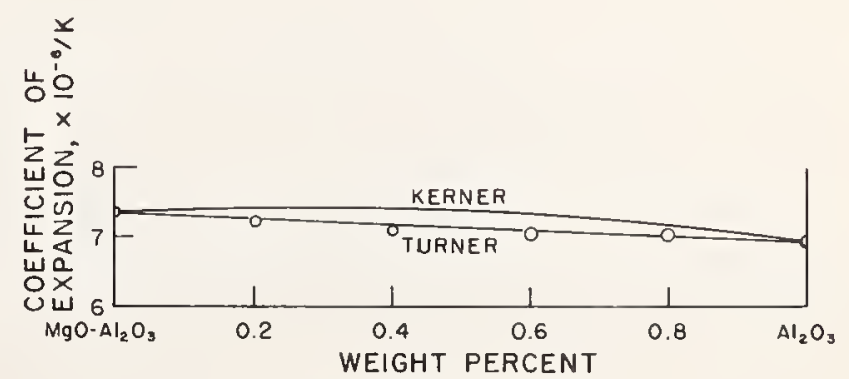

Figure 27. Comparison of the Turner and Kerner equations for calculating expansivity.

Points indicate experimental values for the spinel-alumina composite bodies. 


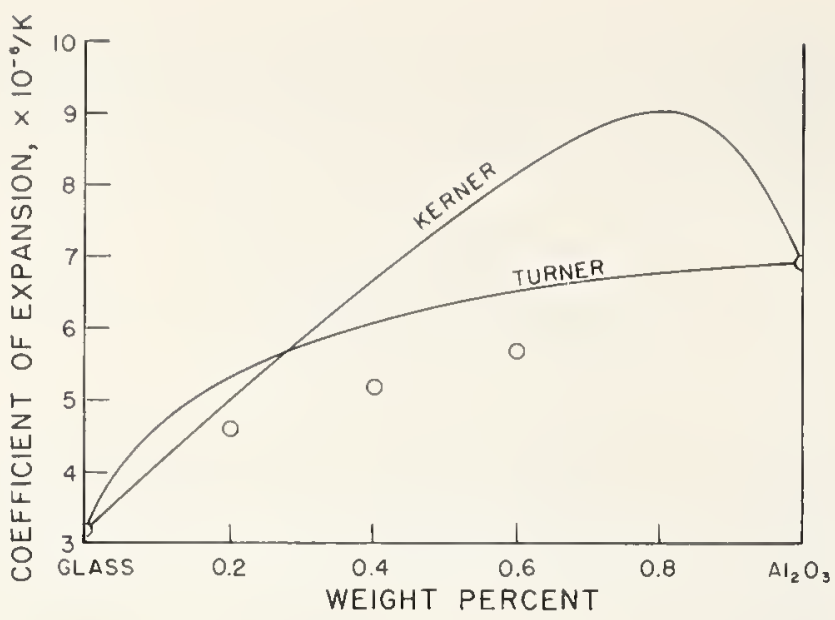

Figure 28. Comparison of the Turner and Kerner equations for calculating expansivity.

Points indicate experimental values for the alumina-borosilicate glass composite bodies.

observed. Fryxell and Chandler [165] found that increasing grain size in $\mathrm{BeO}$ specimens was accompanied by a preferred orientation which caused a change in expansivity. Charvat and Kingery [90], on the other hand, found that large-grained rutile specimens formed microcracks because of grain boundary stresses. This effect caused a hysteresis loop on heating and cooling between 300 and $1400 \mathrm{~K}$. Gillery and Bush [108] observed the same behavior in polycrystalline $\beta$-eucryptite, see figure 29 . Because of the extreme anisotropy of $\beta$-eucryptite, high stresses arise between grains during cooling from the firing temperature and fracture occurs, causing a decrease in density. During reheating the fractures heal and a hysteresis loop is formed.

\subsection{Stress}

From thermodynamic relationships it can be shown that the effect of an elastic stress, $\sigma$ (it does not make any difference whether the stress is applied externally or arises internally from

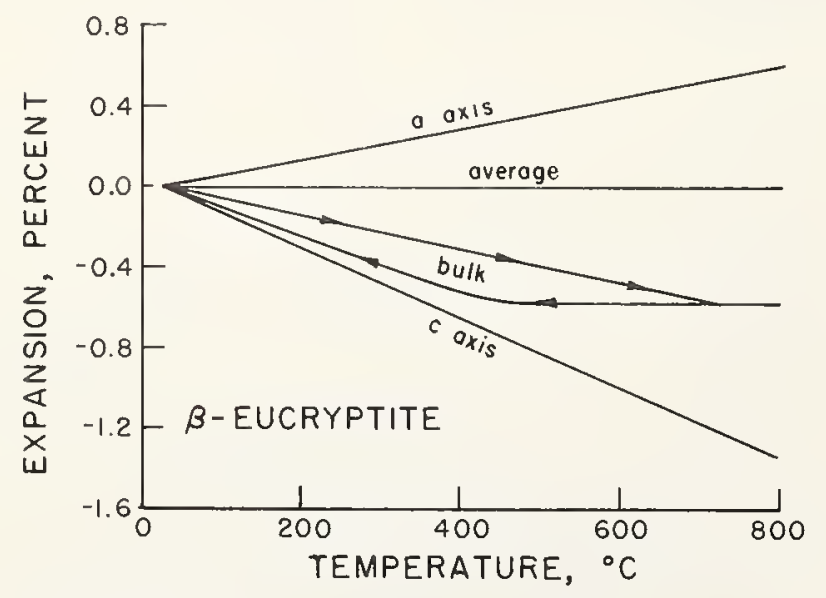

Figure 29. Thermal expansion of $\beta$-eucryptite.

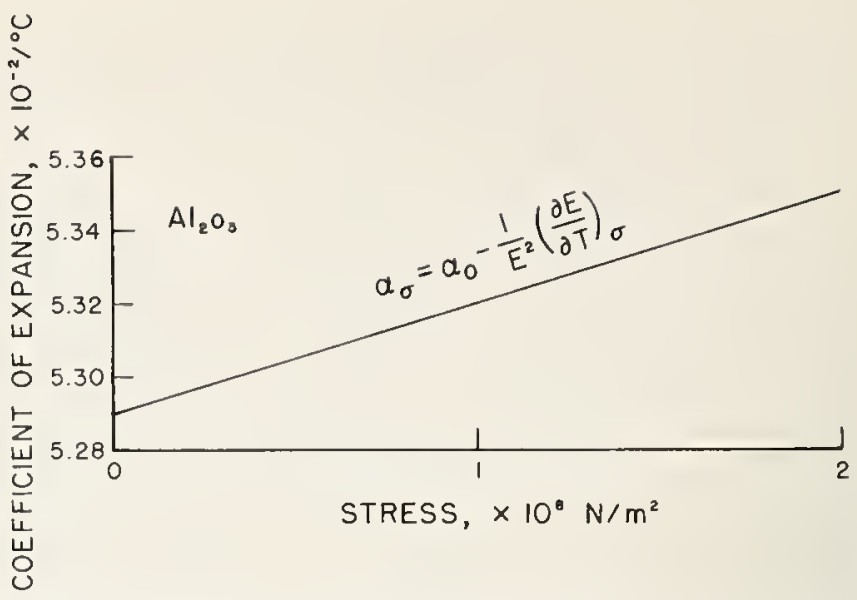

Figure 30. Effect of stress on the expansivity of alumina.

mechanical or thermal causes), on the expansivity of a solid is given by

$$
\left(\frac{\partial \alpha}{\partial \sigma}\right)_{T}=-\frac{1}{E^{2}}\left(\frac{\partial E}{\partial T}\right)_{\sigma}
$$

Rosenfield and Averbach [251] have demonstrated this effect in metals, but there is no indication that anyone has done as much with ceramics. Using a literature value for the temperature coefficient of Young's modulus, a calculation of this effect in alumina was made: the results are shown in figure 30 .

\section{Summary}

The definition of terms used in the field of thermal expansion and a brief review of the theory involved have been given. Four general methods of measuring this thermophysical property have been described, with emphasis placed on the techniques needed to obtain high accuracy. The effects of some characterization parameters on thermal expansion have been discussed. All of the papers published in the Journal of the American Ceramic Society from 1948 through 1967 that contain information on thermal expansion have been referenced. Other survey papers on the expansion of ceramic materials are also referenced $[17,35,252]$.

\section{References}

NoтE: References $1-216$ were published in J. Am. Ceram. Soc.

[1] Staff Owens-Illinois Glass Co., Properties of sodastrontium oxide-alumina-silica glasses, 31, 1 (1948).

[2] Staff Owens-Illinois Glass Co., Effect of boric oxide on properties of soda-dolomitic lime-silica glass, 31, 8 (1948).

[3] Bryant, E. E. and Ammon, M. G., Determination of compressive stress present in porcelain enamel on sheet iron, 31, 28 (1948).

[4] Haystek, H., and Campbell, R. M., Factors involved in production of thin wall tile, 31, 59 (1948).

[5] Plankenhorn, W. J., Refractory ceramic base coats for metal, 31, 145 (1948). 
[6] Tool, A. Q., Effect of heat-treatment on the density and constitution of high-silica glasses of the borosilicate type, 31, 177 (1948).

[7] Shelton, G. R., Creamer, A. S., and Bunting, E. N., Properties of barium-magnesium titanate dielectrics, 31, 205 (1948)

[8] Lamar, R. S., Development of cordierite bodies with sierralite, a new ceramic material, 32, 65 (1949).

[9] Hursh, R. K., Development of a procelain vacuum tube, 32, 75 (1949)

[10] Beck, W. R., Crystallographic inversions of the aluminum orthophosphate polymorphs and their relation to those of silica, 32, 147 (1949).

[11] Gebler, K. A., and Wisely, H. R., Dense cordierite bodies, 32, 163 (1949).

[12] Hummel, F. A., Properties of some substances isostructural with silica, 32, 320 (1949).

[13] Riddle, F. H., Ceramic spark-plug insulators, 32, 333 (1949)

[14] Shevlin, T. S., and Blackburn, A. R., Fundamental study and equipment for sintering and testing of cermet bodies: IV, Development and testing equipment, 32, 363 (1949)

[15] Cook, R. L., and Brunner, C. D., Correlation of glaze-body stresses with thermal properties of whiteware bodies, 32, 401 (1949).

[16] Turnbull, J. C., Recording dilatometer for measuring thermal expansion of solids, 33, 54 (1950).

[17] Hummel, F. A., Observations on the thermal expansion of crystalline and glassy substances, 33, $102(1950)$.

[18] Johnson, A. G., Scholes, S. R., and Simpson, H. E., Volume expansion of glass at high temperatures, 33, $144(1950)$.

[19] Smoke, E. J., Thermally resistant whiteware, 33, $174(1950)$.

[20] Staff Owens-Illinois Glass Co., Effect of $\mathrm{K}_{2} \mathrm{O}$ and $\mathrm{Li}_{2} \mathrm{O}$ on properties of soda-dolomite lime-silica glasses, 33, 181 (1950).

[21] Duwez, P., and Odell, F., Phase relationships in the system zirconia-ceria, 33, 274 (1950).

[22] Gangler, J. J., Some physical properties of eight refractory oxides and carbides, 33, 367 (1950).

[23] Van Gordon, D. V., High-fire opaque glazes for zir 3on bodies, 34, 33 (1951)

[24] Smoke, E. J., Ceramic compositions having negative linear thermal expansion, 34, 87 (1951)

[25] Brackbill, C. E., MeKinstry, H. A., and Hummel, F. A., Thermal expansion of some glasses in the system $\mathrm{Li}_{2} \mathrm{O}-\mathrm{Al}_{2} \mathrm{O}_{3}-\mathrm{SiO}_{2}, 34,107$ (1951).

[26] Hummel, F. A., Thermal expansion properties of some synthetic lithia minerals, 34, 235 (1951).

[27] Ricker, R. W., and Hummel, F. A., Reactions in the system $\mathrm{TiO}_{2}-\mathrm{SiO}_{2}$ revision of the phase diagram, 34, 271 (1951).

[28] Yoshiki, B., and Matsumoto, K., High-temperature modification of barium feldspar, 34, 283 (1951).

[29] Hummel, F. A., and Reid, H. W., Thermal expansion of some glasses in the system $\mathrm{MgO}-\mathrm{Al}_{2} \mathrm{O}_{3}-\mathrm{SiO}_{2}, \mathbf{3 4}$, 319 (1951).

[30] Blackburn, A. R., and Sherlin, T. S., Fundamental study and equipment for sintering and testing of cermet bodies: V Fabrication, testing, and properties of 30 chromium-70 alumina cermets, $\mathbf{3 4}, 327$ (1951).

[31] Beals, R. J., and Cook, R. L., Low-expansion cordierite porcelains, 35, 53 (1952)

[32] Hummel, F. A., Significant aspects of certain ternary compounds and solid solutions, 35, 64 (1952).

[33] Yoshiki, B., and Yoshida, R., Composition of lowalkali glass, 35, 166 (1952)

[34] Karkhanavala, M. D., and Hummel, F. A., Thermal expansion of some simple glasses, 35, 215 (1952).

[35] Austin, J. B., Thermal expansion of nonmetallic crystals, 35, 243 (1952).

[36] Abe, T., Borosilicate glasses, 35, 284 (1952).

[37] Lieberman, A., and Crandall, W. B., Design and construction of a self-calibrating dilatometer for high-temperature use, 35, 304 (1952).
[38] Schwartz, B., The mal stress failure of pure refractory oxides, 35, 325 (1952).

[39] Shartsis, L., Capps, W., and Spinner, S., Density and expansivity of alkali borates and density characteristics of some other binary glasses, $\mathbf{3 6}$, 35 (1953)

[40] Curtis, C. E., and Sowman, H. G., Investigation of the thermal dissociation, reassociation, and synthesis of zircon, 36, 190 (1953).

[41] Comeforo, J. E., Hatch, R. A., Humphrey, R. A., and Eitel, W., Synthetic mica investigation: I, A hot-pressed machinable ceramic dielectric, $\mathbf{3 6}$, 286 (1953).

[42] Walton, J. D., Jr., and Sweo, B. J., Determination of strains between enamel and iron by means of split rings, 36, 335 (1953).

[43] Skinner, K. G., Cook, W. H., Potter, R. A., and Palmour, H., III, Effect of $\mathrm{TiO}_{2}, \mathrm{Fe}_{2} \mathrm{O}_{3}$, and alkali on mineralogical and physical properties of mullite-type and mullite-forming $\mathrm{Al}_{2} \mathrm{O}_{3}-\mathrm{SiO}_{2}$ mixtures: I., 36, 349 (1953).

[44] Goodman, G., Ferroelectric properties of lead metaniobate, 36, 368 (1953).

[45] Karkhanavala, M. D., and Hummel, F. A., The polymorphism of cordierite, 36, 389 (1953).

[46] Quirk, J., and Harman, C. G., Properties of a tin oxide-base ceramic body, 37, 24 (1954).

[47] Walton, J. D., Jr., Study of strains between enamel and iron as related to physical properties of each, 37, 153 (1954)

[48] Mitoff, S. P., and Pask, J. A., Thermal strains during firing of a ceramic body, 37, 168 (1954).

[49] Harrison, D. E., McKinstry, H. A., and Hummel, F. A., High-temperature zirconium phosphates, 37, 277 (1954)

[50] Plankenhorn, W. J., Effect of ceramic coatings on fatigue strength of metal, 3\%, 281 (1954).

[51] Navias, L., Advances in ceramics related to electronic tube developments, 3\%, 329 (1954).

[52] Curtis, C. E., Doney, L. M., and Johnson, J. R., Some properties of hafnium oxide, hafnium silicate, calcium hafnate, and hafnium carbide, $\mathbf{3 7}$, 458 (1954).

[53] Shartsis, L., and Shermer, H. F., Surface tension, density, viscosity, and electrical resistivity of molten binary alkaline-earth borates, 3\%, 544 (1954).

[54] Lamar, R. S., and Warner, M. F., Reaction and fired-property studies of cordierite compositions, 37, 602 (1954)

[55] Kingery, W. D., Factors affecting thermal stress resistance of ceramic materials, 38, 3 (1955).

[56] Buessem, W. R., Thermal shock testing, 38, 15 (1955).

[57] Manson, S. S., and Smith, R. W., Theory of thermal shock resistance of brittle materials based on Weibull's statistical theory of strength, 38, 18 (1955).

[58] Buessem, W. R., and Bush, E. A., Thermal fracture of ceramic materials under quasi-static thermal stresses (ring test), 38, 27 (1955).

[59] Coble, R. L., and Kingery, W. D., Effect of porosity on thermal stress fracture, 38, 33 (1955).

[60] Baroody, E. M., Simons, E. M., and Duckworth, W. H., Effect of shape on thermal fracture, 38, 38 (1955).

[61] Crandall, W. B., and Ging, J., Thermal shock analysis of spherical shapes, 38, 44 (1955).

[62] Brown, F. H., Jr., and Duwez, P., The systems zirconia-lanthana and zirconia-neodymia, 38, 95 (1955).

[63] Smyth, H. T., Thermal expansion of vitreous silica, 38, 140 (1955).

[64] Nadler, M. R., and Fitzsimmons, E. S., Preparation and properties of calcium zirconate, 38, 214 (1955).

[65] Koenig, C. J. Influence of particle-size distribution on the properties of nepheline syenite, $\mathbf{3 8}, 231$ (1955). 
[66] King, B. W., and Suber, L. L., Some properties of the oxides of vanadium and their compounds, 38, 306 (1955).

[67] Knudsen, F. P., Moreland, R. E., and Gcller, R. F., Physical characteristics of titanium carbide type cermets at elevated temperatures, 38, 312 (1955).

[68] Van Gordon, D. V., and Spangenberg, W. C., Adjustment of thermal expansion of cone 8 glazes, 38, 331 (1955).

[69] Wuellner, J. F., and Sweo, B. J., Determination of strains in enameled cast iron, 38, 404 (1955).

[70] Sherlin, T. S., and Hauck, C. A., Fundamental study and equipment for sintering and testing of cermet bodies: VII, Fabrication, testing, and properties of $34 \mathrm{Al}_{2} \mathrm{O}_{3}-66 \mathrm{Cr}-\mathrm{Mo}$ Cermets, 38, 450 (1955).

[71] Sutton, W. H., and Matson, F. R., Factors affecting strength of clays in the temperature range 110 to $800^{\circ} \mathrm{C}, 39,25$ (1956).

[72] Lachman, I. M., and Everhart, J. O., Development of safe cooling schedules for structural clay products, 39, 30 (1956).

[73] Russell, R., Jr., Valencia, C., and Emrich, H. W., Barite in ceramic whitewares, 39, 73 (1956).

[74] Dalton, R. H., Solder glass sealing, 39, 109 (1956).

[75] Burdick, M. D., and Parker, H. S., Effect of particle size on bulk density and strength properties of uranium dioxide specimens, 39, 181 (1956).

[76] Hedden, W. A., and King, B. W., Antimony oxide glasses, 39, 218 (1956).

[77] Godejahn, G. C., Jr., and Cook, R. L., Effect of zircon and zirconia on thermal expansion of lead borosilicate glazes, 39, 256 (1956).

[78] Lauchner, J. H., Cook, R. L., and Andrews, A. I., Fundamental thermal defiection analysis of enamel-metal systems, 39, 281 (1956).

[79] Vukovich, M., Jr., Effect of wollastonite substitutions in a semivitreous dinnerware body, 39, 323 (1956)

[80] Shafer, E. C., and Roy, R., Studies of silica-structure phases: I, $\mathrm{GaPO}_{4}, \mathrm{GaAsO}_{4}$, and $\mathrm{GaSbO}_{4}, \mathbf{3 9}, 330$ (1956).

[81] Kelly, H. J., and Harris, H. M., An automatically recording thermal-expansion apparatus, 39, 344 (1956).

[82] Coble, R. L., and Kingery, W. D., Effect of porosity on physical properties of sintered alumina, $\mathbf{3 9}$, 377 (1956).

[83] Ritland, H. N., Limitations of the fictive temperature concept, 39, 403 (1956).

[84] Lang, S. M., and Kundsen, F. P., Some physical properties of high-density thorium dioxide, 39, 415 (1956).

[85] Whittemore, O. J., Jr., and Ault, N. N., Thermal expansion of various ceramic materials to $1500{ }^{\circ} \mathrm{C}$, 39, 443 (1956).

[86] Curtis, C. E., Properties of yttrium oxide ceramics, 40, 274 (1957).

[87] Belas, R. J., and Cook, R. L. Directional dilatation of crystal lattices at elevated temperatures, 40, 279 (1957).

[88] Juggins, M. L., and Abe, T., Structure of borate glasses, 40, 287 (1957).

[89] Mattyasovszky-Zsoluoy, L., Mechanical strength of porcelain, 40, 299 (1957).

[90] Charvat, F. R., and Kingery, W. D., Thermal conductivity: XIII, Effect of microstructure on conductivity of single-phase ceramics, 40,306 (1957).

[91] Duwez, P., and Loh, E., Phase relationships in the system zirconia-thoria, 40, 321 (1957).

[92] Kingery, W. D., Note on thermal expansion and microstresses in two-phase compositions, 40, 351 (1957).

[93] Lauchner, J. H., Cook, R. L., andAn drews, A. I., Effect of firing schedules on stress-temperature relations in enamel-metal systems, 40, 410 (1957).

[94] Hagy, H. E., and Ritland, H. N., Effect of thermal history on glass expansion characteristics, 40, 436 (1957).
[95] Sastry, B. S. R., and Hummel, F. A., Studies in lithium oxide systems: I, $\mathrm{Li}_{2} \mathrm{O} \cdot \mathrm{B}_{2} \mathrm{O}_{3}-\mathrm{B}_{2} \mathrm{O}_{3}, \mathbf{4 1}, 7$ (1958).

[96] Newkirk, H. W., Jr., and Sisler, H. H., Determination of residual stresses in titanium carbide-base cermets by high-temperature $\mathrm{x}$-ray diffraction, 41, 93 (1958).

[97] Simon, I., Note on thermal expansion of neutronirradiated silica, 41, 116 (1958).

[98] Bush, E. A., and Hummel, F. A., High-temperature mechanical properties of ceramic materials: I, Magnesium dititanate, 41, 189 (1958).

[99] Strimple, J. H., and Giess, E. A., Glass formation and properties of glasses in the system $\mathrm{Na}_{2} \mathrm{O}-\mathrm{B}_{2} \mathrm{O}_{3}-$ $\mathrm{SiO}_{2}-\mathrm{TiO}_{2}, 41,231$ (1958).

[100] Hafner, H. C., Kreidl, N. J., and Weidel, R. H., Optical and physical properties of some calcium aluminate glasses, 41, 315 (1958).

[101] Goughanour, L. W., Shartsis, L., and Shermer, H. F. Viscosity, density, and electrical resistivity of molten alkaline-earth borate glasses with 3 mole $\%$ of potassium oxide, $\mathbf{4 1}, 324$ (1958).

[102] King, B. W., and Kelly, G. D., Infrared-transmitting glasses in the system $\mathrm{K}_{2} \mathrm{O}-\mathrm{Sb}_{2} \mathrm{O}_{3}-\mathrm{Sb}_{2} \mathrm{~S}_{3}, \mathbf{4 1}, 367$ (1958).

[103] Ploetz, G. L., Krystyniak, C. W., and Dumas, H. E., Sintering characteristics of rare-earth oxides, 41, 551 (1958).

[104] Long, G., and Foster, L. M., Aluminum nitride, a refractory for aluminum to $2000^{\circ} \mathrm{C}, 42,53$ (1959).

[105] Ranch, H. W., Commons, C. H., Jr., and Blau, H. H., Exploration of lithia glassforming systems: I, $\mathrm{Li}_{2} \mathrm{O}-\mathrm{CaO}-\mathrm{Al}_{2} \mathrm{O}_{3}-\mathrm{SiO}_{2}$ at different levels from 0 to 30 weight $\% \mathrm{CaO}, \mathbf{4 2}, 113$ (1959).

[106] Lauchner, J. H., and Bennett, D. G., Thermal fracture resistance of ceramic coatings applied to metals I, Elastic deformation, 42, 146 (1959).

[107] Curtis, C. E., and Tharp, A. G., Ceramic properties of europium oxide, 42,151 (1959).

[108] Gillery, F. H., and Bush, E. A., Thermal contraction of $\beta$-eucryptite $\left(\mathrm{Li}_{2} \mathrm{O} \cdot \mathrm{Al}_{2} \mathrm{O}_{3} \cdot 2 \mathrm{SiO}_{2}\right)$ by $\mathrm{x}$-ray and dilatometer methods, 42, 175 (1959).

[109] Schneider, S. J., Effect of heat-treatment on the constitution and mechanical properties of some hydrated aluminous cements, 42, 184 (1959).

[110] Engberg, C. J., and Zehms, E. H., Thermal expansion of $\mathrm{Al}_{2} \mathrm{O}_{3}, \mathrm{BeO}, \mathrm{MgO}, \mathrm{B}_{4} \mathrm{C}, \mathrm{SiC}$ and $\mathrm{TiC}$ above $1000^{\circ} \mathrm{C}, 42,300(1959)$.

[111] Mackenzie, J. D., Density and expansivity of vitreous germania, $\mathbf{4 2}, 310$ (1959).

[112] Carter, R. E., Thermal expansion of $\mathrm{MgFe}_{2} \mathrm{O}_{4}$, FeO and $\mathrm{MgO} \cdot 2 \mathrm{FeO}, 42,324$ (1959).

[113] Bush, E. A., and Hummel, F. A., High-temperature mechanical properties of ceramic materials: II, $\beta$-eucryptite, 42, 388 (1959).

[114] Harrison, D. E., and Hummel, F. A., Reactions in the system $\mathrm{TiO}_{2}-\mathrm{P}_{2} \mathrm{O}_{5}, \mathbf{4 2}, 487$ (1959).

[115] Ramsey, T. H., Jr., Summary of some properties of yttrium iron garnet and study of dislocations observed in an yttrium iron garnet crystal, 42 , 645 (1959)

[116] Tilton, L. W., Structural and thermal expansions in alkali silicate binary glasses, 43, 9 (1960).

[117] Sastry, B. S. R., and Hummel, F. A., Studies in lithium oxide systems: VII, $\mathrm{Li}_{2} \mathrm{O}-\mathrm{B}_{2} \mathrm{O}_{3}-\mathrm{SiO}_{2}, 43$, $23(1960)$.

[118] Wilson, W. B., High-temperature x-ray diffraction investigation of the uranium-carbon system, $\mathbf{4 3}$, 77 (1960).

[119] Goodman, G., Ferroelectric behavior in barium zirconium metaniobate, 43, 105 (1960).

[120] Subbarao, E. C., Studies on lead titanate ceramics containing niobium or tantalum, 43, 119 (1960).

[121] Ploetz, G. L., Muccigrosso, A. T., Osika, L. M., and Jacoby, W. R., Dysprosium oxide ceramies, 43, $154(1960)$.

[122] Flaschen, S. S., Pearson, A. D., and Northover, W. R., Formation and properties of low-melting 
glasses in the ternary systems As-Tl-S, As-Tl-Se and As-Se-S, 43, 274 (1960).

[123] Subbarao, E. C., X-ray study of phase transitions in ferroelectric $\mathrm{PbNb}_{2} \mathrm{O}_{6}$ and related materials, 43, 439 (1960)

[124] Argyle, J. F., and Hummel, F. A., Dialatometric and $x$-ray data for lead compounds: I, 43, 452 (1960).

[125] Baskin, Y., Harada, Y., and Handwerk, J. H., Some physical properties of thoria reinforced by metal fibers, 43, 489 (1960).

[126] Grisaffe, S. J., Thermal expansion of hafnium carbide, 43, 494 (1960).

[127] Subbarao, E. C., Reactions in the system $\mathrm{PbO}-\mathrm{Ta}_{2} \mathrm{O}_{5}$, 44, 92 (1961).

[128] Fenstermacher, J. E., and Hummel, F. A., Hightemperature mechanical properties of ceramic materials: IV, Sintered mullite bodies, 44, 284 (1961).

[129] Dormer, G. J., Norton, G. R., and Payne, B. S., Glassing characteristics of low-alloy steels, 44, 375 (1961).

[130] Warshaw, I., and Roy, R., Thermal-expansion measurements from nonindexed high-temperature x-ray powder patterns, 44, 421 (1961).

[131] Pappis, J., and Blum, S. L., Properties of pyrolytic graphite, 44, 592 (1961).

[132] Hirayama, C., Properties of aluminobarate glasses of group II metal oxides: I, Glass formation and thermal expansion, 44, 602 (1961).

[133] Taylor, R. E., Thermal conductivity and expansion of beryllia at high temperatures, 45, 74 (1962).

[134] Pei-Ching, L., Ghose, A. C., and Gong-Jen, S., High temperature density determination of boron oxide and binary rubidium and cesium borates, 45, 89 (1962)

[135] Sarver, J. F., and Hummel, F. A., Stability relations of magnesium metasilicate polymorphs, 45, 152 (1962).

[136] Studt, P. L., and Fulrath, R. M., Mechanical properties and chemical reactivity in mullite, $\mathbf{4 5}$, $182(1962)$

[137] Thornton, H. R., Bond strength and elastic properties of ceramic adhesives, 45, 201 (1962).

[138] Burk, M., Thermal expansion of ceramic materials at -200 to $0^{\circ} \mathrm{C}, \mathbf{4 5}, 305(1962)$

[139] Wachtman, J. B., Jr., Scuderi, T. G., and Cleek, G. W., Linear thermal expansion of aluminum oxide and thorium oxide from 100 to $1100{ }^{\circ} \mathrm{K}$, 45, 319 (1962).

[140] Kim, K. H., and Hummel, F. A., Studies in lithium oxide systems: $\mathrm{XII}, \mathrm{Li}_{2} \mathrm{O}-\mathrm{B}_{2} \mathrm{O}_{3}-\mathrm{Al}_{2} \mathrm{O}_{3}, 45,487$ (1962)

[141] Merz, K. M., Brown, W. R., and Kirchner, H. P., Thermal-expansion anisotropy of oxide solid solutions, 45, 531 (1962).

[142] Subbarao, E. C., Polymorphism and dielectric properties of $\mathrm{Bi}_{2} \mathrm{Ti}_{4} \mathrm{O}_{11}, 45,564$ (1962).

[143] Murthy, M. K., Thermal-expansion properties of $\mathrm{BaTiGe}_{3} \mathrm{O}_{9}, 45,566$ (1962).

[144] Tien, T. Y., and Carlson, W. G., Effect of additives on properties of lead titanate, 45, 567 (1962).

[145] Pask, J. A., and Fulrath, R. M., Fundamentals of glass-to-metal bonding: VIII, nature of wetting and adherence, 45, 592 (1962).

[146] Chessin, H., and Turkdogan, E. T., A crystallographic investigation of calcium diferrite, 45, 597 (1962).

[147] Murthy, M. K., Thermal-expansion properties of vitreous and crystalline germania, 45, 616 (1962).

[148] Argyle, J. F., and Hummel, F. A., Dialtometric and x-ray data for lead compounds, II, 46, 10 (1963).

[149] Eppler, R. A., Glass formation and recrystallization in the lithium metasilicate region of the system $\mathrm{Li}_{2} \mathrm{O}-\mathrm{Al}_{2} \mathrm{O}_{3}-\mathrm{SiO}_{2}, 46,97$ (1963).

[150] Shaffer, P. T. B., and Mark, S. D., Inert-atmosphere dilatometer for use to $2000^{\circ} \mathrm{C}, \mathbf{4 6}, 104$ (1963).
[151] Rao, Bh. V. J., Influence of $\mathrm{TiO}_{2}$ on properties of glasses in the system $\mathrm{K}_{2} \mathrm{O}-\mathrm{PbO}-\mathrm{SiO}_{2}-\mathrm{TiO}_{2}$ and its relation to structure, $\mathbf{4 6}, 107$ (1963).

[152] Kirchner, H. P., Merz, K. M., and Brown, W. R., Thermal expansion of uranium pyrophosphate and and of ceramic bodies in the system $\mathrm{UO}_{2}-\mathrm{UP}_{2} \mathrm{O}_{7}$, 46, 137 (1963).

[153] Shalek, P. D., Preparation and properties of uranium and thorium monosulfides, 46, 155 (1963).

[154] Sarver, J. F., Thermal-expansion data for rutiletype $\mathrm{GeO}_{2}, \mathbf{4 6}, 195$ (1963).

[155] Takamori, T., Effects of thermomechanical history on properties of glasses, 46, 366 (1963).

[156] Nielsen, T. H., and Leipold, M. H., Thermal expansion in air of ceramic oxides to $2200^{\circ} \mathrm{C}, \mathbf{4 6}$, 381(1963).

[157] Carroll, D. F., Thermal expansion of plutonium nitride, 46, 406 (1963)

[158] Mistler, R. E., Ploetz, G. L., and Smith, J. A. Thermal expansion of polycrystalline lanthanum and neodymium sesquioxides, 46, 561 (1963).

[159] Gillam, E., and Holden, J. P., Structure of nickel oxide containing alumina, 46,601 (1963).

[160] Christensen, J. A., Thermal expansion and changes in volume of uranium dioxide on melting, $\mathbf{4 6}, 607$ (1963).

[161] Mendez-Penalosa, R., and Talyor, R. E., Thermal expansion of uranium monocarbide, 47, 101 (1964).

[162] Herczog, A., Microcrystalline $\mathrm{BaTiO}_{3}$ by crystallization from glass, 4\%, 107 (1964).

[163] Nielsen, T. H., and Leipold, M. H., Thermal expansion of yttria-stabilized zirconia, 4\%, 155 (1964).

[164] Nielsen, T. H., and Leipold, M. H., Thermal expansion of yttrium oxide and of magnesium oxide with yttrium oxide, 4\%, 256 (1964).

[165] Fryxell, R. E., and Chandler, B. H., Creep, strength, expansion, and elastic moduli of sintered $\mathrm{BeO}$ as a function of grain size, porosity, and grain orientation, 4\%, 283 (1964).

[166] Houska, C. R., Thermal expansion of certain group IV and group V carbides at high temperatures, 47, 310 (1964).

[167] Murthy, M. K., and Ip, J., Studies in germanium oxide systems: I, Phase equilibria in the system $\mathrm{Li}_{2} \mathrm{O}-\mathrm{GeO}_{2}, 4 \%, 328$ (1964)

[168] Miccioli, B. R., and Shaffer, P. T. B., High-temperature thermal expansion behavior of refractory materials: I, Selected monocarbides and binary carbides, $4 \%, 351$ (1964) (Correction to paper by B. R. Miccioli and P. T. B. Shaffer, 47, 536 (1964).

[169] Ohnysty, B., and Rose, F. K., Thermal expansion measurements on thoria and hafnia to $4500^{\circ} \mathrm{F}, \mathbf{4}^{\%}$, 398 (1964)

[170] Mendez-Penalosa, R., and Taylor, R. E., Thermal expansion of uranium sesquicarbide, $\mathbf{4 7}, 416$ (1964).

[171] Murthy, M. K., and Agnayo, J., Studies in germanium oxide systems: II, Phase equilibria in the system $\mathrm{Na}_{2} \mathrm{O}-\mathrm{GeO}_{2}, \mathbf{4 7}, 444$ (1964)

[172] Soga, N., Thermal expansion properties of lithium orthogermanate and orthosilicate, $\mathbf{4 7}, 469$ (1964)

[173] Takamori, T., and Tomozawa, M., Thermal expansion of a cubic sodium tungsten bronze, $\mathbf{4 7}, 472$ (1964).

[174] Takamori, T., Thermal expansion characteristics of polycrystalline tungsten oxides, 4\%, 534 (1964)

[175] Floyd, J. R., Effect of secondary crystalline phases on dielectric losses in high-alumina bodies, $4 \%, 539$ (1964).

[176] Tien, T. Y., and Hummel, F. A., Studies in lithium oxide systems: $\mathrm{XIII}, \mathrm{Li}_{2} \mathrm{O} \cdot \mathrm{Al}_{2} \mathrm{O}_{3} \cdot 2 \mathrm{SiO}_{2}-\mathrm{Li}_{2} \mathrm{O} \cdot \mathrm{Al}_{2} \mathrm{O}_{3}$. $2 \mathrm{GeO}_{2}, 4 \%, 582$ (1964).

[177] Lang, S. M., Axial thermal expansion of tetragonal $\mathrm{ZrO}_{2}$ between 1150 and $1700^{\circ} \mathrm{C}, 47,641$ (1964).

[178] Fehrenbacher, L. L., and Jacobson, L. A., Metallographic observation of monoclinic-tetragonal phase transformation in $\mathrm{ZrO}_{2}, 48,157$ (1965).

[179] Nielsen, T. H., and Leipold, M. H., Thermal expansion of nickel oxide, 48,164 (1965). 
[180] Merryman, R. G., and Kempter, C. P., Precise temperature measurement in Debyc-Scherrer spccimens at elevated tcmperatures, 48, 202 (1965)

[181] Takamori, T., Low-temperature thermal expansion characteristics of a cubic sodium tungsten bronze, 48, 277 (1965).

[182] Krochmal, J. J., Comment on paper "High-temperature thermal expansion behavior of refractory materials, I'", 48, 328 (1965).

[183] Corvin, I., and Cartz, L., Anisotropic thermal expansion of $\mathrm{V}_{2} \mathrm{O}_{5}, \mathbf{4 8}, 328$ (1965).

[184] Takamori, T., and Tomozawa, M., Behavior of interlayers of glass-to-tungsten seals, 48, 405 (1965).

[185] Stansfield, O. M., Thermal expansion of polycrystalline $\mathrm{HfO}_{2}-\mathrm{ZrO}_{2}$ solid solutions, 48, 436 (1965).

[186] Branson, D. L., Thermal expansion coefficients of zirconate ceramics, $\mathbf{4 8 , 4 4 1}(\mathbf{1 9 6 5 )}$.

[187] Richardson, J. H., Thermal expansion of three group IV A carbides to $2700^{\circ} \mathrm{C}, 48,497$ (1965).

[188] Tennery, V. J., High-temperature phase transitions in $\mathrm{NaNbO}_{3}, 48,537$ (1965).

[189] Napolitano, A., Macedo, P. B., and Hawkins, E. G., $\mathrm{Viscosity}$ and density of boron trioxide, 48, 613 (1965)

[190] Schreiber, E., and Anderson, O. L., Pressure derivatives of the sound velocities of polycrystalline alumina, 49, 184 (1966).

[191] Ulrich, D. R., and Smoke, E. J., Devitrified barium titanate dielectrics, 49, 210 (1966)

[192] Allen, W. C., Temperature dependence of properties of magnesium ferrite, 49, 257 (1966).

[193] Arias, A., Thermal shock resistance of zirconia with 15 mole \% titanium, 49, 334 (1966).

194] Soga, N., and Anderson, O. L., High-temperature elastic properties of polycrystalline $\mathrm{MgO}$ and $\mathrm{Al}_{2} \mathrm{O}_{3}, \mathbf{4 9}, 355$ (1966).

[195] Anderson, O. L., and Andreatch, P., Jr., Pressure derivatives of elastic constants of single-crystal $\mathrm{MgO}$ at 23 and $-195.8{ }^{\circ} \mathrm{C}, \mathbf{4 9}, 404$ (1966).

[196] Aronson, S., Cisney, E., and Auskern, A. B., Thermal expansion of nonstoichiometric zirconium carbides, 49, 456 (1966).

[197] Tennery, V. J., High-temperature phase transitions in $\mathrm{PbZrO}_{3}, \mathbf{4 9}, 483$ (1966).

[198] Foster, P. K., and Hughes, I. R., Internal stress in ceramics containing cristobalite, 49, 515 (1966).

[199] Baskin, Y., Properties of uranium monophosphide, 49, $541(1966)$.

[200] Kruger, O. L., and Moser, J. B., Sintering characteristics and properties of $\mathrm{PuS}$ and $\mathrm{PuP}, \mathbf{4 9}, 661$ (1966).

[201] Hunter, O., and Brownell, W. E., Thermal expansion and elastic properties of two-phase ceramic bodies, 50, 19 (1967).

[202] Riebling, E. F., Volume relations in $\mathrm{Na}_{2} \mathrm{O} \cdot \mathrm{B}_{2} \mathrm{O}_{3}$ and $\mathrm{Na}_{2} \mathrm{O} \cdot \mathrm{SiO}_{2} \cdot \mathrm{B}_{2} \mathrm{O}_{3}$ melts at $1300{ }^{\circ} \mathrm{C}, \mathbf{5 0}, 46(1967)$.

[203] Vergano, P. J., Hill, D. C., and Uhlmann, D. R., Thermal expansion of feldspar glasses, 50, 59 (1967).

[204] Keihn, F. G., and Keplin, E. J., High-temperature thermal expansion of certain group IV and group $\mathrm{V}$ diborides, 50, 81 (1967).

[205] Karstetter, B. R., and Voss, R. O. ,Chemical strengthening of glass-ceramics in the system $\mathrm{Li}_{2} \mathrm{O}-\mathrm{Al}_{2} \mathrm{O}_{3}-$ $\mathrm{SiO}_{2}, \mathbf{5 0}, 133$ (1967).

[206] Kirchner, H. P, Gruver, R. M., and Walker, R. E., Chemically strengthened, leached alumina and spinel, 50, 169 (1967).

[207] Beall, G. H., Karstetter, B. R., and Rittler, H. L., Crystallization and chemical strengthening of stuffed $\beta$-quartz glass-ceramic, 50, 181 (1967).

[208] Soga, N., and Anderson, O. L., High-temperature elasticity and expansivity of forsterite and steatite, 50, 239 (1967).

[209] Aronson, S., Cisney, E., and Gingerich, K. A., Thermal expansion of some cubic refractory compounds of thorium, $\mathbf{5 0}, 248$ (1967).
[210] Fclty, E. J., and Myers, M. B., Thermal expansion of arsenic-selenium glasses, 50, 335 (1967).

[211] Warshaw, S. I., and Seider, R., Comparison of strength of triaxial porcelains containing alumina and silica, 50, 337 (1967).

[212] Fries, R. J., and Wahman, L. A., Effect of stoichiometry on the thermal expansion of $\mathrm{TaC}_{\mathbf{x}}, \mathbf{5 0}$, 475 (1967).

[213] Provance, J. D., and Wood, D. C., Molybdenum phosphate glasses containing $\mathrm{Ag}_{2} \mathrm{O}$ or $\mathrm{K}_{2} \mathrm{O}, \mathbf{5 0}$, 516 (1967).

[214] Mazdiyasni, K. S., Lynch, C. T., and Smith, J. S., II, Cubic phase stabilization of translucent yttria-zirconia at very low temperatures, 50, 532 (1967).

[215] Riebling, E. F., Blaszyk, P. E., and Smith, D. W., Structure of glasses and melts in the $\mathrm{Na}_{2} \mathrm{O} \cdot \mathrm{GeO}_{2}$. $\mathrm{B}_{2} \mathrm{O}_{3}$ System, 50, 641 (1967).

[216] Weirauch, D. F., and Tennery, V. J., Electrical, $x-r a y$, and thermal expansion studies in the system $\mathrm{KNbO}_{3}-\mathrm{AgNbO}_{3}, \mathbf{5 0}, 671$ (1967).

[217] Hove and Riley, Ceramics for Advanced Technologies, John Wiley \& Sons, Inc., New York, 1965.

[218] Goldsmith, Waterman, and'Hirschhorn, Handbook of Thermophysical Properties of Solid Materials, MacMillan, New York, 1961.

[219] Touloukian, Thermophysical Properties of HighTemperature Solid Materials, Macmillan, New York, 1967.

[220] Lynch, Ruderer, and Duckworth, Engineering Properties of Selected Ceramic Materials, The Am. Ceram. Soc., Inc., Columbus, 1966.

[221] Thielke and Henry, Refractory Materials for Use in High-Temperature Areas of Aircraft, The Pennsylvania State College, AF Technical Report No. 6080, ASTIA, ATI-80745, 1950.

[222] Stutzman, Salvaggi, and Kirchner, Summary Report on an Investigation of the Theoretical and Practical Aspects of the Thermal Expansion of Ceramic Materials, Volume One-Literature Survey, Cornell Aeronautical Laboratory, Inc., Report PI-1273-M-4, ASTIA, AD 220685, 1959.

[223] Campbell, W. J., Thermal Expansion of Magnesium Oxide: An Interlaboratory Study, Bureau of Mines Report of Investigation 6115, 1962.

[224] Gray, American Institute of Physics Handbook, Second Edition, McGraw-Hill, New York, 1963.

[225] Nye, J. F., Physical Properties of Crystals-Their Representation by Tensors and Matrices, Oxford University Press, New York, 1957.

[226] Grüneisen, E., U.S. NASA Publ. No. RE2-18-59W (translation of Handb. Physik, 10, 1 (1926)).

[227] Lechner, R., and Quittner, G., Pressure-induced phonon frequency shifts in lead measured by inelastic neutron scattering, Phys. Rev. Letters $17,1259(1966)$.

[228] Kirby, R. K., Thermal expansion of rutile from 100 to $700^{\circ} \mathrm{K}$, J. Res. NBS 71A, 363 (1967).

[229] Kittel, C., Introduction to Solid State Physics, John Wiley \& Sons, Inc, New York 1956.

[230] ASTM Method of Test, E228-66aT, for Linear Thermal Expansion of Rigid Solids with a Vitreous Silica Dilatometer, 1967 Book of ASTM Standards, Part 31.

[231] Douglas, R. W., and Isard, J. O., Apparatus for the measurement of small differential expansions and its use for the study of fused silica, J. Sci. Instr. 29, 13 (1952).

[232] Meyerhoff, R. W., and Smith, J. F., Anisotropic thermal expansion of single crystals of thallium, yttrium, beryllium, and zinc at low temperatures, J. Appl. Phys. 33, 219 (1962).

[233] Hyde, G. R., Domingues, L. P., and Furlong, L. R., Improved dilatometer, Rev. Sci. Instr. 36, 204 (1965).

[234] Mark, S. D., and Emanuelson, R. C., Thermal expansion apparatus with a silicon carbide 
dilatometer for temperatures. to $1500^{\circ} \mathrm{C}, \mathrm{Am}$. Ceram. Soc. Bull. 37, 193 (1958).

[235] ASTM Method of Test, E289-65T, for Linear Thermal Expansion of Rigid Solids with Interferometry, 1967 Book of ASTM Standards, Part 31.

[236] Saunders, J. B., Improved interferometric procedure with application to expansion measurements, J. Res. NBS 23, 179 (1939).

[237] Nix, F. C., and MacNair, D., An interferometricdilatometer with photographic recording, Rev. Sci. Instr. 12, 66 (1941).

[238] Saunders, J. B., An apparatus for photographing interference phenomena, J. Res. NBS 35, 157 (1945).

[239] Ersner, R. L., Reversible photoelectric fringe counting, Rev. Sci. Instr. 29, 521 (1958).

[240] Willey, L. A., and Fink, W. L., An Interferometer Type of Dialtometer, and Some Typical Results, Am. Inst. Mining Met. Engrs, Technical Publication No. 1859 (1944).

[241] Conway, J. B., and Losekamp, A. C., Thermalexpansion characteristics of several refractory metals to $2500^{\circ} \mathrm{C}$, Trans. Metall. Soc. AIME 236, 702 (1966).

[242] Rothrock, B. D., and Kirby, R. K., An apparatus for measuring thermal expansion at elevated temperatures, J. Res. NBS $71 C, 85$ (1967).

[243] Peiser, Rooksby, and Wilson, X-ray Diffraction by Polycrystalline Materials, Reinhold, New York, 1960.
[244] Fouchaux, R. D., and Simmons, R. O., Measurements of thermal expansion and thermal equilibrium defects in silver chloride, Phys. Rev. 136, A1664 (1964).

[245] Mauer, F. A., and Bolz, L. H., Measurement of Thermal Expansion of Cermet Components by High Temperature X-Ray Diffraction, WADA Technical Report 55-473, Supplement 1, ASTIC Doc. No. AD 155555 (1957).

[246] Campbell, W. J., Stecura, S., and Grain, C., Hightemperature Furnaces for X-ray Diffractometers, Bureau of Mines Report of Investigation 5738, 1961.

[247] Tool, A. Q., Lloyd, D. B., and Merritt, G. E., Dimensional changes caused in glass by heating cycles, J. Res. NBS 5, 627 (1930).

[248] Brueckner, R., Characteristic physical properties of the chief oxide glass formers and their relation to the structure of glasses: II, Mechanical and optical properties as a function of thermal history, Glastech. Ber. 37, 459 (1964).

[249] Turner, P. S., Thermal-expansion stresses in reinforced plastics, J. Res. NBS 37, 239 (1946).

[250] Kerner, E. H., The elastic and thermo-elastic properties of composite media, Proc. Phys. Soc. (London) B69, 808 (1956).

[251] Rosenfield, A. R., and Averbach, B. L., Effect of stress on the expansion coefficient, J. Appl. Phys. 27, 154 (1956).

[252] Kirchner, H. P., Thermal Expansion of Ceramic Crystals, Progress in Solid State Chemistry, Vol. 2, H. Reiss, Editor, Macmillan, 1964. 



\title{
Thermal Conductivity of Ceramics
}

\author{
D. R. Flynn \\ Institute for Applied Technology, \\ National Bureau of Standards, Washington, D.C. 20234
}

\begin{abstract}
A description is given of the underlying phenomena governing thermal conduction in ceramic materials. Heat transfer via lattice vibrations, electronic conduction, and thermal radiation are discussed in some detail. The effects of environmental variables (temperature, atmosphere, electromagnetic fields, pressure and stress, heat flow) on the thermal conductivity of ceramics are discussed. The effects of composition (molecular struccure, impurities, stoichiometry, alloying) and structure (crystallinity, imperfections, additional phases, microstructure) on thermal conductivity are discussed.

It is pointed out that many thermal conductivity data reported in the literature are unreliable, either due to experimental errors or to inadequate sample characterization. A number of methods of measuring thermal conductivity are described, with attention being given to precautions to assist in avoidance of measurement errors. The use of thermal conductivity reference standards is discussed and a list is given of materials which may be used, on an interim basis, as reference standards.
\end{abstract}

Key Words: Ceramics; conductivity; glass; heat conduction; heat transfer; thermal conductivity.

\section{GONTENTS}

2. Definition and phenomenological theory

2.1. Definition

2.2. Phenomenological theory

a. Lattice conduction (phonons)

b. Electronic conduction

c. Radiation "conduction"

d. Gas conduction......... 77

e. Other transport mechanisms....... 78

3. Effects of environment

3.1. Temperature

3.2. Atmosphere..... 81

3.3. Electromagnetic fields

3.4. Pressure and stress__._._.

3.5. Heat flow

4. Directional effects

5. Effects of composition and structure

5.1. Composition

a. Molecular structure

b. Impurities

c. Stoichiometry

\section{Introduction and Scope}

The thermal conductivity of ceramics and other nonmetallic solids is of considerable practical and theoretical importance. Many of the practical applications which require accurate knowledge of thermal conductivity are concerned with devices for conversion of heat into other forms of energy. Furnace and boiler design requires knowledge of the thermal conductivity of insulating bricks and refractories from ambient to high temperatures. In a nuclear reactor, the maximum temperature of the fuel elements is directly related to the thermal conductivity of these fuel elements. The

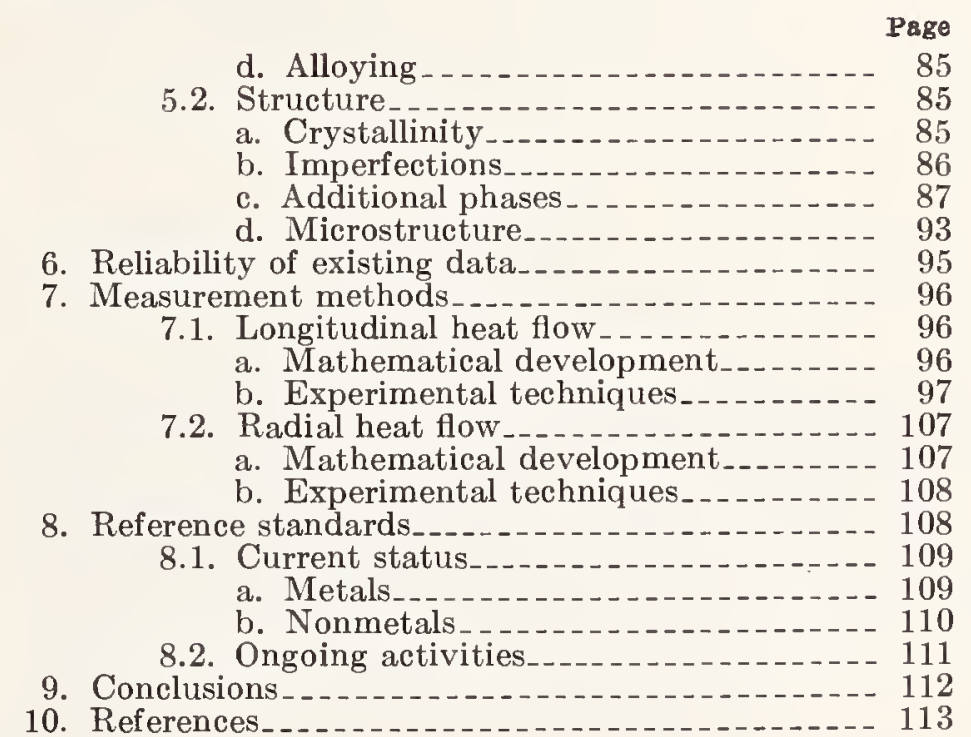

efficiency of a thermoelectric power generator is a function of the thermal conductivity of the thermoelement.

The extreme temperatures encountered in aerospace applications, ranging from containment of cryogenic fuels to ablative heating during reentry, have created yet another area where accurate thermal conductivity data are required.

In many applications of solid-state electronics, materials used as heat sinks or as potting compounds must have as high a thermal conductivity as possible in order to dissipate the heat generated by the device. 
At very low temperatures (i.e., below the boiling point of nitrogen), the thermal conductivity of solids is extremely sensitive to imperfections. Thus thermal conductivity measurement provides a valuable tool for the solid-state physicist.

In this paper we define thermal conductivity and present a brief phenomenological description of the energy transport mechanisms involved. We then discuss the influence of environmental effects, such as temperature, pressure, and stress, on the thermal conductivity of solids. This is followed by a discussion of the effects of composition and structure on the thermal conductivity of ceramics and other nonmetallic solids. Some sources are cited for thermal conductivity data and a few remarks are made regarding the reliability of thermal conductivity data. We describe some of the techniques which are used to measure thermal conductivity and comment on the availability and use of thermal conductivity reference standards.

\section{Definition and Phenomenological Theory}

\subsection{Definition}

The thermal conductivity, $\lambda$, of a substance is related to the rate of heat flow per unit area, $q$, and the temperature gradient, $\nabla T$, by Fourier's law, which for an isotropic medium can be written as

$$
\mathbf{q}=-\lambda \nabla T \text {. }
$$

For one-dimensional heat flow, eq (1) is simply

$$
q_{z}=-\lambda \frac{\partial T}{\partial z}
$$

Equation (1) may be regarded as the definition of thermal conductivity.

If one considers an elemental volume in an isotropic medium which is opaque to thermal radiation, the following second-order differential equation is easily derived:

$$
\nabla \cdot(\lambda \nabla T)+W=C \frac{\partial T}{\partial t}
$$

where $C$ is the heat capacity per unit volume, $t$ is time, and, if heat is produced in the solid, $W$ is the rate of heat production per unit time and unit volume. In general the thermal properties, $\lambda$ and $C$, can be functions of position and of temperature.

For a homogeneous material which has no internal heat production and in which thermal conductivity is independent of temperature, eq (3) reduces to the form

$$
\nabla^{2} T=\frac{1}{\alpha} \frac{\partial T}{\partial t}
$$

where $\alpha=\lambda / C$ is the thermal diffusivity. Note that in order for eq (4) to be valid, $\lambda$ must be independent of temperature and position over the temperature range of interest but $C$ may still be temperature-dependent.

Many engineering applications involving timedependent temperatures require a knowledge of the thermal diffusivity rather than, or in addition to, the thermal conductivity. In this paper we shall confine our attention to thermal conductivity and will not discuss thermal diffusivity. If needed, the thermal diffusivity of a material can be measured directly or can be computed from the thermal conductivity and the volumetric heat capacity. Since heat capacity is relatively insensitive to variations of composition and structure, remarks we make concerning the effects of these on thermal conductivity are applicable to thermal diffusivity as well.

Many applications which require a knowledge of thermal conductivity or thermal diffusivity involve analytical or numerical solutions of some variation of eq (3). The reader who is interested in mathematical solutions of the heat conduction equation should consult, for example, the books by Carslaw and Jaeger [1] $]^{1}$, Schneider [2], and Arpaci [3].

\subsection{Phenomenological Theory}

The theory of thermal conduction is the subject of many research papers and review papers and forms a major portion of the material in quite a few books. Thus we can only briefly touch on the theory of thermal conduction and will limit ourselves to a phenomenological discussion of the processes and principles involved. For detailed discussions of this subject the reader can consult the various books and review papers cited [4-15].

It is important to realize that the form of eq (1) implies that heat conduction is a diffusion process. The thermal energy diffuses through the material. In conduction heat transfer the flow of heat is proportional to the temperature gradient. This may be contrasted with radiative heat transfer through a perfectly transparent medium (e.g., in vacuo) where the thermal flux is related to the temperatures of the surfaces which are exchanging heat. In many cases the heat conducted through a material is directly proportional to the temperature difference-however, these are special cases which arise, for example, because the thermal conductivity is essentially independent of temperature over the temperature range of concern.

A well-known result of the kinetic theory of gases is that the thermal conductivity of a gas is given by

$$
\lambda=1 / 3 C v \Lambda,
$$

where $C$ is the heat capacity per unit volume, $v$ is the average particle velocity (group velocity),

\footnotetext{
1 Figures in brackets indicate the literature references at the end of this paper.
} 
and $\Lambda$ is the mean free path of a gas particle between collisions with another gas particle. It is extremely useful to carry eq (5) over to the case of heat conduction in a solid. Before discussing this it is instructive to consider onedimensional heat flow in an elemental length, $\Delta z$, of material supporting a temperature gradient $\Delta T / \Delta z$. From (2) and (5) the rate of heat flow in the $z$-direction is

$$
q_{z}=-\lambda \frac{\Delta T}{\Delta z}=-\frac{1}{3}(C \Delta T)\left(v \frac{\Lambda}{\Delta z}\right)
$$

The quantity $C \Delta T$ is the excess thermal energy per unit volume at one end of the elemental length with respect to the other end. This excess energy is propagated down the specimen with an effective transport velocity which is just the particle velocity reduced by the ratio of the mean free path to the length of the element.

In a solid material, heat can be transported by a variety of carriers or mobile excitations. For this "gas" of heat carriers we can write, by analogy to eq (5),

$$
\lambda=\frac{1}{3} \sum_{i} C_{i} v_{i} \Lambda_{i}
$$

where the summation is over all carriers. Equation (7) simply states that the heat transported by different carriers may be added to obtain the total heat transported. In a solid where thermal transport is via several types of carriers, the total thermal conductivity is given by

$$
\lambda=\lambda_{l}+\lambda_{e}+\lambda_{r}+\ldots,
$$

where the different subscripts refer to different types of carriers. One definitely should not infer from eqs (7) and (8), however, that the different mechanisms of heat conduction do not interact. The different components of the total thermal conductivity are additive but they are not independent.

Of the three quantities on the right hand side of eqs (5) and (7), the carrier heat capacity and the carrier velocity normally have a relatively well-known temperature dependence and are relatively insensitive to material imperfections and impurities. The carrier mean free path is the troublemaker. It may have a rather complicated and elusive temperature dependence and may be very sensitive to material imperfections and impurities. The mean free path of carriers is determined by the interactions ("collisions") between the carriers and by the interactions between the carriers and material imperfections and impurities.

In the remainder of this section we will discuss the theory of thermal conductivity as it applies to the several mechanisms of heat transfer which take place in ceramic materials.

\section{a. Lattice Conduction (Phonons)}

A solid material may be thought of as a threedimensional array of masses (atoms) held together by springs (interatomic forces). Each atom vibrates with an amplitude which is dependent on temperature; atoms which are at higher temperatures vibrate more vigorously than those at lower temperatures. This thermal motion of the atoms can be resolved into traveling elastic waves which transport thermal energy through the solid. The energy carried by these waves may also be thought of as being carried as a number of quanta of energy, called phonons, which can be treated as particles. This is analogous to the better known practice of treating light waves (or traveling electromagnetic waves) as particles called photons.

In order to better discuss the properties of phonons, it is helpful to consider certain aspects of the vibrational spectrum of a discrete lattice. For this purpose it will suffice to consider a onedimensional lattice with two kinds of atoms equally spaced as shown in figure 1 . If we consider each atom to behave as a classical harmonic oscillator which interacts only with its nearest neighbors, it can be shown that the only waves which can be propagated along this chain of atoms are those having an angular frequency $\left(\mathrm{rad} \mathrm{s}^{-1}\right)$ given by

$$
\omega^{2}=\beta\left(\frac{1}{m}+\frac{1}{M}\right) \pm \beta\left[\left(\frac{1}{m}+\frac{1}{M}\right)^{2}-\frac{4 \sin ^{2} k a}{M m}\right]^{1 / 2},
$$

where $m$ and $M$ are the masses of the two kinds of atoms, $a$ is the separation between adjacent atoms, $\beta$ is the force constant acting to restore each atom to its equilibrium position, and $k$ is the wave vector, which has a sign corresponding to the direction in which the wave travels and a magnitude equal to $2 \pi$ /(wavelength). The two branches of eq (9) are shown plotted in figure 2. The limiting values of eq (9) are shown in figure 3 . The lower branch is called the acoustical branch and the upper branch is called the optical branch.

Phonons transport energy with a velocity (known as the group velocity), $v=d \omega / d k$. For small values of $k$ (long wavelengths), the group velocity in the acoustical branch is

$$
v=\frac{d \omega}{d k}=\left[\frac{2 \beta}{M+m}\right]^{1 / 2} a=\frac{\omega}{k} .
$$

For wavelengths long in comparison to the interatomic spacing, adjacent atoms vibrate in phase in the acoustic branch and our one-dimensional

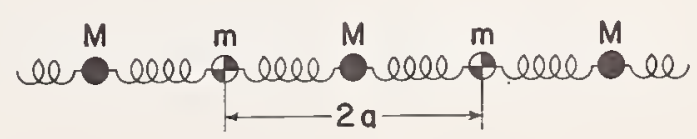

FIGURE 1. Schematic representation of a diatomic linear lattice. 


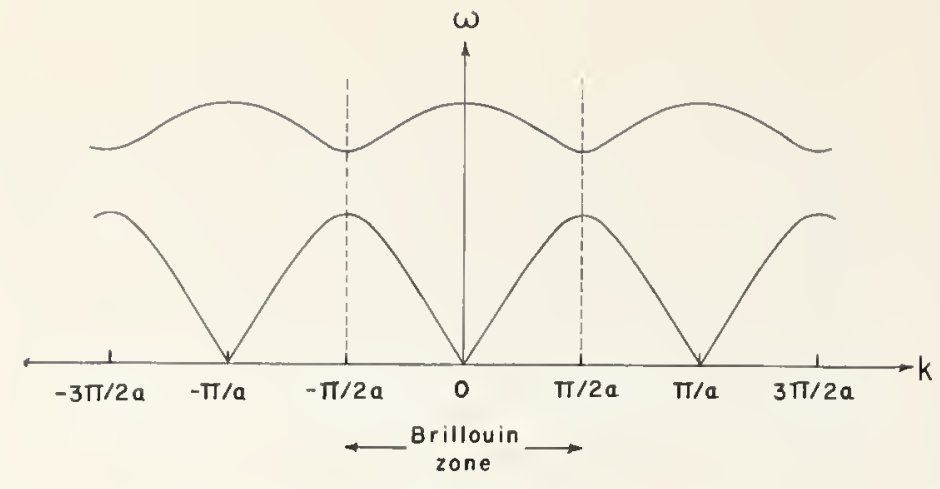

Figure 2. Plot of angular frequency, w, versus wavenumber, $\mathrm{k}$, for a diatomic linear lattice.

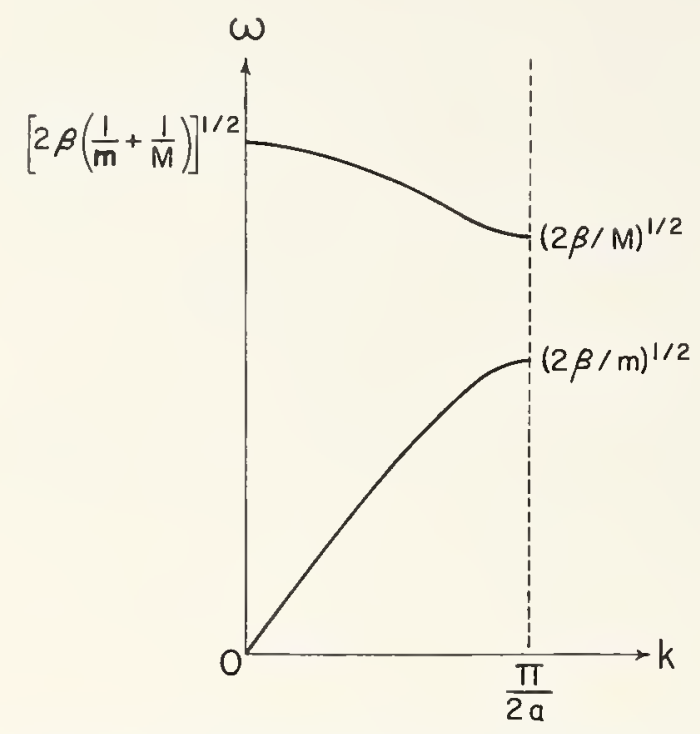

Figure 3. Positive half of the first Brillouin zone for a diatomic linear lattice.

lattice behaves like a continuous elastic string down which phonons propagate with a constant velocity given by eq $(10)^{2}$.

In the higher frequency, or optical branch, the phonons have a constant frequency for long wavelengths. Adjacent atoms vibrate $180^{\circ}$ out of phase for the long-wavelength optical phonons. Since $d \omega / d k=0$ for these phonons, their group velocity is zero and they can transport no energy.

From figure 3 , we see that there is a forbidden band, $2 \beta / m<\omega^{2}<2 \beta / M$, in which no phonons can be excited.

The periodic structure of eq (9), as shown in figure 2, has a very important consequence. Looking at figure 2 and recalling that the energy of a phonon is propagated with the group velocity, $v=d \omega / d k$, we see that a phonon having a wave vector between $\pi / 2 a$ and $\pi / a$ has a group velocity opposite in sign to $k$. Although the wave is traveling to the right, the energy is traveling to the left. As an example consider a phonon of wavelength $8 a / 3$ traveling in the positive direction (i.e., to

2 The "density" (mass per unit length) of our one-dimensional lattice is $d=(M+m) / 2 a$. The "Young's modulus" is $E=f a$. Thus eq (10) is simply the usual expression, $v=\sqrt{E} / \bar{d}$, for the velocity of sound in an elastic continuum. the right) in our one-dimensional lattice; this phonon has a wave vector $+3 \pi / 4 a$. Consider also a phonon of wavelength $8 a$ traveling in the negative direction; this phonon has a wave vector $-\pi / 4 a$. Since $3 \pi / 4 a$ and $-\pi / 4 a$ are completely equivalent in eq (9), we see that these two phonons, of quite different wavelengths, are completely equivalent both as to the frequency of vibration and the direction of energy propagation. In view of this periodicity, we need only be concerned with phonons having wave vectors whose magnitude is less than $\pi / 2 a$ (wavelength greater than $4 a$ ). The region $-\pi / 2 a<k<\pi / 2 a$, indicated by the vertical dashed lines in figure 2 , is known as the Brillouin zone. Wave vectors falling outside the Brillouin zone can be brought back inside by adding or subtracting the appropriate integral multiple of $\pi / a$.

The equivalence of two phonons having wave vectors differing by $n \pi / a$ arises because of constructive or destructive interference effects due to reflections of the phonons from the discrete lattice. Phonons of wave vector equal to $n \pi / a$ cannot propagate at all because their reflections are exactly $180^{\circ}$ out of phase. For phonons of wave vector equal to $(n-1 / 2) \pi / a$, the reflections are in phase and the phonons at the edge of the Brillouin zone correspond to standing waves for which the group velocity vanishes.

It should be noted that all of the aspects discussed so far in this section are classical. We have used the term phonon to represent quanta of energy traveling through the lattice-however, this was done for convenience and not from necessity. Thus, to this point in our discussion, it has not been necessary to invoke any quantum theory.

The extension of the above discussion to a threedimensional lattice of harmonic oscillators presents no particular difficulty. The wave vector $\mathbf{k}$ indicates the direction of propagation and has the magnitude $2 \pi$ (wavelength). For a given $\mathbf{k}$, there are three independent directions in which the atoms can vibrate. For a not-too-complicated lattice, there is a single longitudinal wave having particle displacements parallel to $\mathbf{k}$ and two transverse waves having displacements perpendicular to $\mathbf{k}$. Depending on the symmetry of the crystal, the longitudinal phonon and the two transverse phonons for a given $\mathbf{k}$ can have different frequencies. For a diatomic crystal there may be three acoustical branches and three optical branches for a given direction of propagation.

References [4-15] all contain some discussion of phonons. Other useful references to the theory of lattice vibrations include [16-28].

Having introduced the concept of phonons, we can think of an ideally perfect crystal as an otherwise-empty box containing a "gas" of phonons which collide with one another and with the walls of the box (i.e., the surface of the crystal). These phonons will have different wave- 
vectors and frequencies as discussed above. Bringing in some quantum theory, each phonon transports energy in the amount $\hbar \omega$, where $\hbar=1.05450 \times 10^{-34} \mathrm{Js}^{3}$

In our discussion above, we assumed that the atoms behaved as purely harmonic oscillators. If this were strictly so, the phonons would not interact with one another and the thermal conductivity of a perfect crystal would be infinite. In fact, there are anharmonic terms which allow the phonons to interact and cause thermal resistance. The simplest type of interaction which can occur is when two phonons combine to form a third phonon as shown in figure 4.

Conservation of energy requires that

$$
\hbar \omega_{1}+\hbar \omega_{2}=\hbar \omega_{3}
$$

while conservation of the wave vectors (analogous to conservation of momentum) requires that

$$
\mathbf{k}_{1}+\mathbf{k}_{2}=\mathbf{k}_{3} \text {. }
$$

Phonon interactions such as described by eqs (11) and (12) are known as normal processes or $\mathrm{N}$ processes. Both energy and quasi-momentum (a name sometimes given to $\hbar \mathbf{k}$ ) are conserved and there is no attendant thermal resistance.

Consider, however, an interaction in which $\mathbf{k}_{1}$ and $\mathbf{k}_{2}$ ale roughly in the same direction and their magnitudes are sufficiently large that $\mathbf{k}_{\mathbf{3}}$ lies outside the Brillouin zone and, therefore, corresponds to energy transport in a different direction. For a square, two-dimensional lattice, such an interaction is illustrated in figure 5 . The resultant vector, $\mathbf{k}_{3}$, can be replaced by $\mathbf{k}_{4}=\mathbf{k}_{3}-\mathbf{G}$, where $\mathbf{G}$ is a vector perpendicular to the boundary of the Brillouin zone and equal in magnitude to the width of the zone. For such an interaction, eq (12) can be replaced by

$$
\mathbf{k}_{1}+\mathbf{k}_{2}=\mathbf{k}_{4}=\mathbf{k}_{\mathbf{3}}-\mathbf{G} \text {. }
$$

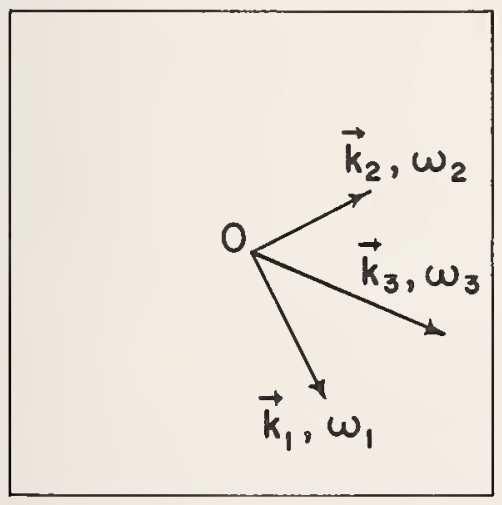

FigUre 4. A normal- or Nprocess in a square, twodimensional lattice.

A phonon of wavevector $\mathbf{k}_{1}\left(\omega_{1}\right)$ combines with a phonon of wavevector $\mathbf{k}_{2}\left(\omega_{2}\right)$ to form a new phonon of wavevector $\mathbf{k}_{3}\left(\omega_{3}\right)$. Both energy and quasi-momentum are conserved.

${ }^{3} h=h / 2 \pi$, where $h$ is Planck's constant.

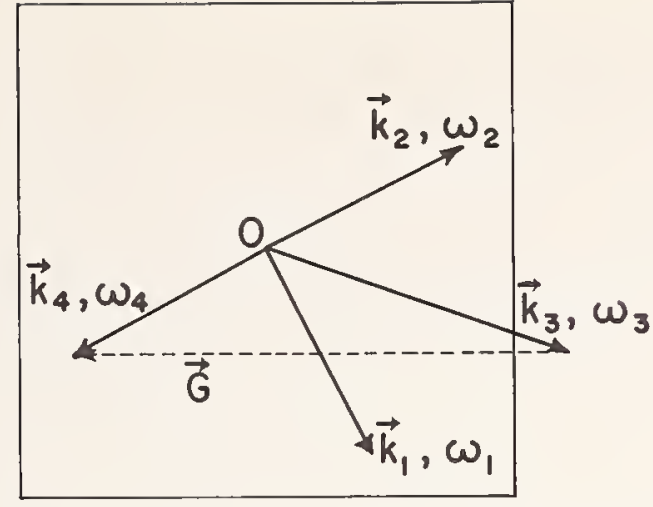

FigURe 5. An umklapp-or U-process in a square, two-dimensional lattice.

A phonon of wavevector $\mathbf{k}_{1}\left(\omega_{1}\right)$ combines with a phonon of wavevectork $\mathbf{k}_{2}\left(\omega_{2}\right)$. Since the resultant vector $\mathbf{k}_{3}\left(\omega_{3}\right)$ crosses the boundary of the first Brillouin zone, the direction of energy flow is changed so that the newly-formed phonon has the wavevector $\mathbf{k}_{4}\left(\omega_{4}\right)$, where $\omega_{4}=\omega_{3}$. In a U-process, energy is conserved but quasimomentum is not conserved.

In such an interaction, known as an umklapp process, or U-process, the direction of energy flow is changed and there is an attendant ther mal resistance. The change in direction of energy flow which accompanies a U-process can be thought of as an interference effect in which the phonons are reflected by the lattice; this is analogous to Bragg reflection of x-rays.

According to quantum theory, the energy of an oscillator can assume only the discrete values

$$
E(\mathbf{k})=\hbar \omega(N+1 / 2),
$$

where $N$ is a positive integer. ${ }^{4}$ The energy corresponding to a given $\mathbf{k}$ thus consists of a zero-point energy, $\hbar \omega / 2$, plus $N$ phonons, each carrying an energy $\hbar \omega$. For a crystal in thermodynamic equilibrium, the average number of phonons in a mode of frequency $\omega$ is

$$
N=N(\omega, T)=\frac{1}{\exp (\hbar \omega / k T)-1},
$$

where $k=1.38054 \times 10^{-23} \mathrm{JK}^{-1}$ is the Boltzmann constant, and $T$ is the absolute temperature. Classically, the energy corresponding to a given temperature would be simply $k T$. The number of units of energy of size $\hbar \omega$ in a classical oscillator is thus $T / \theta$, where $\theta$ is a vibrational temperature defined by $\theta=\hbar \omega / k$. In figure 6 we have plotted the quantity $\theta N / T, N$ being given by eq (15). This is simply the ratio of the number of phonons in a mode of frequency $\omega$ to the number of phononsized units of energy that would exist if there were no quantum effects. We see that at temperatures which are low in comparison to $\theta$, there are very few phonons available.

The paucity of phonons at low temperature affects the lattice thermal conductivity in two

\footnotetext{
4 We can let it be understood that $\mathbf{k}$ specifies the polarization as well as the magnitude and direction of the wave vector.
} 
ways: (1) the specific heat of the solid decreases with decreasing temperatures thus tending to decrease the thermal conductivity; (2) the number of U-processes decreases with decreasing temperature thus increasing the phonon mean free path and tending to increase the thermal conductivity.

It is customary to discuss the heat capacity of a solid in terms of the Debye model; in this model the low temperature heat capacity is proportional to $(T / \theta)^{3}$, where the Debye temperature, $\theta$, is defined by $k \theta=\hbar \omega_{\max }$, where $\omega_{\max }$ is the highest frequency of vibration in the lattice. From our discussion above, it is seen that in order for U-processes to occur it is necessary that the interacting phonons have wave vectors extending about halfway to the boundary of the Brillouin zone. 'This would correspond to phonon energies of about $\hbar \omega=k \theta / 2$. The number of such phonons is given by eq (15); for $T<<\theta$ this is $\exp (-\theta / 2 T)$. The rate at which U-processes occur, then, should be approximately proportional to this factor. Since the mean free path of phonons in an infinitely large ideal crystal is limited only by U-processes, the mean free path should be approximately inversely proportional to this factor. Assuming that the phonon velocity is essentially independent of temperature, the low temperature thermal conductivity of an ideal crystalline lattice is given by

$$
\lambda_{l} \approx a\left(\frac{T}{\theta}\right)^{n} \exp \left(\frac{\theta}{b T}\right), \text { when } T<<\theta,
$$

where $a$ depends on the structure of the crystal, $n$ has a value of 3 or less, and $b$ is of the order of 2 .

At temperatures larger than the Debye temperature, the specific heat of a dielectric crystal becomes essentially constant. At such temperatures, most phonons will have wave vectors of sufficient magnitude to permit U-processes to occur. The number of U-processes will therefore be approximately proportional to the number of phonons; from figure (6) we see that this is ploportional to the temperature. Again assuming that the phonon velocity is essentially independent of temperature, the high temperature thermal conductivity of an ideal crystalline dielectric is given ${ }^{5}$ by

$$
\lambda_{l} \approx A \frac{\Theta}{T} \text {, when } T>>\theta,
$$

At low temperatures, the phonon mean free path would increase exponentially with decreasing temperature in a sufficiently large ideally perfect crystal. In a crystal of finite size, the mean free path of a phonon is limited to a value of the order of the crystal diameter. This was first predicted by Pieirls [30], observed experimentally by de

\footnotetext{
${ }^{5}$ Equation (17) is not necessarily valid for $T \approx \theta$.Leibfried and Schlömann [29] obtain

$$
\lambda_{l}=A \frac{\theta}{T}\left\{1+\bigcirc\left(\frac{\theta}{T}\right)^{2}+\ldots\right\},
$$

where the coefficient of $\left(O / T^{2}\right)^{2}$ is positive and has a magnitude depending on
} the frequency spectrum of the lattice.

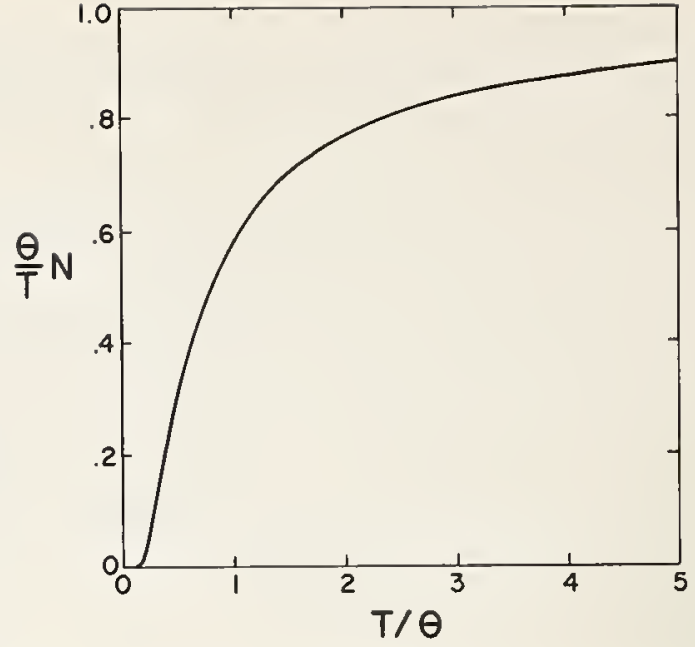

FIGURE 6. The number of phonons in a given mode, as predicted by quantum mechanics, divided by the number of phonon-sized units of energy in a classical oscillator at the same temperature.

Haas and Biermasz [31], and calculated by Casimir [32]. The reflections of phonons from the specimen boundaries is analogous to the flow of a rarified gas in a tube as first discussed by Knudsen [33] and Smoluchowski [34]. Ziman [7, ch. XI] and Klemens [11] discuss boundary scattering of phonons in some detail. Other references include [10,35-43]; Guyer and Krumhansl [42] and Thacher [39] give still more references to theoretical and experimental work on boundary scattering of phonons.

At temperatures low enough that the specific heat varies as $T^{3}$, the thermal conductivity of a finite crystal varies approximately as

$$
\lambda_{l} \propto d T^{3}
$$

where $d$ is the effective diameter of the crystal; the proportionality constant depends on the nature of the crystal surface-a roughened surface which scatters phonons diffusely will result in a lower thermal conductivity than, for example, a cleaved surface which scatters specularly.

Figure 7 shows the data obtained by Thacher [39] for single crystals of isotopically pure LiF of different sizes. Note that this is a log-log plot and the differences among the four curves shown are large.

Qualitatively, at least, we now have discussed the factors which affect the thermal conductivity of a dielectric single crystal which is free from defects. In figure 8 the thermal conductivity of a sample of single-crystal artificial sapphire $\left(\mathrm{A}_{2} \mathrm{O}_{3}\right)$ is shown plotted versus temperature. At higher temperatures, the thermal conductivity is controlled by Umklapp processes and goes approximately as $T^{-1}$ (eq (17)). As the temperature decreases, Umklapp processes are frozen out, the mean free path increases exponentially, and the thermal conductivity varies with temperature 


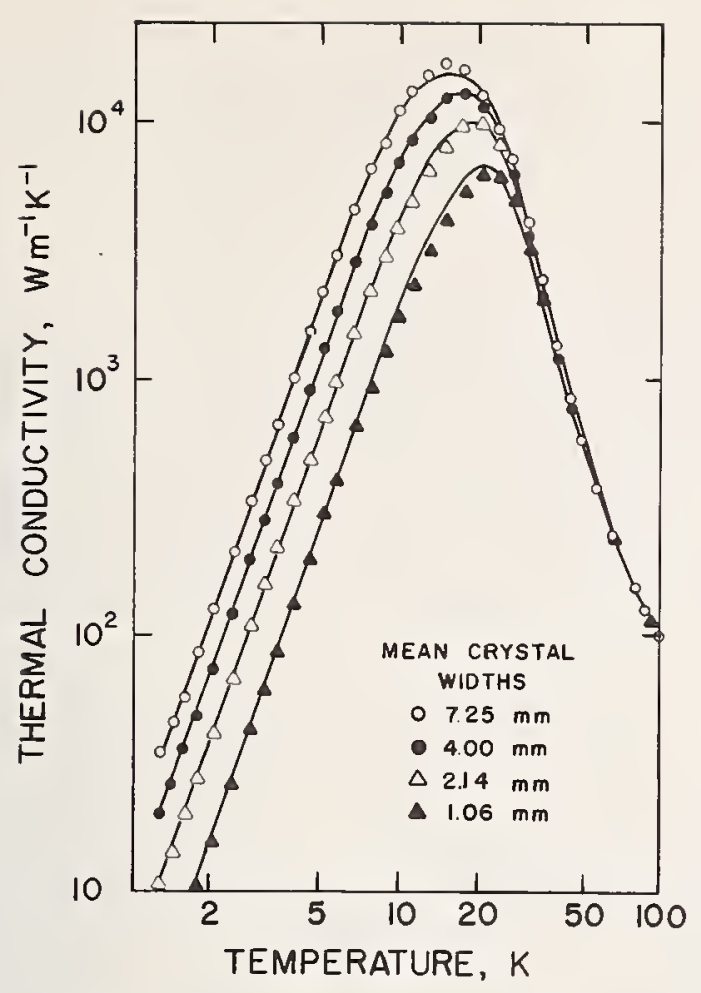

Figure 7. Thermal conductivity of different sized crystals of lithium fluoride, illustrating the effect of boundary scattering of phonons (after Thacher [39]).

according to eq (16). At still lower temperatures, the mean free path becomes constant at a value of the order of the effective crystal diameter and the thermal conductivity varies approximately as $T^{3}$ (eq (18)); in this temperature range it is no longer meaningful to speak of the thermal conductivity of a given material-one can only speak of the thermal conductivity of a given specimen.

In a real crystal, the height of the peak in the thermal conductivity will be reduced, due to scattering of phonons by impurities and imperfections, to a value less than that which would be extant in a perfect crystal. We shall now briefly discuss theoretical predictions of the influence of impurities and imperfections on the thermal conductivity of a dielectric crystal. The very large amount of theoretical work in the area and the complexity of the calculations involved make it impossible for me to do much more than state the results. The interested reader should consult one of the review papers by, for example, Klemens [9-11] or the book by Ziman [7]. Other references include [4-6, 8, 12-15, 29-30, 42, 44-61].

Consider an imperfection (this could be an impurity, vacancy, dislocation, etc.) in an otherwise perfect crystalline lattice. In the vicinity of this imperfection the elastic properties of the lattice will differ from those in the perfect lattice. Consequently, a lattice wave or phonon will be deflected or scattered when it encounters an imperfection. If one thinks of a perfect dielectric

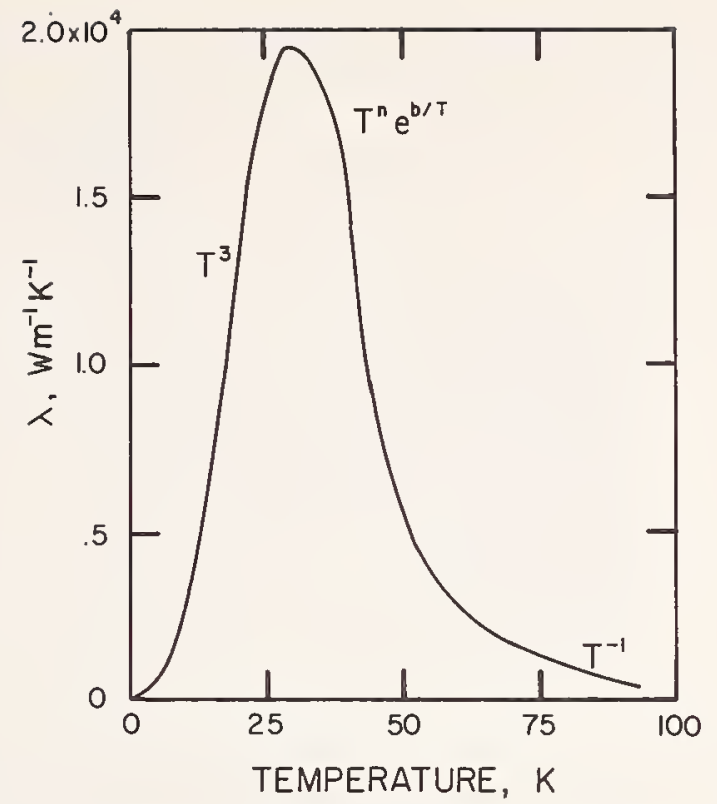

FIgURE 8. Idealized temperature-dependence of thermal conductivity for a single-crystal $\left(\mathrm{Al}_{3} \mathrm{O}_{3}\right)$ in which the only mode of heat conduction is via lattice vibrations (after Ziman [\%, p. 292]).

crystal as an otherwise empty box containing a gas of phonons, then an imperfect crystal can be thought of as a box containing a number of stationary obstacles with which the phonons interact or collide. The effective scattering crosssection of an imperfection is dependent on the extent of the surrounding strain field. If a disordered region is small (point defects) compared to the wavelength of the interacting phonon, the details of the strain field are not important. If a disordered regions is comparable to, or larger than, the wavelength of the interacting phonon, interference effects will enter in and the detailed shape of the strain field must be considered. If a number of impurities or point defects are arranged in some regular array, interference effects again must be considered. In the case of a substitutional impurity, the mass difference between the impurity atom and a normal atom will lead to scattering of phonons, irregardless of the associated strain field.

Quantum-mechanically, one usually considers the Hamiltonian of a system of phonons in the absence of impurities and then applies timedependent perturbation therory to find the effect caused by impurities or imperfections. The references cited above may be consulted for details.

The total lattice thermal resistivity $\left(W_{l}=1 / \lambda_{l}\right)$ can be expressed as the sum of the thermal resistivities due to various scattering mechanisms:

$$
W_{l}=W_{l u}+W_{l b}+W_{l p}+\ldots .
$$

where $W_{l u}$ is the thernial resistivity due to U-processes, $W_{l b}$ is that due to boundary scattering, $W_{l p}$ is that due to point defects, etc. In table 1 we present the temperature dependence 
TABLE 1. Temperature variation of Thermal Resistivity due to Various Interaction Mechanisms (after Klemens [10])

\begin{tabular}{|c|c|}
\hline Scattering mechanism & $\begin{array}{l}\text { Temperature } \\
\text { dependence of } \\
\text { thermal } \\
\text { resistivity }\end{array}$ \\
\hline $\begin{array}{l}\text { Low temperatures }(T<\theta) \text { : } \\
\text { Umklapp processes } \\
\text { Point defects } \\
\text { Long cylinders. } \\
\text { Dislocations (core) } \\
\text { Dislocations (strain field) } \\
\text { Conduction electrons in metals } \\
\text { Stacking faults } \\
\text { Thin sheets embedded in crystal of } \\
\text { continuous orientation } \\
\text { Grain boundaries } \\
\text { External boundaries. } \\
\text { High temperatures }(T>\theta) \\
\text { Umklapp processes. } \\
\text { All imperfections }\end{array}$ & $\begin{array}{l}T^{-3} e^{-\mathrm{e} / \mathrm{b} T} \\
T^{1} \\
T^{0} \\
T^{0} \\
T^{-2} \\
T^{-2} \\
T^{-1} \\
T^{-1} \\
T^{-3} \\
T^{-3} \\
T^{1} \\
T^{0}\end{array}$ \\
\hline
\end{tabular}

of the component of thermal resistivity due to these various scattering mechanisms. If the relative strengths of the different scattering mechanisms are known, eq (19) can be used to obtain the temperature dependence of the total lattice thermal resistivity.

At temperatures well above the Debye temperature, we see that the thermal resistivity due to all imperfections should be independent of temperature and the total thermal resistivity should be simply

$$
W_{l}=1 / \lambda_{l}=A+B T,
$$

where $A$ and $B$ are constants which can be obtained empirically. For fairly pure dielectric crystals, experimental data conform reasonably well to eq (20) in many cases.

In section 5 we shall discuss experimentally obtained thermal conductivity values with respect to the influence of composition and structure.

\section{b. Electronic Conduction}

In many traditional ceramic materials such as metallic oxides, the electrical resistivity is sufficiently high that the electronic component of thermal conductivity is negligible. However, in metallic carbides, graphite, and many semiconductors, for example, the electronic portion of thermal conductivity may be comparable with or larger than the lattice portion. Cermets, or mixtures of metals and ceramics, are also of interest to the ceramic industry; in such two-phase materials, the thermal conductivity of the metallic phase may be almost entirely due to electronic conduction. It is therefore quite appropriate that we briefly discuss the theory of electronic thermal conductivity.

The electrons which conduct heat and electricity may be thought of as a gas of essentially free electrons wandering aimlessly around in the periodic potential field of the crystalline lattice. In the presence of an electric field or temperature gradient, the electrons are motivated in a particular direction and there is a net transport of charge or thermal energy.
These wandering electrons have energies that fall within certain "bands". Between these bands there are gaps, or energy regions which may not be occupied. The two bands which are of interest to us are the valence band and the conduction band. The valence band is analogous to the outermost shell of electrons in a free atom-the electrons are bound to the atom but can be set free if they acquire sufficient kinetic energy. Electrons in the conduction band have sufficient kinetic energy to permit them to travel through the crystal.

In a metal the conduction band is partially filled with electrons even at 0 degrees kelvin. The average number of electrons in a state of energy $E$ is given by the Fermi function:

$$
f(E)=\frac{1}{\exp [(E-\zeta) / k T]+1},
$$

where the Fermi energy, $\zeta$, is determined by the condition that $\Sigma f(E)$ over all quantum states must equal the total number of electrons in the conduction band. ${ }^{6}$ The behavior of the Fermi function is shown in figure 9. At a temperature of absolute zero, all states having energies less than $\zeta$ are filled and none of the higher energy states are filled. As temperature is increased, $f(E)$ no longer is a step function but rather decreases from $f(E)=1$ to $f(E)=0$ over an energy interval of width approximately equal to $k T$ centered at $E=\zeta$. Because of this behavior of the Fermi function, only those electrons having energies near $\zeta$ are affected by temperature change. An electron gas is considered to be "degenerate" when $T<<T_{F}$, where $T_{F}=\zeta / k$ is known as the Fermi temperature. ${ }^{7}$ For a degenerate electron gas, the specific heat of the electrons is proportional to the absolute temperature.

At very low temperatures, electrons could travel essentially unimpeded through a perfect metallic crystal and the mean free path of the electrons would increase very rapidly as the temperature is decreased toward absolute zero. The electronic thermal conductivity of a perfect metallic crystal would thus be limited at low temperatures by boundary scattering as was discussed for the phonon thermal conductivity. However, in a real crystal, as opposed to a perfect

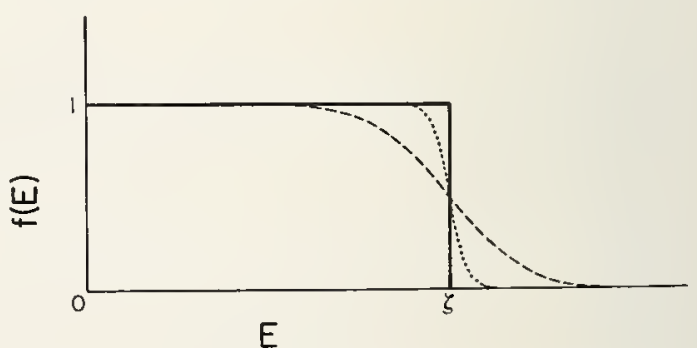

FIGURE 9. The Fermi function, eq(21).

Electrons obey the Pauli exclusion principle so that only two electrons
(of opposite spin) may occupy a given energy level. (of opposite spin) may occupy a given ener
7 Typically, $10^{4} \mathrm{~K}<T_{F}<10^{5} \mathrm{~K}$ for metals. 
one, the limiting factor for electronic thermal conductivity is scattering of electrons by impurities and perfecticns. In the case of very thin films or fine wires, the electronic component of thermal conductivity will be limited by boundary scattering.

At high temperatures, electrons are scattered very effectively by phonons and the mean free path of the electrons is approximately inversely proportional to the number of phonons and thus inversely proportional to temperature. With a heat capacity which is proportional to temperature, an electron velocity (corresponding to $\zeta$ ) which is essentially constant, and a mean free path which is inversely proportional to temperature, we see that eq (7) predicts an essentially constant electronic thermal conductivity for a pure metal at temperatures which are high compared to, say, the Debye temperature, $\theta$, but low compared to the Fermi temperature, $T_{F}$. If impurities are added to a metal, the electronic heat capacity will not be seriously affected and the mean free path will be decreased more at low temperatures than at high. For a completely disordered alloy, the mean free path would be essentially constant and the electronic thermal conductivity would be proportional to the electronic specific heat and hence to temperature.

In discussing electronic thermal conductivity, it is customary and useful to discuss it in terms of the Lorenz function:

$$
L=\frac{\lambda_{e}}{\sigma T}
$$

where $\lambda_{e}$ is the electronic component of thermal conductivity, $\sigma$ is electrical conductivity, and $T$ is absolute temperature. An expression analogous to (19) may be written for the electronic thermal resistivity:

$$
W_{e}=\frac{1}{\lambda_{e}}=W_{e 0}+W_{e l}+\ldots
$$

where $W_{e 0}$ is the thermal resistivity due to scattering of electrons by static imperfections and $W_{e l}$ is the thermal resistivity due to scattering of electrons by phonons [7,60-61]. Similarly, the electrical resistivity may be expressed as

$$
\rho=\frac{1}{\sigma}=\rho_{0}+\rho_{l}+\ldots,
$$

which is exactly analogous to eq (23). We now assume that each component of electronic thermal resistivity is related to the corresponding electrical resistivity by a relation such as eq (22) but with a different characteristic "Lorenz function" for each scattering mechanism. The electronic thermal resistivity may then be written as

$$
W_{e}=\frac{1}{\lambda_{e}}=\frac{1}{T}\left[\frac{\rho_{0}}{L_{0}}+\frac{\rho_{l}}{L_{l}}+\cdots\right] .
$$

These "Lorenz numbers" are not, in general, constant but may vary with temperature. To a good approximation, the structural electronic thermal resistivity (i.e., that due to scattering of electrons by impurities and imperfections) is related to the structural electrical resistivity at all temperatures by a Lorenz relation where $L_{0}$ has the constant theoretical value $L_{0}=2.443 \times 10^{-8}$ $V^{2} K^{-2}{ }^{8}$ (In the present paper, the nomenclature $L_{0}$ will always be used when this theoretical value is meant.) The coefficient, $L_{l}$, associated with the electronic thermal resistivity due to lattice vibrations, is not a constant. At low temperatures, electrons are scattered inelastically and nonisotropically by electron-phonon interactions. Thus the effective mean free path for thermal conduction is smaller than for electrical conduction. Therefore $L_{l}$, as here defined, rises from a value of zero at 0 degrees kelvin and asymptotically approaches the theoretical value, $L_{0}$, at temperatures well above the Debye characteristic temperature, $\theta$.

If there are no additional scattering mechanisms, so that $W_{e}=W_{e 0}+W_{e l}$ and $\rho=\rho_{0}+\rho_{l}$, then the effective Lorenz function is, from eqs (22) and (25),

$$
L=L_{l}\left[1-\frac{\rho_{0}}{\rho}\left(1-\frac{L_{l}}{L_{0}}\right)\right]^{-1}
$$

For a very pure metal where $\rho_{0} / \rho<<1, L \approx L_{l}$; for a highly disordered metal where $\rho \approx \rho_{0}, L \approx L_{0}$. In figure 10 , the theoretical temperature dependence [62-64] of $L / L_{0}$ is shown for various values of $\rho_{0}$. For any real specimen, $\rho_{0}$ is finite so that at sufficiently low temperatures $\rho=\rho_{0}$ and $L=L_{0}$.

At low temperatures, the electronic thermal conductivity of a fairly pure metal or dilute alloy is given closely by

$$
\frac{1}{\lambda_{e}}=\alpha T^{n}+\frac{\beta}{T}, \quad T<<\theta,
$$

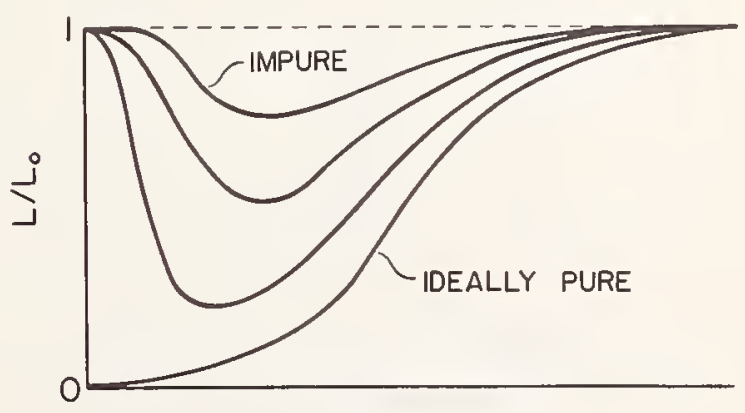

TEMPERATURE

FIgURE 10. The temperature variation of the Lorenz function as a function of purity and temperature.

$L$ is the limiting value of $L$ at low and high temperatures. The top curve relates to the most impure specimen (after Rosenberg [8, p. 120]).

${ }^{8} L_{0}=\frac{\pi^{2}}{3}\left(\frac{k}{e}\right)^{2}$, where $k$ is Boltzmann's constant and $e$ is the charge on an electron. 
where $n>2, \beta \approx \rho_{0} / L_{0}$, and $\alpha$ is an empirical constant which is characteristic of the particular metal. At higher temperatures, electrons are scattered elastically and isotropically by phonons, and $L_{l}=L_{0}$; therefore

$$
\begin{gathered}
W_{e}=\frac{1}{\lambda_{e}} \approx \frac{\rho}{L_{0} T} \\
\approx \frac{1}{L_{0}}\left(\frac{\rho_{0}}{T}+b\right), T>\theta,
\end{gathered}
$$

since $\rho \approx \rho_{0}+b T$ at higher temperatures. For highly pure metals, the electronic thermal conductivity approaches the constant value $\lambda_{e}=L_{0} / b$ at high temperatures. For extremely disordered alloys, the structural resistivity, $\rho_{0}$, dominates and the electronic thermal conductivity increases linearly with temperature.

In figure 11 we see the idealized behavior of the thermal conductivity of copper as derived by Cezairliyan and Touloukian [65-67]. At the lowest temperatures, the thermal conductivities increase linearly with increasing temperature. The thermal conductivity values pass through a maximum value, $\lambda_{m}$, at a different temperature, $T_{m}$, for each sample and then decrease again at higher temperatures to an approximately constant value. These recommended values of Cezairliyan and Touloukian are in only fair agreement with experimental data obtained on copper [68-72].

The electronic component of thermal conductivity for ferromagnetic metals, transition metals, and semi-metals (e.g., bismuth) is less well understood than for more ideal metals and the above is of somewhat restricted applicability. Additional references to the theory of the thermal conductivity of metals include [7-9, 11, 73-79].

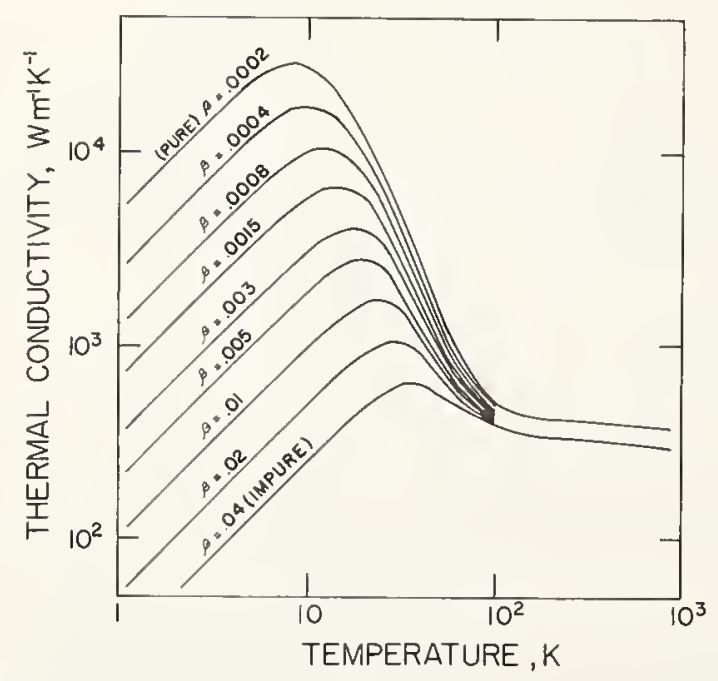

FigURe 11. Thermal conductivity of samples of copper of different purity as a function of temperature.

The parameter $\beta$, expressed in units of $\mathrm{mK}^{2} \mathrm{~W}^{-1}$, (see eq (27)) is approximately equal to the residual electrical resistivity of the sample divided by the theoretical value of the Lorenz function (after Cezairliyan and Touloukian [65-67]).
In an "intrinsic" semiconductor at 0 degrees kelvin, the valence band is completely filled and the conduction band is completely empty; thus the electrical conductivity and the electronic thermal conductivity of an intrinsic semiconductor are zero at absolute zero. As the temperature increases, some electrons attain sufficient thermal energy to get into the conduction band. This results in "holes" being left in the valence band. It is possible for energy and charge to move through the crystal by means of electrons moving from one hole in the valence band to another hole. The semiconductor acts as if there were particles of positive charge moving through it. Thus it has become common to talk about the motion of the holes, which under the influence of a temperature gradient or electric field will result in a net transfer of thermal energy or electric charge.

In an intrinsic semiconductor, the number of electrons in the conduction band and the number of holes in the valence band increase very rapidly with increasing temperature. This results in a rapid increase in electrical conductivity with temperature. The relation between electronic thermal conductivity and electrical conductivity for an intrinsic semiconductor is rather complex $[7,12,14,80-81]$. At higher temperatures, the Lorenz function is given, if certain assumptions are met, by

$$
L=\frac{\lambda_{e}}{\sigma T}=2\left(\frac{k}{e}\right)^{2}\left[1+\frac{\sigma_{h} \sigma_{e}}{2 \sigma^{2}}\left(4+\frac{E_{g}}{k T}\right)^{2}\right],
$$

where $\sigma=\sigma_{e}+\sigma_{h}, \sigma_{e}$ and $\sigma_{h}$ being the portions of the electrical conductivity due to electrons and holes, respectively, and $E_{g}$ is the width of the forbidden gap between the valence and conduction bands. Equation (29) has its maximum value when $\sigma_{h}=\sigma_{e}, L$ then being greater than $6(k / e)^{2}$ by an amount which depends on $E_{g}$.

An "extrinsic" semiconductor, which may in its purest state be an electrical insulator, contains impurities which have either more or fewer valence electrons per atom than the normal atom. This results in the creation of either electrons in the conduction band ( $n$-type semiconductor) or holes in the valence band ( $p$-type semiconductor). Either results in an increase in the electrical conductivity. ${ }^{9}$ In a compound semiconductor, a stoichiometric deficiency of one constituent will act as an impurity. ${ }^{10}$

If the conduction band of an extrinsic semiconductor is only slightly filled, the Fermi energy, and hence the Fermi temperature, will be low so that, at sufficiently high temperatures, $T>>T_{F}$ and the electron gas is nondegenerate. Similarly, if there is a small number of holes in the valence band, the hole gas is nondegenerate. Under such conditions, the Lorenz function is simply $L=$ $2(k / e)^{2}$, which is what eq $(29)$ reduces to if either

\footnotetext{
- For example, 10 ppm of boron in pure silicon will increase the room temper-

ature electrical conductivity by a factor of $10^{3}$.
10 For example, $\mathrm{Cu}_{2} \mathrm{O}$ and $\mathrm{ZnO}$.
} 
$\sigma_{e}$ or $\sigma_{h}$ vanish. If many electrons (or holes) are present so that $T<<T_{F}$, the carrier gas becomes degenerate and $L=L_{0}=1_{3} \pi^{2}(\mathrm{k} / \mathrm{e})^{2}$, as for a metal. The curve in figure 12 shows how the electronic thermal conductivity of a simple extrinsic semiconductor varies with the electrical conductivity.

\section{c. Radiation "Conduction"}

Planck's law [82-84] states that the energy emission per unit time and per unit area $\left(\mathrm{Wm}^{-2}\right)$ from a blackbody in a range of angular frequency $d \omega\left(\mathrm{rad} \mathrm{s}^{-1}\right)$ is given by

$$
e_{b \omega}(T) d \omega=\frac{\hbar \omega^{3} n^{2} d \omega}{4 \pi^{2} c^{2}[\exp (\hbar \omega / k T)-1]},
$$

where $\hbar$ and $k$ have previously been identified, $c$ is the speed of light in vacuum, and $n$ is the index of refraction (at $\omega$ ) of the medium which bounds the blackbody. Expressing eq (30) in terms of frequency rather than wavelength is preferable for our present purpose since $\omega$ does not change when radiation travels from one medium to another medium which has a different index of refraction (i.e., speed of light). Equation (30) can be rearranged as

$$
\frac{e_{b \omega}}{\sigma n^{2} T^{3}}=\frac{15 \hbar}{\pi^{4} k}\left(\frac{\hbar \omega}{k T}\right)^{3} \frac{1}{[\exp (\hbar \omega / k T)-1]},
$$

where $\sigma=\frac{\pi^{2} k^{4}}{60 \hbar^{3} c^{2}}=5.6697 \times 10^{-8} \mathrm{Wm}^{-2} \mathrm{~K}^{-4}$ is known as the Stefan-Boltzmann constant. In this form

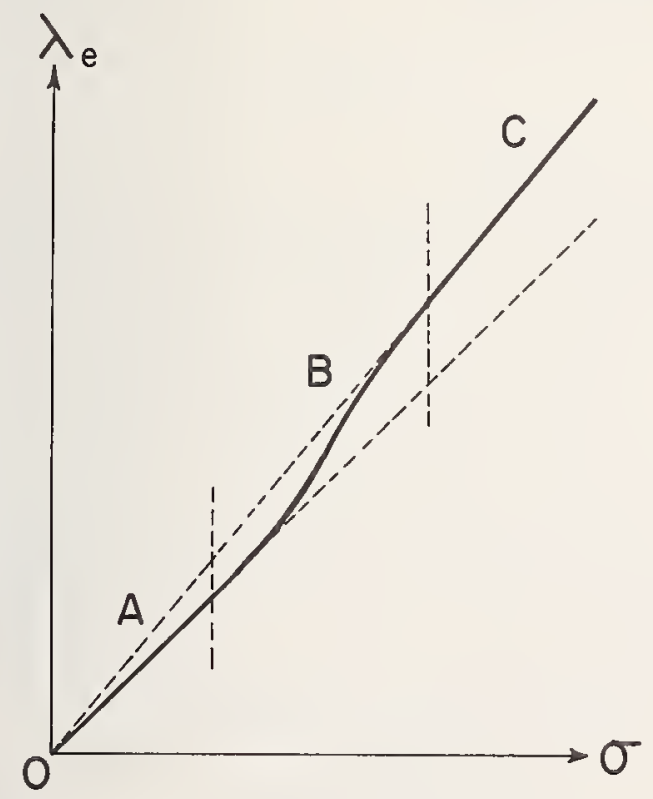

Figure 12. Plot of electronic thermal conductivity versus electrical conductivity for a simple extrinsic semiconductor at constant temperature.

Region $\mathbf{A}$ is the nondegenerate region in which the carriers obey classical statistics and the Lorenz function is given by $L=2(k / e)^{2}$. In the degenerate region, $C$, the carriers obey FermiDirac statistics and $\dot{L}=1 / 3 \pi^{2}(k / e)^{2}$. Region B is intermediate between these two limiting cases. the right hand side of eq (31) is a universal function of $k T / \hbar \omega$ and is so plotted in figure 13.

We note that

$$
\frac{k T}{\hbar \omega}=\frac{k T}{h \nu}=\frac{n l T}{c_{2}},
$$

where $\nu=2 \pi \omega$ is frequency, $l$ is wavelength (in the medium having $n$ as its index of refraction), and $c_{2}=2 \pi \hbar c / k=h c / k=1.43879 \times 10^{-2} \mathrm{~m} \mathrm{~K}$ is known as the second radiation constant. ${ }^{11}$

The total energy radiated is obtained by integrating eq (30) over all frequencies:

$$
e_{b}(T)=\int_{0}^{\infty} e_{b \omega}(T) d \omega=\frac{\hbar}{4 \pi^{2} c^{2}} \int_{0}^{\infty} \frac{n^{2} \omega^{3} d \omega}{[\exp (\hbar \omega / k T)-1]} .
$$

If we assume that the index of refraction of the bounding medium is independent of frequency, eq (33) can be integrated to yield

$$
e_{b}(T)=n^{2} \sigma T^{4} \text {. }
$$

Eq (34) is usually seen without the factor $n^{2}$, since $n=1$ in a vacuum.

The monochromatic energy emitted from a real (non-black) surface is

$$
e_{\omega}=\epsilon_{\omega} e_{b \omega},
$$

where $\epsilon_{\omega}$ is the hemispherical emittance of the surface for radiation of angular frequency $\omega$ (i.e.,

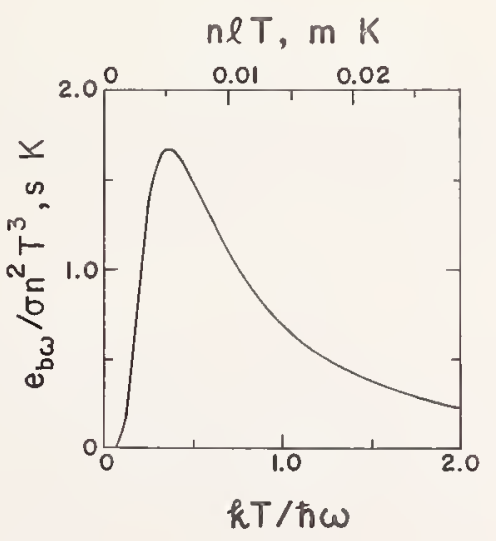

Figdre 13. Spectral emissive power of a blackbody.

it Usually one sees $e_{b l}$, the energy emission in a range of wavelength $d l$, rather than $e_{b \omega}$, which we have diseussed here and plotted in figure 13 . The quantity which is usually plotted is some multiple of

$$
\frac{e_{b l}}{n^{3} T^{5}}=\frac{c_{1}}{(n l T)^{5}\left[\exp \left(c_{2} / n l T\right)-1\right]}
$$

where $c_{1}=2 \pi h c^{2}=3.7405 \times 10^{-16} \mathrm{Wm}^{2}$ is known as the first radiation constant. This is most frequently seen without the $n$ appearing explicitly, $n=1$ being assumed. Even if $n$ is included, this expression of Planck's law in terms of wavelength rather than frequency is not generally valid since it depends on the assumption that $n$ is independent of wavelength. 
photons of energy $\hbar \omega$ ). The total energy emitted from a surface is

$$
e=\int_{0}^{\infty} e_{\omega} d \omega=\int_{0}^{\infty} \epsilon_{\omega} e_{b \omega} d \omega
$$

The total hemispherical emittance is defined as the ratio of the emissive power, $e$, of a given surface to that of a black surface at the same temperature, i.e.,

$$
\epsilon=\frac{e}{e_{b}}
$$

Note that $\epsilon$ is a property of the surface and its temperature.

The monochromatic energy per unit time and area absorbed by a real surface is

$$
\alpha_{\omega} H_{\omega}
$$

where $H_{\omega}$ is the intensity of the incident radiation and $\alpha_{\omega}$ is the monochromatic hemispherical absorptance of the surface. The total hemispherical absorptance is

$$
\alpha=\frac{\int_{0}^{\infty} \alpha_{\omega} H_{\omega} d \omega}{\int_{0}^{\infty} H_{\omega} d \omega} .
$$

Note that $\alpha$ is not a property of the surface alone but is a function of the nature of the incident radiation.

Kirchhoff's law states that

$$
\alpha_{\omega}=\epsilon_{\omega} .
$$

One frequently sees the assumption being made that Kirchhoff's law holds for total absorptance and emittance. This assumption is valid in some cases [84, pp. 41-42] but in general

$$
\alpha \neq \epsilon \text {. }
$$

We now wish to discuss radiative transfer of energy within a diathermanous material; that is, one which is partially transparent to infrared radiation. Radiation (photons) traveling through a medium can suffer attenuation due to absorption and/or scattering. Consider a monochromatic beam of radiation of intensity $I_{\omega}$ (energy per unit time per unit solid angle) as shown in figure 14 . The intensity will be attenuated as

$$
I_{\omega}=I_{0 \omega} e^{-\left(\kappa_{\omega}+\gamma_{\omega}\right) s}=I_{0 \omega} e^{-\beta_{\omega} s}
$$

where $I_{0 \omega}$ is the intensity at $s=0$, the apex of the beam, $\kappa_{\omega}$ is the monochromatic absorption coefficient for photons of energy $\hbar \omega, \gamma_{\omega}$ is the monochromatic scattering coefficient, and $\beta_{\omega}=\kappa_{\omega}+\gamma_{\omega}$ is the monochromatic extinction coefficient.

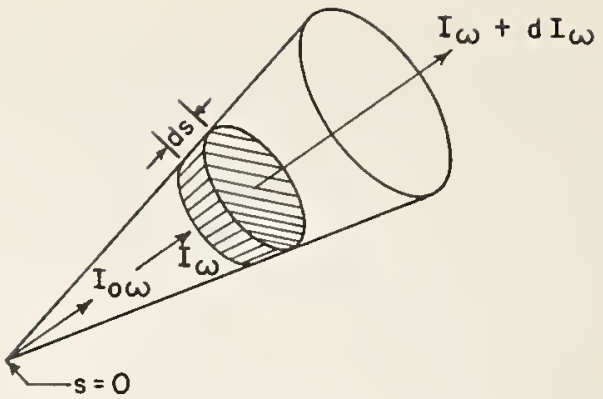

FIgdre 14. Attenuation of radiation due to absorption and scattering (after Sparrow and Cess $[84, p .17]$ ).

There is a fundamental difference between absorption and scattering of photons which should be kept in mind. When a photon is absorbed it is out of the picture, so to speak, and we need no longer be concerned about it. When a photon is scattered, on the other hand, it is still active as an energy carrier and hence we must consider where it goes after being scattered. In isotropic scattering, which is of ten assumed (and probably rarely justified), a photon has no memory of which way it was going before being scattered and hence has equal probability of traveling in any direction. In small-angle scattering the photon is deflected only slightly by interaction with a scattering center so that this type of scattering has much less effect on energy transfer.

From an extension of Kirchoff's law, it is apparent that any medium which absorbs radiation must also emit radiation. For an isotropic medium, the monochromatic emission per unit time and volume is

$$
4 \kappa_{\omega} e_{b \omega} \cdot
$$

The total emission is

$$
4 \kappa_{p} e_{b \omega}
$$

where

$$
\kappa_{p}=\frac{\int_{0}^{\infty} \kappa_{\omega} e_{b \omega} d \omega}{e_{b}} .
$$

Total absorption and scattering coefficients may be defined as

$$
\kappa=\frac{\int_{0}^{\infty} \kappa_{\omega} \int_{4 \pi} I_{\omega} d \Omega d \omega}{\int_{0}^{\infty} \int_{4 \pi} I_{\omega} d \Omega d \omega} .
$$

and

$$
\gamma=\frac{\int_{0}^{\infty} \gamma_{\omega} \int_{4 \pi} I_{\omega} d \Omega d_{\omega}}{\int_{0}^{\infty} \int_{4 \pi} I_{\omega} d \Omega d \omega},
$$


where $d \Omega$ is the element of solid angle and the $4 \pi$ signifies that the integration is to be over all directions. However, since $I_{\omega}$ will depend on conditions throughout the medium and at the surrounding surfaces, $\kappa$ and $\gamma$ are not generally properties of the medium and hence are of rather limited usefulness.

We now have set forth the basic equations which are necessary in order to calculate the heat flux through a diathermanous material. Let us consider one-dimensional heat flow in a slab of thickness L. It is convenient to define a monochromatic optical thickness:

$$
\tau_{\omega}=\int_{0}^{z} \beta_{\omega} d z
$$

where $z$ is a position coordinate measured from the lower surface of the slab. Sparrow and Cess [84, Ch. 7], who cite earlier work, show that the intensity of radiation at any point in the slab can be divided into two components as shown in figure 15 . The monochromatic radiation having a component in the positive $z$-direction is designated as $I_{\omega}{ }^{+}\left(\tau_{\omega}, \mu\right)$; that which has a component in the negative $z$-direction, by $I_{\omega}{ }^{-}\left(\tau_{\omega}, \mu\right)$, where $\mu=\cos \theta$ designates the direction in which the photons are traveling ( $\theta$ is measured relative to the normal to the slab). The component of radiation in the positive direction is given by

$$
\begin{aligned}
& I_{\omega}^{+}\left(\tau_{\omega}, \mu\right)=I_{\omega}^{+}(0, \mu) e^{-\tau_{\omega} / \mu} \\
& \quad+\frac{1}{\pi} \int_{0}^{\tau_{\omega}} \frac{1}{\beta_{\omega}}\left[\kappa_{\omega} e_{b \omega}(t)+\frac{\gamma_{\omega}}{4} G_{\omega}(t)\right] e^{-\left(\tau_{\omega}-t\right) / \mu} \frac{d t}{\mu},
\end{aligned}
$$

where

$$
G_{\omega}\left(\tau_{\omega}\right)=2 \pi \int_{0}^{\pi} I_{\omega}\left(\tau_{\omega}, \mu^{\prime}\right) d \mu^{\prime}
$$

is the incident energy per unit area inside the slab. Both $e_{b \omega}(t)$ and $G_{\omega}(t)$ which appear in eq (48) are unknown functions of position. The first term on the right hand side of eq (48) is the energy that enters at the $z=0$ surface of the slab and is attenuated in accordance with eq (41). The first term in the integral in eq (48) represents energy which is emitted by the medium in the region below $\tau_{\omega}$; the second term in the integral represents energy scattered in the $\theta$ direction in the region below $\tau_{\omega}$. An equation analogous to eq (48) can be written for $I_{\omega}^{-}$, the component in

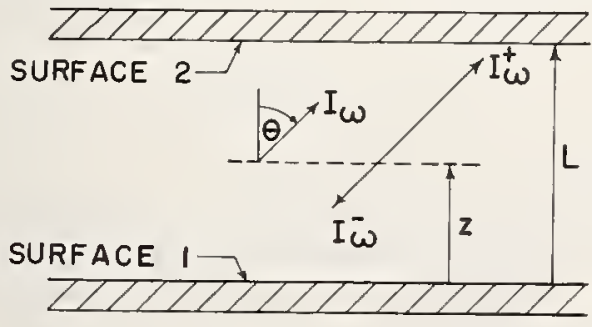

FIGURe 15. Coordinate system for onedimensional radiative transfer (after Sparrow and Cess $[84, p .195]$ ). the negative $z$-direction. The projection of $I_{\omega}=I_{\omega}{ }^{+}+I_{\omega}{ }^{-}$in the $z$-direction gives the total monochromatic radiation flux normal to the slab at any position. Integration over all values of $\omega$ gives the total radiation heat transfer, $q_{T}$.

If both radiative heat transfer and conductive heat transfer are present, conservation of energy requires that, at steady state,

$$
\boldsymbol{\nabla} \cdot(\lambda \boldsymbol{\nabla} T)=\boldsymbol{\nabla} \cdot \boldsymbol{q}_{r},
$$

where $\lambda$ does not include any radiative contribution to the thermal conductivity. Equation (50) implies that there is an interaction between heat transfer by conduction and that by radiation. One cannot, in general, compute the heat flow and temperature distribution due to conduction and, separately, those due to radiation and then add them. As we shall see, this can be done under certain rather restrictive circumstances. In general, the system of equations describing energy transfer in a diathermanous medium consists of two rather formidable simul taneous, nonlinear, in tegrodifferential equations that must be integrated over all photon frequencies. Solution of these equations under various conditions is the subject of a great deal of recent research [85-117]. In this paper we shall only briefly discuss some limiting cases.

Consider first the optically thin limit where the optical thickness of the slab is small, i.e., $\tau_{\omega}(z=L)<<1$, for all $\omega$. In this case, the mean free path of a photon is much larger than the thickness of the slab so that absorption, scattering, and volume emission within the slab are not important. We are only concerned with radiation between the two surfaces. The monochromatic radiation flux between the two surfaces, of emittances $\epsilon_{1 \omega}$ and $\epsilon_{2 \omega}$, is

$$
q_{\tau \omega}=\frac{\left[e_{b \omega}\left(T_{1}\right)-e_{b \omega}\left(T_{2}\right)\right]}{\left(1 / \epsilon_{1_{\omega}}\right)+\left(1 / \epsilon_{2_{\omega}}\right)-1}
$$

where $T_{1}$ and $T_{2}$ are the temperatures of the surface. In general, eq (51) would have to be integrated over all frequencies. For the special case in which the bounding surfaces of the slab are gray, ${ }^{12}$ diffuse ${ }^{13}$ emitters (and absorbers) of energy, the total radiation flux is

where

$$
q_{r}=\sigma n^{2} E\left(T_{1}^{4}-T_{2}^{4}\right),
$$

$$
E \equiv \frac{1}{\frac{1}{\epsilon_{1}}+\frac{1}{\epsilon_{2}}-1},
$$

$\epsilon_{1}$ and $\epsilon_{2}$ being the total hemispherical emittances of the two surfaces. Let us further assume that $\left|T_{1}-T_{2}\right|<<\bar{T}=\left(T_{1}+T_{2}\right) / 2 ;$ then

$$
q_{r}=4 \sigma n^{2} E \bar{T}^{3}\left(T_{1}-T_{2}\right) \text {. }
$$

12 That is, $\epsilon_{1 \omega}$ and $\epsilon_{2 \omega}$ are the same for all $\omega$.

${ }_{13}$ That is, the intensity of the radiation leaving either surface is the same in all angular directions. 
In the optically thin limit it can be shown [84] that the total heat flux is the sum of the radiative flux and the conducted flux; thus for a medium of constant thermal conductivity,

$$
q=q_{c}+q_{r}=\left(\lambda+4 \sigma n^{2} \bar{E}^{3}{ }^{3} L\right) \frac{T_{1}-T_{2}}{L},
$$

where $L$ is the total thickness of the slab. The apparent total thermal conductivity is

$$
\lambda_{\mathrm{app}}=\lambda+4 \sigma n^{2} E \bar{T}^{3} L .
$$

Note that in the optically thin limit, the apparent, or effective, radiative component of thermal conductivity is proportional to thickness and hence is not an intrinsic property of the material.

Consider next the optically thick limit where the optical thickness of the slab is large, i.e., $\tau_{\omega}(z=L)>>1$, for all $\omega$. In this case the mean free path of a photon is much less than the thickness of the slab so that conditions at the surface become unimportant and radiative heat transfer is controlled by absorption, scattering, and volume emission. In the optically thick limit, the monochromatic radiant energy content per unit volume in a frequency interval $d \omega$ is

$$
\frac{4 n e_{b \omega}(T) d \omega}{c}
$$

so that the monochromatic contribution to the volumetric heat capacity is

$$
C_{\omega}=\frac{4 n}{c} \frac{d e_{b \omega}}{d T} d \omega
$$

the velocity of a photon is $v=c / n$; the mean free path of a photon is $\Lambda=1 / \beta_{\omega}$. With these quantities substituted into eq (5) or eq (7), the monochromatic contribution to the thermal conductivity is

$$
\lambda_{\tau \omega}=\frac{4}{3 \beta_{\omega}} \frac{d e_{b \omega}}{d t} d \omega
$$

The total radiative thermal conductivity is

$$
\lambda_{r}=\frac{4}{3 \beta_{R}} \frac{d e_{b}}{d T}=\frac{16 n^{2} \sigma T^{3}}{3 \beta_{R}},
$$

where $\beta_{R}$ is the Rosseland mean extinction coefficient defined by

$$
\frac{1}{\beta_{R}}=\int^{\infty} \frac{1}{\beta_{\omega}} \frac{d e_{b \omega}}{d e_{b}} d \omega
$$

Thus under conditions where the optjcally thick approximation holds, it is meaningful to define a radiative component of thermal conductivity.

In many materials, the extinction coefficient, $\beta_{\omega}$, varies strongly with $\omega$. 'The factor $d e_{b \omega} / d e_{b}$ in the integrand of eq (60) gives greater weight to the values of $\beta_{\omega}$ in the frequency region where there is the most energy. Thus even if $\beta_{\omega}$ were independent of temperature for all $\omega$, the effective extinction coefficient, $\beta_{R}$, could be a very strong function of temperature. For certain materials $\lambda$, could, therefore, increase as $T^{n}$, where $n$ might be as large as 10 .

In figure 16 we show the qualitative behavior of the apparent thermal conductivity of a diathermanous material as a function of thickness. The increase in the apparent thermal conductivity for small thicknesses will vary as $E L$, where $E$ (see eq (54)) is the effective emittance of the bounding surfaces and $L$ is thickness. As the thickness is increased, the increase in the apparent thermal conductivity will asymptotically approach $\lambda_{r}$ as defined in eq (59).

In summary, the radiative contribution to thermal conductivity can be considered as a unique property of a material only when the characteristic dimensions of the sample in question are large compared to the average mean free path for photons. ${ }^{14}$ This is analogous to the situation with the lattice component of thermal conductivity at low temperatures, where we noted that it was necessary to speak of the thermal conductivity of a particular sjze of specimen in the region where the lattice thermal conductivity is limited by boundary scattering of phonons.

Values for the monochromatic extinction coefficient and index of refraction of a number of materials are given in [118]. Most of these correspond to room temperature. Gardon [119] presented values for the spectral absorption coefficient of window glass in the solid and liquid state. Inspection of these values, which are illustrated in figure 17 , reveals that below $600{ }^{\circ} \mathrm{C}$ the monochromatic absorption coefficients are practically independent

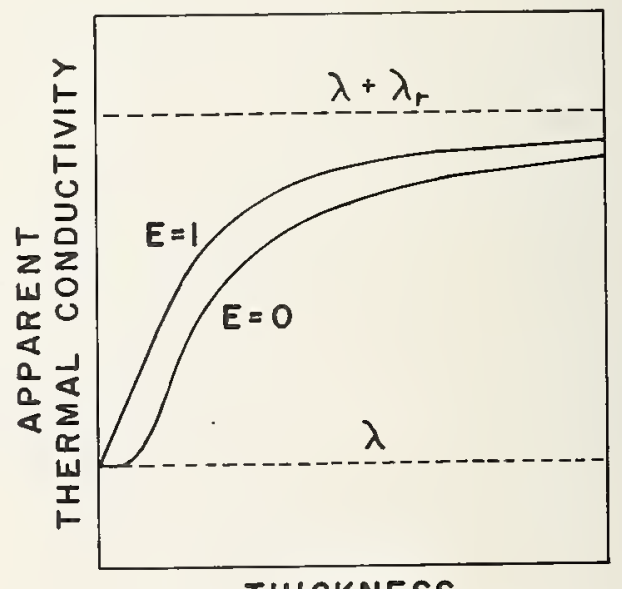

THICKNESS

Figure 16. Apparent thermal conductivity of a diathermanous material as a function of thickness and the effective emittance of the bounding surfaces.

14 The average mean free path for photons is simply $1 / \beta_{R}$, as defined in eq $(60)$. 
of temperature so that one could use room temperature values $[118,120-121]$ of monochromatic absorption coefficients to estimate, using eqs (60) and (61), the radiative component of thermal conductivity for glasses. At temperatures sufficiently high that photons are excited in the wavelength region ${ }^{15}$ shorter than about $2.8 \mu \mathrm{m}$, the weighting factor, $d e_{b w} / d e_{b}$, in eq (60) will cause the Rosseland mean extinction coefficient to decrease very rapidly with increasing temperatures and thus cause a rapid increase in the radiative thermal conductivity. There do not appear to be many high temperature data on the extinction coefficients of ceramics. Folweiler and his group [122124] have carried out high temperature absorption coefficient measurements on single crystal $\mathrm{Al}_{2} \mathrm{O}_{3}$, $\mathrm{MgO}, \mathrm{SrTiO}_{3}$, and $\mathrm{CaF}_{2}$ and on fused $\mathrm{SiO}_{2}$ glass. Chapin and Howe [125] have made measurements on radiant energy transport in $\mathrm{BeO}$.

In general, the radiative thermal conductivity will be reduced below the value in, say, a single crystal by grain boundaries, pores, and any impurities which absorb or scatter photons. In other sections of this paper we will see some experimental results which illustrate certain aspects of radiant energy transfer in ceramics.

Since there are no comprehensive review papers (to my knowledge) which adequately cover the subject of the radiative component of thermal conductivity, I have included a rather large number of references [126-223] to individual papers and books which have bearing on this subject. These references, plus those previously cited [82-125], while not exhaustive, cover a good portion of the pertinent literature.

\section{d. Gas Conduction}

In porous ceramics, it is necessary to be concerned with the thermal conductivity of the gas which fills the pores. For a classical gas, the heat capacity per atom is $f k / 2$, where $f$ is the effective

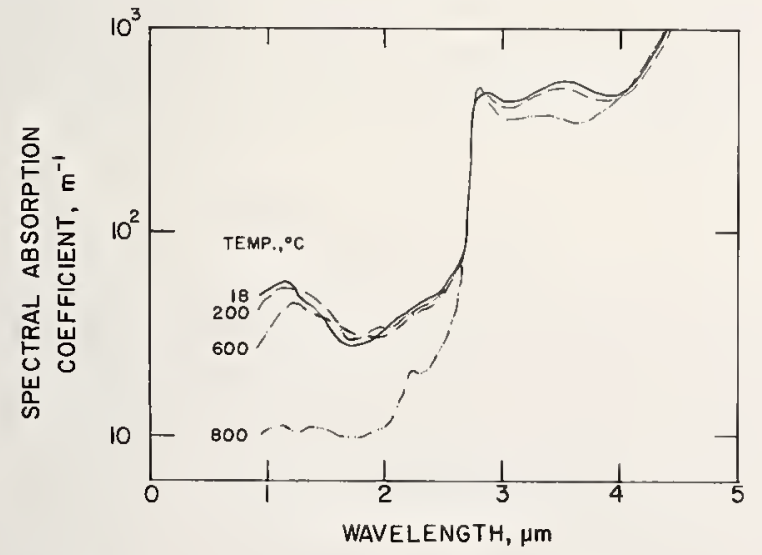

Figdre 17. Monochromatic absorption coefficients of window glass at different temperatures (after Gardon [119]).

The original reference also shows data at 1000 and $1300^{\circ} \mathrm{C}$.

${ }^{15}$ Wavelengths measured in air, not in the glass. number of degrees of freedom (translationalvibrational +rotational) of the gas molecule. For an ideal classical gas, then, the heat capacity per unit volume at constant volume is simply

$$
C=\frac{f p}{2 T}
$$

where $p$ is the pressure of the gas. The mean free path of a gas molecule for a not-too-dense gas is

$$
\Lambda=\frac{k T}{p S}
$$

where $S$ is the effective cross sectional area of a gas molecule. This relation follows easily from the reasonable assumption that the mean free path is inversely proportional to the density of the gas. The average translational velocity of an ideal classical gas is

$$
v=\sqrt{\frac{3 k T}{m}}=\sqrt{\frac{3 R T}{M}},
$$

where $m$ is the weight of the gas molecule, $M$ is the molecular weight of the gas, and $R=8.3143$ $\mathrm{JK}^{-1} \mathrm{~mol}^{-1}$ is the gas constant.

Combining eqs $(5,61,62$, and 63), the thermal conductivity of an ideal classical gas is given by

$$
\lambda=\frac{f_{k}}{6 S} \sqrt{\frac{3 R T}{M}} .
$$

The implications of this equation are as follows:

1. Thermal conductivity is independent of pressure.

2. Thermal conductivity increases as the square root of absolute temperature.

3. Thermal conductivity is proportional to the number of degrees of freedom of the gas molecule.

4. Thermal conductivity decreases with increasing molecular weight. The scattering cross section, $S$, in general increases with molecular weight, so that thermal conductivity decreases more rapidly than $\mathrm{M}^{-1 / 2}$. For gases with the same number of degrees of freedom, thermal conductivity is very roughly inversely proportional to molecular weight.

Perhaps the most surprising thing about the thermal conductivity of a gas is that it is independent of pressure. This happens because the specific heat is proportional to pressure, the mean free path is inversely proportional to pressure, and the average velocity of a molecule is independent of pressure; thus the pressure dependences cancel out. At low pressures, the mean free path becomes large and may be limited by the size of the pores in which it is contained, thus resulting in a decrease in the effective thermal conductivity of the gas. At high pressures, the mean free path 
approaches the size of the molecules and the thermal conductivity increases with pressure.

Real gases depart somewhat from the simplified analysis given above. At low temperatures, particularly in the case of helium, quantum effects enter in. At higher temperatures, additional degrees of freedom may become excited. In general, however, eq (64) provides a fair quantitative description of the thermal conductivity of a gas. For an extended treatment of all the factors which affect the thermal conductivity of gases, the interested reader may consult the treatise by Hirschfelder, Curtis, and Bird [224].

\section{e. Other Transport Mechanisms}

There are some mechanisms, in addition to those discussed above, by which energy may be transported in a ceramic material. In general, these are much less important than phonons, electrons, photons, and gas molecules so we will accordingly only mention them briefly.

In section $2.2 \mathrm{~b}$ we briefly discussed the independent motion of electrons and holes in a semiconductor and the associated thermal conductivity (see eq (29) and accompanying text). It is also possible for an electron and a hole to form a bound state known as an exciton in which an electron and a hole are bound together (in a manner analogous to the formation of a hydrogen atom by a proton and an election) and can travel through a solid as a single entity. Since an exciton is electrically neutral, there is no electrical current associated with its motion.

There has been some speculation in the literature [225-228] regarding the existence of exciton heat transfer in ceramics at high temperatures. To date, however, there is insufficient evidence to prove that this mode of heat conduction is of any practical importance. Recent literature concerning the theory of excitons includes [229-231]; these references cite some of the earlier work.

Ziman [7, p. 319] points out that in some solids the molecules have a significant rotational specific heat which presumably can be transferred between neighboring molecules, thus contributing to the thermal conductivity.

Ziman [7, p. 319] also mentions the possibility of heat conduction by spin waves in which energy is transferred by interaction of spin magnetic moments.

We have not mentioned thermal conductivity in superconductors. In brief, the thermal conductivity of a material will be lower in the superconducting state than in the normal state since the superconducting electrons do not contribute to heat transport. At sufficiently low temperatures, there is no electronic contribution to the thermal conductivity of a superconducting material. Rosenberg [8] discusses the thermal conductivity of superconductors.

If there is mass transport of any kind in a material, there will be an associated energy of transport which can augment heat conduction.
The most obvious mechanism that we have not discussed is gaseous convection. In a fibrous or granular material having a continuous gaseous phase, there can be convection within the material which under certain circumstances can transfer very large quantities of heat. Even in a material having closed pores so that there is no net transfer of gas through the material, convection within the pores can cause increased heat transfer across the pores. As a rule-of-thumb, convection within closed pores is not important unless the pores are larger than, say, $5 \mathrm{~mm}$.

A more subtle type of mass transfer is the diffusion of individual molecules or imperfections under the influence of a potential field. For example, in electromigration, molecules or impurities can migrate under the influence of an applied electric field. In the Soret effect, molecules or impurities can migrate under the influence of the temperature gradient. Since the energy transport is proportional to the temperature gradient, the Soret effect is a legitimate augmentation (or depletion, depending on the sign of the heat of transport) of the thermal conductivity. However, since the Soret effect can result in a redistribution of impurities, vacancies, etc., the thermal conductivity could change with time. Both electromigration and the Soret effect become significant only at temperatures approaching the melting point of the solid. I do not know of any cases where the effect of either mechanism on thermal conductivity has been observed.

If the vapor pressure of a material or one of its constituents becomes significant, a large energy transfer can occur through porous materials due to sublimation (or evaporation) and condensation. Even in nonporous materials, the heat of vaporization could make it very difficult to make an accurate thermal conductivity measurement.

\section{Effects of Environment}

Before discussing the effects of composition and structure on the thermal conductivity of ceramics, it is important to briefly discuss the influences of environment.

\subsection{Temperature}

As we have seen in section 2.2 , thermal conductivity is influenced by temperature for all of the heat conduction mechanisms which were discussed. In later sections, we shall see examples of how the dependence of thermal conductivity on composition or structure varies with temperature. In this section we present several figures showing thermal conductivity versus temperature for a number of ceramic materials and then show a few examples to illustrate the relative importance of different mechanisms of heat conduction at different temperatures.

In figure 18 we compare on a log-log plot the low temperature thermal conductivity of several 


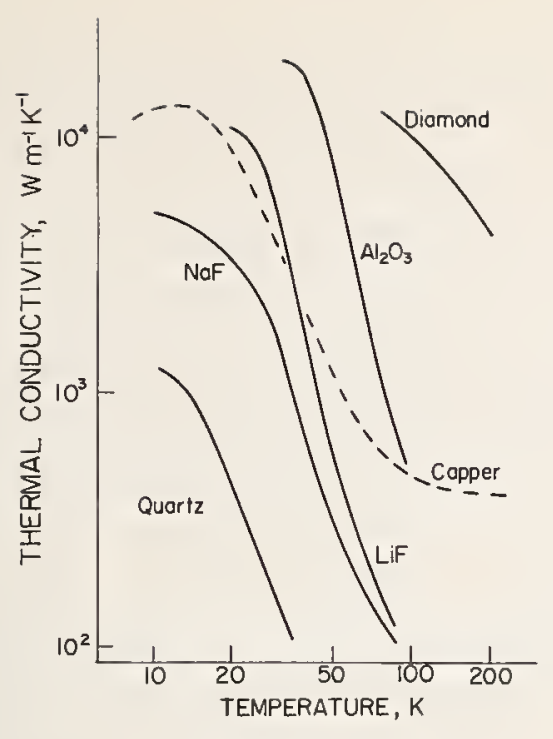

FIGURE 18. Thermal conductivity of some non-metallic crystals compared with very pure copper (after Berman [232]).

nonmetallic single crystals with the thermal conductivity of a very pure copper sample. Heat conduction in these dielectric crystals is entirely due to phonons at low temperatures while the thermal conductivity of copper is chiefly due to electrons. At higher temperatures the thermal conductivity of copper decreases slowly with increasing temperatures, falling from $400 \mathrm{Wm}^{-1} \mathrm{~K}^{-1}$ at room temperature to $340 \mathrm{Wm}^{-1} \mathrm{~K}^{-1}$ at $1200 \mathrm{~K}$. Conversely the thermal conductivity of the dielectric materials continues to decrease fairly rapidly, going roughly as $\mathrm{T}^{-1}$.

In figure 19 we compare the high temperature thermal conductivity of several dense ceramic materials. In figure 20 the thermal conductivities of a number of porous ceramics are shown as functions of mean temperature. We shall discuss the effect of porosity in section 5.2.

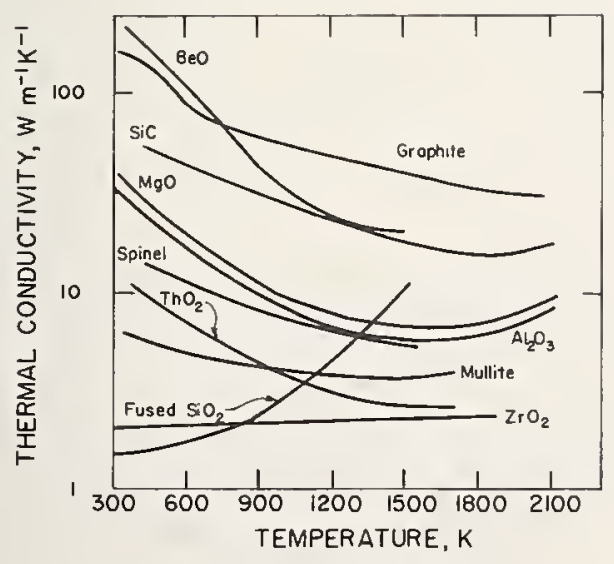

Figure 19. High temperature thermal conductivity of several dense ceramic materials (after Kingery, Klein, and McQuarrie [239]).

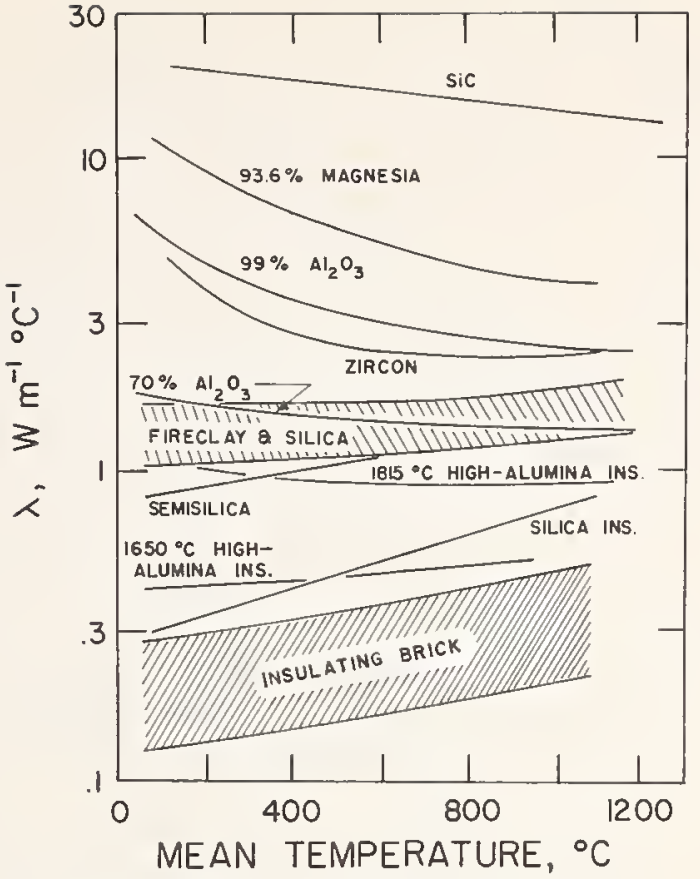

FIgURE 20. Thermal conductivity of several refractory brick (after Ruh and McDowell [234]).

The thermal conductivity of titanium carbide is shown in figure 21. The electronic component of thermal conductivity, computed from eq (22) using $L=L_{0}$ for the Lorenz function, is seen to be about one-half of the total thermal conductivity, the remainder being due to lattice conduction (phonons). This behavior can be contrasted with that of oxide ceramics which generally have a negligible electronic contribution to the thermal conductivity. This separation of thermal conduction into an electronic and a lattice component should not be taken too. seriously since $L$ may not be equal to $L_{0}$ for titanium carbide.

In figure 22, the thermal resistivity data of Taylor [236] for beryllium oxide are shown as a function of temperature. The samples were hotpressed to 99 percent of theoretical density. From 1000 to $1850 \mathrm{~K}$ the thermal resistivity increases

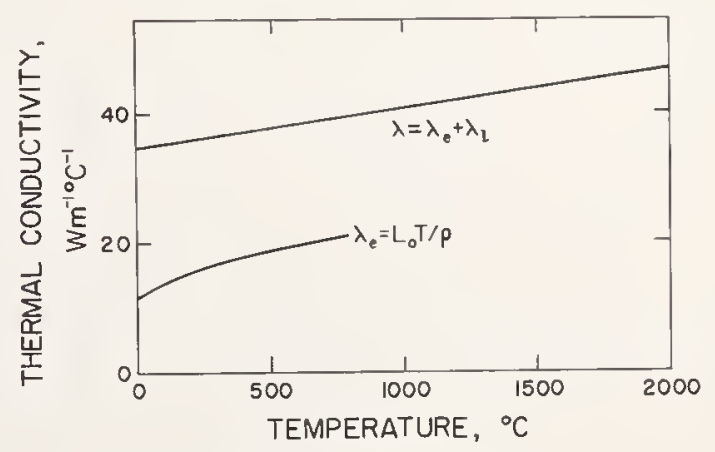

Figure 21. High temperature thermal conductivity of titanium carbide.

The upper curve represents the total measured thermal conductivity, $\lambda=\lambda_{c}+\lambda_{l}$. The lower curve represents the electronic contribution, $\lambda_{\theta}$, computed using the theoretical Sommerfeld value for the Lorenz function (after Williams [235]). 


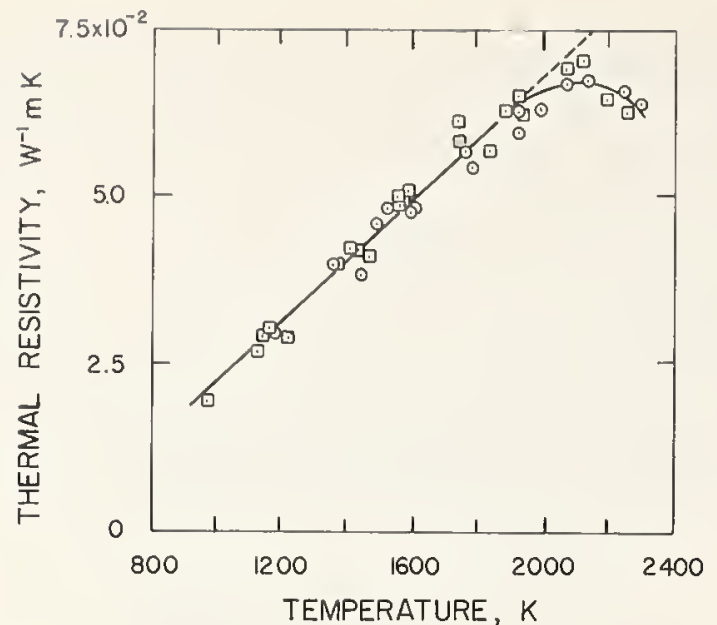

FIGURE 22. High temperature thermal resistivity of two samples of high density beryllium oxide (after Taylor [236]).

linearly with temperature, as predicted by eq (20). At higher temperatures, the thermal resistivity departs from this linear relation, passes through a maximum near $2100 \mathrm{~K}$ and then decreases with increasing temperature. The departure of the high temperature data from the extrapolation of the line through the data below $1850 \mathrm{~K}$ increases as approximately $T^{17}$. There are several possible reasons for this departure. At these very high temperatures, the mean free path of a phonon is becoming very short-of the order of interatomic distances. Thus the mean free path of a phonon may become essentially constant at sufficiently high temperatures. Since specific heat and phonon velocity are also essentially constant at very high temperatures, this would result in a temperature-independent phonon contribution to the thermal conductivity. Such behavior of the mean free path would explain the departure from a linear relation but would not explain the decrease in thermal resistivity (increase in thermal conductivity) at the highest temperatures. To explain this it is necessary to invoke an additional mechanism of heat conduction. The most plausible candidate is radiative heat transfer, or photon conduction. Equation (59) predicts a $T^{3}$ temperature dependence for the total radiative thermal conductivity if the index of refraction and the extinction coefficient are independent of temperature. Chapin and Howe [125] found that beryllia became more opaque to thermal radiation at very high temperatures; if this is so the radiative component of thermal conductivity would increase less rapidly than $T^{3}$. However, the combination of a constant mean free path and a radiative contribution might still lead to the behavior observed in figure 22 . There is also the possibility of some more exotic mechanism such as exciton conduction. At such high temperatures, experimental errors could be quite significant and might account for part of the turn-down in figure 22.
In the case of glasses, the importance of radiative heat transfer is quite clear. Referring back to figure 19 , the thermal conductivity of fused silica is seen to increase slowly with temperature near room temperature and then to increase much more rapidly at higher temperatures. Since fused silica is an amorphous material, the phonon mean free path above room temperature is essentially constant at a value approximating the size of a silicon dioxide tetrahedron [237]. The increase with temperature in the thermal conductivity of fused silica near room temperature is due to the increase in the heat capacity. The spectral absorption coefficient for fused silica is similar to that for window glass as shown in figure 17. Fused silica is essentially opaque to wavelengths (measured in air) longer than $2.8 \mu \mathrm{m}$. At temperatures below, say, $400 \mathrm{~K}$, there is negligible thermal radiation (cf. figure 13) at wavelengths below $2.8 \mu \mathrm{m}$ so that the radiation contribution to thermal conductivity is completely negligible. Above approximately $600 \mathrm{~K}$, there are sufficient short-wavelength photons to transfer significant amounts of energy and the radiative component of thermal conductivity rises very rapidly (perhaps as $T^{4}$ to $T^{6}$ ) with increasing temperature.

Figure 23 shows some values for the apparent radiative contribution to thermal conductivity in a sample of fused silica and in single crystals of three oxide ceramics. These samples were probably not large enough, in comparison to the photon mean free paths, for these measured values to be true properties of the materials.

The temperature dependence of the thermal conductivity of a number of oxide ceramics can be observed from the recommended values given by

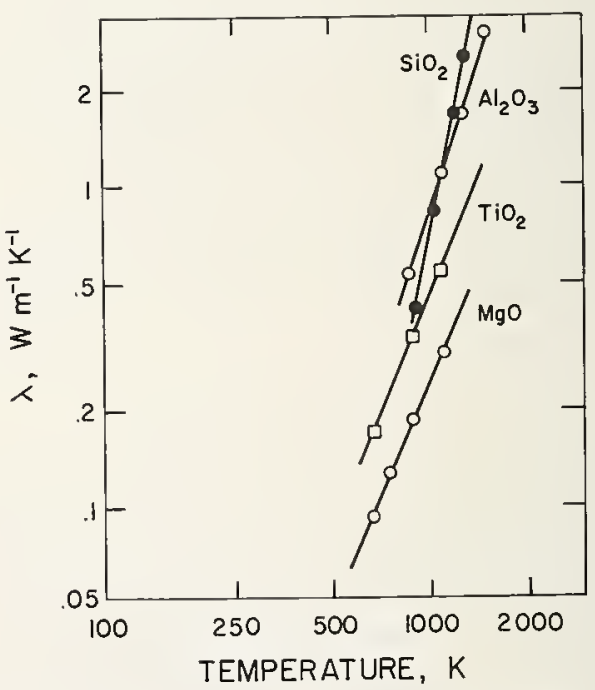

FIGURE 23. Effective radiative component of thermal conductivity in 2.5 $\mathrm{cm}$ samples of silica glass and three single crystal oxide ceramics (after Kingery, Klein, and McQuarrie [233]: the original data are due to Charvat and Kingery [439] and Lee and Kingery [189]). 
Powell, Ho, and Liley [238]. Recommended values for the thermal conductivity of several types of graphite are given by Ho, Powell, and Liley [239]. If one wishes to study the temperature dependence of the thermal conductivity of other ceramic materials, the best place to find the appropriate references is from one of the various data compilations issued by the Thermophysical Properties Research Center [240]. A good review paper, which includes discussion and examples of the temperature dependence of thermal conductivity, has been written by Kingery [241].

\subsection{Atmosphere}

The thermal conductivity of a porous material is dependent on the type of atmosphere pervading the material. In the case of materials having no continuous solid phase, the difference between the thermal conductivity in vacuo and in the presence of, say, atmospheric pressure gas may be several orders of magnitude. Many of the references cited in Section $5.2 \mathrm{~b}$ are concerned with multiphase materials having no continuous solid phase.

In the case of porous ceramic materials, the thermal conductivity will vary in different atmospheres provided the atmosphere can pervade the pores. In figure 24 we show the thermal conductivity of a commercial insulating fire brick in helium, air, and argon. As is apparent from eq (64), the low-molecular-weight gas has the highest thermal conductivity. Reducing the gas pressure would not significantly affect the thermal conductivity values shown in figure 24 until the mean free path of the gas molecules approached the pore size. A reasonably good vacuum would result in thermal conductivity values below those shown for argon. We will return to porous materials in Section $5.2 \mathrm{~b}$.

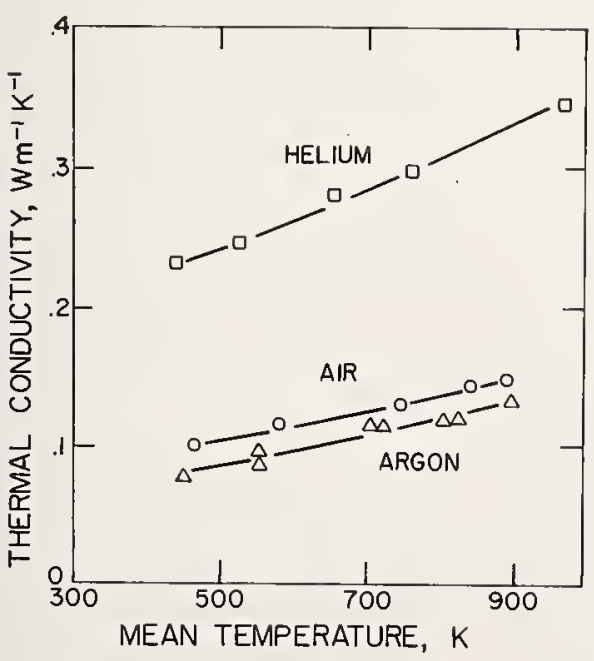

FIgURE 24. Thermal conductivity of a commercial insulating firebrick in various gases (after Young, Hartwig, and Norton [242]).

\subsection{Electromagnetic Fields}

The effect of an electromagnetic field on thermal conductivity is much too complex a subject to discuss in any detail here. Consequently, I shall only make a few brief remarks.

In general, thermal conductivity will not be significantly affected by an electric field of the magnitude normally encountered in engineering applications. In the case of a metal, platinum, Flynn and O'Hagan [243] found no measurable effects on thermal conductivity due to electric current densities of $10^{8} \mathrm{Am}^{-2}$; it should be noted that this corresponded to electric fields of less than $400 \mathrm{Vm}^{-1}$. In semiconductors, or insulators, larger electric fields can be sustained and effects on thermal conductivity might conceivably become significant.

A magnetic field of rather small magnitude exerts a much greater force on an electron than can be exerted by any attainable electric field. In brief, a magnetic field causes an electron (or hole) to follow a curved path so that the effective mean free path in the direction of the temperature gradient is reduced. This can result in a very significant reduction in the electronic component of thermal conductivity. The application of a magnetic field can cause the thermal conductivity to be anisotropic. Ziman [7, pp. 483-523] may be consulted for details. In a dielectric solid, where there is no significant electronic contribution to the thermal conductivity, the application of a magnetic field would normally not affect thermal conductivity.

We have previously discussed the transfer of heat by photons through partially transparent materials. In that discussion, it was tacitly assumed that the spectral distribution of radiation impinging on a volume element of the material was, at least roughly, the same as the spectral distribution of energy emitted by the volume element. If a material is subjected to radiation with an electromagnetic field (photons) of quite different spectral characteristics from those of the field which would be extant in an infinitely large, approximately isothermal, body of the same material (at the same temperature), the radiative heat transfer through the material may be very dependent on the int nsity and spectral distribution of this applied electromagnetic field. For example, at room temperature there is a negligible radiative contribution to the thermal conductivity of window glass. However, high frequency photons due to solar radiation will pass readily through window glass with a large attendant transfer of heat. Under such conditions, it is meaningless to attempt to associate a thermal conductivity with this heat transfer.

\subsection{Pressure and Stress}

In sections $3.1 \mathrm{~d}$ and 3.2 we mentioned briefly the effect of pressure on the thermal conductivity of gases. Near atmospheric pressure, the thermal 
conductivity of a gas is almost independent of pressure.

There has been very little experimental work on the effect of hydrostatic pressure on the thermal conductivity of solids. Bridgman [244, 245] measured the thermal conductivity of a number of rocks and minerals as a function of pressure. Hughes and Sawin [246] have recently measured the pressure dependence of thermal conductivity of several dielectric solids at pressures up to about $1.9 \times 10^{9} \mathrm{Nm}^{-2}\left(\sim 1.9 \times 10^{3} \mathrm{~atm}\right)$ and temperatures to $450{ }^{\circ} \mathrm{C}$. They found the (lattice thermal conductivity to increase by 50 to 100 percent over the range 0 to $2 \times 10^{8} \mathrm{Nm}^{-2}$ ( $\sim 2 \times 10^{3} \mathrm{~atm}$ ) and then to increase less rapidly at higher pressures. This colresponds to less than 0.05 percent for a pressure change of $1 \mathrm{~atm}$.

In a dielectric solid, the increase in thermal conductivity with pressure can be associated with the increase in the Debye temperature (see eqs (16) and (17)). Drickamer et al. [247] review the effect of pressure on the lattice parameters of solids and cite a number of pertinent references. The effect of pressure on the thermal conductivity of a dielectric solid is much greater in the case of solid helium [248-250] where the Debye temperature can be changed very drastically by applied pressure.

At high pressures, of the order of $10^{10} \mathrm{Nm}^{-2}$, many insulators and semiconductors transform to metallic behavior so that the electrical conductivity, and presumably the electronic component of thermal conductivity, increase by several orders of magnitude. Drickamer [251] has surveyed the literature on this increase in the electrical conductivity; I do not know of any experimental thermal conductivity work in this pressure region.

Bridgman [252] found that the Lorenz function, $\lambda_{e} / \sigma T$, of metals could either increase or decrease with increasing pressure. Starr [253], however, found the ratio of thermal to electrical conductivity at constant temperature to remain essentially constant at pressures up to about $10^{9} \mathrm{Nm}^{-2}$. If this is so, one can predict the change in electronic thermal conductivity from the change in electrical conductivity. Review papers on the effect of pressure on the electrical properties of metals or semiconductors include $[245,251,254-258]$. In brief, the electrical conductivity of most metals increases by an amount less than 0.01 percent for each atmosphere of pressure increase. Most semiconductors, and some metals, exhibit a decrease in electrical conductivity with increasing pressure.

In the vast majority of engineering applications, the pressure dependence of thermal conductivity for solid materials can be neglected.

There is also very little information on the effect of elastic strain, due to nonhydrostatic compression, to tension, or to torsion, on thermal conductivity. Any strain, other than hydrostatic, will distort the lattice and thus make it more difficult for energy carriers (e.g., electrons, phonons) to travel through the lattice. This will decrease the thermal conductivity. Bridgman [259] has measured the effect of tensile stress on the thermal conductivity of a number of metals near room temperature and found the decrease in thermal conductivity to be proportional to the stress. As an example, he found that a tensile stress of about $10^{8} \mathrm{Nm}^{-2}\left(\sim 1.5 \times 10^{4} \mathrm{lb}\right.$ in $\left.^{-2}\right)$ decreased the thermal conductivity of copper by about 0.2 percent. The apparent thermal conductivity, however, computed using the unstressed dimensions of the specimen rather than the stressed dimensions, would have increased by about 0.8 percent under this stress. For metals in torsion, the thermal conductivity decrease is approximately proportional to the square of the twist [260-261]. In the case of copper, a twist of 1 $\mathrm{rad} \mathrm{m}^{-1}$ of length reduced the thermal conductivity by about 0.2 percent.

At low temperatures (say, $<100 \mathrm{~K}$ ), the thermal conductivity of relatively pure metals and dielectric crystalline solids might be significantly affected by elastic deformation. Above room temperature, any such effects are probably of no practical importance in most engineering applications. An exception to this would occur if stresses caused microcracks to appear in the material.

If a solid undergoes plastic deformation, its thermal conductivity will be significantly decreased due to the large number of dislocations formed in the lattice. Cold-working of a metal could reduce its thermal conductivity by an order of magnitude. Such a large effect would be much less likely in most ceramic materials due to their brittle nature. We will return to the subject of dislocations and other lattice imperfections in section 5.2 .

\subsection{Heat Flow}

Fourier's law, eq (1), defines thermal conductivity as the negative ratio of the heat flux to the temperature gradient. In practically all applications of thermal conductivity, it is tacitly assum ed that the thermal conductivity of a material does not depend on the heat flux (or the temperature gradient) within the material. In principle, this assumption need not be valid. In practice, no one has yet conclusively proven it is not.

Austin [262] reviewed some of the early attemp ts to find a variation of thermal conductivity with heat flow and concluded that the existence of such an effect had not been established. Recently, Patrassi [263] reported a very large dependence of thermal conductivity on temperature gradient (or heat flow) for uranium dioxide. His experimental procedure and conclusions have been severely criticized by Fulkerson, McElroy, and Moore [264], who made independent measurements of the thermal conductivity of uranium dioxide and found no measureable effect due to temperature gradient. Other laboratories, as a result of Patrassi's article, have attempted to find an effect such as he describes. None of this other work has yet been 
published but as far as I know, there has been no confirmation of Patrassi's results.

There are several possible effects, other than experimental error, which could result in measured thermal conductivity values being dependent on heat flow:

1. Further extension of the formal theory of heat conduction might reveal the necessity of including some higher order terms which would indicate that heat flux is not strictly proportional to temperature gradient.

2. Steep temperature gradients may induce sufficient strain in the solid to scatter energy carriers and lower the thermal conductivity (see section 3.4). Alternatively, the induced strain may open microcracks in the sample.

3. The differential equation for heat flow in a solid may not be valid in the presence of very steep temperature gradients. Examination of the derivation of the diffusion equation reveals that an implicit assumption is made which may not be valid at very high temperature gradients. Without going into detail, it is necussary that

$$
\frac{1}{\lambda} \frac{d \lambda}{d T} \times \frac{d T}{d z} \times \Lambda<<1
$$

Essentially, we are saying that if the diffusion equation is to be valid, it is necessary that there be no significant change in the thermal conductivity over a distance of the order of the mean free path of the energy carriers.

It appears that some further theoretical and experimental work will be required before the issue of the possible dependence of thermal conductivity on heat flux is resolved.

\section{Directional Effects}

Many materials are anisotropic as regards thermal conductivity. All single crystals of other than cubic symmetry may be expected to possess thermal conductivities which are directionaldependent. For single-crystal quartz, the ratio of the thermal conductivity parallel to the $c$-axis to that perpendicular to the $c$-axis drops from 2.0 at $40 \mathrm{~K}$ to 1.4 at $700 \mathrm{~K}$. The thermal conductivity of pyrolytic graphite is extremely anisotropic, typically by a factor of as much as 100 to 200, depending on temperature and details of fabrication.

Polycrystalline materials will generally be more isotropic than single crystals of the some material. Hydrostatically pressed and sintered materials are usually reasonably isotropic. Extruded materials normally retain a significant portion of the anisotropicity characteristic of the single-crystal material.

Carslaw and Jaeger [1] discuss the mathematical theory of heat flow in anisotropic materials. The thermal conductivity of an anisotropic material is best expressed as a tensor rather than a scalar. Drabble and Goldsmid [14, p. 67] discuss the phenomenological theory of heat conduction in anisotropic solids.

In highly-anisotropic materials, it is questionable whether or not the intrinsic thermal conductivity in the lowest-conductivity direction has ever been measured. Kaspar and Zehms [265], who cite a few earlier references, point out that the observed thermal conductivity value for pyrolytic graphite in the $c$-direction is often simply a component of the conductivity perpendicular to the $c$-axis which arises due to departures from ideal crystalline orientation.

In an applicatlon where anisotropicity of thermal conductivity may be important, experimental checks are in order unless previous measurements have been made on the same material produced by the same process.

\section{Effects of Composition and Structure}

It would be possible to write a fairly large book solely concerned with the effects of composition and structure on the thermal conductivity of solids. In this paper I can only attempt to touch on most of the factors of importance and give examples of a number of them.

The influence of compositional and structural variations at low temperatures is covered in some depth, either directly or by reference, in the reviews by Ziman [7], Rosenberg [8], and Klemens [9-11]. At high temperatures, the somewhat more pragmatical reviews by Austin $[78,262]$ and Kingery [233, 241] are very helpful.

\subsection{Composition}

In this section we shall be concerned with the effect of the chemical composition of a solid material on thermal conductivity.

\section{a. Molecular Structure}

The electronic thermal conductivity of a material is related to the number of "free" conduction electrons in the material. As discussed in $2.2 \mathrm{~b}$, the electronic component of thermal conductivity is approximately proportional to the electrical conductivity. Thus copper and silver have very high electronic thermal conductivities as a result of their high electrical conductivities. Many metallic carbides, borides, silicides, nitrides, and sulfides have sufficiently high electrical conductivities to possess significant electronic components of thermal conductivity. The magnitude of the electronic component of thermal conductivity of electrically conductive ceramics can be estimated using the Lorenz relation (eq (22)) with the theoretical Lorenz number, $L_{0}=2.443 \times$ $10^{-8} \mathrm{~V}^{2} \mathrm{~K}^{-2}$.

The lattice component of thermal conductivity in a pure, dense, crystalline material is strongly 
correlated with the complexity of the molecular structure. For example, referring back to figure 19 , the thermal conductivity of alumina is greater than that of spinel which in turn is greater than that of mullite. A rule of thumb is that the simpler structure will have the higher lattice thermal conductivity. For similar molecular structures, the material with the lowest molecular weight will generally have the highest intrinsic thermal conductivity. This trend can be observed for the nonmetallic elements by looking at the data compilation due to $\mathrm{Ho}$ and Powell [266]. In the case of metallic oxides, this trend is seen in figure 25 , where thermal conductivity is plotted versus the atomic weight of the cation.

\section{b. Impurities}

Introduction of impurities into a pure crystalline material normally reduces the thermal conductivity. This is true for metals where the foreign atoms scatter electrons, thus reducing the electronic thermal conductivity. It is true for dielectric materials where the foreign atoms scatter phonons and reduce the lattice thermal conductivity. The thermal resistivities due to impurities are additive for each conduction mechanism (see eqs (19) and (23) for phonons and electrons, respectively).

The additional electronic thermal resistance due to impurities is related to the corresponding increase in electrical resistivity by the Lorenz relation (see eq (25)). Thus we can confine our attention to the effect of impurities on electrical resistivity. The increase in electrical resistivity, due to $x$ atomic percent of a given impurity, is essentially independent of temperature and is given remarkably well by Nordheim's rule [267]:

$$
\Delta \rho(x)=A x(1-x)
$$

where $A$ is a constant which depends upon the parent material (solvent) and the impurity (solute). For small concentrations, the increase in electrical resistivity is proportional to the atomic concentration of impurity atoms. In

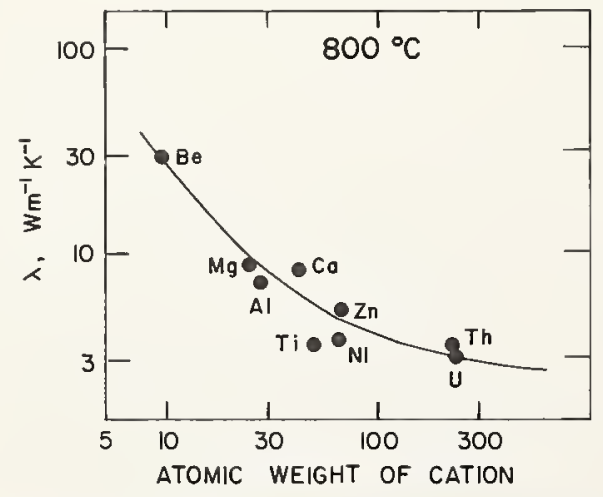

Figure 25. Thermal conductivity of several dense ceramic oxides (after Kingery, Klein and McQuarrie [239]). general, the constant $A$ is eq (65) will be smallest if the impurity atom has the same valency as the parent material. In the case of metals, Linde's rule [268], although not very accurately obeyed, serves as a rule of thumb for the effect of valency:

$$
\Delta \rho=a+b Z^{2}
$$

where $Z$ is the valency difference and $a$ and $b$ are constants for a given solvent for each row in the periodic table from which the solutes are taken.

There is no simple relation, analogous to the Lorenz relation, which permits one to use a more easily measured property to compute the change in the lattice thermal conductivity due to introduction of impurities. In general, the effect of a given atomic percent of impurity on the lattice thermal conductivity will be greatest when the atomic mass difference between solvent and solute is greatest. Except at very low temperatures, the additional thermal resistivity due to impurities is essentially independent of temperature. Since the thermal resistivity due to phonon-phonon interactions (Umklapp-processes) increases approximately linearly with temperature, the relative effect of impurities decreases approximately linearly with temperature in a dielectric crystal. In figure 26 we see the thermal conductivity of a number of specimens of potassium chloride doped with different amounts of potassium nitrite. Increasing the concentration of impurities lowers the peak in the thermal conductivity curve and shif ts it to a higher temperature. Different types of impurities will affect the thermal conductivity by different amounts. The curves shown in figure 26 are typical, however, in that the effect is very

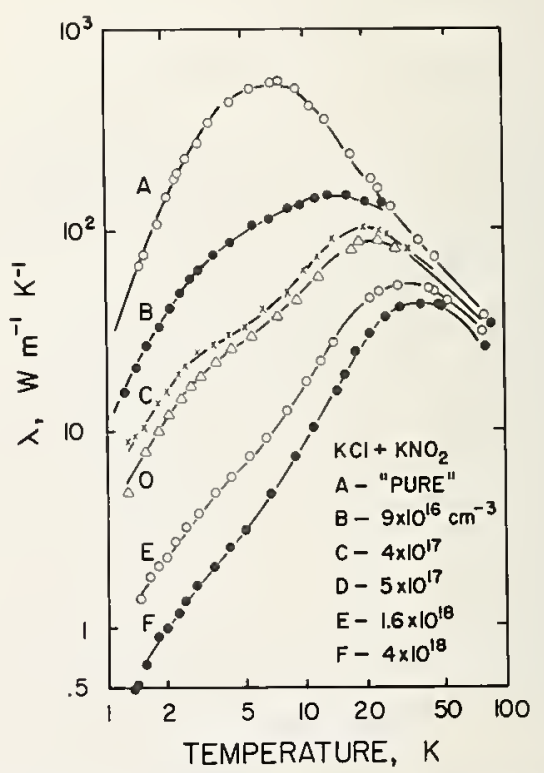

Figure 26. Thermal conductivity of potassium chloride crystals doped with $\mathrm{NO}_{2}$ - ions (after Pohl [286]).

The curves are identified according to the density of $\mathrm{NO}_{2}-$ ions in each sample. 


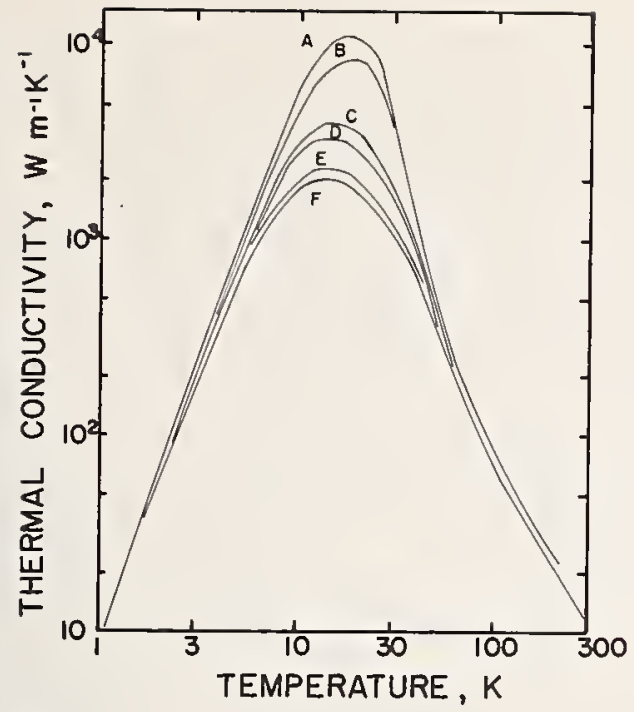

Figure 27. Thermal conductivity of single crystals of $\mathrm{LiF}$ with various ${ }^{\circ} \mathrm{Li}$ contents (after Berman and Brock [269]):

$\begin{array}{cccc}\text { Symbol } & \text { Percent }{ }^{6} \mathrm{LiF} & \text { Symbol } & \text { Percent }{ }^{6} \mathrm{LiF} \\ \text { A } & 0.02 & \mathrm{D} & 90.4 \\ \text { B } & 0.01 & \mathrm{E} & 25.0 \\ \text { C } & 4.6 & \mathrm{~F} & 50.1\end{array}$

pronounced at low temperatures and then decreases rapidly with increasing temperature.

At low temperatures the presence of different isotopes of the same element can cause large differences in thermal conductivity. An example of this is shown in figure 27.

The different survey papers cited [7-15] may be consulted for the many references to the effect of impurities on thermal conductivity. Some references which are too recent to be covered in existing reviews include [39, 270-280].

\section{c. Stoichiometry}

Departures from stoichiometry in dielectric compounds will decrease the lattice thermal conductivity since the interstitials or vacancies present act as impurities. In some cases, departures from stoichiometry will increase the electrical conductivity by a sufficient amount to significantly contribute to the electronic thermal conductivity. The resultant thermal conductivity, arising from the sum of the lattice and electronic components, could, in principle, be either increased or decreased by departures from stoichiometry. Pertinent references to the effect of nonstoichiometry on thermal conductivity include [281-285].

\section{d. Alloying}

Alloying is simply the addition of controlled amounts of "impurities", perhaps to the extent that the solvent and solute lose their identities.

In the case of two metals which form a continuous series of solid-solution disordered alloys, the electronic component of thermal conductivity is related, by the Lorenz function, to the electrical conductivity. In the absence of ordering (e.g., intermetallic "compounds"), Nordheim's rule, eq (65), holds reasonably well over the entire composition range. Austin [78] shows several examples of the thermal conductivity of binary solid solutions of two metals.

In the case of solid solutions of dielectric materials, the thermal conductivity usually varies with composition in a manner similar to that shown in figure 28 for the $\mathrm{KCl}-\mathrm{KBr}$ system and in figure 29 for the $\mathrm{MgO}-\mathrm{NiO}$ system. The chief characteristic feature of these plots in that the thermal conductivity values always fall below the straight line connecting the end points, the departures from this line being roughly parabolic in shape. As shown in figure 29, the relative eff ect of alloying decreases with increasing temperature.

Abeles [289] has presented a phenomenological theory describing the variation of lattice thermal conductivity with alloy composition and temperature. Other pertinent references include [290303].

\subsection{Structure}

\section{a. Crystallinity}

In a highly crystalline material, the mean free path of an energy carrier is much longer, and thus the thermal conductivity much higher, than in a disordered or amorphous solid. This effect is most pronounced at lower temperatures. In figure 30 , the thermal conductivity of fused quartz is contrasted with that of crystalline quartz. The curves shown differ by almost three orders of magnitude near $40 \mathrm{~K}$ and then approach a common value at higher temperatures. As mentioned in section 3.1, the phonon mean free path in fused quartz above room temperature is approximately constant at a value approximating the size of the silica tetrahedron. At some sufficiently high temperature, the increased number of Umklapp processes will

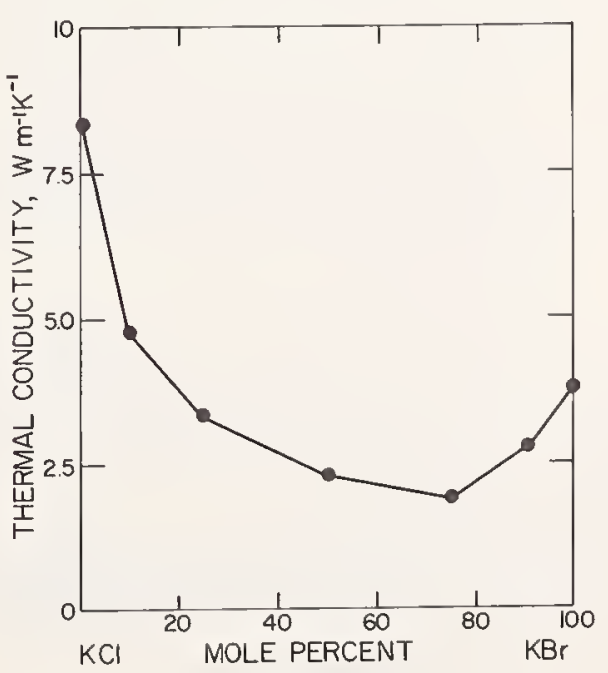

Figure 28. Thermal conductivity of solid solutions of potassium chloride and potassium bromide (after Eucken and Kuhn [287]). 


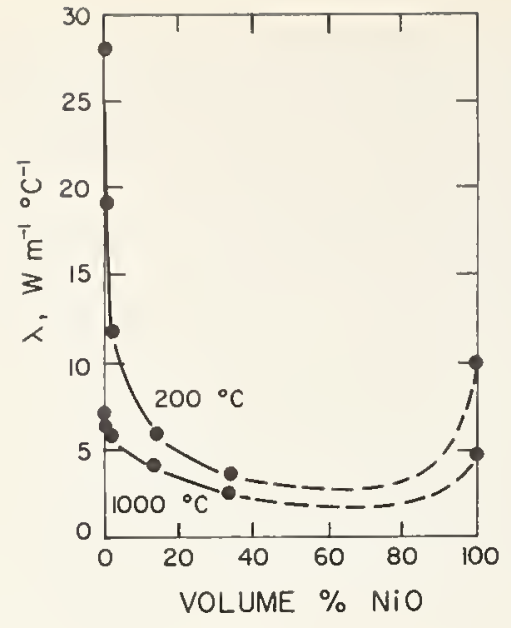

Figure 29. Thermal conductivity of solid solutions of magnesium oxide and nickel oxide (after Kingery [288]).

(The left-hand axis represents pure $\mathrm{MgO}$ ).

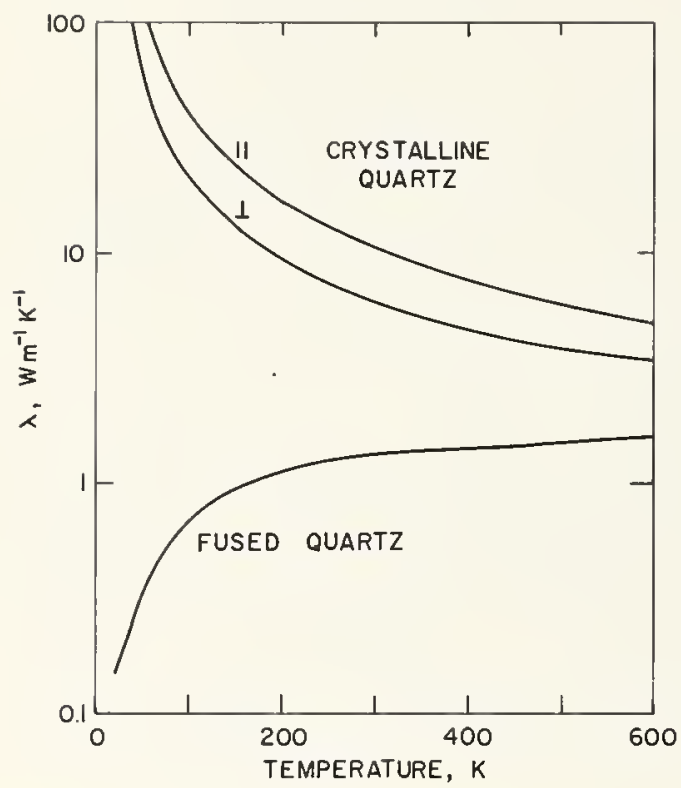

FIGURE 30. Thermal conductivity of crystalline and fused quartz.

The uppermost curve represents the thermal conductivity parallel to the c-axis while the curve just below it represents the conductivity perpendicular to the $c$-axis. These are the recommended values from Powell, Ho, and Liley [238].

decrease the mean free path in crystalline quartz until it reaches the size of the silica tetrahedron. Above this temperature the phonon mean free path will be essentially the same in crystalline and amorphous quartz.

We will see the effect of less drastic departures from crystallinity in the following section.

\section{b. Imperfections}

Dislocations and other imperfections perturb the perfect periodic structure of a crystalline lattice and hence lower the thermal conductivity in much the same manner as we saw for impurities. Table 1 , in section $2.2 \mathrm{a}$, lists the temperature dependence of the thermal resistivity due to different types of imperfections. The various review papers cited [7-15] discuss the effects of imperfections on the thermal conductivity of solids and cite numerous pertinent references. Recent additional references include [304-315].

One class of imperfections that is of particular importance, because of the extensive use of ceramic materials in nuclear applications, is that of the imperfections caused by irradiation. In figure 31, the effect of fast neutron irradiation on the lowtemperature thermal conductivity of single-crystal sapphire is shown. The radiation damage lowers the thermal conductivity, especially near the peak. Part of the damage due to irradiation can be annealed out at high temperatures. Figure 32 shows the thermal conductivity of beryllium oxide at $50^{\circ} \mathrm{C}$ as a function of irradiation dose and irradiation temperature. The effect of a given dose

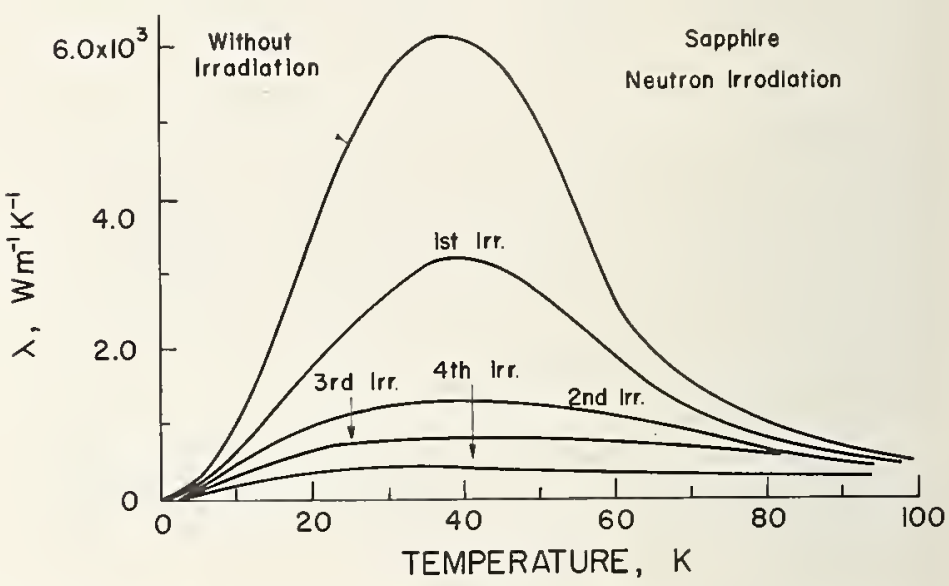

FIgURE 31. Thermal conductivity of synthetic sapphire, $\mathrm{Al}_{2} \mathrm{O}_{3}$, before and after the indicated reactor irradiations.

The doses were $1.5,8.9,20.2$, and $50.2 \times 10^{21}$ fast neutrons $\mathrm{m}^{-2}$, respectively (after Berman, Foster, and Rosenberg [316]).

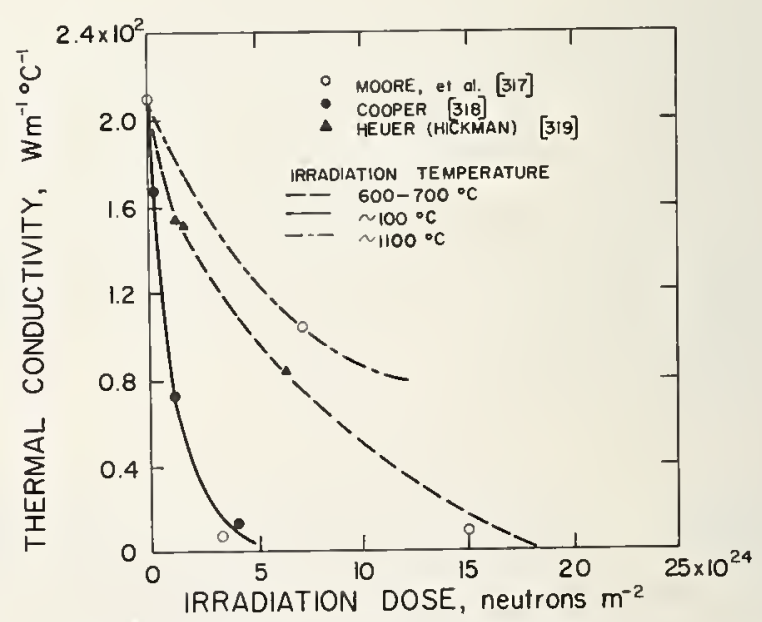

FIGURE 32. The effect of neutron irradiation dose and irradiation temperature on the thermal conductivity of $\mathrm{BeO}$ at $50^{\circ} \mathrm{C}$ (after Moore et al. [317]). 
is less if the irradiation occurs at an elevated temperature.

It is very interesting to compare the effects of neutron irradiation on fused and crystalline quartz. Berman and his co-workers [320-321] found that neutron irradiation lowered the thermal conductivity of crystalline quartz. Crawford and Cohen [322-323] found that irradiation significantly increased the low-temperature thermal conductivity of fused quartz.

References on the effect of neutron and other types of irradiation on thermal conductivity include [324-341]. A few general references on the effects of radiation damage in solids are [342-345].

\section{c. Additional Phases}

All of our previous discussion has been concerned with homogeneous materials. Except for single crystals, most ceramic materials are heterogeneous at least to the extent of having grain boundaries or small porosities. Many ceramic materials have very significant amounts of a second phase present. In this section we will discuss the effects of additional phases on thermal conductivity.

In section 2.1, thermal conductivity was defined as

$$
\lambda=-\frac{q}{\nabla T}
$$

For a heterogeneous material, we extend this definition to

$$
\lambda_{\text {effective }}=-\frac{q}{\langle\nabla T\rangle},
$$

where $\langle\nabla T\rangle$ is the average value of the temperature gradient over a region large in comparison with the size of the inhomogeneities. Unless the sample is large in comparison with the inhomogeneities, it is scarcely meaningful to attempt to define an effective thermal conductivity.

In a multiphase material the effective ther'mal conductivity will depend upon:

1. The thermal conductivity of each phase.

2. The proportions of each phase.

3 . The manner in which the phases are distributed; in particular

a. whether or not the phase is continuous in the direction of heat flow,

b. whether the phase distribution is ordered or random,

c. the size, shape, and orientation of each segment of each phase.

4. The nature of the contacts between the different phases.

5. The emissive and absorptive properties of the phases if there is significant radiative heat transfer through one or more of the phases.

The problem of computing the effective thermal conductivity of a mixture from the thermal conductivities of the components is mathematically the same as the problem of computing the electrical conductivity, dielectric constant, or mag- netic permeability of a heterogeneous mixture. There exists a large body of pertinent literature, much of which is covered in the review articles by Powers [346] and by Meredith and Tobias [347]. Additional references, not given by either [346] or [347], include [78, 140, 149, 162-164, 175, 178, $188,207,213,219,221,223,233-234,241,242$, $262,281,286,288,348-434]$. These references include most of the mathematical analyses of multiphase media and also contain a more limited amount of experimental data on multiphase media. The papers by Everest, et al. [149], Gorring and Churchill [376], Nahas, et al. [400], and Krupiczka [391] contain other reviews of various analytical expressions for predicting conductivity of multiphase media.

We shall now discuss a few of the available mathematical relations for correlating the effective thermal conductivity of a mixture with the thermal conductivities of the individual components. We shall limit our discussion to two-phase systems.

The simplest model for purposes of analysis is that in which the two phases are arrayed in alternative parallel layers as shown in figure 33 . If the heat flow is parallel to the layers, the effective thermal conductivity is given by

$$
\lambda=f_{1} \lambda_{1}+f_{2} \lambda_{2}
$$

where $f_{1}$ and $f_{2}$ are the volume fractions of the phases having thermal conductivities $\lambda_{1}$ and $\lambda_{2}$ respectively. If the heat flow is perpendicular to the phase layers

$$
\lambda=\frac{\lambda_{1} \lambda_{2}}{f_{2} \lambda_{1}+f_{1} \lambda_{2}} .
$$

Equations (68) and (69) represent the extreme limits of the thermal conductivity of a two-phase mixture. These limits are shown in figure 34 for the case $\lambda_{1}=10 \lambda_{2}$. Although both eq (68) and (69) predict thermal conductivity values intermediate between the conductivities of the individual components we see that the conductivity obtained is very different for the two cases. Thus these limits are of relatively little use except for laminated materials.

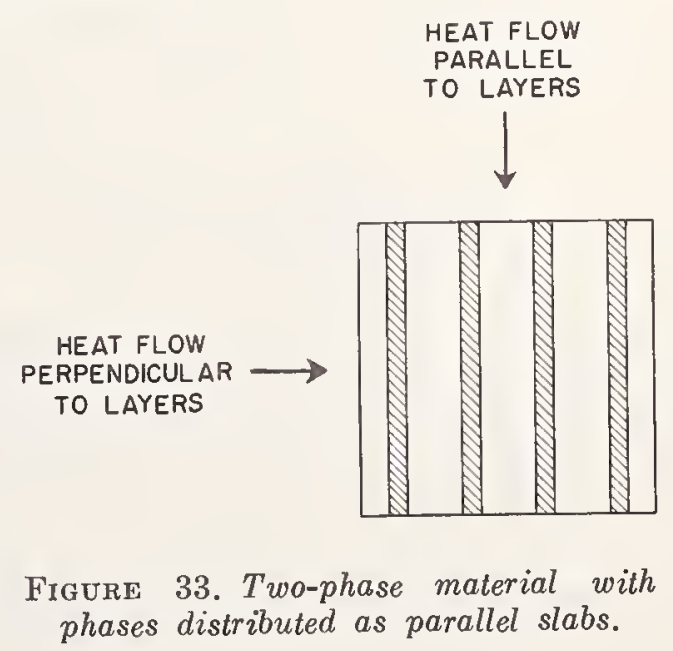




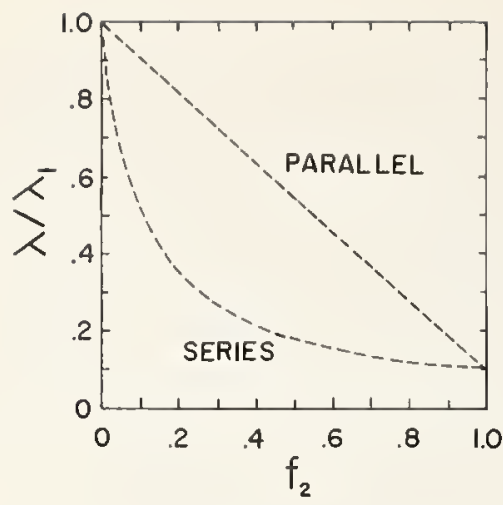

Figure 34. Effective thermal conductivity of a laminated material with heat flow parallel or perpendicular to laminations.

It is possible to obtain tighter limits for the thermal conductivity of a two-phase mixture by calculating the apparent effective conductivity by each of two simple methods:

\section{Series Slabs}

The material is divided into thin slabs perpendicular to the direction of principal heat flow. The effective conductivity of each slab is computed by assuming that the two phases act as conductors in parallel. The effective conductivity of the mixture is then computed by assuming that the slabs act as conductors in series.

\section{Parallel Tubes}

The material is divided into thin tubes or rods parallel to the direction of principal heat flow. The effective conductivity of each tube is computed by assuming that the two phases act as conductors in series. The effective conductivity of the mixture is then computed by assuming that the tubes act as conductors in parallel.

Jackson and Coriell [435] have very recently shown that these two methods provide upper and lower bounds for the true effective conductivity of a mixture. In order to calculate these bounds it is necessary to assume some sort of model representing the manner in which the phases are deployed. Several investigators have represented a disperse second phase by a cubic array of cubes as shown in figure 35 . The two methods described above can then be used to calculate limits for the effective conductivity of the mixture. Since there is some confusion in the literature on these calculations, it is worthwhile to spell out the steps and assumptions involved.

\section{Series Slabs}

1. As shown in figure 35 , the mixture is divided into slabs (A) containing no disperse second phase and into slabs (B) containing both continuous and disperse phases.

2. The effective conductivity of the B-slabs is computed, using eq (68), by assuming the
SERIES SLABS

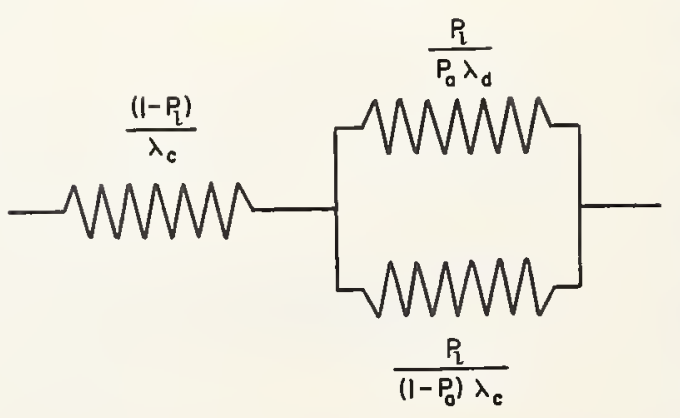

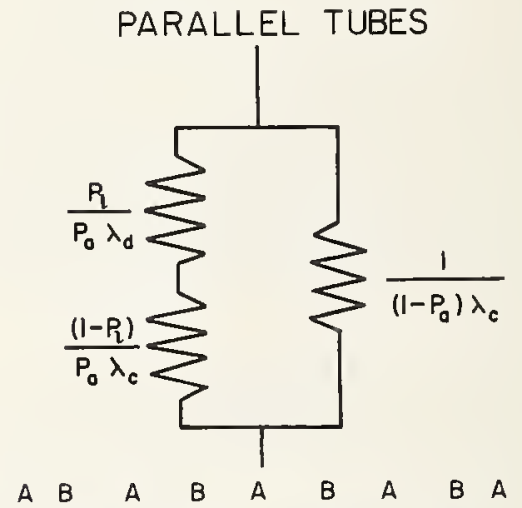

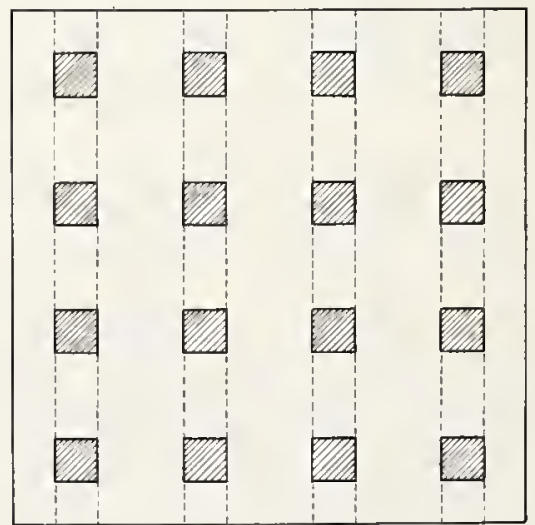

FIGURE 35. Cross-section of the model in which a disperse second phase is considered to be a cubic array of cubes. 
disperse and continuous phases act as conductors in parallel:

$$
\lambda_{\mathrm{B}}=P_{a} \lambda_{d}+\left(1-P_{a}\right) \lambda_{c},
$$

where $P_{a}$ is the fraction of the total area which contains the disperse phase of conductivity $\lambda_{d}$ and $\left(1-P_{a}\right)$ is the fraction of the area which contains the continuous phase of conductivity $\lambda_{c}$.

3. The effective conductivity of the mixture is computed, using eq (69), by taking the A-slabs and the B-slabs in series:

$$
\lambda=\frac{\lambda_{\mathrm{A}} \lambda_{\mathrm{B}}}{P_{l} \lambda_{\mathrm{A}}+\left(1-P_{l}\right) \lambda_{\mathrm{B}}},
$$

where $\lambda_{A}=\lambda_{c}, \lambda_{B}$ was given above, and $P_{l}$ is the fraction of the total length containing the disperse phase. Evaluation of this equation yields

$$
\frac{\lambda}{\lambda_{c}}=\frac{\left(1-P_{a}\right)+P_{a} \lambda_{d} / \lambda_{c}}{\left(1-P_{a}+P_{a} P_{l}\right)+P_{a}\left(1-P_{l}\right) \lambda_{d} / \lambda_{c}} .
$$

\section{Parallel Tubes}

1. In this approximation, the mixture is divided into parallel "tubes" (A) containing no disperse second phase and "tubes" (B) containing both phases.

2. The effective conductivity of the B-tubes is computed, using eq (69), by assuming the disperse and continuous phases to be in series:

$$
\lambda_{\mathrm{B}}=\frac{\lambda_{c} \lambda_{d}}{P_{l} \lambda_{c}+\left(1-P_{l}\right) \lambda_{d}} .
$$

3. The effective conductivity of the mixture is computed, using eq (68), by assuming the A-tubes and B-tubes to be in parallel:

$$
\lambda=P_{a} \lambda_{\mathbf{B}}+\left(1-P_{a}\right) \lambda_{\mathbf{A}} .
$$

Evaluation of this yields:

$$
\frac{\lambda}{\lambda_{c}}=\frac{P_{l}\left(1-P_{a}\right)+\left(1-P_{l}+P_{a} P_{l}\right) \lambda_{d} / \lambda_{c}}{P_{l}+\left(1-P_{l}\right) \lambda_{d} \lambda_{c}}
$$

In the above derivations, we stated that our model was a cubic array of cubes. In fact, it is not necessary to be so restrictive. For eq (70) to be valid, it is only necessary that the model can be divided into two types of slabs (perpendicular to the flow of heat)- one containing no disperse phase and one having a fraction, $P_{a}$, of disperse phase which can be distributed in any manner. For eq (71) to be valid, it is only necessary that the model can be divided into the two types of "tubes" (parallel to the flow of heat). Thus eqs (70) and (71) are, in principle, also applicable to dispersions of, for example, fibers or platelets oriented parallel or perpendicular to the flow of heat. However, these equations will not necessarily bound the true effective conductivity unless both are based on the same phase deployment.

For a disperse phase in the form of cubes, or in which cubes may be used to approximate an isometric disperse phase, equations (70) and (71) may be recast in terms of the volumetric fraction of disperse phase, which we shall designate as $f$. For the model used, it is easily seen that $P_{l}=f^{1 / 3}$ and $P_{l}=f^{2 / 3}$; with these substitutions, we obtain the forms usually seen: ${ }^{16}$

Series Slabs

$$
\frac{\lambda}{\lambda_{c}}=\frac{\left(1-f^{2 / 3}\right)+f^{2 / 3} \lambda_{d} / \lambda_{c}}{\left(1-f^{2 / 3}+f\right)+\left(f^{2 / 3}-f\right) \lambda_{d} / \lambda_{c}},
$$

Parallel Tubes

$$
\frac{\lambda}{\lambda_{c}}=\frac{\left(f^{1 / 3}-f\right)+\left(1-f^{1 / 3}+f\right) \lambda_{d} \lambda_{c} .}{f^{1 / 3}+\left(1-f^{1 / 3}\right) \lambda_{d} / \lambda_{c}} .
$$

Although one would appear to be, on the face of things, considering a fairly complicated model in deriving eqs (70) to (73), a little thought reveals that the model reduces to the two simple electrical networks shown in figure 35 . In the series-slabs model, one effectively assumes that the continuous phase has an infinite thermal conductivity normal to the principal flow of heat; thus this approach, resulting in eqs (70) and (72), always overestimates the effective thermal conductivity. In the parallel-tubes model, one effectively assumes that the continuous phase has zero thermal conductivity normal to the principal flow of heat; thus this approach, resulting in eqs (71) and (73), always underestimates the effective thermal conductivity.

Maxwell [346, 347] derived an expression for the conductivity of a two-phase dispersion of spherical particles of conductivity $\lambda_{d}$ imbedded in a medium of conductivity $\lambda_{c}$. This expression is rigorously valid for dilute dispersions where the average distance between dispersed particles is much larger than the particle size. Maxwell's relation can be written in the form:

$$
\frac{\lambda}{\lambda_{c}}=\frac{2-2 f+(1+2 f) \lambda_{d} / \lambda_{c}}{2+f+(1-f) \lambda_{d} / \lambda_{c}} .
$$

The behavior of eq (74) for small $f$ is more easily seen by expanding it in the form:

$$
\frac{\lambda}{\lambda_{c}}=1-\frac{3 f\left(1-\lambda_{d} / \lambda_{c}\right)}{2+\lambda_{d} / \lambda_{c}}+\frac{3 f^{2}\left(1-\lambda_{d} / \lambda_{c}\right)^{2}}{\left(2+\lambda_{d} / \lambda_{c}\right)^{2}}-\ldots, f<<1 .
$$

In figure 36 , we compare the predictions of the series-slabs expression (eq (72)), the paralleltubes expression (eq (73)), and the Maxwell dilute dispersion expression (eq (74)) for the case $f=0.1$. For values of $\lambda_{d} / \lambda_{c}$ near 1 , all three expres-

19 Equation (4) of Powers [346] and Equation (5) of Nahas, et al. [400], which should agree with our eq (73), are obviously erroneous in that they do not reduce to $\lambda=\lambda_{c}$ when $\lambda_{d}=\lambda_{c}$. 


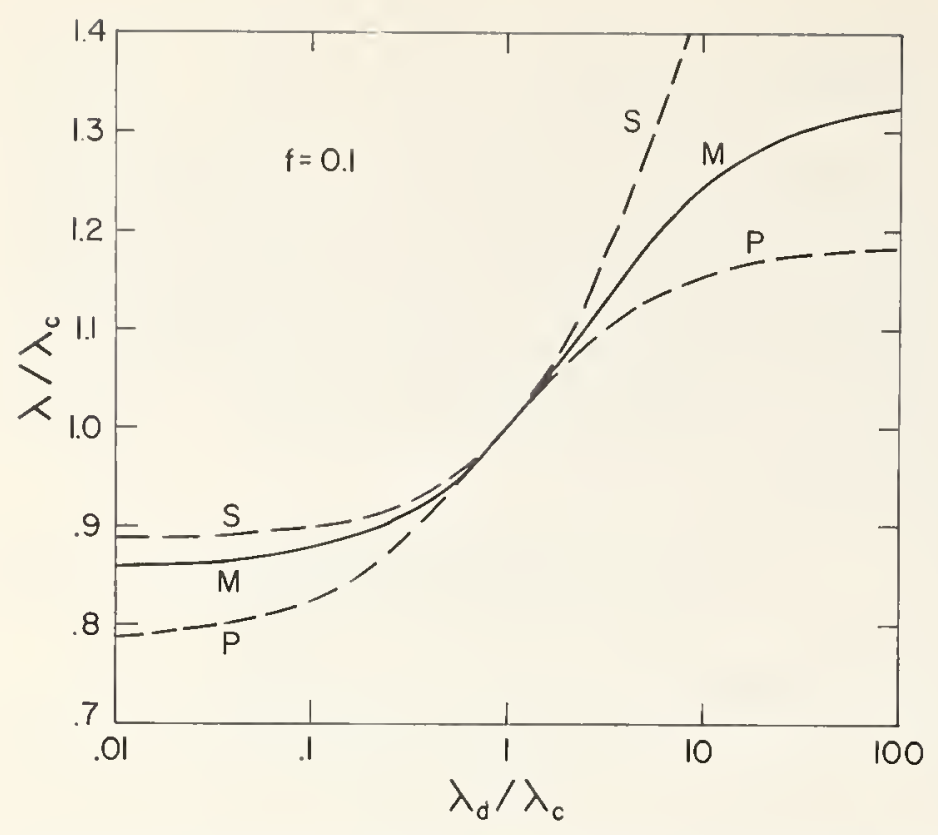

Figure 36. Computed effective conductivity of a dispersion of 0.1 volume fraction of a material of conductivity $\lambda_{\mathrm{d}}$ in a continuous matrix of material of conductivity $\lambda_{\mathrm{c}}$.

$S$ Series-slabs expression, eq (72);

P Parallel-tubes expression, eq (73);

M Maxwell dilute dispersion expression, eq (74).

sions agree. However, if the thermal conductivities of the two phases differ significantly, the seriesslabs and parallel-tubes expressions disagree with the Maxwell expression, which should be very accurate for $f \leq 0.1$.

To predict the thermal conductivity of a dispersion having less than about 0.1 volume fraction of isometric dispersed phase imbedded in an isotropic continuous phase, one should use the Maxwell dilute dispersion expression, eq (74) or (75). The expressions obtained from the cubic array of cubes, eqs (70) to (73), should not be used for dilute dispersions in media having an isotropic continuous phase. For anisotropic media, the Maxwell expression would have to be modified. For highly anisotropic media, such as pyrolytic graphite, the implicit assumptions of either infinite or zero lateral thermal conductivity of the continuous phase might be more nearly met so that one of the series-slabs or the parallel-tubes expressions (eqs (70) to (73)) might be more accurate than in isotropic materials. For heat conduction in the poorest conducting direction, the series-slabs expressions, eqs (70) and (72), should be more accurate while for heat conduction in the best-conducting direction, the paralleltubes expressions, eqs (71) and (73) should be more accurate.

A special case of interest for ceramics is the effect of porosity on thermal conductivity. If the thermal conductivity of the continuous ceramic material is much greater than the effective conductivity of the pores, we can set $\lambda_{d} / \lambda_{c}=0$ and our expressions reduce to:
Series-Slabs

$$
\frac{\lambda}{\lambda_{c}}=\frac{1-f^{3 / s}}{1-f^{2 / 3}+f}, \lambda_{d}<<\lambda_{c}
$$

which for small $f$ reduces to

$$
\frac{\lambda}{\lambda_{c}}=1-f-f^{5 / z}+\ldots, \lambda_{d}<<\lambda_{c} .
$$

\section{Parallel-Tubes}

$$
\frac{\lambda}{\lambda_{c}}=1-f^{2 / 3}, \quad \lambda_{d}<<\lambda_{c} .
$$

\section{Maxwell Dilute Dispersion}

$$
\frac{\lambda}{\lambda_{c}}=\frac{2-2 f}{2+f}, \quad \lambda_{d}<<\lambda_{c}
$$

which for small $f$ reduces to

$$
\frac{\lambda}{\lambda_{c}}=1-\frac{3}{2} f+\frac{3}{2} f^{2}-\ldots, \lambda_{d}<<\lambda_{c} .
$$

In figure 37 the predictions of eqs (76), (78), and (79) are shown for void volume fractions up to 0.1 . Over this porosity range the Maxwell equation should be rather accurate if the porosity is in the form of dispersed, disconnected, isometric pores. In many ceramics this will not be the case and eq (79) should then serve only as an upper limit for the effective thermal conductivity of a porous ceramic material.

For dispersions which are sufficiently dilute for eq (74) to be valid, neither the size distribution of the disperse particles nor the manner in which

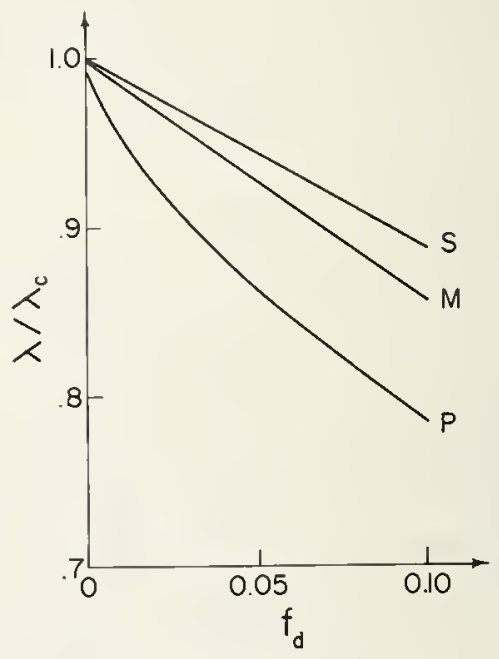

Figdre 37. The effect of porosity on thermal conductivity as computed by:

S Series-slabs expression, eq (76);

P Parallel-tubes expression, eq (78);

M Maxwell dilute dispersion expression, eq (79). 
they are deployed are of consequence. However, these factors must be considered if the concentration of the dispersed phase is increased. Lord Rayleigh treated the case of uniform spheres arrayed in a cubic lattice distribution. Meredith and Tobias extended Rayleigh's derivation by an additional term and obtained [347]:

$$
\frac{\lambda}{\lambda_{c}}=1-\frac{3 f}{\frac{2+\lambda_{d} / \lambda_{c}}{1-\lambda_{d} / \lambda_{c}}+f-\frac{1.315\left(1-\lambda_{d} / \lambda_{c}\right) f^{10 / 3}}{4 / 3+\lambda_{d} / \lambda_{c}+0.409\left(1+\lambda_{d} / \lambda_{c}\right) f^{7 / 3}}}
$$

for the conductivity normal to a side of the cube. If the term involving $f^{10 / 3}$ in the denominator of the right hand side is dropped, this expression reduces to the Maxwell dilute dispersion expression, eq (74). Equation (81) should be more accurate than eq (74) for values of $f$ up to $\pi / 6$ $=0.524$, which is the maximum possible value for a cubic array of spheres.

A rigorous solution for the effective conductivity of a concentrated random array of particles of varying sizes has not been achieved. Several approximations have been developed which are useful in many cases. Bruggeman (see [346-347]) developed an expression,

$$
\frac{\lambda-\lambda_{d}}{\lambda_{c}-\lambda_{d}}\left(\frac{\lambda_{c}}{\lambda}\right)^{1 / 3}=1-f
$$

which has proved rather effective in predicting the conductivity of a dispersion containing a wide range of particle sizes. For $\lambda_{d} \rightarrow 0$, eq (82) reduces to

$$
\frac{\lambda}{\lambda_{c}}=(1-f)^{3 / 2}, \quad \lambda_{d}<<\lambda_{c}
$$

while for $\lambda_{d} \rightarrow \infty$,

$$
\frac{\lambda}{\lambda_{c}}=\frac{1}{(1-f)^{3}}, \quad \lambda_{d}>>\lambda_{c} .
$$

For a concentrated dispersion containing only a narrow range of particle sizes, the Bruggeman variable dispersion expression (eq (82)) tends to. overestimate the effect of the disperse phase while the Maxwell expression (eq (74)) tends to underestimate. Meridith and Tobias [347] suggested an alternative semiempirical expression that predicts conductivity values intermediate between the Bruggeman variable dispersion equation and the Maxwell dilute dispersion equation:

$$
\begin{aligned}
\frac{\lambda}{\lambda_{c}}=\left[\frac{2\left(\lambda_{d} / \lambda_{c}+2\right)+2\left(\lambda_{d} / \lambda_{c}-1\right) f}{2\left(\lambda_{d} / \lambda_{c}+2\right)-\left(\lambda_{d} / \lambda_{c}-1\right) f}\right] \\
\quad \times\left[\frac{(2-f)\left(\lambda_{d} / \lambda_{c}+2\right)+2\left(\lambda_{d} / \lambda_{c}-1\right) f}{(2-f)\left(\lambda_{d} / \lambda_{c}+2\right)-\left(\lambda_{d} / \lambda_{c}-1\right) f}\right]
\end{aligned}
$$

which reduces to:

$$
\frac{\lambda}{\lambda_{c}}=\frac{8(2-f)(1-f)}{(4+f)(4-f)}, \quad \lambda_{d}<<\lambda_{c}
$$

and

$$
\frac{\lambda}{\lambda_{c}}=\frac{(1+f)(2+f)}{(1-f)(2-f)}, \quad \lambda_{d}>>\lambda_{c} \cdot
$$

Meredith and Tobias [347] contrast the predictions of the various expressions we have given above. For a given two-phase system, all of these expressions predict two different conductivities, dependent on which phase is assumed to be disperse. Bruggeman (see [346]) derived another approximate expression that should be applicable to mixtures where neither phase is necessarily continuous:

$$
f_{1} \frac{\lambda_{1}-\lambda}{\lambda_{1}+2 \lambda}+f_{2} \frac{\lambda_{2}-\lambda}{\lambda_{2}+2 \lambda}=0
$$

In figure 38 , the predictions of the Bruggeman mixture equations are compared with those from the Maxwell dilute dispersion equation. We see that for small values of $f_{2}$ the Bruggeman mixture equation is in agreement with a dilute dispersion of particles of conductivity $\lambda_{2}$ in a matrix of conductivity $\lambda_{1}$ while for values of $f_{2}$ approaching unity the mixture equation predicts a conductivity due to particles of conductivity $\lambda_{1}$ dispersed in a matrix of conductivity $\lambda_{2}$.

There have not been enough accurate measurements of the thermal conductivity of well-characterized ceramics to provide adequate experimental confirmation of any of the above equations. For well-defined systems (such as spheres dispersed in a continuous matrix) which are in good correspondence to the models used in deriving these equations, measurements of electrical conductivity or dielectric constant have shown

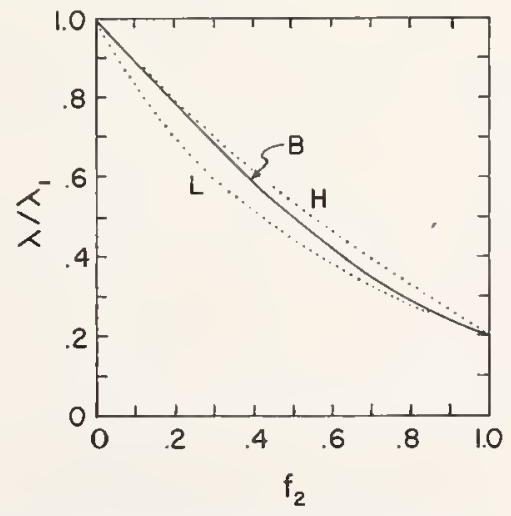

FIgURE 38. Computed effective thermal conductivity of a mixture.

B Bruggeman mixture expression, eq (88);

H Maxwell dilute dispersion expression, eq (74), high-conductivity phase continuous;

L Maxwell dilute dispersion expression, eq (74), low-conductivity phase continuous. 
good agreement with the theoretical predictions of these equations. Two-phase ceramics, in general, cannot be readily described in terms of a simple model such as spheres dispersed in a uniform medium, and lience in many cases the expressions we have cited will indicate qualitatively the effect of an additional phase but should not be relied upon for accurate quantitative predictions.

In the remainder of this section we shall cite a few examples from the literature to illustrate the effect of an additional phase on the thermal conductivity of ceramics. The next section, on microstructure, is also pertinent here.

In figure 39 we show the data of Kingery [288] on six samples of the two-phase system magnesiaforsterite $\left(\mathrm{MgO}-\mathrm{Mg}_{2} \mathrm{SiO}_{4}\right)$ which is reported to form essentially no solid solution. The solid curve shown represents the Bruggeman mixture expression (eq (88)) fitted to the end points. The deviations of the data from the smooth curve could be due to a combination of several factors:

1. The Bruggeman mixture equation is only approximately valid.

2. The data may contain some experimental error.

3. There may be some formation of a solid solution phase which would have a lower conductivity than would a mixture.

4. Errors may have been introduced by the procedure Kingery used to correct his data to correspond to 100 percent dense material.

5. The thermal conductivity of these samples may have been affected by their (perhaps differing) microstructures.

Kingery corrected his data to correspond to theoretically dense material by dividing the measured

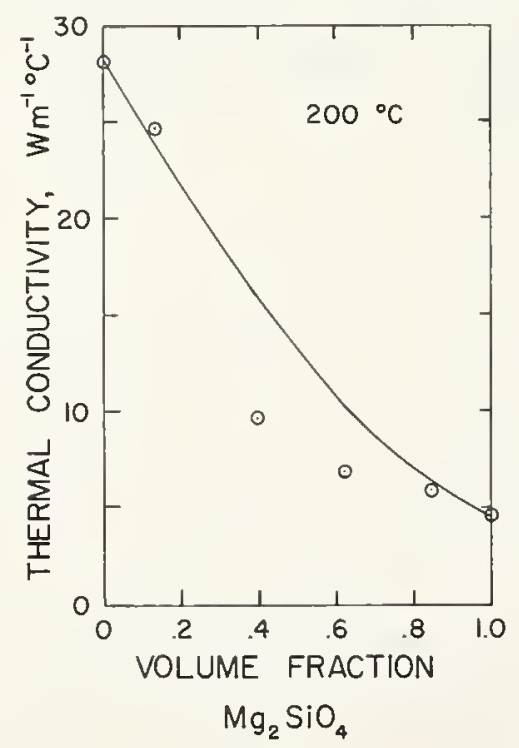

Figure 39. Thermal conductivity of mixtures of magnesia and forsterite as measured by Kingery [288].

The solid curve represents the Bruggeman mixture expression, eq (88). values by $(1-f)$, where $f$ is the volume fraction porosity. As we have seen (e.g., eqs (76 to 80 ) and figure 37 ), porosity would be expected to have a greater effect than was assumed by Kingery. For small values of $f$, it would seem preferable to divide measured conductivity values by $(1-3 f / 2)$ to obtain an estimate of the conductivity of the dense material.

As an example of the effect of porosity on the thermal conductivity of magnesia, we show the data of Moore et al. [317] and of Koenig [436, 437] in figure 40 . The data shown for zero porosity were obtained on single crystals; the other data points correspond to polycrystalline specimens of the porosity shown. Moore, et al. wrote a semiempirical expression to relate conductivity and porosity:

$$
\frac{\lambda}{\lambda_{c}}=\frac{1-f}{1+\beta f}
$$

The three solid curves in figure 40 correspond to different values of $\beta$ in eq (89). For $\beta=0$, eq (89) reduces to the simple expression $\lambda=(1-f) \lambda_{c}$ used by Kingery, among others, for adjusting thermal conductivity data to correspond to theoretical density. For $\beta=0.5$, eq (89) is the same as the Maxwell dilute dispersion expression, eq (79), for the case where the disperse phase has zero conductivity. Moore, et al. found that an empirical value of $\beta=1$ fit the data reasonably well for values of $f$ up to 0.08 but that at higher porosities the conductivity decreased more rapidly.

In section $2.2 \mathrm{c}$ we saw that the apparent contribution to thermal conductivity due to radiation through a transparent slab was proportional to the thickness of the slab in the optically thin limit where there is negligible absorption or emission of radiation within the slab. By analogy to

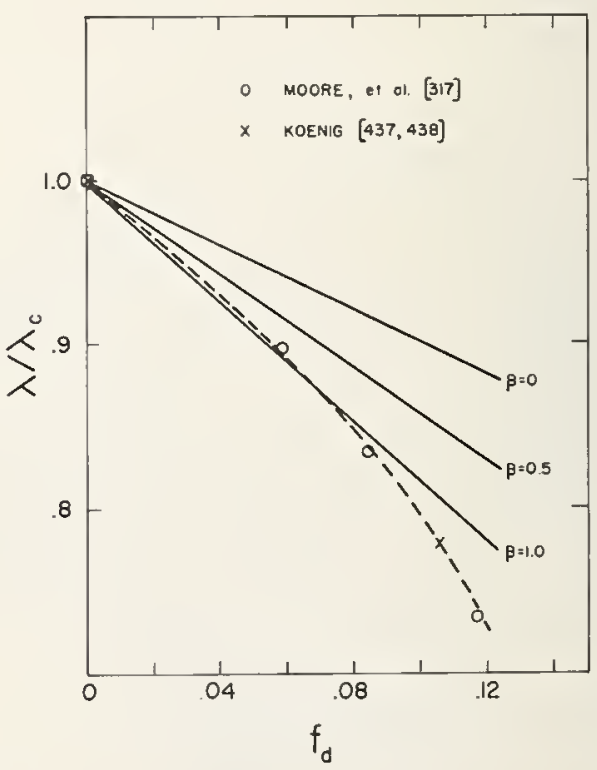

FIgURE 40. Effect of porosity on the thermal conductivity of $\mathrm{MgO}$ at $90{ }^{\circ} \mathrm{C}$ (after Moore, et al. [317]. 
eq (56), the radiative component to the termal conductivity of a pore is

$$
\lambda_{r}=4 \sigma n^{2} E \bar{T}^{3} \cdot g L,
$$

where $\sigma$ is the Stefan-Boltzmann constant, $n$ is the index of refraction of the material filling the pore, $E$ is the effective emittance of the boundary of the pore, $\bar{T}$ is mean temperature, $L$ is the pore size in the direction of heat flow, and $g$ is a geometrical factor dependent on the pore shape. Thus the effective thermal conductivity of a pore is proportional to the size of the pore, all other factors being equal. This effect is shown in figure 41 where the calculated effect of two different pore sizes is shown. For pores less than about $0.01 \mathrm{~cm}$ in diameter, the effect of radiation is slight. At larger pore sizes, however, radiation across the pores can make a very significant contribution to the overall effective thermal conductivity of a porous ceramic, particularly if the emittance is high.

One other effect of porosity, not previously discussed, is to decrease the radiative contribution to heat transfer through diathermanous materials. Even quite small amounts of porosity will act as scattering centers thus reducing the photon mean free path so that the high temperature radiative component of thermal conductivity is less than in single crystals or glasses.

In this section we have specifically discussed only those cases of two-phase media in which the disperse phase was isometric, e.g., spheres or cubes. Powers [346] and Meredith and Tobias [347] also discuss cases in which the disperse phase is anisometric, e.g., ellipsoids or cylinders.

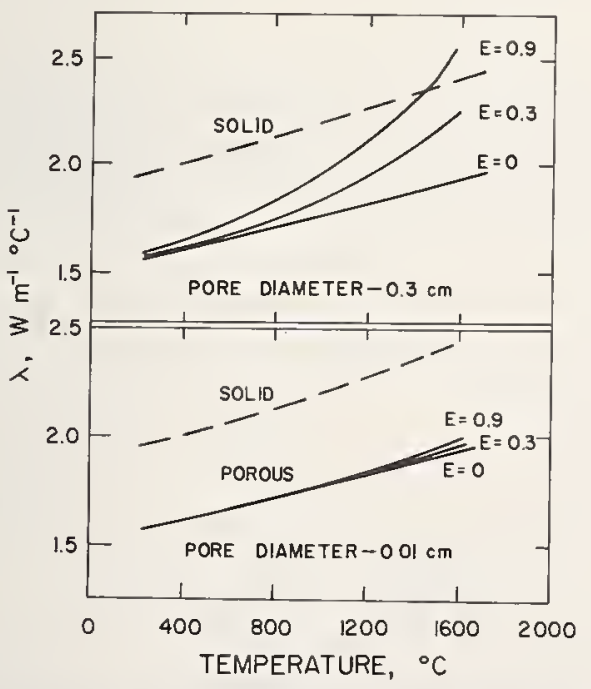

Figdre 41. Thermal conductivity of zirconia sample with 20 percent porosity, assuming various emittances (after Kingery [438].

\section{d. Microstructure}

The microstructure, or internal structure on a microscopic scale, of a ceramic material can have a significant effect on the thermal conductivity of the material. It is helpful to think of a polycrystalline ceramic material as a concentrated dispersion of grains of conductivity $\lambda_{g}$ in a continuous matrix (the grain boundary region) of average conductivity $\lambda_{b}$. In this way, a polycrystalline ceramic may be treated as a two-phase system and the thermal conductivity estimated using the expressions cited in the previous section.

We have seen that the two expressions (eqs (72) and (73)) derived from the model of a cubic array of cubes disagree seriously with one another in the limiting case of small volume fractions of disperse phase. However, for volume fractions of the disperse phase approaching unity, eqs (72) and (73) converge to a common expression which, quite interestingly, is identical to the Maxwell dilute dispersion expression, eq (74). The Maxwell equation can be put in the form

$$
\frac{\lambda}{\lambda_{g}}=\frac{3-2\left(1-\lambda_{b} / \lambda_{g}\right) f_{b}}{3+\left(\lambda_{g} / \lambda_{b}-1\right) f_{b}}
$$

where $f_{b}$ is the volume fraction of the grain boundary material. If the average grain size is $g$ and the average boundary thickness is $b$, the volume fraction of grain boundary material is given by

$$
f_{b}=\frac{3 b}{g}, \quad b<<g .
$$

With this substitution, eq (91) becomes

$$
\frac{\lambda}{\lambda_{g}}=\frac{1-2\left(1-\lambda_{b} / \lambda_{g}\right) b / g}{1+\left(\lambda_{g} / \lambda_{b}-1\right) b / g}
$$

If $b$ and $g$ are measured from a photomicrograph of the ceramic, the approximate effect of grain boundary size on thermal conductivity may be calculated using eq (93), if a reasonable estimate can be made of the conductivity of the grain boundary region. In figure 42 we show a family of curves computed from eq (93). As an example of the use of these curves, if the average grain size is about 200 times the average boundary thickness and the grain boundary conductivity is estimated to be about one-fifth of the conductivity of the grains, then the effective thermal conductivity of the polycrystalline material would be about 97 percent of the conductivity of the grain material. The catch here, of course, is to come up with a reasonable value for the average conductivity of the grain boundary material.

As figure 42 illustrates, thermal conductivity can be very sensitive to both the relative thickness of the grain boundary material and to the effective thermal conductivity of the boundary region. If 


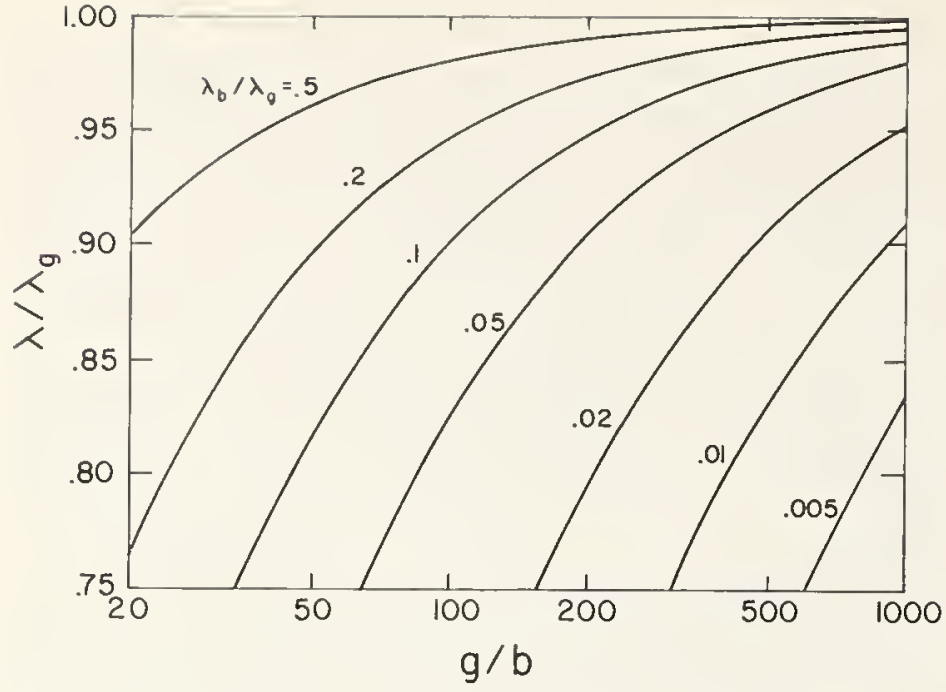

FIGURE 42. Calculated effect of grain size on the effective thermal conductivity, $\lambda$, of polycrystalline materials.

The grains, of average size $g$ in the direction of heat flow, have a thermal conductivity $\lambda_{g}$ while the grain boundary region, of average thickness $b$, has an average conductivity $\lambda_{b}$.

there are no pores or microcracks in the grain boundaries, the thermal conductivity of the boundary region should be at least as high as that of glasses since short-range order persists in the boundary region. The distribution of impurities, pores, and microcracks in a ceramic can be very significant. If such imperfections occur in the grain boundaries they can both lower the conductivity and increase the width of the grain boundary region and hence have a much greater effect than if these same imperfections were dispersed throughout the grains. If enough were known about the degree of crystallinity of the boundary region and the types and amounts of impurities and imperfections concentrated there, it should be possible to estimate the thermal conductivity of the grain boundary region. To my knowledge, no one has attempted this.

For a reasonably pure ceramic material, the grain boundary thickness is probably of the order of $1 \mathrm{~nm}$. At room temperature the thermal conductivity of glasses is about $1 \mathrm{Wm}^{-1} \mathrm{~K}^{-1}$ while that of single crystal ceramics would typically be less than $100 \mathrm{Wm}^{-1} \mathrm{~K}^{-1}$. Inspection of figure 42 shows that for $\lambda_{b} / \lambda_{g}=0.01$ and $g / b=1000$ (corresponding to an average grain size of $1 \mu \mathrm{m}$ ) the effective conductivity would be about 91 percent of that of a single crystal. Evaluation of eq (93) for an average grain size of $10 \mu \mathrm{m}$ yields a corresponding value of 99 percent. Thus, at room temperature and above, one would not expect grain size to have much effect on the thermal conductivity of a dense, pure ceramic unless the grain size were less than $10 \mu \mathrm{m}$. However, if porosities or impurities are present, grain size effects might occur even at larger grain sizes.

One case where microstructure can very significantly affect thermal conductivity is in ma- terials that have different coefficients of thermal expansion in different directions or, in the case of poly-phase materials, between different phases. Microstresses in such materials can cause microcracks in the grain boundaries, thus severely reducing the effective conductivity of the grain boundary region (see e.g., Kingery [241]).

There has been very little definitive work on the effect of microstructure on thermal conductivity. A few pertinent references include [241, $281,285,288,439]$. Three references to the general subject of microstructure are [440-442].

As an example of the effect of microstructure on the thermal conductivity of a ceramic, we show, in figure 43, the data of Moore, et al. [317] on thoria samples prepared by two different processes. The samples prepared from thoria obtained by the sol-gel process consisted of large particles of fairly high density materials suspended in a low density matrix of fine particles; the samples prepared from thoria obtained by an oxalate-precipitate process contained only small pores located predominantly within the grains of a homogenous material. The values of $\beta$ shown on this figure correspond to eq (89).

The thermal history and the method of fabrication of a ceramic material can greatly affect the thermal conductivity since the composition and structure of the ceramic depend on such factors as type of pressing or casting used, maximum firing temperature, time of firing, and, in some cases, rate of cooling. There has been very little work directly relating thermal conductivity with method of fabrication or thermal history. The thermal conductivity of a ceramic is a function of its present composition and structure so that we need not concern ourselves unduly with how that composition and structure were attained. It is useful, however, to list some of the factors affecting thermal

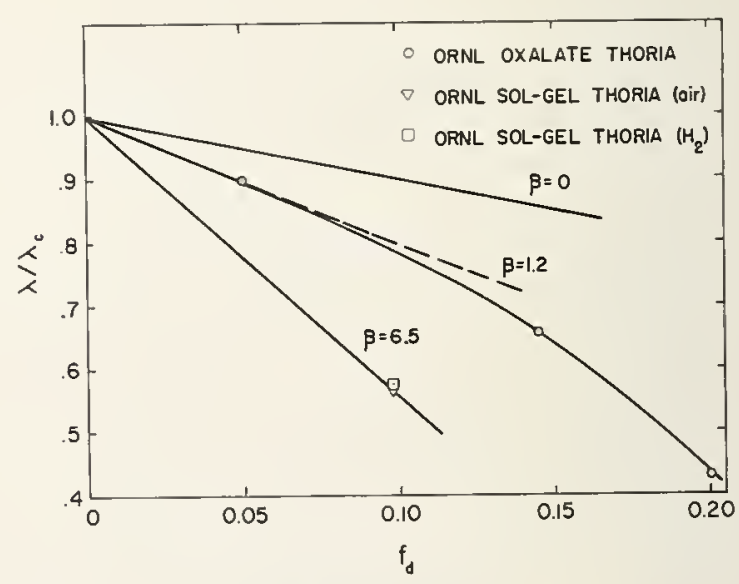

FiguRE 43. Ejfect of porosity on the thermal conductivity (at $75{ }^{\circ} \mathrm{C}$ ) of thoria samples having different microstructures (see text).

The oxalate thoria samples and one of the sol-gel thoria samples were sintered in air at $1650{ }^{\circ} \mathrm{C}$ while the other sol-gel thoria sample was sintered in hydrogen at $1750^{\circ} \mathrm{C}$ (after Moore, et al. [317]). 
conductivity which depend on the fabrication and thermal history:

-quantity and distribution of impurities and whether impurities are in solution or dispersed as additional phases,

-stoichiometry,

-crystallinity,

-quantity and distribution of other phases, including pores,

- grain size and boundary thickness,

-quantity and distribution of imperfections (e.g., dislocations, microcracks).

The most obvious effect of heat treatment is to control the final density, and hence porosity, of a ceramic. Two general references to the sintering process are [443-444].

In figure 43 , we saw the effect of a different starting material on the thermal conductivity of thoria. To illustrate the effect of heat treatment, I have taken several examples from Austin [262]. Figure 44 shows the effect of firing time at $1350^{\circ} \mathrm{C}$ on the thermal conductivity of an initially unfired magnesia brick in which the porosity decreased as a result of sintering; the thermal conductivity increased, asymptotically approaching a value corresponding to an infinitely long firing time. Figure 45 shows the effect of firing time at $1350^{\circ} \mathrm{C}$ on the thermal conductivity of an initially unfired

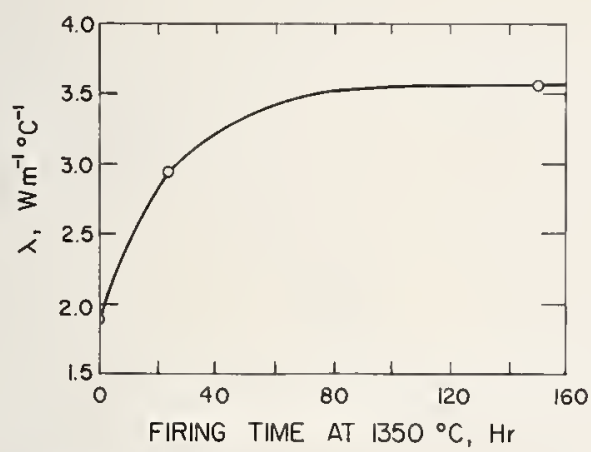

FIGURE 44. Increase with firing period of the thermal conductivity at $800^{\circ} \mathrm{C}$ of a brick in which the chief reaction during firing was sintering (after Austin [262]).

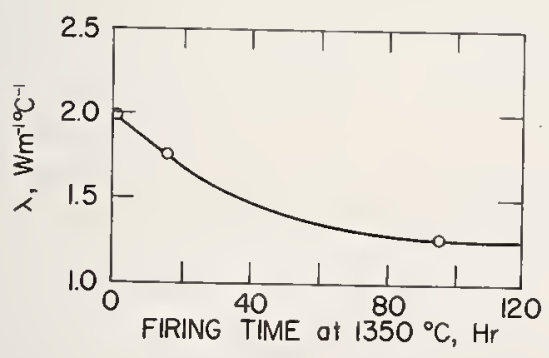

Figure 45. Decrease with firing period of the thermal conductivity at $800^{\circ} \mathrm{C}$ of a brick in which the chief reaction during firing was the removal of combustible or volatile matter (after Austin [262]).

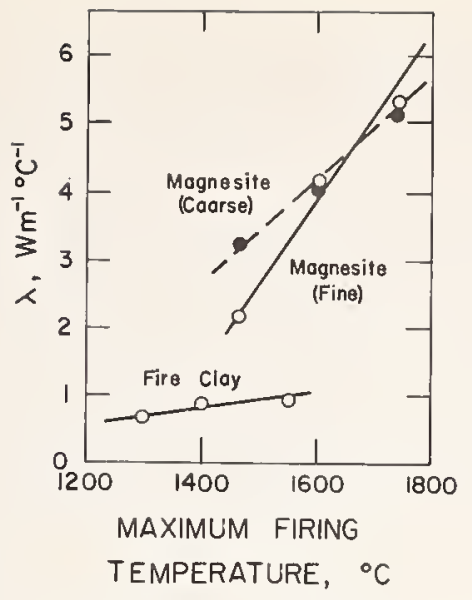

Figure 46. Influence of maximum temperature attained during firing on the thermal conductivity at $800^{\circ} \mathrm{C}$ of fire clay, and of magnesite blocks with different size starting material (after Austin [262]).

brick in which the porosity increased as a result of burning off some of the material of the brick; the thermal conductivity decreased, again asymptotically approaching a value corresponding to an infinitely long firing time. As a final example of the effect of heat treatment, figure 46 illustrates the effect of firing the same material at different maximum temperatures.

\section{Reliability of Existing Data}

In general the accuracy of thermal conductivity values found in the literature is not good. Thermal conductivity values reported for ceramics are, if anything, less accurate than values reported for metals or thermal insulating materials. The reasons for this unhappy state of affairs are several:

1. Thermal conductivity is a difficult property to measure accurately. This is particularly so for materials having intermediate values of thermal conductivity (i.e., neither good conductors nor good insulators).

2. Thermal conductivity reference standards are not readily available nor is the proper use of such reference standards well known or understood.

3. Adequate characterization of thermal conductivity samples seems to be the exception rather than the rule. This is probably particularly so for ceramic materials where the porosity is not always reported, the purity is rarely reported, and the microstructure almost never reported.

Even a cursory examination of the available data compilations [e.g. 238-240] covering thermal conductivity values reveals that the spread in the existing data on a given material is very large, often of the order of 20 to 40 percent. Since many of the samples tested were uncharacterized, it is 
usually difficult, if not impossible, to separate the effects of sample differences from the effects of measurement errors. It is quite apparent, however, that both effects are important. Some of the examples cited in previous sections of this paper clearly illustrate the effects of sample differences, such as purity and porosity, on the thermal conductivity of a given material. As for measurement errors, I have seen cases where reputable, and reasonably experienced, investigators have reported thermal conductivity values (obtained, for example, by an ASTM Standard Method of Test) which were in error by factors of two, three, or even five.

In the above discussion, I do not mean to appear to be an alarmist. There are many good data in the literature. However, to distinguish between good and bad data is not always easy. If you wish to find a suitable value for the thermal conductivity of a particular material, you should approach the available data on similar materials cautiously and critically. Was the specimen adequately characterized? Has the investigator utilized the same equipment to measure the thermal conductivity of reasonably well-defined materials (having a thermal conductivity similar in magnitude to that of the material in question) and, if so, how do the results compare with those of other investigators?

In summary, as regards literature values for thermal conductivity, especially when material characterization data are lacking, caveat emptor!

\section{Measurement Methods}

The technique selected for measuring the thermal conductivity of a particular material depends upon:

1. The thermal conductivity of the material.

2. The temperature range.

3. The physical nature of the material.

4. The accuracy which is required.

5. The availability of existing equipment.

6. Cost.

7. The experience, skills, knowledge, and prejudices of the investigator.

In this section we shall discuss a number of different methods for measuring thermal conductivity. The methods being discussed were selected to illustrate the principles and problems involved as well as to cover, if only in a somewhat cursory manner, many of the types of methods in common use. Where possible, apparatuses used at NBS were selected as examples since I am more familiar with the advantages and disadvantages of our equipment.

In most methods of measuring thermal conductivity, the experimental arrangement is designed to force the flow of heat to be in the direction of one of the coordinate axes. Most methods utilize a cylindrical specimen geometry and force the heat flow to be in either the longitudinal or the radial direction. For anisotropic materials, it is usually preferable to have the heat flow be along one of the principal crystallographic axes.

\subsection{Longitudinal Heat Flow}

\section{a. Mathematical Development}

The total longitudinal heat flow through a specimen of constant cross sectional area is

$$
Q=-\lambda A \frac{\partial v}{\partial z},
$$

where $v$ is the temperature relative to an arbitrary datum plane, and where it is to be understood that $\lambda$ now refers to the thermal conductivity in the $z$-direction. If the temperatures are independent of time, if the specimen is properly insulated or guarded so that there is no transverse gain or loss of heat, and hence $Q$ is a constant, if there is no heat generation within the specimen itself, and if, further, the specimen is homogeneous in the $z$-direction so that $\lambda$ does not depend on $z$, then (94) is easily integrated to yield

$$
Q=\frac{A}{l} \int_{v_{1}}^{v_{2}} \lambda d v
$$

where $v_{1}$ and $v_{2}$ are the values of $v$ at positions $z=l$ and $z=0$, respectively.

Equation (95) is valid under the following restrictions:

1. The specimen is opaque to thermal radiation.

2. The specimen is homogeneous in the direction of heat flow.

3. There is no internal heat production (or absorption).

4. All temperatures in the system are independent of time.

5. The cross-sectional area is constant at all $z$-positions between $z=0$ and $z=l$.

6. The planes $z=0$ and $z=l$ are isothermal surfaces.

7. There are no transverse heat gains or Josses.

Immediately following eq (94), $v$ was defined as the temperature above an arbitrary datum plane. For reasons which will shortly become obvious, it is convenient to designate this datum plane as $T_{0}$ so that $v=T-T_{0}$, where $T$ is the temperature on a fixed scale (e.g., $T$ is expressed on the Celsius or Fahrenheit scale). The temperatures at $z=l$ and $z=0$ are $T_{1}=T_{0}+v_{1}$ and $T_{2}=T_{0}+v_{2}$, respectively.

In order to use eq (95), it is convenient to assume a particular form for the temperature dependence of the thermal conductivity so that the integration can be carried out. Three cases will suffice:

A. The thermal conductivity is independent of temperature so that

$$
\lambda=\lambda_{0} .
$$


In this case eq (95) becomes, after integration and rearrangement,

$$
\lambda_{0}=\frac{Q l}{A\left(T_{2}-T_{1}\right)} .
$$

B. The thermal conductivity is a linear function of temperature, so that

$$
\lambda=\lambda_{0}(1+\beta v) \text {. }
$$

Substitution of eq (98) into eq (95) yields

$$
Q=\lambda_{0} A \frac{T_{2}-T_{1}}{l}\left[1+\frac{\beta}{2}\left(T_{1}+T_{2}-2 T_{0}\right)\right] .
$$

If we define $T_{0}$ as

$$
T_{0}=\frac{T_{1}+T_{2}}{2},
$$

then the term in eq (99) involving $\beta$, the temperature coefficient of thermal conductivity, vanishes so that eq (99) reduces exactly to eq (97). For a material having a thermal conductivity which is a linear function of temperature, the thermal conductivity, $\lambda_{0}$, given by eq (97) corresponds to that at the temperature, $\mathrm{T}_{0}$, given by (100),

C. For many crystalline dielectric solids, the thermal resistivity, or reciprocal of the thermal conductivity, is a linear function of temperature over a considerable temperature range so that

$$
\lambda=\frac{\lambda_{0}}{1+\gamma v} .
$$

Substitution of eq (101) into eq (95) yields

$$
Q=\lambda_{0} A \frac{T_{2}-T_{1}}{l}\left[\frac{1}{\gamma\left(v_{2}-v_{1}\right)} \ln \frac{1+\gamma v_{2}}{1+\gamma v_{1}}\right]
$$

In the present case, the coefficient, $\gamma$, is not so amenable to elimination by choice of a particular reference temperature as was the coefficient, $\beta$, in eq (99). If we retain the definition for $T_{0}$ given by eq (100), then eq (102) becomes

$$
\begin{aligned}
Q=\lambda_{0} A \frac{T_{2}-T_{1}}{l}\left[\frac{2}{\gamma\left(T_{2}-T_{1}\right)} \operatorname{arctanh} \frac{\gamma\left(T_{2}-T_{1}\right)}{2}\right] \\
=\lambda_{0} A \frac{T_{2}-T_{1}}{l}\left[1+\frac{1}{3}\left(\frac{\gamma\left(T_{2}-T_{1}\right)}{2}\right)^{2}\right. \\
\left.+\frac{1}{5}\left(\frac{\gamma\left(T_{2}-T_{1}\right)}{2}\right)^{4}+\cdots\right] . \quad(104)
\end{aligned}
$$

For sufficiently small values of $\gamma\left(T_{2}-T_{1}\right)$, eq (104) reduces to eq (97). Consider a case where $\gamma=.003$
${ }^{\circ} \mathrm{C}^{-1}$, a rather high value; if $\left(T_{2}-T_{1}\right)=100{ }^{\circ} \mathrm{C}$, the second term in the square brackets in eq (104) represents a correction of 0.75 percent; if $\left(T_{2}-T_{1}\right)$ $=300{ }^{\circ} \mathrm{C}$, this term represents a correction of almost 7 percent.

In computing thermal conductivity values for a material whose thermal conductivity is expected to have a temperature dependence such as that in eq (101), one may use eq (100) and eq (97) to compute a mean temperature and the approximate thermal conductivity at that temperature. The first approximations to the thermal conductivity thus obtained for several tests at different mean temperatures can be used to compute values of the coefficient, $\gamma$ which can then be used in eq (103) or (104) to obtain more refined values for thermal conductivity. The resultant values of $\lambda_{0}$ can be used to obtain better values of $\gamma$ etc. The convergence is quite rapid in most practical cases, so that only one or two iterations will normally be required.

If eq (97) is used to compute thermal conductivity, the maximum fractional error in the resultant thermal conductivity value is given by

$$
\begin{aligned}
\left|\frac{\Delta \lambda_{0}}{\lambda_{0}}\right| & \leq\left|\frac{\Delta A}{A}\right|+\left|\frac{\Delta l}{l}\right|+\left|\frac{\Delta\left(T_{2}-T_{1}\right)}{T_{2}-T_{1}}\right|+\left|\frac{\Delta Q}{Q}\right| \\
& +\left|\left(\frac{1}{\lambda} \frac{d \lambda}{d T}\right)_{0} \Delta T_{0}\right|+\left|\begin{array}{l}
\text { errors due to failure to meet } \\
\text { requirements of eq }(97)
\end{array}\right|
\end{aligned}
$$

where $|\triangle A| A \mid$ is the absolute value of the fractional error in the area, etc.; the term in $\Delta T_{0}$ is the fractional error in the thermal conductivity due to the error in the mean temperature; the last term represents errors due to test conditions departing from the assumptions made in deriving eq (97), e.g. temperatures varying with time.

\section{b. Experimental Techniques}

The first concern in most techniques for measuring thermal conductivity is to force the heat flow to be unidirectional, which, for purposes of the present discussion, means longitudinal. Since heat flow is proportional to a geometric factor, a thermal conductivity, and a temperature difference, the direction of heat flow must be controlled by controlling one or more of these variables. ${ }^{16}$ The experimenter's freedom in adjusting these parameters is constrained, sometimes severely, by the often-conflicting requirements of being able to accurately measure total heat flow, area, and temperature differences. Very practical considerations, such as available specimen size, frequently constitute severe constraints on apparatus design.

16 This statement is strictly true only for conductive heat transfer. In some apparatus, transverse heat flows would be by radiation or, rarely, convection. If these transverse heat exchanges are small, as is desired they usually can be represented as being proportional to an "effective" thermal conductivity. 
It is not possible to directly measure the heat flow in a specimen; one must, rather, measure the heat flow into a specimen or out of a specimen. This necessitates that not only must transverse heat losses or gains of the specimen be prevented or accounted for but, further, there must be no unaccounted-for losses or gains between the specimen and the location at which the heat flow is measured. The most common method of measuring heat flow into a thermal conductivity specimen is to measure the electrical power dissipated in a heater at the hotter end of the specimen. The heat flow out of a specimen is sometimes measured by a "flow calorimeter," with which one observes the temperature rise and flow rate in a circulating liquid of known heat capacity, or by a "boil-off calorimeter," with which one observes the boil-off rate of a fluid of known heat of vaporization. Another common method of measuring the heat flow into or out of the specimen is by use of a "heat-flow meter," with which one observes the temperature drop across a known thermal conductance. As most commonly employed, the heatflow meter is a solid material of known thermal conductivity placed in series with the specimen so as to, hopefully, carry the same heat current.

Similarly, it is not possible to directly measure the temperature gradient in a specimen; one must, rather, measure the temperature difference between two or more locations and then compute the gradient. Consider a specimen held between a heat source and a heat sink. If the total thermal resistance of the specimen is large compared with the thermal contact resistances between the source and the specimen and between the specimen and the sink, then the temperature drop across the specimen can be taken as equal to the temperature of the source minus the temperature of the sink and it is not necessary to install temperature sensors in the specimen. However, if the specimen has a low thermal resistance such that thermal contact resistances are not negligible, it is necessary either to correct for these contact resistances or, what is usually done, to install temperature sensors in the specimen.

The apparatus illustrated in figure 47 has recently been used at NBS to measure the thermal conductivity of a specimen of microcrystalline glass over the temperature range 100 to $330 \mathrm{~K}$. The thermal conductivity of the specimen was measured by a steady-state longitudinal heat flow method in which the thermal conductivity was computed from the measured power input to a heater at one end of the specimen, the measured longitudinal temperature distribution along the sample, the measured thermocouple separation, and the cross-sectional area.

The specimen (A) was fastened to a heavy copper "cup" (C) which was thermally connected by three brass legs (E) to a liquid-nitrogen-cooled copper heat sink (F). A heater (I) wound around the copper cup (C) was used, in conjunction with a thermopile and controller, to maintain the

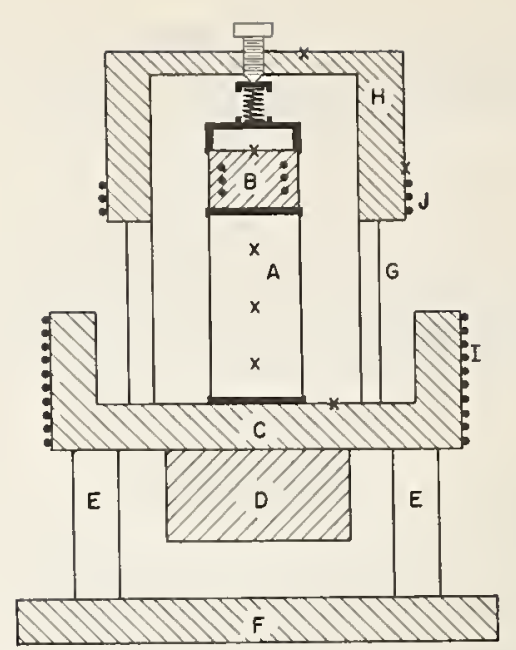

Figure 47. An apparatus utilizing longitudinal heat flow to measure thermal conductivity over the temperature range 100 to $330 \mathrm{~K}$. The components are identified in the text.

copper cup at any temperature from about 90 to $330 \mathrm{~K}$.

A copper block (B) with an internal heater winding provided the measured heat flow down the specimen. The specimen was held between thin copper caps (shown in black) fastened to the adjustable-temperature cup (C) and the heater block (B). In order to achieve good thermal contact, a eutectic alloy of gallium and indium (freezing point $15.7{ }^{\circ} \mathrm{C}$ ) was used in the interfaces between the specimen and the thin copper caps. This assembly was pressed together by means of a loading screw, spring, and stainless steel spider, as shown at the top of figure 47 . The spring was necessary to accommodate differential thermal expansion; it also provided considerable resistance to heat loss from the heater block.

A copper guard cap $(\mathrm{H})$ and radiation shields (G) surrounded the specimen and heater block. Using the heater $(J)$ on the guard cap in conjunction with a thermopile and controller, the temperature of the guard cap $(\mathrm{H})$ was automatically controlled to closely match the temperature of the heater block (B). The space between the specimen and the guard cap and radiation shield was packed with glass wool to minimize radiative heat losses. The entire apparatus was contained in a bell jar which was evacuated to less than $10^{-5}$ torr to further reduce losses from the specimen and the heater block.

Current leads from the specimen heater were thermally grounded to both the heater block and the guard cap so as to minimize conduction of heat along the leads. Because the temperature of the guard cap matched quite closely that of the heater block, the current leads to the specimen heater could be made large enough for heat generation in these leads to be negligible. 
The temperature distribution along the specimen was measured by three butt-welded thernocouples pressed into transverse slits in the convex surface of the specimen. Additional thermocouples, designated by $\mathrm{X}$ in figure 47 , were used to monitor other temperatures in the apparatus. All thermocouples were fabricated from calibrated $0.013 \mathrm{~cm}$ diam copper and constantan wire. Care was taken to thermally temper all thermocouple leads by wrapping them around the specimen in an isothermal region for several centimeters from the junctions. All thermocouple leads were wrapped around and cemented to a copper tempering block (D) to prevent heat from being conducted to the measuring junctions. The thermocouple leads passed through epoxy vacuum seals to individual ice junctions from which copper wires lead to the emf-measuring circuitry.

Power to the specimen heater was provided by a regulated dc power supply operating in a constant voltage mode. The power input was determined by measuring the current through the heater using a standard resistor and the voltage drop across potential taps to the heater using a high-resistance voltbox. Voltages from the standard resistor and the voltbox were read to five significant figures using a precision potentiometer. The emfs of the specimen thermocouples were read to $0.1 \mu \mathrm{V}$. The emfs of other thermocouples were read to $1 \mu \mathrm{V}$.

Each data point was obtained from two tests at essentially the same mean temperature but with different power inputs to the specimen heater in order to minimize systematic errors, especially in temperature measurement.

The thermal equilibriums obtained were such that the specimen temperatures did not drift more than $0.01 \mathrm{~K}$ over a half-hour period.

Several tests were run with a rather large temperature difference between the specimen heater block and the guard cap in order to evaluate the thermal conductance between these regions. Most of this conductance was due to the screw-spring-spider arrangement used to press the heater against the specimen. Using the thermal conductance values thus obtained, all tests were corrected for heat exchanges due to small temperature differences between the specimen heater block and the guard can.

The thermal conductivity values obtained using this apparatus exhibited an estimated standard deviation from a smooth curve of less than onehalf of 1 percent. The uncertainty in the smoothed thermal conductivity values was believed to be not more than 2 percent

The apparatus just described utilizes several features which are not necessary at lower temperatures or in making measurements on materials having higher thermal conductivity values than did the microcrystalline glass $\left(\lambda \approx 5 \mathrm{Wm}^{-10} \mathrm{C}^{-1}\right)$. Further comments are also in order for several other features of the apparatus.
If the heater were wound directly onto the specimen or if the heater block could be clamped or soldered to the specimen, the heater holddown assembly shown in figure 47 would not be necessary. A procedure used by some investigators is to place the heater between two similar specimens as shown in figure 48 . We have recently fabricated an apparatus of this general design for use in measurements on glass samples.

The gallium-indium alloy between the heater and the specimen and between the specimen and the adjustable-temperature cup in the apparatus shown in figure 47 was for the purpose of providing uniform thermal contact and hence promoting plane isotherms in the specimen. If the specimen is longer, relative to its diameter, so that all thermocouples can be placed one to two specimen diameters from both the heat source and the heat sink, the method of introducing and extracting heat from the specimen is not critical since these longer relative lengths allow plane isotherms to become established near the thermocouple locations even if the isotherms are distorted near the source or sink.

At lower temperatures, or in the case of highly conductive specimens, it is not necessary to surround the specimen and heater with thermal insulation-a good vacuum will suffice to preclude unwanted heat exchanges. Similarly, in some applications, particularly below liquid nitrogen temperatures, guard caps or shields are not necessary, it being sufficient to surround the specimen and heater with an essentially isothermal environment not too different in temperature from the temperatures in the specimen. In our application of the apparatus shown in figure 47 , it was necessary to use a guard cap and shield, to install insulation around the specimen, and to evacuate the insulation (to reduce its thermal conductivity) in order to render extraneous heat exchanges essentially negligible. At higher temperatures, as we shall see, it is not always possible to reduce extraneous heat exchanges so as to be negligible and it becomes necessary to evaluate these exchanges and make corrections.

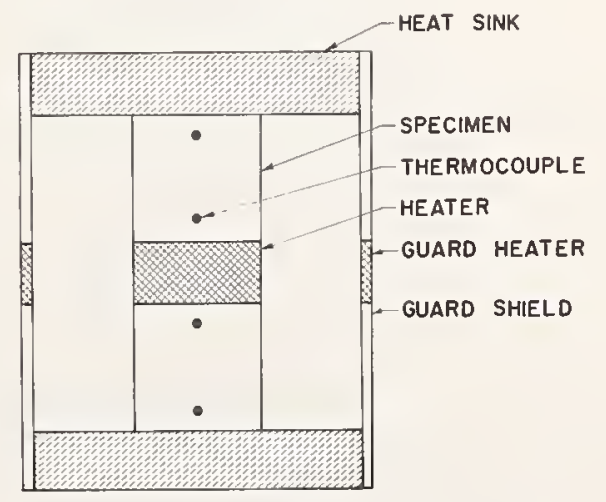

Figure 48. An apparatus to measure thermal conductivity by longitudinal heat flow through two glass specimens with a heater sandwiched between them. 
The method of controlling the temperature of the lieat sink varies considerably among different investigators. Most apparatuses designed for operation below room temperature are immersed in a fluid bath (e.g., liquid nitrogen, liquid helium) rather than having a cryogenic fluid circulated through the apparatus. I feel that the ease-ofaccessibility to the apparatus shown in figure 47 more than offsets the slightly higher costs due to increased liquid nitrogen consumption. Many investigators control the temperature of a cryogenic bath by using a pump to reduce the vapor pressure above the bath. The temperature of the bath is determined from the heat balance condition that at equilibrium the rate of heat flux into the bath equals the latent heat of the fluid times the mass rate of exhaust. The minimum temperature available for a given fluid depends on the pumping speed and the magnitude of the heat input. The temperature of the cryogenic fluid can be raised by decreasing the speed of the pump, which usually is not practical, by increasing the pressure drop between bath and pump, or by increasing the heat flow into the bath. The general problems of providing a controlled-temperature heat sink at cryogenic temperatures are discussed in, for example, the very useful book by White [445].

The apparatus shown in figure 47 utilized copper versus constantan thermocouples fabricated from $.013 \mathrm{~cm}$ diam wire. The cross-sectional area of one wire was only $1 / 40,000$ of the area of the specimen. However, the thermal conductivity of copper is about 100 times that of the microcrystalline glass specimen. Thus the thermal conductance per unit length of one thermocouple wire was about 0.25 percent of that of the specimen. For the accuracy desired, this high a thermal conductance for thermocouple wires is barely tolerable. The options are to use finer wire, which is very delicate to handle; to use an alloy in each leg of the thermocouple so as to have a lower thermal conductivity, with perhaps some sacrifice in the homogeneity of the wire; or to lead the thermocouple wires radially and attach each of them thermally to the guard shield at a point which is at the same temperature as the thermocouple junction on the specimen, a not-too-easy procedure in many apparatuses.

The apparatus shown in figure 49 was used by Ditmars and Ginnings [446] to measure the thermal conductivity of beryllium oxide over the temperature range 40 to $750{ }^{\circ} \mathrm{C}$. This apparatus is similar in principle to, but different in detail from, the apparatus (figure 47) previously discussed. Measured electrical heat, introduced in the sample heater at the top of sample, flowed down the sample and its "adapter" to a heat sink. The sample heater consisted of six small helices of fine wire located in holes in the top of the sample. Anhydrous boric oxide was used to give good thermal contact between the adapter and the sink. 'The sink was cooled with either water or air', depending on the temperature range, and was

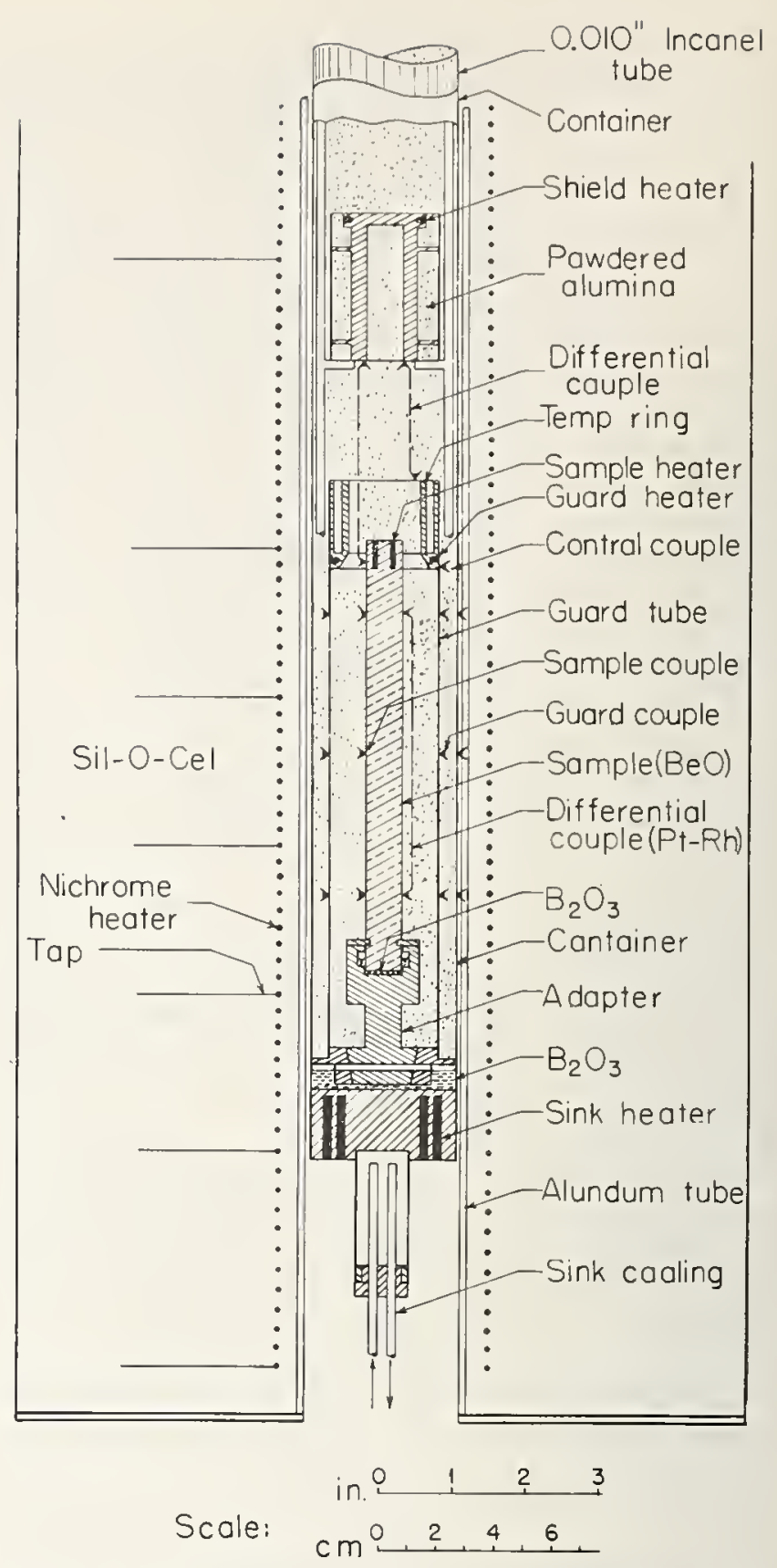

FIGURE 49. An apparatus utilizing longitudinal heat flow to measure thermal conductivity of beryllium oxide over the temperature range 40 to $750{ }^{\circ} \mathrm{C}$ (after Ditmars and Ginnings [446)].

equipped with a heater and a thermocouple so that it could be automatically kept at a constant temperature.

The temperatures along the sample were measured with three thermocouples $(0.013 \mathrm{~cm}$ diam platinum-rhodium versus platinum) having reference junctions at $0^{\circ} \mathrm{C}$ and principal junctions on the sample at the three levels shown in figure 49. In addition, a differential thermocouple was used to measure the temperature difference between the upper and lower levels on the sample. All of the thermocouples on the samples were made with junctions peened into small holes (about 0.06 $\mathrm{cm}$ in diameter and depth) in the cylindrical 
surface of the sample. In order that the temperature difference measured on the sample would correspond to the electric heat put into the top of the sample, precautions were taken to minimize radial heat loss from the sample. For this purpose the sample was surrounded by a metal guard tube provided with thermocouples. The top of the guard tube was automatically controlled at the desired temperature using the guard heater. The top portion (tempering ring) of the guard tube was made of thick nickel to which the electrical leads to the sample were thermally connected, so that it served as a tempering region for bringing the leads to the temperature of the top of the sample, and thus to reduce heat conduction along them.17 A thermal shield and heater, placed above the sample and tempering ring, also served for this purpose and to prevent heat transfer upward through supports and insulation.

The whole assembly, supported by rods extending down to the bottom of the guard, was filled with fine alumina powder for insulation and enclosed in a thin metal tube which served to hold the powder. The apparatus, which was filled with argon during operation, was maintained at the chosen temperature by the surrounding furnace.

Two basic types of experiments were performed for each measurement of thermal conductivity. In the first type, which Ditmars and Ginnings called a conductivity experiment, the furnace temperature was controlled to the desired value, a known constant electric power was put into the sample heater, the temperatures of the guard and shield were adjusted to match those on the sample as closely as possible, and the sink temperature was adjusted to a constant value. In the second type of experiment, which Ditmars and Ginnings called a calibration experiment, no power was put into the sample heater but in other ways the experimental procedure was similar to that in the first type of experiment.' The purpose of this experiment was to correct for errors that did not depend on the power transmitted through the sample. Errors of this type are those due to differences in thermocouples, and those resulting from unknown heat leaks, which presumably were the same in both types of experiments.

Ditmars and Ginnings estimated that their results on beryllia were in error by less than about 3 percent. Laubitz [447] feels this possible inaccuracy could be reduced with only minor modifications.

Laubitz [447] has carefully analyzed extraneous heat flows in thermal conductivity measurement apparatus utilizing longitudinal heat flow methods such as those discussed to this point in this section. His chapter should be required reading for anyone contemplating building an apparatus of this nature. Laubitz and his co-workers at the National Research Council in Canada (see

${ }^{17}$ Laubitz, who recently analyzed this apparatus, [447] feels that the tempering ring caused unwanted heat exchanges between the sample and the guard and hence should not be used. references given in [447]) and our group at the National Bureau of Standards (e.g., see Flynn and O'Hagan [243]) are among the few workers who have undertaken detailed mathematical analyses of this type of apparatus in order to evaluate extraneous heat flows. As Laubitz' recent work [447] clearly illustrates, most apparatus would benefit enormously from such an analysis.

Other useful survey papers pertinent to the type of apparatus we have been discussing include White [445, 448], Drabble and Goldsmid [14], and Mikryukov [449]. The discussion of error sources by Bauerle [450] is also very helpful.

The apparatuses described so far can be used when there is an available insulation, suitable for use between the specimen and guard, which has a thermal conductivity value much lower than that of the specimen. However, if the specimen has a low thermal conductivity, such as is found for example in very porous materials (fibrous or granular materials, insulating firebrick), there may be no insulation which has a significantly lower thermal conductivity. Then the specimen is often used as its own insulation. The first example of an apparatus of this type which we shall discuss is the guarded hot plate. Since this is an ASTM Standard Method of Test, which is adequately described elsewhere [451], I shall only briefly describe the apparatus in order to illustrate the concepts involved.

As shown in figure 50, a guarded hot plate apparatus consists of a heated metering plate, which may be square or circular, separated by a narrow insulating gap from a surrounding coplanar guard plate. Similar specimens are placed on either side of the hot plate; the outside surfaces of the specimens are held between constant temperature cold plates. In operation, the electrical power input to the guard plate is adjusted, usually automatically, so that a mul-

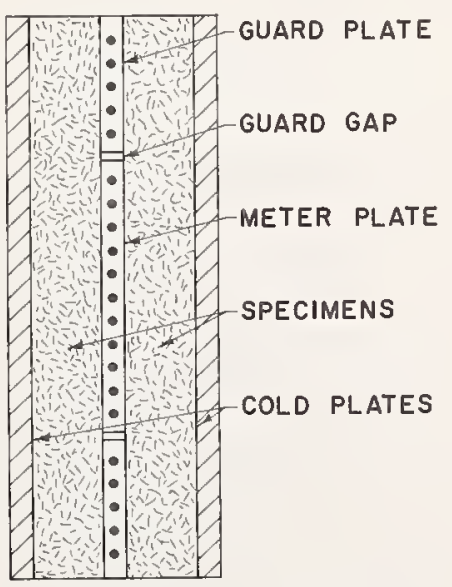

Figure 50. A guarded hot plate apparatus for measuring the thermal conductivity of relatively good insulating materials using ASTM Standard Method of Test C17\%. 
tiple-junction differential thermocouple spanning the guard gap has zero output, indicating that there is no temperature difference across the guard gap. Thus the electrically generated heat input to the metering plate flows perpendicularly from both sides of the plate through the specimens to the cold plates. The average thermal conductivity of the two specimens is determined from the measured power to the metering plate, the temperature drops across the specimens, and the geometry.

In using a guarded hot plate, measurement errors can arise from imperfect guarding, inaccurate determination of temperature differences, or failure to achieve thermal equilibrium, as well as from possible errors in reading instruments or measuring specimen thickness or in thermocouple calibrations.

While it is relatively straightforward to accurately measure the electrical power dissipated in the metering plate heater, it is not at all easy to ascertain that all of this heat is going where it is supposed to. The hot plate must be well designed in order that there be no significant heat exchange between the metering and guard plates. The thermal resistance across the guard gap should be large, the differential thermocouple junctions must be installed so as to appropriately sample the temperature on either side of the gap, and the power to the guard heater must be controlled so as to maintain a sufficiently small temperature difference across the guard gap. If the conductivity of the specimen is high, a corresponding gap may be cut part way or all the way through the specimens. If the guard is too small, or its surfaces are not isothermal, the guarding will not be adequate. In order to ensure adequate guarding, it is common to use edge insulation around the periphery of the guard plate and specimens and/ or to effectively increase the area of the guard plate by using a second (outer) guard plate or by controlling the ambient temperature around the apparatus to a value near the mean temperature of the specimens.

If the thermal resistance of the specimen is sufficiently high, determination of the temperature gradient is easy. The temperature drops between the metering plate and the cold plates are measured using thermocouples (or other temperature-measuring devices) embedded in the metering and cold plates. Contact resistances are neglected and the gradients are computed from these temperature drops and the specimen thicknesses (which preferably should be the same and should be measured under the conditions of testing). If thermal contact resistances may not be neglected, the determination of temperature gradients becomes more difficult. Thermocouples may be installed in the specimen in such a manner as not to disturb the uniform flow of heat. It is frequently advisable, as in the case of rigid specimens, to install a thin resilient pad (rubber, asbestos paper, etc.) on either side of each specimen (i.e., cold plate, resilient pad, specimen, resilient pad, hot plate, resilient pad, etc.). The philosophy here is that if you cannot get good contacts it is better to have uniformly poor ones so that heat flow through the specimens will be uniform. One technique which we have found very useful is, using the thermocouples in the hot plate and cold plates, to measure the effective thermal resistance of the pad-specimen-pad assembly and then, separately, the resistance of the resilient pads without the specimens. By subtraction, the resistance of the specimen is found, providing the contact resistance between the specimen and resilient pads is negligible. In this manner we have obtained good data on, for example, $2.5-\mathrm{cm}$ thick glass specimens using $0.3-\mathrm{cm}$ gum rubber pads.

If contact resistance problems can be handled, a well-designed guarded hot plate will yield results having uncertainties less than 2 percent near room temperature with the uncertainties increasing at both lower and higher temperature. ASTM Committee $\mathrm{C}-16$ is currently studying the problems involved in using a guarded hot plate apparatus to make thermal conductivity measurements at low and high temperatures.

Because of the difficulties of guarding, and because of contact resistance problems, a guarded hot plate is usually not appropriate for measurements on materials having thermal conductivities much higher than that of, say, fused silica.

Useful references to the use of the guarded hot plate, as well as to its associated possible errors, include [452-455].

In all of the thermal conductivity measurement methods we have discussed thus far, the heat flow through the sample has been determined by measuring the electrical power dissipated in a heater. We shall now discuss methods in which the heat flow is determined by other means.

In ASTM Standard Method of Test C201-47, the heat flow through a ceramic specimen, such as a firebrick, is determined by measuring the temperature rise in a stream of water passing at a known rate through the meter section of a guarded water-flow calorimeter. Typically the calorimeter is double-guarded and the water-flowrate through each guard must be carefully adjusted if the guarding is to be effective. This test method is adequately described elsewhere [456] so we will not discuss it further here.

We have been using a somewhat similar test method at NBS intermittently for about 20 years. The NBS guarded steam calorimeter apparatus, which we believe to be simpler and more expeditious in use that a water flow calorimeter apparatus, has not previously been described in the open literature.

Figure 51 shows the arrangement of the assembled apparatus, which consists of a high temperature furnace, the disk-shaped 15.2-cm diam specimen, and the steam calorimeter. The specimen is supported slightly above a circular silicon 


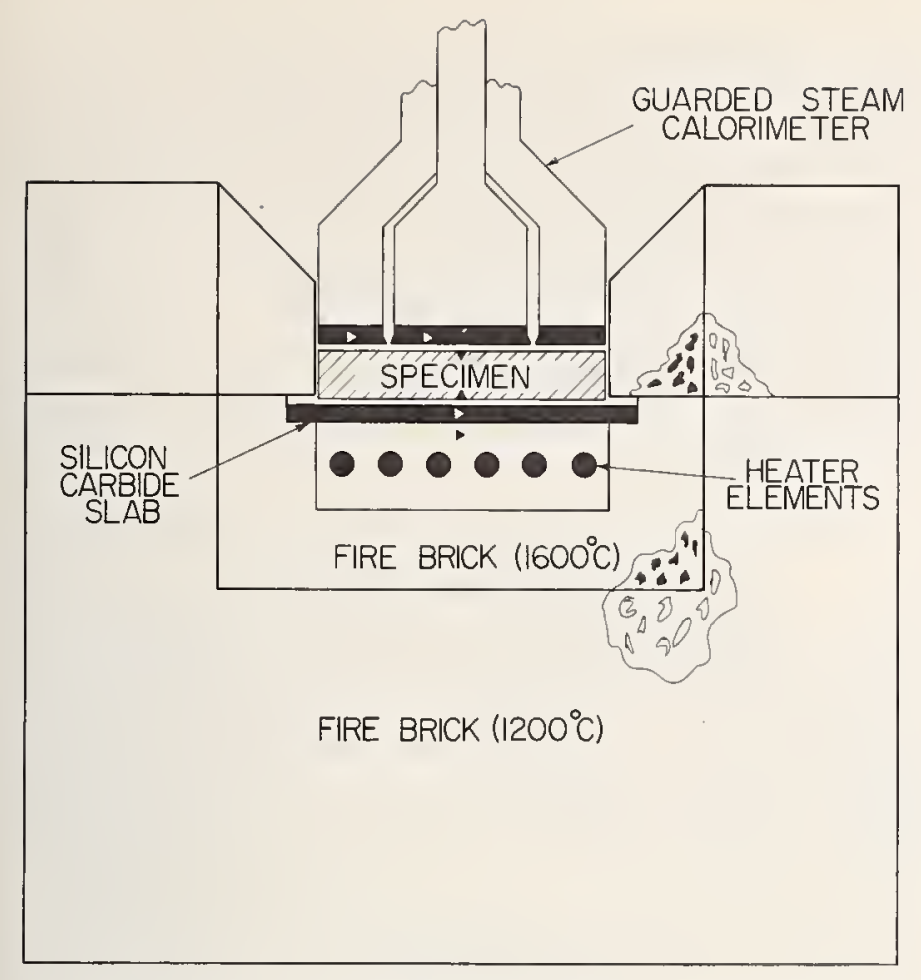

FIgURE 51. The NBS steam calorimeter apparatus for measuring the thermal conductivity of refractories.

carbide plate heated by the furnace, and is heated by transfer of heat across the space between them. At high temperatures, the heat transfer is chiefly by radiation, with a relatively small temperature drop. Heat passes through the specimen, and is transferred from its upper surface, by radiation and conduction, across an air space, to the underside of the steam calorimeter, which is supported above it. The use of thin air spaces on both sides of the specimen is for the purpose of avoiding, or reducing, problems of thermal contact with solid surfaces that arise when a specimen is warped or distorted by expansions resulting from the temperature gradient established in it.

The heat flow through the specimen is determined by means of the steam calorimeter, the metering area of which is the $7.6-\mathrm{cm}$ diam area covered by the metering chamber bottom. The guard chamber of the calorimeter, which operates at the same temperature, acts to protect the metering area of the specimen from edge effects.

The temperature drop through the specimen is measured by means of thermocouples imbedded in its surfaces, as indicated. The relatively high thermal conductivity of the silicon carbide plate promotes substantial uniformity of temperature at its top surface. To promote uniformity of radiant heat emission and absorptance, the surface of the calorimeter bottom, and either one or both surfaces of the specimen, may be painted with a high temperature paint of high emittance.

The high temperature furnace has a fixed cylindrical base, with a removable annulus resting on it which surrounds the specimen and the calorimeter bottom. The base and annulus are fabricated from $1200^{\circ} \mathrm{C}$ insulating firebrick, but each has an inner liner capable of withstanding $1600^{\circ} \mathrm{C}$. The heat source consists of six silicon carbide electrical resistance elements located in the circular chamber in the furnace base. The chamber opening is covered by a $1-\mathrm{cm}$ thick by $18.4-\mathrm{cm}$ diam plate of self-bonded silicon carbide, capable of withstanding $1600{ }^{\circ} \mathrm{C}$ in an oxidizing atmosphere. A heater ring, not shown in figure 50 , surrounding the specimen is used to minimize radial heat flow.

A detailed illustration of the steam calorimeter is given in figure 52. Under test conditions, the heat entering the calorimeter base boils the water in both the metering chamber (A) and guard chamber (B). Steam from the metering chamber rises through the stack $(\mathrm{H})$ from which it passes to the condenser (I) through a connecting tube with a $10 \mathrm{deg}$ downward slope. The condensate is collected in the flask $(F)$. By determining the time required to fill the known volume of the flask between index marks with condensate, it is possible to calculate the heat flow rate represented by the steam condensed. The guard steam passes up on both sides of the radiation shield $(G)$, through the orifice $(K)$, and into the condenser $(L)$. The condensate drains from the base of the condenser back to the guard chamber through the external return tube $(\mathrm{E})$.

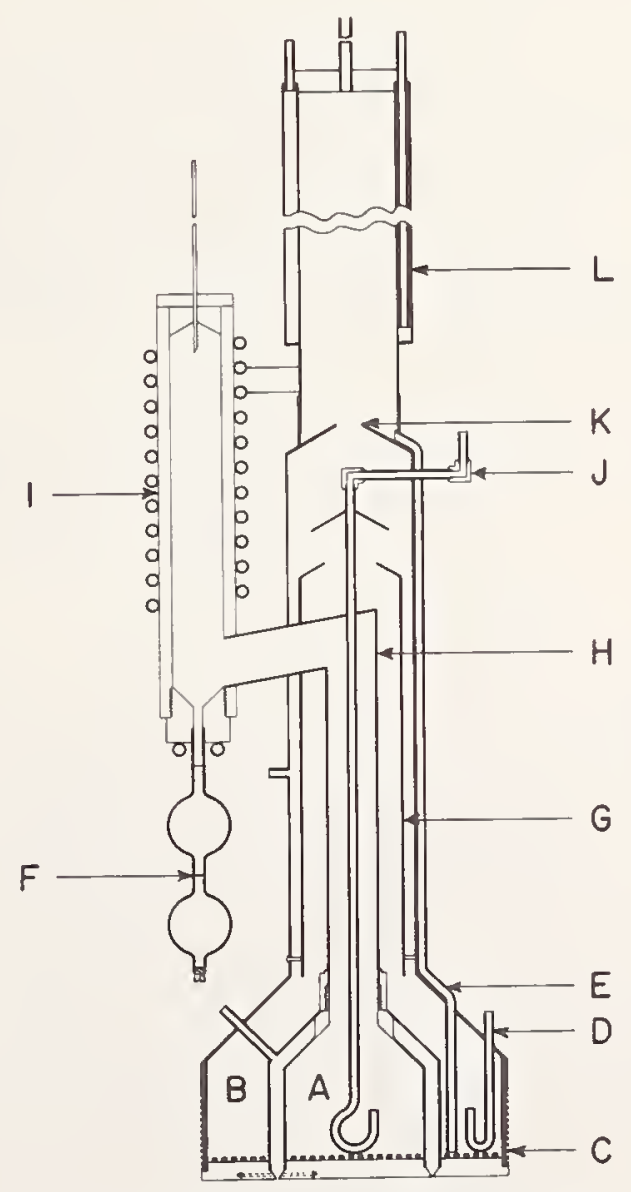

FIGURE 52. Detailed schematic of the NBS steam calorimeter. The components are identified in the text. 
The wetted surfaces of the 0.6 -cm thick copper bottoms are grossly roughened by means of $0.3-\mathrm{cm}$ cubes of brass distributed over and soldered to the surfaces, to aid boiling. The diameter of the metering chamber bottom is $7.6 \mathrm{~cm}$; the gap to the inner diameter of the guard chamber bottom averages $0.02 \mathrm{~cm}$.

Both steam condensers are operated at atmospheric pressure, and boiling in the chambers occurs at substantially this pressure. However, to preclude the possibility of condensation of the metering chamber steam before it reaches the downward sloping tube to the condenser, the temperature of the guard chamber steam is automatically kept about $0.1^{\circ} \mathrm{C}$ higher than that of the metering chamber steam by means of the small pressure drop occurring across the orifice $(K)$, which can be changed in size. Metallic connection of the sloping thin stainless steel tube to the guard chamber wall, and to the brass radiation shield (G), also tends to prevent cooling of the stack $(\mathrm{H})$ by the condenser $(\mathrm{I})$.

An auxiliary electrical heater (C) is located on the outer wall of the guard chamber, for use when necessary in tests at very low flux rates to assure an adequate flow of steam through the orifice (K). Two thermocouples are installed in the bottoms of the metering and guard chambers for monitoring purposes.

In preparation for tests, the two chambers are filled with an excess of water, which is then withdrawn by suction to the level of the ends of the filler tubes $(J, D)$, which are self-trapping. The condensate collected from the flask (F) is returned manually to the metering chamber through the filler tube $(\boldsymbol{J})$. Flasks of a few different volumetric capacities are used, depending on the rate of condensate collection. The volumes between index marks are determined to within $0.01 \mathrm{~cm}^{3}$.

Since the water in both the guard and meter sections boils at nearly the same temperature, the guard section temperature automatically matches that of the meter section without the need for critical adjustments such as are required in using ASTM C201.

We intend to make several changes in the steam calorimeter apparatus in order to improve the accuracy and render it more convenient in operation. These changes include: (a) putting a second guard, of about $30-\mathrm{cm}$ diam, around the existing guard to extend the $100{ }^{\circ} \mathrm{C}$ isothermal surface and thus improve the guarding action, (b) building a new furnace, of lighter construction so as to attain equilibrium more rapidly, with a $30-\mathrm{cm}$ diam hot plate, and (c) investigating the possible advantages of changing to an all-glass condenser for the meter section. Even without these modifjcations, the apparatus is very convenient in operation and yields thermal conductivity values estimated to be uncertain by not more than, say, 3 or 4 percent at specimen hot-side temperatures of $1200^{\circ} \mathrm{C}$ or higher.
The last longitudinal heat flow method we shall discuss is the type of comparative method which is variously known as a comparator, cutbar method, or divided-rod method. Ideally, in this method the same heat flow is caused to pass through both the specimen, of unknown thermal conductivity, and a reference specimen, of known thermal conductivity, which acts as a heat flow meter and hereafter will be referred to as the "meter bar". For constant properties, the heat flow is given by

$$
Q=\frac{\lambda_{s} A_{s} \Delta T_{s}}{l_{s}}=\frac{\lambda_{m} A_{m} \Delta T_{m}}{l_{m}}
$$

where $A, \Delta T$, and $l$ refer to cross-sectional area, temperature difference, and length, respectively, the subscript $s$ designates the specimen, and $m$ designates the meter bar. The thermal conductivity of the specimen, under these ideal conditions, is given by

$$
\lambda_{s}=\lambda_{m} \frac{A_{m} l_{s} \Delta T_{m}}{A_{s} l_{m} \Delta T_{s}}
$$

As normally used with ceramic materials, the cut-bar method is subject to essentially the same uncertainties in determining temperature gradient and area as would be present in other types of longitudinal heat flow methods. However, we are involved with two areas, two lengths, and two temperature differences, so in principle, it would be possible for the total error from these sources to be roughly twice as large. (In practice, certain systematic errors would have the same sign in both the specimen and the meter bar and hence would partially cancel.) In addition, one must add the uncertainty in the thermal conductivity of the "known" meter bar. The additional uncertainties introduced via $\lambda_{m}, A_{m}, l_{m}$, and $\Delta T_{m}$ are almost invariably larger than the error would have been if the heat flow were determined by measuring the electrical power dissipated in a heater.

Probably the largest uncertainty in comparative methods arises from failure to meet the basic underlying requirements of eqs (106) and (107), namely that the heat flow must be the same in the specimen and the meter bar. In the somewhat vain hope of ascertaining compliance with this requirement, many investigators have sandwiched their specimen between two meter bars in a configuration such as that shown in figure 53. The lower meter bar supposedly measures the heat flow into the specimen while the upper meter bar hopefully measures the heat flow out of the specimen. Agreement between the readings obtained with the upper and lower meter bars is a necessary, but not sufficient, condition for the heat flow through the specimen to be the same as that through the meter bars. The heat-flow indications provided by the two meter bars will 


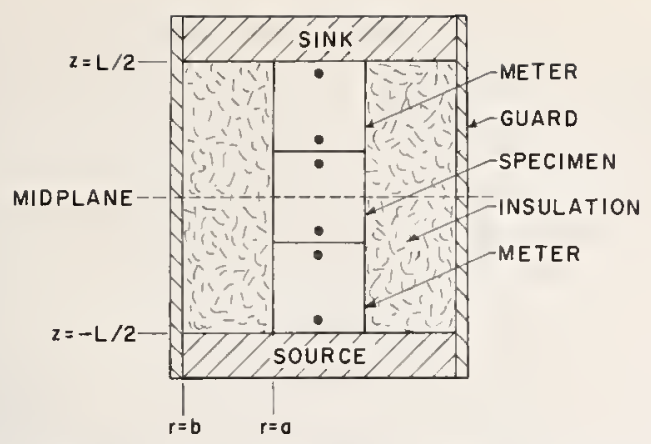

Figure 53. A comparative, or "cut-bar" apparatus in which the heat flow through the unknown specimen is determined from the temperature gradients and thermal conductivities of the "meter-bars" on either side of the specimen.

agree provided the following rather general conditions are met:

(a) The apparatus is geometrically symmetrical about its midplane.

(b) The two meter bars have the same thermal conductivity.

(c) The temperature distribution along the inner surface of the guard cylinder and along the surfaces which bound the outer faces of the meter bars (e.g., the planes $z= \pm L / 2$ and the cylinder $r=b$ shown in figure 53) possesses odd symmetry with respect to the temperatures at the midplane of the apparatus. By this, I mean that $[T(r, \theta, z)+T(r, \theta,-z)] / 2=T(r, \theta, 0)$, where $T$ is temperature and $r, \theta$, and $z$ are cylindrical coordinates ( $z$ being measured relative to the midplane of the apparatus), for all points on these bounding surfaces.

To illustrate the above, let us suppose that the apparatus shown in figure 53 meets conditions (a) and (b). We further suppose that the planes $z= \pm L / 2$ are each isothermal such that condition (c) is satisfied for these two planes. All of these suppositions are very reasonable and, in fact, are goals which investigators often strive for in designing this type of apparatus. In figure 54 we show three possible temperature distributions that might exist along the inner surface of the guard cylinder, $r=b$. The solid curve represents "matched guarding", where the temperature distribution along the guard is the same as that which would be along the meter bar/specimen/ meter bar assembly if the heat flow through this assembly were constant (which is required if eq (106) is to be valid). The dashed curve represents "linear guarding", where the guard temperature varies linearly with longitudinal position from end to end. The hatched curve represents "isothermal guarding", where the guard is isothermal at the specimen mean temperature except very near the ends where it changes rapidly to match the temperatures of the iso-

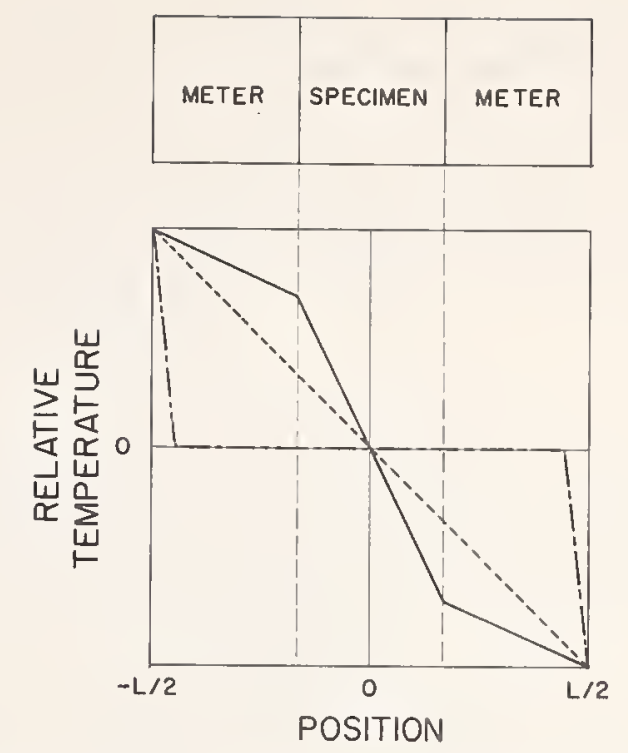

FIgURE 54. Three possible types of guard temperature distribution which will result in agreement between the heat-flow indications of the two meter bars even though the heat flow through the specimen may be different.

thermal surfaces $(z= \pm L / 2)$ which bound the outer faces of the meter bars. All three of these guarding conditions (plus an infinite number of others) satisfy condition (c) given above so that the two meter bars will indicate the same heat flow. However, the heat flowing in the specimen can differ considerably from the heat flow indicated by the meter bars.

In some unpublished work that $\mathrm{H}$. E. Robinson and I carried out in this laboratory in 1961 and 1962 , it was shown that the fractional power change, from that power which would flow through the meter bars and specimen if they were perfectly insulated, can be represented quite closely, for matched or linear guarding, by

$$
\epsilon(z)=\lambda_{i}\left(\frac{1}{\lambda_{m}}-\frac{1}{\lambda_{s}}\right) \cdot F(z)
$$

where $\lambda_{s}, \lambda_{m}$ and $\lambda_{i}$ are the thermal conductivities of the specimen, meter bar, and insulation, respectively, and $F(z)$ is a geometrical factor which depends on the type of guarding. Figure 55 shows $F(z)$ for the case of matched guarding in the apparatus shown in figure 53 . It is informative to evaluate eq (108) for a not-too-unreasonable set of values for the thermal conductivities. Assume we wish to use dense aluminum oxide meter bars $\left(\lambda=36 \mathrm{Wm}^{-1}{ }^{\circ} \mathrm{C}^{-1}\right)$ to measure the thermal conductivity of a borosilicate glass sample $(\lambda=1.1$ $\mathrm{Wm}^{-1}{ }^{\circ} \mathrm{C}^{-1}$ ) near room temperature. As insulation, we use fine aluminum oxide powder in air $(\lambda=0.1$ $\mathrm{Wm}^{-1}{ }^{\circ} \mathrm{C}^{-1}$ corresponds to very low density powder). Substitution of these values into eq (108) yields $E(z)=0.09 F(z)$; at the midplane of the apparatus, $F=2.0$ so that $E(0)=0.18$, or in 


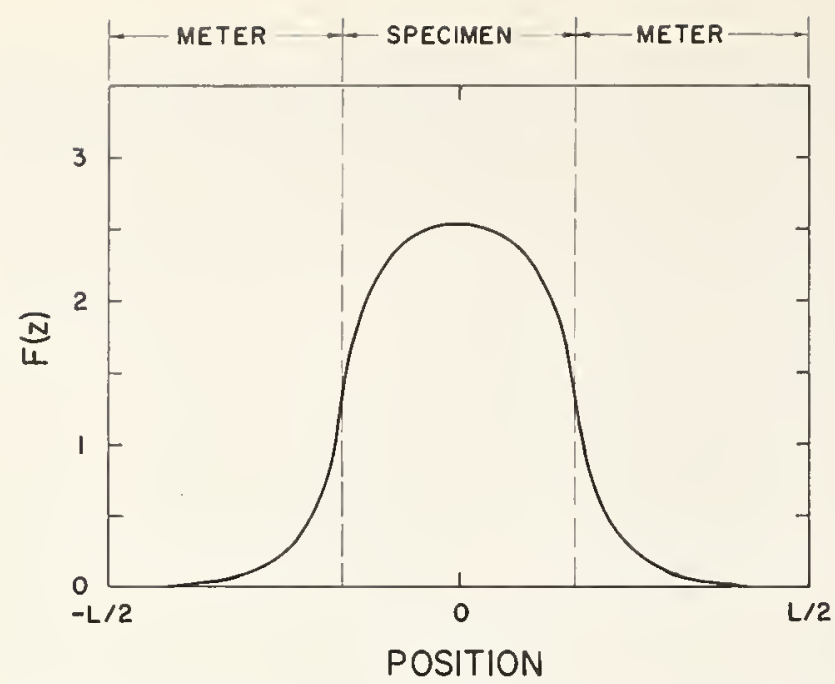

Figure 55. The geometrical factor, $\mathrm{F}(\mathrm{z})$, to be used in eq. (108) for the case of matched guarding in the cut-bar apparatus shown in figure 53.

other words, the heat flow through the midplane of the specimen is 18 percent less than it would be if the specimen and meter bars were perfectly insulated. The error if eq (107) were used to compute $\lambda_{\mathrm{s}}$ would be less than this but still of the order of ten percent. Since the temperature distribution along the guard is assumed to be the same as that along the specimen and meter bars, there are no radial heat exchanges with the guard. For matched guarding the error arises simply due to shunting of heat around the specimen through the insulation. For other than matched guarding, there will be radial heat exchanges between the specimen-meter bars assembly and the guard cylinder in addition to the longitudinal shunting heat flow in the insulation. The attendant error in $\lambda_{s}$, if it is computed using eq (107), can be very large.

For matched guarding, an upper limit for the geometrical factor for shunting heat flow is given by

$$
F \leq\left[\frac{\left(b^{2} / a^{2}\right)-1}{2 \ln (b / a)}-1\right]
$$

where $a$ is the radius of the specimen and meter bars and $b$ is the inner radius of the guard cylinder. For any other type of guarding, there will be a contribution due to radial heat exchanges.

As revealed in eq (108), the error due to shunting heat flow in the insulation is proportional to the difference between the thermal resistivities of the specimen and the meter bars. This error can, therefore, best be minimized by using meter bars having a thermal conductivity as similar as possible to that of the specimen. This also makes guarding easier since no sharp breaks are required in the longitudinal temperature distribution along the guard. A further advantage to having similar thermal conductivities is that the temperature drops in the specimen and meter bars can be made large enough to facilitate accurate measurement while the total temperature drop is kept small.
References which may be consulted for discussions of various aspects of guarding of cut-bar apparatuses including Laubitz [447, 457-458], Bauerle [450], Petrov [459], Didion [460], and Minges [461]. Apparatuses of the type we have been discussion are described by, for example, Francl and Kingery [462], Francis and Tinkelpaugh [463], Mirkovitch [464], and Mahmoodi [465].

A rather different configuration for a comparative cut-bar apparatus is typified by ASTM C40858 , Standard Method of Test for Thermal Conductivity of Whiteware Ceramics [466]. In this apparatus the specimen is soldered between two rather long copper meter bars or "thermodes". There is no guard-the apparatus is contained within an evacuated chamber so that heat loss can occur only by radiation. No thermocouples are placed in the specimen-the temperature drop across the specimen and the contacts with the thermodes is determined by extrapolation of temperatures in each thermode. No correction is made for thermal contact resistance between the specimen and the thermodes. I feel that thermal conductivity values obtained using ASTM C408, in its present form, are liable to large errors. For a material, such as beryllia, which has a high thermal conductivity, errors may arise due to thermal contact resistance. For a material, such as mullite, which has a low thermal conductivity, errors may arise due to heat losses. These factors are considered for a somewhat similar apparatus in the report by Moore, et al. [317].

A number of error sources can be eliminated if a comparative thermal conductivity apparatus is calibrated using specimens of known thermal conductivity. If this is done, it is not necessary to know the thermal conductivity of the meter biars. These considerations led us to suggest an apparatus, which Laubitz [447] has since called an interpolator, such as that shown in figure 56 . The meter bars and specimen are each of length equal to

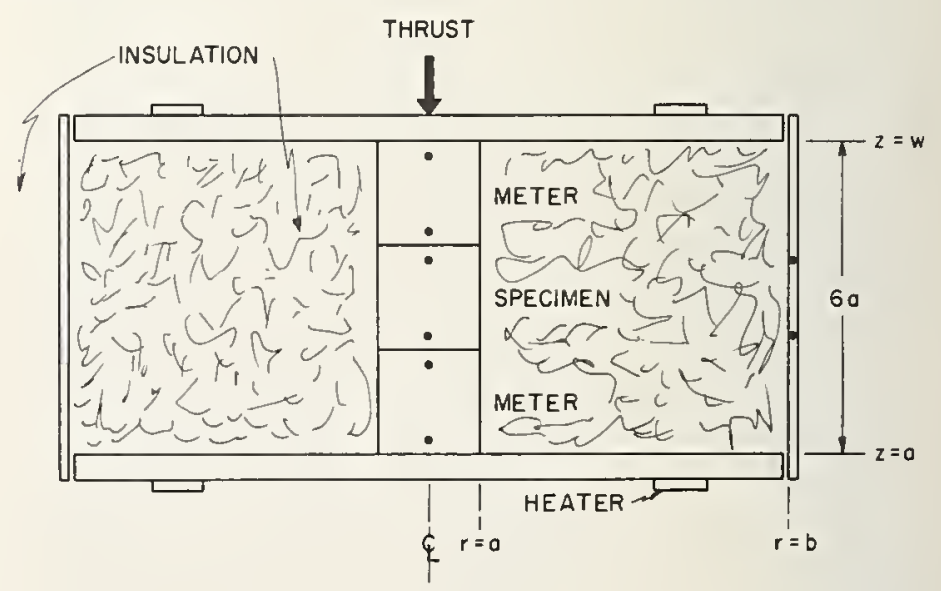

Figure 56. A cut-bar apparatus in which the guard diameter is sufficiently large that the detailed temperature distribution along the guard is unimportant.

Such an apparatus can most accurately be calibrated by the use of substitute specimens. 
twice their radius, $a$. Thick disks of conductive material of radius $b=7 a$, approximately, are used to provide substantially isothermal surfaces at the outer faces of the two meter bars. The "guard", constructed of conductive material, is positioned on poorly conducting supports carried by the lower disk.

With a guard of such large diameter, shunting heat flows are independent of the details of the temperature distribution along the guard. We have calculated the heat exchanges between the meter bars and the insulation and between the specimen and the insulation and found that these exchanges are essentially identical for the three guarding conditions shown in figure 54 . Thus in this apparatus all such extraneous heat exchanges are constant for given meter bars and specimen provided only that the rather general condition (c) described previously is met (i.e., the guard temperature distribution possesses odd symmetry about the midplane of the apparatus). This is easily assured, for example, by making the guard conductive enough for it to be essentially isothermal.

In using this apparatus, the conductivity of the meter bars is assumed to be unknown. A number of specimens of known and differing thermal conductivities should be measured in the apparatus over the intended temperature range. The apparent thermal conductivity, $\lambda_{m}$, of the meter bars should then be computed from eq (107) for each calibration specimen tested. The several $\lambda_{m}$ values obtained will differ somewhat due to shunting heat flows in the insulation surrounding the specimen and meter bars. A plot should be made of these apparent $\lambda_{m}$ values as a function of the known $\lambda_{s}$ values. This constitutes the calibration curve for the apparatus. To compute a thermal conductivity value from data obtained on an unknown specimen, use eq (107) with an approximate value of $\lambda_{m}$ to compute a first approximation of $\lambda_{s}$. Using this value of $\lambda_{s}$, enter the calibration curve and obtain a better value of $\lambda_{m}$ which then is used in eq (107) to compute an improved $\lambda_{s}$. One or two such iterations should suffice.

There are, of course, alternate methods of treating the data. The main point to be made is that the measurements on unknown specimens are made under the same guarding conditions as existed when the apparatus was calibrated using known specimens. In this way, certain systematic errors cancel out and the attainable accuracy can be significantly improved.

Some obvious precautions or arrangements desirable in the use of the envisaged apparatus include the following:

(a) The insulation of conductivity $\lambda_{i}$ should be installed, and if necessary, renewed from a uniform stock, so that $\lambda_{i}$ is substantially the same in all tests at the same temperatures.

(b) Contacting surfaces of the meter bars and specimens should be clean and quite flat to avoid perturbations of the calibration and tests due to altered thermal resistance at contacts.

(c) The reference specimens and the test specimens should always have the same dimensions and thermocouples should be installed at the same locations and in the same manner.

\subsection{Radial Heat Flow}

Although a few investigators have utilized radial heat flow in spherical or spheroidal specimens, the great majority of thermal conductivity measurements utilizing radial heat flow have been carried out on circular cylindrical specimens and we will restrict our discussion to these.

\section{a. Mathematical Development}

The total radial heat flow through a cylindrical element of axial length, $L$, is

$$
Q=-2 \pi L \lambda r \frac{\partial v}{\partial r}
$$

where $v$ is the temperature relative to an arbitrary datum plane, and where it is to be understood that $\lambda$ now refers to the thermal conductivity in the $r$-direction. If temperatures are independent of time, if the specimen is properly insulated or guarded so that there is no axial gain or loss of heat, and hence. $Q$ is constant, if there is no heat generation within the specimen itself, and if, further, the specimen is homogeneous in the $r$-direction so that $\lambda$ does not depend on $r$, then (110) is easily integrated to yield

$$
Q=\frac{2 \pi L}{\ln (b / a)} \int_{v_{b}}^{v_{a}} \lambda d v
$$

where $v_{\mathrm{a}}$ and $v_{\mathrm{b}}$ are the values of $v$ at $r=a$ and $r=b$, respectively. Equation (111) is valid under restrictions analogous to those listed following eq(95).

By analogy with the development of eqs (98) to (100), if $\lambda$ is a linear function of temperature, we obtain

$$
Q=\frac{2 \pi L \lambda_{0}\left(T_{a}-T_{b}\right)}{\ln (b / a)}
$$

where $T_{a}$ and $T_{b}$ are the temperatures at $r=a$ and $r=b$, respectively, and $\lambda_{0}$ corresponds to the temperature $T_{0}=\left(T_{a}+T_{b}\right) / 2$. For materials having a thermal resistivity which is proportional to temperature, expressions analogous to eqs (103) and (104) are easily obtained.

If eq (112) is used to compute thermal conductivity, the maximum fractional error in the resultant thermal conductivity value is given by

$$
\begin{aligned}
\left|\frac{\Delta \lambda_{0}}{\lambda_{0}}\right| & \leq\left|\frac{\Delta b}{b \ln (b / a)}\right|+\left|\frac{\Delta a}{a \ln (b / a)}\right|+\left|\frac{\Delta L}{L}\right| \\
& +\left|\frac{\Delta\left(T_{a}-T_{b}\right)}{T_{a}-T_{b}}\right|+\left|\frac{\Delta Q}{Q}\right|+\left|\left(\frac{1}{\lambda} \frac{d \lambda}{d T}\right)_{0} \Delta T_{0}\right|
\end{aligned}
$$


$+\left|\begin{array}{l}\text { errors due to failure to meet requirements } \\ \text { of equation (112) }\end{array}\right|$ where the terms are analogous to those following eq (105).

\section{b. Experimental Techniques}

Many of the problems and techniques used in radial heat flow methods for measuring thermal conductivity are similar to those we have discussed in conjunction with longitudinal heat flow methods. Accordingly, I will only briefly discuss the configuration usually used in radial heat flow methods and mention a few of the problems encountered with these methods. A very comprehensive discussion of radial heat flow methods is given by McElroy and Moore [467].

A generalized thermal conductivity apparatus utilizing radial heat flow is shown in figure 57. The specimen, in the form of a right circular cylinder, is supported coaxially within a furnace which is used to control the mean temperature of the specimen. A coaxial central hole in the specimen contains either a heater which produces metered power flowing radially outward through the specimen or a heat sink which measures (e.g., by means of a water-flow calorimeter) the power flowing radially inward through the specimen. Temperatures within the specimen, usually at or near the midplane position, are measured by thermocouples or by an optical pyrometer. Guard heaters may or may not be used to control longitudinal heat flows.

The worst errors usually arise from the difficulties of measuring small temperature differences and from nonradial heat flows. Each thermocouple
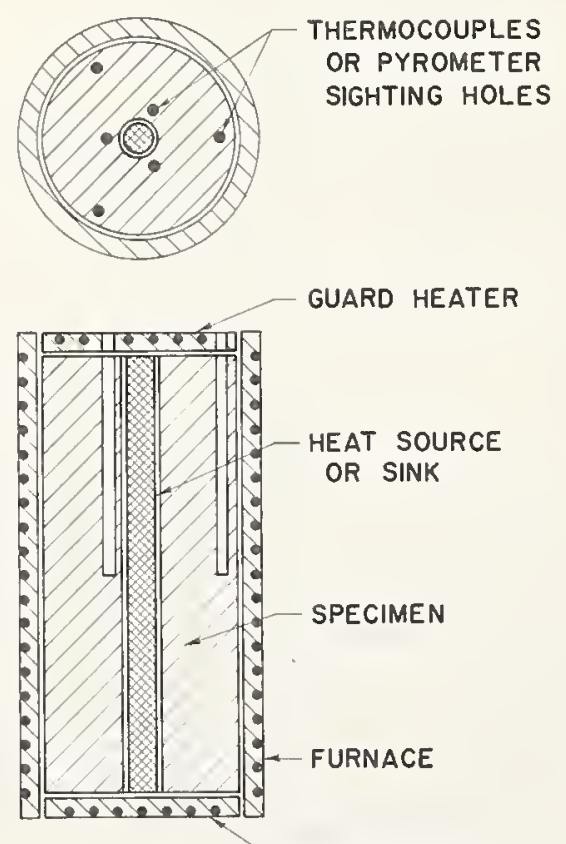

- GUARD HEATER

FigURE 57. A typical configuration for a thermal conductivity apparatus which utilizes radial heat flow in a right circular cylinder. or pyrometer sighting hole should run for some distance along an isotherm. It is usually preferable to have at least three temperature measuring stations at each radius so that the effects of possible eccentric isotherms can be averaged out. Consideration should be given to errors arising from distortion of the heat flow pattern by the finite holes used for temperature sensing. It is good practice to conduct measurements at two different power levels to reduce the effects of certain systematic errors in thermocouples or due to nonradial heat flow. If guard heaters are not used, the specimen should be well-insulated at the ends and should be sufficiently long that the heat flow is radial near the midplane of the specimen. All of these points are discussed by McElroy and Moore [467].

\section{Reference Standards}

Standards and standard reference materials are the basis of a consistent and accurate measuring system. The need for standard reference materials in thermal conductivity measurements is twofold. In the first place, such materials are required for comparative measurements in which the thermal conductivity of the material under test is determined in terms of that of the standard reference material. Secondly, such materials are required in evaluating the accuracy of apparatus designed for direct, as opposed to comparative, thermal conductivity measurements. The degree to which the measured value of the thermal conductivity of the standard reference material agrees with the accepted value is a check on the accuracy of the measurement process.

The basic requirements for any standard reference material are that it be stable, reproducible, and appropriate for the measurements at hand, and that the property in question be uniform throughout the material. The ideal thermal conductivity reference standard should meet the following criteria (also, see [468]) :

1. The thermal conductivity of the reference material should be similar to that of the materials to be measured. This is true whether the reference standard is being used to calibrate a comparative type of apparatus or to check an absolute type of apparatus.

2. The reference material should be homogeneous and isotropic. Preferably it should be reproducible. Four categories of uniformity, in decreasing order of desirability, are as follows:

\section{(a) Reproducible materials}

The most obvious of these would be materials of quite high purity-usually either chemical elements or compounds. A disadvantage here is that the thermal conductivities of such materials are usually markedly affected by the first traces of impurity, at least at low and moderate temperatures. Another, perhaps less obvious, type of reproducible 
material is one in which the manufacturing process is sufficiently refined that one may procure a sample of $\mathrm{X}$ from manufacturer $\mathrm{Y}$ with confidence that the thermal conductivity of this sample is substantially identical to that of any other sample of $\mathrm{X}$ procured, at least from Y, at another time.

(b) Stocked materials of proven uniformity: batch samples.

These are materials known, or found, to be quite uniform in thermal conductivity within a batch or lot, so that measurements made on a relatively few samples of it are sufficient to define the conductivity of the entire batch.

(c) Materials whose thermal conductivity correlates well with some other more easily measured physical property.

An example of this type of material would be certain metal alloys, for which thermal conductivity values may be computed from electrical resistivity data using an empirical correlating equation. A disadvantage of this procedure is the large amount of work that may be necessary in order to adequately define the correlating equation.

(d) Materials not sufficiently uniform to forego need for measuring each reference specimen.

This procedure, although often necessary, is expensive and places quite a work load on the calibrating laboratory, which must maintain an adequate facility indefinitely. In addition, lead-time to obtain a specimen may be onerous.

3. The thermal conductivity of the material should not be affected by age or by environmental or temperature experience; i.e., it must be stable under the conditions of use. Phase transformations, even if completely reversible, are not desirable.

4. The material should be usable over as broad a temperature range as possible, preferably in air, inert gas, or vacuum.

5. The material should be substantially opaque to thermal radiation over the entire temperature range of interest.

6. The material should not react chemically with materials commonly used in constructing thermal conductivity apparatus, especially not with thermocouples.

7. The material should have suitable mechanical properties to permit reasonably economic specimen fabrication while still possessing sufficient strength and hardness to permit normal handling during installation and removal from an apparatus.

8. The cost should not be excessive. "Excessive," however, appears to be defined rather differently by various individuals. Usually the cost of the material is much less than the cost of the measurement.

9. All other things being equal, an electrical insulator offers a slight advantage over an electrical conductor in that thermocouple leads do not need to be insulated from the specimen.

\subsection{Current Status}

With regard to the four categories of uniformity listed above under item 2, a ferw comments are in order. There are a few hopefully reproducible materials, for which thermal conductivity is reasonably well known over a limited temperature range, that can be used as reference materials. We will discuss these shortly. As regards stocked materials, I know of no source where a stock of material of known thermal conductivity is maintained and samples are sold. Efforts to make such materials available are currently underway at the National Bureau of Standards.

Powell [469] has summarized the correlations which exist between thermal and electrical conductivity for a number of alloy systems. For a number of copper alloys having thermal conductivities in the range 50 to $350 \mathrm{Wm}^{-1} \mathrm{~K}^{-1}$, it appears that thermal conductivity can be predicted to within a few percent from measured electrical conductivity values, at least in the temperature range from room temperature to about $500 \mathrm{~K}$. Other systems that exhibit relatively good correlation include aluminum alloys, magnesium alloys, certain steels, and certain nickel-chromium alloys. In as-yet-unpublished work, we have developed a somewhat more complicated correlation expression for ferritic iron-nickel alloys that seems to be good within three percent from 200 to $800 \mathrm{~K}$.

One approach to obtaining thermal conductivity reference materials is to have a particular sample calibrated by an experienced, reliable laboratory. We have carried out a number of such ad hoc measurements in the past and can continue to do so whenever the workload permits.

Returning to the category of reproducible materials, you will find listed below a number of materials that may be used as reference standards on an interim basis. The materials are listed in two categories, metals and nonmetals, in decreasing order of room temperature thermal conductivity within each category. Unless otherwise stated, materials of at least 99.99 percent purity are implied.

\section{a. Metals}

Copper $\left(\lambda \approx 400 \mathrm{Wm}^{-1} \mathrm{~K}^{-1}\right.$ at $\left.300 \mathrm{~K}\right)$

Powell, Ho, and Liley [238] give recommended values for the thermal conductivity of pure copper. They estimate a probable uncertainty in these values of " \pm 3 percent near room tem- 
perature increasing to \pm 5 percent at low and high temperatures."

Their recommended values are in excellent agreement with the very recent experimental data of Laubitz [71] and Moore, McElroy, and Graves [72].

\section{Aluminum $\left(\lambda \approx 240 \mathrm{Wm}^{-1} \mathrm{~K}^{-1}\right.$ at $\left.300 \mathrm{~K}\right)$}

Powell, Ho, and Liley [238] give recommended values for the thermal conductivity of pure aluminum which "are thought to be accurate to within \pm 4 percent below room temperature and \pm 2 percent to \pm 3 percent above." I feel that this uncertainty estimate may be too optimistic.

\section{Tungsten $\left(\lambda \approx 180 \mathrm{Wm}^{-1} \mathrm{~K}^{-1}\right.$ at $\left.300 \mathrm{~K}\right)$}

Powell, Ho, and Liley [238] state that while their recommended values for pure tungsten "are thought to be within \pm 3 to \pm 5 percent of the true values, further confirmatory work on tungsten still seems to be required." As will be briefly discussed at the end of this section, further work on tungsten is currently in progress. Powell and $\mathrm{Ho}$ [470] show some additional data that were not given in [238].

\section{Iron $\left(\lambda \approx 70\right.$ to $80 \mathrm{Wm}^{-1} \mathrm{~K}^{-1}$ at $\left.300 \mathrm{~K}\right)$}

Commercially pure iron, usually produced by the American Rolling Mill Company and known as Armco iron, has been used for many years as a thermal conductivity reference material. The recommended values for Armco iron by Powell, Ho, and Liley [238] "are thought to have an accuracy of \pm 3 percent below room temperature, \pm 2 percent to about $1000 \mathrm{~K}$, the uncertainty probably increasing to about \pm 8 percent at $1600 \mathrm{~K}$ ". C. F. Lucks, of the Battelle Memorial Institute Columbus Laboratories, has been investigating the effects of heat treatment on the conductivity of Armco iron and should be publishing his findings in the near future. In principle, pure iron would be more reproducible and probably less subject to heat treatment effects and hence would be a preferable standard. Powell, Ho, and Liley have also given recommended values for pure iron which they feel are similar in reliability to the values for Armco iron (see also Powell and Tye [476]).

\section{Platinum $\left(\lambda=70 \mathrm{Wm}^{-1} \mathrm{~K}^{-1}\right.$ at $\left.300 \mathrm{~K}\right)$}

The "very tentative" values given by Powell, Ho, and Liley [238] for pure platinum should not be used. Recent measurements by Laubitz and van der Meer [471], Martin Sidles and Danielson [472] and Flynn and O'Hagan [243] have led to a revision of the recommended values (see Powell and Ho [470, figure 16]). Until additional measurements on platinum, currently in progress at the National Bureau of Standards and elsewhere, are completed, I suggest using the averages of the values reported by Laubitz and van der Meer [471] and by Flynn and O'Hagan [243].
Lead $\left(\lambda \approx 35 \mathrm{Wm}^{-1} \mathrm{~K}^{-1}\right.$ at $\left.300 \mathrm{~K}\right)$

The use of lead as a thermal conductivity reference standard at the National Bureau of Standards $[473,474]$ has led to its use in a number of other investigations. Ho, Powell, and Liley [239] give recommended values which "are thought to be accurate to within \pm 3 percent of the true values at moderate temperatures \pm 5 percent at high temperatures and \pm 10 percent at low temperatures .. .". The recent work of Lucks [475] indicates that the thermal conductivity of lead may be very sensitive to small amounts of impurity.

Stainless Steel $\left(\lambda \approx 15 \mathrm{Wm}^{-1} \mathrm{~K}^{-1}\right.$ at $\left.300 \mathrm{~K}\right)$

Powell and Tye [476] have recently reported thermal conductivity values for nine samples of stainless steel of somewhat similar composition (nominally $18 \% \mathrm{Cr}, 8 \% \mathrm{Ni}$ ). The differences found among their samples indicate that the thermal conductivity of an arbitrary sample of $18 / 8$ stainless steel would probably be within \pm 10 percent of their mean value at a given temperature.

Inconel $702\left(\lambda \approx 12 \mathrm{Wm}^{-1} \mathrm{~K}^{-1}\right.$ at $\left.300 \mathrm{~K}\right)$

Measurements of the thermal conductivity of specimens of age-hardened Inconel $702^{18}$ from a single lot of material reported by Laubitz and Cotnam [477], Watson and Flynn [478], and McElroy and Moore [467], as well as other unpublished measurements at the National Bureau of Standards, all agree within \pm 2 percent of a mean curve through these data. As far as I know, there have been no measurements on samples of this alloy from another lot of material. As discussed by Laubitz and Cotnam [477] and Powell and Tye [476], Inconel 702 is subject to changes in conductivity resulting from heat treatment so care is indicated in using this material.

\section{b. Nonmetals}

Beryllium Oxide $\left(\lambda \approx 270 \mathrm{Wm}^{-1} \mathrm{~K}^{-1}\right.$ at $\left.300 \mathrm{~K}\right)$

Powell, Ho, and Liley [238] give recommended thermal conductivity values for 99.5 percent pure, 98 percent dense polycrystalline beryllium oxide. The uncertainty of the recommended values "is though to be within \pm 8 percent at temperatures from 500 to $1000 \mathrm{~K}$ and increases to \pm 15 percent below $300 \mathrm{~K}$ and above $1800 \mathrm{~K}$ ".

\section{Silicon $\left(\lambda \approx 150 \mathrm{Wm}^{-1} \mathrm{~K}^{-1}\right.$ at $\left.300 \mathrm{~K}\right)$}

Silicon is potentially a good reference material due to its availability in large sizes of high purity. Ho and Powell [266] show a plot of a recommended curve for pure silicon. They do not tabulate their recommended values but do state that the proposed curve was drawn intermediately between the data of Glassbrenner and Slack [479] and of Fulkerson, et al. [480].

\footnotetext{
${ }^{18}$ A proprietory alioy of the International Nickel Company; the material used in these measurements was 79.3 percent Ni, 17 percent $\mathrm{Cr}, 2.5$ percent $\mathrm{Al}$, plus other elements in smaller quantities.
} 
Magnesium Oxide $\left(\lambda=50 \mathrm{Wm}^{-1} \mathrm{~K}^{-1}\right.$ at $\left.300 \mathrm{~K}\right)$

Powell, Ho, and Liley [238] give recommended values for 99.5 percent pure, 98 percent dense polycrystalline magnesium oxide. The uncertainty in these values "is though to be within \pm 8 percent at temperatures from 500 to $1000 \mathrm{~K}$ and increases to \pm 15 percent below $300 \mathrm{~K}$ and from 1650 to $2000 \mathrm{~K}$. The uncertainty increases to about \pm 20 percent above $2100 \mathrm{~K}$ ".

\section{Aluminum Oxide $\left(\lambda=40 \mathrm{Wm}^{-1} \mathrm{~K}^{-1}\right.$ at $\left.300 \mathrm{~K}\right)$}

The uncertainty in the recommended values of Powell, Ho, and Liley [238] for 99.5 percent pure, 98 percent dense polycrystalline aluminum oxide "should be within \pm 8 percent at temperatures from 500 to $1000 \mathrm{~K}$ and increases to about \pm 15 percent below $250 \mathrm{~K}$ and above $1800 \mathrm{~K}$."

Crystalline Quartz $\left(\lambda \approx 10 \mathrm{Wm}^{-1} \mathrm{~K}^{-1}\right.$ parallel to $c$ axis and $\lambda \approx 6 \mathrm{Wm}^{-1} \mathrm{~K}^{-1}$ perpendicular to $c$-axis at $300 \mathrm{~K}$ )

Powell, Ho, and Liley [238] give recommended values for the thermal conductivity of quartz single crystals for heat flow both parallel and perpendicular to the $c$-axis. The uncertainty for both sets of values "is thought to be within \pm 5 percent at temperatures from 300 to $500 \mathrm{~K}$ and increases at lower and higher temperatures up to \pm 10 percent at 40 and $800 \mathrm{~K}$ ". Because of infrared transmission of energy, I would not recommend using clear quartz as a reference material above about $600 \mathrm{~K}$.

Pyroceram Code $9606\left(\lambda=4 \mathrm{Wm}^{-1} \mathrm{~K}^{-1}\right.$ at $\left.300 \mathrm{~K}\right)$ Powell, Ho, and Liley [238] state that their recommended values for Pyroceram Code $9606^{19}$ "should be accurate to within \pm 5 percent at temperatures from 200 to $1000 \mathrm{~K}$. The uncertainty increases to \pm 10 percent at $100 \mathrm{~K}$ and $1400 \mathrm{~K}$ ". Of the six sets of data used in deriving these recommended values, two sets were produced by direct measurement of thermal conductivity in two different apparatuses in our laboratory; the other four sets were derived from thermal diffusivity data obtained in three other laboratories. Two of these sets of diffusivity data were obtained in another laboratory at the $\mathrm{Na}$ tional Bureau of Standards on material from the same lot of material as was used in our conductivity measurements. Thus at least four of the six sets of data were obtained on the same lot of material. In more recent measurements, using a third, and different, apparatus, ${ }^{20}$ we have obtained thermal conductivity values on a specimen of Pyroceram Code 9606 from a different lot of material; these values, obtained over the temperature range 100 to $300 \mathrm{~K}$, are about 5 percent higher than our previous data which were used as a basis for the recommended values given in [238]. We are not certain whether the

\footnotetext{
"A product of Corning Glass Works; the material is a "microcrystalline glass."

20 The apparatus shown in figure 47.
}

differences observed are due to experimental difficulties or to sample differences but believe the differences do reflect a variation between samples. Further work is clearly indicated.

Fused Quartz ( $\lambda \approx 1.4 \mathrm{Wm}^{-1} \mathrm{~K}^{-1}$ at $\left.300 \mathrm{~K}\right)$

Powell, Ho, and Liley [238] give recommended thermal conductivity values for high-purity clear fused quartz; the uncertainty "should be within \pm 4 percent at temperatures from 200 to $500 \mathrm{~K}$, and increases to \pm 8 percent at $50 \mathrm{~K}$ and $900 \mathrm{~K}$ and up to \pm 15 percent below $10 \mathrm{~K}$ and at 1400 K'. Carwile and Hoge [481] present independently derived recommended values for the thermal conductivity of "vitreous silica" over the temperature range 80 to $660 \mathrm{~K}$. They state that "the tabulated values of $\lambda$ between 150 and $450 \mathrm{~K}$ are believed to be accurate to \pm 7 percent; near room temperature they may be somewhat better. Below $150 \mathrm{~K}$ the uncertainty increases and may reach \pm 12 percent. Likewise above $450 \mathrm{~K}$ the uncertainty increases and may be as large as \pm 15 percent at the upper limit of the table". These two sets of recommended values agree to within less than 1 percent from 200 to $600 \mathrm{~K}$. I would not recommend using clear fused quartz as a thermal conductivity reference standard at temperatures above $600 \mathrm{~K}$.

\section{Pyrex Glass Code $7740\left(\lambda \approx 1.1 \mathrm{Wm}^{-1} \mathrm{~K}^{-1}\right.$ at $300 \mathrm{~K})$}

The recommended thermal conductivity values given by Powell, Ho, and Liley [238] for Pyrex Glass Code $7740{ }^{21}$ are "thought to be accurate to within \pm 5 percent at moderate temperatures and within \pm 10 percent below $200 \mathrm{~K}$ and above $700 \mathrm{~K}$ ". Again, I would not recommend this as a standard above about $600 \mathrm{~K}$.

\subsection{Ongoing Activities}

In order to make better thermal conductivity reference materials available, the National Bureau of Standards has efforts underway both in our Washington and in our Boulder laboratories. In order that these efforts may be properly directed, you are urged to communicate your needs in this area to R. L. Powell at Boulder (cryogenic temperature range--liquid helium to ambient temperatures) or to me at Washington (liquid nitrogen to high temperatures).

The Air Force Materials Laboratory is sponsoring a program at Arthur D. Little, Inc. to establish high-temperature (above about $1000^{\circ} \mathrm{C}$ ) thermal conductivity and thermal diffusivity standards. Part of this work has already been described [468, 482]; most, if not all, of the work will be included in the Proceedings of the Eighth Conference on Thermal Conductivity held at Purdue University in October, 1968. The materials being investigated are polycrystalline sintered aluminum

21 A borosilicate glass produced by Corning Glass Works. 
oxide and thorium oxide, arc cast tungsten, and two commercial grades of graphite.

In a program under the auspices of AGARD (Advisory Group for Aerospace Research and Development of the North Atlantic Treaty Organization), samples of gold, platinum, tungsten, and aluminum oxide are being distributed to a numbel of laboratories for the purpose of measuring thermal conductivity and ultimately establishing these materials as reference standards.

Several laboratories, notably the groups under M. J. Laubitz at the National Research Council of Canada and under D. L. McElroy at Oak Ridge National Laboratory, have active programs to study the thermal conductivities of well-defined materials over broad temperature ranges. Measurements such as these will contribute greatly to establishing accurate thermal conductivity values on reproducible materials that are suitable as reference standards.

\section{Conclusions}

Many inaccurate thermal conductivity data have been reported on poorly characterized materials-such data are almost useless and, if used in critical applications, may even be harmful. Inaccurate data on well-characterized materials are not much, if any, more useful. Accurate data on poorly characterized materials may be very useful in applications where the identical material is used but are not of much use otherwise. Accurate thermal conductivity data on well-characterized samples, the ultimate desiderata, are characterized by their paucity.

Admittedly, thermal conductivity is a relatively difficult physical property to determine. Accurate thermal conductivity values are expensive to obtain-but not much, if any, more expensive than inaccurate values. And when one considers the possible costs in fuel, efficiency, or safety due to the use of inaccurate thermal conductivity values in designing a system, one is led to the conclusion that the overall cost of an inaccurate measurement is usually larger than the overall cost would have been if a little more effort had been expended to obtain an accurate value. If thermal conductivity is worth measuring, it is worth measuring well.

Similar arguments hold true regarding sample characterization. Adequate identification of a given sample may be expensive, but if everyone did properly characterize his samples, it would eventually be possible, in many cases, to correlate thermal conductivity with density, pore size, impurity content, etc. Such correlations could eliminate the need for many thermal conductivity measurements at a later date. A seemingly unnecessary investment in sample characterization might thus yield future dividends.

In selecting a suitable apparatus for use in measuring thermal conductivity, there are a variety of factors, such as those listed at the beginning of section 7 , which need be considered. I strongly urge that anyone contemplating the construction of a thermal conductivity apparatus study the book "Thermal Conductivity" [483] prior to committing himself to a given type of apparatus. The thirteen chapters in this book, written by some of the top workers in the field, contain a veritable wealth of useful information. A comprehensive error analysis (particularly helpful references include $[243,447,450,467])$ prior to construction of an apparatus can save many difficulties later.

Once an apparatus has been constructed, it is absolutely essential to undertake measurements on a material, such as one of those listed in the previous section, whose thermal conductivity is well known. Ideally the material selected should be as similar as possible, in thermal conductivity and other physical properties, to the material or type of material for which the apparatus was designed.

Prior to undertaking a measurement of the thermal conductivity of a particular material it is important to consider (and, if any doubts exist, check) whether any chemical reactions or material changes might occur during the measurement. Many specimens could contaminate thermocouples or react with apparatus components, especially at elevated temperatures. Before fabricating specimens for conductivity measurements, the sample should be checked for homogeneity to be sure representative specimens are tested.

The material should be characterized as completely as possible. This would include finding out the method and details of material production and any heat treatments or other special treatments given to the material. Minimal characterization should include a statement of the principal chemical composition, the general type of microstructure (single-crystal, crystalline orientation, polycrystalline, amorphous, etc.), the density, and, whenever possible, a detailed chemical analysis. Materials having noncubic structure should be checked for anisotropy. A detailed microstructural analysis should be made to determine grain size, porosity, presence of other phases, and other factors which conceivably could affect the thermal conductivity (see Picklesimer [484]).

If there is any possibility of an electronic contribution to the thermal conductivity, the electrical resistivity should be measured over the temperature range of interest. If there is any possibility of significant radiative heat transfer, it would be helpful to measure optical absorption and scattering coefficients, even if this can only be done at room temperature. Other thermal and mechanical properties (e.g., thermal expansion, thermoelectric power, specific heat, sound velocity) can be very helpful in understanding the results of thermal conductivity measurements, particularly if a phase change or other unusual behavior occurs.

In carrying out high temperature determinations, it is good practice to make measure- 
ments at 50 or $100 \mathrm{deg}$ intervals in increasing ordel of temperature with an adequate number of repeat measurements at lower temperatures to check for changes in the specimen or in some component of the apparatus. If there is any question of change in the specimen, post-test characterization is indicated.

During testing, especially with new apparatus, it is important to make systematic variations in the test conditions (temperature gradients, guard temperatures, type of gas in insulation surrounding specimen, etc.) to enable a more meaningful error analysis. All test conditions which conceivably could affect the results should be recorded.

A detailed error analysis [243, 467] is essential in order to arrive at meaningful estimates of the uncertainties in thermal conductivity values; the estimated uncertainties should be stated clearly and unambiguously [485-486].

\section{References}

[1] Carslaw, H. S., and Jaeger, J. C., Conduction of Heat in Solids, 2nd ed. (Clarendon Press, Oxford, 1959).

[2] Schneider, P. J., Conduction Heat Transfer (Addison-Wesley, Reading, Mass., 1955).

[3] Arpaci, V. S., Conduction Heat Transfer (AddisonWesley, Reading, Mass., 1966).

[4] Seitz, F., Modern Theory of Solids (McGraw-Hill Book Co., Inc., New York, 1940).

[5] Peierls, R., Quantum Theory of Solids (Clarendon Press, Oxford, 1955)

[6] Wannier, G. H., Elements of Solid State Theory (Cambridge University Press, Cambridge, 1959).

[7] Ziman, J. M., Electrons and Phonons (Clarendon Press, Oxford, 1960).

[8] Rosenberg, H. M., Low Temperature Solid State Physics (Clarendon Press, Oxf ord, 1963).

[9] Klemens, P. G., Thermal conductivity of solids at low temperatures, Handbuch der Physik 14, 198 (1956).

[10] Klemens, P. G., Thermal conductivity and lattice vibrational modes, Solid State Phys. 7, 1 (1958).

[11] Klemens, P. G., Theory of the thermal conductivity of solids, ch. 1 in Thermal Conductivity, edited by R. P. Tye (Academic Press, London, in press).

[12] Appel, J., Thermal conductivity of semiconductors, Progress in Semiconductors 5, 143 (1960).

[13] Carruthers, P., Theory of thermal conductivity of solids at low temperatures, Rev. Mod. Phys. 33, 92 (1961).

[14] Drabble, J. R., and Goldsmid, H. J., Thermal Conduction in Semiconductors (Pergamon Press, London, 1961).

[15] Krumhansl, J. A., Thermal conductivity of solids, in Theory and Fundamental Research in Heat Transfer, edited by J. A. Clark (The Macmillan Co., New York, 1963).

[16] Brillouin, L., Wave Propagration in Periodic Structures (McGraw-Hill Book Co., New York, 1946; reprinted with corrections and additions by Dover Publications, New York, 1953)

[17] Louck, J. D., Exact normal modes of oscillation of a linear chain of identical particles, Amer. J. Phys. 30, 585 (1962).

[18] Born, M., Lattice dynamics and x-ray scattering, Proc. Phys. Soc. (London) 54, 362 (1942).

[19] Born, M., Theoretical investigation on the relation between crystal dynamics and x-ray scattering, Rept. Progr. Phys. 9, 294 (1942).

[20] Pines, D., Elementary Excitations in Solids (W. A. Benjamin, Inc., New York, 1963).
[21] Loudon, R., Excitations in crystals, Sci. Prog. (Oxf.) 54, 325 (1966).

[22] Clem, J. R., and Godwin, R. P., Dynamical properties of a one-dimensional "crystal" with free ends, Amer. J. Phys. 34, 460 (1966).

[23] Blackman, M., The specific heat of solids, Handbuch der Physik $y / 1,325$ (1955).

[24] de Launay, J., The theory of specific heats and lattice vibrations, Solid State Phys. 2, 219 (1956).

[25] Keesom, P. H., and Pearlman, N., Low temperature heat capacity of solids, Handbuch der Physik 14, 282 (1956).

[26] Leibfried, G., and Ludwig, W., Theory of anharmonic effects in crystals, Solid State Phys. 12, 276 (1961)

[27[ Mitra, S. S., Vibration spectra of solids, Solid State Phys., 13, 2 (1962).

[28] Cochran, W., and Cowley, R. A., Phonons in perfect crystals, Handbuch der Physik XXV/2a, 59 (1967).

[29] Leibfried, G., and Schlömann, E., Heat conduction in electrically insulating crystals, Nachr. Akad. Wiss. Göttingen Math-physik K1. 2a, 71 (1954).

[30] Pieirls, R., Zur kinetischen Theorie der Wärmeleitung in Kristallen, Ann. Phys. 3, 1055 (1929).

[31] de Haas, W. J., and Biermasz, Th., Die Wärmeleitfähigkeit von Kristallen bei tiefen Temperaturen, Physica 5, 320 (1938).

[32] Casimir, H. B. G., Note on the conduction of heat in crystals, Physica 5, 495 (1938).

[33] Knudsen, M., Die Gesetze der Molekularströmung und der Reibungsströmung der Gase durch Rohren, Ann. Phys. 28, 75 (1909).

[34] Smoluchowski, M., Zur kinetischen Theorie der Transpiration und Diffüsion verdünnter Gase, Ann. Phys. 33, 1559 (1910).

[35] Berman, R., Simon, F. E., and Ziman, J. M., The thermal conductivity of diamond at low temperatures, Proc. Roy. Soc. A220, 171 (1953).

[36] Berman, R., Foster, E. L., and Ziman, J. M., Thermal conduction in artificial sapphire crystals at low temperatures I. Nearly perfect crystals, Proc. Roy. Soc. A231, 130 (1955).

[37] Herring, C., Theory of the thermoelectric power of semiconductors, Phys. Rev. 96, 1163 (1954).

[38] Geballe, T. H., and Hull, G. W., The size dependence of thermal conductivity and Seebeck effect in ptype germanium, Conf. de Phys. des basses Temps., p. 460, (Paris, 1955).

[39] Thacher, P. D., Effect of boundaries and isotopes on thermal conductivity of LiF, Phys. Rev. 156, 975 (1967).

[40] Gereth, R., and Hubner, K., Investigations of surface reflection of relevant phonons in silicon using the transmitted phonon drag effect, Solid State Electronics 10, 935 (1967).

[41] Kazakov, A. E., and Nagaev, E. L., Consideration of partial phonon reflection from boundaries in the calculation of the low temperature thermal conductivity of dielectrics, Soviet Phys.-Solid State 9, 1255 (1967).

[42] Guyer, R. A., and Krumhansl, J. A., Thermal conductivity, second sound, and phonon hydrodynamic phenomena in nonmetallic crystals, Phys. Rev. 148, 778 (1966).

[43] Hurst, W. S., and Frankl, D .R., Thermal conductivity of silicon at low temperatures, Bull. Am. Phys. Soc. (II) 13, 170 (1968).

[44] Callaway, J., Model for lattice thermal conductivity at low temperatures, Phys. Rev. 113, 1046 (1959).

[45] Callaway, J., Low-temperature lattice thermal conductivity, Phys. Rev. 122, 787 (1961).

[46] Carruthers, P., Thermal conductivity of solids. III. Modification of three-phonon processes by isotopic scattering, Phys. Rev. 126, 1448 (1962).

[47] Nettleton, R. E., Foundations of the Callaway theory of thermal conductivity, Phys. Rev. 132. 2032 (1963). 
[48] Berman, R., The thermal conductivity of dielectric solids at low temperatures, Adv. Phys. 2, 103 (1953)

[49] Klemens, P. G., The thermal conductivity of dielectric solids at low temperatures (Theoretical), Proc. Roy. Soc. (London) A208, 108 (1951).

[50] Ziman, J. M., The general variational principle of transport thcory, Can. J. Phys. 34, 1256 (1956).

[51] Krumhansl, J. A., Thermal conductivity of insulating crystals in the presence of normal processes, Proc. Phys. Soc. (London) 85, 921 (1965).

[52] Guyer, R. A., and Krumhansl, J. A., Solution of the linearized phonon Boltzmann equation, Phys. Rev. 148, 766 (1966).

[53] Holland, M. G., Analysis of lattice thermal conductivity, Phys. Rev. 132, 2461 (1963).

[54] Klemens, P. G., Thermal resistance due to point defects at high temperatures, Phys. Rev. 119, 507 $(1960)$.

[55] Greenberg, I. N., Thermal conductivity in solids, USAELRDL Technical Report 2361 (May, 1963), AD 422664.

[56] Klein, M. V., Phonon scattering by lattice defects II, Phys. Rev. 141, 716 (1966).

[57] Berman, R., Heat conduction in non-metallic crystals, Sci. Prog. (Oxford) 55, 357 (1967).

[58] Ranniger, J., Thermal conductivity in nonconducting crystals, Ann. Phys. (N.Y.) 45, 452 (1967)

[59] Tavernier, J., General problems of lattice thermal conductivity in solids (in French), J. Phys. 28 C1, Supp. 2, 40 (1967).

[60] Sham, L. J., and Ziman, J. M., The electron-phonon interaction, Solid State Phys. 15, 223 (1963).

[61] Spector, H. N., Interaction of acoustic waves and conduction electrons, Solid State Phys. 19, 291 (1966).

[62] Makinson, R. E. B., The thermal conductivity of metals, Proc. Cambridge Phil. Soc. 34, 474 (1938).

[63] Sondheimer, E.H.,The theory of the transport phenomena in metals, Proc. Roy. Soc. (London) A203, 75 (1950).

[64] Ziman, J. M., The electrical and thermal conductivities of monovalent metals, Proc. Roy. Soc. (London) A226, 436 (1954)

[65] Cezairliyan. A., Prediction of thermal conductivity of metallic elements and their dilute alloys at cryogenic temperatures, Purdue University, TPRC Rept. 14 (1962).

[66] Cezairliyan, A., and Touloukian, Y. S., Generalization and calculation of the thermal conductivity of metals by means of the law of corresponding states, High Temperature 3, 63 (1965).

[67] Cezairliyan, A., and Touloukian, Y. S., Correlation and prediction of thermal conductivity of metals through the application of the principle of corresponding states, in Advances in Thermophysical Properties at Extreme Temperatures and Pressures, p. 301 (ASME, 1965).

[68] Berman, R., and Macdonald, D. K. C., The thermal and electrical conductivity of copper at low temperatures, Proc. Roy. Soc. (London) A211, 122 (1952).

[69] White, G. K., The thermal and electrical conductivity of copper at low temperatures, Australian J. Phys. 6, 397 (1953).

[70] Powell, R. L., Roder, H. M., and Hall, W. J., Low temperature transport properties of copper and its dilute alloys: Pure copper, annealed and colddrawn, Phys. Rev. 115, 314 (1959).

[71] Laubitz, M. J., Transport properties of pure metals at high temperatures I. Copper, Can. J. Phys. 45, $3677^{(1967)}$.

[72] Moore, J. P., McElroy, D. L., and Graves, R. S., Thermal conductivity and electrical resistivity of high purity copper from 78 to $400^{\circ} \mathrm{K}$, Can. J. Phys. 45, 3849 (1967).

[73] Mendelssohn, K., and Rosenberg, H. M., The thermal conductivity of metals at low temperatures, Solid State Phys. 12, 223 (1961).
[74] Mott, N. F., and Jones, H., The Theory of the Propertics of Metals and Alloys (Clarendon Press, 1936; reprinted by Dover Publications, New York, 1958).

[75] Wilson, A. H., The Theory of Metals, 2nd. ed. (Cambridge Univ. Press, Cambridge, England, 1965).

[76] Olsen, J. L., Electron Transport in Metals (Interscience (Wiley), 1962).

[77] Schleef, D. L., Review of modern theory of the thermal conductivity of metals, in Thermodynamic and Transport Properties of Gases, Liquids and Solids (ASME, 1959).

[78] Austin, J. B., The Flow of Heat in Metals (Amer. Soc. Metals, 1942)

[79] Greenberg, I. N., Heat conduction in solids, Technical Report ECOM-2799 (1967), AD651229.

[80] Steigmeier, E. F., Thermal conductivity of semiconducting materials, ch. 10 in Thermal Conductivity, edited by R. P. Tye (Academic Press, London, in press).

[81] Madelung, O., Halbleiter, Handbuch der Physik 20, 1 (1957).

[82] Planck, M., The Theory of Heat Radiation (Dover, 1959).

[83] Harrison, T. R., Radiation Pyrometry and Its Underlying Principles of Radiant Heat Transfer (John Wiley \& Sons, Inc., New York, 1960).

[84] Sparrow, E. M., and Cess, R. D., Radiation Heat Transfer (Brooks/Cole, 1966).

[85] Bellman, R., Kagiwada, H., Kalaba, R., and Ueno, S., Inverse problems in radiative transfer: layered media, Icarus 4, 119 (1965).

[86] Cess, R. D., The interaction of thermal radiation with conduction and convection heat transfer, in Advances in Heat Transfer, Vol. 1, p. 1 (Academic Press, Inc., New York, 1964).

[87] Chen, J. C., Simultaneous radiative \& convective heat transfer in an absorbing emitting, \& scattering medium in slug flow between parallel plates, A. I. Ch. E. Journal 10, 253 (1964).

[88] Chu, C. M., and Churchill, S. W., Numerical solution of problems in multiple scattering of electromagnetic radiation, J. Phys. Chem. 59, 855 (1955).

[89] Costello, F. A., and Schrenk, G. L., Numerical solution to the heat-transfer equations with combined conduction and radiation, J. Comput. Phys. 1, 541 (1967).

[90] Edwards, R. H., and Bobco, R. P., Radiant heat transfer from isothermal dispersions with isotropic scattering, J. Heat Transfer 89C, 300 (1967).

[91] Einstein, T. H., Radiant heat transfer with flow and conduction, NASA TR R-154 (1963).

[92] Engelmann, F., and Schmidt, H. E., Heat transport by radiation in solids, Nuclear Sci. Engng. 24, 317 (1966).

[93] Goryainov, L. A., Methods of separation of composite heat transfer, Trudy. Mosk. In-ta Inzhen. Zh.-D. Transporta (189), 59 (1965).

[94] Hottel, H. C., Sarofim, A. F., Evans, L. B., and Vasalos I. A., Radiative transfer in anisotropically scattering media: allowance for Fresnel reflection at the boundaries, J. Heat Transfer 90C, 56 (1968).

[95] Hsia, H. M., and Love, T. J., Radiative heat transfer between parallel plates separated by a nonisothermal medium with anisotropic scattering, J. Heat Transfer 89C, 197 (1967).

[96] Landram, C. S., and Greif, R., Semi-isotropic model for radiation heat transfer, AIAA J. 5, 1971 (1967).

[97] Leung, A. T., Simultaneous radiation, conduction, and convection in a spectrally selective, absorbing, emitting, and scattering porous bed, M. S. Thesis in Engineering, University of California, Los Angeles, California, June 1965.

[98] Leung, A. T., and Edwards, D. K., Simultaneous radiation, conduction, and convection in a spectrally selective, emitting and scattering porous bed, J. Heat Transfer 88, 231 (1966). 
[99] Lick, W., Energy transfer by radiation and conduction, Proc. 1963 Heat Transfer and Fluid Mechanics Institute, p. 14 (Stanford Univ. Press, Palo Alto, California, 1963).

[100] Lick, W., Transient energy transfer by conduction and radiation, Intern. J. Heat Mass Transfer $\mathbf{8}$, 119 (1965).

[101] Love, T. J., An investigation of radiation heat transfer in absorbing, emitting, and scattering media, Ph.D. thesis (Purdue University, 1963) ; also ARL63-3, Aeronautical Research Laboratories Report, Office of Aerospace Research, U.S.A.F.

[102] Love, T. J., and Grosh, R. J., Radiative heat transfer in absorbing, emitting, and scattering media, J. Heat Transfer C8\%, 161 (1965).

[103] Mori, Y., and Kurosaki, Y., Heat transport by radiation and other transport mechanisms (1st Rep., Couette flow with simultaneous radiation and conduction), Bull. Jap. SME 9, 560 (1966).

[104] Nixon, J. A., and Monroe, S. G., Heat transfer through diathermanous materials, J. Spacecraft Rockets 2, 907 (1965).

[105] Oppenheimer, A. K., and Bevans, J. T., Geometric factors for radiative heat transfer through an absorbing medium in Cartesian coordinates, J. Heat Transfer C82, 360 (1960).

[106] Sidorov, Yu. P., Heat transfer by heat conduction and radiation in an absorbing medium, Trudy Mosk. In-ta Inzhen. Zh.D. Transporta (189), 109 (1965).

[107] Surinov, Yu. A., The integral equations of the theory of radiation transfer in an anisotropically scattering medium (for the generalized formulation of the three-dimensional problem), High Temperature 3, 385 (1965).

[108] Surinov, Yu. A., Integral equation of radiation transport theory for an absorbing and anisotropically scattering medium, High Temperature 5, 293 (1967).

[109] Tien, C. L., and Abu-Romia, M. M., Perturbation solutions in the differential analysis of radiation interactions with conduction and convection AIAA J. 4, 732(1966).

[110] Viskanta, R., Heat transfer in thermal radiation absorbing and scattering media, Ph.D. thesis (Purdue University, 1960); also ANL-6170.

[111] Viskanta, R., Interaction of heat transfer by conduction, convection, and radiation in a radiating fluid, J. Heat Transfer C85, 318 (1963).

[112] Viskanta, R., Heat transfer by conduction and radiation in absorbing and scattering materials, J. Heat Transfer C8\%, 143 (1965).

[113] Viskanta, R., and Grosh, R. J., Heat transfer in a thermal radiation absorbing and scattering medium, International Heat Transfer Conference, Boulder, Colorado, 1961, p. 820.

[114] Viskanta, R., and Grosh, R. J., Effect of surface emissivity on heat transfer by simultaneous conduction and radiation, Intern. J. Heat Mass Transfer 5, 729 (1962).

[115] Wang, L. S., Differential methods for combined radiation and conduction, $\mathrm{Ph}$. D. thesis (University of California, Berkeley, 1965).

[116] Weiner, M. M., and Edwards, D. K., Simultaneous conduction, convection, and radiation in a porous bed, Proceedings of the 1963 Heat Transfer and Fluid Mechanics Institute, p. 236 (Stanford Univ. Press, Palo Alto, California, 1963.

[117] Wang, Zhu-xi, Theory of heat conduction in the presence of radiation, Scientia Sinica 11, 185 (1962).

[118] American Institute of Physics Handbook, 2nd ed., edited by D. E. Gray (McGraw-Hill Book Co., Inc., New York, 1963).

[119] Gardon, R., A review of radiant heat transfer in

[120] Glaze, F. W. Transmittance of infrared energy by glasses, Bull. Amer. Ceram. Soc. 34, 291 (1955).
[121] Cleek, G. W., Villa, J. J., and Hahner, C. H., Refractive indices and transmittances of several optical glasses in the infrared, J. Opt. Soc. Am. 49, 1090 (1959).

[122] Folweiler, R. C., Thermal radiation characteristics of transparent, semi-transparent, and translucent materials under non-isothermal conditions, ASDTDR-62-719 (April 1964).

[123] Folweiler, R. C., and Mallio, W. J., Thermal radiation characteristics of transparent, semitransparent and translucent materials under non-isothermal conditions, ASD-TDR-62-719, Part II (June 1964).

[124] Hobbs, H. A., and Folweiler, R. C., Thermal radiation characteristics of transparent, semitransparent and translucent materials under non-isothermal conditions, ASD-TDR-62-719, Part III (March 1966).

[125] Chapin, E. J., and Howe, D. G., An exploratory study of radiant energy transport to $\mathrm{BeO}$, Report No. NRL-6195 (March 1965).

[126] All papers in J. Am. Ceram. Soc. 44, No. 7 (July 1961).

[127] Anonymous, The application of high temperature radiative thermal conductivity of minerals and rocks to a model of lunar volcanism, NASA CR-916 (November 1967).

[128] Ambartsumyan, V. A., Theoretical Astrophysics (Pergamon, 1958).

[129] Barnes, R. B., and Bonner, L. G., The Christiansen filter effect in the infrared, Phys. Rev. 49, 732 (1936)

[130] Bosworth, R. C. L., Heat Transfer Phenomena (John Wiley and Sons, Inc., New York, 1952).

[131] Busbridge, I. W., The Mathematics of Radiative Transfer (Cambridge Univ. Press, London, England, 1960).

[132] Busch, G., Fröhlich, C., Hullinger, F, and Steigmeier, E., Struktur, elektrische und thermoelektrische Eigenschaften von $\mathrm{SnSe}_{2}$, Helvetica Physica Acta 34, 359 (1961).

[133] Chandrasekhar, S., On the radiation equilibrium of a stellar atmosphere, XXIV, Astrophys. J. 108, 92 (1948).

[134] Chandrasekhar, S., Radiative Transfer (Oxford University Press, 1950; reprinted by Dover Publications, New York, 1960).

[135] Chandrasekhar, S., Elbert, D., and Franklin, A., The $\mathrm{X}$ and Y-functions for isotropic scattering, Parts I and II, Astrophys. J. 115, 244 (1952).

[136] Chen, J. C., and Churchill, S. W., Radiant heat transfer in packed beds, Amer. Inst. Chem. Eng. J. 9, 35 (1963).

[137] Chromey, F. C., Evaluation of Mie equations for colored spheres, J. Opt. Soc. Am. 50, 730 (1960).

[138] Clark, S. P., Jr., Ábsorption spectra of some silicates in the visible and near infrared, Amer. Mineralogist 42, 732 (1957).

[139] Clark, S. P., Jr., Radiative transfer in the earth's mantle, Trans. Amer. Geophys. Union 38, 931 (1957).

140] Cline, D., and Kropschot, R. H., The thermal properties of powder insulators in the temperature range $300^{\circ}-4^{\circ} \mathrm{K}$, in Radiative Transfer from Solid Materials, edited by H. Blau and H. Fischer, p. 61 (The Macmillan Co., New York, 1962).

[141] Collins, R. J., and Fan, H. Y., Infrared lattice absorption bands in germanium, silicon and diamond, Phys. Rev. 93, 674 (1954).

[142] Czerny, M., and Genzel, L., Energy flux and temperature distribution in the glass baths of melting tanks resulting from conduction and radiation, Glastech. Ber. 25, 387 (1952).

[143] Czerny, M., and Genzel, L., On the depth to which diffuse radiation penetrates glass, Glastech. Ber. 25, 134 (1952).

[144] Czerny, M., Genzel, L., and Heilmann, G., On the flow of radiation in the interior of glass baths, Glastech. Ber. 28, 185 (1955). 
[145] Damköhler, G., Der Chemi-Ingenieur, Eucken-Jacob Vol. III, Part 1, 445 (Akademische Verlagegesellschaft MBH, Leipzig, Germany, 1937).

[146] Davison, B., Neutron Transport Theory (Oxford Univ. Press, Oxford, England, 1957).

[147] Devyatkova, E. D., Ya, M. B., and Smirnov, I. A., Thermal conductivity of tellurium containing different concentrations of additional elements in the temperature range $80-480{ }^{\circ} \mathrm{K}$, Solid State Phys., USSR 1, 555 (1959).

[148] Eckhardt, G., Wärmeleitung und Wärmestrahlung in Gläsern zwischen $100^{\circ}$ and $1200^{\circ} \mathrm{C}$, Glastech. Ber. 32, 373 (1959).

[149] Everest, A., Glaser, P. E., and Wechsler, A. E., Thermal conductivity of non-metallic materials, Summary Report, Contract NAS8-1567, Arthur D. Little, Inc., Cambridge, Mass. (April 1962).

[150] Ewing, C. T., Spann, J. R., and Miller, R. R., Radiant transfer of heat in molten inorganic compounds at high temperatures, J. Chem. Eng. Data 7,246 (1962)

[151] Fritz, W., and Poltz, H., Absolutbestimmung der Wärmeleitfähigkeit von Flüssigkeiten I - Kritische Versuche an einer neuen Plattenapparatur, Int. J. Heat Mass 'Transfer 5, 307 (1962).

[152] Fritz-Schmidt, M., and Gchlhoff, G., Wärmeleitfähigkeitsmessungen an feuerfesten Baustoffen, Glastech. Berichte 8, 206 (1930).

[153] Gardon, R., Emissivity of transparent materials, J. Am. Ceram. Soc. 39, 278 (1956).

[154] Gardon, R., Calculation of temperature distribution in glass plates undergoing heat-treatment, J. Am. Ceram. Soc. 41, 200 (1958).

[155] Geffcken, W., The transmission of heat in glass at high temperatures, Part I, Glastech. Ber. 25, 392 (1952).

[156] Geffcken, W., Zur Forleitung der Wärme in glas bei hohen temperaturen, Glastech. Ber. 29, 42 (1956).

[157] Geffcken, W., Radiale Strahlungsleitung in Langen Zylindern, Glastech. Ber. 32K, VII 28 (1959).

[158] Genzel, L., Measurement of the infrared absorption of glass between $20^{\circ} \mathrm{C}$ and $1360^{\circ} \mathrm{C}$, Glastech $\mathrm{Br}$. 24, 55 (1951).

[159] Genzel, L., Calculation of the radiation conductivity of glasses, Glastech. Ber. 26, 69 (1953).

[160] Genzel, L., The role of heat radiation in the heat conduction process, Z. Physik 135, 177 (1953).

[161] Genzel, L., and Neuroth, N., Alternating illumination method for the measurement of infrared absorption at high temperatures, Z. Phys. 134, 127 (1953)

[162] Godbee, H. W., Ph. D. thesis, Georgia Institute of Technology (July, 1963).

[163] Godbee, H. W., and Ziegler, W. 'T., Thermal conductivities of $\mathrm{MgO}, \mathrm{Al}_{2} \mathrm{O}_{3}$, and $\mathrm{ZrO}_{2}$ powders to $850^{\circ} \mathrm{C}$. I. Experimental, J. Appl. Phys. 37, 40 (1966).

[164] Godbee, H. W., and Ziegler, W. T., Thermal conductivities of $\mathrm{MgO}, \mathrm{Al}_{2} \mathrm{O}_{3}$, and $\mathrm{ZrO}_{2}$ to $850^{\circ} \mathrm{C}$. II. Theoretical, J. Appl. Phys. 3\%, 56 (1966).

[165] Grove, F. J., and Charnock, H., Thermal conductivity of molten glass, Glastech. Ber. 32K, VII 24 (1959).

[166] Grove, F. J., and Jellyman, P. E., The infrared transmission in glass in the range from room temperature to $1400^{\circ}$, J. Soc. Glass Technol. 39, 3 (1955).

[167] Gryvnak, D. A., and Burch, D. E., Optical and infrared properties of $\mathrm{Al}_{2} \mathrm{O}_{3}$ at elevated temperatures, J. Opt. Soc. Am. 55, 625 (1965).

[168] Gumprecht, R. O., and Sliepcevich, C. M., Scattering of light by large spherical particles, J. Phys. Chem. 57, 90 (1953)

[169] Hager, N. E., Jr, and Steere, R. C., Radiant heat transfer in fibrous thermal insulations, J. Appl. Phys. 38, 4663 (1967).

[170] Hamaker, H. C., Radiation and heat conduction in light-scattering material I. Reflection and transmission, Philips Res. Rep. 2, 55 (1947).
[171] Hamakcr, H. C., Radiation and heat conduction in light-scattering material II. General equations including heat conduction, Philips Res. Rep. 2, 103 (1947).

[172] Hamaker, H. C., Radiation and heat conduction in light-scattering material III. Application of the theory, Philips Res. Rep. 2, 112 (1947).

[173] Hamaker, H. C., Radiation and heat conduction in light-scattering material IV. Various extensions and a generalized theory, Philips Res. Rep. 2, 420 (1947).

[174] Henry, R. L., The transmission of powder films in the infra-red, J. Opt. Soc. Am. 38, 775 (1948).

[175] Hill, F. B., and Wilhelm, R. H., Radiative and conductive heat transfer in a quiescent gas-solid bed of particles: Theory and experiment, A.I. Ch. E. Journal 5, 486 (1959).

[176] Huhmann, I., The transport of energy in glass melts, Part II, Huttentechnische Vereingung der Deutschen Glassindustrie (1958).

[177] Joffé, A., Properties of various semiconductors, J. Phys. Chem. Solids 8, 6 (1959).

[178] Jones, T. T., The effect of thickness and temperature on heat transfer through foamed polymers, Proc. Seventh Conference on Thermal Conductivity, edited by D. R. Flynn and B. A. Peavy, p. 737 (NBS Special Publication 302, 1968).

[179] Kellet, B. S., Steady flow of heat through hot glass, J. Opt. Soc. Am. 42, 339 (1952).

[180] Kellet, B. S., Transmission of radiation through glass in tank furnaces, J. Soc. Glass Technol. 36, 115 (1952); for correction see J. Soc. Glass Technol. 37, 268 (1953).

[181] Klein, J. D., Heat transfer, by radiation, in powders, Ph.D. Thesis, Mass. Inst. Tech. (June 1960).

[182] Kohler, M., Einfluss der Strahlung auf den Wärmetransport durch eine Flüssigkeitsschicht, Z. Angew. Phys. 18, 356 (1965).

[183] Kourganoff, V., Basic Methods in Transfer Problems (Oxford University Press, Oxford, England, 1952).

[184] Kräger, C., and Eligehausen, H., Úber das Warmeleituermögen des einschmelzenden Glasgemengls, Glastech. Ber. 32, 362 (1959).

[185] Krumhansl, J. A., Thermal conductivity in semiconductors, J. Phys. Chem. Solids 8, 343 (1959).

[186] Kubelka, P., and Munk, F., Ein Beitrag zer Optik der Farbanstriche, Z. tech. Phys. 12, 593 (1931).

[187] Larkin, B. K., and Churchill, S. W., Heat transfer by radiation through porous insulations, Amer. Inst. Chem. Eng. J. 5, 467 (1959).

[188] Laubitz, M. J., Thermal conductivity of powders, Can. J. Phys. 37, 798 (1959).

[189] Lee, D. W., and Kingery, W. D., Radiation energy transfer and thermal conductivity of ceramic oxides, J. Am. Ceram. Soc. 43, 594 (1960).

[190] Lucks, C. F., Matolich, J., and Van Velzor, J. A., Experimental measurement of thermal conductivities, specific heats and densities of metallic, transparent, and protective materials, Part III, U.S. Air Force Tech. Rept., No. 6145, Wright Air Development Center (1954).

[191] McConnell, R. K., Jr., McClaine, L. A., Lee, D. W., Aronson, J. R., and Allen, R. V., A model for planetary igneous differentiation, Rev. Geophys. 5, 109 (1967).

[192] McMahon, H. O., Thermal radiation from partially transparent reflecting bodies, J. Opt. Soc. Am. 40, 376 (1950).

[193] Milne, E. A., Radiative equilibrium in the outer layers of a star, Monthly Notices Roy. Astron. Soc. 81, 361 (1921).

[194] Monroe, J. E., Jr., Infrared transmission and radiation conductivity of alumina from room temperature to $1800 \mathrm{C}$, in Study of Heat Transfer of Ceramic Materials, Part 2, pp. 38-66 (Alfred Univ., Jan. 1955 to April 1957). 
[195] Neuroth, N., The effect of temperature on the spectral absorption of glasses in the infrared, Glastech. Ber. 25, 242 (1952).

[196] Poltz, H., Einfluss der Wärmestrahlung auf die Isolierwirkung poröser Dämmschichten, Allg. Warmetechnik 11, 64 (1962).

[197] Poltz, H., Die Wärmeleitfähigkeit von Flüssigkeiten II. Der Strahlungsanteil der effektiven Wärmeleitfähigkeit, Int. J. Heat Mass Transfer 8, 515 (1965).

[198] Poltz, H., Die Wärmeleitfähigkeit von Flüssigkeiten III. Abhängigkeit der Wärmeleitfähigkeit von der Schichtdicke bei organischen Flüssigkeiten, Int. J. Heat Mass Transfer 8, 609 (1965).

[199] Poltz, H., Some aspects concerning thermal conductivity data of liquids and proposals for new standard reference materials, Proc. Seventh Conf. on Thermal Conductivity, edited by D. R. Flynn and B. A. Peavy, p. 47 (NBS Special Publication 302, 1968).

[200] Poltz, H., and Jugel, R., The thermal conductivity of liquids IV-Temperature dependence of thermal conductivity, Int. J. Heat Mass Transfer 10, 1075 (1967).

[201] Reynolds, M. M., Brown, J. D., Fulk, M. M., Park, O. E., and Curtis, G. W., Vacuum-powder insulation, in Advances in Cryogenic Engineering, Vol. 1, p. 216 (Plenum, 1954).

[202] Richards, P. I., Multiple isotropic scattering, Phys. Rev. 100, 517 (1955).

[203] Rolinski, E. J., and Purcell, G. V., Investigation of radiation and conduction heat transfer in fibrous high temperature insulation, AFML-TR-67-251 (December, 1967).

[204] Rosseland, S., Astrophysik auf Atom-Theoretischer Grundlage (Julius Springer Verlag, Berlin, Germany, 1931).

[205] Rosseland, S., Theoretical Astrophysics (Oxford Univ. Press, Oxford, England, 1936).

[206] Russell, H. W., Principles of heat flow in porous insulators, J. Am. Ceram. Soc. 18, 1 (1935).

[207] Schotte, W., Thermal conductivity of packed beds, A. I. Ch. E. Journal 6, 63 (1960).

[208] Schuster, A., The influence of radiation on the transmission of heat, Phil. Mag. 5, 243 (1903).

[209] Schuster, A., Radiation through a foggy atmosphere, Astrophys. J. 21, 1 (1905).

[210] Slack, G. A., and Glassbrenner, C., Thermal conductivity of germanium from $3^{\circ} \mathrm{K}$ to $1020{ }^{\circ} \mathrm{K}$, Phys. Rev. 120, 782 (1960).

[211] Sobelev, V. V., A Treatise on Radiative Transfer (D. Van Nostrand Co., New York, 1963).

[212] Spitzer, W. G., and Fan, H.Y., Infrared absorption in indium antimonide, Phys. Rev. 99, 1893 (1955).

[213] Strong, H. M., Bundy, F. P., and Bovenkirk, H. P., Flat panel vacuum thermal insulation, J. Appl. Phys. 31, 39 (1960).

[214] Van De Hulst, H. C., Light Scattering by Small Particles (John Wiley \& Sons, Inc., New York, 1957).

[215] Van De Hulst, H. C., Scattering in a planetary atmosphere, Astrophys. J. 10\%, 220 (1948).

[216] Van der Held, E. F. M., The contribution of radiation to the conduction of heat, Appl. Sci. Res. A3, 237 (1952)

[217] Van der Held, E. F. M., The contribution of radiation to the conduction of heat II. Boundary conditions, Appl. Sci. Res. A4, 77 (1953)

[218] Van Zee, A. F., and Babcock, C. L., A method for the measurement of thermal diffusivity of molten glass, J. Am. Ceram. Soc. 34, 244 (1951).

[219] Verschoor, J. D., and Greebler, P., Heat transfer by gas conduction and radiation in fibrous insulations, Trans. ASME 74, 961 (1952).

[220] Walther, A., Door, J., and Eller, E., Mathematical calculation of the temperature distribution in a glass melt due to conduction and radiation, Glastech. Ber. 26, 133 (1953).
[221] Wechsler, A. E., and Glaser, P. E., Investigation of the thermal properties of high-temperature insulation materials, ASD-TDR-63-574 (July 1963), AD 420193.

[222] Wray, K. I., and Connolly, T. J., Thermal conductivity of clear fused silica at high temperatures, J. Appl. Phys. 30, 1702 (1959).

[223] Yagi, S., and Kunii, D., Studies on effective thermal conductivities in packed beds, A. I. Ch. E. Journal 3, 373 (1957).

[224] Hirschfelder, J. O., Curtiss, D. F., and Bird, R. B., Molecular Theory of Gases and Liquids, Second edition (John Wiley \& Sons, Inc., 1964).

[225] Lawson, A. W., On the high temperature heat conductivity of cylinders, J. Phys. Chem. Solids 3, 155 (1957)

[226] Jamieson, J. C., and Lawson, A. W., High-temperature heat conductivity of some metal oxides, J. Appl. Phys. 29, 1313 (1958).

[227] Whitmore, D. H., Excitation processes in ceramics and the anomalous increase in thermal conductivity at elevated temperatures, J. Appl. Phys. 31, 1109 (1960).

[228] Whitmore, D. H., Excitation processes in ce amics and the anomalous increase in thermal conductivity at elevated temperatures, WADD TR 60-866 (July, 1962), AD 285283.

[229] Jerome, D., Rice, T. M., and Kohn, W., Excitonic insulator, Phys. Rev. 158, 462 (1967).

[230] Zittartz, J., Transport properties of the "excitonic insulator" I. Electrical conductivity, Univ. of Calif. (San Diego) Technical Report No. 47 (Contract NONR-2216-(11)), AD 657439.

[231] Zittartz, J., Transport properties of the "excitonic insulator" II. Thermal conductivity, Univ. of Calif. (San Diego) Technical Report No. 48 (Contract NONR-2216-(11)), AD 657440.

[232] Berman, R., Heat transfer in solids, Bull. I.I.F./ I.I.R., 23 (Annexe 1965-2).

[233] Kingery, W. D., Klein, J. D., and McQuarrie, M. C., Development of ceramic insulating materials for high temperature use, Trans. Amer. Soc. Mech. Engrs. 80,705 (1958)

[234] Ruh, E., and McDowell, J. S., Thermal conductivity of refractory brick, J. Am. Ceram. Soc. 45, 189 (1962).

[235] Williams, W. S., High-temperature thermal conductivity of transition metal carbides and nitrides, J. Am. Ceram. Soc. 49, 156 (1966).

[236] Taylor, R. E., Thermal conductivity and expansion of beryllia at high temperatures, J. Am. Ceram. Soc. 45, 74 (1962).

[237] Kittel, C., Interpretation of the thermal conductivity of glasses, Phys. Rev. 75, 972 (1949).

[238] Powell, R. W., Ho, C. Y., and Liley, P. E., Thermal conductivity of selected materials, NSRDS-NBS 8 (November 1966).

[239] Ho, C. Y., Powell, R. W., and Liley, P. E., Thermal conductivity of selected materials, Part 2, NSRDSNBS 16 (February 1968).

[240] For a listing of these, contact the Thermophysical Properties Research Center, Purdue University, 2595 Yeager Road, West Lafayette, Indiana 47906.

[241] Kingery, W. D., The thermal conductivity of ceramic dielectrics, in Progress in Ceramic Science, Vol. 2, edited by J. E. Burke, p. 181, (Pergamon Press, Inc., New York, 1962).

[242] Young, R. C., Hartwig, F. J., and Norton, C. L., Effect of various atmospheres on thermal conductance of refractories, J. Am. Ceram. Soc. 47, 205 (1964)

[243] Flynn, D. R., and O'Hagan, M. E., Measurements of the thermal conductivity and electrical resistivity of platinum from 100 to $900^{\circ} \mathrm{C}$, J. Research NBS y1 C (Engr. and Instr.) No. 4, 255 (1967). 
244] Bridgman, P. W., The thermal conductivity and compressibility of several rocks under high presssures, Amer. J. Science, VII, 81 (1924).

[245] Bridgman, P. W., The Physics of High Pressure, (The Macmillan Co., New York, 1931).

[246] Hughes, D. S., and Sawin, F., Thermal conductivity of dielectric solids at high pressure, Phys. Rev. 161, 861 (1967).

[247] Drickamer, H. G., Lynch, R. W., Clendenen, R. L., and Perez-Albuerne, E. A., X-ray diffraction studies of the lattice parameters of solids under very high pressure, Solid State Physics 19, 135 (1966).

[248] Berman, R., Simon, F. E., and Wilks, J., Thermal conductivity of dielectric crystals: the "Umklapp" process, Nature 168, 277 (1951).

[249] Webb, F J., Wilkinson, K. R., and Wilks, J., The thermal conductivity of solid helium, Proc. Roy. Soc. (London) A214, 546 (1952).

[250] Webb, F J., and Wilks, J., The thermal conductivity of solid helium at high densities, Phil. Mag. 44, 664 (1953).

[251] Drickamer, H. G., The effect of high pressure on the electronic structure of solids, Solid State Physics 17, 1 (1965).

[252] Bridgman, P. W., The effect of pressure on the thermal conductivity of metals, Proc. Amer. Acad. Arts Sci. 5\%, 77 (1921).

[253] Starr, C., The pressure coefficient of thermal conductivity of metals, Phys. Rev. 54, 210 (1938).

[254] Lawson, A. W., The effect of hydrostatic pressure on the electrical resistivity of metals, in Metal Physics, Vol. 6, edited by B. Chalmers and R. King, p. 1 (Pergamon Press, Inc., New York, 1956).

[255] Swenson, C. A., Physics at high pressure, Solid State Physics 11, 41 (1960).

[256] Bundy, F. P., and Strong, H. M., Behavior of metals at high temperatures and pressures, Solid State Physics 13, 81 (1962).

[257] Paul, W., The electrical properties of metals and semiconductors, in High Pressure Physics and Chemistry, Vol. 1, edited by R. S. Bradley, p. 299 (Academic Press, Inc., New York, 1963).

[258] Landwehr, G., Electrical properties of metals and semiconductors under high pressure, in Physics of High Pressures and the Condensed Phase, edited by A. Van Itterbeek, p. 556 (NorthHolland Publishing Co., Amsterdam, Netherlands 1965).

[259] Bridgman, P. W., The effect of tension on the thermal and electrical conductivity of metals, Proc. Amer. Acad. Arts Sci. 59, 119 (1923).

[260] Lees, C. H., and Calthrop, J. E., The effect of torsion on the thermal and electrical conductivities of metals, Proc. Phys. Soc. (London) 35, 225 (1923).

[261] Calthrop, J. E., The effects of torsion on the thermal and electrical conductivities of metals, Proc. Phys. Soc. (London) 36, 168 (1924).

[262] Austin, J. B., Factors influencing the thermal conductivity of non-metallic materials, Symposium on Thermal Insulating Materials, p. 3 (Ämer. Soc. for Testing Materials, 1939).

[263] Patrassi, E., Die Wärmeleitfähigkeit von Urandioxid bei sehr hohen Temperaturgradienten, J. Nucl. Mater. 22, 311 (1967).

[264] Fulkerson, W., McElroy, D. L., Moore, J. P., Comments on a paper by E. Patrassi, Die Wärmeleitfähigkeit von Urandioxid bei sehr hohen Temperaturgradienten (The thermal conductivity of $\mathrm{UO}_{2}$ at very high temperature gradients), J. Nucl. Mater. 26, 223 (1968).

[265] Kaspar, J., and Zehms, E. H., Thermal diffusivity of carbons and graphites in the temperature range from 1800 to $3300^{\circ} \mathrm{K}$, SSD-T R-67-116 (June, 1967) AD 655786.

[266] Ho, C. Y., and Powell, R. W., The state of knowledge regarding the thermal conductivity of the non-metallic elements-those solid at normal temperature, Proc. Seventh Confercnce on Thermal Conductivity, edited by D. R. Flynn and B. A. Peavy, p. 33 (NBS Special Publication 302, 1968).

[267] Nordheim, L., Die Theorie der thermoelektrischen Effckte, Sci. et Ind., No. 131 (Paris, 1934).

[268] Linde, J. O., Elektrische Eigenschaften verdünnter Mischkristallegierungen, Ann. Phys. 10, 52 (1931); 15, 219 (1932).

(269] Berman, R., and Brock, J. C. F., The effect of isotopes on lattice heat conduction, I. Lithium fluoride, Proc. Roy. Soc. 289A, 46 (1965).

[270] Aldrich, R. E., Burke, W. J., and McCarthy, K. A., Resonant phonon scattering in colored sodium chloride crystals, Solid State Commun. 5, 899 (1967).

[271] Baumann, F. C., Harrison, J. P., Pohl, R. O., and Seward W. D., Thermal conductivity in mixed alkali halides: $\mathrm{KCl}$ :Li and $\mathrm{KBr}$ :Li, Phys. Rev. 159, 691 (1967).

[272] Baumann, F. C., and Pohl, R. O., Scattering of phonons by monatomic impurities in potassium halides, Phys. Rev. 163, 843 (1967).

[273] Kutasov, V. A., and Smirnov, I. A., Effect of surplus antistructural $\mathrm{Bi}$ on the thermal conductivity of crystal lattices of $\mathrm{Bi}_{2} \mathrm{Te}_{3}, \mathrm{Bi}_{2} \mathrm{Te}_{3}-\mathrm{Sb}_{2} \mathrm{Te}_{3}$ and $\mathrm{Bi}_{2} \mathrm{Te}_{3}-\mathrm{Bi}_{2} \mathrm{~S}_{3}$ with iodine admixtures, Phys. Status Solidi 18, 479 (1966) (in German).

[274] Mirlin, D. N., and Smirnov, I. A., Thermal conductivity of additively colored $\mathrm{KCl}$ crystals Soviet Phys. Solid State 9, 1452 (1967).

[275] Schwartz, J. W., and Walker, C. J., Thermal conductivity of some alkali halides containing divalent impurities, I. Phonon resonances, Phys. Rev. 155, 959 (1967).

[276] Schwartz, J. W., and Walker, C. J., Thermal conductivity of some alkali halides containing divalent impurities, II. Precipitate scattering, Phys. Rev. 155, 969 (1967).

[277] Smirnov, I. A., Thermal conductivity of $\mathrm{NaF}$ single crystals containing $\mathrm{K}, \mathrm{Li}$, and $\mathrm{Cl}$ impurities $\left(80-400^{\circ} \mathrm{K}\right)$, Soviet Phys. Solid State 9, 1454 (1967).

[278] Walton, D., Scattering of phonons by a square-well potential and the effect of colloids on the thermal conductivity, I. Experimental, Phys. Rev. 157, 720 (1967).

[279] Walton, D., and Lee, E. J., Scattering of phonons by a square-well potential and the effect of colloids on the thermal conductivity, II. Theoretical, Phys. Rev. 15\%, 724 (1967).

[280] Worlock, J. M., Thermal conductivity in sodium chloride crystals containing silver colloids, Phys. Rev. 14\%, 636 (1966).

[281] Ross, A. M., Dependence of the thermal conductivity of uranium dioxide on density, microstructure, stoichiometry, and thermal-neutron irradiation, Atomic Energy of Canada, Ltd. Report No. CRFD-817 (September 1960).

[282] May, J. E., and Stoute, R. L., Observations on the thermal conductivities of certain partially-reduced ceramic oxides, Atomic Energy of Canada, Ltd., Report No. AECL-2169 (January 1965).

[283] Godfrey, T. G., Fulkerson, W., Kollie, T. G., Moore, J. P., and McElroy, D. L., Thermal conductivity of uranium dioxide from $-57^{\circ}$ to $1100{ }^{\circ} \mathrm{C}$ by a radial heat flow technique, J. Am. Ceram. Soc. 48, 297 (1965).

[284] Thurber, W. R., and Mante, A. J. H., Thermal conductivity and thermoelectric power of rutile $\left(\mathrm{TiO}_{2}\right)$, Phys. Rev. 139, A1655 (1965).

[285] Hetzler, F. J., and Zebroski, E. L., Thermal conductivity of stoichiometric and hypostoichiometric uranium oxide at high temperatures, Trans. Amer. Nucl. Soc. 7, 392 (1964).

[286] Pohl, R. O., Thermal conductivity and phonon resonance scattering, Phys. Rev. Letters 8, 481 (1962). 
[287] Eucken, A., and Kuhn, E., Ergebnisse neuer Messungen der Wärmeteilfähigkeit fester krystallisierter Stoffe bei 0 und $-190 \mathrm{C}, \mathrm{Z}$. physik. Chemie 134, 193 (1928).

[288] Kingery, W. D., Thermal conductivity: XIV, conductivity of multicomponent systems, J. Am. Ceram. Soc. 42, 617 (1959).

[289] Abeles, B., Lattice thermal conductivity of disordered semiconductor alloys at high temperatures, Phys. Rev. 131, 1906 (1963).

[290] Abdurakhmanova, A. A., and Aliev, M. I., On the mechanism of thermal conductivity in ( $\mathrm{GaSb})_{3 x}$ $\left(\mathrm{Ga}_{2} \mathrm{Te}_{3}\right)_{1-x}$ and ( GaDSb $)_{x}-(\mathrm{GaTe})_{1-x}$ solid solutions, Phys. Status Solidi 20, 777 (1967).

[291] Aliev, M. I., and Dzhangirov, A. Yu., Investigation of the thermal and electrical properties of InSb$\mathrm{In}_{2} \mathrm{Te}_{3}$ alloys, Soviet Phys.-Solid State 6, 1916 (1965).

[292] Bhandari, C. M., and Verma, G. S., Thermal conductivity of Ge-Si alloys at high temperatures, Phys. Rev. 138, A288 (1965).

[293] Drabble, J. R., The lattice thermal conductivity of alloys, Adv. Energy Conv. 3, 441 (1963).

[294] Hockings, E. F., Kudman, I., Seidel, T. E., Schmelz, C. M., and Steigmeier, E. F., Thermal and electrical transport in InAs-GaAs alloys, J. Appl. Phys. 37, 2879 (1966).

[295] Kelemen, F., Neda, A., Niculescu, D., and Cruceanu, E., On the thermal conductivity of CdTe and some $\mathrm{CdTe}_{1-x} \mathrm{Sn}_{x}$ and $\mathrm{CdTe}_{1-x} \mathrm{Se}_{x}$ solid solutions, Phys. Status Solidi 21, 557 (1967).

[296] Kudman, I., Lattice thermal conductivity of semiconductor alloys, Proc. Seventh Conference on Thermal Conductivity, edited by D. R. Flynn and B. A. Peavy, p. 97 (NBS Special Publication 302, 1968).

[297] Kudman, I., Ekstrom, L., and Seidel, T., Hightemperature thermal and electrical properties of GaSb-InSb alloys, J. Appl. Phys. 38, 4641 (1967).

[298] Parrott, J. E., The high temperature thermal conductivity of semiconductor alloys, Proc. Phys. Soc. (London) 81, 726 (1963).

[299] Remoissenet, M., and Godefroy, L., Thermal diffusivity of barium titanate and of strontium titanate solid solutions, Comt. Rend. 262B, 56 (1966).

[300] Spitzer, D. P., Castellion, G. A., and Haacke, G., Anomalous thermal conductivity of $\mathrm{Cd}_{3} \mathrm{As}_{2}$ and the $\mathrm{Cd}_{3} \mathrm{As}_{2}-\mathrm{Zn}_{3} \mathrm{As}_{2}$ alloys, J. Appl. Phys. 3\%, 3795 (1966).

[301] Williams, W. S., Phonon scattering in $\mathrm{KC} 1-\mathrm{KBr}$ solid solutions at low temperatures, Phys. Rev. 119, 1021 (1960).

[302] Zeinalov, S. A., and Aliev, M. I., Investigation of the thermal conductivity of InSb-GaSb solid solutions, Phys. Status Solidi 22, 153 (1967).

[303] Zerfang, R., The lattice thermal conductivity through dilution in alloys at low temperatures, Phys. Status Solidi 24, 221 (1967).

[304] Bar'yakhtar, V. G., Savchenko, M. A., and Tarasenko, V. V., Relaxation and thermal conductivity in magnetic substances with dislocations, Soviet Physics-JETP 24, 623 (1966).

[305] Bross, H., The influence of defect ordering on the lattice heat conductivity of insulators, $Z$. Phys. 189, 33 (1966).

[306] Brown, R. A., Resonance scattering and the electrical and thermal resistivities associated with extended defects in crystals, Phys. Rev. 156, 692 (1967).

[307] Caldwell, R. F., and Klein, N. V., Experimental and theoretical studies of phonon scattering from simple point defects in $\mathrm{NaCl}$, Phys. Rev. 158, 851 (1967)

[308] Callaway, J., Localized defects in semiconductors, Phys. Rev. 156, 860 (1967).

[309] Moss, M., Effect of plastic deformation on the thermal conductivity of $\mathrm{CaF}$ and LiF, J. Appl. Phys. 36, 3308 (1965).
[310] Moss, M., Scattering of phonons by dislocations, J. Appl. Phys. 37, 4168 (1966).

[311] Payton, D. N., III, Rich, M., and Visscher, Wm. M., Lattice thermal conductivity in disordered harmonic and anharmonic crystal models, Phys. Rev. 160, 706 (1967)

[312] Petrov, A. V., Scattering of phonons by large lattice distortions, Soviet Phys. Solid State 8, 2988 (1966).

[313] Smirnov, I. A., The effect of plastic deformation on the thermal conductivity of $\mathrm{NaCl}$ and $\mathrm{KCl}$ single crystals, Soviet Phys. Solid State 8, 21 (1966).

[314] Taylor, A., Albers, H. R., and Pohl, R. O., Effect of plastic deformation on the thermal conductivity of various ionic crystals, J. Appl. Phys. 36, 2270 (1965)

[315] Zavt, G. S., A heat conduction theory of dielectrics at low temperatures. A case of arbitrary interaction between phonons and defects, Trudy In-ta Fiz. i Astronom, Akad. Nauk Estonsk. SSR (29), 95 (1965).

[316] Berman, R., Foster, E. L., and Rosenberg, H. M., The thermal conductivity of irradiated dielectric crystals at low temperatures, in Rept. Conf. Defects in Crystalline Solids, p. 321 (Physical Society, London, 1955).

[317] Moore, J. P., Kollie, T. G., Graves, R. S., and McElroy, D. L., Thermal conductivity measurements on solids between 20 and $150{ }^{\circ} \mathrm{C}$ using a comparative-longitudinal apparatus: results on $\mathrm{MgO}, \mathrm{BeO}, \mathrm{ThO}_{2}, \mathrm{Th}_{\mathrm{x}} \mathrm{U}_{1-\mathrm{x}} \mathrm{O}_{2+\mathrm{y}}$ and $\mathrm{Al}-\mathrm{UO}_{2}$ cermets, ORNL-4121 (June 1967).

[318] Cooper, M. K., Palmer, A. R., and Stolarski, G. Z. A., The effect of neutron irradiation on the thermal conductivity of beryllium oxide, J. Nucl. Mater. 9, 320 (1963).

[319] Hickman, B. S., The effect of neutron irradiation on beryllium oxide, AAEC/E99, (October 1962).

[320] Berman, R., Simon, F. E., Klemens, P. G., and Fry, T. M., Effect of neutron irradiation on the thermal conductivity of a quartz crystal at low temperature, Nature 166, 864 (1950).

[321] Berman, R., The thermal conductivities of some dielectric solids at low temperatures (Experimental), Proc. Roy. Soc. A208, 90 (1951).

[322] Crawford, J. M., Jr., and Cohen, A. F., Effect of fast neutron bombardment on the thermal conductivity of silica glass at low temperature, p. 43 in ORNL-2614 (November 1958).

[323] Cohen, A. F., Low-temperature thermal conductivity in neutron irradiated vitreous silica, J. Appl. Phys. 29, 591 (1958).

[324] Albany, H. J., and Vandevyver, M., Effects of electron irradiation on the thermal conductivity of $\mathrm{n}$ - and p-type germanium, Phys. Rev. 160, 633 (1967).

[325] Albany, H. J., and Vandevyver, M., Low-temperature thermal conductivity of fast-neutron irradiated silicon and germanium, J. Appl. Phys. 38, 425 (1967).

[326] Berman, R., Foster, E. L., Schneidmesser, B., and Tirmizi, S. M. A., Effect of irradiation on the thermal conductivity of synthetic sapphire, J. Appl. Phys. 31, 2156 (1960).

[327] Clough, D. J., and Sayers, J. B., The measurement of the thermal conductivity of $\mathrm{UO}_{2}$ under irradiation in the temperature range $150-1600{ }^{\circ} \mathrm{C}$, AERE-R-4690 (United Kingdom Atomic Energy Authority, Harwell, December, 1964).

[328] De Goer, A. M., and Dreyfus, B., $\gamma$-irradiation effect on the thermal conductivity of $\mathrm{Al}_{2} \mathrm{O}_{3}$ with $\mathrm{Cr}$ or Mn impurities at low temperatures, Phys. Status Solidi 22, 77 (1967).

[329] Freeman, R. J., Thermal diffusivity measurements on pre- and post-irradiated $\mathrm{BeO}, \mathrm{GEM} \mathrm{P}-452$ (General Electric Company, NMPO, Cincinnati, Ohio, November 1966). 
[330] Henson, R. W., and Reynolds, W. N., 'Lattice parameter changes in irradiated graphite, Carbon 3, 277 (1965).

[331] Hunter, L. P., Effect of fission recoil fragments on the thermal conductivity of graphite, J. Appl. Phys. 30, 1969 (1959).

[332] Jaeger, R. E., The thermal conductivity of neutron irradiated $\mathrm{Al}_{2} \mathrm{O}_{3}, \mathrm{BeO}$, and $\mathrm{MgO}$ at low temperatures, Ph. D. Dissertation, Rutgers University, March 1967.

[333] Jaeger, R. E., Thermal conductivity of neutron irradiated $\mathrm{Al}_{2} \mathrm{O}_{3}, \mathrm{BeO}$, and $\mathrm{MgO}$ at low temperatures, Paper No. 13-N-67F, Nuclear Division of Amer. Ceram. Soc. (September 19-22, 1967).

[334] MeDonald, D. L., Low-temperature irradiation of beryllium oxide, Appl. Phys. Letters 2, 175 (1963).

[335] Pryor, A. W., Tainsh, R. J., and White, G. K., Thermal conductivity at low temperature of neutron-irradiated 'BeO, J. Nucl. Mater. 14, 208 (1964).

[336] Seyfert, P., Influence of neutron irradiation at the temperature of $80^{\circ} \mathrm{K}$ on the thermal conductivity of silicon, Compt. Rend. 265B, 609 (1967).

[337] van Dong, Nguyen, Effect of fast neutrons on thermal conductivity and the thermoelectric power of germanium, J. Phys. (Paris) 27, 128 (1966).

[338] Vook, F. L., Thermal conductivity of electronirradiated germanium, Phys. Rev. 138, A1234 (1965).

[339] Vook, F. L., Thermal conductivity of electronirradiated silicon, Phys. Rev. 140, A2014 (1965).

[340] Vook, F. L., Thermal conductivity of electronirradiated InSb, Phys. Rev. 149, 631 (1966).

[341] Vorozheikina, L. F., Igitkhanishvili, D. D., Politov, N. G., and Kvachadze, V. G., Electric and thermal conductivities and optical absorption of neutron irradiated alkali halides, Bull. Acad. Sci. USSR, Phys. Ser. 29, 75 (1965).

[342] Billington, D. S., and Crawford, J. H., Jr., Radiation Damage in Solids (Princeton University Press, Princeton, New Jersey, 1961).

[343] Kirchner, J. F., and Bowman, R. E., Effects of Radiation on Materials and Components (Reinhold Publishing Corp., New York, 1964).

[344] Strumane, R., Nichoul, J., Gevers, R., and Amelinckx, S., The Interaction of Radiation with Solids (North Holland Publishing Co., Amsterdam, Netherlands, 1964).

[345] Nuclear Science and Technology for Ceramists, NBS Misc. Publ. 285 (May 1967).

[346] Powers, A. E., Conductivity in aggregates, KAPL2145, Knolls Atomic Power Laboratory (March 1961).

[347] Meredith, R. E., and Tobias, C. W., Conduction in heterogeneous systems, in Advances in Electrochemistry and Electrochemical Engineering, Vol. 2, edited by C. W. Tobias, p. 15 (John Wiley \& Sons, Inc., New York, 1962).

[348] Argo, W. B., and Smith, J. M., Heat transfer in packed beds, Chem. Eng. Prog. 49, 443 (1953).

[349] Awbery, J. W., Note on heat-flow through a granulated material, Phil. Mag. 12, 1152 (1931)

[350] Babanov, A. A., Methods for calculation of thermal conduction coefficients of capillary-porous materials, Soviet Phys.-Tech. Phys. 2, 476 (1957).

[351] Baldwin, D. E., Jr., Beckman, R. B., Rothfus, R. R., and Kermode, R. I., Heat transfer in beds of oriented spheres, I/EC Process. Des. Dev. 5, 281 (1966)

[352] Barrett, L. B., Heat transfer in refractory insulating materials, Part I. Texture and insulating power, Trans. Brit. Ceram. Soc. 48, 235 (1948-49).

[353] Baxley, A. L., and Couper, J. R., Thermal conductivity of two-phase systems: Thermal conductivity of suspensions, Univ. Arkansas Eng. Exp. Station Research Report No. 8 (November 1966).

[354] Baxley, A. I., Nahas, N. C., and Couper, J. R., Thermal conductivity of two-phase systems, Proc. Seventh Conf. on Thermal Conductivity, edited by D. R. Flynn and B. A. Peavy, p. 685 (NBS Special Publication 302, 1968).

[355] Bischoff, K. B., Axial thermal conductivities in packed beds, Can. J. Chem. Eng. 40, 161 (1962).

[356] Boegli, J. S., and Deissler, R. G., Measured effective thermal conductivity of uranium dioxide powder in various gases and gas mixtures, NACA RM E54L10 (March 1955).

[357] Brailsford, A. D., and Major, K. G., The thermal conductivity of aggregates of several phases, including porous materials, Brit. J. Appl. Phys. 15, 313 (1964).

[358] Bretsznajder, S., and Ziolkowski, D., The effective thermal conductivity of granular beds, Intern. Chem. Eng. 6, 85 (1966).

[359] Brodie, D. E., and Mate, C. F., Heat transfer in gas-filled powders at low temperatures, Can. J. Phys. 43, 2344 (1965).

[360] Chaudhary, D. R., Bhandari, R. C., Heat transfer through dispersed system having irregular pore sizes, Indian J. Pure Appl. Phys. 5, 413 (1967).

[361] Chapoton, A., Wacrenier, J. M., and Lebrun, A., Contribution to the determination of conductivities and permittivities at the level of the cavities of porous solids, Compt. Rend. 265B, 989 (1967).

[362] Luikov, A. V., Shashkov, A. G., Vasiliev, L. L., and Fraiman, Yu. E., Thermal conductivity of porous systems, Int. J. Heat Mass Transfer 11, 117 (1968).

[363] Deissler, R. G., and Boegli, J. S., An investigation of effective thermal conductivities of powders in various gases, Trans. ASME 80, 1417 (1958).

[364] de Vries, D. A., Het warmtegeleidingsvermogen van grand, Mededel. Landbouwhogeschool Wageningen/Nederland 52, 1 (1952).

[365] Dul'nev, G. N., Heat transfer through solid disperse systems, Inzh.-Fiz. Zh. 9, 399 (1965).

[366] Dul'nev, G. N., Heat conduction of statistical mixtures, Inzh.-Fiz. Zh. 9, 538 (1965).

[367] Dul'nev, G. N., and Komkova, L. A., Analysis of the experimental studies of thermal conductivity of solid porous systems, Inzh.-Fiz. Zh. 9, 517 (1965).

[368] Dul'nev, G. N., and Zarichnyak, V. P., Thermal conductivity of multicomponent mixtures, Inzh.Fiz. Zh. 12, 419 (1967).

[369] Dul'nev, G. N., and Sigalova, Z. V., The thermal conductivity of granular systems, Intern. Chem. Eng. 5, 218 (1965).

[370] Dunsky, V. D., and Tamarin, A. I., On heat conductivity of disperse material in vacuum, in Heat and Mass Transfer in Dispersed Systems, p. 160, (Minsk, 1965).

[371] Eucken, A., Allgemeine Gesetzmässigkeiten fur das Wärmeleitvermögen verschiedener Stoffarten und Aggregatzustände, Forsch. Gebiete Ingenieurw. 11, 6 (1940).

[372] Euler, F., Simple geometrical model for the effect of porosity on material constants, J. Appl. Phys. 28, 1342 (1957).

[373] Francis, R. K., and Tinklepaugh, J. R., Thermal conductivity in ceramic-metal laminates, J. Am. Ceram. Soc. 43, 560 (1960).

[374] Francl, J., and Kingery, W. D., Thermal conductivity: IX, Experimental investigation of effect of porosity on thermal conductivity, J. Am. Ceram. Soc. 37, 99 (1954).

[375] Gopalarathnan, C. D., Effective thermal conductivity in packed beds, A. I. Chem. E. J. 7, 249 (1961)

[376] Gorring, R. L., and Churchill, S. W., Thermal conductivity of heterogeneous materials, Chem. Eng. Prog. 57, 53 (1961).

[377] Grekila, R. B., and Tien, T. Y., Conductivity discontinuity in a two-phase system, J. Am. Ceram. Soc. 48, 22 (1965).

[378] Hamilton, R. L., and Crosser, O. K., Thermal conductivity of heterogeneous two component 
systems, Ind. Eng. Chem. Fundamentals 1, 187 (1962).

[379] Jakob, M., Heat Transfer, Vol. I, p. 88 (John Wiley \& Sons, Inc., New York, 1950).

[380] Jefferson, T. B., Thermal conductivity determinations for suspensions of graphite in water and oil, Ph. D. thesis (Purdue University, 1955).

[381] Jefferson, T. B., Witzell, O. W., and Sibbitt, W. L., Thermal conductivity of graphite-silicone oil and graphite-water suspensions, Ind. Eng. Chem. 50, 1589 (1958)

[382] Joy, F. A., Thermal conductivity of insulation containing moisture, Symposium on Thermal Conductivity Measurements and Applications of Thermal Insulations, ASTM STP 217 (1957).

[383] Kaganer, A. M. G., Contact heat transfer in granular material in vacuum, Inzh.-Fiz. Zh. 10, 30 (1966).

[384] Kaganer, A. M. G., and Semenova, R. I., Thermal conductivity and thermal diffusivity of insulating powders at atmospheric pressure and in vacuum studied by various methods, Inzh.-Fiz. Zh. 13, 24 (1967).

[385] Kannuluik, W. G., and Martin, L. II., Conduction of heat in powders, Proc. Roy. Soc. (London) A141, 58 (1933).

[386] Kharlamov, A. G., Thermal conductivity of packing of ceramic pellets, Inzh.-Fiz. Zh. 9, 48 (1965).

[387] Keller, J. B., Conductivity of a medium containing a dense array of perfectly conducting spheres or cylinders or nonconducting cylinders, J. Appl. Phys. 34, 991 (1963).

[388] Keller, H. B., and Sachs, D., Calculations of the conductivity of a medium containing cylindrical inclusions, J. Appl. Phys. 35, 537 (1964).

389] Kimura, M., Effective thermal conductivity of wetted packed beds, Kagaku Kogaku (Chem. Eng., Japan) 23, 502 (1959).

[390] Krischer, O., and Esdorn, H., Die Wärmeübertragung in feuchten, porigen Stoffen verschiedener Struktur, Forsch. Gebiete Ingenieurw. A22, 1 (1956).

[391] Krupiczka, R., Analysis of thermal conductivity in granular materials, Intern. Chem. Eng. 7,122 (1967).

[392] Kunii, D., and Smith, J. M., Heat transfer characteristics of porous rocks, A. I. Ch. E. Journal 6, 71 (1960).

[393] Lees, C. H., On the thermal conductivity of single and mixed solids and liquids and their variation with temperature, Proc. Roy. Soc. (London) A191, 339 (1898).

[394] Levine, H., The effective conductivity of a regular composite medium, Stanford University TR-6 (July 1965), AD 622870.

[395] Loeb, A. L., Thermal conductivity: VIII, A theory of thermal conductivity of porous materials, J. Am. Ceram. Soc. 3\%, 96 (1954).

[396] Little, R. C., Carpenter, F. G., and Deitz, V. R., Heat transfer in intensively outgassed powders, J. Chem. Phys. 37, 1896 (1962).

[397] Masamune, S., and Smith, J. M., Thermal conductivity of beds of spherical particles, Ind. and Eng. Chem. Fundamentals 2,136 (1963).

[398] Miller, J. V., Predicting thermal conductivity of tungsten-uranium dioxide dispersions, NASA TM X-1145 (1965).

[399] Miller, J. V., Estimating thermal conductivity of cermet fuel materials for nuclear fuel application, NASA TN D-3898 (April 1967).

[400] Nahas, N. C., Griffin, C. L., and Couper, J. R., Thermal conductivity of two-phase systems: A survey and critical evaluation of the literature, Univ. Arkansas Eng. Exp. Station Research Report No. 5 (April 1964).

[401] Nahas, N. C., and Couper, J. R., Thermal conductivity of two-phase systems: Thermal conductivity of emulsions, Univ. Arkansas Eng. Exp. Station Research Report No. 7 (March 1966).
[402] Neimark, L. A., Kittel, J. H., and Hoenig, C. L., Irradiation of metal-fiber-reinforced thoria-urania, Report No. ANL-6397 (December 1961).

[403] Nusselt, E. K. W., Wärmeübertragg. b.d. BoneSchnabelfeuerg, Z. Bayer, Revis.-Ver., (1913).

[404] Pliat, Sh. N., Extension of Odelevskii's theory of generalized conductivity to thermal conductivity, Soviet Physics--Tech. Phys. Z, 2588 (1957).

[405] Preston, F. W., Mechanism of heat transfer in unconsolidated porous media at low flow rates, Ph. D. thesis, The Pennsylvania State University (1957), (see also Dissertation Abstracts 17, 2593 (1957)).

[406] Rabinowitz, P., Calculations of the conductivity of a medium containing cylindrical inclusions by the method of orthogonalized particular solutions, Technical Report, Brown University, (June 1965), AD 618052 .

[407] Ribaud, M., Conductibilité thermique des matériaux poreaux et pulvérulents, étude théorique, Chaleur \& Ind. 18, 36 (1937).

[408] Rieman, G. M. H. W. Die Partiellen DifferentialGleichunger der Mathematischen Physik, Band 1, p. 474 (F. Vieweg und Sohn, Braunschweig, 1919).

[409] Schroeder, J. B., Thermal conductivity in two-phase alloys, J. Appl. Phys. 32, 1798 (1961), (see discussion by R. W. Ure, Jr., J. Appl. Phys. 33, 2290 (1962)).

[410] Shimokawa, J., Deionization processes with ion exchange and with electromigration, Japan Atomic Energy Research Institute, JAERI 1038 (June 1962).

[411] Shudnovskii, A. F., Thermophysical Characteristics of Disperse Materials (Fizmatgiz (USSR), 1962).

[412] Somerton, W. H. Some thermal characteristics of porous rocks, J. Petrol. Technol. 10, 61 (May 1958).

[413] Strong, M., II, Bundy, F. P., and Bovenkirk, M. P., Flat panel vacuum thermal insulation, J. Appl. Phys. 31, 39 (1960).

[414] Sugawara, A., and Yoshizawa, Y., An investigation on the thermal conductivity of porous materials and its application to porous rocks, Australian J. Phys. 14, 469 (1961).

[415] Sugawara, A., and Yoshizawa, Y., An experimental investigation on the thermal conductivity of consolidated porous materials, J. Appl. Phys. 33, 3135 (1962).

[416] Swift, D. L., The thermal conductivity of spherical metal powders including the effect of an oxide coating, Intern. J. Heat Mass Transfer 9, 1061 (1966).

[417] Tadokro, Y., On the effect of porosity upon thermal conductivity, diffusivity, and heat capacity at high temperatures, Science Report, Tohuku Imp. Univ. I, 15, 567 (1926)

[418] Tsao, G. T.-N., Thermal conductivity of two-phase materials, Ind. Eng. Chem. 53, 395 (1961).

[419] van Bueren, H. G., Imperfections in Crystals, (North Holland Publishing Company, Amsterdam, Netherlands, 1961).

[420] Vasil'ev, L. L., and Fraiman, Iu. E., Investigation of the thermophysical properties of the chamotte ceramics in the $80-1200^{\circ} \mathrm{K}$ range. Inzh-Phys. Zh. 9, 762 (1965) (Translation TT-66-61303 available from Clearinghouse for Fed. Sci. and Tech. Inf.).

[421] Waddams, A. L., Flow of heat through granular materials, J. Soc. Chem. Ind. 63, 337 (1944).

[422] Wakao, N., Omura, S., and Fukuda, M., Effective thermal conductivity of packed beds in low pressure range, Chem. Engng. Japan 30, 1119 (1966).

[423] Wang, D. I-J., Multiple layer insulation, in Aerodynamically Heated Structures, edited by P. E. Glaser (Prentice-Hall, Inc., New York, 1962).

[424] Warren, J. E., and Messmer, J. H., Thermal conductivity of two-phase materials, Ind. and Eng. Chem. Fundamentals, 1, 222 (1962). 
[425] Watson, K., The thermal conductivity measurements of selected silicate powders in vacuum from $150^{\circ}-350^{\circ} \mathrm{K}, \mathrm{Ph}, \mathrm{D}$. thesis (California Inst. of Tech., 1964).

[426] Webb, J., Thermal conductivity of soil, Nature 17\%, 989 (1956).

[427] Wechsler, A. E., Glass beads-a standard for the low thermal conductivity range, Proc. Seventh Conf. on Thermal Conductivity, edited by D. R. Flynn and B. A. Peavy, p. 89 (NBS Special Publication 302, 1968).

[428] Weiner, J. H., Transient heat conduction in multiphase media, Brit. J. Appl. Phys. 6, 361 (1955).

[429] Whitmore, D. H., Apparent thermal conductivity of a heterogeneous ceramic body under isothermal conditions, J. Am. Ceram. Soc. 43, 281 (1960).

[430] Wilhelm, R. H., Johnson, W. C., Wynkoop, R., and Collier, D. W., Reaction rate, heat transfer, and temperature distribution in fixed-bed catalytic converters-solution by electrical network, Chem. Eng. Prog. 44, 105 (1948).

[431] Willhite, G. P., Kunii, D., and Smith, J. M., Heat transfer in beds of fine particles (heat transfer perpendicular to flow), A. I. Ch. E. Journal 8, 340 (1962).

[432] Woodside, W., Calculation of the thermal conductivity of porous media, Can. J. Phys. 36, 815 (1958).

[433] Woodside, W., and Messmer, J. H., Thermal conductivity of porous media. I. Unconsolidated sands, J. Appl. Phys. 32, 1688 (1961)

[434] Wyllie, M. R. J., and Southwick, P. F., An experimental investigation of $\mathrm{S}$. P. and resistivity phenomena in dirty sands, J. Petroleum Tech. 6, T. P. 3750 (1954).

[435] Jackson, J. L., and Coriell, S. R., Transport coefficients of composite materials, J. Appl. Phys. 39, 2349 (1968). (See also p. 4733).

[436] Koenig, J. H., Ceramic Bodies with High Thermal Conductivity, Progress Report No. VI (Rutgers University, 1952) ATI 163519.

[437] Koenig, J. H., Ceramic Bodies with High Thermal Conductivity, Progress Report No. II (Rutgers University, 1953) AD 13154.

[438] Kingery, W. D., Thermal conductivity: XII, Temperature dependence of conductivity for singlephase ceramics, J. Am. Ceram. Soc. 38, 251 (1955).

[439] Charvat, F. R., and Kingery, W. D., Thermal conductivity: XIII, Effect of microstructure on conductivity of single-phase ceramics, J. Amer. Ceram. Soc. 40, 306 (1957).

[440] Amelinckx, S., and Dekeyser, W., The structure and properties of grain boundaries, Solid State Physics 8, 327 (1959).

[441] Microstructure of Ceramic Materials, NBS Misc. Publ. 257 (April 1964).

[442] Kingery, W. D., Effect of microstructure on the properties of ceramics, in Physics and Chemistry of Ceramics, edited by C. Klingsberg, p. 286 (Gordon and Breach Science Publ., Inc., New York, 1963).

[443] Coble, R. L., and Burke, J. E., Sintering in ceramics, in Progress in Ceramic Science, Vol. 3 , edited by J. E. Burke (The Macmillan Co., New York, 1963).

[444] Burke, J. E., The science and technology of sintering, in Physics and Chemistry of Ceramics, edited by C. Klingsberg, p. 165 (Gordon and Breach Science Publ., Inc., New York, 1963).

[445] White, G. K., Experimental Techniques in LowTemperature Physics (Oxford Univ. Press, Oxford, 1959).

[446] Ditmars, D. A., and Ginnings, D. C., Thermal conductivity of beryllium oxide from $40^{\circ}$ to $750{ }^{\circ} \mathrm{C}$, J. Research NBS 59, No. 2, 93 (1957) RP2775.

[447] Laubitz, M. J., Measurement of the thermal conductivity of solids at high temperatures using steady-state linear and quasi-linear heat flow, ch. 3 in Thermal Conductivity, edited by R. P. Tye (Academic Press, Inc., London, in press).

[448] White, G. K., Measurement of solid conductors at low temperatures, ch. 2 in Thermal Conductivity, edited by R. P. Tye (Academic Press, London, in press).

[449] Mikryukov, V. E., Teploprovodnost i Elektroprovodnost Metalov i Splavov (Metallurgizdat, Moscow, 1959).

[450] Bauerle, J. E., Thermal conductivity, in chapter on Measurements of properties of thermoelectric materials, p. 285 in Thermoelectricity, edited by R. R. Heikes and R. W. Ure (Interscience Publishers, Inc., New York, 1961).

[451] Standard method of test for thermal conductivity of materials by means of the guarded hot plate, ASTM Designation: C177-63, in 1967 Book of ASTM Standards, Part 14, p. 17 (American Society for Testing and Materials, Philadelphia, 1967).

[452] International comparative measurements of thermal conductivity, Bull. I.I.F./I.I.R. XLVIII, 29 (1968).

[453] Rouselle, J.-C., A guarded hot plate apparatus for measuring thermal conductivity from -80 to $+100{ }^{\circ} \mathrm{C}$, Proc. Seventh Conf. on Thermal Conductivity, edited by D. R. Flynn and B. A. Peavy, p. 513 (NBS Special Publication 302, 1968).

[454] Orr, H. W., A study of the effects of edge insulation and ambient temperatures on errors in guarded hot-plate measurements, Proc. Seventh Conf. on Thermal Conductivity, edited by D. R. Flynn and B. A. Peavy, p. 521 (NBS Special Publication 302, 1968).

[455] Pratt, A. W., Heat transmission in thermal insulating materials, ch. 6 in Thermal Conductivity, edited by R. P. Tye (Academic Press, London, in press).

[456] Standard method of test for thermal conductivity of refractories, ASTM Designation: C201-47, in 1967 Book of ASTM Standards, Part 13, p. 170 (American Society for Testing and Materials, Philadelphia, 1967).

[457] Laubitz, M. J., On measurement of thermal conductivity at high temperatures, Can. J. Phys. 39, 1029 (1961)

[458] Laubitz, M. J., On the series comparator methods of measuring thermal conductivity, Proc. Black Hills Summer Conference on Transport Phenomena, p. 8 (South Dakota School of Mines and Technology, 1962).

[459] Petrov, A. V., Methods of measuring the thermal conductivity of semiconductors at high temperatures, in Thermoelectric Properties of Semiconductors (Proc. First and Second Conferences on Thermoelectricity), edited by V. A. Kutasov, p. 17 (Consultants Bureau, 1964).

[460] Didion, D. A., An analysis and design of a linear guarded cut-bar apparatus for thermal conductivity measurements (Catholic University of America, 1968) AD 665789.

[461] Minges, M. L., Heat losses in a cut-bar apparatus: experimental-analytical comparisons, Proc. Seventh Conf. on Thermal Conductivity, edited by D. R. Flynn and B. A. Peavy, p. 197 (NBS Special Publication 302, 1968).

[462] Francl, J., and Kingery, W. D., Thermal conductivity: IV, Apparatus for determining thermal conductivity by a comparative method; Data for $\mathrm{Pb}, \mathrm{Al}_{2} \mathrm{O}_{3}$, and $\mathrm{MgO}$, J. Am. Ceram. Soc. 3\%, 80 (1954).

[463] Francis, R. K., and Tinkelpaugh, J. R., Thermal conductivity in ceramic-metal laminates, J. Am. Ceram. Soc. 43, 560 (1960).

[464] Mirkovitch, V. V., Comparative method and choice of standards for thermal conductivity determinations, J. Am. Ceram. Soc. 48, 387 (1965).

[465] Mahmoodi, P., Apparatus for determining thermal conductivity, U.S. Patent No. 3,263,485 (August $2,1966)$. 
466] Standard method of test for thermal conductivity of whiteware ceramics, ASTM Designation: C408-58, in 1967 Book of ASTM Standards, Part 13, p. 348 (American Society for Testing and Materials, Philadelphia, 1967).

[467] McElroy, D. L., and Moore, J. P., Radial heat flow methods for the measurement of the thermal conductitity of solids, ch. 4 in Thermal Conductivity, edited by R. P. Tye (Academic Press, Inc., London, in press).

[468] Development of high temperature thermal conductivity reference standards (Arthur D. Little, Inc., Cambridge, Mass.,), Tech. Report AFMLTR-66-415 (January 1967).

[469] Powell, R. W., Correlation of metallic thermal and electrical conductivities for both solid and liquid phases, Int. J. Heat Mass Transfer 8, 1033 (1965).

[470] Powell, R. W., and Ho, C. Y., The state of knowledge regarding the thermal conductivity of the metallic elements, Proc. Seventh Conf. on Thermal Conductivity, edited by D. R. Flynn and B. A. Peavy, p. 1 (NBS Special Publication 302, 1968).

[471] Laubitz, M. J., and van der Meer, M. P., The thermal conductivity of platinum between 300 and $1000^{\circ} \mathrm{K}$, Can. J. Phys. 44, 3173 (1966).

[472] Martin, J. J., Sidles, P. H., and Danielson, G. C., Thermal diffusivity of platinum from 300 to 1200 ${ }^{\circ} \mathrm{K}, \mathrm{J}$. Appl. Phys. 38, 3075 (1967).

473] Shelton, S. M., and Swanger, W. H., Thermal conductivity of irons and steels and some other metals in the temperature range $0-600^{\circ}$, Trans. Amer. Soc. Steel Treating 21, 1061 (1933).

474] Van Dusen, M. S., and Shelton, S. M., Apparatus for measuring thermal conductivity of metals up to 600 C, NBS J. Research 12, No. 4, 429 (1934) RP668.

[475] Lucks, C. F., Pyrometric standard lead as a thermal conductivity reference material, J. Appl. Phys. 38, 1973 (1967).
[476] Powell, R. W., and Tye, R. P., New measurements on thermal conductivity reference materials, Int. J. Heat Mass Transfer 10, 581 (1967).

[477] Laubitz, M. J., and Cotnam, K. D., Thermal and electrical properties of Inconel 702 at high temperatures, Can. J. Phys. 42, 131 (1964).

[478] Watson, T. W., and Flynn, D. R., Thermal conductivity of four heat-resistant alloys, Trans. Metall. Soc. AIME 242, 844 (1968)

[479] Glassbrenner, C. J., and Slack, G. A., Thermal conductivity of silicon and germanium from $3 \mathrm{~K}$ to the melting point, Phys. Rev. 134, A 1058 (1964).

[480] Fulkerson, W., Moore, J. P., Williams, R. K., Graves, R. S., and McElroy, D. L., Thermal conductivity, electrical resistivity, and Seebeck coefficient of silicon from 100 to $1300^{\circ} \mathrm{K}$, Phys. Rev. 167, 765 (1968).

[481] Carwile, L. C. K., and Hoge, H. J., Thermal conductivity of vitreous silica: selected values, Proc. Seventh Conf. on Thermal Conductivity, edited by D. R. Flynn and B. A. Peavy, p. 59 (NBS Special Publication 302, 1968).

[482] Wechsler, A. E., and Minges, M. L., Development of high temperature thermal conductivity standards, Proc. Seventh Conf. on Thermal Conductivity, edited by D. R. Flynn and B. A. Peavy, p. 77 (NBS Special Publication 302, 1968).

[483] Tye, R. P., (editor), Thermal Conductivity (Academic Press, Inc., London, in press).

[484] Picklesimer, M. L., Microstructural Examination, ORNL-TM-1338 (January, 1966).

[485] Eisenhart, C., Realistic evaluation of the precision and accuracy of instrument calibration systems, J. Research NBS 67C, (Engr. and Instr.) No. 2, 161 (1963).

[486] Eisenhart, C., Expression of the uncertainties of final results, Science 160, 1201 (1968). 



\title{
Thermal Radiation Properties of Ceramic Materials
}

\author{
Joseph C. Richmond \\ National Bureau of Standards, Washington, D.C.: 20234
}

\begin{abstract}
The thermal radiation properties of materials are defined, and the basic physical laws governing the amount of thermal radiation emitted by a blackbody radiator, and its geometric and spectral distribution, are reviewed. Equations are given relating the thermal radiation properties of emittance, reflectance, transmittance, and absorptance to the optical properties of index of refraction, absorption coefficient, and scattering coefficient. The effect of impurities and processing variables such as grain size and porosity on the optical properties is discussed. The influence of thermal gradients normal to the surface of ceramic specimens on the observed thermal radiation properties is reviewed. Several methods of measuring thermal radiation properties of ceramic materials are discussed briefly, and representative normal spectral emittance data for alumina, thoria, magnesia, and zirconia are given.
\end{abstract}

Key Words: Alumina; ceramics; emittance; magnesia; reflectance; temperature coefficient of emittance; thermal radiation properties; thoria, zirconia.

\section{Introduction}

Thermal radiation is electromagnetic radiation that is generated by the thermal motion of the particles of which a material is composed, such as molecules, atoms, ions, and electrons. All matter is constantly emitting thermal radiation, and in turn is constantly being irradiated by thermal radiation from its surroundings. Both processes occur simultaneously, and the net rate of heat transfer to or from a body is determined by the relative rates of the two processes.

The thermal radiation properties of a body are those properties that determine the rates of absorption and emission of thermal radiation, and hence the rates of heat transfer to or from the body by radiation. When thermal radiation is incident on a body, it is either reflected, absorbed or transmitted. Nothing else can happen to it. Hence, we can write

$$
\Phi_{r}+\Phi_{a}+\Phi_{t}=\Phi_{i},
$$

where $\Phi$ is flux, the time rate of flow of radiant energy, and the subscripts $r, a, t$ and $i$ designate the reflected, absorbed, transmitted, and incident flux, respectively. If we divide both sides of equation (1) by $\Phi_{i}$ and define the reflectance, $\rho$, as $\Phi_{r} / \Phi_{i}$, the absorptance, $\alpha$, as $\Phi_{a} / \Phi_{i}$ and the transmittance, $\tau$, as $\Phi_{t} / \Phi_{i}$, we get

$$
\rho+\alpha+\tau=1 \text {. }
$$

For an opaque specimen, $\tau=0$, hence

$$
\rho+\alpha=1 \text {. }
$$

Kirchhoff's law states that the absorptance, $\alpha$, is equal to the emittance, $\epsilon$,

$$
\alpha=\epsilon,
$$

hence, for an opaque body the reflectance and the emittance sum to one

$$
\rho+\epsilon=1 .
$$

We thus see that for an opaque body the thermal radiation properties are fully defined by either the emittance or the reflectance.

Fluorescent and phosphorescent materials absorb radiant energy at one wavelength and reemit it at another, usually longer, wavelength. Because of this behavior, they may appear to deviate from the above relationships when irradiated with heterochromatic flux, since at the wavelength of fluorescent or phosphorescent emission the reflected or transmitted flux may exceed the incident flux. For such materials it is necessary to measure the spectral reflectance or transmittance by a technique in which the specimen is irradiated by monochromatic flux, and the reflected or transmitted flux is measured by means of a detector that senses only flux of the same wavelength as the incident flux. The flux emitted by phosphorescent or fluorescent emission is not considered to be reflected flux.

\section{Definition of Terms}

Up to this point we have described thermal radiation, thermal radiation properties, and flux. It will be appropriate at this time to more rigor- 
ously define a number of additional terms that we shall be using. ${ }^{1}$

Reflectance, $\rho$, is the fraction of the incident flux that is reflected without change in frequency.

Absorptance, $\alpha$, is the fraction of the incident flux that is absorbed and converted to another form of energy.

Transmittance, $\tau$, is the fraction of the incident flux that is transmitted without change in frequency.

All of these properties are influenced by the wavelength, direction of incidence, and degree and direction of polarization relative to the plane of incidence of the incident flux, and, for reflectance and transmittance, by the size and direction of the solid angle over which the reflected or transmitted flux is collected for measurement. They may also vary somewhat with the temperature of the specimen. Hence, these terms are modified to indicate these conditions.

Spectral means at a given wavelength, or in a narrow wavelength band centered about a given wavelength.

Directional means in a given direction, usually measured from the normal to the surface.

Normal is a special case of directional, and means in a direction normal to the surface.

Specular, referring to reflectance, means in the direction of mirror reflection.

Diffuse, or hemispherical, referring to incident flux, means incident uniformly from all possible directions.

Hemispherical, referring to reflected, transmitted, or emitted flux, refers to flux summed over a hemisphere.

The terms reflectance, absorptance, and transmittance are defined as fractions of incident flux, and are dimensionless numbers. The numerical value, being a ratio, is independent of the magnitude of the incident flux, but for equations (2) and (3) to be valid, the conditions of irradiation must be the same for each term in the equation. This means that the spectral or wavelength composition and state of polarization of the incident flux and its direction of incidence must be the same for each term.

A blackbody radiator absorbs all radiant energy incident upon it, and emits radiant energy at the maximum possible rate for any body at its temperature. The amount of flux emitted by a blackbody radiator is given by the StefanBoltzmann equation, its spectral distribution by the Planck equation, and its geometric distribution by Lambert's cosine law. These are the fundamental laws of thermal radiation.

The Stefan-Boltsmann equation is

$$
M=\sigma T^{4}
$$

1 There has been much confusion in nomenclature in this area. Judd [1] has published a recommended system of nomenclature for reflectance terms. has published a recommended system of nomenclature for reflectance terms.
(Figures in brackets indicate the literature references at the end of this paper). where $M$ is the radiant exitance, or total flux emitted per unit surface area by a blackbody emitter in units of $\mathrm{Wm}^{-2}, \sigma$ is the StefanBoltzmann constant, $5.6697 \times 10^{-8} \mathrm{Wm}^{-2} \mathrm{~K}^{-4}$, and $T$ is the absolute temperature in kelvins.

Lambert's cosine law may be expressed as follows:

$$
I_{\theta}=I_{0} \cos \theta
$$

in which $I_{\theta}$ is the intensity per unit area in units of $\mathrm{Wsr}^{-1} \mathrm{~cm}^{-2}$ of a blackbody radiator in a direction $\theta, \theta$ being the angle between the given direction and the normal to the emitting surface, and $I_{n}$ is the in tensity per unit area of the blackbody radiator normal to its surface.

Planck's radiation equation is

$$
M_{\lambda}=c_{1} \lambda^{-5}\left[\exp \left(c_{2} / \lambda T\right)-1\right]^{-1}
$$

where $M_{\lambda}$ is the spectral radiant exitance of a blackbody radiator per unit wavelength interval at wavelength $\lambda$, in units of watts per square meter and meter wavelength interval, $c_{1}$ is the first radiation constant, $3.7415 \times 10^{-16} \mathrm{Wm}^{2}, \lambda$ is the wavelength in meters, $c_{2}$ is the second radiation constant, $1.43879 \times 10^{-2} \mathrm{mK}$, and $T$ is the absolute temperature, in kelvins.

No real material is a true blackbody radiator; hence, for all real materials, the flux emitted is less than that emitted by a balckbody radiator. However, the radiation laws can still be used for real materials by using the emittance as a proportionality factor.

Emittance, $\epsilon$, is defined as the ratio of the radiant exitance of a specimen (radiant flux per unit area emitted) to that of a blackbody radiator at the same temperature and under the same conditions.

Emissivity is defined as the emittance of a specimen having an optically smooth surface and a thickness sufficient to be completely opaque, and is a property of the material of which the specimen is composed.

Emittance varies with the direction of emission relative to the surface of the specimen, the wavelength of the emitted radiation, and the temperature of the specimen. The emitted radiation may be elliptically polarized, particularly if emitted at large angles from the normal to the surface of the specimen. The terms directional, normal, hemispherical and spectral apply to emittance as well as to all of the other terms previously defined. The term total, referring to emittance, means at all wavelengths. This term has no specific meaning for reflectance, absorptance, and transmittance, unless the spectral distribution of the incident flux, or source, is specified. For emittance, however, it is only necessary to specify the temperature, since the spectral distribution of flux emitted by a blackbody radiator is given by the radiation laws as a function of temperature.

For Kirchhoff's law, equation (4), to be rigorously correct, ${ }^{2}$. it is necessary that the spectral

\footnotetext{
2See reference [2] for a proof of Kirchhoff's law
} 
composition, degree of polarization, and direction of incidence of the incident flux for the absorptance evaluation should be the same as the spectral composition, degree of polarization, and direction of emission for the emitted flux in the emittance evaluation. This would appear to impose a severe restriction upon the general applicability of Kirchhoff's law. However, there are no polarization effects for normally incident flux, and the effects become of appreciable significance only for specularly reflecting materials at angles near the Brewster's angle. This point will be discussed in more detail later. For spectral incident flux the spectral distribution within the narrow wavelength interval approximates that of blackbody radiation sufficiently closely that the effect of the deviation is negligible. Hence equation (3) applies to normal spectral values of absorptance and emittance, and can be used to convert one to the other.

Normal spectral emittance over a wide wavelength range, at a given temperature, can be converted to total emittance at the same temperature by multiplying the spectral values, wavelength by wavelength, by the value of the Planck distribution function at the same wavelength to produce a curve of emitted flux as a function of wavelength. This curve is integrated to obtain the total emitted flux, which is then divided by the total integrated flux for a black body at the same temperature to obtain the total normal emittance. For most nonconducting materials, the emittance, either total or spectral, does not change appreciably with angle of emission at angles of emission less than about $50^{\circ}$ from the normal. For conductors such as metals, the effect begins to appear at angles greater than 10 to $15^{\circ}$ from the normal.

Spectral emittance and absorptance are not strong functions of temperature, hence values obtained at one temperature can be used to predict values at other temperatures with only small errors, if the difference in temperature is not great. Total emittance, on the other hand, may or may not vary appreciably with temperature depending upon the shape of the spectral emittance curve.

Figure 1 shows the variation with temperature of the total hemispherical emittance of Inconel coated with two different ceramic coatings, and figure 2 shows the variation with temperature of total hemispherical emittance of electropolished and of oxidized type 321 stainless steel. Figure 3 shows variation with temperature of normal spectral emittance of alumina, thoria, magnesia and zirconia.

Reflectance is usually easier to measure than absorptance, and spectral absorptance is frequently computed from spectral reflectance by use of equation (3). In this case, the reflectance must be measured under conditions of hemispherical viewing, and the resulting absorptance is for

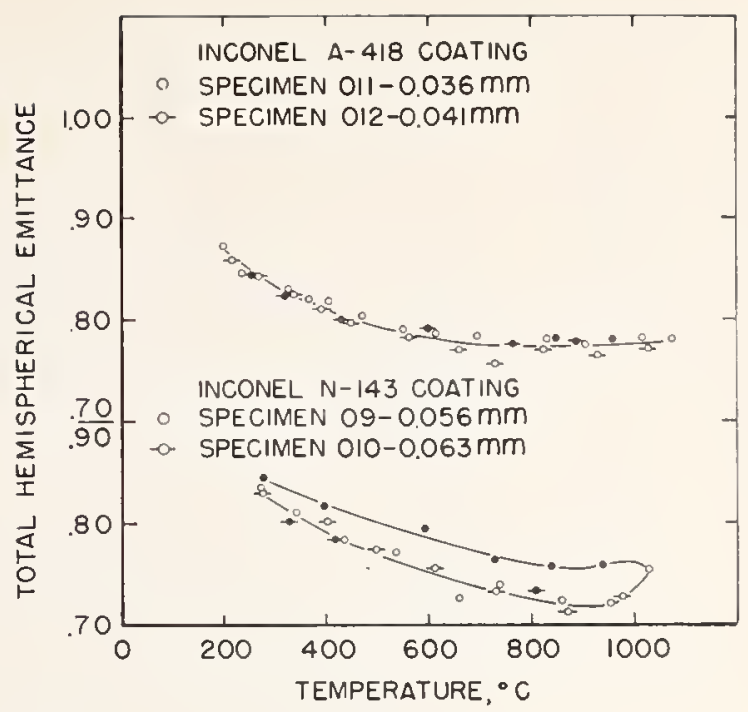

Figure 1. Total hemispherical emittance of composite specimens consisting of Inconel coated with NBS coating $A-418$, top curve, and NBS coating $N-143$, bottom curve.

Open points represent values obtained during heating; closed points, during cooling.

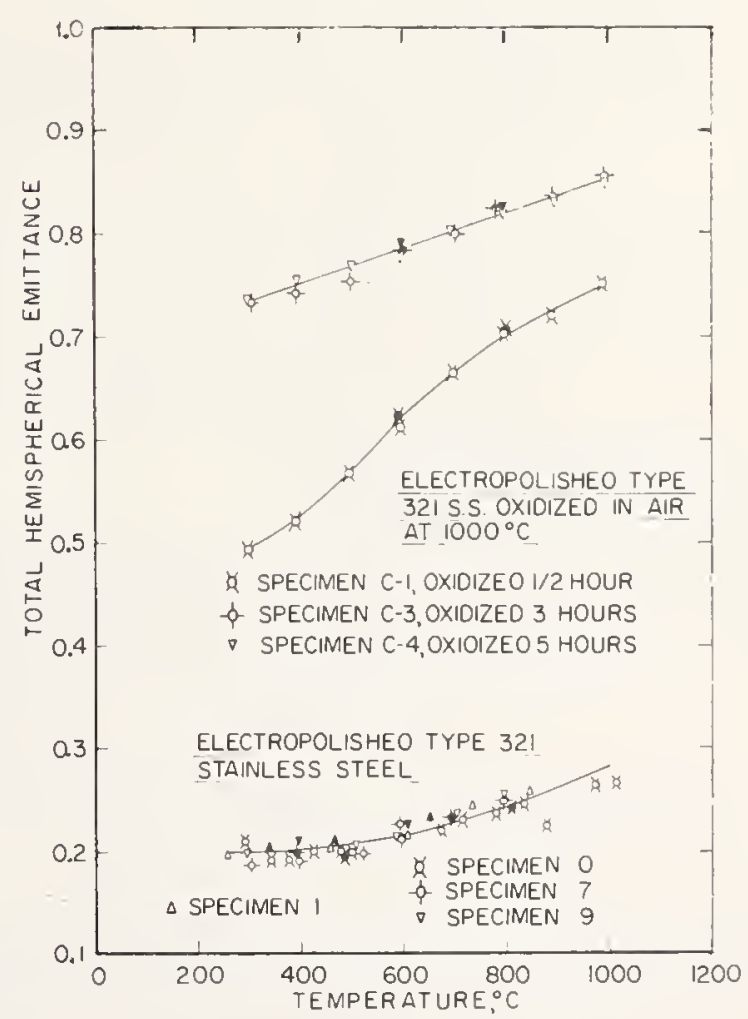

FIGURE 2. Total hemispherical emittance of type 321 stainless steel specimens in the electropolished condition, bottom curve, and after oxidation in air at $1000^{\circ} \mathrm{C}$ for various times, top curves.

Open points represent values obtained during heating; closed points, during cooling.

the same conditions of irradiation as the reflectance.

Thermal radiation properties are frequently referred to as surface properties. This is obviously 


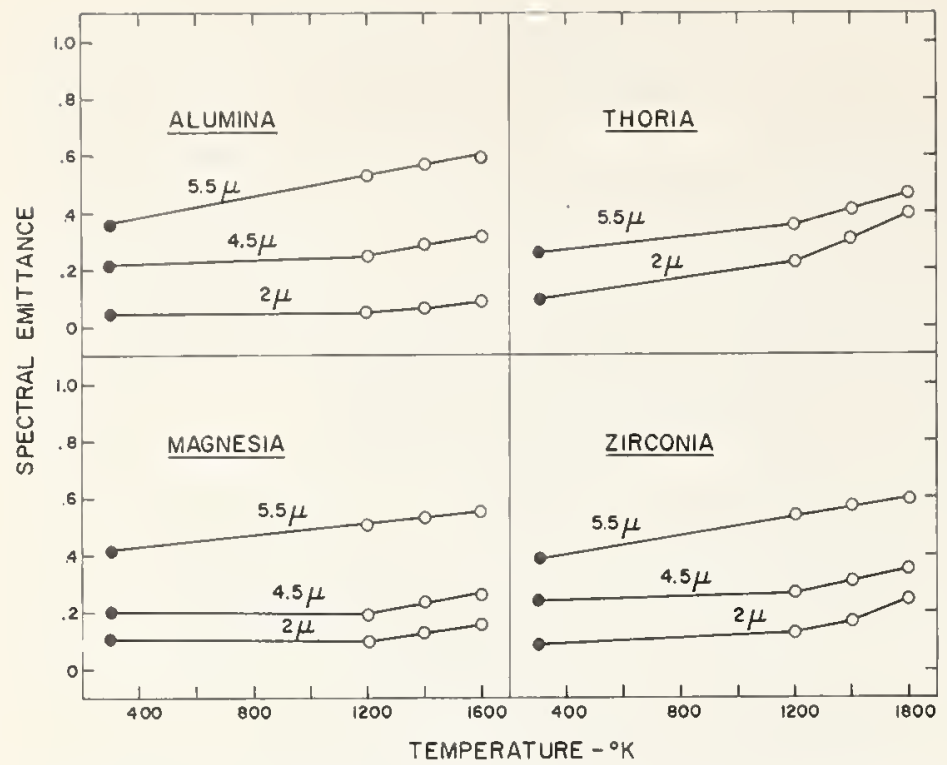

FIGURE 3. Temperature dependence of normal spectral emittance of four polycrystalline ceramic oxides.

a gross approximation, and is true only to the extent that the thermal radiation properties can be markedly affected by the surface condition of the specimen being measured, particularly its roughness. The processes of absorption and emission involve interaction of the electromagnetic waves with material particles, and hence cannot occur at a surface, which is only a twodimensional interface. While in one sense reflection occurs at an interface, even this process involves penetration of the incident electromagnetic waves beyond the interface to a depth that may be several times the wavelength.

The function of the surface, or interface, in determining thermal radiation properties is that it affects the amount and geometric distribution of the reflected flux, and the amount and distribution of the flux that penetrates the interface to where it can be absorbed and transmitted. It also affects the fraction of the internally generated flux that penetrates the interface and is emitted, and to a lesser extent, its geometric distribution. This will be discussed in more detail later.

Surface contamination, even in amounts that are difficult to detect by normal chemical means, can also significantly affect thermal radiation properties.

For metals, all of which have a very high absorption coefficient over most of the wavelength range of thermal radiation, the processes of emission, absorption and reflection occur in a very thin surface layer, perhaps only a few hundred atomic diameters in thickness. Hence for metals, it is a fair approximation to say that the thermal radiation properties are surface properties. Ceramic materials, on the other hand, usually have much lower absorption coefficients than do metals, at least at some wavelengths, and for such materials the thermal radiation properties must be considered to be volume properties.
The thermal radiation properties of a specimen can be affected by the contour of its surface. The specimen will have the highest reflectance or the lowest emittance if its surface is completely smooth. Increasing surface roughness will decrease reflectance and increase emittance. In the case of a metal, the effect can be large. For instance the total hemispherical emittance of type 321 stainless steel at $400{ }^{\circ} \mathrm{C}$ increased from about 0.2 in the electropolished condition to about 0.55 when sandblasted [3]. The normal spectral emittance of polycrystalline ceramics, on the other hand, has been shown to be nearly independent of surface roughness [4].

Bennett [5] has derived the following equation relating reflectance of a metal at normal incidence to surface roughness:

$$
\rho / \rho_{0}=\exp -(4 \pi \sigma / \lambda)^{2}+32 \pi^{4}(\sigma / \lambda)^{4}(\Delta \alpha / m)^{2},
$$

in which $\rho$ is the reflectance of a rough metal surface for normally incident flux reflected into an optical system having an acceptance solid angle with a half angle $\Delta \alpha(\Delta \alpha=\pi / 2$ for hemispherical collection), $\rho_{0}$ is the reflectance of a perfectly smooth surface of the same metal under the same conditions, $\sigma$ is the root-mean-square (rms) roughness of the surface, $\lambda$ is the wavelength of the incident radiation, and $m$ is the rms slope of the surface.

The first term, $\exp -(4 \pi \sigma / \lambda)^{2}$, accounts for interference effects, and predominates when $\sigma / \lambda$ $<<1.0$. The second term accounts for geometric effects, and predominates when $\sigma / \lambda>>1.0$.

The treatment becomes almost hopelessly complex at oblique incidence, because of polarization effects. If such effects are neglected, equation (9) can still be used by substituting for $\sigma$ the term $\sigma$ $\cos \theta$, where $\theta$ is the angle of incidence, measured from the normal to the gross plane of the surface.

\section{Wavelength Range of Interest}

The spectral distribution of the radiance (the flux per unit wavelength interval, unit solid angle and unit area of source projected normal to the direction of emission) of a blackbody radiator is given by the Planck equation:

$$
\begin{aligned}
L_{\lambda}=1.19096 \lambda^{-5} & \\
\times & {[\exp (1.43879 / \lambda T)-1]^{-1} \times 10^{-16}, }
\end{aligned}
$$

where $L_{\lambda}$ is the radiance in watts per square meter, steradian, and meter wavelength interval $\left(\mathrm{Wm}^{-2} \mathrm{sr}^{-1}\right) \mathrm{m}^{-1}, \lambda$ is wavelength in meters and $T$ is temperature in kelvins. A plot of $L_{\lambda}$ as a function of $\lambda$ for a blackbody at $1000 \mathrm{~K}$ is shown in figure 4 .

The nature of equation (10) is such that there is some energy emitted at all wavelengths between zero and infinity, but the amount at very short and very long wavelengths is so small that it can be neglected. At any temperature, 99 percent of 


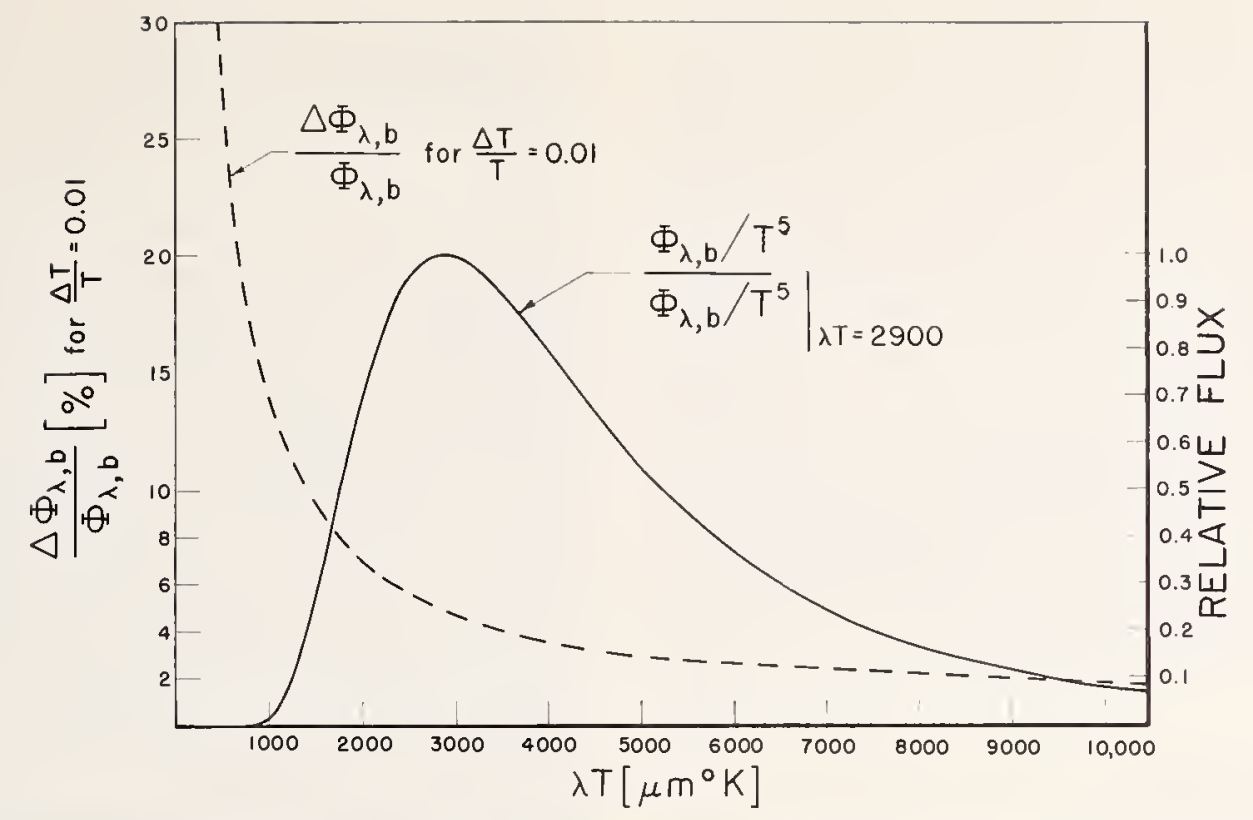

FIGURE 4. Relative spectral radiance of a blackbody radiator, plotted as a function of $\lambda \mathrm{T}$ in $\mathrm{cmK}$, curve $A$, and percentage increase in spectral radiance produced by $a$ one percent increase in temperature, plotted as a function of $\lambda \mathrm{T}$ in $\mathrm{cm} K$, curve $B$.

the energy is emitted between wavelengths represented by values of $\lambda T$ of 1322 and $29372 \mu \mathrm{mK}$, and about 25 percent of the energy is at wavelengths shorter than the peak. At room temperature $(300 \mathrm{~K}) 99$ percent of the energy is at wavelengths between 4.4 and $97.9 \mu \mathrm{m}$. As a practical matter, most determinations of thermal radiation properties cover the wavelength range of 1 to $15 \mu \mathrm{m}$, with others covering the extended range from about 0.25 to $40 \mu \mathrm{m}$. There is considerable interest in spectral emittance at 0.65 $\mu \mathrm{m}$, the wavelength of ten used in optical pyrometry. Most of our discussion will be confined to the 1 - to $15-\mu \mathrm{m}$ range. Figure 5 shows how the wavelength limits for 99 percent of the radiant energy change with temperature.

\section{Theoretical Considerations}

When two transparent media of different indices of refraction are separated by an optically smooth interface, a beam of radiation striking the interface will be refracted according to Snell's law, and reflected according to Fresnel's law. Snell's law is expressed as:

$$
n_{1} \sin \theta_{i}=n_{2} \sin \theta_{r}
$$

where $n_{1}$ is the index of refraction of the medium from which the beam is incident, $\theta_{i}$ is the angle of incidence, measured from the normal to the interface, $n_{2}$ is the index of refraction of the medium into which the beam is refracted, and $\theta_{r}$ is the angle of refraction, again measured from the normal to the surface. The equation is valid over the entire range of $\theta_{1}$ when $n_{2}>n_{1}$. When $n_{1}>n_{2}$, the equation is still valid at values of $\theta_{i}$ less than the critical angle. At angles greater than the critical angle, total internal reflection will occur. The critical angle, $\theta_{c}$, is defined by the equation

$$
\theta_{c}=\sin ^{-1}\left(n_{2} / n_{1}\right)
$$

Fresnel's law, in its general form, is expressed as:

$$
\begin{aligned}
\rho=1 / 2\left[\frac{n_{1} \cos \theta_{i}-n_{2} \cos \theta_{r}}{n_{1} \cos \theta_{i}+n_{2} \cos \theta_{r}}\right]^{2} \\
+1 / 2\left[\frac{n_{2} \cos \theta_{i}-n_{1} \cos \theta_{r}}{n_{2} \cos \theta_{i}+n_{1} \cos \theta_{r}}\right]^{2}
\end{aligned}
$$

where $\rho$ is the reflectance of the interface for unpolarized incident flux. Equation (13) can be reduced, by substituting for $n_{1}$ or $n_{2}$ from equation (11), to

$$
\rho=1 / 2\left[\frac{\sin ^{2}\left(\theta_{i}-\theta_{r}\right)}{\sin ^{2}\left(\theta_{i}+\theta_{r}\right)}\right]+1 / 2\left[\frac{\tan ^{2}\left(\theta_{i}-\theta_{r}\right)}{\tan ^{2}\left(\theta_{i}+\theta_{r}\right)}\right]
$$

In equations (13) and (14) the first term gives the reflectance for radiation polarized perpendicular to the plane of incidence, and the second term that for radiation polarized parallel to the plane of incidence. The place of incidence is the plane containing the direction of incidence and the normal to the interface (surface). There is some disagreement in the literature as to what constitutes the plane of polarization. In this paper it is defined as the plane containing the $\mathbf{E}$ (electric) vector.

It can be seem from equation (14) that when $\left(\theta_{i}+\theta_{r}\right)=\pi / 2, \tan \left(\theta_{i}+\theta_{r}\right)$ goes to infinity, and the reflectance for radiation polarized parallel to the plane of incidence goes to zero. The angle at 

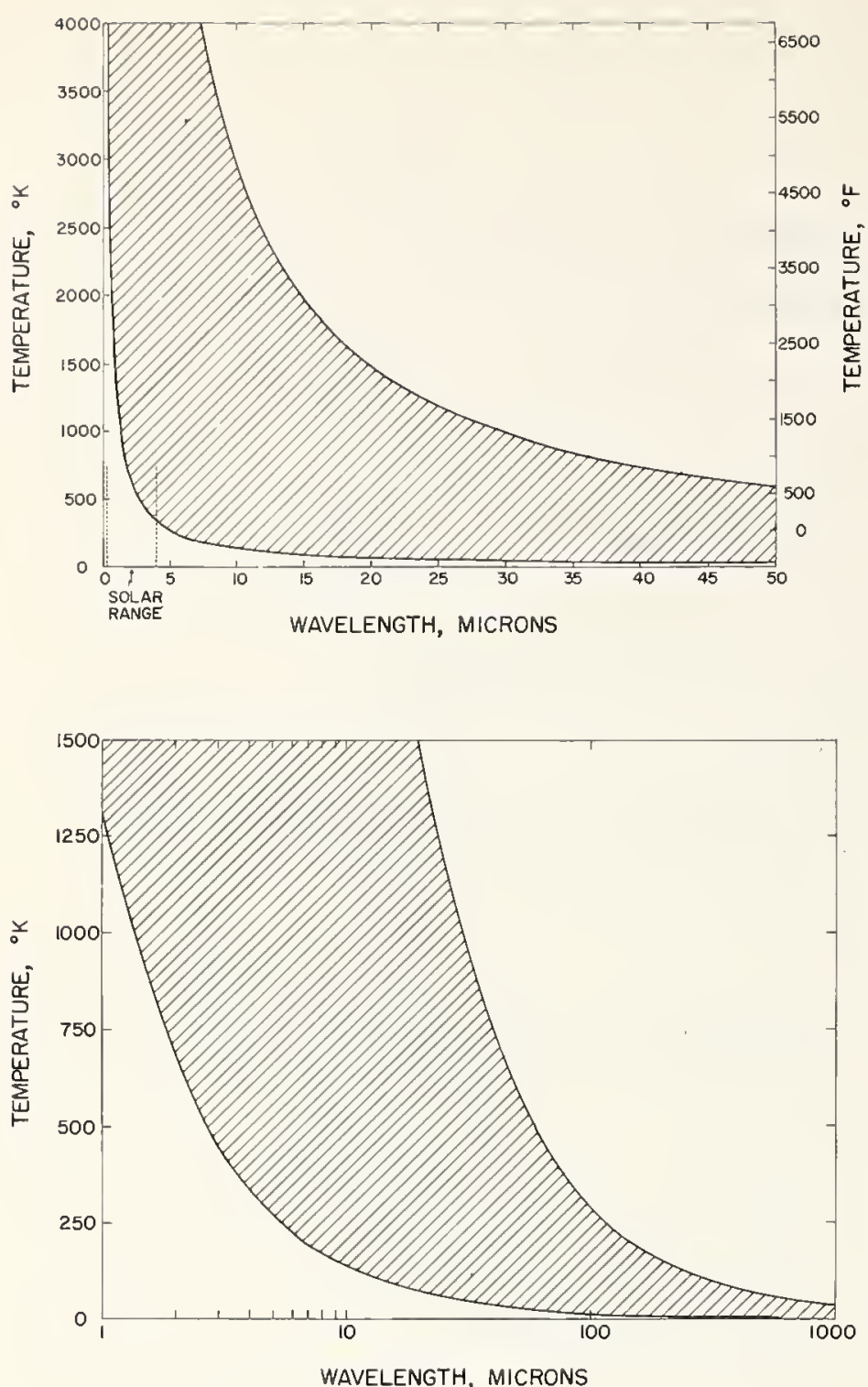

FIGURE 5. Wavelength limits encompassing 99 percent of the flux emitted by a blackbody radiator at the indicated temperature, for high temperatures, top curve; and low temperatures, bottom curve.

which this occurs is known as the Brewster's angle, $\theta_{B}$, and is defined as:

$$
\theta_{B}=\tan ^{-1} n_{2} / n_{1}
$$

Equations (11) through (15) were derived for a perfect dielectric that is optically homogeneous. Such a material has no absorption. Although no real material conforms completely to this model, the equations can be applied with no significant error to transparent dielectric materials in general. For materials of which the absorption coefficient is not negligible, the complex index of refraction, $n^{*}$, must be substituted for the simple index of refraction, $n$, in the equations.

$$
n^{*}=n+i k
$$

where $i=\sqrt{ }-1$, and $k$ is the absorption index.

$$
k=a \lambda / 4 \pi
$$

where $a$ is the absorption coefficient, and $\lambda$ is wavelength.

The absorption coefficient, $a$, is defined by the equation

$$
\Phi_{x}=\Phi_{0} \exp (-a x)
$$

where $\Phi_{x}$ is the flux remaining after traversing a thickness $x$ of nonscattering material, and $\Phi_{0}$ is the flux at $x=0$.

Let us assume that we have an optically homogeneous specimen of a dielectric material, having two parallel faces, $A$ and B, that are optically smooth and essentially infinite in extent, so that edge effects can be neglected. We will further assume that interference effects can be neglected. This is a reasonable model for a sheet of polished plate glass, or a single crystal of quartz or sodium chloride, for instance. When a beam of radiation is incident on surface $A$ of such a specimen, it is partly reflected and partly refracted, according to the Fresnel equation (13) or (14). The radiation that is refracted is transmitted undeviated until it hits the second surface, $\mathrm{B}$, where it again undergoes reflection and refraction according to the Fresnel equation. In its course through the material, it is partially absorbed according to equation (18). By following the beam until it is completely absorbed or has left the specimen, we can compute the reflectance, transmittance, and absorptance of the specimen. All of the flux initially reflected by surface A plus that leaving the specimen through surface A makes up the reflected portion, that leaving through surface B makes up the transmitted portion, and that absorbed makes up the absorbed portion. These portions divided by the incident flux are the reflectance, transmittance, and absorptance.

Gardon [6] gives a very complete account of the emittance of isothermal transparent materials. He derives a spectral volume emissive power, $j_{\lambda}$, defined by the equation:

$$
j_{\lambda}=a_{\lambda} n^{2} M_{B, \lambda} \pi^{-1}
$$

where $M_{B, \lambda}$ is the spectral exitance of a blackbody radiator at the temperature of the specimen. $M_{B, \lambda}$ can be obtained from the Planck equation. His final equation for the spectral radiance on the inside of the surface, $L_{i \lambda} \varphi_{X}$, at wavelength $\lambda$ and angle $\varphi$ for a specimen of thickness $X$ is:

$$
L_{\mathrm{I} \lambda \varphi \mathrm{X}}=n_{2} \frac{M_{B}, \lambda}{\pi}[1-\exp (-a X / \cos \varphi) \cos \varphi]
$$

and for the internal spectral irradiance on the inside of the surface, $E_{i \lambda x}$, at wavelength $\lambda$ for a specimen of thickness $X$,

$$
E_{\imath \lambda \mathrm{X}}=n^{2} M_{b, \lambda}\left[2-\exp \left(-a_{\lambda} X\right)-\left(a_{\lambda} X\right)^{2}-\xi_{1}\left(-a_{\lambda} X\right)\right]
$$


where the exponential integral $\xi_{i}(-p)$ is defined as $\xi_{i}(-p)=\int_{p}^{\infty} e^{-q} q^{-1} d q$. The flux that is incident internally on the surface of the specimen undergoes reflection and refraction. All of the flux that is incident at angles greater than the critical angle is totally reflected. For a specimen thick enough to be opaque, this is a fraction $1-1 / n^{2}$ of the total flux. For a transparent specimen, the fraction is larger. The critical angle, $\theta_{c}$, is defined by equation (12). The fraction of the internally incident flux that is incident at angles less than $\theta_{c}$ is refracted to fill the entire hemisphere on the external side of the surface, and the fraction transmitted at any angle can be computed from equation (14) by setting $\theta_{r}$ as the internal angle of incidence and $\theta_{i}$ as the external angle of refraction.

For transparent birefringent single crystals each ray appears as two plane polarized components with their planes of polarization normal to each other, and the fraction of each component is determined by the orientation of the ray relative to the crystal axes of the specimen. For such a specimen the index of refraction will be different for the two portions of the ray, and because the two portions are plane polarized, the two parts of equation (14) must be used separately. As a further complication, unless the plane of incidence happens to include a crystallographic axis of the crystal, neither of the planes of polarization will be in the plane of incidence. The situation where there is a thermal gradient normal to the surface of the specimen has been treated in a second paper by Gardon [7].

For an optically inhomogeneous specimen, the situation becomes so complex that Gardon's approach is no longer valid. A single ray undergoes so many internal reflections and refractions that it soon loses its identity. Practically all polycrystalline materials fall into the category of intensely scattering materials, which have been treated by Kubelka [8] and by Hamaker [9]. The scattering occurs by reflection and refraction whenever the index of refraction of the material changes along the path of the ray, primarily at pores, grain boundaries, or inclusions. For materials of this type, an entirely different mathematical treatment is required.

Consider a specimen of very large area, so that edge effects may be neglected, that is irradiated diffusely over its entire area. Under steady-state conditions, the incident flux not reflected at the surface will penetrate into the specimen, diminishing with the depth of penetration; the amount of the diminution at any point along a plane parallel to the surface will be a function only of the distance of the plane from the surface. Under these conditions a one-dimensional analysis can be used. All flux lost by lateral scattering will be compensated by an equal gain through similar scattering from the surrounding area.
The flux is separated into two portions, I, traveling outward from the interior of the specimen, and $J$, traveling inward from the surface, both traveling normal to the plane of the surface. Both are attenuated by scattering and absorption. A diffuse absorption coefficient, $K$, is defined by equating $K I d x$ to the reduction in $I$ by absorption within an infinitesimal layer $d a$. It should be noted that the diffuse absorption coefficient, $K$, is exactly twice the absorption coefficient, $a$, referred to previously. A diffuse backscattering coefficient $S$ is similarly defined by equating $S I d x$ to the flux scattered backwards from $I$ and added to $J$ in the layer $d x$.

Within the distance $d x$ the flux $I$ will be diminished by absorption and backscattering, and reinforced by the flux backscattered by $J$. Hence we can write:

$$
\begin{aligned}
& d I / d x=-(K+S) I+S J, \text { and } \\
& d J / d x=(K+S) J-S I .
\end{aligned}
$$

These are the basic differential equations used by Kubelka [8], Hamaker [9], and others.

Klein [10] derives the following equations for those materials where there is essentially no specular reflection at the surface. These equations should apply to most unglazed ceramic specimens, particularly if the surface is not polished:

$$
\begin{aligned}
& \rho=\frac{\left(1-\beta_{0}{ }^{2}\right) \sinh \sigma_{0} D}{\left(1+\beta^{2}\right) \sinh \sigma_{0} D+2 \beta_{0} \cosh \sigma_{0} D} \\
& \alpha=\frac{2 \beta_{0}\left(B_{0} \sinh \sigma_{0} D+\cosh \sigma_{0} D-1\right)}{\left(1+\beta^{2}\right) \sinh \sigma_{0} D+2 \beta_{0} \cosh \sigma_{0} D} \\
& \tau=\frac{2 \beta_{0}}{\left(1+\beta_{0}{ }^{2}\right) \sinh \sigma_{0} D+2 \beta_{0} \cosh \sigma_{0} D}
\end{aligned}
$$

in which $\beta_{0}=[K /(K+2 S)]^{1 / 2}$,

$$
\begin{aligned}
\sigma_{0} & =[K(K+2 S)]^{1 / 2}, \text { and } \\
D & =\text { thickness of specimen. }
\end{aligned}
$$

Equation (24) has been further developed [11] to include the effects of external specular reflectance of the specimen and internal specular reflection at both surfaces of the specimen. The final equation is:

$$
\begin{aligned}
\rho(2 \pi ; 2 \pi)= & \rho_{e}+\left(1-\rho_{e}\right)\left(1-\rho_{i}\right) \\
& \times \frac{\left(1-\beta_{0}\right) M e^{\sigma_{0} D}-\left(1+\beta_{0}\right) O e^{-\sigma_{0} D}}{M N e^{\sigma_{0} D}-O P e^{-\sigma_{0} D}}
\end{aligned}
$$

where $\rho(2 \pi ; 2 \pi)$ is the hemispherical reflectance of the specimen for completely diffuse irradiation, $\rho_{e}$ is the specular reflectance of the specimen front surface for externally incident diffuse flux, $\rho_{i}$ is the specular reflectance of the specimen front surface for internally incident diffuse flux, $\rho_{s}$ is 
the specular reflectance of the specimen back surface for internally incident diffuse flux, and

$$
\begin{gathered}
M=\left(1+\beta_{0}\right)-\rho_{s}\left(1-\beta_{0}\right), \\
N=\left(1+\beta_{0}\right)-\rho_{i}\left(1-\beta_{0}\right), \\
O=\left(1-\beta_{0}\right)-\rho_{s}\left(1+\beta_{0}\right), \\
P=\left(1-\beta_{0}\right)-\rho_{i}\left(1+\beta_{0}\right) .
\end{gathered}
$$

For a specimen thick enough to be completely opaque, equation (27) reduces to:

$$
\rho(2 \pi ; 2 \pi)=\rho_{e}+\frac{\left(1-\rho_{e}\right)\left(1-\rho_{i}\right)\left(1-\beta_{0}\right)}{\left(1+\beta_{0}\right)-\rho_{i}\left(1-\beta_{0}\right)}
$$

and for such a specimen:

$$
\epsilon(2 \pi)=1-\rho(2 \pi ; 2 \pi),
$$

which states that the hemispherical emittance is equal to one minus the hemispherical reflectance for diffuse irradiation.

The absorption coefficient, either $a$ or $K$, is primarily a function of composition, and is usually not greatly affected by the structure. However, for some impurities, the effect on the absorption coefficient will depend on whether they are present in solution in the crystals of the primary constituents or as separate crystallites at grain boundaries, and if present as crystallites, by their size. The scattering coefficient, on the other hand, is markedly affected by the structure of the specimen, particularly the grain size and porosity.

A few years ago we observed an interesting example of the effect of the form in which impurities are present on the thermal radiation properties of a ceramic material. We are making some measurements on sintered magnesium oxide. The material as received was a dirty brown color, which we assumed was due to contamination by iron oxide. A specimen was treated with hydrochloric acid in an effort to remove the contaminant, then washed and heated with a torch to drive off any remaining chlorides. After this treatment the specimen was white, and we assumed that the contaminant had been removed. However, when the specimen was heated in a furnace for measurement of its emittance, it regained its dirty brown color on cooling to room temperature. We then discovered that the color change was reversible. When the specimen was cooled rapidly from a high temperature, it was white; when cooled slowly, it was dirty brown. The explanation offered was that the impurity, presumably iron oxide, went into solution in the $\mathrm{MgO}$ crystals at high temperature, and remained in solution on rapid cooling, where it had little effect on reflectance. On slow cooling, the impurity precipitated out at grain boundaries, where it had an appreciable effect on reflectance.
Typical values of $\beta_{0}, \sigma_{0}, S$ and $K$ are as follows. For a highly opaque white porcelain enamel with a reflectivity of about $0.9, \beta_{0}=0.05$ and $\sigma_{0}=50$ $\mathrm{mm}^{-1}, K=2.5 \mathrm{~mm}^{-1}$ and $S=500 \mathrm{~mm}^{-1}$. For a polycrystalline ceramic with a reflectivity of about 0.82 that is essentially opaque at a thickness of $3 \mathrm{~mm}$, the values would be $\beta_{0}=0.1$, $\sigma_{0}=1.67 \mathrm{~mm}^{-1}, K=0.167 \mathrm{~mm}^{-1}$ and $S=8.25$ $\mathrm{mm}^{-1}$. For a material with a reflectivity of about 0.6 that is essentially opaque at a thickness of $25 \mathrm{~mm}, \beta_{0}=0.25, \sigma_{0}=0.2 \mathrm{~mm}^{-1}, K=0.05 \mathrm{~mm}^{-1}$ and $S=0.375 \mathrm{~mm}^{-1}$. A material such as an opalescent glass might have these properties. In general, $K$ can vary from near zero for materials composed of highly transparent crystals, to a value as high as $50,000 \mathrm{~mm}^{-1}$ for some metals at some wavelengths. $S$ will vary from near zero for an optically homogeneous material such as an optical glass or a pure metal to a high of perhaps $1000 \mathrm{~mm}^{-1}$ for some white paints. It should be emphasized that for light-scattering materials the reflectivity is related to $\beta_{0}$, which is a function of the ratio of $K$ to $S$, and not to the absolute value of either $K$ or $S$, while the extinction coefficient, which determines the opacity of a material, is related to the product $S K$.

The usual laws of reflection and refraction at an interface between two materials of different indices of refraction, equations (14) and (11) above, apply only if there is a finite thickness of material on each side of the interface. This finite thickness is of the same order of magnitude as the wavelength of the radiation involved. The phenonemon can be explained quantitatively in terms of frustrated internal reflectance [12]. If two $45^{\circ}$ totally reflecting prisms are placed so that their totally reflecting surfaces are parallel, and are then brought slowly together, the totally reflecting surfaces will begin to transmit as they approach each other. The transmission for polarized radiation with its electric vector in the plane of incidence is given by

$$
\frac{\rho}{\tau}=\frac{\left(\mathrm{N}^{2}-1\right)^{2}}{N^{2}\left(N^{2}-2\right)} \sinh ^{2}\left[\pi \frac{\sqrt{2 d}}{\lambda} \sqrt{N^{2}-2}\right]
$$

where $\rho$ is the reflectance of the interface, $\tau$ is its transmittance $(\rho+\tau=1), N$ is the index of refraction ratio, $d$ is the distance separating the prisms and $\lambda$ is the wavelength of the radiation involved. For these conditions, $\rho / \tau=1 / 4$ that given in equation (30) when the electric vector is normal to the plane of incidence. The index of refraction ratio is $N=n_{1} / n_{2}$, where $n_{1}$ is the index of the prisms and $n_{2}$ is that of the layer. When the layer is vacuum, $N=n_{1}$. When it is air, $N=n_{1}$ with an error of about 3 parts in 10,000 .

Because of this phenomenon, there is a marked effect of the size of particles such as inclusions, crystallites, or pores on the scattering efficiency of each particle. The scattering efficiency per particle will decrease markedly with diameter 
below a critical diameter. As a rough approximation, the total scattering power will be proportional to the total interface area, and from this standpoint the scattering particles should be as small as possible. The net result of the two effects is that there is an optimum size for scattering efficiency. A study of rutile pigments in paint [13] has shown that in this case the optimum diameter of the pigment particles is about 0.4 times the wavelength of the light being scattered.

The scattering power of a particle diminishes as the distance separating it from its nearest neighbors becomes less than the critical distance mentioned above. On this basis the maximum scattering power per particle would occur when particles of the optimum size are separated by a distance at least equal to their diameter. If spherical particles were arranged in a cubic array, this would occur at volume concentrations of particles of less than about 6.6 percent. Actually the total scattering for all particles increases as the number of particles increases, because of the increase in total interface area, until the particles get so closely packed that the decrease in effective interface area is greater than the increase in total interface area. Reference [13] indicates that for the rutile pigment studied, the maximum total scattering occurred at a pigment volume concentration of about 25 percent.

Radiant heat transfer within and from optically inhomogeneous materials with no specular reflection at the surface is covered by Hamaker [9] and Klein [10], for the case where there is a thermal gradient in the material.

\section{Thermal Gradient Problems}

The general physical properties of ceramic materials combine to make thermal gradients a serious problem in the evaluation and use of thermal radiation properties of ceramics. Ceramic materials in general tend to be somewhat translucent, and hence emit and absorb thermal radiation within a surface layer of appreciable thickness. Ceramic materials in general also have low thermal conductivity and high total emittance as compared to metals. These properties combine to produce th ermal gradients within a heated specimen unless careful precautions are taken to minimize such gradients. The gradients tend to be normal to a surface that is emitting or absorbing thermal radiation. As a further complication, the gradients tend to be nonlinear near such a surface.

When a specimen is emitting from a surface layer of appreciable thickness with a large thermal gradient normal to the surface, it has no unique temperature, and it is difficult to define an effective temperature for the emitting layer. Emittance is defined as the ratio of the flux emitted by a specimen to that emitted by a blackbody radiator at the same temperature and under the same conditions. It is thus necessary to define an effective temperature for the nonisothermal specimen before its emittance can be evaluated. If the effective temperature is defined as that of the surface, a specimen with a positive thermal gradient (surface cooler than interior) will emit at a greater rate than an isothermal specimen at the same temperature, and in some cases may even have an "emittance" greater than 1.0. If the gradient is negative (surface hotter than interior) it will emit at a lesser rate. If the "effective temperature" is defined as that of an isothermal specimen that emits at the same rate as the nonisothermal specimen, we find that the "effective temperature" is difficult to evaluate even if the extinction coefficient and thermal gradient are accurately known. Also, if spectral emittance is desired, we find that the extinction coefficient, and hence the thickness of the emitting layer, varies with wavelength, and we have the anomolous situation of a specimen whose effective temperature varies with wavelength. There is no completely satisfactory solution to this problem. The Planck equation does not apply to a nonisothermal specimen, and any attempt to apply it to such specimens will result in errors. Because of these considerations, all measurements of emittance of ceramic specimens should be made under conditions where thermal gradients within the specimen are as small as possible.

\section{Methods of Measurement}

The properties of reflectance, absorptance, and transmittance for most materials, and particularly ceramic materials, are not strong functions of temperature, and hence these properties can be measured on nonisothermal specimens with but little error. However, such measurements are not easy to make on hot specimens. First, all of the reflected or transmitted flux must be measured, which involves collection over a hemisphere. Such measurements are difficult at wavelengths beyond about $2.5 \mu \mathrm{m}$, where conventional integrating sphere coatings decrease markedly in reflectance. Second, the small amount of reflected or transmitted flux must be measured in the presence of a much larger amount of flux emitted by the hot specimen. Such measurements require the use of chopped incident flux and synchronous amplification of the signal from the detector.

Because of the difficulties mentioned above, most measurements of thermal radiation properties of ceramic materials have been direct emittance measurements, where the flux emitted by a hot specimen is measured and compared to that emitted by a blackbody reference at the same temperature.

Any temperature difference between the specimen and reference blackbody will cause an error in the measured emittance that is much larger than the percentage difference in absolute temperature. For total emittance measurements, the percentage error in emittance is approximately four times the percentage difference in tempera- 
ture. This is illustrated in figure 4 for a one percent difference in temperature. As seen in figure 4, curve $B$, the error in spectral emittance can be much larger at wavelengths shorter than the peak in the Planck curve, $A$.

In most cases where direct emittance measurements of ceramic specimens have been made, an effort has been made to heat the specimen under conditions where the thermal gradients are kept as small as possible. This may involve alternate heating and viewing of a moving specimen. The specimen is enclosed in a furnace where it is heated then moved in front of a cooled port where it is viewed briefly. It may also involve alternate heating by radiation and viewing of a stationary specimen, where the periods of heating and viewing are controlled by rotating shutters. Movable specimens have been tested in the form of a rotating disc $[14,15,16,17]$, a rotating cylinder [18,19], or as dises mounted on an oscillating beam [20]. Stationary specimens have been heated in an arcimage furnace $[21,22,23,24]$ and in a solar furnace [25].

In the case of a moving specimen that is alternately heated and cooled, the specimen will reach a steady-rate temperature, and if the motion is rapid enough that the viewing time is short, the temperature fluctuation of the viewed surface will be so small that it can be neglected.

Photon conduction does not generate a thermal gradient in a material. The photons pass through the material without affecting it in any way until they are absorbed. As a result, a specimen that is heated by radiation tends to have a small thermal gradient normal to the heated surface. The radiation is absorbed within the surface layer from which the emitted radiation originates, and after the specimen has reached a steady state condition, there is little phonon conduction within the surface layer. If the time periods during which the specimen is alternately heated and viewed are brief, the temperature fluctuation of the specimen will be small. Usually these periods are only a fraction of a second. With both moving specimens and radiantly heated stationary specimens the thermal gradients normal to the surface can be reduced to a point where they do not introduce a significant error into the measurement.

The rotating cylinder method used at the $\mathrm{Na}$ tional Bureau of Standards [18] will be described briefly. The specimen was a hollow cylinder $25 \mathrm{~mm}$ (one inch) in diameter and $25 \mathrm{~mm}$ (one inch) high, with an outer surface ground to be round to about $.05 \mathrm{~mm}(0.002 \mathrm{in})$. A cross sectional view of the furnace is shown in figure 6 . The specimen was mounted on top of an alumina pedestal, which revolved inside a platinum-wound resistance furnace. The winding of the furnace was designed so that there was no axial thermal gradient along the specimen. The specimen revolved in front of a water-cooled viewing port. A theoretical analysis [26], confirmed by temperature measurements at a point near the surface of a rotating specimen, indicated (1) that the temperature drop at a point on the surface of the rotating specimen as it passed in front of the viewing port was inversely proportional to the speed of rotation of the specimen, and was only about $2 \mathrm{~K}$ at a speed of $50 \mathrm{rmp}$, and (2) that the temperature measured by a stationary, radiation-shielded, thermocouple located at the center of the hollow rotating specimen was the same within about $1 K$ as that measured by the thermocouple imbedded near the surface of the rotating specimen.

The flux from the hot specimen was focused on the entrance slit of one beam of a double beam infrared spectrometer, and the flux from a laboratory blackbody furnace was focused on the entrance slit for the second beam of the spectrometer. The blackbody furnace was controlled to the same temperature as the specimen. The spectrometer automatically scanned over the spectral range of 1 to $15 \mu \mathrm{m}$, and plotted the ratio of the signals from the two beams, which after correction for spectrometer errors, was the normal spectral emittance of the specimen.

Typical data at $1400 \mathrm{~K}$ for alumina, thoria, magnesia and zirconia are shown in figures 7, 8, 9, and 10 . The curves are similar in several respects. The emittance is low at short wavelengths, where the absorption coefficient for these materials is low, and high at long wavelengths, where the absorption coefficient is high. The location of the absorption edge, the steep part of the curve connecting the high and low portion, and its slope, is different for different materials. The height of the low-emittance and high-emittance portions of the curve is also different for the different materials. The effect of temperature on the spectral emittance at several wavelengths for these materials is shown in figure 3 . In general, the spectral emittance increases with an increase in temperature, and the percentage increase is greater at short wavelengths, where the emittance is low, than at long wavelengths, where the emittance is high. The absorption edge also tends to move toward a shorter wavelength as the temperature is increased, but the change is not large.

\section{Summary}

The terminology in the field of thermal radiation properties and radiant heat transfer has been reviewed, and the equations relating the thermal radiation properties of ceramics to their optical properties of index of refraction, absorption coefficient, and scattering coefficient have been given. The problems involved in measuring the thermal radiation properties of ceramic materials have been discussed, one specific method has been briefly described, and data on alumina, thoria, magnesia, and zirconia presented. 


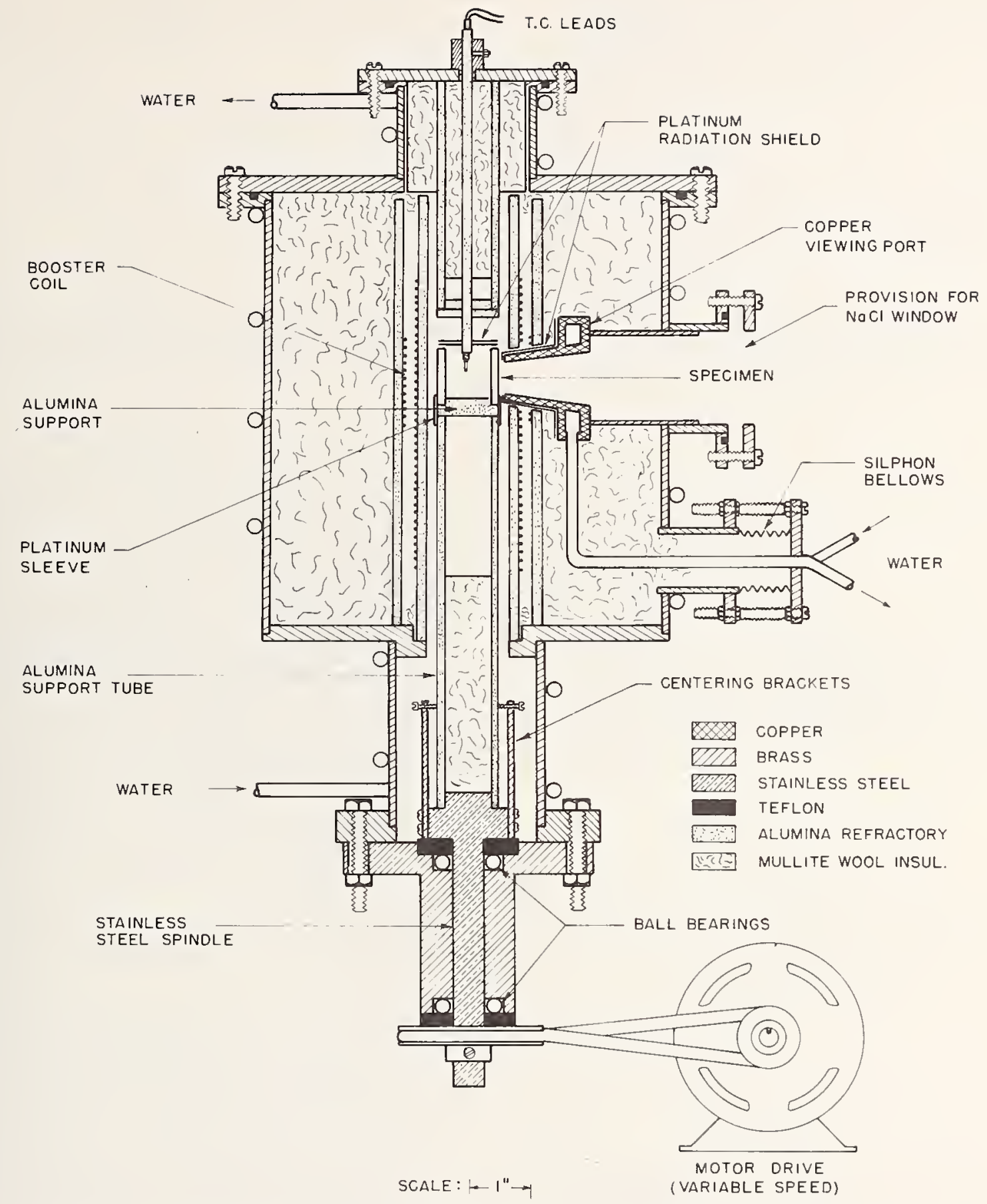

FIGURE 6. Cross-sectional view of the rotating cylinder specimen furnace used in measuring normal spectral emittance of ceramic materials. 


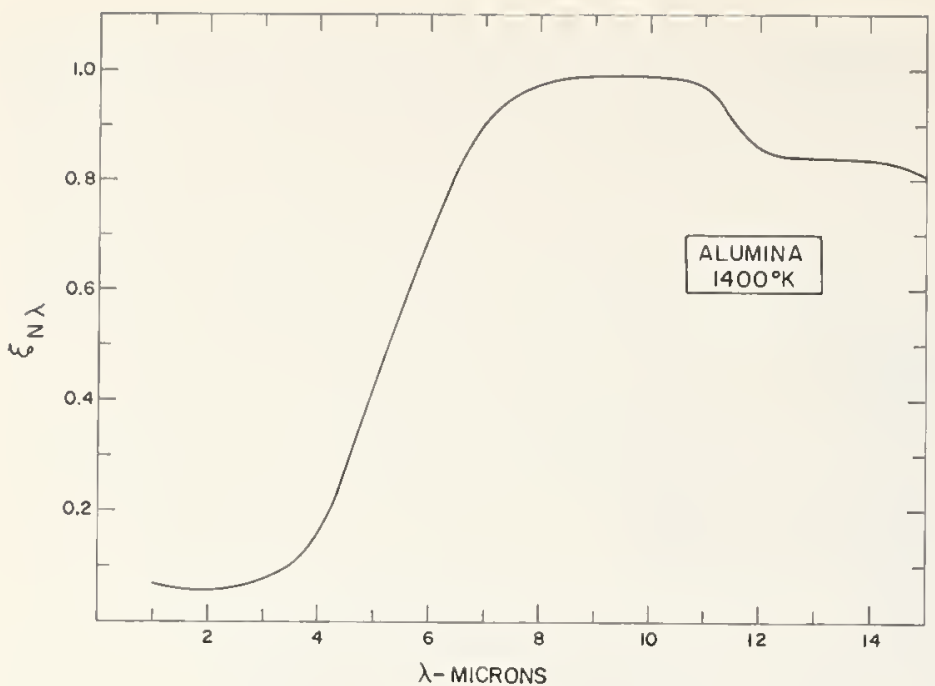

Figure 7. Normal spectral emittance of commercially pure alumina at $1400 \mathrm{~K}$.

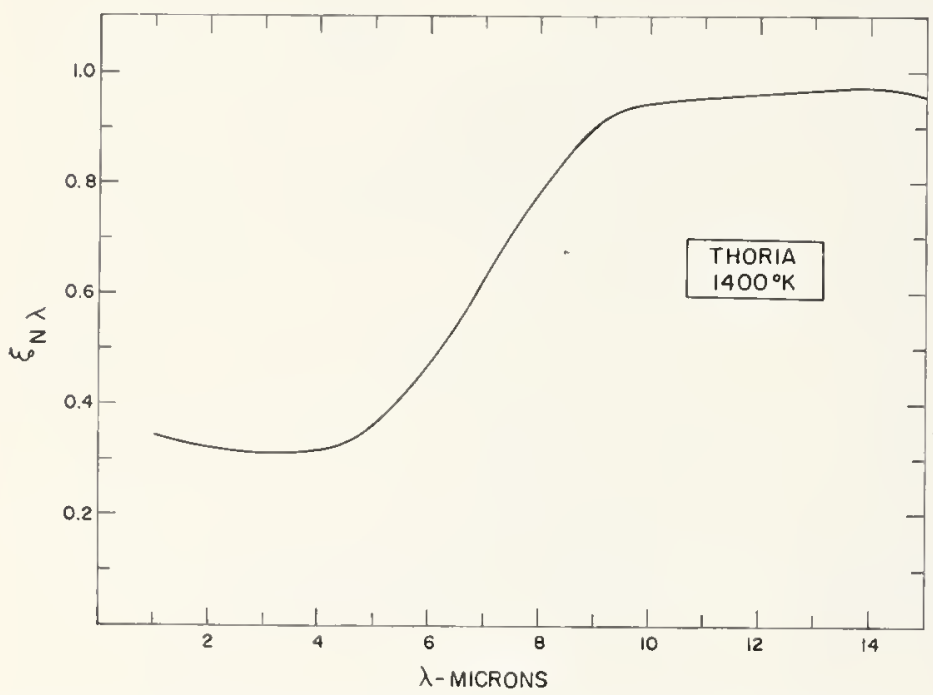

Figdre 8. Normal spectral emittance of commercially pure thoria at $1400 K$.

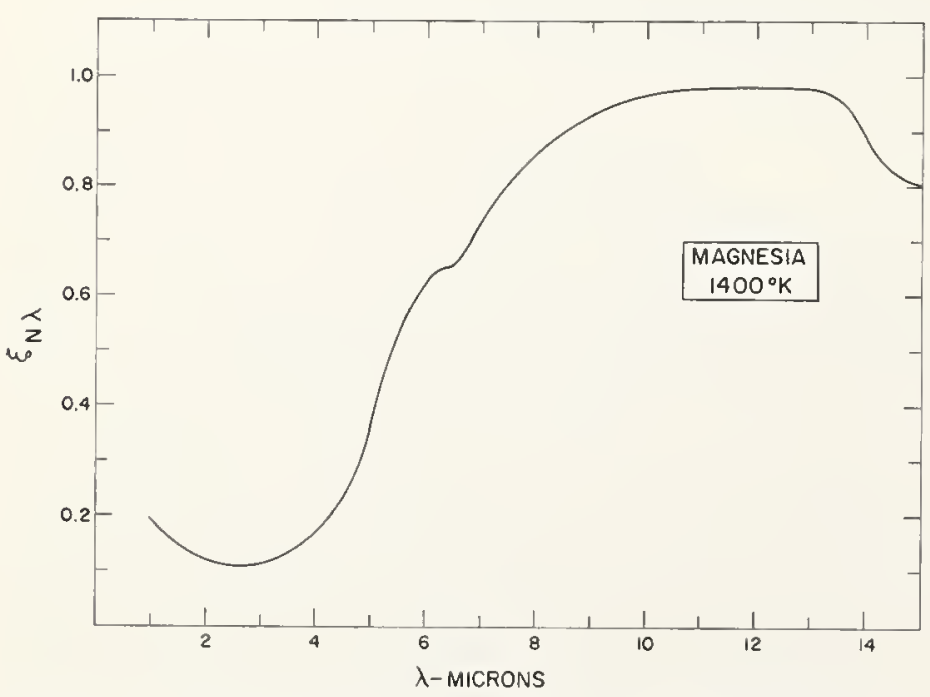

Figure 9. Normal spectral emittance of commercially pure magnesia at $1400 \mathrm{~K}$.

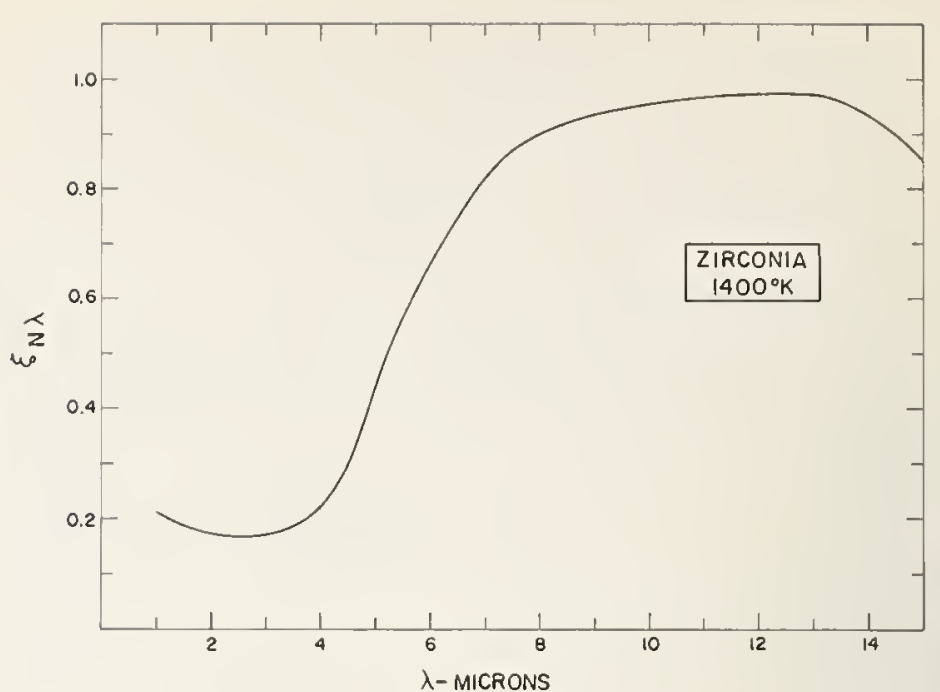

Figure 10. Normal spectral emittance of commercially pure zirconia at $1400 \mathrm{~K}$.

\section{References}

[1] Judd, D. B., Terms, definitions, and symbols in reflectometry, J. Opt. Soc. Am. 57, No. 4, 545-552 (April 1967).

[2] Kneissl, G. J., and Richmond, J. C., A laser-source integrating sphere reflectometer, NBS Tech. Note $439,1968$.

[3] Richmond, J. C., and Harrison, W. N., Total hemispherical emittance of coated and uncoated Inconel and types 321 and 430 stainless steel, J. Res. NBS 66C (Engineering and Instrumentation), No. 3, 261-269 (1962)

[4] Richmond, J. C., Effect of surface roughness on emittance of nonmetals, J. Opt. Soc. Am. 56, No. 2, 253-254 (Feb. 1966).

[5] Bennett, H. E., Influence of surface roughness, surface damage, and oxide films on emittance, Proceedings of Symposium on Thermal Radiation of Solids, San Francisco, 1964, ed. by S. Katzoff (NASA Special Publication SP-55, Washington, Superintendent of Documents, GPO 1965) pp. 145152.

[6] Gardon, R., The emissivity of transparent materials, J. Am. Ceram. Soc. 39, No. 8, 278-287 (August 1956).

17] Gardon, R., A review of radiant heat transfer in glass, J. Am. Ceram. Soc. 44, No. 7, 305-312 (July 1961).

[8] Kubelka, P., New contributions to the optics of intensely light-scattering materials, J. Opt. Soc. Am. 38, No. 5, 448-457 (May 1948).

[9] Hamaker, H. C., Radiation and heat conduction in light scattering materials, Philips Research Reports 2, 55-67, 103-111, 112-125, 420-425 (1947).

[10] Klein, J. D., Heat transfer, by radiation, in powders, Doctoral Dissertation, Massachusetts Institute of Technology (1960) Available from University Microfilms.

[11] Richmond, J. C., Relation of emittance to other optical properties, J. Res. NBS 6rC (Engin eering and Instrumentation), No. 3, 217-226 (1963).

[12] Strong, John, Concepts of Classical Optics, p. 124 (W. H. Freeman and Co., San Francisco, 1958).

[13] Bruehlman, R. J., Thomas, L. W., and Gomek, E., Effect of particle size and pigment volume concentration on hiding power of titanium dioxide, Federation of Societies for Paint Technology Official Digest, 33, No. 433, 252-267 (Feb. 1961).

[14] Slemp, W. S., and Wade, W. R., A method for measuring the spectral normal emittance in air of a variety of materials having stable emittance characteristics, Proceedings of Symposium on Measurement of Thermal Radiation Properties of Solids, Dayton, 
O., 1962, ed. by J. C. Richmond (NASA Special Publication SP-31, Washington, Superintendent of Documents, GPO, 1963) pp. 433-439.

[15] Clayton, W. A., A $500^{\circ}$ to $4500^{\circ} \mathrm{F}$ thermal radiation test facility for transparent materials, Proceedings of Symposium on Measurement of Thermal Radiation Properties of Solids, Dayton, O., 1962, ed. bv J. C. Richmond (NASA Special Publication SP-31, Washington, Superintendent of Documents, GPO, 1963) pp. 445-460.

[16] Kjelby, A. S., Emittance measurement capability for temperatures up to $3000^{\circ} \mathrm{F}$, Proceedings of Symposium on Measurement of Thermal Radiation Properties of Solids, Dayton, O., 1962, ed. by J. C. Richmond (NASA Special Publication SP-31, Washington, Superintendent of Documents, GPO, 1963).

[17] McMahon, H. O., Thermal radiation characteristics of some glasses, J. Am. Ceram. Soc. 34 No. 3, 91-96 (1951).

[18] Clark, H. E., and Moore, D. G., A rotating cylinder method for measuring normal spectral emittance of ceramic oxide specimens from $1200^{\circ}$ to $1600^{\circ} \mathrm{K}$, J. Res. NBS 70A (Physies and Chemistry), No. 5, 393-415 (1966).

[19] Folweiler, R. C., Thermal radiation characteristics of transparent, semi-transparent and translucent material under non-isothermal conditions, Air Force Systems Command, Wright-Patterson Air Force Base, Aeronautical Systems Division Technical Documentary Report ASD-TDR-62-719 (April, 1964).

[20] Betz, H. T. Olson, O. H., Schurin, B. D., and Morris, J. C., Determination of emissivlty and reflectivity data on aircraft structural materials, Part II, Techniques for measurement of total normal emissivity, solar absorptivity and presentation of results, Wright Air Development Center Technical Report TR 56-222 Part II (Oct. 1958).

[21] Comstock, D. F., Jr., A radiation technique for determining the emittance of refractory oxides Proceedings of Symposium on Measurement of
Thermal Radiation Properties of Solids, Dayton, O., 1962, ed. by J. C. Richmond (NASA Special Publication SP-31, Washington, Superintendent of Documents, GPO, 1963) pp. 461-468.

[22] Evans, R. J., Clayton, W. A. and Fries, M. A very rapid $3000^{\circ} \mathrm{F}$ technique for measuring emittance of opaque solid materials, Proceedings of Symposium on Measurement of Thermal Radiation Properties of Solids, Dayton, O., 1962, ed. by J. C. Richmond (NASA Special Publication SP-31, Washington, Superintendent of Documents, GPO, 1963) pp. 483-488.

[23] Null, M. R., and Lozier, W. W., Measurement of reflectance and emittance at high temperature with a carbon arc image furnace, Proceedings of Symposium on Measurement of Thermal Radiation Properties of Solids, Dayton, O., 1962, ed. by J. C. Richmond (NASA Special Publication SP-31, Washington, Superintendent of Documents, GPO, 1963) pp. $535-539$.

[24] Wilson, R. G., Hemispherical spectral emittance of ablation chars, carbon and zirconia (to $3700^{\circ} \mathrm{K}$ ), Proceedings of Symposium on Thermal Radiation of Solids, San Francisco, 1964, ed. by S. Katzoff (NASA Special Publication SP-55, Washington, Superintendent of Documents, GPO, 1965) pp. $259-275$.

[25] Laszlo, T. S., Gannon, R. E. and Sheehan, P. J., Emittance measurements of solids above $2000{ }^{\circ} \mathrm{C}$, Proceedings of Symposium on Thermal Radiation of Solids, San Francisco, 1964, ed. by S. Katzoff, (NASA Special Publication SP-55, Washington, Superintendent of Documents, GPO, 1965) pp. $277-286$.

[26] Peavy, B. A., and Eubanks, A. G., Periodic heat flow in a hollow cylinder rotating in a furnace with a viewing port, Proceedings of Symposium on Measurement of Thermal Radiation Properties of Solids, Dayton, O., 1962, ed. by J. C. Richmond, (NASA Special Publication SP-31, Washington, Superintendent of Documents, GPO, 1965) pp. $553-563$. 



\title{
Elastic Deformation of Ceramics and Other Refractory Materials
}

\author{
J. B. Wachtman, Jr. \\ Institute for Materials Research, \\ National Bureau of Standards, Washington, D.C. 20234
}

\begin{abstract}
Elastic deformation of ceramics is reviewed first from a continuum, thermodynamic point of view; nonlinear effects and pressure dependence of elastic moduli are included. The results of several important technological problems, including wave propagation, resonance, and the effects of gross microstructure on elastic moduli, are discussed in terms of continuum elasticity. The atomic theory of elastic moduli is then briefly sketched from the lattice dynamics point of view and correlations of other physical properties with elastic moduli are discussed. Finally the measurement of elastic moduli and the characterization of ceramics with respect to elastic moduli are considered.
\end{abstract}

Key Words: Ceramics; elastic constants; lattice dynamics; porosity; resonance; wave propagation.

\section{GONTENTS}

1. Introduction

2. Continuum elasticity

2.1. Thermodynamics and simple pressurevolume relations..................

2.2. Stress

2.3. Strain

2.4. Linear elasticity

2.5. Nonlinear elasticity $\ldots \ldots \ldots$

2.6. Generalizations of linear elasticity

3. Specific problems involving elastic deformation.

3.1. General discussion

3.2. Elastic waves in isotropic materials

3.3. Elastic waves in anisotropic materials . . .

3.4. Attenuation of elastic waves by scattering and nonlinear effects.

3.5. Resonance of isotropic materials

3.6. Resonance of anisotropic materials

3.7. Effect of porosity on elastic moduli of isotropic materials

3.8. Relation of elastic moduli of polycrystalline materials to the moduli of the single-crystal grains

\section{Introduction}

Anyone reading in modern elasticity theory might well obtain the impression of a vast and complicated field with little contact with laboratory experiment or technological practice. Yet much of that theory is based on sound analysis. Its applicability and relevance of some of its. results cannot be seriously doubted. The more experimentally inclined and the more technologically interested should be given a clear statement of the principal conclusions derived. This then is such an attempt at a review of elastic theory for the ceramist.

Elastic properties of ceramics are of interest to a variety of scientists and engineers in relation.
3.9. Elastic moduli of composites_._._.

4. Atomic theory of elasticity

4.1. Lattice statics__._.

4.2. Linear lattice dynamics: Relation of elastic constants to sound velocity, specific heat, optical absorption, and dielectric constant -

4.3. Nonlinear lattice dynamies: Thermal expansion and temperature dependence of

4.4. Effect of solid solution and phase changes on elastic constants _._._.

4.5. Internal friction and the $\Delta \mathrm{E}$ effect

5. Special topies $\ldots$

5.1. Correlations of other properties with elastic constants $\ldots \ldots$

5.2. Elastic moduli of graphite $\ldots \ldots \ldots$

5.3. Measurement of elastic moduli

5.4. Characterization of ceramics with respect to elastic moduli

6. References . 然 0 .

to a wide range of problems. They provide one of the most direct measurements of chemical bonding and accordingly are needed as basic data in calculations of cohesive energy, surface energy, theoretical strength of crystals, a wide range of properties such as specific heat which are interrelated by lattice dynamics, point defect properties, and dislocation properties. They are needed for direct engineering use in calculating the stress distribution and deflection of structures under static load and their resonances under dynamic loads, the values of thermal stresses, and the stress distribution in composites. They are needed in designs of electromechanical transducers and of various devices employing ultrasonic wave propagation. 
Elastic properties are needed to interpret wave propagation in the earth as used in fundamental studies of its composition, in prospecting, and in study of soils. Elastic moduli, or quantities directly dependent upon elastic moduli, are sometimes measured as a quality control technique. We seek here to provide sufficient background knowledge on the elastic properties of ceramics and the factors upon which they depend to facilitate their use in such applications. We hope also to convey some impressions of the interesting and active research going on in many areas of elasticity.

Solids respond to the application of stress by undergoing deformation which may be either elastic (i.e. recoverable) or plastic (i.e. not recoverable). This definition of elastic deformation includes not only instantaneous deformation which is linearly proportional to the stress, but also includes deformation which is time dependent and/or nonlinearly related to stress, provided the deformation is fully recoverable in time, however long. The subject of elasticity is frequently developed by introducing empirical relations between stress and strain, but may also be developed from the point of view of thermodynamics in which a generalized form of the Helmholtz free energy is written as a function of the strain and temperature. The usual stress-strain relation at constant temperature is then obtained by differentiating the free energy; the second derivatives are the isothermal elastic constants. This thermodynamic approach is useful in treating the relation of the elastic properties of solids to other properties; solids also respond to stress by changes in their thermal, electrical, and magnetic properties. The latter are not properly a part of the subject of elasticity, but a number of equations can be derived from thermodynamics which express relationships between the value of an elastic property measured under one condition, such as constant electric field, to the value of the same property measured under a different condition, such as constant electric displacement.

In the usual thermodynamic development of elasticity one treats a solid as a continuous material and the resulting treatment is strictly applicable only to volumes containing many atoms. Within this limitation many important scientific and technical problems can be treated. Section 2 of the present paper outlines the development of continuum elasticity including a brief discussion of single crystal effects (anisotropy) and nonlinear effects.

In section 3 the use of the continuum theory to solve various problems of technological importance is considered. The application of this theory to resonance, wave propagation, and the relation of the texture and microstructure of ceramics to their elastic properties is briefly discussed.

Section 4 discusses the relation between the atomic structure of solids (including defects) and their elastic properties. On one level this involves lattice dynamics in which each atom is treated as a point mass (or as a point mass and concentric shell) connected by springs to other atoms; the resulting theory leads to useful relations between elastic properties and a variety of other properties such as specific heat, index of refraction, dielectric constant at low frequency, cohesive energy, and surface energy. If nonlinear springs are used, relations to anharmonic properties such as thermal expansion, thermal conductivity, and high frequency scattering result. On a deeper level elastic properties should be derivable by detailed calculation starting from fundamental constants; recent work in this area is beyond the scope of this paper. Some physical properties, such as phase changes and solid solution effects, are discussed in section 4. The effect of structural defects in producing anelastic (time dependent but recoverable) deformation is then discussed.

In section 5 special topics including elastic moduli of graphite and the correlation of other physical properties with elastic moduli are discussed. Experimental techniques for determining elastic properties have been greatly improved by modern electronic instrumentation. These techniques are described and estimates of their accuracy are given.

The paper is concluded with a discussion of the features of composition and microstructure upon which elastic properties of ceramics depend. Throughout the paper, qualitative features are described and just enough mathematics is introduced to indicate the manner in which the quantitative treatment is developed; the mathematic details are left to the references as much as possible. The subject, however, demands the introduction of an appreciable number of symbols and the use of a subscript notation; the symbols approved by IRE [1] ${ }^{1}$ are used (except that we retain $S$ and $T$ without subscript as entropy and absolute temperature respectively), and are summarized in table 1 . Table 2 lists the International System of Units [2] and their equivalents in some of the customary engineering units.

Tables listing numerical values of various elastic properties are given as the properties are introduced. In selecting data for each table, the contemporary definition of ceramics as inorganic, non-metallic materials was used, and accordingly data on some important semiconductor materials is included, but data on alkali halides are not included because these are extensively covered elsewhere [3].

Data on some refractory materials (here considered as materials melting above $1000{ }^{\circ} \mathrm{C}$ ) other than ceramics are also included because one of the major applications of ceramics is at high temperatures so that a comparison with other high temperature materials is of interest. The literature on single crystal elastic constants through 1964 has been surveyed in a pair of

\footnotetext{
1 Figures in brackets indicate the literature references at the end of this paper.
} 
Table 1. Symbols

Symbol

$A=U-T S$

$A, A^{*}$

$A(i j k)$

$\mathbf{a}=\left(a_{1}, a_{2}, a_{3}\right)$

$B$

$C_{i j k l m n}$

$C_{i j k}$

$c_{i j k l}$

$c_{i j}$

$c$

$c_{p}, c_{o}$

d

E

$\mathbf{F}=\left(F_{1}, F_{2}, F_{3}\right)$

$f$

$G=H-T S$

$G=\mu$

$H=U+p V$

$K_{n}$

$k$

$\mathbf{I}=\left(l_{1}, l_{2}, l_{3}\right)$

$l$

$\bar{m}$

$n$

$P$

$p$

$R$

$r$

$S$

$S_{i j}$

$S$

$s_{i j k l}$

$s_{i j}$

$T$

$T_{i j}$

$T_{i}$

Anisotropy factors.
Quantity

Helmholtz free energy per unit mass.

Constants in nonlinear stressstrain relation.

Initial position vector.

Bulk modulus.

Third-order elastic constants in tensor notation.

Third-order elastic constants in matrix notation.

Second-order elastic constants in tensor notation.

Second-order elastic constants in matrix notation.

Velocity of light.

Specific heat at constant pressure or volume.

Diameter of cylinder.

Thermal energy.

Force per unit area.

Frequency.

Gibbs free energy per unit mass.

Shear modulus.

Enthalpy per unit mass.

Thickness correction factor for longitudinal vibration of a slender cylinder.

Boltzmann's constant, also wave number.

Unit normal to plane.

Length of rod or bar.

Reduced mass.

Index of refraction, also mode number for resonance.

Porosity.

Pressure, also number of atoms per molecule.

Gas constant.

Interatomic spacing:

Entropy per unit mass.

Infinitesimal strain in tensor notation, also spheroidal mode of sphere.

Infinitesimal strain in matrix notation.

Second-order elastic compliances in tensor notation.

Second-order elastic compliances in matrix notation.

Absolute temperature, also correction factor in flexural vibration of slender bar or rod.

Stress components in tensor notation, also torsional modes of sphere.

Stess components in matrix notation.

Thickness of plate.
TABLE 1. Symbols-Continued

$\begin{array}{ll}\text { Symbol } & \text { Quantity } \\ t_{i j} & \text { Thermodynamic tension in } \\ & \text { tensor notation. } \\ t_{i} & \text { Thermodynamic tension in } \\ & \text { matrix notation. }\end{array}$

$U \quad$ Internal energy per unit mass.

$\mathbf{u}=\left(u_{1}, u_{2}, u_{3}\right) \quad$ Motion vector.

$V \quad$ Volume.

$v$

$\mathbf{x}=\left(x_{1}, x_{2}, x_{3}\right)$

$Y$

ze

$a$

$\beta$

$\gamma$

$\delta$

$\epsilon_{i j}$

Velocity of sound; $v_{l}$ for longitudinal, $v_{s}$ for transverse.

Final position vector.

Young's modulus of elasticity.

Ionic charge $; e=$ electronic charge.

Linear thermal expansion coefficient.

Volume thermal expansion coefficient.

Grüneisen's constant.

Grüneisen's second constant.

Finite strain components in tensor notation and spatial description (sometimes called an Eulerian description).

$\epsilon_{i}$

Finite strain components in matrix notation and spatial description.

Static dielectric constant. $\epsilon_{0}$

$\eta_{i j}$

$\eta_{i}$

$\lambda$

$\mu=G$

$\nu$

$\nu_{0}$

$\rho$

$\chi$

$\omega$
Finite strain components in tensor notation and material description (sometimes called a Lagrangian description).

Finite strain components in matrix notation and material description.

Lamé second-order elastic constant for elasticially isotropic material, also wavelength.

Lamé second-order elastic constant for elastically isotropic material.

Poisson's ratio.

Long wavelength limit of the frequency of the transverse optical mode of vibration.

Density.

Compressibility.

Angular frequency.

TaBLe 2. Units

\begin{tabular}{|c|c|c|}
\hline Quantity & $\begin{array}{l}\text { Inter- } \\
\text { national } \\
\text { unit }\end{array}$ & Value in other commonly used units \\
\hline Volume....... & $\mathrm{m}^{3}$ & $10^{6} \mathrm{~cm}^{3}=35.314 \mathrm{ft}^{3}=6.1024 \times 10^{4} \mathrm{in}^{3}$ \\
\hline Pressure...... & $\mathrm{N} / \mathrm{m}^{2}$ & $\begin{array}{l}10 \mathrm{dyn} / \mathrm{cm}^{2}=1.450 \times 10^{-4} \mathrm{psi}=1.020 \times 10^{-8} \\
\mathrm{~kg} / \mathrm{cm}^{2}\end{array}$ \\
\hline Energy & $\mathbf{J}$ & $0.239 \mathrm{Cal}(\mathrm{gm})$ \\
\hline
\end{tabular}


excellent papers $[3,4]$ which draw on a number of previous surveys [5-9]. We have accordingly concentrated on listing values published since 1964, but have included some of the older data for comparison. 'The engineering data on elastic moduli of polycrystalline ceramics has been surveyed [10] and several other useful compilations of elastic properties and related properties of ceramics have recently been published [11-13].

\section{Continuum Elasticity}

\subsection{Thermodynamics and Simple Pressure- Volume Relations}

The simplest type of elastic behavior is the change of volume (and sometimes of shape) in response to pressure (i.e., to isostatic stress). If the solid is isotropic (i.e., if its properties do not vary with direction), its shape will not change under pressure and its equation of state is simply its volume as a function of pressure and temperature. The exact functional form of the equation of state is not known for any solid; a power series approximation is commonly used. Overton [14] has discussed the equivalence of Bridgman's form of the equation of state,

$$
V(T, p)=V(T, p=0)\left[1-a_{1}(T) p+a_{2}(T) p^{2}+. .\right],
$$

to that of Slater,

$$
\begin{aligned}
& V(T, p)=V(T=0, p=0)\left[1+A_{0}(T)-a_{1}^{\mathrm{s}}(T) p\right. \\
& \left.+{a_{2}}^{\mathrm{s}}(T) p^{2}+. .\right] \text {, }
\end{aligned}
$$

where $V=$ volume, $p=$ pressure, $T=$ absolute temperature, superscript $s$ refers to Slater's parameters, $a_{0}$ is a function of temperature which gives the volume expansion at zero pressure, and the remaining $a$ 's are empirical functions of temperature which express the elastic properties. In general, contribution of higher terms is small. To give a complete thermodynamic description, these elastic properties are needed together with the thermal expansion and specific heat. These data permit the internal energy, $U$, and the entropy, $S$, to be calculated from which the enthalpy, $H=U+p V$, the Helmholtz free energy, $A=U-T S$, and the Gibbs free energy, $G=U+p V-T S$ can be calculated.

Randomly oriented polycrystalline ceramics and glass are isotropic so that either equation (1) or (2) provides a complete description of their elastic response to isostatic pressure, but the behavior of anisotropic ceramics such as single crystals requires the introduction of strain and stress with a corresponding increase in complexity of notation. Even for isotropic ceramics, however, strain and stress must be introduced to describe their elastic response in other situations such as in bending. Before going on to do so it is helpful to use the conceptually simple pressure-volume relationship to illustrate some points concerning the distinc- tions between adiabatic and isothermal values and between linear and nonlinear behavior.

The isothermal bulk modulus, $B_{T}$, is defined as the reciprocal of the isothermal compressibility, $\chi_{r}$, defined by

$$
\chi_{r}=-\left(\frac{\partial V}{\partial p}\right)_{T} / V
$$

From equation (1) the compressibility is given by

$$
\chi_{T}(T, p)=\left[a_{1}(T)-2 a_{2}(T) p_{j}^{\prime} V(T, p=0) / V(T, p) .\right.
$$

For small pressures, the second term can be neglected and the volume ratio taken to be unity so that $\chi_{T}(T, p=0)=a_{1}(T)$. This is the quantity commonly measured in static tests at relatively low pressures. Even at modest pressures the second term can be determined, however, by using highaccuracy ultrasonic velocity measurements and the results used to predict behavior at high pressure. The calculations involve some complexity in converting from transit time data to elastic properties which we will not consider here, and also necessitate careful handling of the values of bulk modulus measured at different pressures because a pressure dependent volume enters into equation (4). Another complication arises because ultrasonic measurements give adiabatic rather than isothermal values. The conversion equation from the adiabatic to the isothermal compressibility is

$$
\chi_{r}=\chi_{S}+T \beta^{2} / \rho C_{p}
$$

where $\beta$ is the volume thermal expansion coefficient, $\rho$ is the density, and $C_{p}$ is the specific heat at constant pressure [15]. These latter values are all to be taken at the pressure of measurement. Cook [16] has derived equations which permit one to combine specific heat and density measured as a function of temperature at a single, fixed pressure with ultrasonic data measured as a function of pressure and temperature to calculate $\chi_{r}(T, p)$. A modified form of these equations, due to McSkimin [17] was used by Anderson [18] to obtain the isothermal compressibility of vitreous silica over the range 0 to $300 \mathrm{~K}$ and 1 atm to $8000 \mathrm{~atm}$. Thurston $[15,19]$ has reviewed the calculations necessary if the solid being studied is anisotropic as well as nonlinear. Anderson [20, 21] has followed Murnaghan in assuming that the bulk modulus is a linear function of pressure given by

$$
B(p, T)=B_{0}(T)+B^{\prime}{ }_{0}\left(T^{\prime}\right) p
$$

and has applied the resulting Murnaghan logarithmic equation [21]

$$
\ln \left(V_{0} / V\right)=\left(B^{\prime}{ }_{0}\right)^{-1} \ln \left[B^{\prime}{ }_{0}\left(p / B_{0}\right)+1\right]
$$

and a similar equation due to Birch to a variety of metals and ceramics. He evaluated the param- 
eters $B_{0}$ and $B^{\prime}{ }_{0}$ from ultrasonic data taken in the pressure range 0 to $4 \mathrm{kbar}$ and found that the resulting equation was in reasonable agreement up to quite high pressures, for example up to 1500 kbar for $\mathrm{Al}_{2} \mathrm{O}_{3}$ as shown in figure 1 .

Simple thermodynamic manipulation leads to the relation

$$
B_{T}=V\left(\frac{\partial^{2} A}{\partial V^{2}}\right)
$$

This is a useful expression for the isothermal bulk modulus which involves only the internal energy as a function of volume and does not involve the pressure explicitly. Similar expressions give various other isothermal elastic constants as second derivatives of the Helmholtz free energy with respect to various strain components, as we shall see. These expressions are useful in discussing the coupling of elastic properties to electrical and magnetic properties. For the elastic properties to be accurately defined, the electrical and magnetic state must be specified during the deformation. This may be accomplished by holding the electric and magnetic fields constant, or, as one of several other possibilities, by holding the electric polarization and the magnetic field constant. The resulting elastic properties will generally have slightly different values; equations relating the two sets of

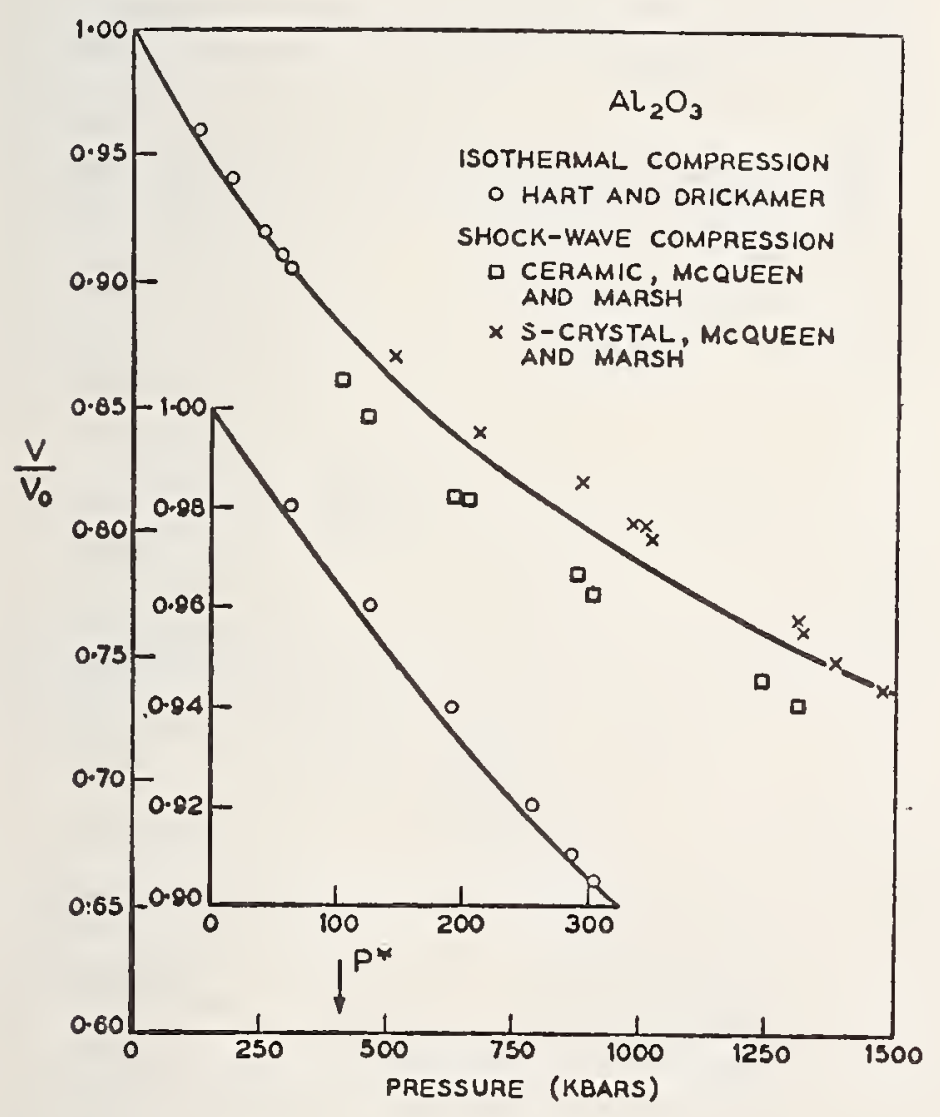

Figure 1. Compressibility of $\mathrm{Al}_{2} \mathrm{O}_{3}$.

Solid line calculated from data taken at pressures below 2 kbar using the Murnaghan equation (7). See Anderson [21] for details of calculations and references to experimental work. values can be derived by using the appropriate expressions similar to equation (8) as described in detail by Nye [22].

In the following sections we sketch the mathematics necessary to extend the foregoing to stresses other than simple pressure and to deformations which involve change of shape as well as change of volume. It is simplest to first introduce the stress and strain in a form suitable for description of small deformations; this is the subject of infinitesimal elasticity and is the level on which engineering problems in elasticity are usually solved. A complication arises because the strains and stresses are second rank tensors and so would normally be written with two subscripts, but are instead usually written with one subscript because they possess a symmetry which allows this to be done. A second complication arises from the fact that some of the engineering strains differ by a factor of two from the strains in tensor notation commonly used in modern treatments of elasticity because of the convenience of the latter in expressing the orientation dependence of elastic moduli. For elastically isotropic bodies, such as glass and randomly oriented polycrystalline ceramics, the equations relating stress and strain take a simple form. For all single crystals, including cubic crystals, the equations take on a similar form but have more terms which increase in number for less symmetric crystals. Another complication arises for single crystals; the value of the elastic constants depends on the orientation of the coordinate axes used to describe the deformation. In using single crystal elastic constants one must bear this in mind.

When the strain is large and accurate treatment is required, finite elasticity must be used. A new definition of strain must be introduced and a distinction must be made between the use of coordinate axes fixed in space (giving a spatial or Eulerian description) and coordinate axes which move with the deforming material (giving a material or Lagrangian description). The stress previously defined in infinitesimal elasticity is appropriate to a spatial description; for a material description a related concept, the thermodynamic tension, is appropriate. Throughout the treatment of either infinitesimal or finite elasticity the distinctions previously mentioned concerning the difference between adiabatic and isothermal behavior and the specification of electrical and magnetic state remain necessary.

\subsection{Stress}

Stress is simply the force per unit area with which the material on one side of a chosen surface acts on the material on the other side. We see from figure 2 that two vectors are required to specify stress for a particular surface. One vector is required to describe the orientation of the surface and is drawn perpendicular to the surface; the other describes the force per unit area on the 


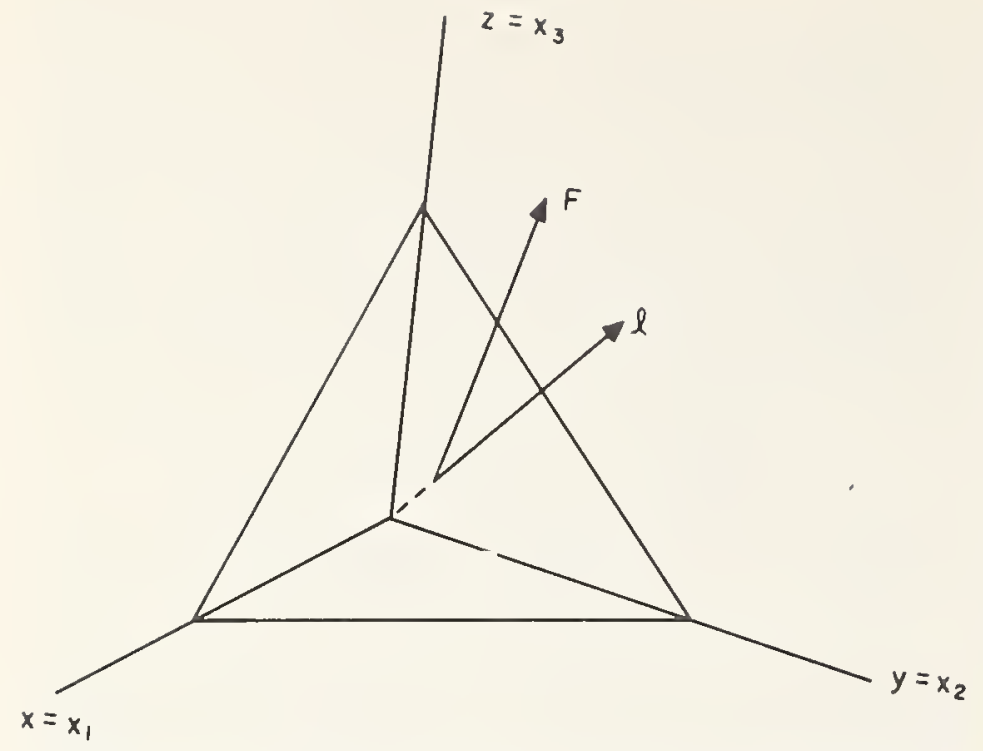

FIGURE 2. The force, F, acting on a surface with unit normal vector 1 .

surface and can have any orientation. The surface can be chosen through a given point in an infinite number of orientations; for each there will be a different value of the force vector even though the same stress is acting at the point. It can be shown [22] that the stress acting at a point is completely specified by giving six numbers; the six stress components $T_{11}, T_{22}, T_{33}, T_{12}=T_{21}, T_{13}=T_{31}$, and $T_{23}=T_{32}$. The force (with components $F_{1}, F_{2}$, $F_{3}$ ) acting on a surface with unit normal (with components $l_{1}, l_{2}, l_{3}$ ) is given by [22]

and

$$
\begin{aligned}
& F_{1}=T_{11} l_{1}+T_{12} l_{2}+T_{13} l_{3}, \\
& F_{2}=T_{21} l_{1}+T_{22} l_{2}+T_{23} l_{3},
\end{aligned}
$$

$$
F_{3}=T_{31} l_{1}+T_{32} l_{2}+T_{33} l_{3}
$$

The same equation can be written

$$
F_{i}=\sum_{j=1}^{3} T_{i j} l_{j}, i=1,2,3 .
$$

The stress components have the following physical meaning; $T_{i j}$ is the force in the $x_{i}$ direction acting on a unit surface area whose normal is in the $x$, direction.

These stress components written with two subscripts are said to be in tensor notation. It is convenient to introduce a single subscript notation, called matrix notation, by defining $T_{1}=T_{11}$, $T_{2}=T_{22}, T_{3}=\mathrm{T}_{33}, T_{4}=T_{23}, T_{5}=T_{13}$, and $T_{6}=T_{12}$.

\subsection{Strain}

The components of strain at a chosen point in a solid body are introduced to specify the deformation of a small region around the point. The strain components are deliberately chosen to specify only the deformation of this region and do not include translation or rigid body rotation. Figure 3 is an illustration of the deformation of a body which carries a portion initially at $A_{0} B_{0} C_{0} D_{0}$ into $A B C D$. We specify the position of $A_{0}$ by the vector a; after being moved along the vector $\mathbf{u}, A_{0}$ becomes $A$ at position $\mathbf{x}$. If the whole portion $A_{0} B_{0} C_{0} D_{0}$ moves by the same amount $\mathbf{u}$, the motion is simply one of rigid body translation. We are therefore interested not in $\boldsymbol{u}$ itself, but in how $\mathbf{u}$ varies as we examine different points, such as $D_{0}$, near $A_{0}$ and so we wish to know the values of the set of 9 quantities $\frac{\partial u_{i}}{\partial a_{j}}$ at $A_{0}(i, j=1,2,3)$. The same information is also provided by the set of 9 quantities $\frac{\partial x_{i}}{\partial a_{j}}$ which have the same value as $\frac{\partial u_{i}}{\partial a_{j}}$ if $i \neq j$ and are greater by one if $i=j$; these quantities are called the deformation gradients and the whole description of strain can be written in terms of them as an alternative to using $\frac{\partial u_{i}}{\partial a_{j}}[19,23]$. Either set of quantities is satisfactory in that rigid body translation has no effect on their values, but both sets are still affected by rigid body rotation. We can, however, take combinations of either set which will exclude rigid body rotation and so give a proper description of strain.

In most engineering applications of ceramics, the elastic strain is small enough to justify the use of infinitesimal elasticity although some exceptions exist for very high strength materials, for isostatic pressure applications, for devices which exploit nonlinear behavior, etc. A strain is considered infinitesimal if the largest of the

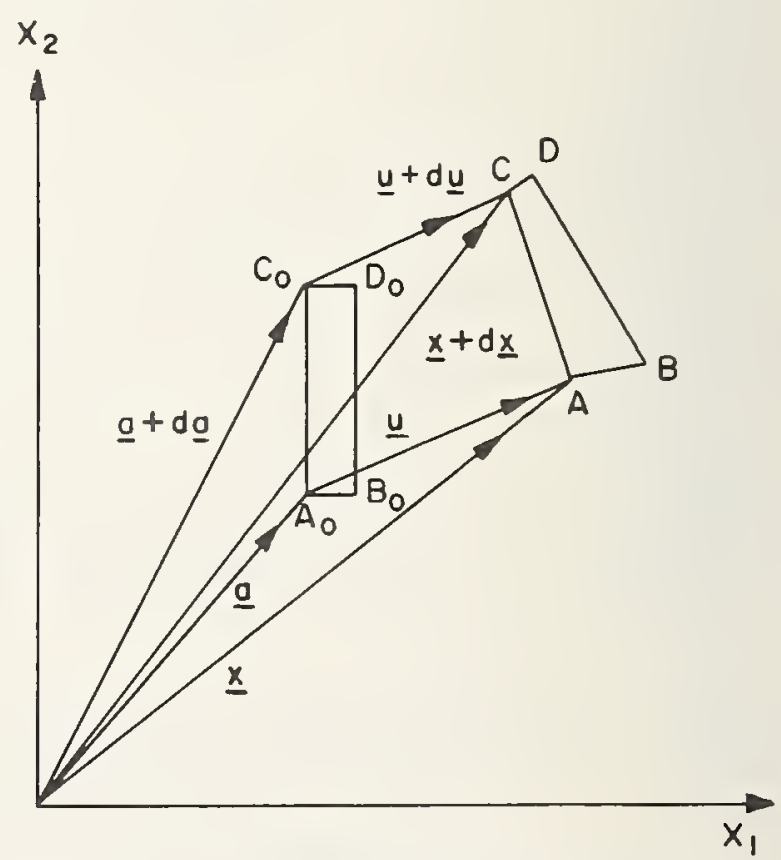

Figure 3. Deformation of a portion $\mathrm{A}_{0} \mathrm{~B}_{0} \mathrm{C}_{0} \mathrm{D}_{0}$ of a body into $\mathrm{ABCD}$ by a motion vector $\mathrm{u}$ which is a function of position. 
quantities $\frac{\partial u_{i}}{\partial a_{j}}$ is small (typically less than 0.01) so that powers and products of the quantities $\frac{\partial u_{i}}{\partial a_{j}}$ can be neglected. Then the infinitesimal strain component $\mathrm{S}_{i j}$ is defined by

$$
S_{i j}=\frac{1}{2}\left(\frac{\partial u_{i}}{\partial a_{j}}+\frac{\partial u_{j}}{\partial a_{i}}\right), i, j=1,2,3 .
$$

The same symmetry holds here as for the stress so that $S_{12}=S_{21}, S_{13}=S_{31}$, and $S_{23}=S_{32}$. As in the case of the stress, these strain components written with two subscripts are said to be in tensor notation. As with the stress, it is customary to introduce a single subscript matrix notation, but factors of 2 are introduced into the definition of the shear strains in matrix notation so that the whole set of strain components in matrix notation is just the usual engineering strains: $S_{1}=S_{11}, S_{2}=S_{22}$, $S_{3}=S_{33}, S_{4}=2 S_{23}, S_{5}=2 S_{13}$, and $S_{6}=2 S_{12}$.

The physical meaning of the infinitesimal strains is illustrated in figure 4 . The strain $S_{1}$ refers to an increase from initial length $l$ to $l+\Delta l$ in the $x_{1}$ direction with other dimensions remaining constant. Its value is $S_{1}=\Delta l / l$. The strains $S_{2}$ and $S_{3}$ refer to the same type of deformation along the $x_{2}$ and $x_{3}$ axes respectively. The strain $S_{6}$ refers to a shear in the $x_{1} x_{2}$ plane and also has the value $\Delta l / l$, but now $\Delta l$ is measured at a right angle to $l$. It thus corresponds to changing a square into a parellelogram as shown. $S_{4}$ and $S_{5}$ refer to the same type of deformation in the $x_{2} x_{3}$ and $x_{1} x_{3}$ planes respectively.

These definitions of infinitesimal strain suffice for infinitesimal elasticity and hence for most engineering problems. When large deformations or nonlinear effects must be treated, the definition of strain in equation (11) is replaced by defining the finite strain components given by

$$
\eta_{i j}=\frac{1}{2}\left(\frac{\partial u_{i}}{\partial a_{j}}+\frac{\partial u_{j}}{\partial a_{i}}+\frac{\partial u_{k}}{\partial a_{i}} \frac{\partial u_{k}}{\partial a_{j}}\right), i, j=1,2,3 .
$$

In this equation, the last term contains a repeated subscript $k$, which is understood to mean a sum; that is

$$
\frac{\partial u_{k}}{\partial a_{i}} \frac{\partial u_{k}}{\partial a_{j}}=\sum_{k=1}^{3} \frac{\partial u_{k}}{\partial a_{i}} \frac{\partial u_{k}}{\partial a_{j}}
$$
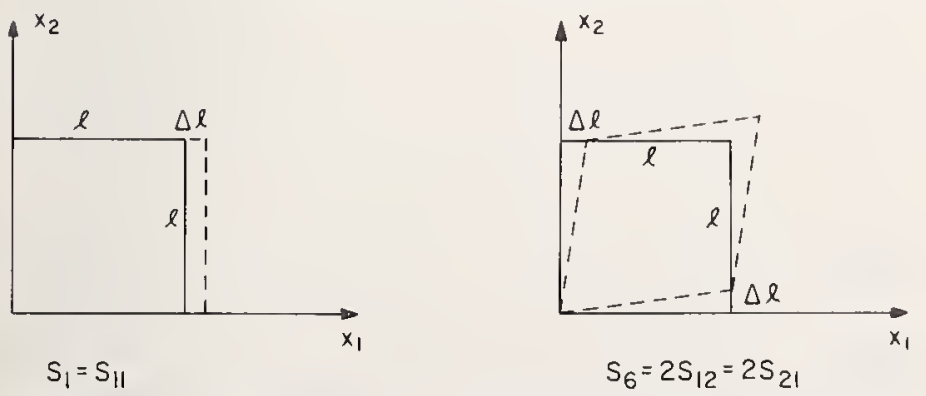

FIGURE 4. Illustration of the strain components $\mathrm{S}_{1}$ and $\mathrm{S}_{6}$.
When the partial derivatives are small, this term can be neglected and the finite strain of equation (12) reduces to the infinitesimal strain of equation (11). A new feature arises here, however. The finite strain of equation (12) is called the Lagrangian strain in a material description because the independent variables, $a_{i}$ refer to points fixed in the material. An alternate and equally valid definition of finite strain is that of the Eulerian strain or strain in a spatial description which is defined by

$$
\epsilon_{i j}=\frac{1}{2}\left(\frac{\partial u_{i}}{\partial x_{j}}+\frac{\partial u_{j}}{\partial x_{i}}-\frac{\partial u_{k}}{\partial x_{i}} \frac{\partial u_{k}}{\partial x_{j}}\right)
$$

As the partial derivatives become small $\frac{\partial u_{i}}{\partial x_{j}}$ approaches $\frac{\partial u_{i}}{\partial a_{j}}$, so that the strain of equation (14) also approaches the infinitesimal strain of equation (11). When the strain is finite, however, $\eta_{i j}$ is not the same as $\epsilon_{i j}$. Either can be used, but one must carefully distinguish which is being used as we shall see. The physical meaning of these strain components in terms of the local rotation and stretch of line elements in a deforming body is discussed elsewhere [19, 23].

\subsection{Linear Elasticity}

For stresses small enough to allow use of infinitesimal strains, it is found that strain is proportional to stress to a good approximation (Hooke's law). Thus-a stress $T_{1}$ will produce six strain components for a body with no symmetry and all can have different constants of proportionality which we call the elastic compliances, $s_{i j}$. These strains are given by

and

$$
\begin{aligned}
& S_{1}=s_{11} T_{1}, \\
& S_{2}=s_{21} T_{1}, \\
& S_{3}=s_{31} T_{1}, \\
& S_{4}=s_{41} T_{1}, \\
& S_{5}=s_{51} T_{1}, \\
& S_{6}=s_{61} T_{1},
\end{aligned}
$$

If all six independent stress components are acting simultaneously, these equations become the generalized Hooke's law, which is

$$
\begin{gathered}
\qquad \begin{aligned}
S_{1} & =s_{11} T_{1}+s_{12} T_{2}+s_{13} T_{3}+s_{14} T_{4}+s_{15} T_{5}+s_{16} T_{6}, \\
S_{2} & =s_{21} T_{1}+s_{22} T_{2}+s_{23} T_{3}+s_{24} T_{4}+s_{25} T_{5}+s_{26} T_{6}, \\
S_{3} & =s_{31} T_{1}+s_{32} T_{2}+s_{33} T_{3}+s_{34} T_{4}+s_{35} T_{5}+s_{36} T_{6}, \\
S_{4} & =s_{41} T_{1}+s_{42} T_{2}+s_{43} T_{3}+s_{44} T_{4}+s_{45} T_{5}+s_{46} T_{6}, \\
S_{5} & =s_{51} T_{1}+s_{52} T_{2}+s_{53} T_{3}+s_{54} T_{4}+s_{55} T_{5}+s_{56} T_{6},
\end{aligned} \\
\text { and } \\
S_{6}=s_{61} T_{1}+s_{62} T_{2}+s_{63} T_{3}+s_{64} T_{4}+s_{65} T_{5}+s_{66} T_{6}
\end{gathered}
$$

These equations can all be written in one line as

$$
S_{i}=\sum_{j=1}^{6} s_{i j} T_{j}, i=1,2 \ldots 6 .
$$

These are six equations giving the six strains in terms of the six stresses. Alternately we can solve 
these equations for the stresses in terms of the strains and get

$$
T_{i}=\sum_{j=1}^{6} c_{i j} S_{j}, i=1,2, \ldots 6
$$

where $c_{i j}$ are called the elastic constants and can be calculated from the $s_{i j}$ and conversely [22]. For any material, it can be shown that $s_{i j}=s_{j i}$ and $c_{i j}=c_{j i}$. This means that for a crystal of very low symmetry (triclinic) there are 21 independent elastic compliances or constants. Fortunately this number is much reduced for higher symmetry crystals being only 3 for cubic crystals and 2 for isotropic materials. For each of the 32 crystal classes, the scheme of elastic constants and compliances has been worked out from the symmetry elements possessed by the class and the results are listed in many texts $[3,5,7,22]$. In table 3 the numerical values of the constants and compliances are listed for a number of ceramics and some other refractory materials. These data are the fundamental description of the linear elastic properties of crystals. They are independent except that conditions of crystal stability require some to be larger than others [24, 25].

For cubic crystals the generalized Hooke's law becomes

$$
\begin{aligned}
& S_{1}=s_{11} T_{1}+s_{12} T_{2}+s_{12} T_{3}+0+0+0, \\
& S_{2}=s_{12} T_{1}+s_{11} T_{2}+s_{12} T_{3}+0+0+0, \\
& S_{3}=s_{12} T_{1}+s_{12} T_{2}+s_{11} T_{3}+0+0+0, \\
& S_{4}=0+0+0+s_{44} T_{4}+0+0, \\
& S_{5}=0+0+0+0+s_{44} T_{5}+0, \\
& \text { and } \quad S_{6}=0+0+0+0+0+s_{44} T_{6} .
\end{aligned}
$$

Thus, for example, the application of a tensile stress, $T_{1}$, along the $x_{1}$ axis causes a tensile strain, $S_{1}$, along the $x_{1}$ axis and perpendicular tensile strains, $S_{2}$ and $S_{3}$, which make up the contraction expressed by Poisson's ratio. This ratio is defined by $\nu=-S_{2} / S_{1}$ when only $T_{1}$ is acting and so is given by $-s_{12} / s_{11}$. It is important to note that the form of equation (19) depends on the reference axes $x_{1} x_{2} x_{3}$ being chosen along the usual axes of a cubic crystal. If the axes are rotated equation (19) goes over into equation (16) (usually written with primes on the $s_{i j}$ to indicate a rotated system of coordinates) even for a cubic crystal; in this case each of the $s^{\prime}{ }_{i j}$ of (16) is a complicated trigonometrical function of the orientation of the axes and the three independent compliances $s_{11}, s_{44}$, and $s_{12}$ of equation (19). For example:

$s^{\prime}{ }_{11}=s_{11}-\left[2\left(s_{11}-s_{12}\right)-s_{44}\right]\left[\sin ^{2} \theta \cos ^{2} \theta+.25 \sin ^{4} \theta \sin ^{2} 2 \phi\right]$

where $\theta$ and $\phi$ express the position of the $x^{\prime}{ }_{3}$ axis in the usual spherical polar angles. The compliance
TABLE. 3. Single crystal elastic constants of some nonpiezoelectric ceramics and some metallic refractory crystals

\begin{tabular}{l|c|c}
\multicolumn{2}{c}{ In units of $10^{10} \mathrm{~N} / \mathrm{m}^{2}$ for $c_{i j}$ and $10^{-12} \mathrm{~m}^{2} / \mathrm{N}$ for $s_{i j}$. } \\
\hline Substance & Subscripts of $c_{i j}$ or $s_{i j}$ & $\begin{array}{l}\text { Refer } \\
\text { ence }\end{array}$
\end{tabular}

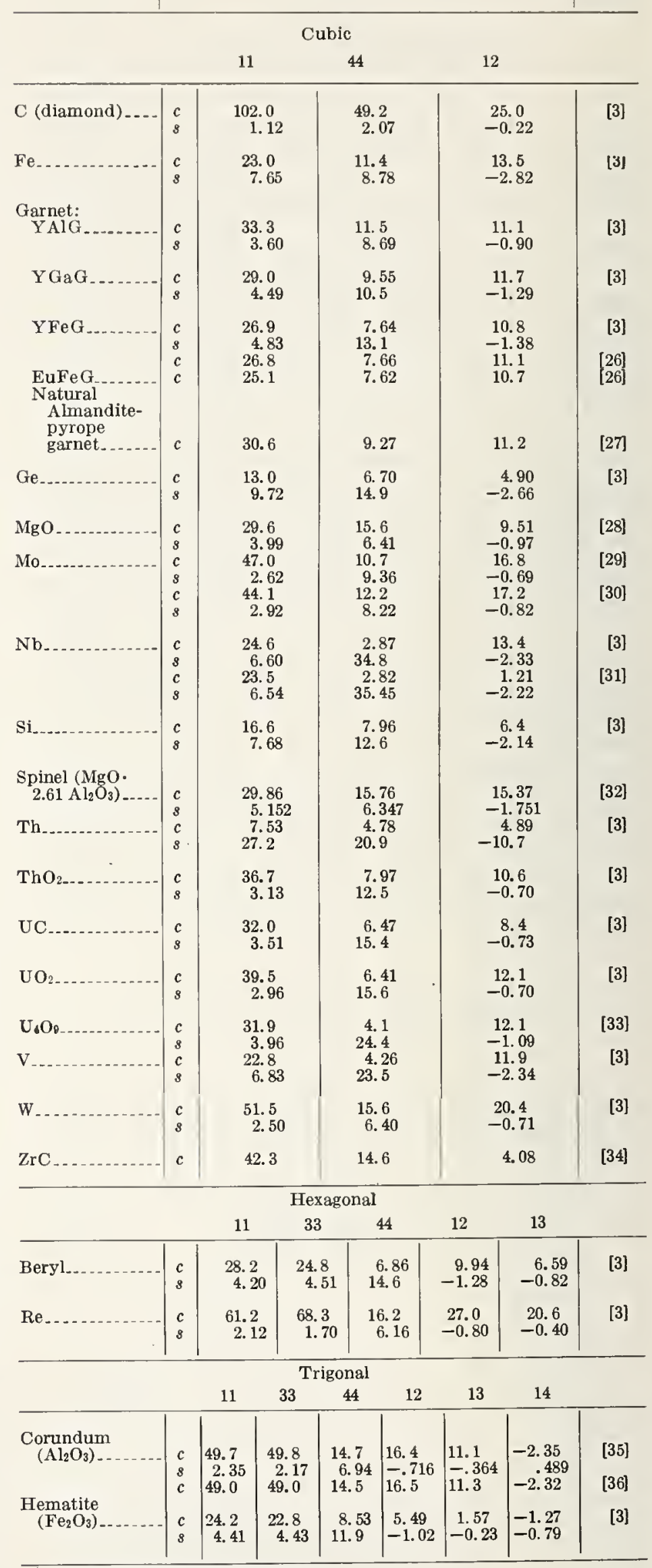

See footnote at end of table. 
TABLE 3. Single crystal elastic constants of some nonpiezoelectric ceramics and some metallic refractory crystals-Continued

\begin{tabular}{l|l|l}
\hline Substance & Subscripts of $c_{i j}$ or $s_{i j}$ & $\begin{array}{l}\text { Refer- } \\
\text { ence* }\end{array}$ \\
\hline
\end{tabular}

Tetragonal, higher symmetry

$\begin{array}{llllll}11 & 33 & 44 & 66 & 12 & 13\end{array}$

\begin{tabular}{|c|c|c|c|c|c|c|c|c|}
\hline Rutile $\left(\mathrm{TiO}_{2}\right)_{\ldots} \ldots$ & $\begin{array}{l}c \\
s \\
c \\
s\end{array}$ & $\begin{aligned} 27.3 \\
6.55 \\
26.6 \\
6.79\end{aligned}$ & $\begin{array}{c}48.4 \\
2.59 \\
47.0 \\
2.59\end{array}$ & $\begin{array}{c}12.5 \\
8.00 \\
12.4 \\
8.07\end{array}$ & $\begin{array}{r}19.4 \\
5.16 \\
18.9 \\
5.30\end{array}$ & $\begin{array}{c}17.6 \\
-3.76 \\
17.3 \\
-4.02\end{array}$ & $\begin{array}{c}14.9 \\
-0.86 \\
13.6 \\
-0.80\end{array}$ & $\begin{array}{l}{[37]} \\
{[38]}\end{array}$ \\
\hline Zircon...... & $\begin{array}{l}c \\
s\end{array}$ & $\begin{array}{l}7.35 \\
13.9\end{array}$ & $\begin{array}{c}4.60 \\
22.1\end{array}$ & $\begin{array}{c}1.38 \\
72.0\end{array}$ & $\begin{array}{l}1.60 \\
62.0\end{array}$ & $\begin{array}{r}0.90 \\
-1.6\end{array}$ & $\begin{array}{c}-0.54 \\
1.4\end{array}$ & [3] \\
\hline
\end{tabular}

Tetragonal, lower symmetry

$\begin{array}{lllllll}11 & 33 & 44 & 66 & 12 & 13 & 16\end{array}$

\begin{tabular}{l|l|r|r|r|r|r|r|r|r}
\hline $\mathrm{CaMOO}_{4} \ldots \ldots . .$. & $c$ & 14.47 & 12.65 & 3.69 & 4.51 & 6.64 & 4.46 & 1.34 & [39] \\
& s & 9.89 & 9.29 & 27.10 & 24.68 & -4.32 & -1.96 & -4.22 & \\
& $c$ & $\mathbf{1 4 . 5 0}$ & 12.82 & 3.67 & 4.56 & 6.18 & 4.96 & -1.46 & [40] \\
& 8 & 9.74 & 9.58 & 27.22 & 24.71 & -3.80 & -2.30 & 4.33 &
\end{tabular}

* References used by Hearmon [3] are not repeated in our list of references except in a few cases where comparison with other data on the same material is involved.

$s_{11}$ is the reciprocal of Young's modulus (defined as the ratio of an applied tensile stress to the resulting parallel tensile strain) and $s^{\prime}{ }_{11}$ continues to have this meaning when the coordinates are rotated. Thus, Young's modulus of cubic crystals varies with orientation as do all the other elastic moduli. It is therefore of importance to avoid misunderstanding in dealing with the elastic moduli of crystals, including cubic crystals, to refer all measurements to a standard set of axes $[41,42]$.

If $2\left(s_{11}-s_{12}\right)$ is equal to $s_{44}$, Young's modulus and all other elastic moduli become independent of orientation. Zener [43] has defined $A=2\left(s_{11}-\right.$ $\left.s_{12}\right) / s_{44}$, which is also equal to $2 c_{44} /\left(c_{11}-c_{12}\right)$, as the anisotropy factor for cubic crystals. It has the physical significance of expressing the ratio of the resistance to shear deformation in the [010] direction on the (100) plane to that in the [1 10$]$ direction on the (110) plane. A modified anisotropy factor for cubic crystals, $A^{*}$, has been introduced by Chung and Buessem [44]. The factor $A$ is found to vary with composition from 0.25 for $\mathrm{RbI}$ to about 8 for $\mathrm{Li}$ [44].

For elastically isotropic materials, the generalized Hooke's law takes the form

$$
\begin{aligned}
& S_{1}=s_{11} T_{1}+s_{12} T_{2}+s_{12} T_{3}+0+0+0, \\
& S_{2}=s_{12} T_{1}+s_{11} T_{2}+s_{12} T_{3}+0+0+0, \\
& S_{3}=s_{12} T_{1}+s_{12} T_{2}+s_{11} T_{3}+0+0+0, \\
& S_{4}=0+0+0+2\left(s_{11}-s_{12}\right) T_{4}+0+0, \\
& S_{5}=0+0+0+0+2\left(s_{11}-s_{12}\right) T_{5}+0,
\end{aligned}
$$$$
\text { and } S_{6}=0+0+0+0+0+2\left(s_{11}-s_{12}\right) T_{6} \text {. }
$$

That is, for isotropic materials $s_{44}=2\left(s_{11}-s_{12}\right)$. It is, however, more usual to describe an elastically isotropic body in terms of any two of the five quantities $Y=$ Young's modulus, $G=$ shear modulus (sometimes called one of Lamé's constants and designated by $\mu$ ), $\nu=$ Poisson's ratio, $B=$ bulk modulus, and $\lambda=$ the other Lamé constant. Table 4 gives the relations between these isotropic moduli; for example, if $B$ and $G$ are chosen as the independent pair, the remaining moduli are given by $Y=9 B G /(3 B+G), \quad \nu=(3 B-2 G) /(6 B+$ $2 G)$, and $\lambda=B-(2 G / 3)$. A connection to the compliances for elastically isotropic material is provided by $Y=1 / s_{11}$ and $G=1 / s_{44}=1 / 2\left(s_{11}-s_{12}\right)$,

\begin{tabular}{|c|c|c|c|c|}
\hline & \multicolumn{4}{|c|}{ Independent Pair of Moduli } \\
\hline & $Y, G=\mu$ & $B, G=\mu$ & $B, \nu$ & $\lambda, G=\mu$ \\
\hline$Y$ & $Y$ & $9 B G /(3 B+G)$ & $3 B(1-2 \nu)$ & $\begin{array}{c}G(3 \lambda+2 G) / \\
(\lambda+G)\end{array}$ \\
\hline$G=\mu$ & G & $G$ & $\begin{array}{c}3 B(1-2 \nu) / \\
2(1+\nu)\end{array}$ & $G$ \\
\hline$B$ & $Y G / 3(3 G-Y)$ & $B$ & $B$ & $\lambda+(2 G / 3)$ \\
\hline$\nu$ & $(Y / 2 G)-1$ & $\begin{array}{c}(3 B-2 G) / \\
(6 B+2 G)\end{array}$ & $\nu$ & $\lambda / 2(\lambda+G)$ \\
\hline$\lambda$ & $\begin{array}{l}(Y-2 G) G / \\
(3 G-Y)\end{array}$ & $B-(2 G / 3)$ & $3 \nu B(1+\nu)$ & $\lambda$ \\
\hline
\end{tabular}
$\nu=-s_{12} / s_{11}, B=1 / 3\left(s_{11}+2 s_{12}\right)$, and a connection to the elastic constants by $G=c_{44}, \lambda=c_{12}$.

TABLE 4. Expressions for various isotropic elastic moduli in terms of various pairs of moduli

Both glass (treated elsewhere in this volume [45]) and randomly oriented, fine grained polycrystalline ceramics are isotropically elastic. Values of isotropic moduli for polycrystalline ceramics are presented in table 5. This table gives values for pore-free ceramics obtained by measuring at various porosities and extrapolating to zero porosity. The elastic moduli of many porous ceramics have been measured [10]; we defer a

\begin{tabular}{|c|c|c|c|c|c|c|}
\hline \multirow[t]{2}{*}{ Substance } & \multirow[t]{2}{*}{ Porosity } & $Y$ & $G$ & $B$ & \multirow[t]{2}{*}{$\nu$} & \multirow[t]{2}{*}{ Reference } \\
\hline & & \multicolumn{3}{|c|}{$100 \mathrm{bar}=10^{10} \mathrm{~N} / \mathrm{m}^{2}$} & & \\
\hline $\mathrm{Al}_{2} \mathrm{O}_{3--}$ & 0.0 & 41.02 & & & & [46] \\
\hline $\mathrm{Al}_{2} \mathrm{O}_{3-}$ & .0 & 40.68 & 16.33 & $(26.65)$ & $(0.246)$ & {$[47,48]$} \\
\hline $\mathrm{Al}_{2} \mathrm{O}_{3--}$ & .0 & 40.20 & 16.40 & $(24.42)$ & $(.226)$ & [12] \\
\hline $\mathrm{Al}_{2} \mathrm{O}_{3}{ }^{*}$ & .004 & 39.86 & 16.14 & 25.02 & $(.235)$ & {$[12]$} \\
\hline $\mathrm{Al}_{2} \mathrm{O}_{3} \ldots$ & .0 & 40.39 & 16.35 & $(25.42)$ & $(.235)$ & [49] \\
\hline $\mathrm{Dy}_{2} \mathrm{O}_{3} \ldots$ & .0 & 17.05 & -..... & - n & $\ldots$ & {$[50]$} \\
\hline $\mathrm{Er}_{2} \mathrm{O}_{3} \ldots$ & .0 & 18.63 & & & & {$[50]$} \\
\hline $\mathrm{MgO}$ & .0 & 30.50 & 12.90 & $(15.99)$ & $(.182)$ & [51] \\
\hline MgO... & .0 & 31.77 & 13.96 & $(14.62)$ & $(.138)$ & [52] \\
\hline MgO.- & .0 & 31.00 & 13.15 & (16.08) & $(.179)$ & [12] \\
\hline $\mathrm{ThO}_{2}$ & .0 & 26. 10 & 10.06 & $(21.45)$ & $(.297)$ & [53] \\
\hline $\mathrm{TiO}_{2} \ldots$ & .0 & 28.42 & 11.15 & $(21.00)$ & $(.274)$ & [49] \\
\hline $\mathrm{Y}_{2} \mathrm{O}_{3}$ & .0 & 13.83 & & & & {$[54]$} \\
\hline $\mathrm{ZnO}$ & .0 & 12.35 & 4.56 & (14.11) & $(.354)$ & [49] \\
\hline
\end{tabular}
discussion of the effect of porosity on elastic moduli to section 3.7 .

TABLE 5. Elastic moduli of isotropic polycrystalline ceramics at or near zero porosity

Values in parentheses calculated from other values using expressions in table 4; $B$ value is quite sensitive to error in $Y$ or $G$.

*Values for all other materials extrapolated to zero porosity. These values taken at $\rho=3.972 \mathrm{~g} / \mathrm{cm}^{3}$ on alumina containing trace of $\mathrm{MgO}$; density of pure alumina from $\mathrm{x}$-ray lattice constants is $3.986 \mathrm{~g} / \mathrm{cm}^{3}$. 


\subsection{Nonlinear Elasticity}

'There has been much work done in recent years on nonlinear mechanics and on nonlinear elasticity in particular. 'The subject is large and complex; we can only give a brief introduction supported by references for further reading. Much of the recent work is directed toward attempting to describe the properties of fluids; Truesdell and Toupin [23] have given a general survey and Fung's book provides a readable introduction [55]. Within the very general field on nonlinear continuum mechanics we shall restrict our attention to the finite elasticity of solids.

In discussing finite strain we introduced the Lagrangian strain, $\eta_{i}$, and the Eulerian strain, $\epsilon_{i}$, as being appropriate for use with coordinate systems fixed in the material and in space respectively. Finite elasticity can be developed in terms of either but most of the experimental work is reported in terms of Lagrangian strain. The stress, $T_{k}$, which we discussed is referred to axes fixed in space. The corresponding quantity for axes fixed in the material is called "the thermodynamic tension," $t_{i j}$ in tensor notation or $t_{i}$ in matrix notation with the same rule relating $t_{i}$ to $t_{i j}$ as was given for relating $T_{i}$ to $T_{i j}$. The thermodynamic tension is related to the stress by [19]

$$
t_{i j}=J \sum_{k, l=1}^{3} T_{k l} \frac{\partial a_{i}}{\partial x_{k}} \frac{\partial a_{j}}{\partial x_{l}}
$$

where $J=$ determinant $\left(\frac{\partial x_{k}}{\partial a_{i}}\right)$ For infinitesimal strains $t_{i j}$ becomes equal to $T_{i j}$.

In introducing infinitesimal elasticity we considered the stress to be applied and developed equation (17) to express the resulting strain. Much of the basic data on nonlinear elasticity is determined in plane wave propagation experiments in which a strain is imposed by the wave motion and the resulting stress (for infinitesimal elasticity) would be given by equation (18). The literature on nonlinear elasticity of solids is accordingly written in terms of the $c_{i j}$ of equation (18) and their generalization rather than the $s_{i j}$ of equation (17).

To generalize equation (18) one first replaces the infinitesimal stress and strain by their Lagrangian counterparts for finite strain;

$$
t_{i}=\sum_{j=1}^{6} C_{i j} \eta_{j}
$$

One then adds a nonlinear term of the form

$$
\sum_{j, k=1}^{6} A(i j k) C_{i j k} \eta_{j} \eta_{k}
$$

to express the nonlinear contribution to the thermodynamic tension. The $A(i j k)$ are numerical constants which various authors have introduced dfferently leading to definitions of the third order elastic constants, $C_{i j k}$, which differ from one another by numerical factors. The definition which is usually used today is due to Brugger [56]. It is easiest to express Brugger's definition by recalling the relation that the stress is the derivative of the internal energy per unit volume with respect to the strain and using the corresponding relation for finite strain:

$$
t_{i}=\rho_{0}\left(\frac{\partial U}{\partial \eta_{i}}\right)_{s}=\rho_{0}\left(\frac{\partial A}{\partial \eta_{i}}\right)_{T} .
$$

The internal energy is taken per unit mass for finite strain and the factor $\rho_{0}$ (which is the density of the unstrained solid) is introduced to give the proper limit of energy per unit volume for infinitesimal strain. The internal energy is then given by [56]

$$
\rho_{0} U=\frac{1}{2} \sum_{i, j=1}^{6} c_{i j} \eta_{i} \eta_{j}+\frac{1}{6} \sum_{i, j, k=1}^{6} C_{i j k} \eta_{i} \eta_{j} \eta_{k}
$$

and the elastic constants are given by

$$
c_{i j}=\rho_{\circ}\left(\frac{\partial^{2} U}{\partial \eta_{i} \partial \eta_{j}}\right)_{s}
$$

and

$$
C_{i j k}=\rho_{\circ}\left(\frac{\partial^{3} U}{\partial \eta_{i} \partial \eta_{j} \partial \eta_{k}}\right) \cdot
$$

By using (25) and (26) one obtains the generalized form of (23) including the term (24) and the numbers $A(i j k)$. In using the original literature in which the third order elastic constants were introduced and enumerated for various crystal symmetries $[6,57,58,59,60]$ care must be used because of the different definitions used for the $c_{i j k}$. The relations between the different definitions are summarized by Brugger [56].

For cubic crystals there are three independent second-order elastic constants as mentioned earlier: $c_{11}=c_{22}=c_{33}, c_{12}=c_{13}=c_{23}, c_{44}=c_{55}=c_{66}$ and all others zero. The five cubic crystal classes split into one set of three with six independent thirdorder constants and a set of two with eight independent third-order elastic constants. Cubic crystals with point group $m 3 m, 432$, or $\overline{4} 32$ have the following six independent third-order elastic constants: $C_{111}=C_{222}=C_{333}, \quad C_{144}=C_{255}=C_{366}, C_{112}=$ $C_{223}=C_{133}=C_{113}=C_{122}=C_{233}, \quad C_{155}=C_{244}=C_{344}=C_{166}$ $=C_{266}=C_{355}, \quad C_{123}, C_{456}$, and all others zero. These third-order constants are determined experimentally by measuring the variation of the velocity of ultrasonic waves with application of homogeneous static stress as we shall discuss in a later section. This procedure causes some complexity in the analysis of the data; the experimental results give the isothermal variation of combinations of adiabatic effective second-order elastic constants with pressure. The resulting "mixed derivatives" must be corrected either to purely adiabatic or purely isothermal conditions 
using appropriate thermodynamic relations $[61$, $62,63,64]$. The term "effective second-order constants" is used to make a distinction from the thermodynamic second-order elastic constants which are defined as second derivatives of the energy. The effects of pressure enter the thermodynamic formulation through the third-order and higherorder elastic constants all of which are defined by (27) and (28) evaluated at zero strain and so are pressure independent. One may, however, choose to express the effect of pressure by allowing the second-order thermodynamic coefficient to be evaluated as a function of strain and so giving its pressure derivative. On the other hand, the effect of pressure may be described by using only the second-order constants which enter wave velocity expressions and allowing these to vary with pressure; in this case they are termed effective elastic constants. One may calculate either set of data given the other complete set, but the calculations are quite compex. This type of calculation is considered for various crystal symmetries and types of experiments in many papers; we refer to some of the more recent from which earlier references can be obtained $[65,66$, 67]. Both types of data are useful. One may be interested in the response of solids to isostatic pressure in which case a table of $\partial c_{i j} / \partial p$ is useful. Data for a number of solids is presented in table 6 .

For isotropic materials, as mentioned previously, the number of independent second-order elastic constants is reduced from three for cubic crystals to two. The three constants $c_{11}, c_{44}$, and $c_{12}$ are no longer independent; $c_{11}=2 c_{44}+c_{12}$. The independent pair are sometimes given the symbols $\lambda$ and $\mu$ and are termed the second-order Lainé constants with $\lambda=c_{12}$ and $\mu=c_{44}$. Similarly the six independent third-order elastic constants for cubic crystals reduce to three which are sometimes $[65,74]$ written $\nu_{1}, \nu_{2}, \nu_{3}$ and called the third-order Lamé constants with $\nu_{1}=C_{123}$, $\nu_{2}=C_{144}, \nu_{3}=C_{456}, \quad C_{112}=\nu_{1}+2 \nu_{2}, C_{155}=\nu_{2}+2 \nu_{3}$, and $C_{111}=\nu_{1}+6 \nu_{2}+8 \nu_{3}$. Some numerical values of third-order elastic constants for ceramic materials are given in table 7 and isotropic materials in table 8.

Recent developments in nonlinear elasticity theory include the calculation of lattice parameter changes with pressure in terms of second and thirdorder moduli [76]. Ghate [77, 78] has introduced fourth-order constants and calculated the pressure dependence of the effective second-order constants in terms of these; Chung has also considered fourth-ordered effects for cubic crystals [79]. 'Truesdell and Toupin [80] have considered conditions on elastic constants in finite elasticity similar to those previously mentioned for infinitesimal elasticity [24, 25].

TABLE 7. Third-order elastic constants of some ceramic crystals

All $C_{i j k}$ in units of $10^{10} \mathrm{~N} / \mathrm{m}^{2}$.

\begin{tabular}{|c|c|c|c|c|c|c|c|}
\hline Substance & $C_{111}$ & $C_{144}$ & $C_{112}$ & $C_{166}$ & $C_{123}$ & $C_{456}$ & $\begin{array}{l}\text { Refer- } \\
\text { ence }\end{array}$ \\
\hline $\begin{array}{l}\text { GaAs. } \\
\text { Ge } \\
\text { Ge... } \\
\text { MgO. } \\
\text { Yi } \\
\text { Y FeG }\end{array}$ & $\begin{array}{c}-62.2 \\
-71.0 \\
-71.6 \\
-489.5 \\
-82.5 \\
-233\end{array}$ & $\begin{array}{r}0.2 \\
-2.3 \\
-5.3 \\
11.3 \\
1.2 \\
-14.8\end{array}$ & $\begin{array}{r}-38.7 \\
-38.9 \\
-40.3 \\
-9.5 \\
-45.1 \\
-71.7\end{array}$ & $\begin{array}{l}-26.9 \\
-29.2 \\
-31.5 \\
-65.9 \\
-31.0 \\
-30.6\end{array}$ & $\begin{array}{l}-5.7 \\
-1.8 \\
-1.8 \\
-6.9 \\
-6.4 \\
-3.3\end{array}$ & $\begin{array}{l}-3.9 \\
-5.3 \\
-4.7 \\
14.7 \\
-6.4 \\
-9.7\end{array}$ & $\begin{array}{l}{[70]} \\
{[71]} \\
{[72]} \\
{[72]} \\
{[71]} \\
{[73]}\end{array}$ \\
\hline
\end{tabular}

TABLE 8. Third-order elastic constants of isotropic materials All $C_{i j k}$ in units of $10^{10} \mathrm{~N} / \mathrm{m}^{2}$.

\begin{tabular}{|c|c|c|c|c|}
\hline Substance & $\nu_{1}=C_{123}$ & $\nu_{2}=C_{144}$ & $\nu_{3}=C_{456}$ & Reference \\
\hline $\begin{array}{l}\text { Polycrystalline } \mathrm{Mo}_{0}{ }^{1} \\
\text { Polycrystalline } \mathrm{W} \\
\text { Vitreous } \mathrm{SiO}_{2}\end{array}$ & $\begin{array}{r}19.4 \\
-42.9 \\
54\end{array}$ & $\begin{array}{l}-39.8 \\
-25.8 \\
93\end{array}$ & $\begin{array}{l}-22.7 \\
-26.7 \\
-11\end{array}$ & $\begin{array}{l}{[75]} \\
{[75]} \\
{[72]}\end{array}$ \\
\hline
\end{tabular}

1 Specimens were sintered and had some porosity.

\subsection{Generalizations of Linear Elasticity}

Mindlin and Tierstein [81, 82] have proposed a generalization of linear elasticity in which couplestresses acting on a unit area are taken into account as well as the usual stress. A proportionality is assumed between couple-stress (couple per unit area) and curvature (gradient of rotation). The ratio of couple-stress to curvature is then a new type of modulus of elasticity with the dimensions of force. The theory has been generalized to take into account nonlinear effects [83, 84]. The linear theory has been applied to the calculation of stress distribution around a hole [82] and to the bending and torsion of bars [85]. Schijve [86] has

TABLE 6. Dependence of effective second-order elastic constants upon pressure, $\left(\frac{\mathrm{dc}_{\mathrm{ij}}{ }^{8}}{\mathrm{dp}}\right)$, at zero pressure and their values, $\mathrm{c}_{\mathrm{ij}}{ }^{\mathrm{s}}$ for Some Ceramics at $25^{\circ} \mathrm{C}$.

Superscript $S$ indicates constant entropy.

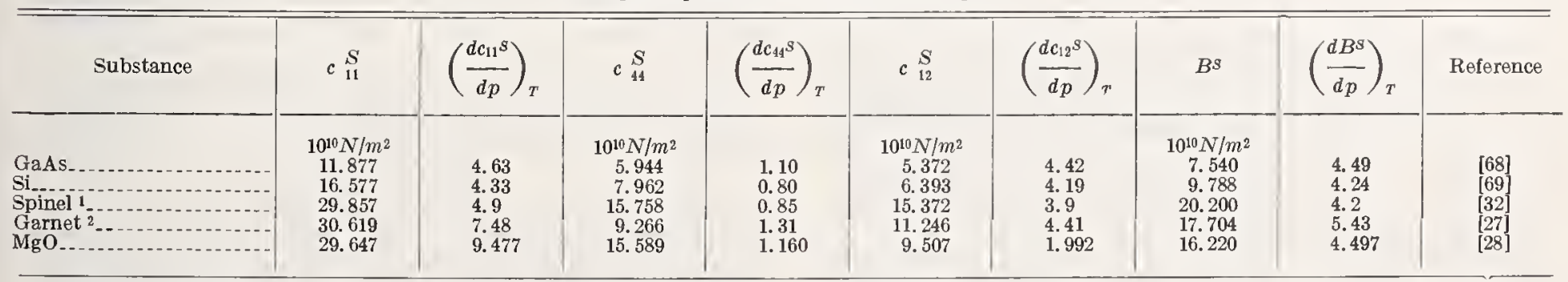

$1 \mathrm{MgO} \cdot 2.61 \mathrm{Al}_{2} \mathrm{O}_{3}$.

2 Almandite-pyrope type. 
carried out bending experiments which suggest that the new couple-stress elastic moduli for aluminum are small (he could not detect them) and that stress distributions around holes are not likely to be in significant error if couple-stress effects are neglected. It should be noted that the couple-stress theory appears to be on a firm theoretical foundation; only the size of the effect is in question.

A different attempt to generalize linear elasticity was previously made by Laval [87] and by Raman and his co-workers [88-90]. This point of view has been developed by LeCorre [91] and by Joel and Wooster [92]. Some data from the literature were examined [90, 93] and it was claimed that the classical theory of infinitesimal elasticity was inadequate. Recently, however, a careful experimental test of Raman's version [89] of this theory was made [94]; the results support the classical theory. In any event, it appears that the proper way to introduce rotations into elasticity is that discussed in the preceeding paragraph and that the effects are small, at least for small strains. Further discussion of the Laval-Raman approach can be found in Huntington's review paper [7].

\section{Specific Problems Involving Elastic Deformation}

\subsection{General Discussion}

So far we have been concerned with elasticity as a way of describing a property of material; we have assumed the stress and strain to be uniform throughout the material. In many practical situations the stress and strain vary from point to point in a specimen being tested for its properties or in a material in service. Many materials are inhomogeneous so that the stress varies within the body even though the external loading may be uniform. We must therefore be concerned with some problems involving stress distribution within materials even though it is the properties of materials and not the solution of elasticity problems that is our subject.

The propagation of elastic waves is the basis for the most accurate methods of determination of elastic properties as well as being of great importance to the study of many other physical properties (physical acoustics), to nondestructive testing, to military applications, and to geological studies. Accordingly, we sketch some of the elementary results of the theory of wave propagation in solids.

Resonance of solid shapes is another technique used to measure elastic properties. Resonance is also a behavior of considerable engineering importance. We therefore give the relations between resonance frequencies and elastic properties for a few simple shapes.

Polycrystalline ceramics are aggregates of elastically anisotropic crystals and frequently include pores. The effect of porosity and the relation between bulk elastic properties and the properties of the grains are therefore discussed. Ceramics are increasingly being fabricated as composites so that the elastic properties of composites are also of interest.

\subsection{Elastic Waves in Isotropic Materials}

The simplest and most widely used type of elastic wave is a plane wave in an infinite medium (one whose dimensions are many times the wavelength). The general result (as derived, for example, by Kolsky [95]) is that two types of plane wave can be propagated in an isotropic medium. The first is called longitudinal (or irrotational or dilational) and has its direction of vibration parallel to the direction of propagation. The second is called transverse (or equivoluminal or distorsional) and has its direction of vibration perpendicular to the direction of propagation. For each wave the velocity squared times the density is equal to an elastic modulus or combination of elastic moduli. Table 9 lists expressions for $\rho \mathrm{v}^{2}$ in terms of various choices of elastic moduli; see Anderson et al. [12, 96] for expressions in terms of the pairs $B, Y$, and $Y, v$ as well as most of the present pairs. If the velocities and density are known, one can, for example, solve for $G$ and for $\lambda+2 G$ (see the last column of table 9) and then obtain other elastic moduli by using the expressions in terms of $\lambda$ and $G$ given in table 4.

When the body is not large compared with the wavelength, these expressions no longer hold; the velocities become dependent on frequency and approximation methods must be used in most cases. One case which can be solved exactly is that of a very thin cylindrical rod (circular cross section). The equations for $\rho v_{t}^{2}$ are unchanged but the longitudinal waves have a different velocity, termed the rod velocity, $v_{r}$, which is given by $\rho v_{r}{ }^{2}=Y$. If the diameter of an initially thin rod is continuously increased, the value of $\rho v_{\tau}{ }^{2}=Y$ should change to $\rho v_{l}^{2}=Y(1-\nu) /(1+\nu)(1-2 \nu)$ which is equivalent to the forms of table 9 . The transition is not continuous, however, surface waves are involved; the two values of $\rho v^{2}$ are correct for the limits of small and large diameters, respectively [97-102]. The same transition occurs if the dimensions of a slender rod are kept constant but the frequency is changed from a low value to a high one. At sufficiently low frequency the wavelength (given by $\lambda=v / f$ where $f$ is the frequency) will be large compared with the diam-

TABLE 9. Expressions for $\rho v^{2}$ for Longitudinal $\left(v_{1}\right)$ and Transverse Waves $\left(\mathrm{v}_{\mathrm{t}}\right)$ in an Infinite, Isotropic Medium

\begin{tabular}{c|c|c|c|c}
\hline \hline & \multicolumn{3}{|c}{ Independent Pair of Moduli } \\
\hline $\begin{array}{c}\rho v_{l}^{2} \\
\rho v^{2}\end{array}$ & $\begin{array}{c}G(4 G-Y) /(3 G-Y) \\
G\end{array}$ & $B, G=\mu$ & $B, \nu$ \\
\hline
\end{tabular}


eter of the rod and $\rho v_{r}^{2}$ should approach the value $Y$. It is therefore convenient in the resonance method of determining Young's modulus to use slender rods; correction factors for finite thickness are discussed in a subsequent section. At sufficiently high frequency, the wavelength becomes small compared with the rod diameter and $\rho v^{2}$ should approach $\rho v_{l}^{2}$. In the high-frequency methods of determining elastic moduli it is therefore convenient to use cylinders of large diameter. Experimental work [102] supported the theoretical prediction that as the diameter-to-wavelength ratio increased the bulk velocity was approached; for ratio values above five no difference in velocity was detected. Modern, high-precision methods are capable of precision of velocity measurement exceeding one part in $10^{5}$, however, and diameter-towavelength ratios of 50 or more appear necessary when working to this precision. For aluminum oxide $v_{l}$ is about $10^{4} \mathrm{~m} / \mathrm{sec}$ so that a diameter of $5 \mathrm{~cm}$ would be required for a frequency of $10 \mathrm{Mhz}$ or $0.5 \mathrm{~cm}$ for $100 \mathrm{MHz}$ for high precision work.

The propagation of elastic compressional waves in rectangular bars [103], in impulsively loaded cylindrical rods [104], and in explosively loaded plates [105] has also been studied. Truesdell [106] has discussed the propagation of waves in an isotropic elastic material subject to a finite static strain.

\subsection{Elastic Waves in Anisotropic Materials}

Single crystals are elastically anisotropic as are most composite materials. The symmetry of many composite materials is the same as that of certain crystals so that the results derived for single crystals can sometimes be applied to certain composites. For this reason a qualitative understanding of the elastic behavior is useful in discussing behavior of engineering materials even though single crystals may not be directly involved.

A simple example of wave propagation in an elastically anisotropic material is that of plane wave propagation along the [110] direction in a cubic crystal. It is found [8] that a longitudinal wave (vibration in the [110] direction) travels with velocity given by

$$
\rho v_{l}^{2}=\left(2 c_{44}+c_{11}+c_{12}\right) / 2
$$

In addition, there are two transverse waves: $v_{t 1}$ with vibration in the $[1 \overline{1} 0]$ direction and $v_{t 2}$ with vibration in the [001] direction. Their velocities are given by

$$
\rho v_{t 1}^{2}=\left(c_{11}-c_{12}\right) / 2,
$$

and

$$
\rho v_{t 2}^{2}=c_{44} \text {. }
$$

This example illustrates one feature of a general result for small-amplitude elastic waves in an unstrained body. For any given direction of prop- agation there are three waves whose directions of vibration are at right angles to each other. In the above example one direction of vibration coincides with the direction of propagation so that one wave is purely longitudinal and the other two are purely transverse. In general, however, one wave is quasi-longitudinal (vibration at a small angle to the direction of propagation) and the other two are quasi-transverse. If the wave is propagating along an axis of rotational symmetry one wave is required by symmetry to be purely longitudinal; the other two are accordingly purely transverse. In addition to these puremode directions, each type of crystal symmetry permits some additional special directions whose number and orientation depend on the numerical values of the elastic constants. These special directions have been tabulated for all crystal symmetries except monoclinic and triclinic by Borgnis [107] and Brugger [108]. These directions are useful because a plate cut perpendicular to such a direction will vibrate in a pure thickness mode [107] and because the use of these directions for the propagation of waves simplifies the determination of the third-order elastic constants [65, 66]. A second class of special directions is one for which a pure transverse mode may be propagated but for which the other two modes are quasi-transverse and quasi-longitudinal; equations for these directions have been derived for several classes of crystal symmetry [109].

The expressions for the velocity of propagation in directions for which crystal symmetry requires pure longitudinal and transverse wave propagation are easily derived and many have been tabulated [8]. Unfortunately these do not provide enough information to completely determine the elastic constants for low-symmetry crystals. A complete determination for low-symmetry tetragonal crystals has, however, been carried out [39] and equations for monoclinic crystals have been derived [110].

An interesting feature of wave propagation in anisotropic crystals is the fact that the ray direction need not be parallel to the direction of propagation of a plane wave [111-115]. This effect occurs for light waves as well as sound waves. Thus, if a mechanical transducer (such as a quartz crystal) is cemented on an arbitrary flat face of a crystal specimen, three plane waves (one quasilongitudinal and two quasi-transverse) will be excited in the crystal. Each will have its direction of propagation normal to the face of the specimen but the energy will travel along inclined directions (ray direction) and each of the three rays will reach the opposite side of the specimen at a region not directly opposite the transducer. It is desirable when possible to avoid this complication by choosing directions for which the ray direction coincides with the wave normal, but even when this is not done the velocity expressions mentioned previously are still correct. One must arrange the experiment to measure the wave 
velocity even though the ray is traveling at an angle [116]. A method of calculating the elastic constants by successive approximations using relocities measured in an arbitrary direction has been developed $[117,118]$.

\subsection{Attenuation of Elastic Waves by Scattering and Nonlinear Effects}

Elastic waves lose amplitude by gradual conversion of their energy into heat. This occurs by many mechanisms; this subject is generally termed "Physical Acoustics" and is a large subject in itself $[119,120]$. This attenuation in solids frequently is caused by nonelastic behavior associated with impurities and defects; these effects are briefly discussed in a subsequent section. Attenuation also takes place in purely elastic materials by scattering in inhomogeneous materials and as a result of nonlinearity. Mason and McSkimin [121] have discussed scattering in polycrystals; the process of Rayleigh scattering which takes place when the wavelength is greater than three times the grain size gives a loss proportional to grain volume. At higher frequencies the type of loss process changes and becomes a diffusion process with the loss inversely proportional to grain diameter when the wavelength is one third the grain diameter or less.

In pure and highly perfect single crystals the attenuation is typically very low at frequencies of a few megahertz but increases with increasing frequency due to interaction between the elastic waves and thermal phonons through the thirdorder elastic constants [122-124].

The velocities of elastic waves in rocks and minerals have been compiled $[12,125]$ and the attenuation observed in seismology has been discussed [126].

\subsection{Resonance of Isotropic Materials}

A simple type of resonance is the thickness vibration of a thin plate. The motion can be described by a pattern of standing waves resulting from the reflection of traveling waves at the large flat surface of a plate. Constructive interference will occur whenever the thickness of the plate equals an integral number of half wave-lengths; $t=n \lambda / 2=n v / 2 f$. If the lateral dimensions of the plate are large, the velocity of waves in an infinite medium can be substituted. Thus, for thicknesslongitudinal resonance of a thin flat plate of large extent

$$
\lambda+2 G=4 \rho f_{l}^{2} t^{2} / n^{2}
$$

and for thickness-shear resonance

$$
G=4 \rho f_{t}^{2} t^{2} / n^{2} .
$$

For a cylinder of circular cross section the last equation holds unchanged and results from an analysis of torsional resonance so that a cylinder of any diameter-to-length ratio is suitable for the determination of the shear modulus from torsional resonance. The longitudinal resonance frequency of a very slender cylinder can also be deduced from the condition for standing waves and the resulting equation is

$$
Y=4 \rho f_{l}^{2} t^{2} / n^{2} K_{n}
$$

where $K_{n}$ approaches one exactly as the diameterto-length ratio approaches zero. Rayleigh derived an approximate expression for $K_{n}$ valid for small diameter-to-length ratios which is

$$
K_{n}=1-n^{2} \pi^{2} \nu^{2} d^{2} / 8 l^{2}
$$

More accurate $K_{n}$ values can be obtained from Bancroft's [97] velocity results; a table is given by Spinner and Tefft [127] who also summarize theoretical and experimental resonance equations for torsional, longitudinal, and flexural vibrations of cylinders and bars of rectangular cross section. For the fundamental mode of flexure of a cylinder

$$
Y=1.261886 \rho l^{4} f^{2} T / d^{2}
$$

where $T$ is a correction factor [128-130] which approaches one exactly as $d / l$ approaches zero and which is tabulated up to $d / l=0.60$ [128]. Equations for the vibration of thick-walled hollow cylinders have also been derived [131]. Lucey [132] has proposed approximate equations for thick discs and thick cylinders derived by requiring the first order corrections for thin discs and slender rods to be compatible.

Resonance equations for bars of rectangular cross section are available [128] but are accurate only for slender bars.

A sphere displays two types of vibrational modes [133, 134]; one, designated $T_{n m}$ is torsional and involves only shear deformation so that the shear modulus can be calculated from a frequency measurement. The other type, designated $S_{n m}$, is spheroidal and involves both shear and dilation so that a frequency measurement combined with a knowledge of the shear modulus completely determines the properties of an isotropic material.

All of the discussion of resonance refers to free vibration. In practice care must be taken not to constrain the vibration if the equations for free vibration are to be used.

\subsection{Resonance of Anisotropic Materials}

The resonance of thin plates of large extent can be treated as before by using $t=n v / 2 f$ but for anisotropic materials the velocity is a function of the orientation and the single crystal elastic constants as previously discussed. Many single crystals are piezoelectric so that the electrical boundary conditions must be taken into account. Tiersten treats this problem [135]; vibration of plates has been used to determine the elastic constants of several piezoelectric crystals [136]. 
The vibration of slender single crystal cylinders has been treated by Voigt [137] and Goens [138]; the use of this theory has been clarified by Kimura [139] and Brown [140]. The general result is that the flexural and torsional modes are coupled except when the axis of the cylinder lies along certain special orientations in the crystal. This coupling changes the flexural and torsional resonance frequencies in general. If, however, the cylinder is very slender, the flexural resonance frequency is unchanged and the torsional frequency is shifted by an amount which can be calculated. Measurements of flexural and torsional resonance frequencies can therefore be used to determine the single crystal elastic constants as has been done, for example, for $\mathrm{CaMoO}_{4}[40]$.

\subsection{Effect of Porosity on Elastic Moduli of Isotropic Materials}

Most polycrystalline ceramics are porous; we shall define porosity by $P=$ fractional pore volume. Elastic moduli (bulk, shear, or Young's modulus) all decrease with increasing porosity; the initial decrease is rapid but becomes less rapid at higher porosity. Many authors have proposed equations to account for this effect; these equations are listed in table 10. Equations 2, 3, 6, 7, 13, and 14 of this table are theoretical equations and should agree at small porosity values. In fact they do; all reduce to the form $G / G_{0}=1-b p$ and similarly for $B$ and $Y$ at small porosity with the same values of $b$ from any of the equations for the same modulus [53]. For a Poisson's ratio value $\nu_{0}=0.20$ all give $b=2.00$ [149]. The predicted slope agrees with experiment for alumina with large, artificially made spherical pores [144] and for glass with spherical pores [149]. At larger values of porosity

TABLE 10. Equations proposed by various authors for porosity dependence of elastic moduli of isotropic materials

Subscript zero indicates value at zero porosity.

\begin{tabular}{|c|c|c|}
\hline Number & Equation & Reference \\
\hline $\begin{array}{l}1 \\
2 \ldots \\
3 \\
4 \\
5 a \\
6 \\
7 \\
8{ }^{b} \\
9 b \\
10^{b} \\
11^{d} \\
12^{d} \\
13 \\
14 \\
15 \\
15\end{array}$ & $\begin{aligned} & G= G_{0}[1-5 P / 3] \\
& 1 / B= 1 / B_{0}(1-P)+3 P / 4 G_{0}(1-P)+\text { terms of } \\
& \text { order } P 3 \\
& G= G_{0}\left[1-5 P\left(3 B_{0}+4 G_{0}\right) /\left(9 B_{0}+8 G_{0}\right)+\text { terms }\right. \\
&\left.\text { of order } P^{2}\right] \\
& d Y / d P= 2.36 \\
& G=G_{0}\left[1-5 P\left(3 B_{0}+4 G_{0}\right) /\left(9 B_{0}+8 G_{0}\right)+A P^{2}\right] \\
& B=B_{0}[1-P(1+3 B / 4 G)] \\
& G=G_{0}[1-5 P(3 B+G) /(9 B+8 G)] \\
& Y=Y_{0} \exp (-b P) \\
& G=G_{0} \exp (-b P) \\
& Y=Y_{0} \exp \left(-b_{0} P_{0}-b_{c} P c\right) \\
& B=B_{0}[1-A P /(1-(A+1) P)] \\
& G=G_{0}[1-A P /(1-(A+1) P)] \\
& B=B_{0}(1-P) /\left[1+\left(1+\nu_{0}\right) / 2 P\left(1-2 \nu_{0}\right)\right] \\
& G=G_{0}(1-P) /\left[1+2\left(4-5 \nu_{0}\right) / P\left(7-5 \nu_{0}\right)\right] \\
& Y=Y_{0}(1-P) /\left[1+\left(1+\nu_{0}\right)\left(13-15 \nu_{0}\right) /\right. \\
&\left.2 P\left(7-5 \nu_{0}\right)\right] \\
& G=G_{0}\left(1-b P+c P^{2}\right) \\
& Y=Y_{0}\left(1-b P+c P^{2}\right) \\
& G=G_{0}\left[1-15 P\left(1-\nu_{0}\right) /\left(7-5 \nu_{0}\right)\right] \\
& Y=Y_{0}\left[1-3 P\left(9+5 \nu_{0}\right)\left(1-\nu_{0}\right) / 2\left(7-5 \nu_{0}\right)\right]\end{aligned}$ & $\begin{array}{r}{[141]} \\
{[142]} \\
{[142]} \\
{[143]} \\
{[144]} \\
{[145]} \\
{[145]} \\
{[47]} \\
{[48]} \\
{[146]} \\
{[147]} \\
{[147]} \\
{[148]} \\
{[148]} \\
{[148]} \\
{[53]} \\
{[53]} \\
{[153]} \\
{[153]}\end{array}$ \\
\hline
\end{tabular}

a Parameter $A$ to be determined by setting $G=0$ at $P=1$.

- Parameter $b$ to be determined experimentally.

$c$ Parameters $b_{0}$ and $b_{c}$ to be determined experimentally. Subscripts $o$ and

c refer to open and closed pores.

¿ Parameter $A$ to be determined empirically.

- Parameters $b$ and $c$ to be determined experimentally. the slope becomes smaller in absolute value. The equations of Hashin as modified by Hasselman [147], equations 11 and 12 of table 10, and by Weil [148], equations 13 and 14 of table 10, appear preferable in combining a theoretical foundation with a proper form, but the empirical equations $8,9,16$, and 17 appear to give a satisfactory fit to the data out to 40 or 50 percent porosity.

Although the theoretical equations correctly predict the slope at low porosity for specimens with spherical pores, the slope in normal polycrystalline ceramics is larger numerically [46]. Table 11 gives slope values for a number of polycrystalline ceramics; the values vary widely for some materials and generally depart from the theoretically expected value of about 2.0. Spriggs [146] proposed that open and closed pores affect elastic moduli to a different extent (see equation 10 of table 10). It has long been suspected that $b$ values greater than 2.0 (see table 11 for examples) are the result of pores not being spherical. Hasselman and Fulrath [151] used the solution by Hashin and Rosen [152] for cylindrical pores perpendicular to the direction of measurement and found that the data on alumina compiled by Knudsen [46] fall within the upper and lower bounds predicted by Hashin and Rosen's theory.

TABLE 11. Slope of elastic modulus versus porosity curve at zero porosity

\begin{tabular}{|c|c|c|c|c|}
\hline Material & Modulus & $\begin{array}{l}\text { Type of } \\
\text { equation } \\
\text { fitted to } \\
\text { determine } \\
\text { slope }\end{array}$ & Slope $=b$ & References \\
\hline $\begin{array}{l}\mathrm{Al}_{2} \mathrm{O}_{3} \\
\mathrm{Al}_{2} \mathrm{O}_{3} \\
\mathrm{Al}_{2} \mathrm{O}_{3} \\
\mathrm{BeO} \\
\mathrm{BeO} \\
\mathrm{Dy}_{2} \mathrm{O}_{3} \\
\mathrm{Er}_{2} \mathrm{O}_{3} \\
\mathrm{Glass}^{*} \\
\mathrm{Glass}^{*} \\
\mathrm{MgO} \mathrm{M} \\
\mathrm{MgO} \\
\mathrm{ThO}_{2} \\
\mathrm{ThO}_{2} \\
\mathrm{Y}_{2} \mathrm{O}_{3}\end{array}$ & $\begin{array}{l}Y \\
Y \\
G \\
Y \\
G \\
Y \\
Y \\
Y \\
G \\
Y \\
G \\
Y \\
G \\
Y\end{array}$ & $\begin{array}{l}\text { A } \\
\text { A } \\
\text { A } \\
\text { B } \\
\text { B } \\
\text { C } \\
\text { C } \\
\text { C } \\
\text { C } \\
\text { A } \\
\text { A } \\
\text { D } \\
\text { D } \\
\text { C }\end{array}$ & $\begin{array}{c}1.61 \text { to } 4.35 \\
3.95 \\
1.72 \text { to } 3.26 \\
1.99 \text { to } 2.81 \\
2.00 \text { to } 2.80 \\
2.44 \\
2.36 \\
2.06 \\
1.94 \\
4.74 \\
3.90 \\
2.34 \text { to } 2.68 \\
2.52 \text { to } 2.88 \\
2.49\end{array}$ & $\begin{array}{r}{[47]} \\
{[46]} \\
{[146]} \\
{[150]} \\
{[150]} \\
{[50]} \\
{[50]} \\
{[149]} \\
{[149]} \\
{[52]} \\
{[52]} \\
{[53]} \\
{[53]} \\
{[54]}\end{array}$ \\
\hline
\end{tabular}

A: $Y=Y_{0} \exp (-b P)$.

B: $Y=Y_{0}[1-b P /(1-(b+1) P)]$.

C: $Y=Y_{0}(1-b P)$.

D: $Y=Y_{0}\left(1-b P+c P^{2}\right)$

${ }^{*} 16 \% \mathrm{Na} \mathrm{Na}_{2} \mathrm{O}, 14 \% \mathrm{~B}_{2} \mathrm{O}_{3}, 70 \% \mathrm{SiO}_{2}$.

\subsection{Relation of Elastic Moduli of Poly. crystalline Materials to the Moduli of the Single-Crystal Grains}

The elastic moduli of isotropic aggregates of anisotropic crystals in terms of the moduli of the crystals has been calculated by a number of writers. There are two general approaches. In the first approach upper and lower bounds are rigorously derived; in the second approach approximations are introduced to calculate an average value whose reliability is then difficult to assess.

The first approach was introduced by Hill [153] who showed that averages previously in- 
troduced by Voigt and Reuss were in fact upper and lower bounds respectively; i.e., $B_{v} \geq B \geq B_{r}$ and $G_{0} \geq G \geq G_{r}$. The values for these bounds are given in table 12. Hashin and Shtrikman developed a variational method giving closer bounds and used it to obtain specific expressions for cubic crystals [154-156]. Peselnick and Meister [157, 158] have used this method to determine bounds for crystals of hexagonal, higher trigonal, and higher tetragonal symmetry. The Hashin-Shtrikman bounds are always better and sometimes much better than the Voigt-Reuss-Hill bounds as shown in a number of compilations $[11,13,157$, 158].

Table 12. Expressions for Bounds and Estimates on Elastic Moduli of an Isotropic Aggregate of Anisotropic Crystals

\begin{tabular}{lc}
\hline \hline & Crystals of Any Symmetry \\
\hline$B_{0} \geq B \geq B_{r}$ and & (Voigt-Reuss-Hill) $[7,152]$ \\
$G_{0} \geq G \geq B_{r}$ & \\
where: & \\
$B_{0}$ & $=\left(c_{11}+c_{22}+c_{33}+2 c_{12}+2 c_{23}+2 c_{13}\right) / 9$ \\
$G_{0}$ & $=\left(c_{11}+c_{22}+c_{33}-c_{12}-c_{23}-c_{13}+3 c_{44}+3 c_{55}+3 c_{86}\right) / 15$ \\
$B_{r}$ & $=\left[s_{11}+s_{22}+s_{33}+2 s_{12}+2 s_{23}+2 s_{13}\right]^{-1}$ \\
$G_{0}$ & $=\left[\left(4 s_{11}+4 s_{22}+4 s_{33}-4 s_{12}-4 s_{23}-4 s_{13}+3 s_{44}+3 s_{55}+3 s_{66}\right) / 15\right]^{-1}$
\end{tabular}

Cubic Crystals

$B=\left(c_{11}+2 c_{12}\right) / 3 \quad \begin{aligned} & \text { (Exact expression) } \\ & G \text { lies between } G_{1}^{*} \text { and } G_{2}^{*} \quad \text { (Hashin and Shtrikman as expressed by } \\ & \text { Simmons [13]) }\end{aligned}$
$\begin{aligned} \text { Where: } & \\ G_{1}^{*} & =G_{1}+3\left[-4 \beta_{1}+5 /\left(G_{2}-G_{1}\right)\right]^{-1} \\ G_{2}^{*} & =G_{2}+2\left[-6 \beta_{2}+5 /\left(G_{1}-G_{2}\right)\right]^{-1} \\ \beta_{1} & =-3\left(B+2 G_{1}\right) / 5 G_{1}\left(3 B+4 G_{1}\right) \\ \beta_{2} & =-3\left(B+2 G_{2}\right) / 5 G_{2}\left(3 B+4 G_{2}\right) \\ G_{1} & =\left(c_{11}-c_{12}\right) / 2 \\ G_{2} & =c_{49}\end{aligned}$

$G$ is conjectured by Kroner [160] to be exactly equal to a root of the equation: where:

$a=3 \mu(k+4 \nu) / 6 k \nu$

$b=\mu(9 k+4 \nu) / 6 k \nu$

$c=4 \mu^{2} / 3 k \nu$

$k=\left(c_{11}+2 c_{12}\right) / 3$

$\mu=c_{44}$

$\nu=\left(c_{11}-c_{12}\right) / 2$

The second approach was followed by Hershey and by Kroner [159, 160] who used a self-consistent method. Kroner et al. [161] have shown that his solution for the shear modulus of an aggregate of cubic crystals lies within the Hashin-Shtrickman bounds and Kroner suggests that his result is exact provided the orientations are completely random. Kroner [160] subsequently derived another expression for the shear modulus using the correlation function method; this answer is also said to be exact but is too complex to give here. Walpole $[162,163]$ has given another proof of the Hashin-Shtrickman bounds and confirmed that Kroner's first solution does lie within the HashinShtrikman bounds for cubic crystals.

Polycrystalline ceramics sometimes display sufficient preferred orientation to significally affect elastic moduli. Extruded $\mathrm{BeO}$ shows varying degrees of preferred orientation depending on the starting materials and the degree of sintering [150]. The variation of Young's modulus with degree of preferred orientation has been used to draw con- clusions about the single crystal elastic constants [164]. Rocks usually have a preferred orientation of the grains; the linear compressibility has been studied as a function of preferred orientation [165].

\subsection{Elastic Moduli of Composites}

The engineering importance of a wide variety of composite materials has led to extensive work on means of describing and measuring their bulk properties and on the relationship between these bulk properties and the properties of their constituents. One important type of composite is typified by a plywood sheet. This is usually treated as a problem in two-dimensional elasticity and orthorhombic symmetry is assumed; the material is said to have orthotropic symmetry. Formulas for bending, twisting, etc. are available [166].

Another important class of composites consists of slender fibers dispersed in a matrix. The case in which all the fibers are aligned parallel to each other and are so long that end effects can be neglected has been treated in detail. Such a composite has five independent elastic moduli and is equivalent to a single crystal with hexagonal point group. A convenient description of elastic properties is provided by using the plane strain bulk modulus, the tranverse shear modulus, the axial shear modulus, the axial Young's modulus, and the corresponding Poisson's ratio. Upper and lower bounds in terms of the elastic moduli and volume fraction of the fibers and matrix have been derived [167, 168]. Hill [169] has derived estimates for these five moduli, using the selfconsistent method, which lie within these bounds. Similar estimates have been given for aligned ellipsoids or spheres in a matrix [170-171]. Comparisons with some of these theoretical results have been made for the systems graphite in zirconium carbide [172], alumina in glass [149], and tungsten in glass [173].

A number of special problems involving the elastic response of composites have been treated with varying degrees of approximation. We mention concrete [174], plaster and procelain [175], and rocks containing cracks [176-178].

\section{Atomic Theory of Elasticity}

\subsection{Lattice Statics}

A treatment of the internal energy of a crystal in terms of elastic constants and a simple atomic model was given by Born in 1918 [179; 180]. The theory has subsequently been elaborately developed but the original simple treatment still gives insight into the dependence of elastic constants on interatomic distance and on a unique characteristic of many ceramic oxides. In this simple model one assumes the energy is given by

$$
U=-A / r^{m}+B^{\prime} / r^{n}
$$


where $r$ is the interatomic spacing. The first term is the contribution due to attractive force; for ionic crystals having ions with charge $z$ the exponent $m$ is one and the addition of all the Coulomb forces gives $A=a(z e)^{2}$ where $a$ is a numerical constant $(a=1.7476$ for $\mathrm{MgO})$ obtained from this sum. The second term is the contribution of the repulsive forces; the constants $B^{\prime}$ and $n$ must be determined by fitting the equation to experimental data in the following manner. We have from thermodynamics.

$$
\begin{gathered}
p=-\frac{d U}{d v} \\
B=-v \frac{d p}{d v} .
\end{gathered}
$$

Since $v=$ constant times $r^{3}$, we have $3 v d r=r d v$ and so obtain

and

$$
p=\left(-A / r+n B^{\prime} / r^{n}\right) / 3 v
$$

We now use $r=r_{0}$ at $p=0$ and obtain

which gives

$$
n B^{\prime} / r_{0}{ }^{n}=A / r_{0}
$$

$$
B=p(1+n / 3)+(n-1) A / 9 r v .
$$

The parameter $n$ is determined by setting $p=0$ and requiring this expression to give the measured value of the bulk modulus. The resulting value of $n$ ranges from 6 to 12 for various alkali halides and from 6 to 9.5 for a small number of oxides considered [181]. The internal energy calculated from $n$ usually agrees with the measured cohesive energy within 10 percent. This simple theory has a further success in predicting how the bulk modulus varies with lattice parameter in a family of ionic compounds with the same crystal structure. Taking $p=0$ and $r=r_{0}$ we have

$$
B=a\left(z e^{2}\right)(n-1) / 9 r_{0} v_{0} .
$$

Since $r_{0} v_{0}$ is proportional to $a^{4}$ (where $a$ is the lattice parameter) we expect plots of $\log B$ as a function of $a$ to have a slope of -4 and plots of both the bulk modulus and the shear modulus do show this slope for alkali halides [182, 183]. The above treatment also provides a basis for equation (6) which is the basic assumption needed for the successful Murnaghan equation, number (7).

Oxides do not generally obey the predicted $1 / a^{4}$ variation of bulk modulus, however. The variation is more nearly described by $1 / a^{9}[183$, 184], see figure 5. Anderson [183] has given a qualitative explanation in terms of a modification of the above model in which another term of

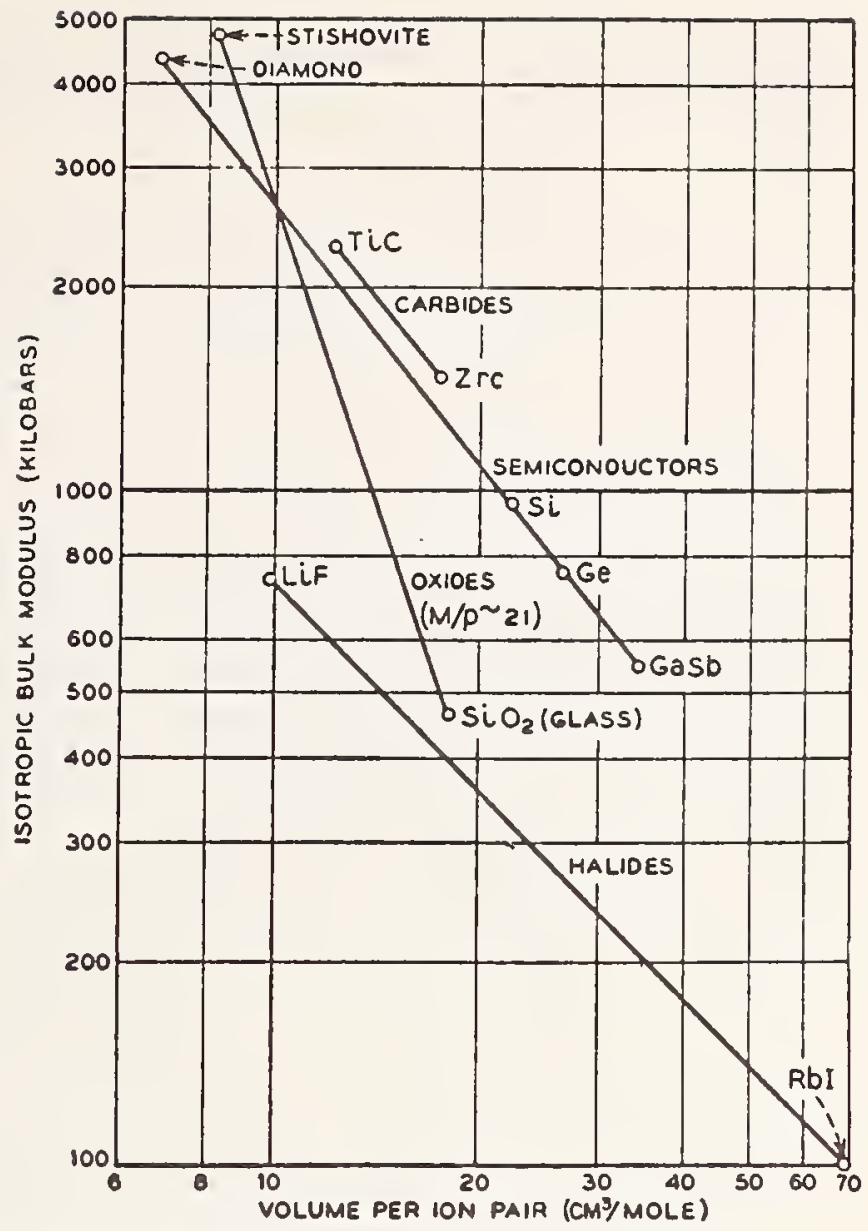

FIgURE 5. The variation of the bulk modulus with volume for several classes of materials.

(Oxides have a slope of -3 on this plot.) After
Anderson [183].

the form $C / R^{m}$ is introduced to account for the repulsion of the large anions which will be in direct contact with each other for a cation-anion radius ratio less than 0.414 . Many oxides have smaller ratio values and are essentially structures made up of close packed oxygen with small cations in the interstices. The attractive forces draw the oxygen into a state of compression and it is the form of the oxygen-oxygen repulsive term which causes the $1 / a^{9}$ variation of the bulk modulus. Anderson [185] has proposed that the change of bulk modulus of oxides with temperature or pressure is approximately accounted for by the change of volume through the $1 / a^{9}$ law. That is, the bulk modulus of a pore-free oxide made up of close packed oxygen ions can be calculated approximately simply from its specific volume, even though changes of volume may result from change of cations, of pressure, or of temperature. General approximation formulas are given [183] for the large class of oxides (including most oxides of technological interest) having a mean atomic weight of about 21 ; these permit estimation of averaged $B, G$, and related elastic properties from the density. 
4.2. Linear Lattice Dynamics: Relation of Elastic Constants to Sound Velocity, Specific Heat, Optical Absorption, and Dielectric Constant.

The foregoing static model permits the calculation of the internal energy, $U$, and the enthalpy, $U+p V$, as a function of pressure. By considering the vibrations of the atoms one can introduce temperature as a variable and calculate many properties. The subject of lattice dynamics is large and mathematically complex. We shall quote a few results to show how some thermal and electrical properties depend on the elastic constants and other quantities. The simplest lattice dynamics treatments are based on the $\mathrm{MgO}$ structure or other simple structures; the resulting equations cannot be expected to apply exactly to other structures. The simplest treatment assumes linear springs between atoms (the harmonic approximation) and successfully treats sound velocity, specific heat, optical absorption, and ionic contributions to the dielectric constant. We defer to the next section a discussion of such properties as specific heat which depend on the introduction of nonlinear springs (the third-order elastic constants).

We have already given the relation of sound velocity to elastic constants in discussing continuum mechanics. The equations for $v_{l}$ and $v_{s}$ are compatible with the atomic model for crystals and hold unchanged for wavelengths large compared to atomic spacings. As the wavelength decreases the velocity eventually begins to decrease also. This effect is usually shown on plots of frequency as a function of wave number $(k=2 \pi / \lambda)$. This plot has two curves emerging from the origin with slopes equal to the velocity of lingitudinal and transverse waves respectively as shown in figure 6 .

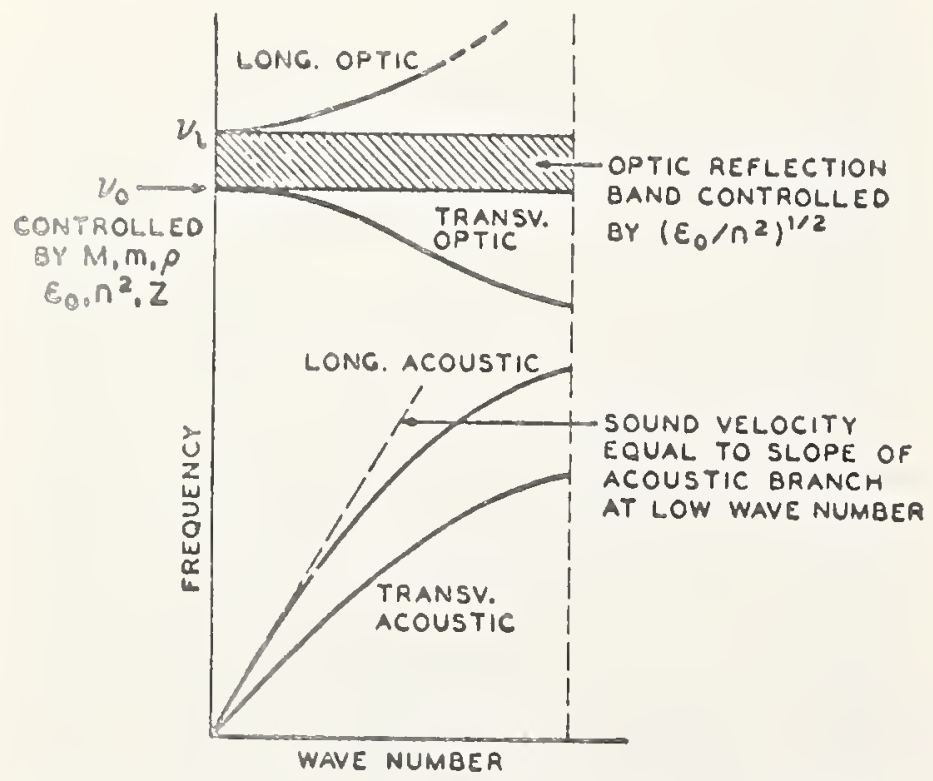

Figure 6. Frequency-wave number plot for a three-dimensional isotropic body.

After Anderson [186].
There are also two optical branches of this plot corresponding to vibrations in which ions of opposite sign move in opposition. It is possible to choose light of such a frequency (usually in the infrared) that it equals $\nu_{0}$ of figure 6 with the result that a strong absorption occurs. Szigeti $[187,188]$ has derived an equation giving this frequency in terms of the isothermal bulk modulus, $B_{T}$, the lattice parameter, $a$, for the $\mathrm{MgO}$ structure, the static dielectric constant, $\epsilon_{0}$, the index of refraction, $n$, and the reduced mass, $\bar{m}$ :

$$
2 \pi \nu_{0}=\left[6\left(n^{2}+2\right) B_{T} a / \bar{m}\left(\epsilon_{0}+2\right)\right]^{1 / 2} .
$$

Anderson and Glynn [189] have used the measured absorption frequency to calculate the bulk modulus successfully for $\mathrm{MgO}$.

The dielectric constant at low frequency (below $10^{10} \mathrm{~Hz}$ ) is the sum of a contribution due to electronic motion and ionic motion; its value at optical frequencies (which equals the square of the index of refraction, $n$ ) is due to electronic motion alone. Szigetti has shown that the difference, $\Delta \epsilon$, due to the ionic contribution is given by

$$
\Delta \epsilon=\left(n^{2}+2\right)^{2} c^{2} / 6 \pi \nu_{0}^{2} a^{3} \bar{m}
$$

for the $\mathrm{MgO}$ structure. We see that this depends on the bulk modulus through $\nu_{0}$.

The specific heat of a crystal is obtained from counting up all the modes of vibration using the frequency-wave number plot mentioned above. Debye approximated this complex problem by taking the sound velocity to be independent of wave number and obtained a useful approximate equation giving the specific heat as a function (tables of which are available [190]) of $T / \theta$ where $\theta$ is the Debye temperature and is given by

$$
\theta=(h / k)(3 p N \rho / 4 \pi M)^{1 / 3} v_{m}
$$

where $h=$ Planck's constant, $k=$ Boltzmann's constant, $p=$ number of atoms per molecule, $N=$ Avogadro's number, $\rho=$ density, and $M=$ molecular weight. The velocity $v_{m}$ is a special type of average over all directions in the crystal given by

$$
v_{m}=\left[\text { ave. of }\left[\left(1 / v_{l}^{3}\right)+\left(1 / v_{t 1}{ }^{3}\right)+\left(1 / v_{t 2}{ }^{3}\right)\right] / 3\right]^{-1 / 3}
$$

The problem of calculating $v_{m}$ and hence $\theta$ and the specific heat at any temperature is simple in principle; for any direction the three velocities can be calculated from the elastic constants and a numerical average over a large number of directions can be taken. Alers [191] has reviewed a number of procedures for doing this. Anderson has shown that approximating a single crystal by an isotropic material with elastic constants given by averaging the Voigt and Reuss bounds leads to a simple expression for $v_{m}$ which agrees with the accurately calculated value within a few percent for many crystals [192]. The calculation of Debye temperatures from elastic constants continues to be an active field [193-196]. 
Detailed treatments of the vibrational spectrum and related properties have been given for a number of structures besides $\mathrm{MgO}$ including rutile $\left(\mathrm{TiO}_{2}\right)[197,198]$ and the fluorite structure $\left(\mathrm{UO}_{2}, \mathrm{ThO}_{2}\right.$, etc.) $[199,200]$.

\subsection{Nonlinear Lattice Dynamics: Thermal Expansion and Temperature Dependence of Elastic Constants}

The forces between atoms in a crystal are not simply linear functions of displacement but include nonlinear terms as well. This leads to great complexity in the treatment of lattice vibrations and properties dependent upon these vibrations $[9,179]$. Some properties, such as thermal expansion and temperature dependence of the elastic constants, depend for their very existence upon the nonlinear terms in the interatomic force laws. These properties are usually treated in the socalled quasi-harmonic approximation in which the forces are taken to be linear but with a vibrational frequency, $\omega$, for the atoms which depends upon the average interatomic distance. This dependence is expressed by a number $\gamma$, called Grüneisen's constant, defined by $\gamma=d l n$ $\omega / d l n V$ which is assumed to be the same for all vibrations and in the simplest treatment is iridependent of temperature. It can be shown $[179,201,202]$ that the volume thermal expansion coefficient, $\beta$, is given by

$$
\beta=\gamma c_{v} / B_{T} V
$$

where $c_{n}$ is the specific heat at constant volume. The use of an integrated form of this equation has been discussed [203] and it has been shown [204] that $\gamma$ is not truly constant but may vary by about 10 percent as the temperature is increased from absolute zero to the melting point. We shall leave further discussion of thermal expansion to the companion paper in this volume on thermal expansion and simply point out that in principle one can calculate the thermal expansion from knowledge of elastic properties alone. We have already discussed the calculation of $c_{v}$ from elastic constant data; $\gamma$ can be calculated using both the second-order and third-order constants [205, 206].

The elastic constants change with temperature because of the presence of nonlinear terms in the force expressions. Leibfried and Ludwig [9] give

$$
B=B_{0}(1-D E+d p)
$$

where $B_{0}$ is the value in the absence of nonlinear terms and $D$ and $d$ are complicated functions of the nonlinear terms. The temperature dependence comes in through the thermal energy, $E$, which increases with the temperature. Anderson [207] has derived the following equation for the temperature dependence of the bulk modulus:

$$
B=B_{0}-3 R \gamma \delta E / V_{0}
$$

where $R$ is the gas constant, subscript zero indicates value at absolute zero and $\delta$ is a second Grüneisen constant (approximately independent of the temperature) defined by

$$
\delta=-(1 / a)(\partial \ln B / \partial T)_{p} .
$$

This proportionality of the temperature dependent part of the bulk modulus to the thermal energy leads to a variation proportional to $T^{4}$ at low temperature (where the specific heat goes as $T^{3}$ in the Debye theory) and proportional to $T$ at high temperature (where the specific heat is approximately constant). It is sometimes said that elastic constants vary with temperature because of thermal expansion and this is true in the sense that both depend on nonlinear terms in the potential energy. However, Stern [208] points out that thermal expansion depends only on the odd terms (corresponding to third-order elastic constants) but the temperature dependence of elastic constants depends on both odd and even terms (corresponding to third-order and fourthorder elastic constants). The transition from zero slope at absolute zero to a linear decrease with increasing temperature predicted by theory is found in plots of Young's modulus or the shear modulus for many crystalline oxides and is shown in figure 7 for $\mathrm{Al}_{2} \mathrm{O}_{3}$. This behavior is well described by the empirical equation

$$
Y=Y_{0}-b T \exp \left(-T_{0} / T\right)
$$

in which $Y_{0}$ is Young's modulus at absolute zero and $b$ and $T_{0}$ are empirical constants [209]. Anderson [207] has shown that at high temperatures $T_{0}$ corresponds approximately to one-half the Debye theta. Tables 13 and 14 give temperature coefficients for elastic moduli of various single-crystal and polycrystalline ceramics respectively. These coefficients are actually functions of temperature, usually changing smoothly from a value of zero at low temperature to a constant at high temperature, but the value calculated from any temperature interval above $\theta / 2$ will generally approximate the high temperature limit. An order-of-magnitude rule is that at high temperatures, elastic moduli of crystalline ceramics decrease one percent with each increase of $100^{\circ} \mathrm{C}$. Extrapolations of elastic moduli to very high temperatures has been given for several oxides [215].

Barker [216] has shown that the quantity $a^{2} Y$ is approximately constant for 68 of 79 materials examined. This implies a correlation between the anharmonic properties and the elastic moduli.

\subsection{Effect of Solid Solution and Phase Changes on Elastic Constants}

The effect of solid solution on elastic moduli has been studied in a number of metal alloys but little appears to have been done in ceramic systems. Slagle and McKinstry [217] have studied the system $\mathrm{KCl}-\mathrm{KBr}$ and found that plots of 


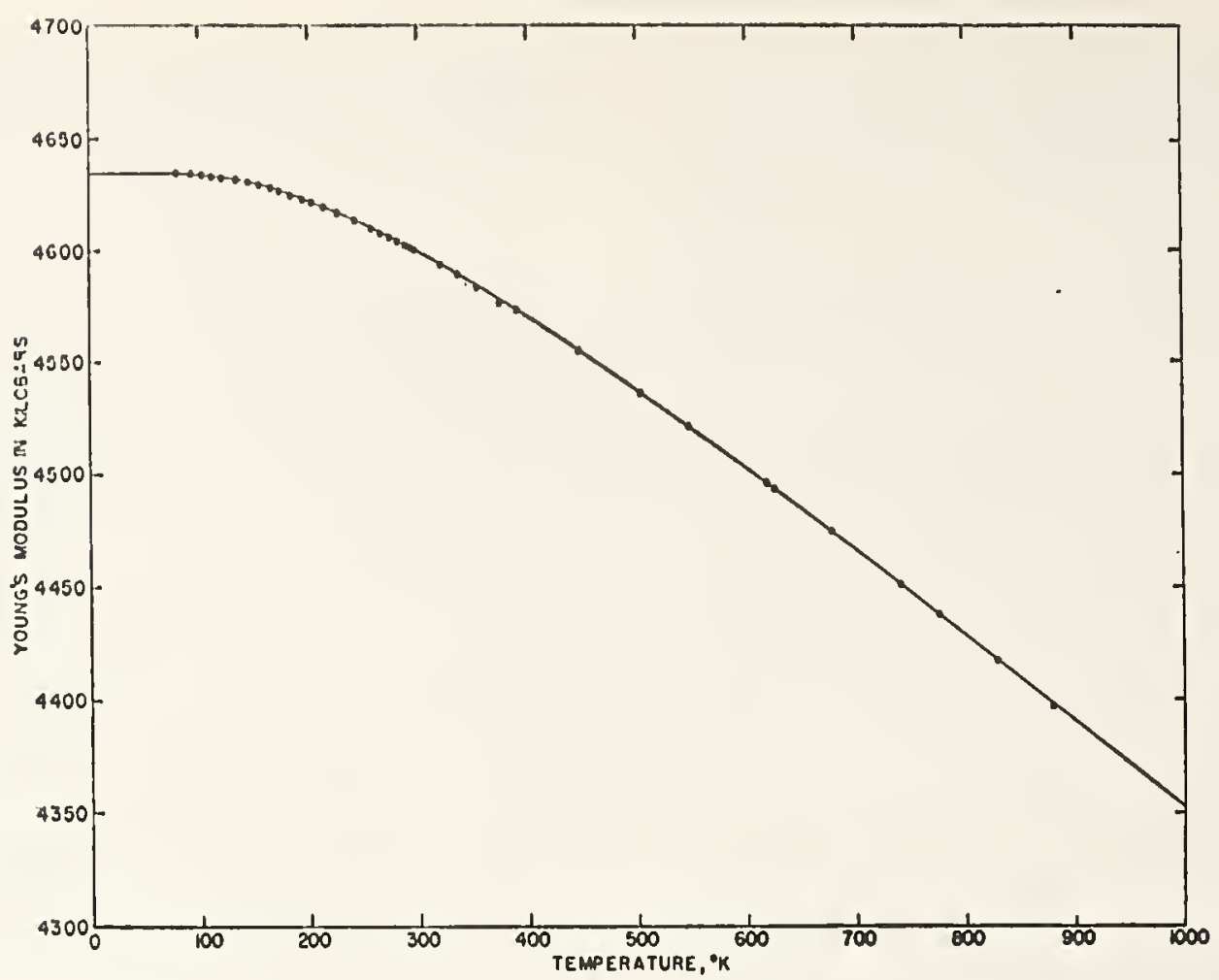

FIGURE 7. Young's modulus of single-crystal aluminum oxide as a function of temperature.

After Wachtman ot al. [209].

TABLE 13. Temperature coefficients for some single crystal elastic constants

\begin{tabular}{|c|c|c|c|c|c|c|}
\hline Material & Quantity & Value & $\begin{array}{l}\text { Tempera- } \\
\text { ture range }\end{array}$ & $\begin{array}{c}\text { Refer- } \\
\text { ence }\end{array}$ & $\begin{array}{l}\text { Debye } \\
\text { theta }\end{array}$ & $\begin{array}{l}\text { Refer- } \\
\text { ence }\end{array}$ \\
\hline $\begin{array}{l}\mathrm{Al}_{2} \mathrm{O}^{3} \\
\mathrm{YAlG} \\
\quad \text { (garnet) } \\
\mathrm{MgO} \\
\mathrm{UC}\end{array}$ & $\begin{array}{l}\left(\Delta B_{s} / \Delta T\right) / B_{s} \\
\left(\Delta c_{11} / \Delta T\right) / c_{11} \\
\left(\Delta c_{44} / \Delta T\right) / c_{44} \\
\left(\Delta c_{12} / \Delta T\right) / c_{12} \\
\left(\partial B_{s} / \partial T\right) / B_{s} \\
\left(\Delta c_{11} / \Delta T\right) / c_{11} \\
\left(\Delta c_{44} / \Delta T\right) / c_{44} \\
\left(\Delta c_{12} / \Delta T\right) / c_{12}\end{array}$ & $\begin{array}{l}10^{-4} / K \\
-0.68 \\
-0.9 \\
-0.7 \\
-0.5 \\
-0.97 \\
-1.0 \\
+2.0 \\
-0.75\end{array}$ & $\begin{array}{c}K \\
300 \text { to } 800 \\
200 \text { to } 300 \\
200 \text { to } 300 \\
200 \text { to } 300 \\
300 \\
150 \text { to } 300 \\
150 \text { to } 300 \\
150 \text { to } 300\end{array}$ & $\begin{array}{l}{[210]} \\
{[211]} \\
{[211]} \\
{[211]} \\
{[28]} \\
{[212]} \\
{[212]} \\
{[212]}\end{array}$ & 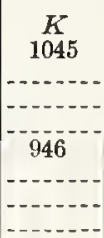 & [209] \\
\hline
\end{tabular}

TABLE 14. Temperature coefficients for elastic moduli of some polycrystalline ceramics

\begin{tabular}{|c|c|c|c|c|c|c|}
\hline Material & Quantity & Value & $\begin{array}{l}\text { Tempera- } \\
\text { ture range }\end{array}$ & $\begin{array}{r}\text { Refer- } \\
\text { ence }\end{array}$ & $\begin{array}{l}\text { Debye } \\
\text { theta }\end{array}$ & $\begin{array}{l}\text { Refer- } \\
\text { ence }\end{array}$ \\
\hline $\begin{array}{l}\mathrm{Mg}_{2} \mathrm{SiO}_{4} \\
\text { (forsterite). } \\
\mathrm{Mg}_{2} \mathrm{SiO}_{3} \\
\\
\mathrm{ThO}_{2} \\
\mathrm{Y}_{2} \mathrm{O}_{3} \ldots\end{array}$ & $\begin{array}{l}(d Y / d T) / Y \\
(d Y / d T) / Y \\
(d G / d T) / G \\
(d B / d T) / B \\
(d Y / d T) / Y \\
(d Y / d T) / Y \\
(d Y / d T) / Y \\
(d / / d T) / Y \\
(d G / d T) / G \\
(d B / d T) / B \\
(d Y / d T) / Y \\
(d G / d T) / G \\
(d B / d T) / B \\
(d Y / d T) / Y \\
(d G / d T) / G \\
(d B / d T) / B \\
(d Y / d T) / Y \\
(d Y / d T) / Y\end{array}$ & $\begin{array}{l}10^{-4} / K \\
-1.4 \\
-1.3 \\
-1.4 \\
-0.84 \\
-1.1 \\
-1.1 \\
-1.9 \\
-1.8 \\
-2.0 \\
-1.1 \\
-1.6 \\
-1.7 \\
-1.3 \\
-1.2 \\
-1.2 \\
-1.2 \\
-1.4 \\
-1.1\end{array}$ & $\begin{array}{c}K \\
(\mathrm{a}) \\
300-1400 \\
300-1400 \\
300-1400 \\
300-100 \\
300-1100 \\
(\mathrm{a}) \\
300-1100 \\
300-1100 \\
300-100 \\
300-900 \\
300-900 \\
300-900 \\
300-900 \\
300-900 \\
300-900 \\
(\mathrm{a}) \\
300-1300\end{array}$ & $\begin{array}{r}{[209]} \\
{[213]} \\
{[213]} \\
{[213]} \\
{[50]} \\
{[50]} \\
{[209]} \\
{[213]} \\
{[213]} \\
{[213]} \\
{[214]} \\
{[214]} \\
{[214]} \\
{[214]} \\
{[214]} \\
{[214]} \\
{[209]} \\
{[54]}\end{array}$ & $\begin{array}{c}\begin{array}{c}K \\
1045\end{array} \\
\\
946 \\
\end{array}$ & [209] \\
\hline
\end{tabular}

${ }^{a}$ Fit of $B$ in $Y=Y_{0}-B T \exp \left(-T_{0} / T\right)$ over 78 to $873 \mathrm{~K}$. $b$ Incorrectly listed as 782 rather than 363 in reference [209]. $c_{11}$ and $c_{44}$ fall below the straight line predicted by a simple method-of-mixtures rule; i.e. the curves are concave upward. Examples of departures from straight line behavior in both directions (either concave upward or concave downward) are known in various metal systems [7] so that no simple rule can be given.

The behavior of elastic constants near the melting point of materials with the $\mathrm{MgO}$ structure has been extensively studied [217]. It is proposed that melting occurs when vibrations corresponding to the shear associated with $c_{11}-$ $c_{12}$ become so large that the structure becomes unstable. One might therefore expect $c_{11}-c_{12}$ to approach zero or at least to become small as the melting temperature is approached. The most recent measurements show a smooth decrease of $c_{11}-c_{12}$ with temperature to a value just before melting of about one-quarter of the value at $25^{\circ} \mathrm{C}$ for $\mathrm{NaCl}, \mathrm{KCl}, \mathrm{KBr}$. In the system $\mathrm{KCl}$ $\mathrm{KBr}$ the values of $c_{11}-c_{12}$ measured at $700{ }^{\circ} \mathrm{C}$ vary with composition in the same way as the melting point.

In solid-to-solid phase changes the elastic constants change as would generally be expected for physical properties in a first-order phase change. In second-order phase changes the usual thermodynamic state functions are continuous but their derivatives, including elastic constants, may be discontinuous. Second-order phase changes are important in connection with order-disorder processes, change from nonferroelectric to ferroelectric state, etc. Some phase changes of this type were first discovered by measuring elastic 
constants as a function of temperature; this was the case for $\mathrm{SrTiO}_{3}$ [218]. Typically some of the elastic constants may show a smooth change with temperature while others show a large variation and may approach zero. Presumably the latter correspond to a type of vibration whose amplitude is becoming large as the transition temperature is approached and which corresponds to the type of atomic rearrangement involved in the phase change. Thus the phase change of first or second order may be signaled by the behavior of the elastic constants at temperatures well removed (e.g., $20{ }^{\circ} \mathrm{C}$ ) from the transition temperature. Examples are the change at $184 \mathrm{~K}$ in $\mathrm{KMnF}_{3}$ [219], the paramagnetic-antiferromagnetic transition at $30.8 \mathrm{~K}$ in $\mathrm{UO}_{2}$ [220], and the order-disorder transition at $243 \mathrm{~K}_{2}$ in $\mathrm{NH}_{4} \mathrm{Cl}$ [221].

\subsection{Internal Friction and the $\Delta \mathrm{E}$ Effect}

We have previously emphasized that elastic deformation (in our definition) is characterized by complete recovery when the stress is removed even though the recovery may require a long time. Time dependent behavior of this type causes a portion of the energy used in producing the deformation to be converted into heat. This loss is said to be due to internal friction and is usually a function of temperature and of frequency in cyclic deformation. Loss also occurs in cyclic plastic deformation and as a result of other irreversible processes (such as domain wall motion) induced by stress. Only the type of internal friction associated with recoverable deformation is an appropriate subject for discussion in a treatment of elasticity but the two types are sometimes difficult to separate experimentally and even conceptually for certain processes which have long recovery times. Extensive reviews of internal friction are available [222-225] and we limit our treatment to a brief discussion of two types which are particularly useful in throwing light on the properties of point defects and dislocations respectively.

A point defect (such as a vacancy, an interstitial, or an impurity atom) generally causes a distorsion of its neighborhood. If this distorsion has a symmetry lower than the point group of the crystal, it can occur in more than one way. The different ways of occurring will have equal energy and equal probability in the absence of stress or other influence such as electric field which lowers the symmetry of the crystal. Under stress the different ways can become inequivalent leading to the development of a preferred distribution if time is allowed for the necessary thermally activated jumps. This jumping is accompanied by an additional strain (causing the appropriate elastic modulus to decrease) and an energy loss. This subject has been worked out in detail [226-238]. Measurement of this internal friction can be used to obtain several different types of information concerning defects in crystals. First, the variation of the amplitude of the internal friction peak with type of stress applied gives information on the symmetry of the defect and can sometimes be used to reduce the number of possible models for an unknown defect. This type of investigation is most useful when it can be combined with measurements of other properties such as dielectric loss, optical spectra, and electron spin resonance which also give information on the symmetry of the defect. Second, study of the temperature dependence of the internal friction with varying stress components at constant frequency can give information on the number of relaxation times involved in point defect motion which can then be interpreted in terms of a specific atomic model of the defect. Third, the anisotropy of distortion around a defect can be deduced, although one other piece of information such as the lattice parameter dependence on defect concentration is needed to completely specify the strain caused by a defect. Fourth, studies of the size of the internal friction peak in quenched specimens as a function of annealing temperature can sometimes be used to measure the association energy for pairing of point defects. Fifth, the jump frequency for the motion involved in diffusion can sometimes be measured so that diffusion measurements can then be made at temperatures much lower than is possible with direct diffusion measurements [239]. Ceramics in which point defect properties have been studied by internal friction measurements include $\mathrm{CaF}_{2}$ [240, 241], $\mathrm{CeO}_{2}$ [242], $\mathrm{MgO}$ [243, 244], $\mathrm{NiO}$ [245], $\mathrm{SiO}_{2}$ (quartz) [246], $\mathrm{ThO}_{2}$ [231], $\mathrm{TiO}_{2}$ [247-250], $\mathrm{UO}_{2}$ [251], and $\mathrm{ZrO}_{2}$ [252].

Dislocations in crystals are usually pinned at various points along their length by impurities or point defects; they are pinned more firmly at intersections with other dislocations. Under small stress the free segments bow out, but the ends remain attached to the pinning points if the stress is not too great; the dislocations return to their original positions when the stress is removed. This inelastic, recoverable deformation gives rise to a frequency-dependent but amplitude-independent internal friction and a corresponding decrease in elastic moduli. If greater stress is used, the dislocations break away from their less firm pinning points producing a loss which increases with increasing amplitude. These effects have been extensively studied in metals in which plastic deformation may - produce such a high density of dislocations that reduction of the elastic moduli by a few percent occurs. This is termed the $\Delta E$ effect. The elastic moduli can be returned to their original value by annealing or by pinning the dislocations by radiation damage. The theory of dislocation damping has been extensively developed [253] and can be used to determine the resistance to dislocation motion if the other parameters of the theory are determined. The theory has been applied to explain internal friction in $\mathrm{Al}_{2} \mathrm{O}_{3}$ [254, 255], $\mathrm{MgO}[254,256,257]$, and $\mathrm{TiO}_{2}$ [258]. 
Polycrystalline ceramics may exhibit damping caused by point defects and dislocations, but other effects such as porosity, viscous phases, and phase changes are frequently predominant in causing damping [259].

\section{Special Topics}

\subsection{Correlations of Other Properties With Elastic Constants}

Several physical properties in addition to those already discussed are related to elastic moduli. The presence of interstitials and vacancies should cause a change in the bulk modulus [260]; accurate measurements of bulk modulus might be useful as a measure of high densities of Frenkel defects. Solid solution causes a change of lattice parameter; the lattice parameter of ruby has been calculated as a function of chromium content in terms of elastic moduli [261]. The change of lattice parameter is usually proportional to the concentration of the solute in a solid solution (Vegard's law), but deviations occur which have been treated in terms of elastic moduli [262]. The elastic constants of many valley semiconductors depend somewhat on the population of electrons in the conduction band and hence on the doping [263]. We have previously mentioned therelation between elastic moduli and volume for isostructural compounds; a similar correlation involving both the volume and cohesive energy has been proposed [264]. Finally, a correlation between elastic moduli and strength is often noted as, for example, for a series of whitewares [265]. There is an apparent justification for this relation; approximate treatments of theoretical strength typically give values of the order of one-tenth of Young's modulus. Empirical correlations, however, usually involve much lower strengths under circumstances in which fracture starts at some flaw. The correlation of strength with elastic moduli is often due to having both bulk elastic moduli and strength affected in the same way by a variation of microstructure such as porosity. This type of correlation must not be expected to hold in general, however. A series of alumina bodies showed a variation in strength of a factor of 500 [266]; their elastic moduli vary by only about a factor of 2 .

\subsection{Elastic Moduli of Graphite}

Graphite is not a unique material but a family of materials differing in crystal structure, microstructure, and preferred orientation. Single crystals are usually hexagonal with a layer structure and have a very much greater stiffness parallel to the base plane than perpendicular to it. Spence [267] gives the single crystal elastic constants as $c_{11}=116, c_{33}=4.7, c_{44}=0.23, c_{12}=29$ and $c_{13}=10.9$ $\times 10^{10} \mathrm{~N} / \mathrm{m}^{2}$. A typical extruded polycrystalline graphite has Young's modulus values of 1.06 and $0.47 \times 10^{10} \mathrm{~N} / \mathrm{m}^{2}$ parallel and perpendicular respec- tively to the direction of extrusion [268]. Measurements of Young's modulus on various pyrolytic graphites gave values ranging from 56 to $0.8 \times 10^{10}$ $\mathrm{N} / \mathrm{m}^{2}$ where dislocations were pinned by light neutron irradiation [269] to eliminate the $\Delta E$ effect which is large in graphite [270-273]. The temperature dependence of Young's modulus from 25 to $1000^{\circ} \mathrm{C}$ has been reported as a function of various heat treatments at higher temperatures [274].

\subsection{Measurement of Elastic Moduli}

Elastic moduli were originally determined by static tests in which relatively large loads were applied and the resulting deflection was measured. These static tests are less accurate than dynamic tests but are still of great engineering importance. They are covered in the paper on mechanical testing in this volume. 'The dynamic tests are of two basic types: measurement of the velocity of propagation of waves, and measurement of the resonance frequency. We have previously discussed the relation of elastic constants to wave velocity or resonance frequency; it remains now to briefly sketch the experimental procedures.

The principles of wave velocity measurement and the calculation of elastic moduli from the velocities have long been understood. The availability of good megahertz oscilloscopes and other electronic apparatus following World War II has led to much work in this field. We shall mention a few papers which are important to the development of this technique to its present highly refined state and some which consider its application specifically to ceramics.

The basic technique is to bond a piezoelectric generator onto one flat face of a specimen cyrstal, excite sound waves, and measure the transit time to a flat and parallel opposite face. Price and Huntington [275] measured the echo time for ultrasonic pulses in single crystals and studied some of the phenomena of acoustical birefringence, mode coupling, and beam spreading. Eros and Reitz [276] discussed the problem of transit time error arising from change of shape of the pulse upon reflection from the interface between two media. McSkimin $[277,278]$ has summarized (and greatly developed) methods in which reflected but unrectified pulses are compared with an identical later pulse whose delay is controlled by a continuous wave oscillator. This is essentially an interference technique capable of a precision approaching one part in $10^{6}$. Application of accurate methods to high temperatures [279] and low temperatures [280] has been discussed. The application of these techniques to rocks and minerals has been reviewed [12, 281]. Neighbours and Schacher [282] have presented a general perturbation method for calculating second-order elastic moduli from measurements made in directions other than those for which pure modes are propagated. 
The accuracy of elastic constant measurements generally much less than the precision with which measurements can be repeated in the same apparatus or with which changes of elastic constants with temperature or pressure can be measured. Einspruck and Truell [283] conducted a survey in which nine laboratories made measurements on the same vitreous silica specimen; seven of the nine agreed within 0.3 percent in values of transit time. An error in absolute value of 0.3 percent is much greater than precision of 1 in $10^{6}$ achievable in relative measurements but is still better than the accuracy of static tests which is rarely better than a few percent. In addition to experimental problems, the comparison of dynamic and static test results may be affected by the different stress level and the different effects of defects such as cracks [284].

The resonance method of determining elastic moduli is capable of achieving absolute accuracy of 0.1 percent or better when care is taken to choose a shape for which the vibrational equations are accurately known and when the dimensions can be held to a corresponding tolerance. It can be used to measure changes with temperature of 1 in $10^{4}$ or better when care is taken to minimize coupling to the apparatus and the atmosphere. Methods of exciting and measuring resonances have been extensively reviewed [127, 285, 286] and specialized apparatus has been described for use at low temperature [287], high temperature [288], constant strain amplitude [289], very low strains [290], and for materials with very high damping [291]. Techniques for direct mechanical excitation and detection of resonance up to 300 $\mathrm{kHz}$ have been developed [292]. Resonance of spheres at frequencies up to $2 \mathrm{MHz}_{\mathrm{z}}$ has been used [134].

A method of measuring elastic constants using diffuse reflection of $\mathrm{x}$-rays has been developed $[293,294]$ but accuracies of only 5 to 45 percent were reported in a recent evaluation of the method [295]. A technique for detecting mechanical resonance and measuring sound velocity by nuclear magnetic resonance techniques has been described [296].

Measurement of the elastic moduli of fibers presents special problems; experimental techniques have been reviewed [297].

\subsection{Characterization of Ceramics With Respect to Elastic Moduli}

It is sometimes desired to reproduce a given physical property closely in a whole series of ceramic parts. The manufacturer should then seek to control the aspects of composition and microstructure upon which the property of interest depends. If property reproducibility is not sufficiently well achieved, it is then of interest to characterize the specimens, i.e. to measure appropriate aspects of composition and microstructure, and to determine which are varying.
For elastic moduli the characterization required is usually relatively straightforward for single crystals, but more complicated for polycrystals and composites. Our whole discussion of elastic moduli has been aimed at explaining the factors upon which elastic moduli depend; in conclusion we briefly summarize the more important ones.

For single crystals the presence of impurities is usually of little consequence to elastic moduli provided the solid solution limit is not exceeded. Within the range of solid solution a simple linear method-of-mixtures calculation, though not exactly correct, will give a resonable estimate. If the solid solution limit is exceeded and precipitate particles are included within the crystal the system may be treated as a composite using one of the theories previously discussed provided the shape of the precipitate corresponds to the model assumed. Care is necessary, however, because if the precipitate shrinks more than the crystal during cooling and breaks away from the matrix the specimen may actually be a single crystal with pores so far as elastic behavior is concerned. If the precipitate shrinks less than the crystal, internal cracking or dislocation generation may occur so that the elastic moduli are reduced more than would be expected from the volume of second phase. Single crystals are usually sufficiently pure, however, that these problems do not occur. A more common problem is lack of accurate measurement of orientation and sometimes lack of understanding that elastic moduli of single crystals depend on orientation even for cubic crystals and that for trigonal and tetragonal crystals they depend on the angle around the optic axis as well as the angle to the optic axis. The elastic moduli of relatively ductile ceramics such as single crystal $\mathrm{MgO}$ can be reduced by the presence of a high density of unpinned dis]ocations through the $\Delta \mathrm{E}$ effect.

In single-phase polycrystalline ceramics the factors most likely to affect elastic moduli at room temperature are porosity (including cracking from thermal stresses) and preferred orientation. As mentioned previously equations are known which correctly predict the change in elastic moduli as a function of a small volume fraction of spherical pores, but porosity usually is not even approximately spherical and causes a greater reduction in elastic moduli. Polycrystalline ceramics prepared by sintering of a powder made up of equiaxed particles are likely not to have a significant degree of preferred orientation. Ceramics prepared by extrusion or hot pressing or ceramics prepared from unequally axed powder particles are likely to show appreciable preferred orientation and this can have a pronounced effect on elastic moduli.

Polyphase polycrystalline ceramics are subject to the same sources of variability, but may in extreme cases of thermal mismatch exhibit severe internal cracking causing a large reduction in elastic moduli. Such a body may undergo a degree of crack healing when heated and develop a new 
pattern of cracks upon recooling. This type of body has no well defined values of elastic moduli; the measured values will change erratically as a result of thermal cycling.

Ceramic composites may possess any of the above sources of variability but the degree of orientation of the reinforcing fibers or plates and the degree of bonding between components are likely to be the predominant factors.

\section{References}

[1] Mason, W. P., Crystal Physics of Interaction Processes, Academic Press, New York (1966).

[2] Chisholm, L. J., Units of Weight and Measure, NBS Misc. Publ. 286, U.S. Government Printing Office (1967).

[3] Hearmon, R. F. S., The Elastic Constants of Nonpiezoelectric Crystals, Landolt-Bornstein, New Series, Group III, Volume 1 (1966).

[4] Beckmann, R., The Elastic, Piezoelectric, and Dielectric Constants of Piezoclectric Crystals, Landolt-Bornstein, New Series, Grolip III, Volume 1 (1966).

[5] Hearmon, R. F. S., The Elastic Constants of Anisotropic Materials, Rev. Mod. Phys. 18, 409 (1946).

[6] Hearmon, R. F. S., The Elastic Constants of Anisotropic Materials, II, Advan. Phys. 5, 323 (1956)

[7] Huntington, H. B., The Elastic Constants of Crystals, Solid State Phys. 7, 214 (1958), edited by F. Seitz and D. Turnbull, Academic Press.

[8] Aleksandrov, K. S., and Ryzhova, T. V., The Elastic Properties of Crystals, Soviet Phys.Cryst. 6, 228 (1961). Translated from Kristallografia 6, 289 (1961).

[9] Leibfried, G., and Ludwig, W., Theory of Anharmonic Effects in Crystals, Solid State Phys. 12, 276 (1961), edited by F. Seitz and D. Turnbull, Academic Press.

[10] Lynch, J. F., Ruderer, C. G., and Duckworth, W. H., Engineering Properties of Ceramies, published by the American Ceramic Society, Columbus, Ohio (1966).

[11] Simmons, G., Single Crystal Elastic Constants and Calculated Elastic Properties, Journal of the Graduate Research Center, Southern Methodist University Press, 34, 1 (March 1965).

[12] Anderson, O. L., and Lieberman, R. C., Sound Velocities in Rocks and Minerals, Vesiac State-ofthe-Art Report 7885-4-X, The University of Michigan.

[13] Simmons, G., Hashin Bounds for Aggregates of Cubic Crystals, Journal of the Graduate Research Center, Southern Methodist University Press, 36, 1 (January 1967).

[14] Overton, W. C., Jr., Relation Between Ultrasonically Mcasured Properties and the Coefficients in the Solid Equation of State, J. Chem. Phys. 37, 116 (1962).

[15] Thurston, R. N., Ultrasonic Data and the Thermodynamics of Solids, Proc. IEEE 53, 1320 (1965).

[16] Cook, R. K., Variations of Elastic Constants and Static Strains with Hydrostatic Pressure: A Method for Calculation from Ultrasonic Measurements, J. Acoust. Soc. Am. 29, 445 (1957).

[17] MeSkimin, H. J., Elastic Moduli of Single Crystal Germanium as a Function of Hydrostatic Pressure, J. Acoust. Soc. Am. 30, 314 (1957).

[18] Anderson, O. L., An Accurate Determination of the Equation of State by Ultrasonic Measurements, Proceedings of the Conference on High Pressure Research, p. 225, E. Bundy, Editor, John Wiley and Sons, Inc., New York (1963).
[19] Thurston, R. N., Wave propagation in fluids and normal solids, p. 1 in Physical Acoustics, Volume IA, W. P. Mason, editor, Academic Press, New York (1965)

[20] Anderson, O. L., Two methods for estimating compression and sound velocity at very high pressures, Proc. Natl. Acad. Sci. 54, 667 (1965).

[21] Anderson, O. L., The use of ultrasonic measurements under modest pressure to estimate compression at high pressure, J. Phys. Chem. Solids 27, 547 (1966).

[22] Nye, J. F., Physical Properties of Crystals, Oxford University Press, Oxford, England (1957).

[23] Truesdell, C., and Toupin, R. A. The classical field theories, in Handbuch der Physik Volume III/1, S. Flugge, editor, Springer, Bcrlin (1960).

[24] Bechtcrew, P., Analytical investigation of the general Hooke's Law. Use of the rule of potential energy and the principal of minimum work, Z . Krist. 64, 373 (1926).

[25] Alers, G. A., and Neighbours, J. R., Crystal stability and elastic constants, J. Appl. Phys. 28, 1514 (1957).

[26] Bateman, T. B., Elastic moduli of single-crystal europium iron garnet and yttrium iron garnet, J. Appl. Phys. 37, 1294 (1966).

[27] Soga, N., Elastic constants of garnet under pressure and temperature, J. Gcophys. Research 72,4227 (1967).

[28] Anderson, O. L., and Andreatch, P., Jr., Pressure derivatives of clastic constants of single-crystal $\mathrm{MgO}$ at $23^{\circ} \mathrm{C}$ and $-195.8^{\circ} \mathrm{C}, \mathrm{J}$. Am. Ceram. Soc. 49. 404 (1966).

[29] Bolef, D. I., and de Klerk, J., Elastic constants of single-crystal Mo and $\mathrm{W}$ between $77^{\circ} \mathrm{K}$ and 500 ${ }^{\circ}$ K, J. Appl. Phys. 33, 2311 (1962).

[30] Featherstone,-F. H., and Neighbours, J. R., Elastic constants of tantalum, tungsten, and molybdenum, Phys. Rev. 130, 1324 (1963).

[31] Wasilewski, R. J., Elastic constants of $\mathrm{Nb}$ by resonance at sonic frequencies, J. Phys. Chem. Solids 26, 1643 (1965).

[32] Schreiber, E., Elastic moduli of single-crystal spinel at $25^{\circ} \mathrm{C}$ and to $2 \mathrm{kbar}$, J. Appl. Phys. 38, 2508 (1967).

[33] de Batist, R., Room temperature elastic constants of single crystal $\mathrm{U}_{4} \mathrm{O}_{9}$, Materials Research Bullntin $1,75(1966)$.

[34] Lowrie, R., quoted by H. L. Brown and C. P. Kempter, "Elastic properties of zirconium carbide" Phys. Stat. Sol. 18, K21 (1966).

[35] Wachtman, J. B., Jr., Tefft, W. E., Lam, D. G., Jr., Stinchfield, R. P., Elastic constants of synthetic single crystal corundum at room temperature", J. Res. NBS 64A (Phys. and Chem.) No. 3, 213 (1960).

[36] Bernstein, B. T., Elastic constants of synthetic sapphire at $27^{\circ} \mathrm{C}$, J. Appl. Phys. 34, $169(1963)$.

[37] Birch, F., Elastic constants of rutile - a correction to a paper by R. K. Verma, Elasticity of some highdensity crystals., J. Geophys. Res. 65, 3855 (1960).

[38] Wachtman, J. B., Jr., Teff t, W. E., and Lam, D. G., Jr., Elastic constants of rutile $\left(\mathrm{TiO}_{2}\right)$, J. Res. NBS 66A, (Phys. and Chem.) No. 6, 465 (1962).

[39] Alton, W. J., and Barlow, A. J., Acoustic-wave propagation in tetragonal crystals and measurements of the elastic constants of calcium molybdate, J. Appl. Phys. 38, 3817 (1967).

[40] Wachtman, J. B., Jr., Brower, W. S., and Farabaugh, E. N., Elastic constants of single crystal calcium molybdate $\left(\mathrm{CaMoO}_{4}\right)$, J. Am. Ceram. Soc. 51, 341 (1968).

[41] J. G. Brainerd (Chairman of Standards Committee), IRE standards on piezoelectric crystals, 1945, Proc IRE 37, 1378 (1949).

[42] H. Jaffe (Chairman of Subcommittee), IRE standards on piezoelectric crystals: Measurement of piezoelectric ceramies, 1961, Proc IRE 49, 1161 (1961). 
[43] Zener, C., Elasticity and Anelasticity of Metals, University of Chicago Press, Chicago, Illinois (1948).

[44] Chung, D. H., and Buessem, W. R., The elastic anisotropy of crystals, J. Appl. Phys. 38, 2010 (1967).

[45] De Macedo, P. B., Viscoelasticity of glasses, p. 169, this volume.

[46] Knudsen, F. P., Effect of porosity on Young's modulus of alumina, J. Am. Ceram. Soc. 45, 94 (1962).

[47] Spriggs, R. M., Expression for effect of porosity on elastic modulus of polycrystalline refractory materials, particularly aluminum oxide, J. Am. Ceram. Soc. 44, 628 (1961).

[48] Spriggs, R. M., and Brissette, L. A., Expressions for shear modulus and Poisson's ratio of porous refactory oxides, J. Am. Ceram. Soc. 45, 198 (1962).

[49] Chung, D. H., and Buessem, W. R., The VoigtReuss-Hill (VRH) approximation and the elastic moduli of polyerystalline $\mathrm{ZnO}, \mathrm{TiO}_{2}$ (rutile), and $\alpha-\mathrm{Al}_{2} \mathrm{O}_{3}$, J. Appl. Phys. (in press).

[50] Manning, W. R., Marlowe, M. O., and Wilder, D. R., Temperature and porosity dependence of Young's modulus of polycrystalline dysprosium oxide and erbium oxide, J. Am. Ceram. Soc. 49, 227 (1966).

[51] Chung, D. H., Elastic moduli of single crystal and polyerystalline MgO, Phil. Mag. 8, 833 (1963).

[52] Spriggs, R. M., Brissette, L. A., and Vasilos, T. Effect of porosity on elastic and shear moduli of polycrystalline magnesium oxide, J. Am. Ceram. Soc. 45, 400 (1962).

153] Spinner, S., Knudsen, F. P., and Stone, L., Elastic constant-porosity relations for polycrystalline thoria, J. Research NBS 67C (Engr. and Instr.) No. 1, 39 (1963).

[54] Marlowe, M. O., and Wilder, D. R., Elasticity and internal friction of polycrystalline yttrium oxide, J. Am. Ceram. Soc. 48, 227 (1965).

[55] Fung, Y. C., Foundations of Solid Mechanics, Prentice-Hall, Inc., New York, (1965).

[56] Brugger, $K$., Thermodynamic of higher order elastic coefficients, Phys. Rev. 133, A1611 (1964).

[57] Murnaghan, F. D., Finite deformations of an elastic solid, Am. J. Math. 59, 235 (1937).

[58] Birch, F., Finite elastic strain of cubic crystals, Phys. Rev. 72, 809 (1947).

[59] Murnaghan, F. D., Finite Deformation of an Elastic Solid, John Wiley and Sons, Inc., New York (1951).

[60] Hearmon, R. F. S., Third-order elastic coefficients, Acta. Cryst. 6, 331 (1953).

[61] Skove, M. J., and Powell, B. E., Symmetry of mixed third-order elastic constants, J. Appl. Phys. 38, 404 (1967).

[62] Powell, B. E., and Skove, M. J., Relation between isothermal and mixed third-order elastic constants, J. Appl. Phys. 38, 404 (1967).

[63] Barsch, G. R., Adiabatic, isothermal, and intermediate pressure derivatives of the elastic constants for cubic symmetry, I. Basic formulae, Phys. Stat. Sol. 19, 129 (1967).

[64] Barsch, G. R., and Chang, Z. P., Adiabatic, isothermal, and intermediate pressure derivatives of the elastic constants for cubic symmetry, II. Numerical results for 25 materials, Phys. Stat. Sol. 19, 139 (1967)

[65] Thurston, R. N., and Brugger, K., Third-order elastic constants and the velocity of small amplitude elastic waves in homogeneously stressed media, Phys. Rev. 133, A 1604 (1964). See also R. N. Thurston, Effective elastic coefficients for wave propagation in crystals under stress, J. Acoust. Soc. Am. 37, 348 (1968).

[66] Brugger, K., Determination of third-order elastic coefficients in crystals, J. Appl. Phys. 36, 768 (1965).
[67] Chung, D. H., The first pressure derivatives of polycrystalline elastic moduli: Their relation to singlecrystal acoustic data and thermodynamic relations, J. Appl. Phys. 38, 5104 (1967).

[68] MeSkimin, H. J., Jayaraman, A., and Andreatch, P., Jr., Elastic moduli of GaAs at moderate pressures and the evaluation of compression to 250 kbar, J. Appl. Phys. 38, 2362 (1967).

[69] MeSkimin, H. J., and Andreateh, P., Jr., Elastic moduli of silicon vs. hydrostatic pressure at 25.0 ${ }^{\circ} \mathrm{C}$ and $-195.8^{\circ} \mathrm{C}$, J. Appl. Phys. 35, 2161 (1964).

[70] MeSkimin, H. J., and Andreatch, P., Jr., Thirdorder elastic moduli of gallium arsenide, J. Appl. Phys. 38, 2610 (1967).

[71] MeSkimin, H. J., and Andreatch, P., Jr., Measurement of third-order moduli of silicon and germanium, J. Appl. Phys. 35, 3312 (1964).

[72] Bogardus, E. H., Third-order elastic constants of Ge, MgO, and fused $\mathrm{SiO}_{2}$, J. Appl. Phys. 36, 2504 (1965).

[73] Eastman, D. E., Measurement of third-order elastic moduli of yttrium iron garnet, J. Appl. Phys. 37, $2312(1966)$.

[74] Toupin, R. A., and Bernstein, B., Sound waves in deformed perfectly elastic materials. Acoustoelastic effect, J. Acoust. Soc. Am. 33, 216 (1961).

[75] Smith, R. T., Stern, R., and Stephens, R. B. W., Third-order elastic moduli of polycrystalline metals from ultrasonic velocity measurements, J. Acoust. Soc. Am. 40, 1002 (1966).

[76] Thurston, R. N., Calculation of lattice-parameter changes with hydrostatic pressure from third-order elastic constants, J. Acoust. Soc. Am. 41, 1093 (1967).

[77] Ghate, P. B., Fourth-order elastic coefficients, J. Appl. Phys. 35. 337 (1964).

[78] Ghate, P. B., Effect of hydrostatic pressure on the elastic behavior of cubic crystals, Phys. Stat. Sol. 14, 325 (1966)

[79] Chung, D. H., The elastic constants of a cubic crystal subjected to moderately high hydrostatic pressure, J. Phys. Chem. Solids, 29, 417 (1968).

[80] Truesdell, C., and Toupin, R., Static grounds for inequalities in finite strain of elastic materials, Arch. Ratl. Mech. Anal. 12, 1 (1963).

[81] Mindlin, R. D., and Tiersten, H. F., Effects of couple-stresses in linear elasticity, Arch. Ratl. Mech. Anal. 11, 415 (1962).

[82] Mindlin, R. D., Influence of couple-stresses on stress concentrations, Experimental Mechanics, 3, 1 (1963).

[83] Toupin, R. A., Elastic materials with couple-stresses, Arch. Ratl. Mech. Anal. 11, 385 (1962).

[84] Green, A. E., and Rivlin, R. S., Multipolar continuum mechanies: Functional theory I, Proc. Roy. Soc. (London) A284, 303 (1965).

[85] Koiter, W. T., Couple-stresses in the theory of elasticity, Proc. Kon. Ned. Akad. V. Wetensch B67, 17 and 30 (1964).

[86] Schijve, B., Note on couple-stresses, J. Mech. Phys. Solids 14, 113 (1966).

[87] Laval, J., "Elasticité des cristaux, Compt. Rend. 232, 1947 (1951).

[88] Raman, C. V., and Viswanathan, K. S., The elastic behavior of isotropic solids, Proc. Indian Acad. Sci. 42, 1 (1955).

[89] Raman, C. V., and Viswanathan, K. S., On the theory of the elasticity of crystals, Proc. Indian Acad. Sci. 42, 51 (1955).

[90] Raman, C. V., and Krishnamurti, D., Evaluation of the four elastic constants of some cubic crystals, Proc. Indian Acad. Sci. 42, 111 (1955).

[91] LeCorre, Y., Les densites de couple et les pseudorotations dans la theorie de l'elasticité de Laval, J. phys radium 19, 36 (1958).

[92] Joel, N., and Wooster, W. A., Number of elastic constants required in erystal elasticity, 182, 1078 (1958). 
[93] Joel, N., and Wooster, W. A., On the validity of the classical theory of erystal elasticity as applied to ammonium di-hydrogen phosphate, Acta. Cryst. $11,575(1958)$

[94] Swartz, K. D., and Granato, A. V., Experimental test of the Laval-Raman-Viswanathan theory of elasticity, J. Acoust. Soc. Am. 38, 824 (1965).

[95] Kolsky, I., Stress Waves in Solids, Oxford University Press, Oxford, England (1953).

[96] Soga, N., and Anderson, O. L., Simplified method for calculating elastic moduli of ceramic powder from compressibility and Debye temperature data, J. Am. Ceram. Soc. 49, 318 (1966).

[97] Bancroft, D., The velocity of longitudinal waves in cylindrical bars, Phys. Rev. 59, 588 (1941).

[98] Hudson, G. E., Dispersion of elastic waves in solid circular cylinders, Phys. Rev. 63, 46 (1943).

[99] Cooper, J. L. B., The propagation of elastic waves in a rod, Phil. Mag. 38, 1 (1947).

[100] Davies, R. M., A critical study of the Hopkinson pressure bar, Trans. Roy. Soc. (London) A240, 375 (1948).

[101] Stanford, E. G., A contribution on the velocity of longitudinal elastic vibrations in cylindrical rods, and on the relationship between Young's modulus and temperature for aluminum, Nuovo Cimento supplement to Volume $\boldsymbol{7}, 332$ (1950).

[102] Tu, L. Y., Brennan, J. N., and Sauer, J. A., Dispersion of ultrasonic pulse velocity in cylindrical rods, J. Acoust. Soc. Am. 27, 550 (1955).

[103] Morse, R. W., The velocity of compressional waves in rods of rectangular cross section, J. Acoust. Soc. Am. 22, 219 (1950).

[104] Miklowitz, J., The propagation of compressional waves in a dispersive elastic rod, J. Appl. Mech. 24, 231 (1957)

[105] Lee, E. H., Finite-strain elastic-plastic theory with application to plane-wave analysis, J. Appl. Phys. 38, 19 (1967).

[106] Truesdell, C., General and exact theory of waves in finite elastic strain, Arch. Ratl. Mech. Anal. 8, 263 (1961).

[107] Borgnis, R. E., Specific directions of longitudinal wave propagation in anisotropic media, Phys. Rev. 98, $1000(1955)$.

[108] Brugger, K., Pure modes for elastic waves in crystals, J. Appl. Phys. 36, 759 (1965).

[109] Khatkevich, A. G., Special directions for elastic waves in crystals, Soviet Phys.-Cryst. 9, 579 (1965). Translated from Kristallografiya 9, 690 (1964).

[110] Aleksandrov, K. S., An ultrasonic pulse method of measuring the elastic moduli of monoclinic crystals, Soviet Phys.-Cryst. 3, 630 (1958).

[111] Musgrave, M. J. P., On the propagation of elastic waves in aeolotropic media. I. General principles, Proc. Roy. Soc. (London) A226, 339 (1954).

[112] Musgrave, M. J. P., On the propagation of elastic waves in aeolotropic media. II. Media of hexagonal symmetry, Proc. Roy. Soc. (London) A226, 356 (1954).

[113] Miller, G. F., and Musgrave, M. J. P., On the propagation of elastic waves in aeolotropic media. III. Media of cubic symmetry, Proc. Roy. Soc. (London) A236, 352 (1956).

[114] Musgrave, M. J. P., On whether elastic wave surfaces possess cuspidal edges, Proc. Cambridge Phil. Soc. 53, 897 (1957).

[115] Musgrave, M. J. P., On an elliptic cone of internal refraction for quasi-transverse waves in tetragonal crystals, Acta Cryst. 10, 316 (1957).

[116] Markham, M. F., Measurement of elastic constants by the ultrasonic pulse method, Brit. J. Appl. Phys., Supplement No. 6, 56 (1957).

[117] Neighbours, J. R., and Smith, C. S., An approximation method for the determination of the elastic constants of cubic single crystals, J. Appl. Phys. 21, 1338 (1950).
[118] Neighbours, J. R., An approximation method for the determination of the elastic constants of single crystals, J. Acoust. Soc. Am. 26, 865 (1954).

[119] Herzfeld, K. F., Fifty years of physical ultrasonics, J. Acoust. Soc. Am. 39, 813 (1966).

[120] Mason, W. P., Physical Acoustics, a series of volumes published by Academic Press, New York.

[121] Mason, W. P., and McSkimin, H. J., Energy losses of sound waves in metals due to scattering and diffusion, J. Appl. Phys. 19, 940 (1948).

[122] Fitzgerald, T. M., Chick, B. B., and Truell, R., Ultrasonic attenuation in $\mathrm{Al}_{2} \mathrm{O}_{3}$ at ultrahigh frequencies and low temperatures, J. Appl. Phys. 35,2647 (1964).

[123] Maris, H. J., The absorption of sound waves in perfect dielectric crystals, Phil. Mag. 12, 89 (1965).

[124] Oliver, D. W., and Slack, G. A., Ultrasonic attenuation in insulators at room temperature, J. Appl. Phys. 37, 1542 (1966).

[125] Simmons, G., Velocity of shear waves in rocks to 10 kilobars, I, J. Geophys. Res. 69, 1123 (1964).

[126] Knopoff, L., and MacDonald, G. J. F., Attenuation of small amplitude stress waves in solids, Rev. Modern Phys. 30, 1178 (1958).

[127] Spinner, S., and Tefft, W. E., A method for determining mechanical resonance frequencies and for calculating elastic moduli from these frequencies, Proc. ASTM 61, 1221 (1961).

[128] Pickett, G., Equations for computing elastic constants from flexural and torsional resonant frequencies of vibration of prisms and cylinders, Proc. Am. Soc. Testing Mater. 45, 846 (1945).

[129] Pickett, G., Flexural vibration of unrestrained cylinders and discs, J. Appl. Phys. 16, 820 (1945).

[130] Tefft, W. E., Numerical solution of the frequency equations for the flexural vibration of cylindrical rods, J. Research NBS 64B (Math. Sci.) No. 4, 237 (1960).

[131] Gazis, D. C., Exact analysis of the plane-strain vibrations of thick-walled hollow cylinders, J. Acoust. Soc. Am. 30, 786 (1958).

[132] Lucey, G. K., Jr., Resonant and antiresonant frequencies of thick discs and rods, J. Acoust. Soc. Am., to be published.

[133] Love, A. E. H., A treatise on the mathematical theory of elasticity, Reprinted by Dover Publications, New York \& London (1944).

[134] Frazer, D. B., and LeCraw, R. C., Novel method of measuring elastic and anelastic properties of solids, Rev. Sci. Inst. 35, 1113 (1964).

[135] Tiersten, H. F., Thickness vibrations of piezoelectric plates, J. Acoust. Soc. Am. 35, 53 (1963).

[136] Warner, A. W., Onoe, M., and Coquin, G. A., Determination of elastic and piezoelectric constants for crystals in class $(3 \mathrm{~m})$, J. Acoust. Soc. Am. 39, 42 (1967).

[137] Voigt, W., Lehrbuch der kristallphysik, Teubner, Berlin (1928).

[138] Goens, E., Uber die Biegungs-und Drillungsschwinigungen eines dünnen kreiszylindrischen Kristallstabes von beliebiger kristallographischer Orientierung, Ann. Physik 15, 455 (1932).

[139] Kimura, R., On the elastic moduli of ferromagnetic materials, Part I. Dynamical measurements of the elastic moduli of iron crystals, Proc. Phys.-Math. Soc. Japan 21, 686 (1939).

[140] Brown, W. F., Jr., Interpretation of torsional frequencies of crystal specimens, Phys. Rev. 58, 998 (1940).

[141] Dewey, J. M., The elastic constants of materials loaded with non-rigid fillers, J. Appl. Phys. 18, 578 (1947).

[142] Mackenzie, J. K., The elastic constants of a solid containing spherical holes, Proc. Phys. Soc. B63, $2(1950)$.

[143] Gatto, F., Influence of small cavities on velocity of sound in metals, Allumino 19, 19 (1950). 
[144] Coble, R. L., and Kingery, W. D., Effect of porosity on physical properties of sintered alumina, J. Am. Ceram. Soc. 39, 377 (1956).

[145] Kerner, E. H., The elastic and thermo-elastic properties of composite media, Proc. Phys. Soc. (London) 69B, 808 (1956).

[146] Spriggs, R. M., Effect of open and closed pores on elastic moduli of polycrystalline alumina, J. Am. Ceram. Soc. 45, 454 (1962).

[147] Hasselman, D. P. H., On the porosity dependence of the elastic moduli of polycrystalline refractory materials, J. Am. Ceram. Soc. 45, 452 (1962).

[148] Weil, N. A., Parametric effects governing the mechanics of ceramic materials, Chapter in "Proceedings of an International Symposium on High Temperature Technology", Butterworths, London (1964).

[149] Hasselman, D. P. H., and Fulrath, R. M., Effect of small fraction of spherical porosity on elastic moduli of glass, J. Am. Ceram. Soc. 47, 52 (1964).

[150] Fryxell, R. E., and Chandler, B. A., Creep, strength, expansion, and elastic moduli of sintered $\mathrm{BeO}$ as a function of grain size, porosity, and grain orientation, J. Am. Ceram. Soc. 47, 283 (1964).

[151] Hasselman, D. P. H., and Fulrath, R. M., Effect of cylindrical porosity on Young's modulus of polycrystalline materials, J. Am. Ceram. Soc. 48, 545 (1965).

[152] Hashin, Z., and Rosen, B. W., Elastic moduli of fiber-reinforced materials, J. Appl. Mech. 31, 223 (1964).

[153] Hill, R., The elastic behavior of a crystalline aggregate, Proc. Phys. Soc. (London) 65A, 349 (1952).

[154] Hashin, Z., The elastic moduli of heterogeneous materials, J. Appl. Mech. 29, 143 (1962).

[155] Hashin, Z., and Shtrikman, S., On some variational principles in anisotropic and nonhomogeneous elasticity, J. Mech. Phys. Solids 10, 335 (1962).

[156] Hashin, Z., and Shtrikman, S., A variational approach to the theory of the elastic behavior of polycrystals, J. Mech. Phys. Solids 10, 343 (1962).

[157] Peselnick, L., and Meister, R., Variational method of determining effective moduli of polycrystals: (A) hexagonal symmetry, (B) trigonal symmetry, J. Appl. Phys. 36, 2879 (1965).

[158] Meister, R., and Peselnick, L., Variational method of determining effective moduli of polycrystals with tetragonal symmetry, J. Appl. Phys. 37, 4121 (1966).

[159] Hershey, A. V., The elasticity of an iostropic aggregate of anisotropic cubic crystals, J. Appl. Mech. 21, 236 (1954).

[160] Kroner, E., Elastic moduli of perfectly disordered composite materials, J. Mech. Phys. Solids 15, 319 (1967).

[161] Kroner, E., Datta, B. K., and Kessel, D., On the bounds of the shear modulus of macroscopically isotropic aggregates of cubic crystals, J. Mech. Phys. Solids 14, 21 (1966).

[162] Walpole, L. J., On bounds for the overall elastic moduli of inhomogeneous systems-I. J. Mech. Phys. Solids 14, 151 (1966).

[163] Walpole, L. J., On bounds for the overall elastic moduli of inhomogeneous systems-II. J. Mech. Phys. Solids 14, ¿89 (1966).

[164] Sjodahl, L. H., and Chandler, B. A., Single-crystal elastic constants of $\mathrm{BeO}$ from polycrystalline measurements, J. Am. Ceram. Soc. 46, 351 (1963).

[165] Brace, W. F., Relation of elastic properties of rocks to fabric, J. Geophys. Res. 70, 5657 (1965).

[166] Hearmon, R. F. S., Applied Anisotropic Elasticity, Oxford University Press, Oxford, England (1961).

[167] Hill, R., Theory of mechanical properties of fibrestrengthened materials; I. Elastic behavior, J. Mech. Phys. Solids 12, 199 (1964).

[168] Hashin, Z., On elastic behavior of fibre reinforced materials of arbitrary transverse phase geometry, J. Mech. Phys. Solids 13, 119 (1965).
[169] Hill, R., Theory of mechanical properties of fibrestrengthened materials-III. Self consistent model, J. Mech. Phys. Solids 13, 189 (1965).

[170] Hill, R., A self-consistent mechanics of composite materials, J. Mech. Phys. Solids 13, 213 (1965).

[171] Budiansky, B., On the elastic moduli of some heterogeneous materials, J. Mech. Phys. Solids 13, 223 (1965).

[172] Hasselman, D. P. H., Experimental and calculated Young's modulus of zirconium carbide containing a dispersed phase of graphite, J. Am. Ceram. Soc. 46, 103 (1963).

[173] Hasselman, D. P. H., and Fulrath, R. M., Effect of spherical tungsten dispersions on Young's modulus of a glass, J. Am. Ceram. Soc. 48, 548 (1965).

[174] Hirsch, T. J., Modulus of elasticity of concrete affected by elastic moduli of cement paste matrix and aggregate, J. Am. Concrete Inst. 59, 427 (1962).

[175] Rath, J., Modulus of elasticity of a two-component material, Silikaty 10, 4 (1966).

[176] Walsh, J. B., The effect of cracks on the compressibility of rock, J. Geophys. Res. 70, 381 (1965).

[177] Walsh, J. B., The effect of cracks on the uniaxial elastic compression of rocks, J. Geophys. Res. 70, 399 (1965).

[178] Walsh, J. B., The effect of cracks in rocks on Poisson's ratio, J. Geophys. Res. 70, 5249 (1965).

[179] Born, M., and Huang, K., Dynamical Theory of Crystal Lattices, Oxford University Press, Oxford, England (1954).

[180] Born, M., Reminiscences of my work on the dynamics of crystal lattices, in Lattice Dynamics, edited by R. F. Wallis, Pergamon Press, New York (1965).

[181] Seitz, F., The Modern Theory of Solids, McGrawHill, New York (1940).

[182] Gilman, J. J., The strength of ceramic crystals, in The Physics and Chemistry of Ceramics, ed. by C. Klingsberg (Gordon and Breach, New York, 1963).

[183] Anderson, O. L., Determination and some uses of isotropic elastic constants of polycrystalline aggregates using single-crystal data, in Physical Acoustics, edited by W. P. Mason, Academic Press, New York (1965).

[184] Anderson, O. L., and Nafe, J. E., The bulk modulusvolume relationship for oxide compounds and related geophysical problems, J. Geophys. Res. 70, 3951 (1965).

[185] Anderson, O. L., A proposed law of corresponding states for oxide compounds, J. Geophys. Res. 71, 4963 (1966).

[186] Anderson, O. L., Lattice dynamics in geophysics, Trans. N.Y. Acad. of Sci. 27, 298 (1965).

[187] Szigeti, B., Polarizability and dielectric constant of ionic crystals, Trans. Faraday Soc. 45, 155 (1949).

[188] Szigeti, B., Compressibility and absorption frequency of ionic crystals, Proc. Roy. Soc. (London) A204, $51(1950)$.

[189] Anderson, O. L., and Glynn, P., Measurement of compressibility in polycrystalline $\mathrm{MgO}$ using the reflectivity method, J. Phys. Chem. Solids 26, $1961(1965)$

[190] Gray, D. E., Coordinating Editor, American Institute of Physics Handbook, McGraw-Hill, New York (1963).

[191] Alers, G. A., Use of sound velocity measurements in determining the Debye temperature of solids, in Physical Acoustics IIIB, edited by W. P. Mason, Academic Press, New York (1965).

[192] Anderson, O. L., A simplified method for calculating the Debye temperature from elastic constants, J. Phys. Chem. Solids 24, 909 (1963).

[193] Fedorov, F. I., The Debye temperature of a crystal, Soviet Phys.-Cryst. 10, 125 (1965). Translated from Kristallografiya 10, 167 (1965).

[194] Fedorov, F. I., and Bystrova, J. G., Debye temperatures of cubic crystals, Soviet Phys.-Cryst. 11, 
333 (1966). Translated from Kristallografiya 11, 368 (1966).

[195] Robie, R. R., and Edwards, J. L., Some Debye temperatures from single-crystal elastic constant data, J. Appl. Phys. 37, 2659 (1966)

[196] Ali, M., and Nagels, P., Evaluation of the Debye temperature of thorium dioxidc, Phys. Stat. Solidi 21, 113 (1967).

[197] Dayal, B., and Appalonarasimham, N., The evaluation of the elastic constants of rutile from spcctroscopic data, J. Sci. Research of the Benares Hindu University 1, 26 (1950).

[198] Pandey, H. N., The theoretical elastic constants and specific heats of rutile, Phys. Stat. Solidi 11, 743 (1965).

[199] Dolling, G., Cowley, R. A., and Woods, A. D. B., The crystal dynamics of uranium dioxide, Can. J. Phys. 43, 1397 (1965).

[200] Axe, J. D., and Pettit, G. D., Infrared dielectric dispersion and lattice dynamics of uranium dioxide and thorium dioxide, Phys. Rev. 151, 676 (1966).

[201] Grüneisen, E., The state of a solid body, NASA republication $\mathrm{RE} 2-18-59 \mathrm{~W}$ of a translation of "Zustand des festen Körpers", Handbuch der Phys. Bd. 10, pages 1-52, Springer, Berlin (1926).

[202] Slater, J. C., Introduction to Chemical Physics, McGraw-Hill (1939).

[203] Hume-Rothery, W., On Grüneisen's equation for thermal expansion, Proc. Phys. Soc. (London) 57, 209 (1945).

[204] Barron, T. H. K., On the thermal expansion of solids at low temperatures, Phil. Mag. 46, 720 (1955).

[205] Sheard, F. W., Calculation of the thermal expansion of solids from the third-order elastic constants, Phil. Mag. 3, 1381 (1958).

[206] Swartz, K. D., Anharmonicity in sodium chloride, J. Acoust. Soc. Am. 41, 1084 (1967).

[207] Anderson, O. L., Derivation of Wachtman's equation for the temperature dependence of elastic moduli of oxide compounds, Phys. Rev. 144, 553 (1966).

[208] Stern, E. A., Theory of anharmonic properties of solids, Phys. Rev. 111, 786 (1958).

[209] Wachtman, J. B., Jr., Tefft, W. E., Lam, D. G., Jr., and Apstein, C. S., Exponential temperature dependence of Young's modulus for several oxides, Phys. Rev. 122, 1754 (1961).

[210] Tefft, W. E., Elastic constants of synthetic single crystal corundum, J. Research NBS 70A (Phys. and Chem.) No. 4, 277 (1966).

[211] Alton, W. J., and Barlow, A. J., Temperature dependence of the elastic constants of yttrium aluminum garnet, J. Appl. Phys. 38, 3023 (1967).

[212] Graham, L. J., Nadler, H., and Chang, R., Elastic constants of UC, J. Appl. Phys. 34, 1572 (1963).

[213] Soga, N., and Anderson, O. L., High-temperature elastic properties of polycrystalline $\mathrm{MgO}$ and $\mathrm{Al}_{2} \mathrm{O}_{3}$, J. Am. Ceram. Soc. 49, 355 (1966).

[214] Soga, N., and Anderson, O. L., High-temperature elasticity and expansivity of fosterite and steatite, J. Am. Ceram. Soc. 50, 239 (1967).

[215] Soga, N., Schreiber, E., and Anderson, O. L., Estimation of bulk modulus and sound velocities of oxides at very high temperatures, J. Geophys. Res. 71, 5315 (1966).

[216] Barker, R. E., Jr., An approximate relation between elastic moduli and thermal expansivities, J. Appl. Phys. 34, 107 (1963).

[217] Slagle, O. D., and McKinstry, H. A., Temperature dependence of the elastic constants of the alkali halides. I. $\mathrm{NaCl}, \mathrm{KCl}$, and $\mathrm{KBr}$. II. The solid solution $\mathrm{KCl}-\mathrm{KBr}$. III. $\mathrm{CsCl}, \mathrm{CsBr}$, and $\mathrm{CsI}$, J. Appl. Phys. 38, 437 (1967).

[218] Bell, R. O., and Rupprecht, G., Elastic constants of strontium titanate, Phys. Rev. 129, 90 (1963).

[219] Aleksandrov, K. S., Reshchikova, L. M., and Beznosikov, B. V., Behavior of the elastic constants of $\mathrm{KMnF}_{3}$ single crystals near the transition of puckering, Phys. Stat. Solidi 18, K17 (1966).
[220] Brandt, O. G., and Walker, C. T., Temperature dependence of elastic constants and thermal expansion for $\mathrm{UO}_{2}$, Phys. Rev. Letters 18, 11 (1967).

[221] Garland, C. W., and Jones, J. S., Elastic constants of ammonium chloride near the lambda point, $J$. Chem. Phys. 39, 2874 (1963).

[222] Leurgans, P. J., Editor, Conference on internal friction due to crystal lattice imperfections, Acta Met. 10 [4] (1962).

[223] Lazan, B. J., and Wyman, L. L., Symposium Chairman, Internal friction, damping and cyclic plasticity, ASTM special technical publication No. 378, 1965.

[224] Mason, W. P., Editor, Physical acoustics, IIIA, Effect of imperfections, Academic Press, New York (1965).

[225] Mason, W. P., Editor, Physical acoustics, IVA, Applications to quantum and solid state physics, Academic Press New York (1966).

[226] Haven, Y., and van Santen, J. H., Electrically and elastically active relaxation modes, J. Chem. Phys. 22, 1146 (1954).

[227] Haven, Y., and van Santen, J. H., Dielectric relaxation of lattice defects in crystals, Nuovo Cimento 7 (suppl.), 605, (1958).

[228] Wachtman, J. B., Jr., and Peiser, H. S., Symmetry conditions for internal friction caused by jumping of point defects in crystals, Appl. Phys. Letters 1, 20 (1962)

[229] Nowick, A. S., and Heller, W. R., Anelasticity and stress-induced ordering of point defects in crystals, Advan. Phys. 12, 251 (1963).

[230] Franklin, A. D., Relaxation modes for trapped crystal point defects, J. Research NBS, 67A (Phys. and Chem.) No. 4, 291 (1963).

[231] Wachtman, J. B., Jr., Mechanical and electrical relaxation in $\mathrm{ThO}_{2}$ containing $\mathrm{CaO}$, Phys. Rev. 131, 517 (1963).

[232] Wachtman, J. B., Jr., Peiser, H. S., and Levine, E. P., Symmetry splitting of equivalent sites in oxide crystals and related mechanical effects, J. Research NBS 67A (Phys. and Chem.) No. 4, 281 (1963).

[233] Bhagvantam, S., and Pantulu, P. V., Point defects and relaxation phenomena in crystals, Proc. Indian Acad. Sci. 58, 183 (1963).

[234] Chang, R., Mechanical relaxation associated with paired point defects in cubic lattices of $\mathrm{O}_{h}$ point group symmetry, J. Phys. Chem. Solids 25, 1081 (1964).

[235] Franklin, A. D., Shorb, A., and Wachtman, J. B., Jr., Relaxation modes of trapped crystal point defects: The three-neighbor shells model in $\mathrm{NaCl}, \mathrm{J}$. Research NBS 68A (Phys. and Chem.) No. 5, 425 (1964).

[236] Nowick, A. S., and Heller, W. R., Dielectric and anelastic relaxation of crystals containing point defects, Advan. Phys. 14, 101 (1965).

[237] Wachtman, J. B., Jr., and Peiser, H. S., Symmetry conditions on jump rates occurring in relaxation times associated with point defect motions between equivalent general sites in crystals, J. Phys. Chem Solids 27, 975 (1966).

[238] Nowick, A. S., Dielectric and anelastic relaxation of crystals containing point defects. II, Advan. Phys. 16, 1, (1967).

[239] Wert, C., Determination of the diffusion coefficient of impurities by anelastic methods, in Physical Acoustics, Vol IIIA, edited by W. P. Mason, Academic Press, New York (1966).

[240] Johnson, H. B., Tolar, N. J., Miller, G. R., and Cutler, Ivan B., Dipole relaxation in $\mathrm{CaF}_{2}$ doped with NaF, J. Am. Ceram. Soc. 49, 458 (1966).

[241] Southgate, P. D., Anelastic and dielectric loss in yttrium-doped calcium fluoride, J. Phys. Chem. Solids 27, 1623 (1966). 
[242] Lay, K. W., and Whitmore, D. H., Dielectric and mechanical losses due to calcium-oxygen vacancy pairs in cerium dioxide, Proc. Fifth Rare Earth Conference, Ames, Iowa (1965).

[243] Southgate, P. D., Mechanical relaxation of a point defect in magnesium oxide, J. Appl. Phys. 36, 2696 (1965).

[244] Southgate, P. D., Internal friction due to chromium and iron in magnesium oxide, J. Phys. Chem. Solids 27, 1263 (1966).

[245] van Houten, S., Mechanical losses in Li-doped $\mathrm{NiO}$ semiconductors, J. Phys. Chem. Solids 23, 1045 (1962)

[246] Dodd, D. M., and Frazer, D. B., The 3000-9000 $\mathrm{cm}^{-1}$ absorption bands and anelasticity in crystalline $\alpha$-quartz, J. Phys. Chem. Solids 26, 673 (1965).

[247] Carnahan, R. D., and Brittain, J. O., Point-defect relaxation in rutile single crystals, J. Appl. Phys. 34, 3095 (1963).

[248] Wachtman, J. B., Jr., and Doyle, L. R., Internal friction in rutile containing point defects, Phys. Rev. 135, A276 (1964).

[249] Carnahan, R. D., and Brittain, J. O., Low-temperature internal friction peak in rutile, J. Appl. Phys. 37, 1808 (1966).

[250] Wachtman, J. B., Jr., Spinner, S., Brower, W. S., Fridinger, T., and Dickson, R. W., Internal friction in rutile containing $\mathrm{Ni}$ or $\mathrm{Cr}$, Phys. Rev. 148, 811 (1966).

[251] Sochino, G., de Batist, R., and Gevers, R., Relaxation processes in single-crystal $\mathrm{UO}_{2}$, Proc. British Ceram. Soc. 9, 73 (1967).

[252] Wachtman, J. B., Jr., and Corwin, W. C., Internal friction in $\mathrm{ZrO}_{2}$ containing $\mathrm{CaO}$, J. Research NBS 69A (Phys. and Chem.) No. 5, 457 (1965).

[253] Granato, A. V., and Lucke, K., The vibrating string model of dislocation damping, in Physical Acoustics, Vol. IVA, edited by W. P. Mason, Academic Press, New York (1966).

[254] Chang, R., Dislocation relaxation phenomena in oxide crystals, J. Appl. Phys. 32, 1127 (1961).

[255] Huber, R. J., Baker, G. S., and Gibbs, P., Hightemperature kilocycle internal friction in $\mathrm{Al}_{2} \mathrm{O}_{3}$ single crystals, J. Appl. Phys. 32, 2573 (1961).

[256] Dahlberg, P., Carnahan, R. D., and Brittain, J. O., dislocation damping in magnesium oxide crystals at low frequencies, J. Appl. Phys. 33, 3493 (1961).

[257] Southgate, P. D., Mendelson, K. S., and DePerro P. L., Kilocycle-range dislocation damping in magnesium oxide, J. Appl. Phys. 37, 206 (1966).

[258] Carnahan, R. D., and Brittain, J. O., Dislocacion damping in rutile single crystals, J. Am. Ceram. Soc. 48, 15 (1966).

[259] Astbury, N. F., and Davis, W. R., Internal friction in ceramics, Trans. Brit. Ceram. Soc. 63, 1 (1964).

[260] Melngailis, J., A model for the change in elastic modulus of a solid caused by point defects, Phys. Stat. Solidi 16, 247 (1966).

[261] Jan, J. P., Steinemann, S., and Dinichert, P., The density and lattice parameters of ruby, J. Phys. Chem. Solids 12, 349 (1960).

[262] Muñoz, R., On the elastic model for the deviations from Vegard's law, Phil. Mag. 14, 1105 (1966).

[263] Csavinsky, P., Effect of the thermal population of the $(000)$ and (100) conduction bands on the elastic constants of heavily doped n-type germanium, J. Appl. Phys. 37, 1967 (1966).

[264] Plendl, J. N., Mitra, S. S., and Gielisse, P. J., Compressibility, cohesive energy, and hardness of non-metallic solids, Phys. Stat. Solidi 12, 367 (1965).

[265] Kalnin, I. L., Strength and elasticity of whitewares: Part I, Relation between flexural strength and elasticity, Bull. Am. Ceram. Soc. 46, 1174 (1967).
[266] Wachtman, J. B., Jr., Mechanical properties of ceramics-an introductory survey, Bull. Am. Ceram. Soc. 46, 756 (1967).

[267] Spence, G. B., Extended dislocations in the anisotropic elastic continuum approximation, Proc. of the Fifth Conference on Carbon, Volume 2, Pergamon Press, New York (1961).

[268] Reynolds, W. N., The mechanical properties of reactor graphite, Phil. Mag. 11, 357 (1965).

[269] Price, R. J., Young's modulus of pyrolytic carbon in relation to preferred orientation, Phil. Mag. 12, 561 (1965).

[270] Baker, C., and Kelly, A., The effect of neutron irradiation on the elastic moduli of graphite single crystals, Phil. Mag. 9, 927 (1964).

[271] Kelly, B. T., The elastic constants of polycrystalline carbons and graphites, Phil. Mag. 9, 721 (1964).

[272] Woolley, R. L., The elasticity of polycrystalline graphite, Phil. Mag. 11, 475 (1965).

[273] Summers, L., Walker, D. C. B., and Kelly, B. T., The effect of fast neutron irradiation on the $c_{33}$ elastic modulus of highly oriented pyrolytic graphite, Phil. Mag. 14, 317 (1966).

[274] Mason, I. B., and Knibbs, R. H., Variation with temperature of Young's modulus of polycrystalline graphite, Nature 188, 33 (1960).

[275] Price, W. J., and Huntington, H. B., Acoustical properties of anisotropic materials, J. Acoust. Soc. Am. 22, 32 (1950).

[276] Eros, S., and Reitz, J. R., Elastic constants by the ultrasonic pulse echo method, J. Appl. Phys. 29, 683 (1958).

[277] McSkimin, H. J., Notes and references for the measurement of elastic moduli by means of ultrasonic waves, J. Acoust. Soc. Am. 33, 606 (1961).

[278] Mcskimin, H. J., Variations of the ultrasonic pulse-superposition method for increasing the sensitivity of delay-time measurements, J. Acoust. Soc. Am. 37, 864 (1965).

[279] McSkimin, H. J., Measurement of ultrasonic wave velocities and elastic moduli for small solid specimens at high temperatures, J. Acoust. Soc. Am. 31, 287 (1959).

[280] Alers, G. A., The measurement of very small sound velocity changes and their use in the study of solids, in Physical Acoustics IVA, edited by W. P. Mason, Academic Press, New York (1966).

[281] Simmons, G., Ultrasonics in Geology, Proc. IEEE 53, 1337 (1965).

[282] Neighbours, J. R., and Schacher, G. E., Determination of elastic constants from sound-velocity measurements in crystals of general symmetry, J. Appl. Phys. 38, 5366 (1967).

[283] Einspruch, N. G., and Truell, R., Results of an ultrasonic measurement survey, J. Appl. Phys. 33, 3087 (1962).

[284] Simmons, G., Comparison of static and dynamic measurements of compressibility of rocks, J. Geophys. Res. 70, 5649 (1965).

[285] Fine, M. E., Dynamic methods for determining the elastic constants and their temperature variation in metals, ASTM Bulletin No. 181, page 20 (1952).

[286] Kesler, C. E., and Chang, T. S., Review of sonic methods for the determination of mechanical properties of solid materials, University of Illinois T. and A. M. report 54, September 1954.

[287] Fine, M. E., Apparatus for measuring the elastic moduli and internal friction of solids from 1.7 to above $77^{\circ} \mathrm{K}$ and some values for $a$-quartz, Rev. Sci. Inst. 25, 1188 (1954).

[288] Fine, M. E., Apparatus for precise determination of dynamic Young's modulus and internal friction at elevated temperatures, Rev. Sci. Inst. 29, 643 (1957).

[289] Thompson, D. O., and Glass, F. M., Elastic constant-internal friction spectrometer, Rev. Sci. Inst. 29, 1034 (1958). 
[290] Vernon, E. V., An apparatus for the determination of dynamic elastic moduli at low strains, J. Sci. Inst. 35, 28 (1958).

[291] Simott, K. M., Apparatus for the mcasurement of shear modulus and internal friction between 4.2 and $100^{\circ} \mathrm{K}$, J. Appl. Phys. 29, 1433 (1958).

[292] Watts, C. R., and Carnahan, R. D., Determination of clastic constants of solids, Acrospace Corporation report TR-1001 (2250-10)-2, Air Force report number SSD-TR-67-4, Sept. 1966.

[293] Ramachandran, G. N., and Wooster, W. A., Determination of elastic constants of crystals from diffuse reflexions of x-rays. I. Theory of method, Acta. Cryst. 4, 335 (1951).

[294] Ramachandran, G. N., and Wooster, W. A., Determination of elastic constants of crystals from diffuse reflexions of x-rays. II. Application to some cubic crystals, Acta. Cryst. 4, 431 (1951).

[295] Sumer, A., and Smith, J. F., A comparison of the elastic constants of chromium as determined from diffuse $x$-ray and ultrasonic techniques, J. Appl. Phys. 34, 2691 (1963).

[296] Mahlcr, R. J., and Tanttila, W. H., Ultrasonic determination of crystalline resonances and sound velocities, using NMR techniques, J. Acoust. Soc. Am. 38, 429 (1965).

[297] Ross, J. H., Approving Officer, Evaluation techniques for fibers and yarns used by the Fiberous Matcrials Branch, Nonmetallic Materials Division, Air Force Materials Laboratory, Air Force Materials Laboratory, Technical Report AFMLTR-67-159, Sept. 1967. 


\title{
Viscoelasticity of Glasses*
}

\section{Pedro Buarque de Macedo}

\author{
The Catholic University of America, Washington, D.C. 20017 \\ and \\ Institute for Materials Research, \\ National Bureau of Standards, Washington, D.C. 20234
}

\begin{abstract}
Viscosity measurement techniques such as rotating cylinder, beam bending, fiber elongation, etc., are reviewed and compared to each other as to their viscosity range and experimental uncertainty. Thermal expansion coefficient and density measurements are discussed. Since the high-temperature melt density values in the literature do not agree with each other to within their respective standard deviations, a detailed discussion of such measurements is given. The high-temperature ultrasonic interferometer used to make sound absorption and velocity measurements is described along with its elaborate electronic circuitry.

Viscoelasticity provides a linear mathematical framework to analyze both the time and the frequency dependence of the mechanical parameters in the glass transition. The deformation, annealing, and strain points are analyzed as to the particular physical process (viscous flow, volume relaxation, shear relaxation) involved.

The limits of validity of the empirical (technological) experiments used to characterize these processes are considered. Volume relaxation is distinguished from shear relaxation. Non-Newtonian flow is considered to be due to immiscible glasses.

Since the viscosity governs most manufacturing processes, such as the ability to stir a melt or to shape an object, its temperature dependence is of utmost importance. Not only is the least squares method to fit data to the Fulcher equation discussed, but also the various theories which lead to the equation. The validity of these theories is tested with recent data on $\mathrm{B}_{2} \mathrm{O}_{3}$, and they are found to be deficient.
\end{abstract}

Key Words: Annealing density; glasses; glass transformation; modulus; viscoelasticity; viscosity.

\section{Introduction}

In the first part of this paper a review of the best techniques for making viscoelastic measurements in molten glasses will be made. There will be a detailed description of viscosity measurements, since viscosity plays such a critical role in the manufacture and fabrication of glass. A shorter discussion of density, sound absorption, and phase velocity measurements will also be given.

Secondly, a review of annealing and relaxation theory as well as a discussion of the interpretations of experimental data which are still controversial, such as the role of microstructure in viscous flow, will be made. These reviews are planned to help both the quality control man and the researcher.

Thirdly, a review of viscosity theories will be made. The material in this section is taken from a recent paper by Napolitano and Macedo [1]. ${ }^{1}$ Since this area is highly controversial, the opinions expressed here are those of the author and may or may not be widely accepted throughout the scientific community. Unlike the first two sections, this last section will be of primary interest to the researcher, but the engineer should be aware of existing viscosity theories.

*Work at Cotholic University of America supported by Air Force Office of Scientific Research Grant No. AFOSR-68-1376.

1 Figures in brackets indicate the literature references at the ${ }^{-}$end of this paper.

\section{Measurement of Physical Properties of Molten Glasses}

\subsection{Viscosity}

Between the boiling point and the glass transition a liquid may exhibit viscosities ranging from $10^{-2}$ to $10^{+15}$ poise. [One poise is the cgs unit of viscosity. A newton-s $/ \mathrm{m}^{2}$, the mks unit, is equal to 10 poise.] In most molten silicate glasses, the difficulty in readily obtaining temperatures in excess of $1500{ }^{\circ} \mathrm{C}$ rules out the measurement of viscosities much below $10^{2} \mathrm{P}$. Thus, for molten glasses one is interested in measuring viscosities between $10^{2}$ and $10^{15}$ poise. At present this cannot be done with a single technique. Table 1 gives the most common techniques used for molten oxides, as well as the range of viscosities and the approximate error. Most of the techniques require calibration of the equipment with liquids of known viscosity at room temperature. In fact all the high-temperature viscosity measurements below $10^{7}$ poise rely on the calibration made with NBS standard viscosity oils.

The most reliable high-temperature viscometer is the rotating cylinder one $[2,3,4,5,6,7]$. The latest version of this type of apparatus is described in detail by Napolitano et al. [2], and can measure 
TABLE 1. Viscosity measurement techniques

\begin{tabular}{|c|c|c|c|}
\hline Technique & $\begin{array}{l}\text { Viscosity } \\
\text { range }\end{array}$ & Error : & References \\
\hline $\begin{array}{l}\text { Rotating cylinder } \\
\text { Counterbalance-sphere. } \\
\text { Penetrometer } \\
\text { Fiber elongation. } \\
\text { Beam bending } \\
\text { Softening point } \\
\text { Annealing point } \\
\text { Strain point }\end{array}$ & $\begin{array}{c}\text { Poises } \\
10^{0}-10^{10} \\
10^{1}-10^{5} \\
10^{5}-10^{9} \\
10^{5}-10^{15} \\
10^{8}-10^{15} \\
\sim 10^{7.6} \\
\sim 10^{13} \\
\sim 10^{14.8}\end{array}$ & $\begin{array}{l}\text { Percent } \\
2 \\
5 \\
5 \\
10 \\
10 \\
2^{\circ} \mathrm{C} \\
2^{\circ} \mathrm{C} \\
4^{\circ} \mathrm{C}\end{array}$ & $\begin{array}{l}2,3,4,5,6,7 \\
8,9 \\
10,11 \\
3,4,12,13,14 \\
15,16 \\
3,19 \\
3,16,19 \\
3,16,19\end{array}$ \\
\hline
\end{tabular}

n The estimated proportional standard deviation of viscosity will vary betwcen various authors using the same technique.

viscosities from the centipoise range up to $10^{10}$ poise. The instrument is capable of measuring viscosities to within 1 percent, but the reported errors are at least double that because of the inability to eliminate temperature gradients and fluctuations during measurements. The rotating cylinder viscometer is based on the general equation of motion:

$$
I \frac{d^{2} \theta}{d t^{2}}+\eta K_{1} \frac{d \theta}{d t}+K_{2} \theta=K_{3} i
$$

where

$$
\begin{aligned}
& \mathrm{I}=\text { moment of inertia, } \\
& \theta=\text { angular displacement }, \\
& \mathrm{t}=\text { time, } \\
& \eta=\text { viscosity }
\end{aligned}
$$

$K_{1}, K_{2}$, and $K_{3}$ are constants of the apparatus; and $i$ is current through the coil. Thus the first term of the equation is the torque from inertia. The second term is the torque from viscous drag. The third term is an elastic torque due to an angular deflection of the suspension, and the last term is the magnetic torque applied to the system.

Method 1. Over the lowest viscosity range log $\eta=0$ to $\log \eta=5$ ( $\log$ stands for $\log 10$ ) the outer cylinder is rotated at a fixed angular velocity. The viscous drag of the liquid produces a torque on the inner cylinder. A measure of this torque is made by applying an appropriate current to the coil such that the resulting magnetic torque will bring the inner cylinder back to its rest position at $\theta=0$.

Therefore the first term of eq (1) is zero, because there is no angular acceleration in the system when measurements are being made. The second term is present and significant. It is retained in the equation of motion for Method 1, but since the inner cylinder is held at rest, the angular velocity of the outer cylinder is used in this term. The third term is zero when the system is balanced, since the angle across the suspension is zero. Even so, it controls the sensitivity. By changing suspensions the value of $K_{2}$ can be changed and with it, the sensitivity. Since the fourth term is significant, it is kept. Thus the equation of motion for Method 1 is

$$
\eta K_{1} \frac{d \theta}{d t}=K_{3} i
$$

or

$$
\eta=C_{1} i / \omega
$$

where $C_{1}=\frac{K_{3}}{K_{1}}$ and $\omega=\frac{d \theta}{d t}$ (angular velocity). $C_{1}$ in eq (3) was obtained by calibrating the viscometer with NBS standard oils. Thermal expansion corrections on $C_{1}$, known for room temperatures, have to be made for accurate viscosity measurements at temperature, $T$, up to $1400^{\circ} \mathrm{C}$ according to the following equation:

$$
C_{1}(T)=C_{1_{25^{\circ} \mathrm{C}}}\left(1+\beta_{p}[T-25]\right)^{-3}
$$

where $\beta_{p}$ is the linear expansion coefficient of platinum.

Method 2. For viscosities from about $\log$ $\eta=4.5$ to $\log \eta=7.5$ the aperiodic method [4] is used. In this decay mode of operation, the outer cylinder remains at rest while the inner cylinder is displaced by an angle $\theta$ and allowed to return to its zero position under the force of the torsion wire. Since the system is an over-damped harmonic oscillator, one observes an exponentially decaying angular velocity as the inner cylinder approaches its equilibrium position. The term involving the moment of inertia in eq (1) is extremely small and can be neglected, giving rise to the following equation:

$$
\eta \frac{d \theta}{d t}=-C_{2} \theta
$$

where

$$
C_{2}=K_{2} / K_{1}
$$

Integrating

$$
\int_{\theta_{1}}^{\theta_{2}} \eta \frac{d \theta}{\theta}=-\int_{i_{1}}^{t_{2}} C_{2} d t
$$

we have

$$
\eta=\frac{C_{2}\left(t_{2}-t_{1}\right)}{\ln \theta_{1} / \theta_{2}}
$$

The calibration constant $\left(C_{2}\right)$ for Method 2 can be calculated once the viscometer has been calibrated by Method 1. First an independent measurement of the current required for a given angular deflection of the inner cylinder is made. This gives us:

$$
\theta=\frac{K_{3}}{K_{2}} i=C_{4} i
$$

From eqs (3), (5), and (7) one may obtain $C_{2}$ from $C_{1}$ and $C_{4}$ according to the following:

$$
C_{2}=\left(C_{1} / C_{4}\right) \text {. }
$$

Method 3. For viscosities from about log $\eta=5.5$ to $\log \eta=10$ the following method was used. The outer cylinder again remains at rest while a constant torque is applied to the inner cylinder by means of the magnetic field interaction, and the angular velocity is measured about $\theta=0$. Thus the term $K_{2} \theta$ is negligible and can be omitted. Since 
measurements are made in a steady state the acceleration is zero, and we have the following:

$$
\eta \frac{d \theta}{d t}=\frac{K_{3}}{K_{1}} i=C_{3} i
$$

integrating

$$
\int_{\theta_{1}}^{\theta_{2}} \eta d \theta=\int_{t_{1}}^{t_{2}} C_{3} i d t
$$

we have

$$
\eta=C_{3} i \frac{t_{2}-t_{1}}{\theta_{2}-\theta_{1}}
$$

$C_{3}$ and $C_{1}$ are equal.

An easier way of obtaining the torque involved in the measurement is with a Brookfield viscometer after adapting it for high temperatures. This was done by Eppler [5], Gruber and Litovitz [6], and Tiede [7]. Eppler [5] not only used the Method 1 operation, which is the one recommended by the manufacturer, but also Method 2, with a similar increase in viscosity range.

For viscosities higher than $10^{10}$ poise two techniques are commonly used, the fiber elongation and the beam bending techniques. Both of the techniques represent absolute methods of measuring viscosity and, unlike the rotation methods, require no calibration.

The fiber elongation technique has been used by many authors $[3,4,12,13,14]$. The recent work of Napolitano and Hawkins [3] gives one of the best descriptions of the apparatus described below:

Weights ranging from 5 to $1000 \mathrm{~g}$, depending on the test temperature and viscosity of the glass, were used to apply tension to fibers of uniform diameter $(\sim 0.75 \mathrm{~mm})$ and $10 \mathrm{~cm}$ long. An optical lever with a multiplication factor of 50 was used to observe the resulting elongation with a telescope equipped with a vertical scale. The viscosity, $\eta$, can be calculated from [14].

$$
\eta=F l /(3 \dot{A} d l / d t)
$$

where

$$
\begin{aligned}
F & =\text { extending force }(\text { dyne }) \\
l & =\text { fiber length }(\mathrm{cm}) \\
A & =\text { fiber cross section area }\left(\mathrm{cm}^{2}\right) \\
\frac{d l}{d t} & =\text { elongation rate }(\mathrm{cm} / \mathrm{s})
\end{aligned}
$$

Some glasses cannot be made into fibers because of devitrification, phase separation, surface volatilization, etc. For many of these, samples can be cut and ground to form beams of simple geometry. The beam-bending method, devised by Jones [15] and perfected by Hagy [16], gives viscosity results derived from the rate of viscous deflection of a beam according to the equation

$$
\eta=\frac{g L^{3}}{2.4 I_{c^{\nu}}}\left[M+\frac{\rho A L}{1.6}\right]
$$

where

$g=$ acceleration due to gravity $\left(\mathrm{cm} / \mathrm{s}^{2}\right)$

$I_{c}=$ cross section moment of inertia $\left(\mathrm{cm}^{4}\right)$

$\nu=$ midpoint deflection rate $(\mathrm{cm} / \mathrm{min})$

$M=$ centrally applied load $(\mathrm{g})$

$\rho=$ density of glass $\left(\mathrm{g} / \mathrm{cm}^{3}\right)$

$A=$ cross section area of beam $\left(\mathrm{cm}^{2}\right)$

$L=$ suport span (cm).

The second term within the brackets accounts for the contribution of the weight of the beam to bending and is usually neglected.

Both techniques can measure between $10^{8}$ and $10^{15}$ P. Figure 1 of Napolitano and Macedo [17] shows an Arrhenius plot $(\log \eta$ versus $1 / T)$ of the viscosity of $\mathrm{GeO}_{2}$ glass in the annealing region. As can be seen from the scatter of points both fiber elongation [17] and the beam bending [18] data not only give the same values but also have about the same uncertainty.

Table 1 also shows other viscosity measurement techniques, and the interested reader can look up the appropriate reference. When building a viscometer it is highly recommended that the apparatus be tested at or about the temperature one plans to use it. For this, one can now buy NBS Standard Reference Materials with certified viscosities.

In industry complete viscosity runs are made only to evaluate a new glass. For quality control

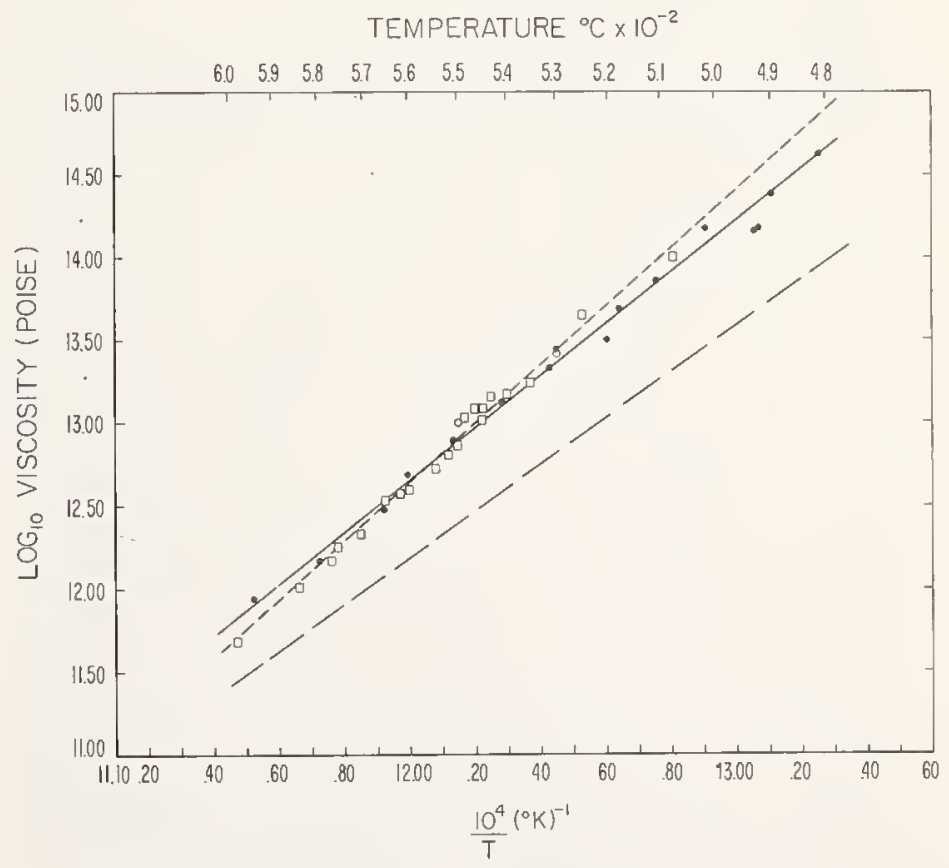

Figure 1. Arrhenius plot of viscosity of $\mathrm{GeO}_{2}$.

$\square$ F \& P ref. [18]

- Flame drawn fibers $\}$ from N \& $M$, ref. [17]

Fiber drawn from crucible trom

. . . - least squares fit to $F \&$ P data

- least squares fit of combined data

extrapolation of high temperature Arrhenius least square fit of F \& P data 
one uses fixed point measurements such as softening point, annealing point, and strain point. These three points represent empirical measurements which not only involve viscosity but also other physical quantities. They are described in the ASTM part 13 [19]. NBS also provides five reference glasses for the calibration of furnaces used in measuring these fixed points.

\subsection{Density}

Density measurements of glasses are usually made by Archimedes' principle either by direct weighing or by flotation. These measurements are now routine and require no further discussion. The expansion coefficient methods are also generally accepted and can be made either by interferometric technique [20] or by dial technique [19]. Measurements of the density of molten glasses, on the other hand, have not been standardized and the disagreement between various authors $[21,22,23,24,25,26]$ is outside their respective standard deviations. The technique universally used is to weigh a restrained platinum sphere suspended by a platinum wire in a melt. The possible sources of error are:

(a) homogeneity of the melt; if there are seeds (bubbles) in the melts, the measured density will be lower.

(b) difficulty in finding the null point, because of viscous drag.

(c) the role of surface tension on the suspension wire; it has either been ignored or miscalculated. Shartsis [8] made an error in the calculation of the surface tension effect which was extensively copied by other authors.

(d) convection currents caused by an updraft from the furnace may give erroneous weighings.

I will describe the technique Napolitano et al. used in a previous publication [21] to measure the density of $\mathrm{B}_{2} \mathrm{O}_{3}$.

The density was measured by Archimedes' principle using a restrained sphere. Equilibrium was obtained by letting a one-arm deflection balance settle. Thus it was necessary only to adjust the weights to $0.1 \mathrm{~g}$, whereas the next decimals were obtained by deflection and required only periodic observations. Densities were measured on glasses with viscosities up to $10^{6}$ poise. At the aforementioned value, the balance took $92 \mathrm{hr}$ to come to equilibrium. To estimate the uncertainty of these measurements after the first equilibrium was obtained, the cup was lowered $1 \mathrm{~mm}$ so that an upper uncertainty limit could be obtained in the weight; then it was raised $1 \mathrm{~mm}$ for the lowered limit. It took $92 \mathrm{hr}$ for the balance to come to equilibrium at each of the subsequent weighings. These limits were within $\pm 0.001 \mathrm{~g} / \mathrm{cm}^{3}$.

To calculate the density from the data, the effects of surface tension on the suspension wire had to be considered. Surface tension force was not reproducible at room temperature with a platinum wire $0.081 \mathrm{~cm}$ in diameter because of variation in contact angle; therefore, a gold- plated tungsten wire $0.010 \mathrm{~cm}$ in diameter was used. The volumes of three spheres $(2.0,1.5$, and $1.0 \mathrm{~cm}$ in diameter), as well as the platinum suspension wire $(0.081 \mathrm{~cm}$ in diameter), were calculated from the difference in weight in air and in $4 \mathrm{C}$ distilled water. The combined effects of surface tension and buoyancy of the tungsten wire were computed to be less than $1 \mathrm{mg}$ in weight and therefore were neglected.

At high temperatures the surface tension effect was reproducible in molten $\mathrm{B}_{2} \mathrm{O}_{3}$. This was demonstrated by weighing each sphere at three depths. From the difference in volume and weight between the spheres an absolute calculation of the density can be made. In order not to make measurements at each temperature with three balls, the effect of surface tension was calculated assuming 100 percent wettability and disregarding the Wilhelmy [27] correction (for the effect of surface tension on thin wires). The authors found that Wilhelmy gave experimental evidence that this correction, reported in the early part of his publication, was not correct. Thus the density was calculated according to the following:

$$
d=\left(W_{\mathrm{a} \mid \mathrm{r}}-W_{m}+2 \pi r \sigma / g\right)\left[V_{0}\left(1+\beta_{p} T\right)^{3}\right]^{-1}
$$

where $W_{\mathrm{alr}}=$ weight of sphere in air $(\mathrm{g})$ $W_{m}=$ weight of sphere in melt (g) $V_{0}=$ volume of sphere in distilled water at $4^{\circ} \mathrm{C}\left(\mathrm{cm}^{3}\right)$

$r=$ radius of suspension wire $(\mathrm{cm})$

$\sigma=$ surface tension of $\mathrm{B}_{2} \mathrm{O}_{3}\left(\right.$ dynes $\left./ \mathrm{cm}^{2}\right)$

$g=$ gravitational constant $\left(\mathrm{cm} / \mathrm{s}^{2}\right)$

$\beta_{p}=$ linear expansion coefficient of platinum $/{ }^{\circ} \mathrm{C}$

$T=$ temperature $\left({ }^{\circ} \mathrm{C}\right)$

Density values from this calculation, using spheres 2.0 and $1.5 \mathrm{~cm}$ in diameter, fall within $\pm 0.002 \mathrm{~g} / \mathrm{cm}^{3}$ of the average values obtained by the difference in volume calculations.

In comparing the data [21] in figure 2 with other authors, our data approximately parallel those of Mackenzie [23] and of Shartsis et al. [25] but are about 1 percent higher. Our data are more nearly in agreement with the results of $\mathrm{Li}$ et al. [26] and Riebling [22]. I believe that most of the disagreement lies in the surface tension correction. The best way to minimize such errors is to have as large a sphere as possible, since in this case the surface tension correction is a minimum.

A more difficult problem is to make density measurements on melts having viscosities higher than $10^{6}$ poise. In this region it becomes prohibitively long to obtain balance by the Archimedes method. Thus a new technique was developed by Macedo, Capps, and Litovitz [18] to operate at high temperatures. A picnometer was fashioned which had a ground-glass ball joint, which made it possible to add or remove large specimens without affecting the internal volume of the picnometer. Cylindrical specimens of boron trioxide glass 


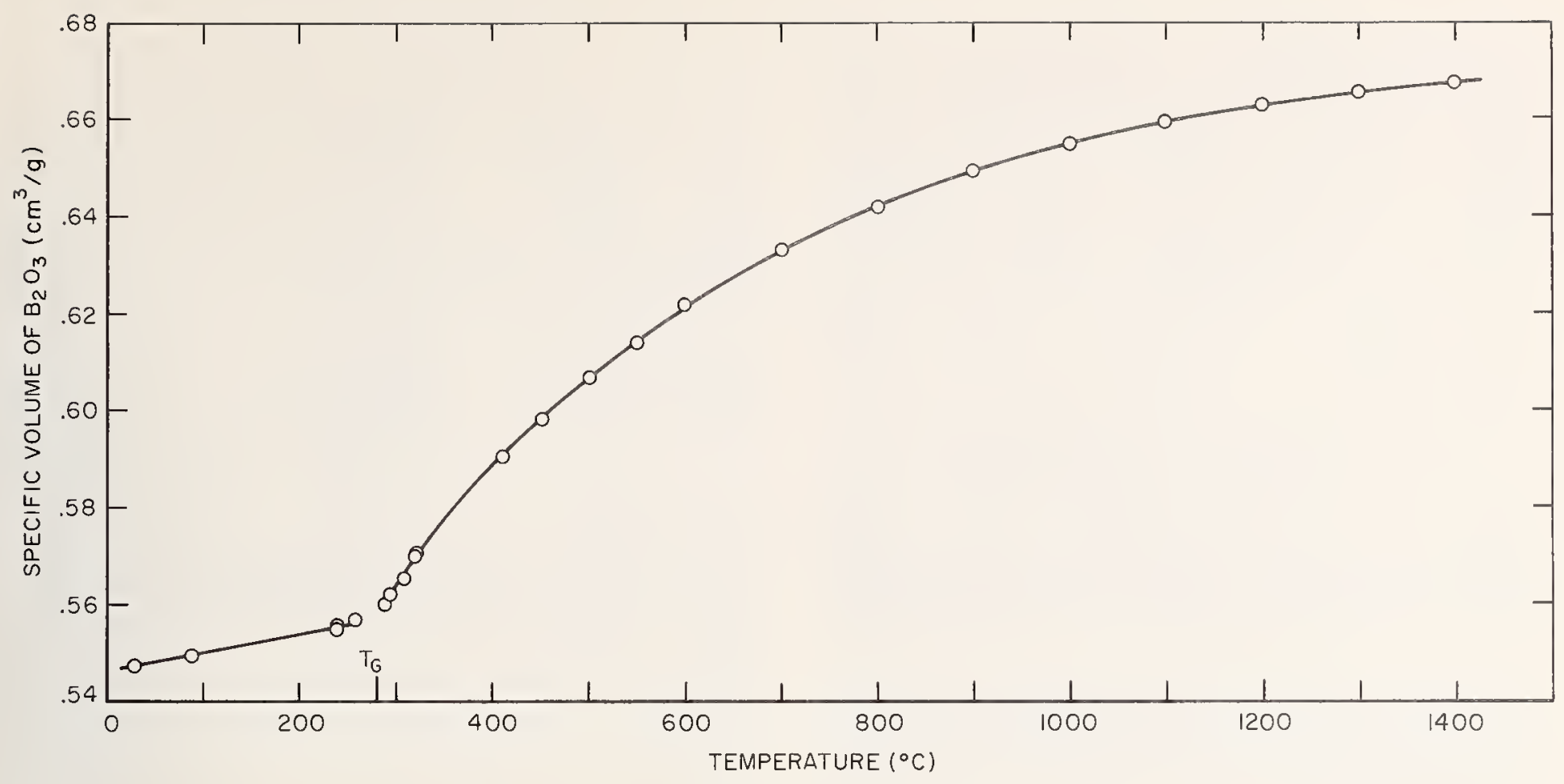

FrguRE 2. Specific volume versus temperature for vitreous $\mathrm{B}_{2} \mathrm{O}_{3}$.

Data above $400 \mathrm{C}$ taken from $\mathrm{NMH}$, ref. [21], below $400^{\circ} \mathrm{C}$ from $\mathrm{MCL}$, ref. [28].

were cast with a diameter of about $2 \mathrm{~cm}$ and a length of about $7 \mathrm{~cm}$. A Teflon stopcock in the filling tube made it unnecessary to use any grease. Mercury was used as the picnometer fluid which on heating was allowed to overflow into a weighing bottle outside of the furnace. The overflow spout was immersed below the mercury level in the weighing bottle, permitting intake of mercury when the system contracted on cooling. The bottle was removed from the assembly for weighing. Temperature changes were made in discrete steps. After thermal equilibrium was reached at a new temperature, the overflow bottle was weighed and then replaced; all of the overflow accumulated from step to step, and the total amount was always weighed to prevent an accumulation of weighing errors. This weight gain or loss of the picnometer system between temperatures represented the amount of mercury displaced by the expanding or contracting system. Comparing the expansion rate of the mercury system with the expansion rate of the mercury-plus-sample system enables one to determine the density $\rho$ of the sample alone. Figure 3 shows the weight of mercury displaced in both runs. The solid points represent the picnometer filled with mercury. They are numbered in chronological order to show reproducibility. The open circles represent the picnometer with $\mathrm{B}_{2} \mathrm{O}_{3}$ sample and $\mathrm{Hg}$. The first three points are high due to the fact that the original sample was highly quenched. Upon heating we have annealed our sample, and only points after No. 4 were used in our calculation. The agreement of Nos. 6 and 11 shows that once the sample is annealed the agreement is comparable to that of the mercury curve.

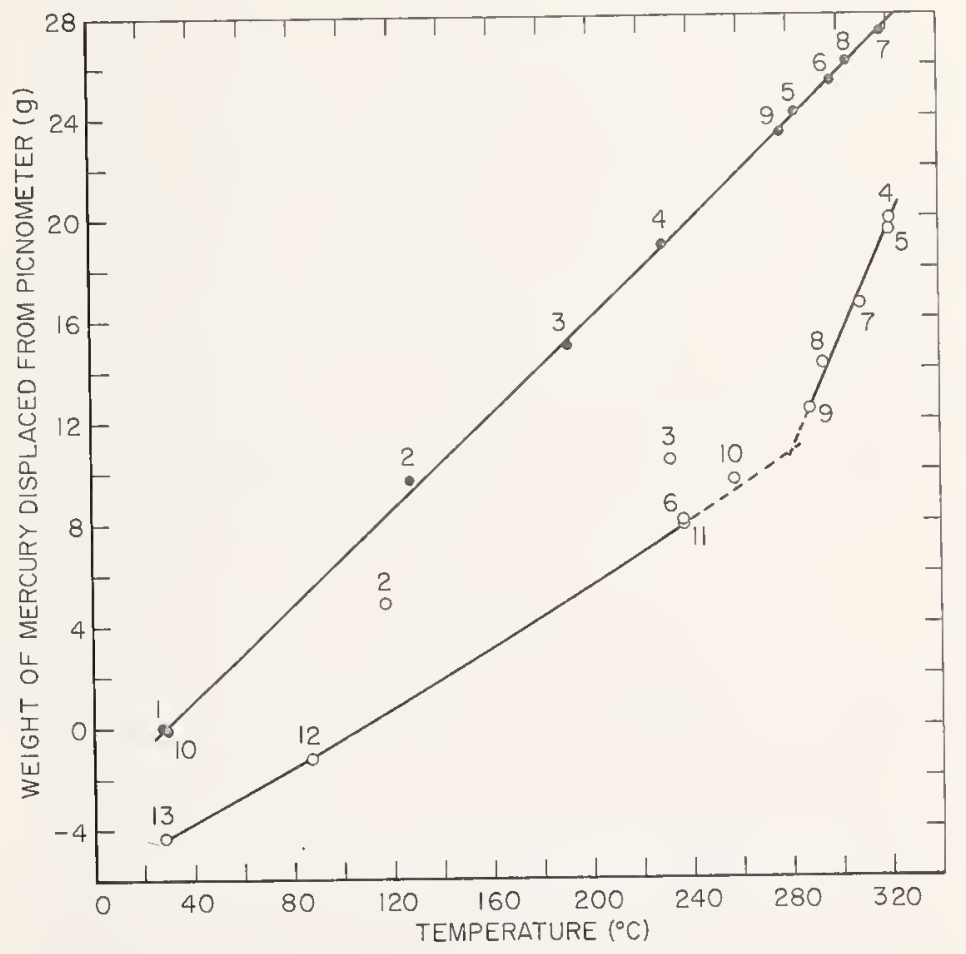

FIgURE 3. Weight of mercury displaced from picnometer as a function of temperature.

- picnometer filled with mercury; $O$, picnometer filled with sample

plus mercury.

The greatest source of error comes from the degassing of mercury at high temperatures and can be estimated from the scatter of the data points about the smooth curve. In both cases this 
scatter is less than $0.5 \mathrm{~g}$ which corresponds to a fractional uncertainty in the volume, of 0.15 percent. Thus the maximum error of the reported density or specific volume is \pm 0.2 percent. This data was used together with that of Napolitano, Macedo, and Hawkins [21] to produce a complete volume versus temperature curve in figure 2 . Interpolation was necessary between 325 and 411 ${ }^{\circ} \mathrm{C}$, but this was easily carried out because of the smooth nature of the specific volume versus temperature curve.

An upper limit of $325^{\circ} \mathrm{C}$ for picnometer experiments was a practical limit. Degassing of the mercury was a troublesome and imperfect process as some of the bubbles released would cling tenaciously to the vessel walls. This problem increased with temperature. Under ideal conditions the upper limit would be the boiling point of the mercury $\left(356-357{ }^{\circ} \mathrm{C}\right)$ but because it has a high vapor pressure well below this, safety considerations also forced a compromise. This technique can be used at higher temperatures if one substitutes for mercury other molten metals such as Wood's metal or tin.

\subsection{Ultrasonic Propagation}

Mason's volumes on physical acoustics [29] describe ultrasonic measurements very well. Therefore, I will only describe a new technique which has not been included yet in this series. It was developed by Litovitz [30], Tauke [31], Simmons [32], and myself. Prior to this work high-temperature ultrasonic measurements were made in such a way that the sound was generated at room temperature and transmitted to the hightemperature section of the oven through fused silica or polycrystalline alumina rods. The difficulties of this technique for measurements in corrosive liquids such as the molten oxides have been clearly outlined by Bockris and Kojonen [33]. Two main problems appear: firstly, the temperature range desired is above the softening point of fused silica $\left(1100^{\circ} \mathrm{C}\right)$ and secondly, both fused silica and alumina are soluble in the molten oxides. To overcome this difficulty Bockris and Kojonen glued molybdenum crucibles on the ends of alumina rods. This technique has two disadvantages: firstly, there is a large acoustic loss at the junction of the crucible and rod, and secondly, polycrystalline alumina exhibits large grain boundary loss at high temperatures. The ultrasonic attenuation in the bond was so high in this system that the top alumina rod had to be directly immersed in the liquid. The resultant rapid corrosion caused considerable experimental difficulty.

To avoid these difficulties we [30] used molybdenum acoustic transmission lines. This metal is superior to alumina for several reasons. It is not corroded by molten oxides and melts at a much higher temperature $\left(2700^{\circ} \mathrm{C}\right)$. It withstands high thermal gradients and is mechanically strong.

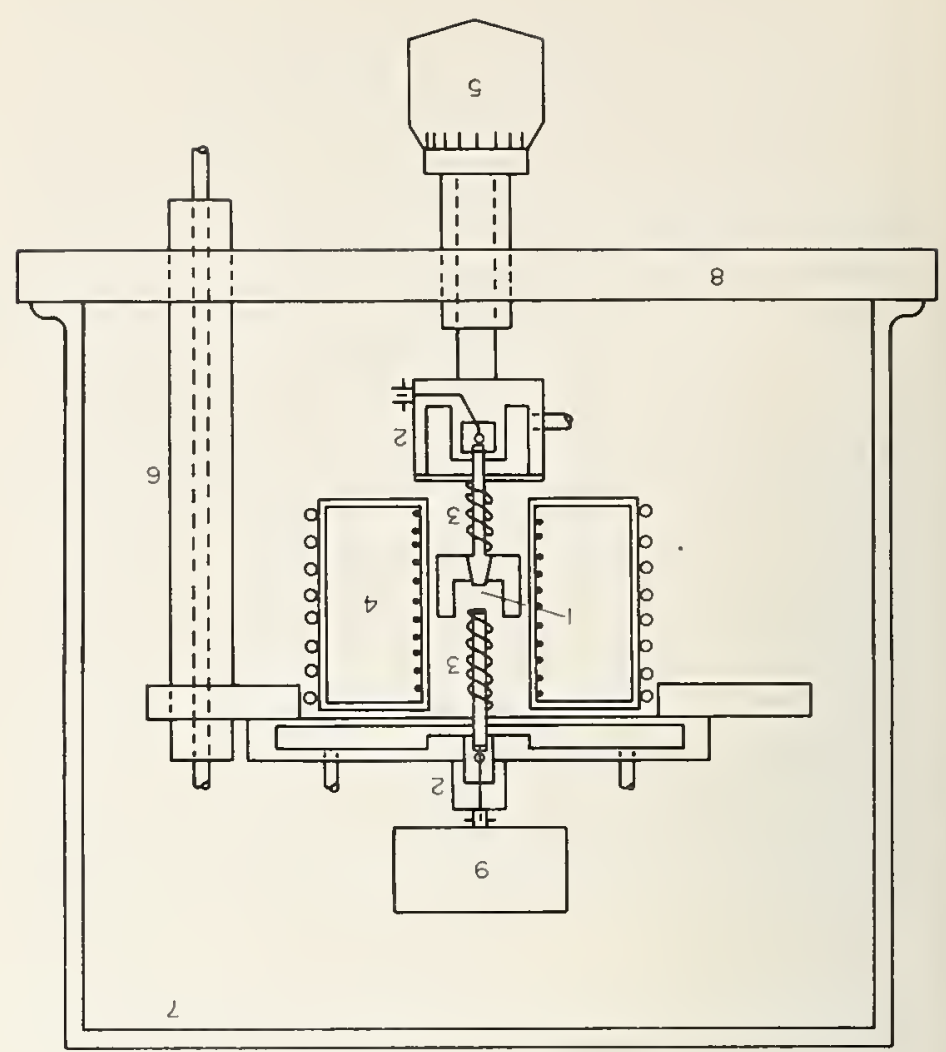

FIGURE 4. High-temperature interferometer.

1, melt sample; 2 , water cooled shear transducer holders; 3 , single crystal molybdenum rods oriented with their axes along (110) and $1.27 \mathrm{~cm}$ in diameter by $20 \mathrm{~cm}$ in length; 4, furnace wound with molybdenum wires; 5 , micrometer screw; 6 , water cooled alignment columns; 7 , metal belljar used as both a shield against stray pickup of electrical signals and a chamber for a controlled atmosphere of $95 \% \mathrm{~N}_{2}$ and $5 \% \mathrm{H}_{2}$ gas; 8 , base plate and support; 9 , tuned cathode follower (from ref. [32]).

An obvious disadvantage of molybdenum is its high thermal conductivity. This was surprisingly easy to overcome. The heating element was taken from the walls of the oven and put right on the rods. Polycrystalline molybdenum has a higher value of ultrasonic absorption than quartz owing to scattering from crystalline boundaries. To diminish this loss, arc cast molybdenum was used because of its smaller crystal size with upper frequency limit of $15 \mathrm{MHz}$. Single crystals of molybdenum are readily available and permit measurements up to $85 \mathrm{MHz}$.

The alumina rods used by Bockris and Kojonen have a disadvantage compared with either polycrystalline or single crystals of molybdenum in that they have a significantly higher absorption at temperatures about $1100^{\circ} \mathrm{C}$ due to grain boundary losses. This temperature-dependent increased loss is not observed in molybdenum.

This interferometer shown in figure 4 can be used for both shear and longitudinal ultrasonic measurements in the megahertz range ( 1 to 85 $\mathrm{MHz}$ ). All that is required to change from one measurement to another is to replace the quartz transducers, which convert electromagnetic energy to accoustical energy and back to electromagnetic. 


\section{a. Electronic Apparatus}

Tauke, Litovitz, and Macedo [31], using the standard pulse echo technique (described below) with a 50-dB dynamic range (difference between maximum signal and noise), were able to make shear measurements through a melt of $\mathrm{B}_{2} \mathrm{O}_{3}$, only in cases of absorption per cycle less than $2 \mathrm{~Np} / \mathrm{cycle}$. This restricted their measurements to the region $\omega \tau>1$ ( $\tau$ is relaxation time), so that they could obtain only half of the relaxation curve. In order to make shear transmission measurements over a larger range of $\omega \tau$, other methods were investigated by Simmons and Macedo [32].

The use of a lock-in amplifier was considered to reduce noise. Normally such an amplifier is used with "continuous wave" operation and reduces the frequency bandpass of the receiver to less than $1 \mathrm{~Hz}$ with much lower noise levels. Unfortunately the dynamic range gained at the receiver by the "continuous wave" operation, in the case of the high-temperature interferometer, is lost at the transmitter. Due to the continuuus excitation of the crystal, the peak power must be lowered to keep the average power level constant and avoid damaging the crystal and bond. In addition crosstalk, which is the receiving of transmitted signals by electrical pickup rather than ultrasonic transmission, cannot be discriminated against in the "continuous wave" application of the lock-in amplifier.

In pulse operation however, crosstalk is separated by time discrimination since such signals travel with the speed of light and arrive at the receiver promptly, while the ultrasonic signal is delayed. Simmons and Macedo [32] showed how one could advantageously combine the pulse technique and the lock-in amplifier technique, while still using a commercial amplifier. This is done by using the triggering rate of the transmitter in pulse operation as the reference frequency for the lock-in amplifier.

(i) Standard Pulse Operation. The standard equipment for pulse-echo measurements in wide use today is shown in figure 5. The transmitter generates a radiofrequency pulse of the order of $1 \mathrm{~kW}$ peak power which is applied to the lower transducer through a matching network. The signal after passing through both rods and the liquid is received by the upper transducer, amplified using a tuned cathode follower at the crystal holder, and then fed into a narrow band preamplifier. It is added to the comparison signal and then fed into the wide-band amplifier. The amplifier has a wide enough band to allow the use of the comparison signal generator at its lowest frequency (10 $\mathrm{MHz}$ ) to measure signals of much lower frequencies without distortion. This was checked using a step attenuator. The video output from the amplifier is displayed on an oscilloscope.

(ii) Pulsed Phase-Lock Detection. Figure 6 shows the circuit diagram of Simmons and Macedo [32]

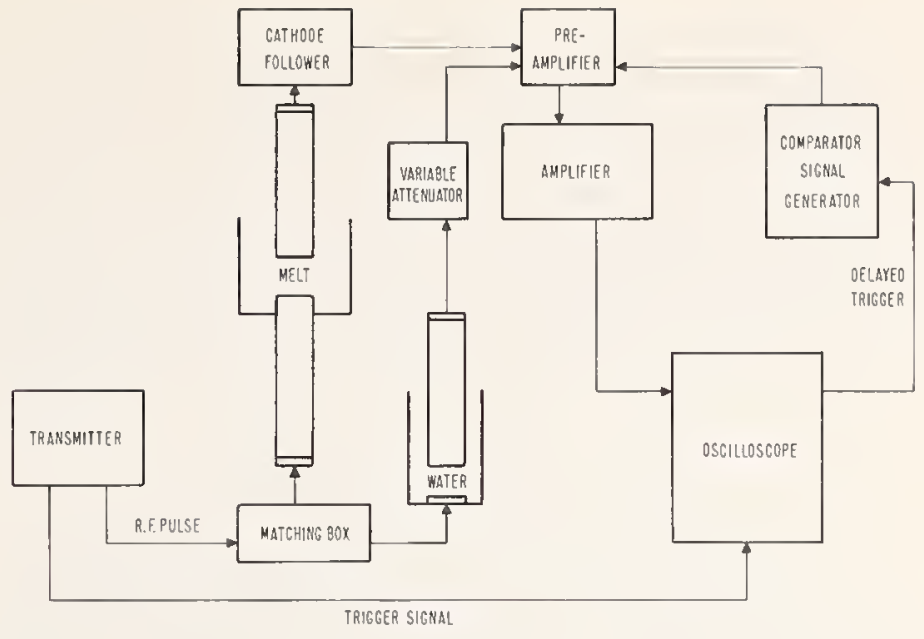

Figure 5. Standard equipment for pulse-echo measurements showing both the water and high temperature interferometers (from ref. [32]).

for the operation of the pulsed ultrasonic system with the lock-in amplifier. 'The details of the ultrasonic system are shown in figure 5. It should be noted in examining figure 6 that since the lockin amplifier detection system is used on the video signal from the first amplifier, it can be added to any ultrasonic apparatus in which the rf signal is rectified.

The triggering system provides a series of pulses which alternately trigger the ultrasonic system and the comparator pulse. These rf pulses are combined, amplified, and rectified in the receiver. Five hundred times a second the following train of video pulses leaves the receiver: the first pulse is due to the electronic crosstalk (it carries no ultrasonic information); the second pulse represents the straight through transition of sound energy, which has the desired ultrasonic information; subsequently a series of echoes arrive, which for this experiment are not interesting. After the

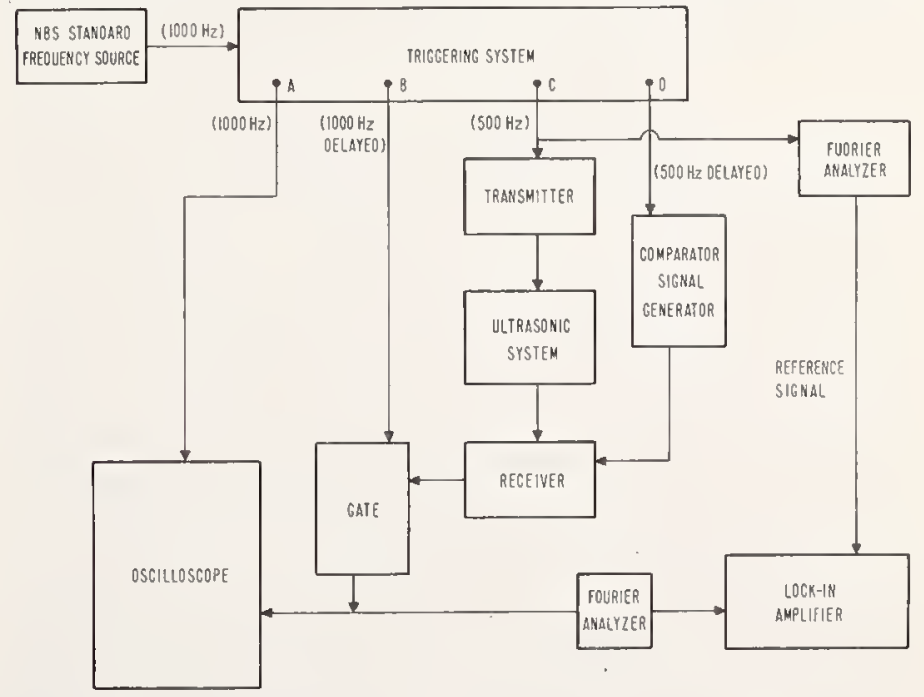

Figure 6. Modifications of the pulsed ultrasonic equipment necessary for use of the phase-lock detection system.

For simplicity, the section of figure 5 between the transmitter and amplifier, which remains unchanged, has been included into one "ultrasonic system." (from ref. [32]). 
echoes have died out (have been attenuated in time till their amplitude is much less than the noise of the system) approximately $1 \mathrm{~ms}$ later the comparison pulse arrives. The gate width and trigger are chosen to allow transmission of only the maximum amplitude sections of the echo of interest and the comparator pulse. In this manner the information fed into the Fourier analyzer and the lock-in amplifier is made up of two nearly square pulses. The Fourier analyzer passes only the $500 \mathrm{~Hz}$ component of these pulses. This not only cancels out the gate pedestal which has a $1000 \mathrm{~Hz}$ repetition rate, but also subtracts the comparison signal from the echo since they are out of phase. The output $C$ at $500 \mathrm{~Hz}$ is used as comparison signal for the lock-in amplifier, which therefore analyzes the echo pulse as an in-phase signal and the comparison pulse as an out-of-phase signal, thus changing phase by $180^{\circ}$ according to which signal is largest. Intensity measurements are made by matching both signals and obtaining a null.

The lock-in amplifier uses its narrow band pass with an integration network and produces an enhancement in signal to noise ratio of around 25 $\mathrm{dB}$ over the video display of the pulse. The null detector feature of this system permits comparison with a high degree of sensitivity (less than $0.1 \mathrm{~dB}$ ) provided the original signal is 10 to $15 \mathrm{~dB}$ above noise.

\section{b. Measurement Technique}

Methods used for measuring absorption and velocity of ultrasonic waves in liquids are discussed below using the apparatus described in the preceding sections. I am summarizing previously used methods [30,31] as described by Simmons et al. [32].

i. Absorption. The absorption measurements are made by stepwise decreasing the path length in the melt with a micrometer screw and recording the amplitude of the comparison pulse necessary to produce a null at the lock-in amplifier for each step.

ii. Velocity. Depending on the magnitude of the absorption coefficient and the viscosity of the melt, various techniques can be used to measure the ultrasonic velocity in the sample. For low absorption and viscosity below $2 \times 10^{5}$ poise, the signal coming through the high-temperature ultrasonic system is beaten against a pulse coming through a water interferometer at room temperature. The position of the rods in the water remains fixed. As the high-temperature path length is changed, a series of maxima and minima can be observed on the oscilloscope. The slope of a plot of minima versus rod separation in the melt is the ultrasonic wavelength, $\lambda$, in the sample. Knowing the frequency $f$, the velocity, $V=\lambda f$, can be calculated. As the absorption increases, this measurement becomes progressively more difficult as fewer and fewer minima are measured, and the accuracy consequently goes down.
The technique used in the high attenuation regions is similar to the preceding one, in that two ultrasonic systems are used in parallel, one with water, the other with the sample at high temperatures. The smallest detectable signal is found by closing the gap between the two rods in the high-temperature path. Then an attenuator in the water path must be adjusted so that the intensities of both signals match each other. By adjusting the path length in the water, the signals can then be superimposed, a minimum attained by phase cancellation; and the respective positions of the interferometers recorded. The melt gap is then decreased by steps of $0.0 \mathrm{C} 5$ or $0.010 \mathrm{~cm}$, depending on the available range of travel, and the gap in the water is reduced until a minimum is again observed. After the positions of the interferometers are again recorded the above procedure is repeated (see fig. 7). To obtain good phase cancellation one must adjust the attenuator in the water line until both signals have approximately the same strength. Whenever this is not possible (i.e., large melt signal) the comparison pulse must be adjusted to cancel out the excess signal and emphasize the minimum at the lock-in. Since the velocity of sound in water is known, the velocity in the sample can be calculated by the relation

$$
V_{s}=\frac{X_{s}}{X_{H_{2} \mathrm{O}}} V_{\mathrm{H}_{2} \mathrm{O}}
$$

where $V_{s}$ is the velocity in the sample, $V_{\mathrm{H}_{2} \mathrm{O}}$ the velocity in water, and $X_{\mathrm{H}_{2} \mathrm{O}}$ the separation in water corresponding to $X_{s}$ in the melt.

For viscosities above $2 \times 10^{5}$ poise (temperatures below $840^{\circ} \mathrm{C}$ in the alkali borosilicate glass tested) it becomes difficult to move the two rods with

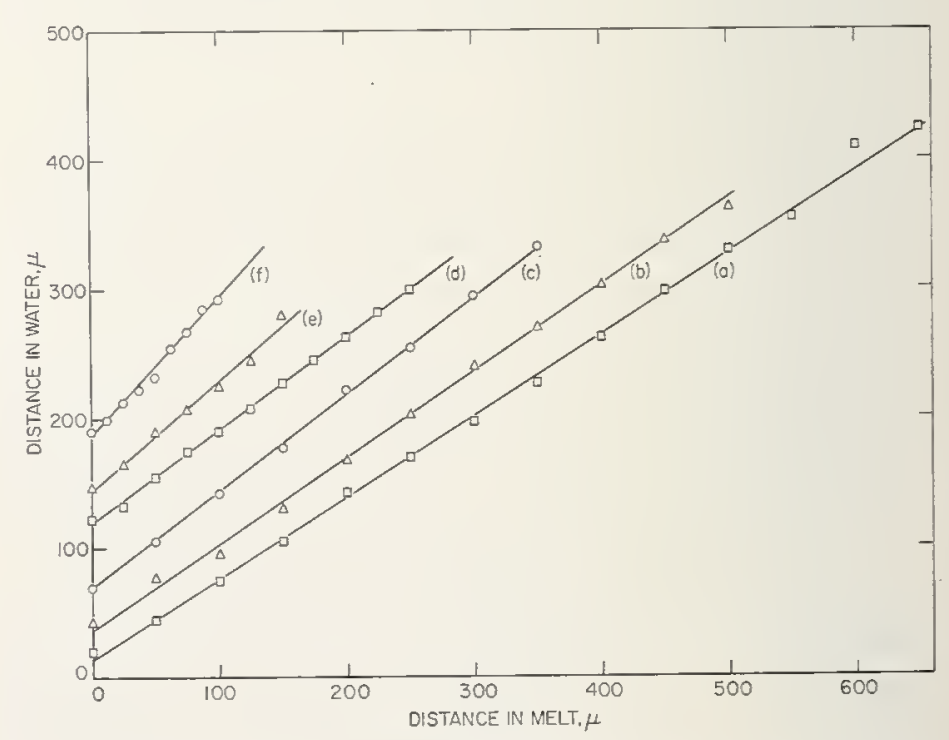

FIGURE 7. Velocity measurements at $15 \mathrm{MHz}$ in alkali borosilicate glass over the temperature range $100 \mathrm{~s}^{\circ} \mathrm{C}$ to $1264^{\circ} \mathrm{C}$ and having absorption per wavelength between $1.38 \mathrm{~Np} /$ cycle and $4.87 \mathrm{~Np} /$ cycle from ref. [32]. 
respect to each other without noticeable slippage in the micrometer screw driving linkage. In this case, Simmons et al. [32] devised a new technique for measuring the sound velocity. The temperature induced change in transit time of the shear wave through the two transmission lines separated by a fixed distance in liquid is first measured. The change in transit time in the liquid alone is then obtained by subtracting the temperature-induced change in transit time of the wave through the transmission lines with the two rods touching. Since the measurements are made for a fixed distance of travel in the liquid, the temperatureinduced change in transit time can be related to a temperature dependence of the velocity.

This technique was used on the molten sodaborosilicate glass below $830^{\circ} \mathrm{C}$. The first echo of the transmitted wave in the sample was beaten against the water echo, but in this case the travel distance in the water (whose temperature was kept constant) was adjusted to remain out of phase with the sample echo while the sample temperature was varied and the sample distance remained fixed. Thus, one could keep track of the temperature-induced changes in transit time by recording the changes in the travel distance in water necessary to keep the two echoes out of phase. Plots of the temperature dependence of the travel through the water are shown in figure 8 [32]. Curve (a) represents the distance traveled in water versus temperature when the two molybdenum rods are touching. Curve (b) is the same plot except that the rods are separated by 0.254 $\mathrm{cm}$ of melt, and the added slope represents the temperature dependence of the sound velocity in the sample. The water temperature was kept

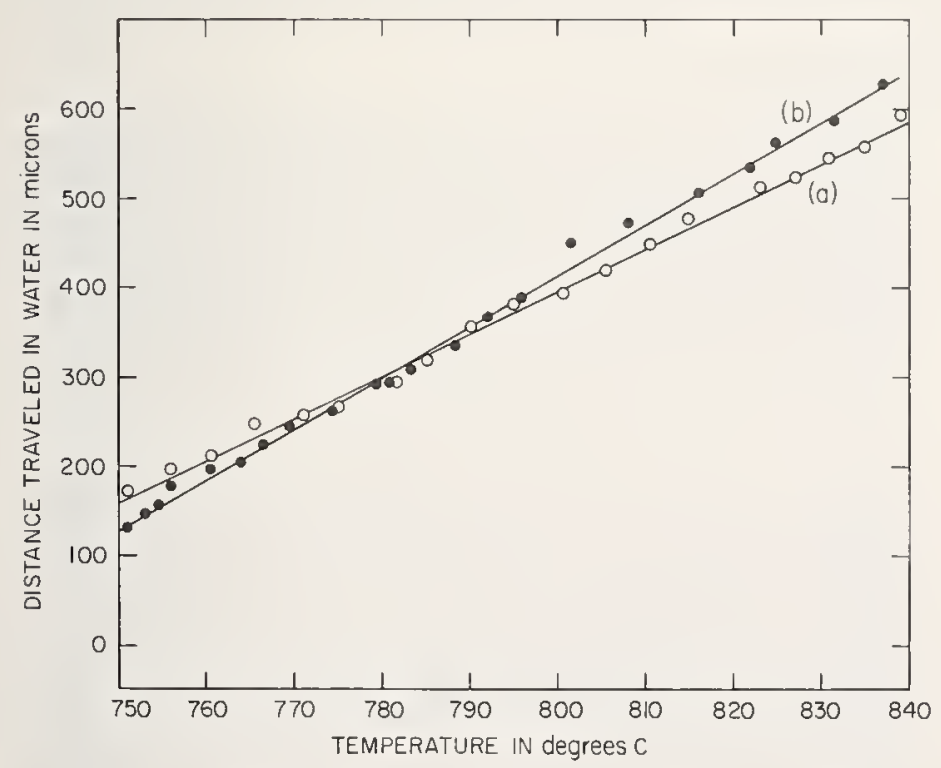

FIGURE 8. Temperature dependence of distance traveled in water necessary to keep the water echo out of phase with the sample echo in the measurements of velocity at viscosities above $2 \times 10^{5}$ poise.

Curve (a) is the temperature dependence corresponding to the transmission lines only, and curve (b) is the dependence corresponding to the transmission lines and the melt from ref. [32]. constant; thus the change in transit time could be directly related to a change in travel distance in the water through the known sound velocity in water. The shear velocity outside the relaxation region (temperatures lower than $830^{\circ} \mathrm{C}$ in this liquid sample) was then calculated by adding the changes in velocity to a directly measured value at a higher temperature. This was done using the following equation:

$$
V(T)=V_{0}\left[1+\frac{A V_{0}}{X_{\mathrm{m}} V_{\mathrm{H}_{2} \mathrm{O}}}\left(T_{0}-T\right)\right]
$$

where $V_{0}$, is the velocity of the melt at $T_{0}, X_{\mathrm{m}}$ is the fixed distance of travel in the melt, $V_{\mathrm{H}_{2} \mathrm{O}}$ the velocity in water, and $A$ the difference between the slopes of curves (b) and (a) in figure 7.

\section{Viscoelastic Considerations}

The fundamental question in viscoelastic behavior is: What and where is the glass transition? In figure 9 from ref. [17], the increase in length is plotted versus temperature for a $\mathrm{GeO}_{2}$ glass sample. In this experiment the temperature was raised at $2^{\circ} \mathrm{C} / \mathrm{min}$. The region where the expansion coefficient increases drastically is known as the transformation range. Above this range one has a liquid in metastable thermal equilibrium. By metastable it is meant that the state of lowest free energy is the crystalline, but this transformation requires nucleation and as long as there are no nuclei present the liquid is in thermal equilibrium. The larger expansion coefficient above $\mathrm{Tg}$ is characteristic of the liquid state and is due to the change in order as one changes temperature. This order has been characterized by various authors as the "number of holes," or "free volume," or "excess entropy," or "number of broken bonds," etc.

In a glass the degree of order does not change as one raises or lowers the temperature; thus all the second-order thermodynamic parameters (thermal expansion, $\beta=(1 / V)(\partial V / \partial T)_{p}$; specific heat $C p=(\partial H / \partial T)_{p}$; compressibility $\kappa_{s}=-(1 / V)$ $\left.(\partial V / \partial P)_{s}\right)$ have a value lower than the corresponding parameters in the liquid. This lack in change of the degree of order makes the glass in a thermodynamic non-equilibrium or unstable situation. If one observes a glass for long periods of time one would expect to see the degree of order change as it tries to approach equilibrium. This process is known as relaxation and measurements of its time dependence are commonly called annealing. In order to mathematically describe the annealing process, one has to study the time dependence of the approach to equilibrium as a function of both temperature and one or more order parameters. This work can be separated into two parts: firstly, the study of the time dependence of the structural rearrangements, which will be characterized in terms of a spectrum of relaxation 


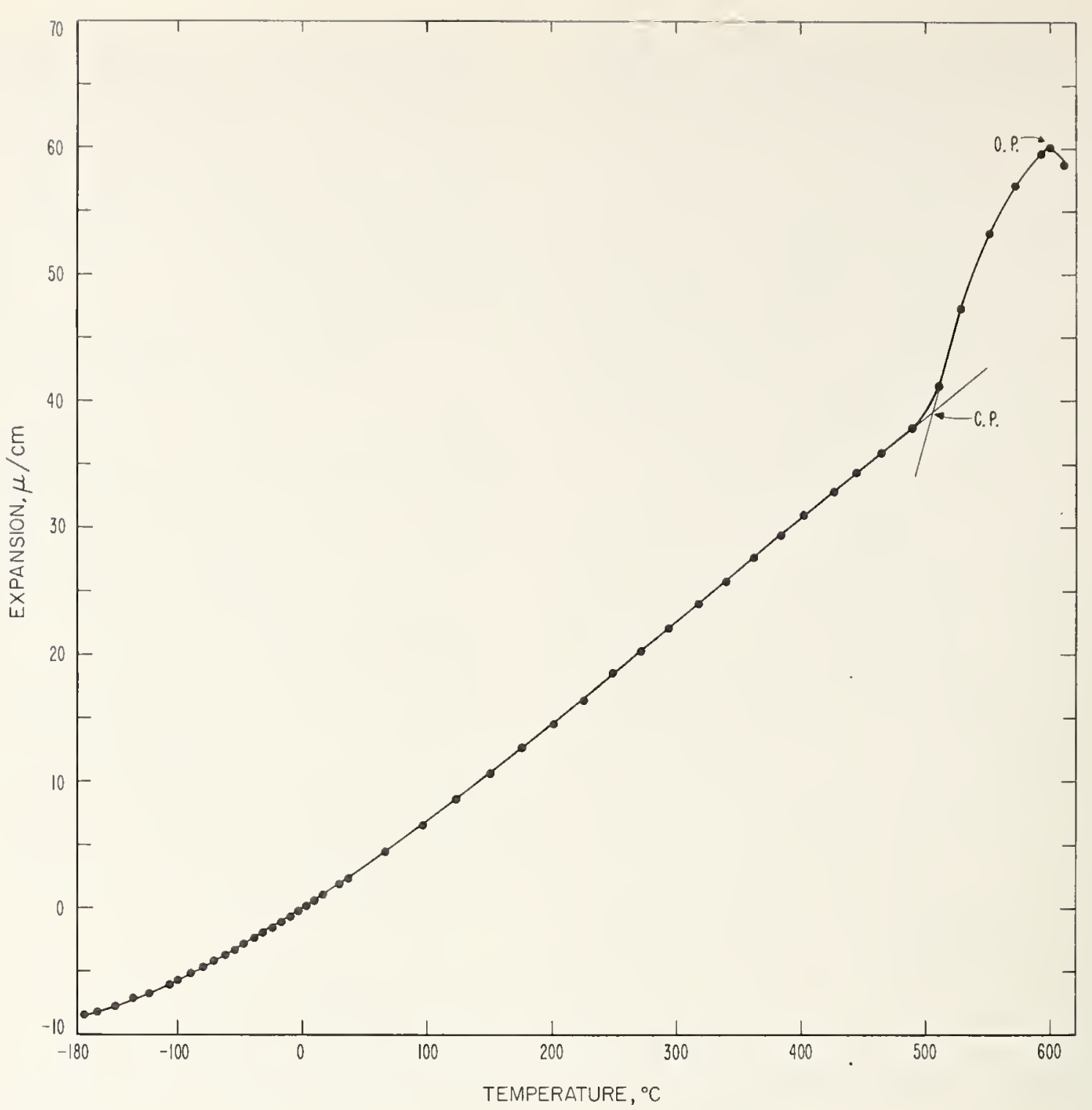

FIGURE 9. Thermal expansion of vitreous $\mathrm{GeO}_{2}$ showing the critical point and the deformation point.

Figure taken from $\mathrm{N} \& \mathrm{M}$, ref. [17].

times; secondly, the temperature and structure dependence of this spectrum.

\subsection{Measurement of the Spectrum of Relaxation Times}

Let us define as linear an experiment where the time dependence of the normalized stress or strain relaxation is independent of the initial stress or strain. Of course, a nonlinear experiment is one which is not linear. One of the major problems is to design an annealing experiment which is linear. Ultrasonics provides probably the best solution to this problem. Sound waves can be generated and detected (see sec. 2) with stresses and strains which may be treated as infinitesimal. From the sound absorption, $\alpha$, and velocity, $V$, the real and imaginary parts of the complex modulus can be calculated according to

$$
\begin{aligned}
m^{*}=m^{\prime}+i m^{\prime \prime}= & \frac{\rho V^{2}\left(1-(\alpha V / \omega)^{2}\right)}{\left(1+(\alpha V / \omega)^{2}\right)^{2}} \\
& +i \frac{2 \rho V^{2}(\alpha V / \omega)}{\left(1+(\alpha V / \omega)^{2}\right)^{2}}
\end{aligned}
$$

where $m^{*}$ can either be the complex shear modulus $G^{*}$, or the complex longitudinal modulus $M^{*}$, depending whether the shear or longitudinal sound absorption and velocity are used. The longitudinal modulus can be calculated from $G^{*}$ and the complex compression modulus, $K^{*}$, according to

$$
M^{*}=K^{*}+4 / 3 G^{*} .
$$

Figure 10(a) shows a plot of the real and imaginary components of the longitudinal modulus, $M^{*}$, versus frequency. At low frequencies where the structure of the liquid has plenty of time to rearrange itself between successive crests of the sound wave, $M^{*}$ is real and frequency independent and represents the modulus of compression of the liquid (the reciprocal of the liquid compressibility). As the sound period becomes shorter and shorter the structure cannot keep up with the cycling pressure, and the liquid becomes progressively less and less compressible. Finally, at high frequencies the liquid exhibits glasslike moduli, $M_{\infty}\left(M_{\infty}=K_{\infty}\right.$ $+4 / 3 G_{\infty}$ where $K_{\infty}$ is the unrelaxed modulus of 

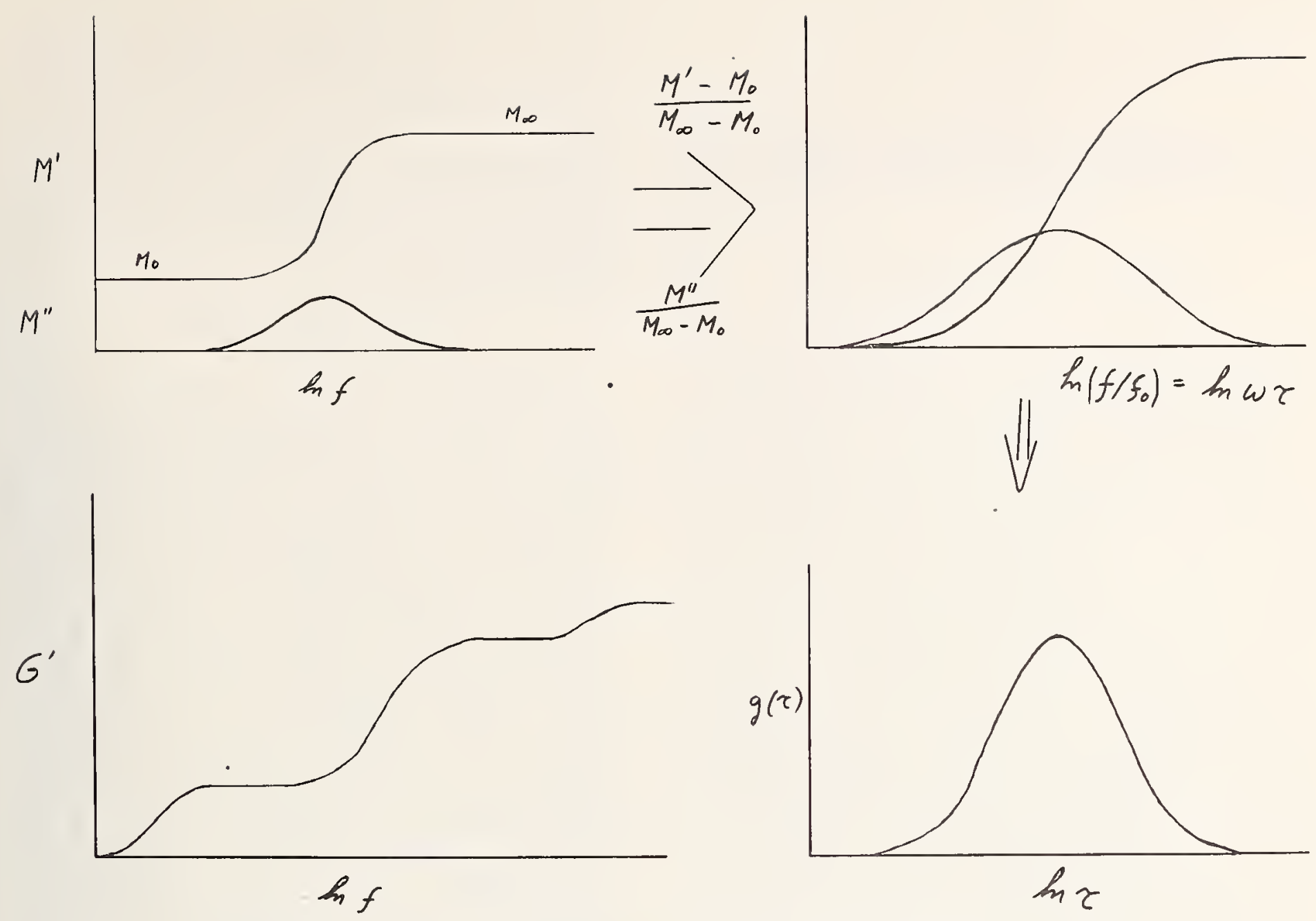

Figure 10. (a) Plot of real and imaginary parts of modulus versus log frequency.

(b) Reduced plot.

(c) Plot of shear modulus versus log frequency.

This plot is exaggerated to show all three relaxation mechanisms:

(1) entropy or polymer like relaxation not observed in molten oxides; (2) pseudo glass transition; (3) delay elastic effects; in silicate and borate melts the relative strengths are $0 \% ; 90 \% ; \sim 10 \%$ respectively.

(d) Spectrum of relaxation times.

compression and $G_{\infty}$ is the shear modulus). By definition liquids do not exhibit equilibrium shear rigidity; thus the high-frequency behavior is definitely a solidlike property. Actually, it is the glass modulus that one is observing, and by changing the frequency one can go through the glass transition. Associated with the dispersion of the real part of the modulus there is an absorption of the sound wave which is related to the imaginary part of the modulus. Just as the low-frequency real part of the modulus was the liquid modulus of compression (reciprocal of the compressibility), the low-frequency imaginary part of the modulus is given by $\omega \eta_{L}$, where $\omega$ is the angular frequency and $\eta_{L}$ is the longitudinal viscosity. In a liquid there are two elementary and independent stressstrain processes, one associated with shear and the other with compression. The shear has the wellknown shear viscosity, $\eta_{s}$ the measurement of which is described in section 2 , while the compression exhibits an analogous volume viscosity, $\eta_{v}$. The longitudinal viscosity represents the following sum of these viscosities:

$$
\eta_{L}=\eta_{0}+4 / 3 \eta_{s^{*}}
$$

By measuring the propagation of both shear and longitudinal sound waves, one can subtract $G^{*}$ from $M^{*}$ and obtain the relaxation curves for pure compression. By calculating the reduced modulus for compression

$$
m^{\prime}=\frac{K^{\prime}-K_{0}}{K_{\infty}-K_{0}} ; m^{\prime \prime}=\frac{K^{\prime \prime}}{K_{\infty}-K_{0}}
$$

or for shear $\quad M^{\prime}=G^{\prime} / G_{\infty} ; M^{\prime \prime}=G^{\prime \prime} / G_{\infty}$

at each frequency one can compare the experimental curve with theoretical results. For a single relaxation time, $\tau$, one has

$$
m^{\prime}+i m^{\prime \prime}=\frac{(\omega \tau)^{2}}{1+(\omega \tau)^{2}}+i \frac{\omega \tau}{1+(\omega \tau)^{2}} .
$$

In general, the data do not fit, and one has to assume a distribution of relaxation times $g(\tau)$. Thus, one has integrals of the form

$$
m^{\prime}+i m^{\prime \prime}=\int_{0}^{\infty}\left[\frac{(\omega \tau)^{2}}{1+(\omega \tau)^{2}}+i \frac{\omega \tau}{1+(\omega \tau)^{2}}\right] g(\tau) d \tau
$$


where for molten oxides $g(\tau)$ is taken to be

$$
g(\tau) d \tau=(b / \sqrt{\pi}) \exp \left[-b^{2} \ln ^{2}\left(\tau / \tau^{\prime}\right)\right] d \ln \tau
$$

where $\tau^{\prime}$ is the most probable $\tau$, and $b$ is the width. Schematically $g(\tau)$ is given in figure $10(d)$. From eqs (21) and (22) one obtains a very useful expression

$$
\eta_{s}=\lim _{\omega \tau \rightarrow 0}\left(\frac{G^{\prime \prime}}{\omega}\right)=G_{\infty} \bar{\tau}=G_{\infty} \int_{0}^{\infty} \tau g(\tau) d \tau
$$

where $\bar{\tau}$ is the average shear relaxation time. This is the reason why $\eta_{s}$ is used to define the annealing range (annealing point and strain point).

Working with glass one needs to specify the time-temperature relationship for three basic processes (deformation, annealing, and strain points). The upper limit of the annealing range is determined by the deformation point, where within minutes the glass will sag under gravity. This process is directly related to the viscosity itself and can be well characterized by viscosity measurements. Next, one is interested in the glass transition itself, where the volume relaxation time becomes comparable with the effective experimental times. A priori the volume relaxation time has nothing to do with the shear viscosity, and a measurement of shear viscosity would be useless to characterize this process. Fortunately, there are some relationships which we found to hold for silicate glasses between the shear and volume relaxation times. The average volume relaxation time at constant pressure and temperature is about six times larger than the average shear relaxation time. Also the shear modulus of rigidity $G_{\infty}$ is about $2 \times 10^{11}$ dynes $/ \mathrm{cm}^{2}$. Thus, using eq (23) for a viscosity of $10^{13}$ poise one calculates the average shear relaxation time to be $\sim 50$ sec and the average volume relaxation time $\sim 5 \mathrm{~min}$. If one measures the expansion coefficient of a glass by rate heating at $2^{\circ} \mathrm{C} / \mathrm{min}$, the first kink (critical point in fig. 9) corresponds to a temperature having an average volume relaxation time of a few minutes. For silicate glasses the sample has $\sim 10^{13}$ poise at the critical point, but this is not a viscosity measurement; and for the same volume relaxation time the shear viscosity varies from $10^{14}$ poise for $\mathrm{GeO}_{2}$ to $10^{12}$ poise for $\mathrm{B}_{2} \mathrm{O}_{3}$. A second and sometimes worse error is the use of the average relaxation times rather than the spectrum, since the very small relaxation times often play the predominant role in annealing. This introduces at least a factor of 10 error in estimates of annealing times.

To better understand the effects of a spectrum of volume relaxation times let us examine figure 11 [17]. In the relaxation curves shown, the structure changes enough to affect the relaxation time spectrum during an approach curve (nonlinear effect). The existence of both nonlinear effects and a distribution of relaxation times make the calculation of the relaxation time spectrum very difficult, if not impossible. In order to cir- cumvent this problem the crossover technique $[34,35]$ was developed. The thermal history involved in the crossover can best be understood if one first understands the mathematical model used to represent the spectrum. The analysis is simplified by assuming that the spectrum is given by two relaxation mechanisms of equal strength, each having a single relaxation time. Thus the average index $N$ is given as a function of time by the following relation

$$
N=1 / 2\left[M_{1}(t)+M_{2}(t)\right]
$$

where $M_{i}(t)=N_{\infty}+N_{i} e^{-t / \tau_{i}}$, in which $N_{\infty}$ is the equilibrium value of index; $N_{i}$, the initial deviation of index from $N_{\infty}$ associated with mechanism $i$ having relaxation times $\tau_{i}$. This formulation can be thought of as describing the relaxation (change of volume) to a final equilibrium value following a change in temperature (analogous to an isothermal recovery function) in terms of two separate mechanisms, the first of which relaxes appreciably faster than the second. $N_{1}$ and $N_{2}$ are measures of the total relaxation undergone by the two separate mechanisms in reaching equilibrium. If the starting point is one of equilibrium $\left(M_{1}(0)=M_{2}(0)\right)$, the two mechanisms will have to relax the same total amount $N_{1}=$ $N_{2}=\left(N_{\infty}\left(T_{1}\right)-N_{\infty}\left(T_{2}\right)\right)$. However, when starting from a nonequilibrium approach curve, $N_{1}$ and $\mathrm{N}_{2}$ will depend on the particular approach curve being followed. Figure 12 due to Macedo and Napolitano [34] shows schematically the time dependence of $N, M_{1}$, and $M_{2}$ at $T_{2}$ for a glass initially at equilibrium at $T_{1}$, where $T_{1}>T_{2}$. As the structure rearranges itself to the new equilibrium, $M_{1}$ will rise faster since it is associated with the shorter relaxation time $\tau_{1}$; while $M_{2}$ will change more slowly. Thus, around the bend of the approach curve, there will be the largest difference between $M_{1}$ and $M_{2}$ in the glass. As time progresses, $M_{1}$ will reach its equilibrium value for $T_{2}$ first $\left(\tau_{2}>\tau_{1}\right)$ and stay there. From then on the spread between $M_{1}$ and $M_{2}$ narrows because the slow relaxation process continuously approaches the same equilibrium value at a slower rate. Finally, when the structure is completely, at the new equilibrium the spread becomes zero and one has again an equilibrium glass $\left(M_{1}=M_{2}\right)$.

The crossover experiment involves taking a glass having index, $a$ (see fig. 12) from the approach curve and introducing it into a furnace whose temperature is $T_{x} . T_{x}$ is preselected such that the equilibrium index at $T_{x}, N_{\infty}\left(T_{x}\right)$, is equal to $a$. Thus at a crossover, $a$, the fast relaxation time $\left(\tau_{1}\right)$ corresponds to an index $M_{1}$ higher than the (average) measured value, $N$, and the slow relaxation time $\left(\tau_{2}\right)$ corresponds to an index $M_{2}$ lower than $N$.

If the index at the point of crossover, $a$, is equal to the equilibrium index at the crossover temperature $T_{x}$, then $N_{2}=-N_{1}$. A prerequisite for a minimum in the crossover experiment is 


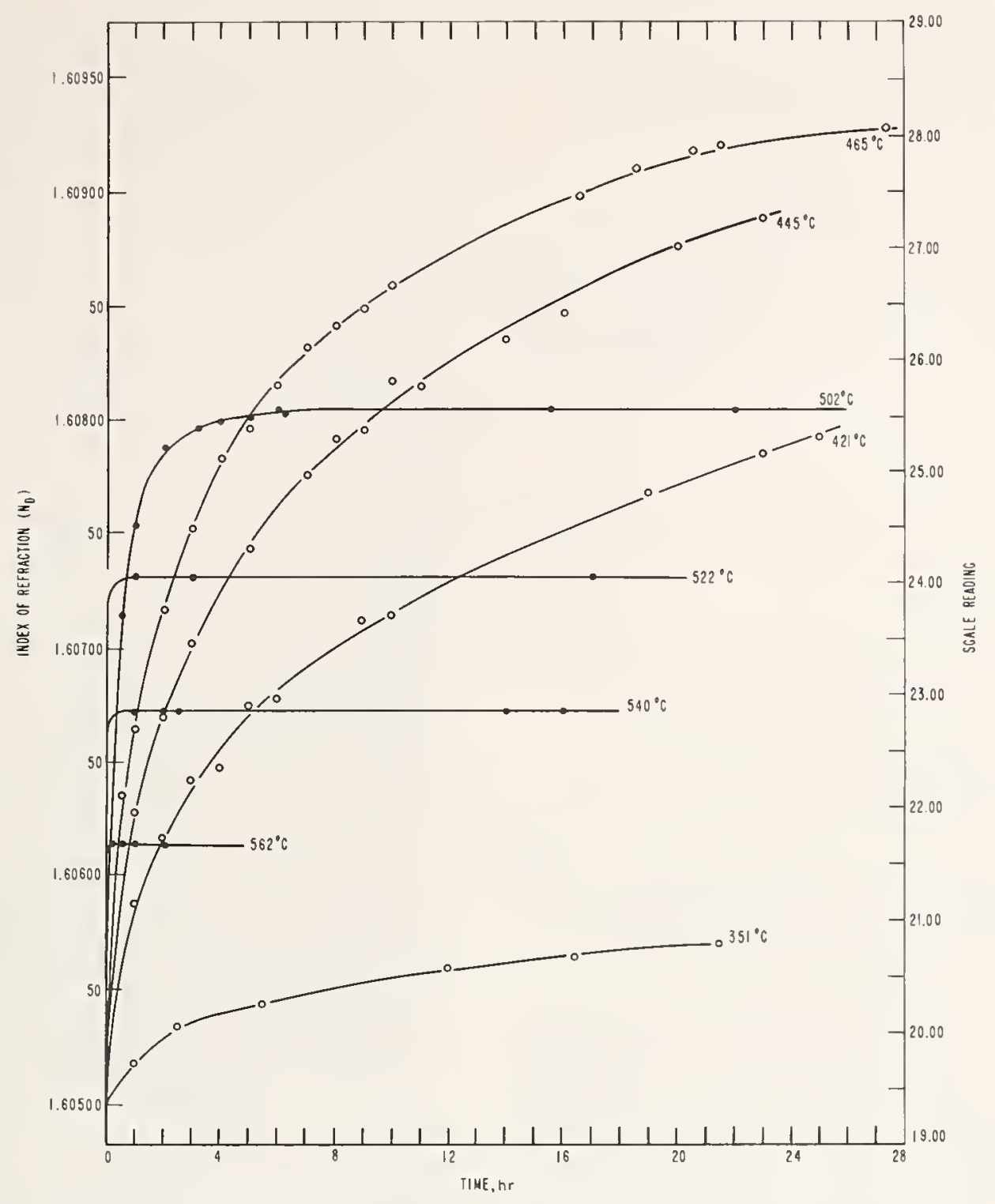

FigURE 11. Index of refraction versus time isotherms for vitreous $\mathrm{GeO}_{2}$.

Figure taken from N\&M, ref. [17].

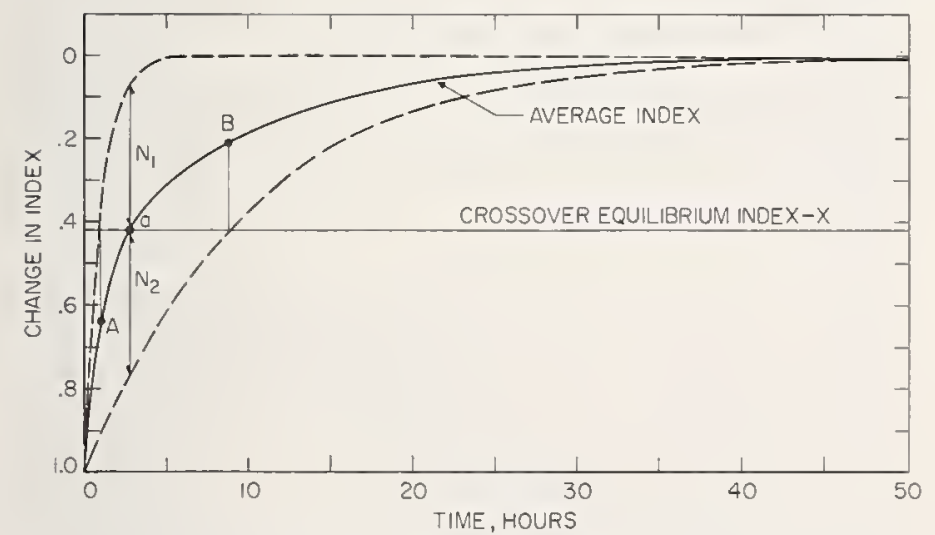

FIGURE 12. Illustration of the two relaxation time model (ref. 34) represented by the upper and lower dashed curves.

The average or measured index is given by solid line. The crossover equilibrium index (x) and the transfer point (a) are also shown.

that $N_{1}$ and $N_{2}$ have opposite signs. This is fulfilled when $N$ has a value between $A$ and $B$ in figure 12. Figure 13, from Macedo and Napolitano [34], shows $M_{1}$ (the upper curve), $M_{2}$ (the lower curve) and $\mathrm{N}$ the solid line (calculated index). The agreement of the calculated curve with the actual experimental points for a borosilicate crown glass is seen to be good.

From this discussion there are two points one should consider. First, because of the spectrum of relaxation times, two glasses with the same composition and same index of refraction or fictive temperature can have vastly different structures (compare glasses in the beginning and end of figure 13). Second, the two relaxation time model should only be considered as a mathematical convenience; the spectrum of relaxation times physically can be represented better as a ydussian. This explains the existence of a very small number of relaxation times, orders of magnitude smaller than the average relaxation times. These very fast relaxation times are responsible for the annealing observed at $351{ }^{\circ} \mathrm{C}$ in figure 11 . 


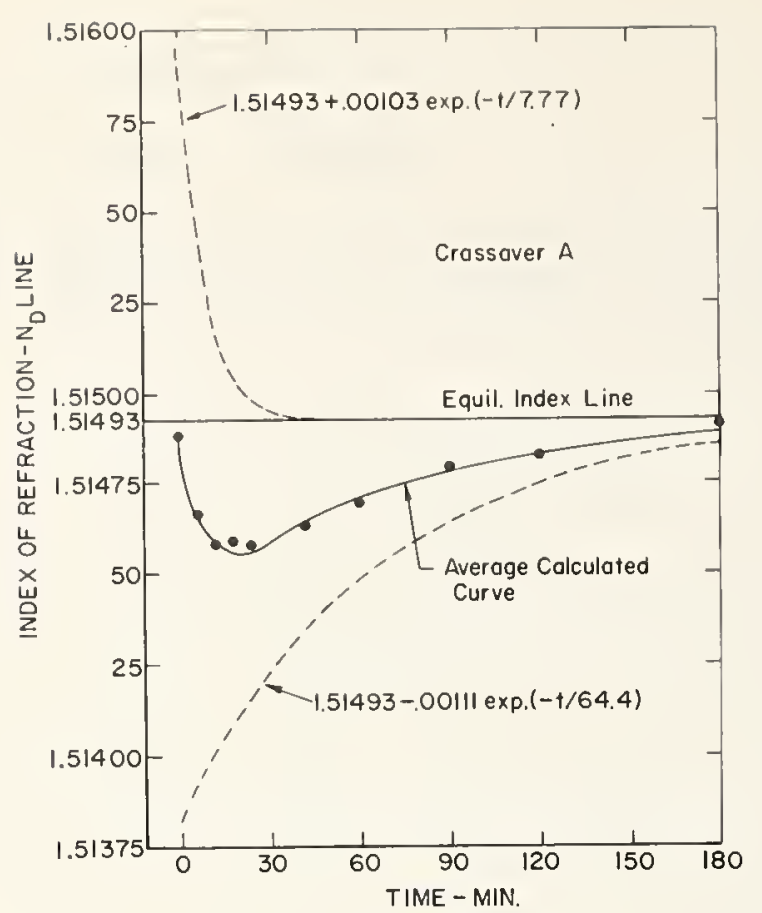

Figure 13. Crossover A for BSC glass (ref. 34 , 35), showing the average of the fast and slow relaxation processes.

\subsection{Strain Point and Viscosity in Nonequilibrium Structures}

Glasses that are cooled through the glass transformation region with a temperature gradient across the sample have frozen (thermally arrested) strains. These strains produce optical birefringence. Such glasses are brought to the "bottom" of the annealing range and there strain relaxation will eliminate birefringence. This relaxation is due to shear flow and shear viscosity controls relaxation rates. The problem is that glasses are not at thermal equilibrium, because complete volume relaxation takes too long at this temperature region. The viscosity is not a unique function of temperature, but also depends on the structure. Thus, in order to characterize strain relaxation one needs to know how to extrapolate the viscosity to lower temperature for a glass at fixed structure. This area is in a major controversy between theoretical arguments, experiments, and technical applications. The theoretical arguments will be discussed in section 4 . The problem with making a viscosity measurement as a function of volume relaxation is that viscosity can not be measured instantaneously. Upon applying a load the elongation rate is much larger than one would expect from viscous flow. This occurs because glasses are complicated materials and there are several different relaxation mechanisms acting at the same time. If the load is removed there will be a time-dependent contraction, but when the process is completed not all of the deformation will have been recovered. Those relaxation processes whose deformation can be recovered are called delayed elastic effects, while the irreversible deformations are associated with viscous flow. In fact one does not have to remove the load and measure the permanent part of the deformation, but one need only wait till all the delayed elastic effect has worked itself out and the elongation rate is no longer a function of time. Zijlstra [36] made measurements of elongation rate versus time after temperature jumps and erroneously associated all the elongation rate with a viscous process. From these measurements he concluded that the viscosity of the nonequilibrium frozen structure glass was almost temperature independent (fig. 14). On the other hand, Kurkjian [37] showed that by the time that the delayed elastic effects are worked out the glass is at or almost at thermal equilibrium (volume relaxation is almost completed). Kurkjian not only questioned Zijlstra's work, but also indicated that he considered that type of measurement would be impossible to make.

Lillie [13], studying the technical problem of strain relaxation in glass, had in effect solved the theoretical problem 30 years before it was proposed. He loaded the sample at high temperature where both volume relaxation and delayed elastic eflects take a reasonable time. Then the sample was cooled at a constant rate. For awhile volume relaxation kept up with the cooling rate but as volume relaxation time became longer and longer its structure lagged the equilibrium structure. That could be seen from the viscosity-temperature curve as it changed slope. However the change in slope was only 25 percent, far less than Zijlstra's value (95 percent). This rate cooling measurement is the now Annealing-Strain Point ASTM procedure. As it will be seen in the next section, this relatively small change in slope contradicts most modern theories of viscosity. A sample run of the viscosity versus temperature at constant cooling rate is shown in figure 15 for NBS Standard glass 710 due to Hagy [15].

\subsection{Nonlinear Viscoelasticity}

All the viscoelastic theory thus far presented rested on the fact that one can linearize the equa-

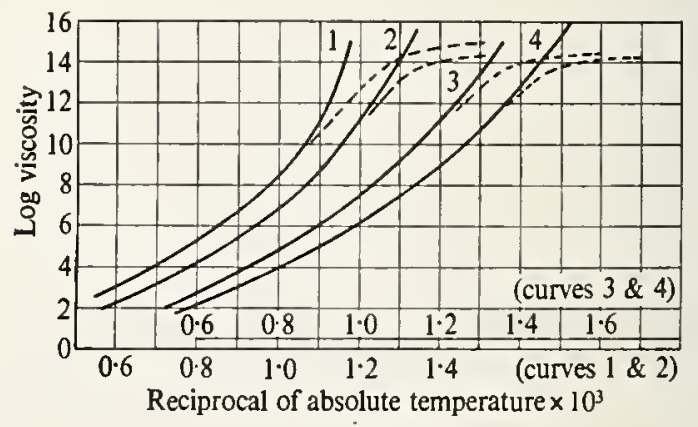

Figure 14. Viscosity-temperature curves taken from Zijlstra [36].

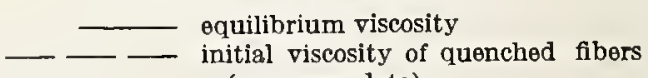
(erroneous data). 


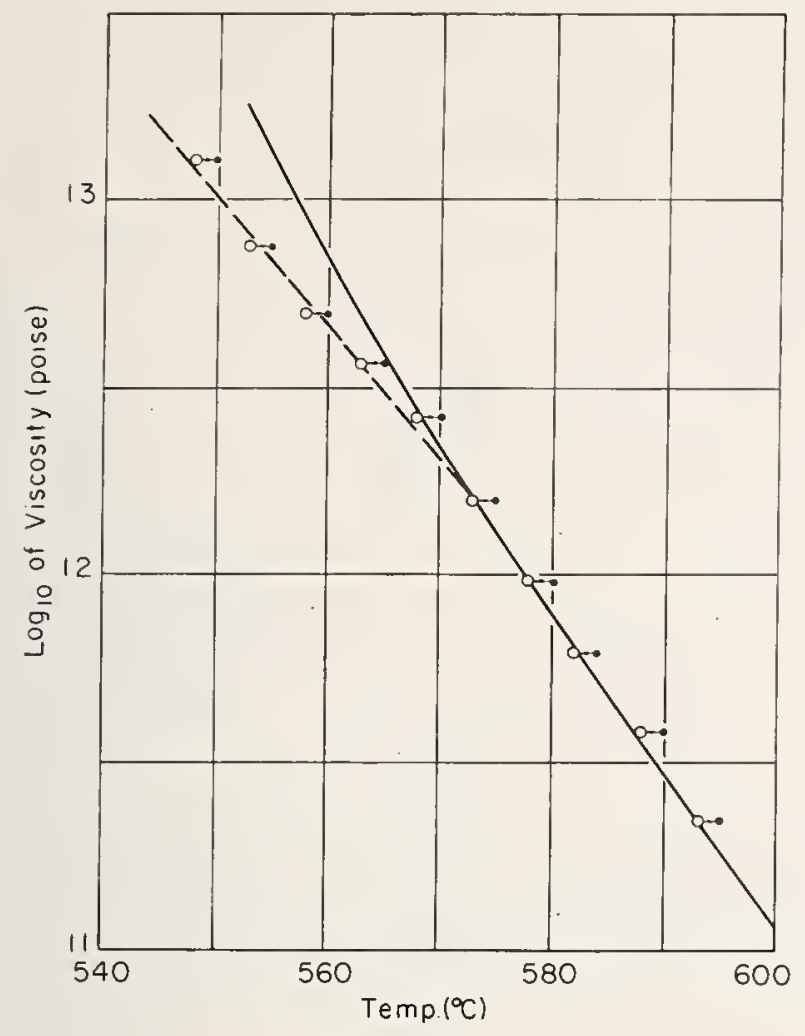

Figure 15. Data for NBS Standard Glass No. 710 from Hagy [15], sample cooled at $4{ }^{\circ} \mathrm{C}$ per minute for determination of annealing and strain points.

observed data

data corrected by $2^{\circ} \mathrm{C}$ for thermocouple log. Equilibrium viscosities for glass shown by solid line.

tions. For viscosity that means that the shear strain rate is proportional to the shear stress. Recently authors [38, 39, 40] have shown experimental proof of nonlinear behavior in the annealing region of several glasses. Since the experimental uncertainty of these measurements were comparable with the effect, the results were generally attributed to bad data and ignored. However, the effect is probably real and must be accounted for before it destroys our whole theoretical framework. Bartenev [38] states that the nonlinear effects are most pronounced when the glasses were observed to phase separate. Lack of linear behavior of two liquid-phase composites is expected, and does not contradict our theoretical structure.

This existence of microphase in melts and glasses does cause unexpected and otherwise unexplained behaviors. Whenever one is working with a glass, one should keep track of its microstructure by electron microscopy. Glasses must now be characterized not only by composition but also by detailed thermal history, since it will affect both its volume relaxation (fictive temperature) and its microstructure.

\section{Temperature Dependence of the Vis- cosity and Relaxation Time}

\subsection{Fulcher Equation}

In order to interpolate and extrapolate viscosity measurements, one would like to have an analytical expression for the temperature dependence of the viscosity. The most widely used expression, is an empirical equation proposed by Fulcher [41],

$$
\log \eta=A+B /\left(T-T_{0}\right)
$$

in which $A, B$ and $T_{0}$ are arbitrary constants. In order to fit this equation to data by the least squares method, it has to be linearized to the form

$$
T \log \eta=B-A T_{0}+A T+T_{0} \log \eta .
$$

One needs a program subroutine for least squares fitting a function of three variables (vectors)

$$
Y_{i}=C_{1} X_{1 i}+C_{2} X_{2 i}+C_{3} X_{3 i}
$$

where $Y_{i}$ are the unknowns, $X_{t i}$ are the variables, and $C_{l}$ are the desired coefficients. Note " $i$ " differentiates each set of data. Matching equations (26) and (27) one has

$$
\begin{aligned}
& Y_{i}=T_{i} \log \eta_{i} \\
& X_{1 i}=1 \\
& X_{2 i}=T_{i} \\
& X_{3 i}=\log \eta_{i} \\
& T_{0}=C_{3} \\
& B=C_{1}+C_{2} C_{3} \text {. }
\end{aligned}
$$

We have minimized the error on $T \log \eta$ which favors the high-temperature data. This is the usual procedure since the low viscosity measurements at high temperatures are more accurate than the high viscosity data at low temperatures. Because of the linearization of the Fulcher equation the standard deviations calculated for $C_{1}, C_{2}$ and $C_{3}$ are not applicable to $A, B$, and $T_{0}$. If one wants the standard deviation of the data about the Fulcher curve, $\sigma$, one can calculate it

$$
\sigma^{2}=(N-3)^{-1} \Sigma_{i}\left(\log \eta_{\exp t}-\log \eta_{\mathrm{ca} i t}\right)^{2}
$$

where $N$ is the number of data points. If, on the other hand, the uncertainties on $A, B$, and $T_{0}$ are desired, a second calculation has to be performed. This time we assume a set of parameters $\left(A^{\prime}, B^{\prime}\right.$, and $T_{0}^{\prime}$ from the above calculation) and proceed to calculate an improved set $\left(A, B\right.$, and $\left.T_{0}\right)$ by the following iterative procedure. A difference in $\log \eta$ can be caused by:

$$
\begin{aligned}
\Delta \log \eta=\sum_{i} & \frac{\partial \log \eta}{\partial X i} \Delta X_{1}=\Delta A \\
& +\left(T_{i}-T_{0}\right)^{-1} \Delta B+\frac{B^{\prime}}{\left(T_{i}-T_{0}^{\prime}\right)^{2}} \Delta T_{0} .
\end{aligned}
$$


Matching eq (29) and (27) we have

$$
\begin{aligned}
& Y_{i}=\log \eta_{i \exp }-\log \eta_{i \text { cal }}=\log \eta_{i \exp } \\
& \quad-A^{\prime}-B^{\prime}\left(T_{i}-T_{0}{ }^{\prime}\right)^{-1} \\
& X_{1 i}=1 \\
& X_{2 i}=\left(\mathrm{T}_{i}-\mathrm{T}_{0}\right)^{-1} \\
& X_{3 i}=\mathrm{B}\left(T_{i}-T_{0}\right)^{-2} .
\end{aligned}
$$

After the least squares fit one has: $\Delta A=C_{1}$; $\Delta B=C_{2} ; \Delta T_{0}=C_{3}$.

The best values then become

$$
\begin{gathered}
A=A^{\prime}+\Delta A=A^{\prime}+C_{1} \\
B=B^{\prime}+\Delta B=B^{\prime}+C_{2} \\
T_{0}=T_{0}^{\prime}+\Delta T_{0}=T_{0}^{\prime}+C_{3}
\end{gathered}
$$

and the standard deviations of $A, B$, and $T_{0}$ are those of $C_{1}, C_{2}$, and $C_{3}$ respectively. If the initial values of the parameters are taken from a least squares fit to eq (26), the $C_{i}$ are comparable to their standard deviations and only one iteration is necessary. Notice, that eq (29) minimizes the error in $\log \eta$ rather than $T \log \eta$. If the reader is interested in the latter he can multiply all the equations by $T$. The rest of this section is taken from Macedo and Napolitano [1].

The extensive measurements carried out at the National Bureau of Standards and several participating laboratories on standard glasses No. 710 [3] and 711 [42] have shown that the Fulcher equation does indeed fit the viscosity of these glasses within experimental accuracy between $10^{2}$ and $10^{12}$ poise. A plot of the deviations between the experimental points and a least squares fit to the Fulcher equation in this range for both of the standard glasses is shown in figure 16 from reference [2].

For a comparison a plot of the deviations from a least squares fit to the Fulcher equation for a

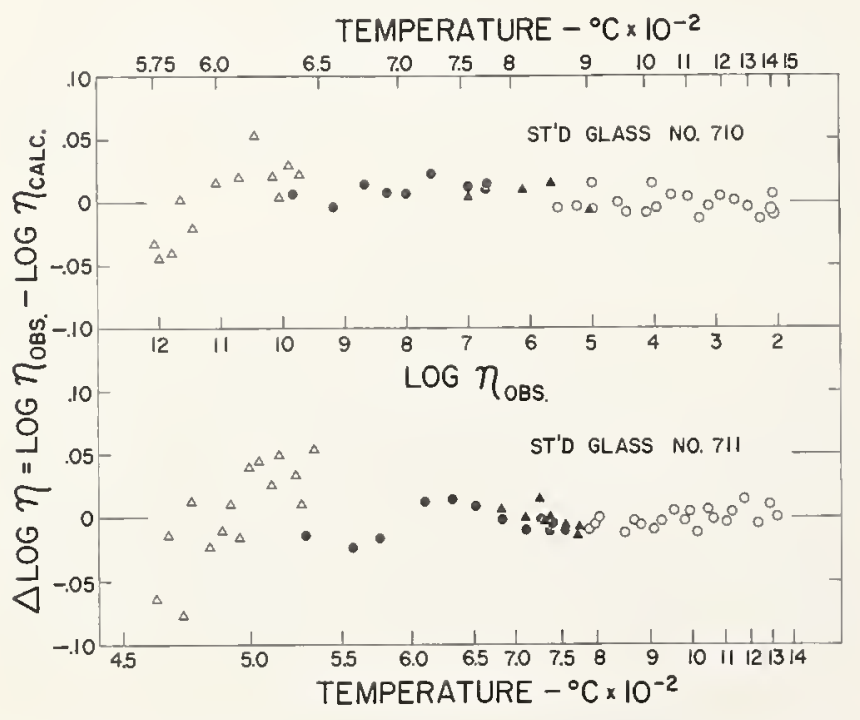

Figure 16. Deviations between the observed log viscosity values and those calculated from the least squares fit to the Fulcher equation for NBS standard glasses Nos. 710 and 711 [2, 3, 42].
BSC glass (Ritland's) [43] where viscosity measurements have been made to $10^{14.72}$ poise is shown in figure 17 from reference [1]. The deviations are well outside experimental error but still reasonable. In general, if the data are limited to viscosity values below $10^{12}$ a good fit can be obtained to the Fulcher equation [41]. In extrapolating this fit to (low temperature) higher viscosity values, the Fulcher equation predicts higher values than the actual measured values, whereas, if (as shown in fig. 17) a least squares fit is made to the Fulcher equation with all of the data (up to $10^{14.72}$ poise) an "s" shape type of curve is obtained for the deviations.

In view of the great empirical success of the Fulcher equation, many different theoretical bases have been proposed for it. In all of these models approximating assumptions had to be made to reduce the theoretical equation to the form of the Fulcher equation. In the hope that one of these theoretical models will be an improvement over the Fulcher equation we will review theories in terms of all the available viscosity data in vitreous $\mathrm{B}_{2} \mathrm{O}_{3}$. For the sake of presentation, the smooth data taken from reference [1] will be used and plots will be given of the differences between the predicted and experimental values.

These approximating assumptions are especially bad for $\mathrm{B}_{2} \mathrm{O}_{3}$ because of the peculiar temperature dependence of the expansion coefficient [44] and the specific heat [45]. Thus, it can be expected that the Fulcher equation will be a poor fit to the $\mathrm{B}_{2} \mathrm{O}_{3}$ data. In fact, as shown in figure 18 , curve $\mathrm{A}$, the equation is a very poor fit, having a proportional standard deviation of 40 percent and deviating by as much as a factor of 2.2. The calculated con-

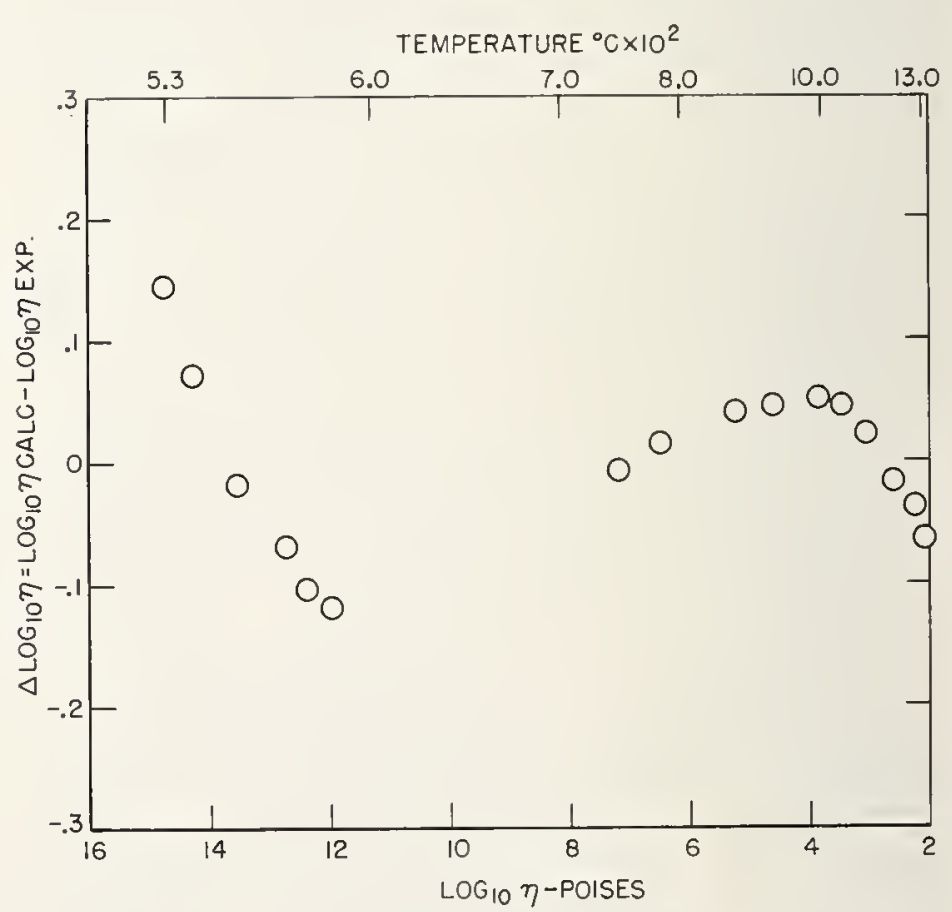

Figure 17. Deviations between the observed log viscosity values and those calculated from the least squares fit to the Fulcher equation for BSC glass [1]. 
stants for this equation and those that follow are given in table 2 .

TABLE 2. Calculated constants for each equation for $\mathrm{B}_{2} \mathrm{O}_{3}$ glass from ref. [1].

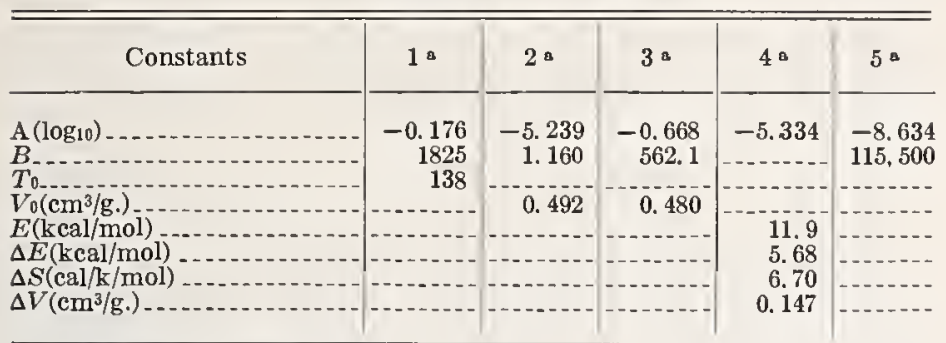

a 1-Fulcher equation (ref. 41).

2-WLF equation (ref. 46).

3-R RE equation (ref. 51).

4-Hybrid equation (ref. 52).

5-A \& $G$ equation (ref. 56).

\subsection{The Free Volume Approach}

\section{a. Doolittle Equation}

Williams, Landel, and Ferry (WLF) [46] have shown that the Fulcher equation can be considered identical (assuming the expansion coefficient is

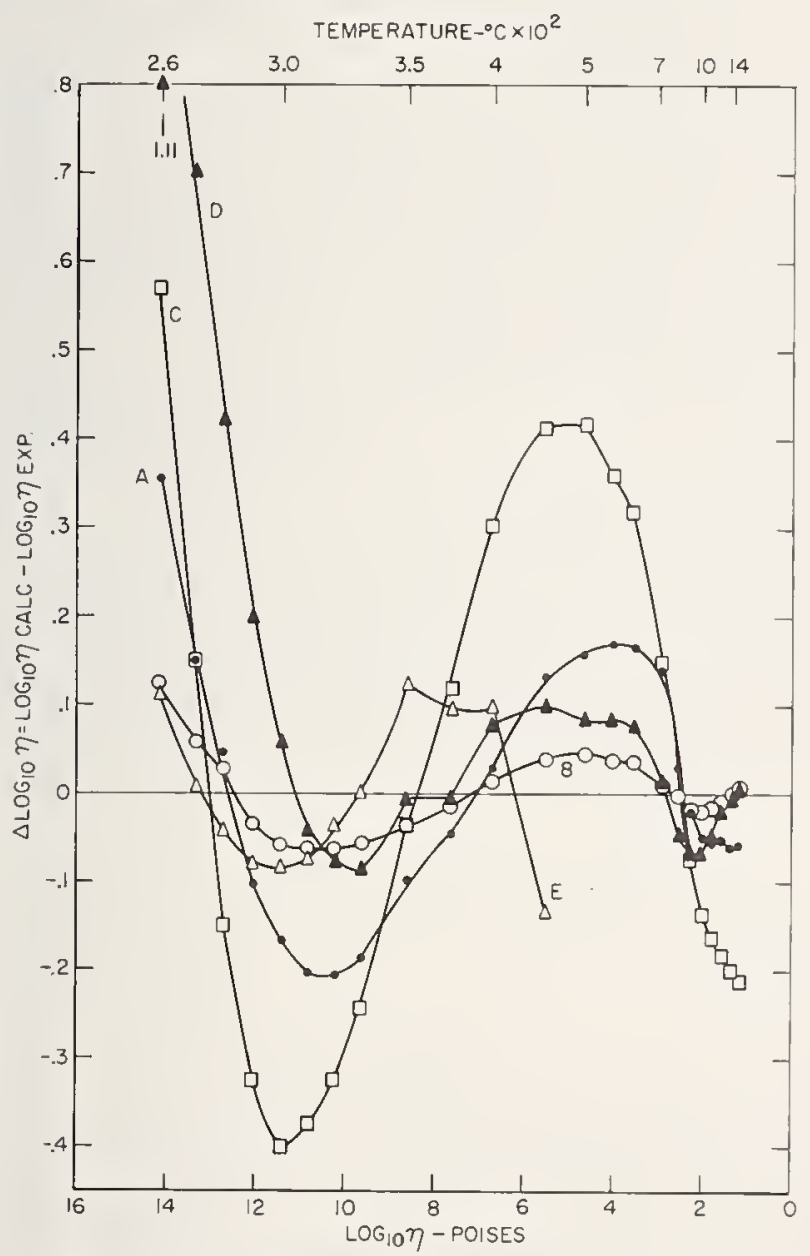

Figure 18. Deviations between the observed log viscosity values and those calculated from the least squares fit to several models for $\mathrm{B}_{2} \mathrm{O}_{3}$ glass [1].

A-Fulcher [41].

O B-WLF [46]

$\square \mathrm{C}-$ Ree, Ree and Eyring [51]

$\Delta$ D-Macedo and Litovitz [52]

$\triangle \mathrm{E}-\mathrm{Adam}$ and Gibbs $[56]$ temperature independent) to the free volume equation (in the Doolittle [47] equation) where

$$
\log \eta=A+B /\left(V-V_{0}\right)
$$

in which $V-V_{0}$ is the free volume per unit mass, and $A, B$, and $V_{0}$ are arbitrary parameters. This equation was later derived by Cohen and Turnbull [48] and Bueche [49]. Even though a constant expansion coefficient would be a good assumption for most liquids, it would be especially bad for $\mathrm{B}_{2} \mathrm{O}_{3}$ since $\beta$ changes by a factor $\sim 20$ between 270 and $1300{ }^{\circ} \mathrm{C}$. Curve $\mathrm{B}$, figure 18 , represents a fit to the free volume equation, and, as can be seen even though the deviations are well above experimental uncertainty, the fit is much better than the Fulcher equation fit.

The free volume equation not only predicts the temperature dependence of the viscosity through the volume but also the pressure. In fact, it predicts that if one changes the pressure appropriately to maintain constant volume, the viscosity should be temperature independent. Sperry and Mackenzie [50] have observed strong temperature dependence of the viscosity at constant volume, and found the activation energy at constant volume to be not only large as in most other liquids but almost equal to the activation energy at constant pressure. Thus, the free volume approach can not explain the viscositypressure dependence, suggesting that the success in fitting the viscosity-temperatue dependence is probably fortuitous.

\section{b. Significant Structure Theory}

In an effort to introduce a temperature dependence of the viscosity at constant free volume Ree, Ree, and Eyring [51] proposed an equation of the form

$$
\log \eta=A+B /\left[T\left(V-V_{0}\right)\right]
$$

in which the activation energy $\left[B /\left(V-V_{0}\right)\right]$ is a function of the free volume. Curve $\mathrm{C}$, figure 18 , shows the deviations of the least squares fit of this equation to the data.

\section{c. Hybrid Equation}

In the hybrid equation [52] the probability of a molecule obtaining simultaneously enough energy to break its bonds and free volume to perform a jump is calculated. This equation has the form:

$$
\ln \eta=\mathrm{A}+V_{0} /\left(V-V_{0}\right)+E /(R T)+\ln T .
$$

It became evident that this equation, similar to the free volume equation (31), overestimates the pressure dependence of the viscosity. Among investigators in this field there has grown a general sentiment that $V_{0}$ is pressure dependent. For example, Matheson [53] solved a similar problem with the viscosity of glycerine by making the 
reasonable assumption that $V_{0}$ is temperature and pressure dependent. Such a calculation has been made for $\mathrm{B}_{2} \mathrm{O}_{3}$ [54] where a two-state model for the free volume of $\mathrm{B}_{2} \mathrm{O}_{3}$ was assumed. In this case the hybrid equation takes the form

$$
\ln \eta=A+\left(V-V_{f}\right) / V_{f}+E_{v} /(R T)
$$

in which $V_{f}$ is the free volume given by a two-state calculation,

$$
V_{f}=\Delta V \cdot(1+\exp [(\Delta H-T \Delta S) / R T])
$$

where $\Delta V, \Delta S$, and $\Delta H(\Delta H=\Delta E+P \Delta V)$ are respectively the differences in volume, entropy, and enthalpy between states 1 and 2. It was also shown that these equations not only predicted the temperature dependence of the volume and viscosity, but also the pressure dependence of the viscosity as measured by Dane and Birch [55]. Figure 19 shows a plot of $\partial \log \eta / \partial P$ versus temperature where the two extreme data points are from Dane and Birch, while data between the extremes is the more recent work of Sperry and Mackenzie [50]. As can be seen in figure 19 the two sets of data agree well with each other, and since the hybrid equation was shown to be consistent with the Dane and Birch data it also agrees with the Sperry and Mackenzie data.

In figure 18 , curve $D$, the hybrid equation is tested with Macedo and Napolitano's [1] new lowter _ erature data and it also fails by predicting a too fast increase in viscosity in the glass transition region. The failure can be attributed to either eqs (33) and (34) (viscosity theory) or eq (35) (improper calculation of the free volume). This point is discussed at length in ref [1], the conclusions of which are given in section 4.4 .

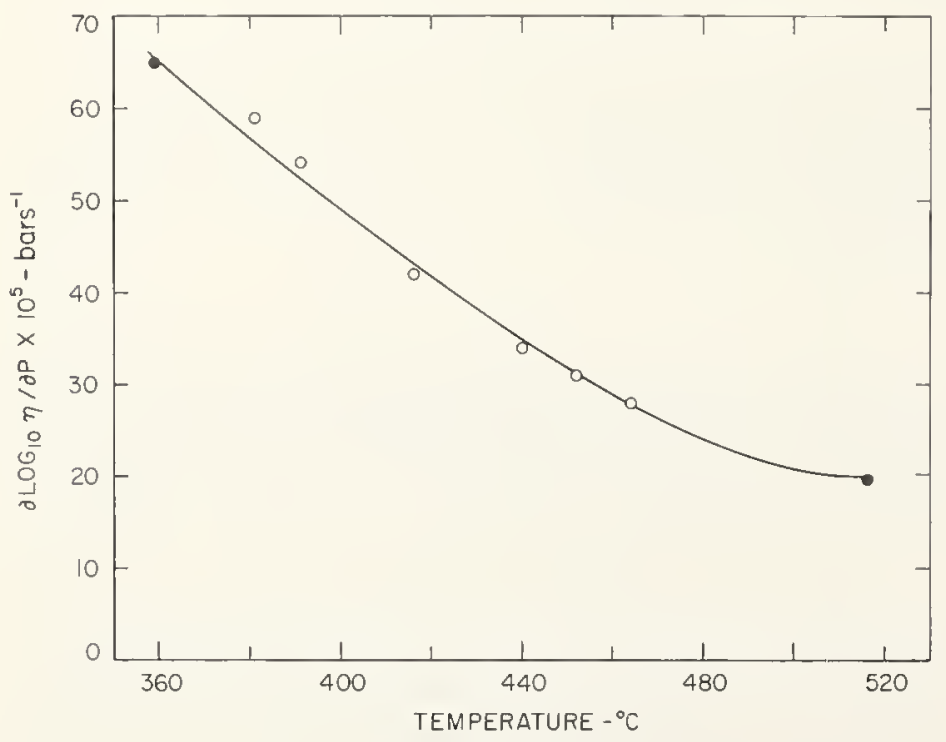

FigURe 19. Plot of $\partial \log \eta / \partial p$ versus temperature from ref. [1].

-Dane and Birch [55]

- - Sperry and Mackenzie [50]

\subsection{Configurational Entropy Model}

Adam and Gibbs [56] proposed that in order to have viscous flow a flow unit has to overcome a potential barrier. In addition, depending upon the configurational entropy, more than one unit may have to perform the jump at a time. This gives an equation of the form

$$
\log \eta=A+B /\left[T\left(S-S_{0}\right)\right]
$$

in which $\left(S-S_{0}\right)$ is the configurational entropy, $S_{c}$. Araujo [57] equating the temperature dependence of $S_{c}$ to the difference between the liquid and the crystal temperature dependence of the entropy, was able to fit the viscosity data for $\mathrm{B}_{2} \mathrm{O}_{3}$ between $10^{6}$ and $10^{1.4}$ poise very well. Unfortunately, there must have been an arithmetical mistake since over this temperature range the extrapolation of crystal specific heats of Kelly [58] and Thomas and Parks [45] (which Araujo claims to use) crosses the liquid values, see figure 20. This would predict increasing viscosity with increasing temperatures at a temperature above the crossover point but still within the measurement range. This phenomenon was not observed. Thus, one must assume, the specific heat extrapolation is incorrect. The viscosity data was cut off at $500^{\circ} \mathrm{C}$ and refitted where $T_{0}$, $A$, and $B$ were varied for best results. Curve $\mathrm{E}$, figure 18, shows the deviation from the fit. The fit is still poor, but better than average. This appearance of improvement is due to a much smaller temperature range.

\subsection{Conclusions of Equation of Viscosity}

Having considered the best known viscosity theories, in view of their new additional viscosity data, and finding no reasonable fits, Macedo and Napolitano [1] examined the validity of the basic assumptions underlying these theories. They found that the temperature dependence of the viscosity is not controlled by structural effects such as free

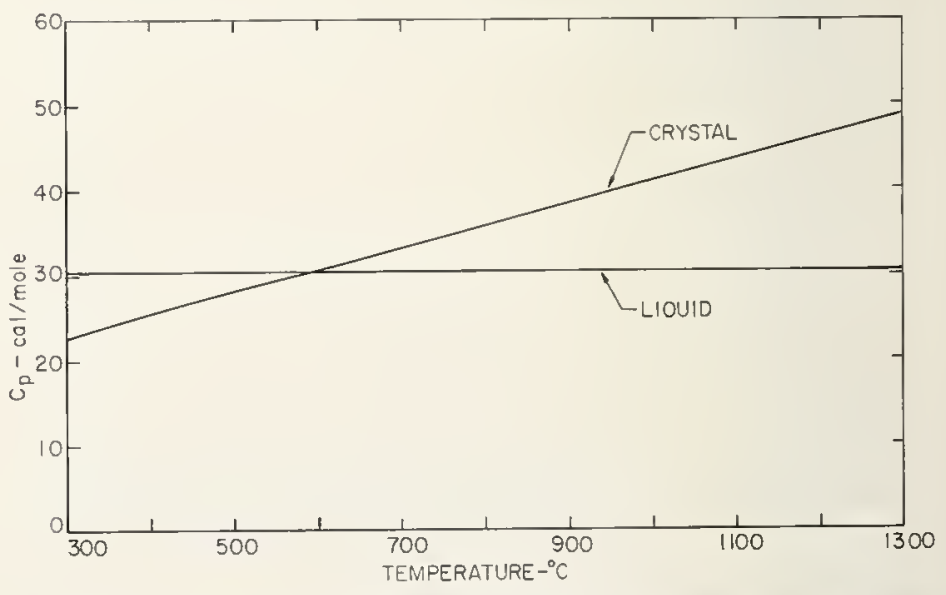

Figdre 20. Plot of specific heats versus temperature from Kelly and Thomas and Parks for $\mathrm{B}_{2} \mathrm{O}_{3}$ from ref. [1]. 
volume, configurational entropy, etc., but by activation energy effects which are probably caused by the necessity of bonds to be broken in the viscous flow process. Further, the onset of the non-Arrhenius region is a direct consequence of the appearance of a distribution of relaxation times and/or a distribution of activation energies (as shown by Tauke, Litovitz, and Macedo [31]). The appearance of a symmetric distribution of relaxation times (activation energies) cannot be explained by existing viscosity theories.

In the annealing range all the theories are in error in that they predict that the viscosity should go to infinity much faster than experimental data shows $(\partial E / \partial(1 / T)$ should be an ever-increasing function and it is not (see fig. 21)). In fact, $\mathrm{B}_{2} \mathrm{O}_{3}$ viscosity was again Arrhenius at low temperatures for over five decades. Therefore, one must conclude that the present theories of viscosity are inadequate, in particular, to fit the viscosity data and, in general, to give physical significance to the calculation of shear relaxation and its spectrum times.

A naive, yet better, model for the viscosity of $\mathrm{B}_{2} \mathrm{O}_{3}$ is one similar to that of Ottar and Ruigh [59] in which the liquid has a "disassociated" state at high temperature $(T>800)$ in the Arrhenius region. Then, there is an intermediate region where association takes place, finally, almost completely associated, the liquid again becomes Arrhenius. This model has to be made much more specific in order to explain the distribution of relaxation time behavior, and to be generalized for other liquids. However, this must await the more extensive data on the distribution of relaxation times.

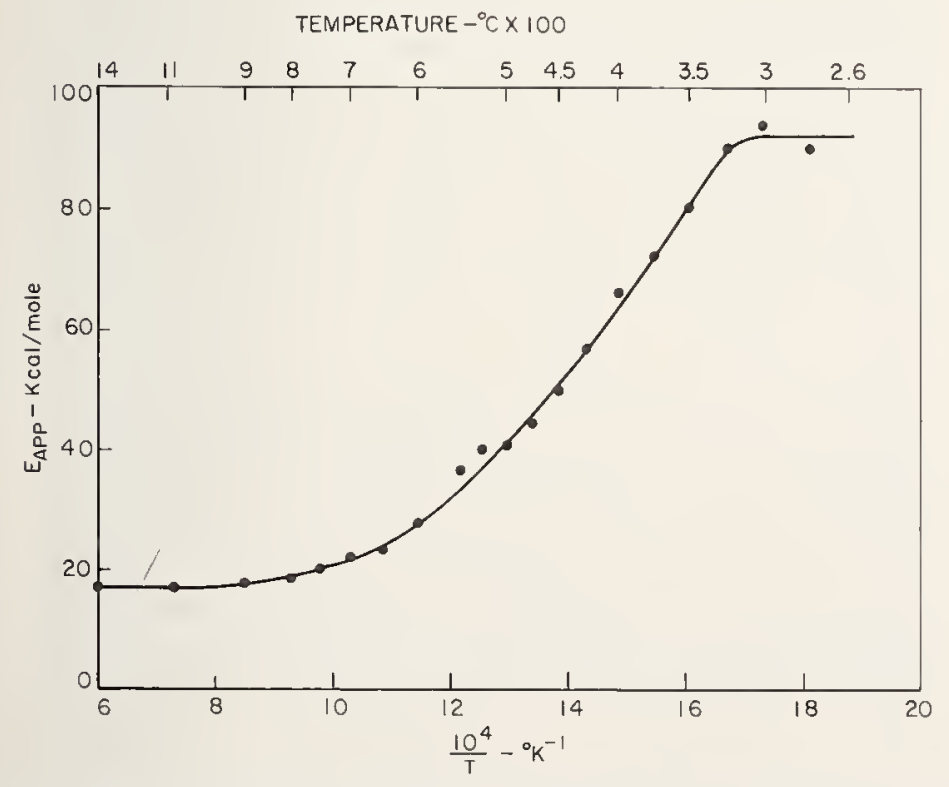

Figure 21. Plot of apparent activation energies versus temperature for $\mathrm{B}_{2} \mathrm{O}_{3}$ glass from ref. [1].

\section{References}

[1] Macedo, P. B., and Napolitano, A., J. Chem. Phys. 49, 1887-95 (1968).

[2] Napolitano, A., Macedo, P. B., and Hawkins, E. G., J. Res. NBS 69A (Phys. and Chem.), No. 5, 449-55 (1965).

[3] Napolitano, A., and Hawkins, E. G., J. Res. NBS 68A (Phys. and Chem.), No. 5, 439-448 (1964).

[4] Robinson, H. A., and Peterson, C. A., J. Am. Ceram. Soc. 27, 129 (1944).

[5] Eppler, R. A., J. Am. Ceram. Soc. 49, No. 12, 579-80 (1966).

[6] Gruber, G., and Litovitz, T. A., J. Chem. Phys. 40, 13-26 (1964).

[7] Tiede, R. L., J. Am. Ceram. Soc. 42, 537 (1959).

[8] Shartsis, L., and Spinner, S., J. Res. NBS 46, No. 3, 176-194 (1951).

[9] Riebling, E., Rev. Sci. Instr. 34, 568-72 (1963).

[10] Kelley, J. E., Roberts, T. D., and Harris, H. M., Bur. Mines Report No. 6358 (1963).

[11] Boow, J., Phys. Chem. Glasses 8, 45-55 (1967).

[12] Poole, J. P., J. Am. Ceram. Soc. 32, 215 (1949).

[13] Lillie, H. R., J. Am. Ceram. Soc. 14, 502 (1931).

[14] Lewis, W. K., L. Squire, and G. Broughton, Industrial chemistry of colloidal and amoprhous materials, p. 24 (MacMillan Co., New York, 1942).

[15] Hagy, H. E., J. Am. Ceram. Soc. 46, 93-97 (1963).

[16] Jones, G. O., J. Soc. Glass Tech. 28, [130] 432 (1944).

[17] Napolitano, A., and Macedo, P. B., J. Res. NBS 72A (Phys. and Chem.), No. 4, 425-33 (1968).

[18] Fontana, E. H., and Plummer, W. A., Phys. Chem. Glasses 7, 139-146 (1966).

[19] 1966 Book of ASTM Standards Part 13-Softening point page 305 , Annealing and strain points page 295, Average Linear Expansion of Glass page 301 .

[20] Saunders, J. B., J. Res. NBS 35, 157-186 (1945) RP 1668.

[21] Napolitano, A., Macedo, P. B., and Hawkins, E. G., J. Am. Ceram.' Soc. 48, 613-616 (1965).

[22] Riebling, E. F., J. Am. Ceram. Soc. 49, 19-23 (1966).

[23] Mackenzie, J. D., J. Phys. Chem. 63, 1875-78 (1959).

[24] Mackenzie, J. D., Rev. Sci. Instr. 27, 297-99 (1956).

[25] Shartsis, L., Capps, W., and Spinner, S., J. Am. Ceram. Soc. 36, 35-43 (1953).

[26] Li, P.-C., Ghose, A. C., and Su, G.-J., J. Am. Ceram. Soc. 45, 89-91 (1962).

[27] Wilhelmy, L., Pogg. Ann. 119, 177-217 (1863).

[28] Macedo, P. B., Capps, W., and Litovitz, T. A., J. Chem Phys. 44, 3357-64 (1966).

[29] Mason, W. P., Physical Acoustics, Vol. I Methods and Devices; Vol. IIA Properties of Gases Liquids and Solutions; Vol. IIB Properties of Polymers Glasses and Nonlinear Acoustics (Academic Press, New York and London, 1965).

[30] Macedo, P. B., and Litovitz, T. A., J. Phys. Chem. Glasses 6, 69-80 (1965).

[31] Tauke, J., Litovitz, T. A., and Macedo, P. B., J. Am. Ceram. Soc. 51, No. 3, 158-163 (1968).

[32] Simmons, J. H., and Macedo, P. B., High temperature shear ultrasonic interferometer using sensitive phase-lock detection system, J. Acoust. Soc. Am. 43, 1295 (1968).

[33] Bockris, J. O'M., and Kojonen, E., J. Am. Chem. Soc. 82, 4493 (1960).

[34] Macedo, P. B., and Napolitano, A., J. Res. NBS $71 \mathbf{A}$ (Phys, and Chem.), No. 3, 231-38 (1967).

[35] Spinner, S., and Napolitano, A., J. Res. NBS $70 A$ (Phys. and Chem.), No. 2, 147-152 (1966).

[36] Zijlstra, A. L., Phys. Chem. Glasses 4, 143-151 (1963).

[37] Kurkjian, C. R., Phys. Chem. Glasses 4, 128-136 (1963).

[38] Bartenev, G. M., Physics of Non-Crystalline Solids (Delft Conference, edited by J. A. Prins) (NorthHolland Publ. Corp., Amsterdam, 1964).

[39] Stevels, J. M., Verres et Réfractaires \%, 91 (1953); Glass Ind. 35, 657 (1954). 
[40] Tarasov, V. V., New Problems in the Physics of Glass (Moscow, USSR, 1963).

[41] Fulcher, G. S., J. Am. Ceram. Soc. 8, 339-55 (1925).

[42] Napolitano, A., and Hawkins, E. G., NBS Misc. Publ. 260-11, 24 pp (1966).

[43] Ritland, H. N., J. Am. Ceram. Soc. 39, 403-6 (1956).

[44] Napolitano, A., Macedo, P. B., and Hawkins, E. G., J. Am. Ceram. Soc. 48, 613-16 (1965).

[45] Thomas, S. B., and Parks, G. S., J. Phys. Chem. 35, 2091 (1931).

[46] Williams, M. L., Landel, R. F., and Ferry, J. D., J. Am. Chem. Soc. 77, 3701-07 (1955).

[47] Doolittle, A. K., J. Appl. Phys. 22, 1031-35 (1951); J. Appl. Phys. 22, 1471-75 (1951); J. Appl. Phys. 23, 236-39 (1952).

[48] Cohen, M. H., and Turnbull, D., J. Chem. Phys. 31, 1164-69 (1959).

[49] Bueche, F., J. Chem. Phys. 30, 748-52 (1959).

[50] Sperry, L. L., and Mackenzie, J. D., Phys. Chem. of glasses 9, 91 (1968).
[51] Ree, T. S., Ree, T., and Eyring, A., Proc. Natl. Acad. Sci. U.S. 48, 501-16 (1962).

[52] Macedo, P. B., and Litovitz, T. A., J. Chem. Phys. 42, 245-56 (1965).

[53] Matheson, A. J., J. Chem. Phys. 44, 695-699 (1966).

[54] Macedo, P. B., Capps, W., and Litovitz, T. A., J. Chem. Phys. 44, 3357-64 (1966).

[55] Dane, E. B., and Birch, F., J. Appl. Phys. 9, 669-74 (1938).

[56] Adam, G., and Gibbs, J. H., J. Chem. Phys. 43, 139-146 (1965).

[57] Araujo, R. J., J. Chem. Phys. 44, 1299-1300 (1966).

[58] Kelley, K. K., U.S. Bur. of Mines Bull. 584 (1960), Contributions to the data on theoretical metallurgy, XIII. High temperature heat-content, heat capacity, and entropy data for the elements and inorganic compounds.

[59] Ottar, B., and Ruigh, W. L., Phys. Chem. Glasses 3, 95-8 (1962). 


\title{
Inelastic Deformation of Oxide Ceramics
}

\author{
Richard M. Spriggs* \\ Physical Ceramics Laboratory, Materials Research Center, Lehigh University, \\ Bethlehem, Pennsylvania 18015
}

\begin{abstract}
The current understanding of the inelastic deformation of oxide ceramics is reviewed. Special emphasis is given to the permanent deformation caused by stress-enhanced diffusional creep, dislocation motion, and grain-boundary sliding. Brief reference is also made to recoverable inelastic deformation caused by point defect motion, dislocations, and grain boundary sliding. It is recognized that the observed permanent deformation is usually the result of more than one process, with complicating interactions frequently occurring, and with the relative contribution of each mechanism depending upon a host of structural, compositional, and experimental factors. Detailed information is available for only a few oxide ceramics and the need for additional research is evident.
\end{abstract}

Key Words: Anelasticity; creep; deformation; dislocations; grain boundaries; inelastic (plastic); oxide ceramics; point defects.

\section{Introduction}

When ceramic materials are subjected to a stress at ambient or elevated temperatures, they respond by deforming. This deformation frequently is the result of more than one deformation process. Furthermore, interactions among simultaneous processes often occur, making it difficult to assess quantitatively the effects of each process. However, the individual processes can frequently be separated or indentified and qualitatively or semiquantatively evaluated.

The deformation experienced by a ceramic material may be elastic and hence recoverable (i.e., strain which is fully and instantaneously recovered when the stress is removed). Alternatively, the deformation may be inelastic and may or may not be recoverable. Recoverable inelastic deformation can be considered to be time-dependent strain which is fully recovered when the external load is removed, even though recovery may take infinite time, i.e., observations over experimentally practical times indicate complete recovery upon extrapolation [1] ${ }^{1}$. Nonrecoverable inelastic deformation, on the other hand, is permanent strain or deformation which is not recovered in any length of time when the load is removed; it is frequently referred to as plastic deformation.

The subject of the present discussion is restricted to inelastic deformation, primarily the permanent deformation caused by phenomena such as the introduction of microcracks, disloca-

-The author is professor of metallurgy and materials science, director of the Physical Ceramics Laboratory, and associate director, Materials Research Center, Lehigh University, Bethlehem, Pennsylvania 18015.

1 Figures in brackets indicate the literature references at the end of this paper. tion motion, grain boundary sliding, and stressenhanced diffusional creep. Furthermore, it deals largely with oxide ceramics. Brief reference is also made to recoverable inelastic deformation. The reader is referred to other papers in this Symposium for a complete description of elastic deformation of crystalline ceramics and other refractory materials [2], and inelastic deformation of non-oxide ceramics [3]. An unusually complete introductory survey by Wachtman [1] on the mechanical properties of ceramics serves as a starting point for much of the following discussion.

\section{Recoverable Inelastic Deformation}

Wachtman [1] cites several important examples of recoverable inelastic deformation which are summarized below. Included are anelasticity caused by point defect motion, anelasticity in amorphous glasses, and recoverable inelastic deformation caused by either dislocations or grain boundary sliding. In each of these instances, it is important to keep in mind that recoverable inelasticity implies that when a load is applied to a specimen, the free energy is increased in such a way that when the load is removed the deformation is eventually fully recovered as the free energy returns to a minimum under the action of thermal vibrations [1].

\subsection{Anelasticity Caused by Point Defect Motion}

Small inelastic deformation which is linear in the stress, i.e., anelasticity, is produced by the 
stress-driven motion of point defects such as interstitial ions or vacancies. Anelastic deformation is usually and more easily studied in dynamic experiments in which the stress varies periodically with time and the internal friction (fractional energy dissipated per cycle) is measured.

While most of the work on anelasticity caused by point defects has been conducted with metals, some work has been done on ceramics and such studies promise to be quite useful in identifying types of point defects in ceramic crystals and measuring their jump rates [1]. Wachtman [1] cites work which has been reported on $\mathrm{MgO}[4,5]$, $\mathrm{ZrO}_{2}[6,7], \mathrm{ThO}_{2}[8], \mathrm{TiO}_{2}[9-11], \mathrm{CaF}_{2}$ [12], and $\mathrm{SiO}_{2}$ [13]. Under certain circumstances, electronic motion can also contribute to anelasticity in ceramics, as in lithium-doped $\mathrm{NiO}$ semiconductors [14]. In addition, typical internal friction values have been tabulated for commercial ceramics [15]; such values tend to be variable, however, and depend upon factors such as trace impurities, phase changes, etc. [1].

\subsection{Anelasticity in Amorphous Glasses}

According to Wachtman [1], internal friction measurements in glass may show either the motion of alkali ions or the viscoelastic behavior of the glass network, or even a combination of the two effects [16, 17]. The motion of alkali ions gives rise to a pronounced "elastic after effect" in chemically strengthened glass; an anelastic strain equal to 11 percent of the elastic strain has been observed after $15 \mathrm{~min}$ at $25 \mathrm{C}$ [18]. The viscous relaxation of the glass network itself at high temperatures gives rise to large differences in elastic moduli measured at frequencies low enough to permit relaxation [1]. For $\mathrm{B}_{2} \mathrm{O}_{3}$ at $800 \mathrm{C}$, for example, the longitudinal modulus at high frequency is three times the low-frequency value [19].

\subsection{Recoverable Inelastic Deformation Caused by Dislocations}

The movement of dislocations is the most important mechanism of inelastic deformation in metals at room temperature and in many glassfree ceramics of large grain size at high temperatures [1]. Dislocation mobility is thus very important to an understanding of the deformation of ceramics and depends strongly on the presence of other imperfections such as point defects, precipitates, and other dislocations [20].

Under lower stresses, segments of pinned dislocation bow outward while the ends remain attached to the pinning points. At greater stresses, the dislocation breaks away from some of the pinning points. With the former situation, where bowing only occurs, the dislocations return to their original positions when the stress is removed; this inelastic, recoverable deformation gives rise to a frequency-dependent but amplitude-independent internal friction. By contrast, the latter occurrence, where dislocations break away, produces amplitude-dependent internal friction [1]. Dislocation mobility can be related to measurable internal friction by theory [21-23], but other factors such as dislocation loop length and dislocation density are also involved.

Chang [24] analyzed data for $\mathrm{MgO}$ and $\mathrm{Al}_{2} \mathrm{O}_{3}$, but Carnahan and Brittain [25] found that for $\mathrm{TiO}_{2}$ (rutile) the experimental results showed qualitative disagreement with the theory, as was also found for $\mathrm{MgO}$ [26] and $\mathrm{Cu}-\mathrm{Ge}$ alloys [27]. Wachtman [1] concluded that while the study of internal friction caused by dislocations in ceramic oxides is a promising area, the analysis is not yet completely reliable.

The ordering of point defects in the stress field of a moving dislocation should represent still another source of inelastic deformation in ceramics, but apparently has not yet been investigated [1].

\subsection{Recoverable Inelastic Deformation Caused by Grain Boundary Sliding}

Inelastic deformation due to grain boundary sliding appears to be a general phenomenon in polycrystalline ceramics [1], and the role played by impurities is probably of critical importance. However, grain boundary properties and their effect on the deformation behavior of ceramics are not well understood at present. The frequent occurrence of concurrent recoverable and nonrecoverable phenomena tends to make it difficult to separate effects. Some inelastic deformation effects attributable to grain boundary sliding, however, have been cited by Wachtman [1].

A rapid decrease in Young's modulus of polycrystalline alumina above $950 \mathrm{C}$, accompanied by an increase in internal friction over the single crystal values, has been attributed to grain boundary sliding [28]. Other studies of alumina show qualitatively the same effects [29-31], with increasing purity increasing the temperatures at which these effects occur by as much as $400 \mathrm{C}$. Thus the introduction of grain boundaries appears to raise the damping capacity (i.e., internal friction) in a certain characteristic temperature range; this range is broadened and usually extended to lower temperatures by the addition of impurities.

Creep measurements with $\mathrm{Cr}_{2} \mathrm{O}_{3}$-doped alumina at 1000 to $1200 \mathrm{C}$ have revealed decreasing creep rates which were slowly recovered when the load was removed $[32,33]$. This recovery was attributed to viscous grain boundary sliding accompanied by elastic deformation of the grains. The elastically deformed grains reversed the grain boundary sliding when the external load was removed. Higher stresses, higher temperatures, or longer times permit plastic deformation within grains, so that only part of the inelastic strain can then be recovered [1]. 


\section{Nonrecoverable Inelastic Deformation}

The deformation of a ceramic material may, in certain circumstances, be completely recoverable (e.g., a small amount of grain boundary sliding accompanied by elastic deformation), partly recoverable, or completely plastic, depending upon the mechanisms operating [1]. The latter behavior is of particular interest in this section, especially creep deformation under constant load.

At elevated temperatures, two factors become important to change the deformation behavior of ceramics. First, dislocations become more mobile on existing slip systems and new slip systems become possible, and second, the diffusion of point defects is enhanced to the point where they begin to contribute to the deformation [34].

Unfortunately, the temperature range where a multiplicity of slip systems becomes available in most ceramic oxides is so high that additional complications come into play and the occurrence of nonrecoverable inelastic deformation by the simple movement of dislocations is never observed [34]. Elevated temperature deformation is always enhanced by the diffusion of point defects and by the onset of viscous sliding at grain boundaries. The diffusion of vacancies (or interstitial ions) contributes to each of the three basic mechanisms considered responsible for creep: vacancy migration under stress, dislocation motion, and grain boundary sliding. (The first and third processes, it should be noted, apply specifically to polycrystalline materials.) In the sections that follow, each of these mechanisms is discussed. Additional factors are considered, including the viscous deformation of glass and combined deformation processes.

The mechanisms responsible for deformation depend upon a host of structural and compositional factors as well as stress, temperature, and other test parameters. Structural variables include crystallography, grain size, subgrain structure, and porosity. Insofar as possible, the dependence of deformation on each of these factors is reviewed.

\subsection{Vacancy Migration Under Stress (Diffusional Creep)}

In vacancy migration under stress, deformation results from diffusional flow within each grain away from those boundaries where there is a high local compressive stress toward those boundaries having a high local tensile stress. This is generally referred to as Nabarro-Herring creep [35, 36] and has been found to be the primary deformation process for many ceramics at high temperatures, relatively low stresses, and fine grain sizes, with a temperature dependence similar to that for volume diffusion.

In Nabarro-Herring creep, self-diffusion within the grains of a polycrystalline solid permits the solid to deform under a shear stress, leading to viscous flow with the diffusional viscosity, $\eta$, given by:

$$
\eta=\frac{k T r^{2}}{10 D_{l} \Omega}
$$

where $k$ is Boltzmann's constant, $T$ is the absolute temperature, $r$ is the average grain radius, $D_{l}$ is the bulk self-diffusion constant, and $\Omega$ is the volume of the diffusing species. The diffusion coefficient, $D_{l}$, is exponentially related to temperature as follows:

$$
D_{l}=D_{0} \exp (-\Delta H / R T) .
$$

Under a tensile stres, $\sigma$, the tensile creep rate is given in terms of the average grain diameter, $G S$, by [37]:

$$
\dot{\epsilon}=13.3 \sigma D \Omega / k T(G S)^{2} \text {. }
$$

In a similar mechanism proposed by Coble [38], the changes in grain shape are attributed to grain boundary diffusion rather than lattice diffusion, and the creep rate is described by:

$$
\dot{\epsilon}=47 \sigma D_{b} w \Omega / k T(G S)^{3},
$$

where $D_{b}$ is the diffusion coefficient for the slowest moving species in the grain boundary and $w$ is the width of the grain boundary.

In a very recent brief review of diffusional creep mechanisms, Gifkins [39] compared both NabarroHerring creep and Coble creep with the suggestion that creep can occur by grain boundary sliding, also controlled by diffusion. Gifkins creep leads to movement along the boundary of grainboundary protrusions and can be accommodated by diffusion around triple points. The original diffusion-controlled mechanism for grain-boundary sliding was proposed by Gifkins and Snowden [40] and is based on the idea of the movement of double ledges or protrusions along the boundary. The rate of sliding, $\dot{S}_{b}$, for a grain boundary at $45 \mathrm{deg}$ to the applied stress is then:

$$
\dot{S}_{b} \simeq \alpha \sigma D_{b} \Omega / L k T,
$$

where $\alpha$ is a geometrical constant, approximately equal to $2 ; L$ is the length of the grain boundary protrusion $\left(\sim 10^{-6} \mathrm{~cm}\right.$ in tungsten [31]); and $\sigma$, $D_{b}, \Omega, k$, and $T$ have their former meanings.

The predicted creep rates, utilizing eq (5), have been 100 times too fast to match certain experimental results [42]; this has been attributed to factors which prevented continuous sliding on all boundaries. An equation for the accommodation of sliding by diffusion around triple points, one possible reason for sliding pauses, has also been derived by Gifkins [43]; the rate of accommodation, $\dot{S}_{a}$, for a boundary at $45^{\circ}$ to the applied stress, is:

$$
\dot{S}_{a} \simeq 40 \Omega D_{b} \sigma w /(G S)^{2} k T,
$$


where $w$ is the grain boundary width and $G S$ is the grain diameter.

By combining the mechanisms represented by eq (5) and (6) with some grain boundary migraticn, Gifkins envisions creep extension which occurs entirely by diffusional mechanisms but which does not result in permanent change of grain shape [39], as is required by Nabarro-Herring and Coble creep. The predominating mechanism would depend upon grain size; coarse grain sizes would be controlled by accommodation (eq (6)), while fine grain sizes would be controlled by sliding (eq (5)).

To compare eqs (5) and (6) with NabarroHerring creep and Coble creep, Gifkins [39] makes certain further approximations and assumptions and derives the proportionalities between creep rate and stress, temperature, grain size, and numerical constants shown in table 1.

The suggestion by Gifkins [39] of diffusioncontrolled grain-boundary-sliding-type creep is too recent to have been critically examined in relation to the creep of polycrystalline ceramic oxides. The most promising evidence offered to date has been the very recent work of Hensler and Cullen [42] on grain shape change during the creep of 98 percent dense $\mathrm{MgO}$ at 1200 to $1500 \mathrm{C}$ and 1000 to 6000 psi. Hensler and Cullen found no change in grain shape after compressive creep deformation up to 30 to 44 percent compression; these results where qualitatively interpreted in terms of Gifkins grain boundary sliding creep [39].

As previously mentioned, Nabarro-Herring intragranular diffusional creep has been found to be the primary deformation process for many ceramics at high temperatures, relatively low stresses, and fine grain sizes; eq (3) has been confirmed for $\mathrm{Al}_{2} \mathrm{O}_{3}$ [45-49], $\mathrm{MgO}$ [50], $\mathrm{BeO}$ [5153], $\mathrm{UO}_{2}-\mathrm{BeO}$ mixtures [54], and $\mathrm{NaCl}$ [55]. For $\mathrm{Al}_{2} \mathrm{O}_{3}$, eq (3) is obeyed using $D_{l}$ for aluminum in aluminum oxide despite the fact that $D_{l}$ for oxygen is smaller and should be rate controlling; this is thought to be due to enhanced diffusion of oxygen along the grain boundaries [56].

A brief summary of the major findings concerning $\mathrm{Al}_{2} \mathrm{O}_{3}, \mathrm{BeO}, \mathrm{MgO}$, and $\mathrm{SiC}$ is presented below.

\section{a. Creep of Alumina}

As cited by Vasilos and Passmore [57], the available creep data at high temperatures for poly- crystalline alumina generally support the Nabarro Herring diffusional creep model over a range of stress (100 to $4,000 \mathrm{psi}$ ) and temperature (1200 to $1800 \mathrm{C})$. Studies of high-density, fine-grained $\mathrm{Al}_{2} \mathrm{O}_{3}$ (LUCALOX) by Warshaw and Norton [46] and Folweiler [45] illustrate this point. Both found the creep rate to vary linearly with stress between 1500 and $1800 \mathrm{C}$, and the activation energy was measured to be $130 \mathrm{kcal} / \mathrm{mole}$. Similarly, Chang [58] also found a viscous behavior, although with a higher activation energy (200 $\mathrm{kcal} /$ mole) in this temperature range.

Recently, Passmore et al. [49, 59] determined the variation of creep rate in alumina with stress and temperature by using the incremental stress and temperature method introduced by Dorn [60] and illustrated in figure 1. Specimens were first crept to a strain of 0.32 percent at the relatively high stress of $9000 \mathrm{psi} \pm 50 \mathrm{psi}$; the stress was then decreased to a lower level. After further creep at constant temperature and the lower stress, the

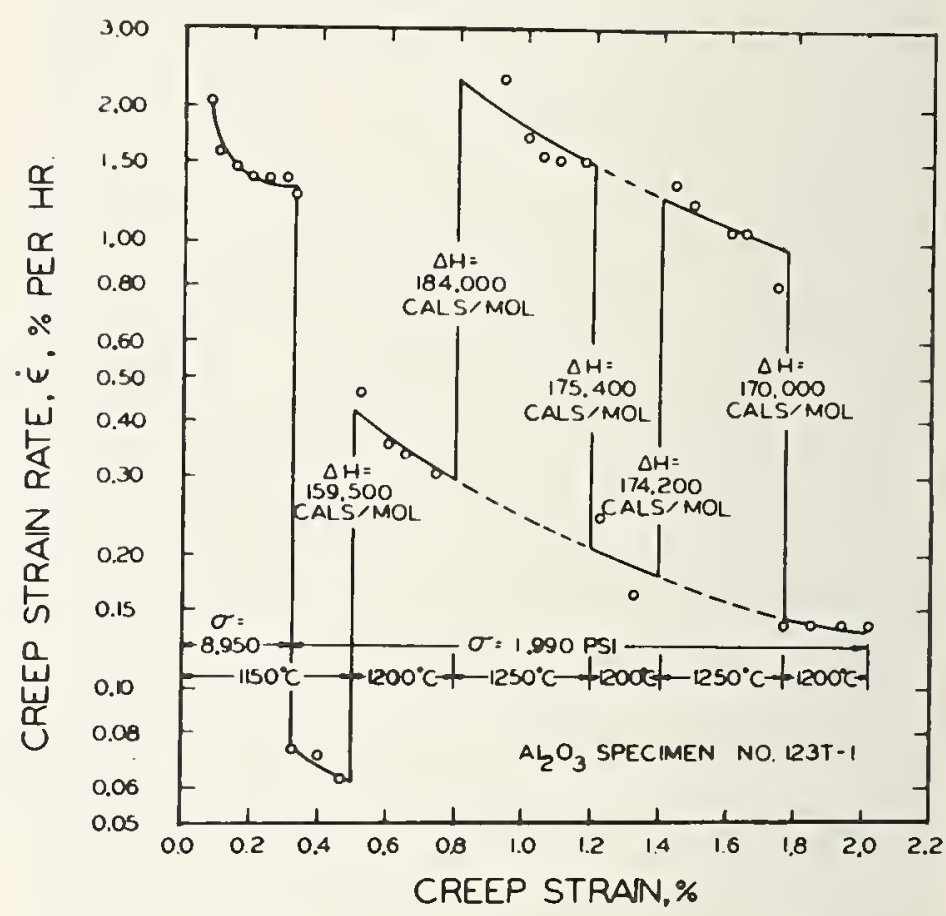

FiguRE 1. Typical creep rate-creep strain curve used in the evaluation of the stress and temperature dependence of creep for pure, dense, fine-grained aluminum oxide.

(After Vasilos and Passmore. Courtesy of Syracuse University Press)

TABLe 1. Dependence of creep rate (e) on variables in equations based on diffusion-controlled creep mechanisms [39]

\begin{tabular}{|c|c|c|c|c|c|c|}
\hline \multirow{2}{*}{ Creep type } & \multirow{2}{*}{ Mechanism characteristic } & \multirow{2}{*}{ Stress } & \multirow{2}{*}{ Temp. } & \multicolumn{2}{|c|}{ Grain size } & \multirow{2}{*}{$\begin{array}{l}\text { Numerical } \\
\text { constants }\end{array}$} \\
\hline & & & & (A) 1 & $(B)^{2}$ & \\
\hline Nabarro-Herring [36] - & Grain elongation & $\sigma$ & $D_{l}$ & \multirow{4}{*}{\multicolumn{2}{|c|}{$\begin{array}{l}(\mathrm{GS})^{-2} \\
(\mathrm{GS})^{-3} \\
(\mathrm{GS})^{-1}(\mathrm{GS})^{-2} \\
(\mathrm{GS})^{-3}\end{array}$}} & 13.3 \\
\hline Coble $[38] \ldots$ & Grain elongation...... & $\sigma$ & $D_{b}$ & & & $347 w$ \\
\hline Gifkins-Snowden [40].. & Grain-boundary sliding; some grain elongation. ............. & $\sigma$ & $D_{b}$ & & & $42 / x$ \\
\hline Gifkins [43] .... & Grain-boundary sliding, accommodated; no grain elongation... . & $\sigma$ & $D_{b}$ & & & $40 w$ \\
\hline
\end{tabular}

1Assuming $L$ in eq (5), grain boundary protrusion length, is independent of $(G S)$ for Gifkins-Snowden creep.

Assuming $L$ depends on $(G S)$

${ }^{3} w$ (grain boundary width) $\simeq 10^{-7} \mathrm{~cm}$.

${ }^{4} \chi$ is a factor introduced to account for pauses other than the necessity for triple point accommodation, and may have a value of $\sim 100$. 
temperature was rapidly changed to a new constant level. This process was repeated several times with periods of creep at constant temperature being alternated with rapid temperature changes. This procedure yields several estimates of the activation energy for creep. Rapid stress changes at constant temperature can likewise be employed to determine the creep rate dependency on stress.

In contrast to the observation of Warshaw and Norton [46], Passmore et al. [49, 59] observed no region of steady-state creep. As indicated in figure 1 , the creep rate continued to decrease with increasing creep strain up to strains of 1.5 to 2.0 percent. Warshaw and Norton, on the other hand, reported constant, steady-state creep in bending at strains of 0.2 percent and below. Vasilos and Passmore [57] concluded that the different creep rate-strain dependences may have resulted from the different temperatures employed. At the high temperatures (1600 to $1800 \mathrm{C})$ used by Warshaw and Norton, recovery processes may have been rapid enough to offset any strain hardening, whereas the latter process predominated at the lower temperatures used by Passmore et al.

Creep rates determined graphically at a strain of 0.32 percent are shown as a function of stress

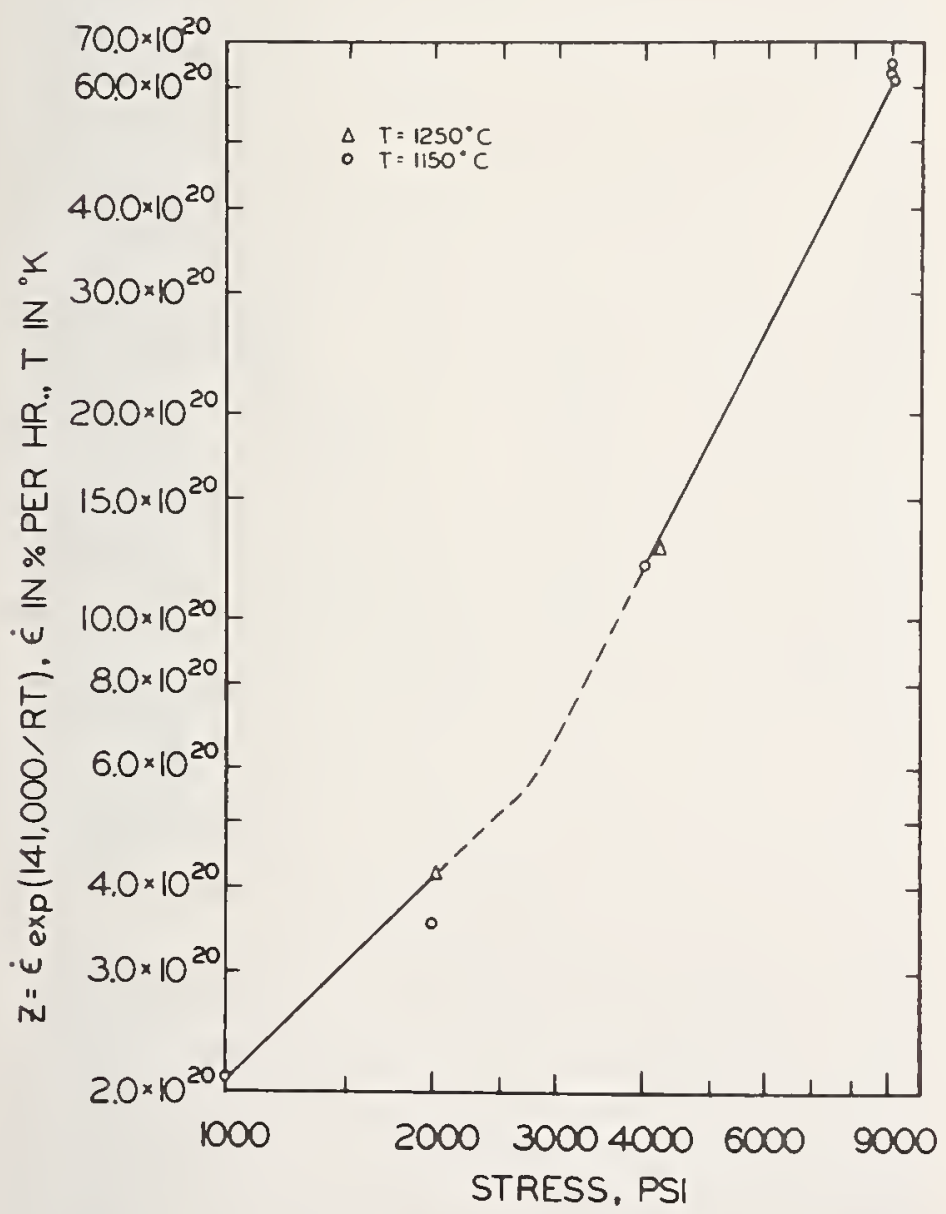

FIGURE 2. Effect of stress on the temperature-compensated creep rate of dense, fine-grained alumina at constant strain of 0.32 percent.

(After Vasilos and Passmore. Courtesy of Syracuse University Press) in the range of 1,000 to 9,000 psi in figure 2. After Passmore et al., creep rates for the two temperatures used, 1150 and $1250 \mathrm{C}$, are expressed in terms of the temperature-compensated creep rate, commonly called the Zener-Hollomon parameter, $Z=\dot{\epsilon} \exp (\Delta H / R T)$, using a value of 141 $\mathrm{kcal} / \mathrm{mole}$ for $\Delta H$. At low stresses (up to about 2000 psi), a linear relation between creep rate and stress was observed. At higher stresses, howeve., the creep rate dependence of stress increased to a square dependence, which suggests a transition from predominantly viscous creep mechanisms of the Nabarro-Herring type to a more complex one, probably involving plastic flow. A nonlinear stress dependence $\left(\dot{\epsilon} \propto \sigma^{4}\right)$ was also observed by Warshaw and Norton [46] in the 650 to 1,300 psi stress range for coarse-grained $(50$ to $100 \mu \mathrm{m})$ alumina. The results of Passmore, et al. [49, 59] appear to be consistent with the interpretation of Warshaw and Norton and are considered more definitive, because possible effects of variations in substructure and intergranular separation were minimized or eliminated by carrying out the creep-rate experiments at low stresses immediately after creep at a constant level of higher stress $(9000 \mathrm{psi})$ to a constant creep strain (0.32 percent). Vasilos and Passmore [57] thus believed that the enhanced creep rates observed above 2,000 psi by Passmore et al. were, in fact, indicative of a transition in the creep mechanism.

The Nabarro-Herring creep mechanism (eq (3)) predicts an inverse proportionality between creep rate and grain size, i.e., $\dot{\epsilon} \propto 1 /(G S)^{2}$. This has been confirmed by several investigators, the first for alumina being Folweiler [45]. Thus, largegrained material has a higher creep resistance than fine-grained material.

Table 2 summarizes the high-temperature deformation behavior of pure and doped polycrystalline alumina with regard to temperature range, rate-controlling mechanism, and activation energy [57].

Several investigators have compared diffusion coefficients calculated from creep rates using eq (3) with directly measured diffusion coefficients, but there has been no general agreement. The creep-diffusion coefficients have generally been greater than self-diffusion values, although Paladino and Coble [56] have reported very good agreement with aluminum ion diffusion, which is the faster moving species. Enhanced diffusion of oxygen along grain boundaries has been invoked to explain this finding [56].

\section{b. Creep of Beryllia}

Chang [51] reported good agreement between creep deformation rate and measured diffusion coefficients for $\mathrm{BeO}$, thus supporting the diffusional creep model. In further work on the compressive creep behavior of beryllia, Vandervoort and Barmore [52] also found that the creep rate was 
TABLE 2. High temperature deformation and creep of polycrystalline alumina (after Vasilos and Passmore)

\begin{tabular}{|c|c|c|c|c|c|}
\hline $\begin{array}{l}\text { Result } \\
\text { No. }\end{array}$ & Material & $\begin{array}{c}\text { Temperature } \\
\text { range }\end{array}$ & Rate-controlling deformation mechanism & Energy & Investigators \\
\hline $1 \ldots . . .$. & High-density Lucalox, 5-10 $\mu$ m grain size. & $\begin{array}{l}\text { Centigrade } \\
\quad 1600-1800\end{array}$ & Vacancy migration & $\begin{array}{r}\mathrm{kcal} / \mathrm{mole} \\
130\end{array}$ & Warshaw and Norton [46]. \\
\hline $2 \ldots \ldots$ & High-density Lucalox, 5-35 $\mu \mathrm{m}$ GS.... & $1600-1800$ & Vacancy migration & 130 & Folweiler [45]. \\
\hline $3 \ldots$ & $97 \%$ dense, $50-100 \mu \mathrm{m}$ GS............. & $1600-1800$ & Grain-boundary sliding & 185 & Warshaw and Norton [46]. \\
\hline $4 \ldots \ldots$ & High-density, $100 \mu \mathrm{m}$ GS. $\ldots$. & $1750-1900$ & Grain-boundary sliding & 280 & Coble and Guerard [47]. \\
\hline $5 . \ldots$ & Same as 4 plus $\mathrm{Cr}_{2} \mathrm{O}_{3} \ldots \ldots$. & $1750-1900$ & Grain-boundary sliding & 280 & Coble and Guerard [47]. \\
\hline $6 \ldots$ & Polycrystalline & $1400-1700$ & Vacancy migration plus grain-boundary sliding...- & 200 & Chang [51]. \\
\hline $7 \ldots \ldots$ & Same as 6 plus $1 \% \mathrm{Cr}_{2} \mathrm{O}_{3}{ }^{1} \ldots \ldots$ & $1400-1700$ & Vacancy migration plus grain-boundary sliding-..- & 200 & Chang [51]. \\
\hline $8 \ldots \ldots$ & Same as 6 plus $1 \% \mathrm{~L}_{\mathrm{a} 2} \mathrm{O}_{3} 1 \ldots$ & $1400-1700$ & Vacancy migration plus grain-boundary sliding & 200 & Chang [51]. \\
\hline $9 . \ldots . . .$. & $99 \%$ Dense, $2 \mu \mathrm{m}$ GS & $1200-1375$ & Vacancy migration & 154 & Passmore et al. [49, 59]. \\
\hline $10 \ldots \ldots$ & Same as 9 plus $1 \mathrm{~m} / 0 \mathrm{Cr}_{2} \mathrm{O}_{3}{ }^{2}$ & $1300-1375$ & Vacancy migration and formation..- & 151 & Passmore et al. $[49,59]$. \\
\hline
\end{tabular}

$1 \mathrm{o} / \mathrm{o}=$ weight percent

${ }^{2} \mathrm{~m} / \mathrm{o}=$ mole percent

linearly dependent on the applied stress (up to 6,000 psi) over the temperature range of 1370 to $1510 \mathrm{C}$, with an apparent creep activation energy of $96 \mathrm{kcal} / \mathrm{mole}$. In addition, a comparison of calculated diffusion coefficients with directlymeasured beryllium ion coefficients revealed fair agreement. In work of Fryxell and Chandler [53] on the compressive creep of $\mathrm{BeO}$, a linear relation was also found between stress and creep rate up to a stress of $6,000 \mathrm{psi}$ at $1200 \mathrm{C}$. Above 6,000 psi, however, the creep rate depended upon stress raised to a power greater than unity. In addition, for small-grained specimens, they observed a creep rate which decreased with both time and strain considerably more than for larger $(10 \mu \mathrm{m}$ and larger) grain sized material. These latter observations are consistent with the previously mentioned findings of Passmore and Vasilos for fine-grained alumina.

\section{c. Creep of Magnesia}

As is the case with alumina and beryllia, the available creep data for polycrystalline magnesia at low stresses also generally support a diffusional creep model $[50,61]$. Perhaps because of the more plastic behavior of magnesia, however, the results for magnesia are more complicated, with a creep activation energy which is not invariant with grain size, a grain size dependence which is intermediate between the Nabarro-Herring and Coble creep models, and a change in the stresscreep rate dependency from unity (viscous creep) to 1.5 below $5 \mu \mathrm{m}$ grain size.

Passmore et al. [61], in a comprehensive investigation, recently determined the creep of $\mathrm{MgO}$ over a range of temperature, stress, and grain size. A typical creep rate-strain curve for dense, fine-grained ( 2 to $3 \mu \mathrm{m}$ ) magnesia is shown in figure 3 [61]. The creep behavior of $\mathrm{MgO}$ is similar to other polycrystalline oxides in that the strain rate decreases with increasing strain. Typical changes in creep rate corresponding to changes in temperature, are also shown in figure 3. Vasilos and Passmore [57] attributed the changes in creep rate in figure 3 solely to temperature changes, since stress was maintained constant and substructural effects were eliminated by extrapolating creep rates to the same value of strain for each temperature.

The activation energy values from Passmore's work [61] are plotted versus grain size in figure 4 for grain sizes from 2 to $20 \mu \mathrm{m}$. As seen, $\Delta H$ at first decreases sharply with increasing grain size from about $96 \mathrm{kcal} / \mathrm{mole}$ for a grain size of $2 \mu \mathrm{m}$ to $54.1 \mathrm{kcal} / \mathrm{mole}$ at $5.5 \mu \mathrm{m}$ and then remains constant with further grain size increases. Passmore et al. [61] observed no variations in $\Delta H$ with stress, strain, or temperature. The observed energy of $54.1 \mathrm{kcal} / \mathrm{mole}$ for the larger grain sizes was held to be consistent with a diffusional creep mechanism in which the creep rate is controlled by extrinsic oxygen ion diffusion $(\Delta H=62.4$ $\mathrm{kcal} / \mathrm{mole}$ according to Oishi and Kingery [62]). For grain sizes below $5.5 \mu \mathrm{m}$, the increasing $\Delta H$ may indicate a transition to control by intrinsic magnesium ion diffusion $(\Delta H=70 \mathrm{kcal} / \mathrm{mole}$ according to Lindner and Parfitt [63]).

Passmore et al. [61] observed a linear relationship between creep rate and stress for grain sizes of 5.5 to $20 \mu \mathrm{m}$. There was, however, a transition to a higher-order stress function, i.e., from 1.0 to 1.5, with decreasing grain size, which Passmore took as further evidence of a transition in creep mechanism for the finer grain sizes.

The grain size dependence of the creep rate below $5.5 \mu \mathrm{m}$ could not be determined [61] because of the variations in $\Delta H$ with grain size, which made it impossible to compensate the creep rates satisfactorily for variations in temperature over a significant range of grain sizes. The observed grain size dependence for the range from 5.5 to $20 \mu \mathrm{m}$ was described by an exponent of -2.5 (i.e., $\dot{\epsilon} \propto 1 /(G S)^{2.5}$ ), which is intermediate between those predicted by the Nabarro-Herring and Coble models for diffusional creep. 


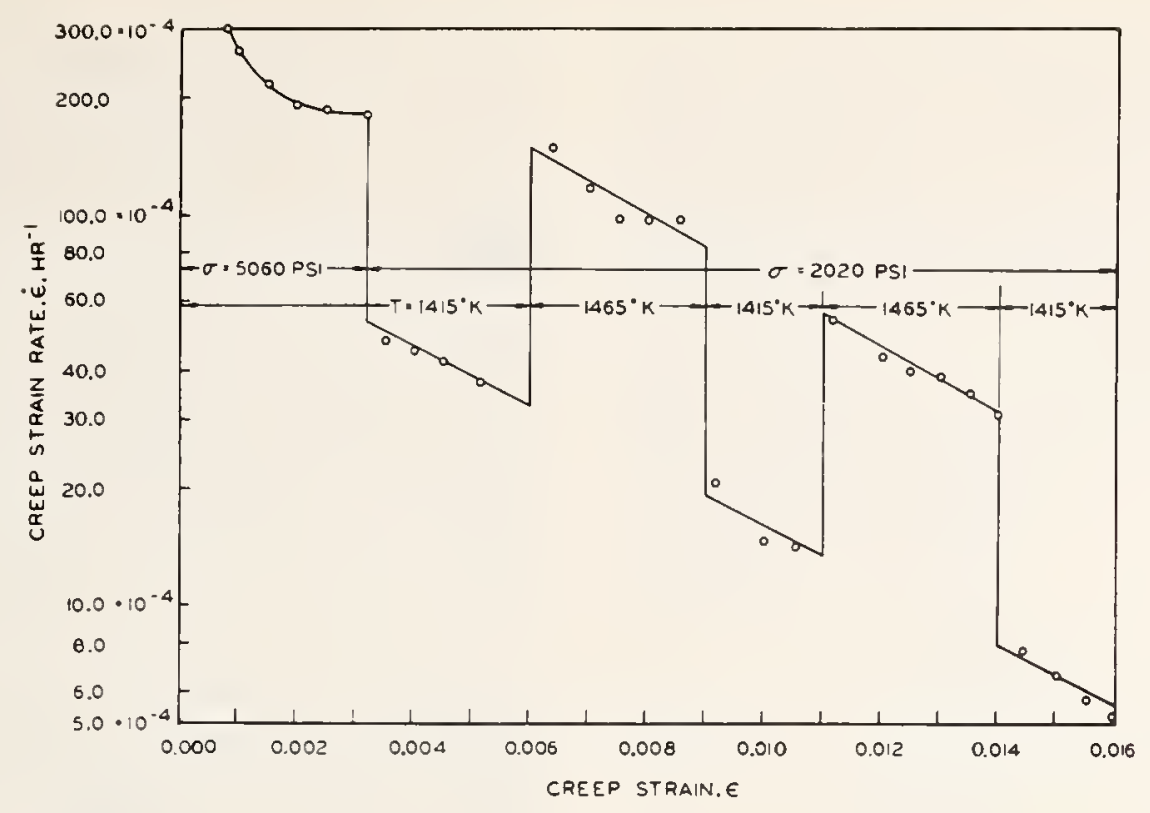

FIGURE 3. Typical creep rate-creep strain curve for dense, fine-grained (2 to $3 \mu \mathrm{m}$ ) magnesium oxide.

(After Vasilos and Passmore. Courtesy of Syracuse University Press)

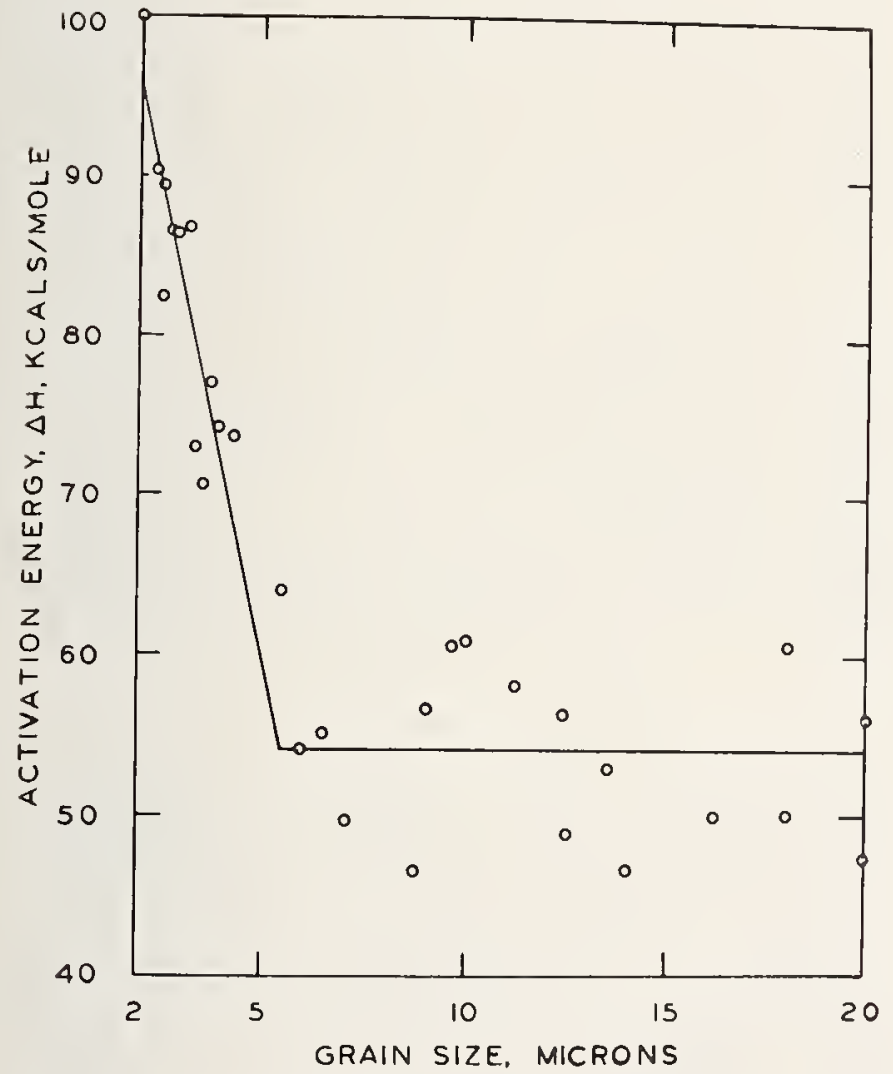

FIGURE 4. Effect of grain size on the activation energy for creep of dense magnesia.

(After Vasilos and Passmore. Courtesy of Syracuse University Press)

Impurities such as $1 \mathrm{~m} / \mathrm{o} \mathrm{ZrO}_{2}$ or $\mathrm{CaO}$ added to the $\mathrm{MgO}$ had no significant effects on creep rate but did increase the activation energy by 20 percent [57].

Passmore et al. [61] attributed creep in $\mathrm{MgO}$ in part to a stress-directed diffusional mechanism controlled by extrinsic oxygen ion diffusion in the
5.5 to $20 \mu \mathrm{m}$ grain size range, although the calculated diffusion coefficients were higher than those predicted by the Nabarro-Herring model. It was suggested that this discrepancy may be due to a vacancy formation mechanism, which was consistent with the observed formation of dislocation substructure and preferentially distributed porosity during creep, as well as with the observed decrease in creep rate with increasing creep strain [61].

\section{d. Creep of Silicon Carbide}

Farnsworth and Coble [37] concluded that diffusional creep was also the most probable ratecontrolling mechanism for the deformation of polycrystalline silicon carbide. Viscous flow was observed in dense $\mathrm{SiC}$ at temperatures from 1900 to $2200 \mathrm{C}$, at stresses from 3,000 to 30,000 psi, and at strain rates up to $10^{-3} / \mathrm{min}$.

\subsection{Creep by Dislocation Motion}

The deformation of polycrystalline ceramic oxides by plastic flow processes is less common than deformation by stress-directed diffusion. This fact results largely from the lack of sufficient slip modes in most polycrystalline oxides. Taylor [64] has shown that for plastic deformation to occur by dislocation movement in polycrystalline bodies, slip must occur on five independent slip systems to allow a general change in shape of individual grains without void formation. While metals, in general, meet the foregoing criterion, many ceramics do not, especially at reasonable temperatures. For example, Groves and Kelly [65] have examined the observed crystallographic slip systems for a number of common ceramic materials; their results are shown in table 3 . 
It is concluded that none of the common ceramics is capable of a great amount of plastic deformation at low temperatures, although at elevated temperatures materials with the sodium chloride and calcium fluoride structures should possess the potential for such deformation. At brief summary of results for oxides such as magnesia and alumina is given below.

TABLE 3. Number of independent slip systems for some common ceramic crystal structures [after Groves \& $\_$elly]

\begin{tabular}{|c|c|c|}
\hline Structure & $\begin{array}{l}\text { Crystallographic } \\
\text { slip systems }\end{array}$ & $\begin{array}{l}\text { Number of } \\
\text { independent } \\
\text { systems }\end{array}$ \\
\hline $\begin{array}{l}\mathrm{NaCl} \text { (low temps.) (e.g., } \mathrm{MgO}) \\
\mathrm{NaCl} \text { (high temps.) (e.g., MgO) }\end{array}$ & \multirow{3}{*}{$\begin{array}{l}\{10\}<1 \overline{1} 0> \\
110\}<110>\text { and } \\
001\}<110> \\
0001\}<1120> \\
100\}<010> \\
001\}<110> \\
001\}<1 \overline{1} 0>\text { and } \\
110\}<1 \overline{1} 0> \\
101\}<10 \overline{1}>\text { and } \\
110\}<001>\end{array}$} & \\
\hline $\begin{array}{l}\mathrm{Hexagonal} \text { (e.g., } \mathrm{Al}_{2} \mathrm{O}_{3}, \text { graphite) } \\
\mathrm{CsCl} \\
\mathrm{CaF}_{2} \text { (low temps.) (e.g., } \mathrm{UO}_{2} \text { )........ } \\
\mathrm{CaF}_{2} \text { (high temps.) }\end{array}$ & & $\begin{array}{l}2 \\
3 \\
3 \\
5\end{array}$ \\
\hline $\mathrm{TiO}_{2}$ & & \\
\hline
\end{tabular}

\section{a. Magnesia}

As cited by Vasilos and Passmore [57], the requirements for plastic flow in magnesia are relatively severe as compared to simple halides. The stress required to promote slip in $\mathrm{MgO}$ on the $\{001\}\langle 1 \overline{1} 0\rangle$ system at room temperature is approximately 100 times higher than that to cause slip on the $\{110\}<1 \overline{10}\rangle$ system, and extremely high stresses are required even for local deformation. Therefore, polycrystalline magnesia exhibits virtually no plastic deformation at room temperature, even though dislocations are quite mobile and equivalent single crystals are ductile. The difference in stress level for slip on the two sets of systems decreases with increasing temperature, and at $1000 \mathrm{C}$ is down to a factor of 10 (equivalent to LiF at room temperature). A slight amount of plastic deformation in $\mathrm{MgO}$ has been observed by Copley and Pask [66] in compression at temperatures up to $1000 \mathrm{C}$. At room temperature, the strain at fracture was about 0.6 percent for polycrystalline $\mathrm{MgO}$ and about 2 percent above $600 \mathrm{C}[66]$.

Slip in magnesia also tends to be nonuniform. Slip bands are usually few and randomly spaced before yielding at room temperature. Further straining causes the widening of these already existing slip bands, rather than the appearance of new ones. Such slip localization into wide bands causes stress concentration and the formation and growth of crack nuclei where slip bands intersect grain boundaries.

An important additional consequence of nonuniform slip is that a grain cannot change shape as required. For such a change to be possible, slip must occur on closely spaced planes, the amount of shear varying continuously from one phase to the next. With nonuniform slip, therefore, large elastic strains necessarily occur in the grains of a deformed polycrystalline specimen.
Such strains, again, favor the growth of crack nuclei and help propogate cleavage fracture [57].

As the temperature is increased, conditions become less favorable for cleavage fracture to occur. Self-diffusion becomes more rapid and, as a result, stress-induced climb can occur, increasing the difficulty of nucleating cracks. Dislocations also become increasingly mobile at higher temperatures and stress relaxation by plastic deformation becomes more important [57].

Day and Stokes [67] have shown that plastic deformation of polycrystalline magnesia under tension is possible only when the temperature exceeds $1700 \mathrm{C}$. Below $1700 \mathrm{C}$, an insufficient number of the available independent slip systems are capable of interpenetrating and operating concurrently within a given volume of material to satisfy the Taylor-Groves-Kelly criteria for deformation of polycrystalline bodies [67]. Therefore, grain boundary constraints arise which, when relaxed by intergranular sliding, lead to intergranular flaws and eventually intergranular rupture.

Day and Stokes [67] observed a slight amount of slip below $1700 \mathrm{C}$ in the vicinity of the fracture. Passmore, et al. [61] also observed a slight amount of slip at temperatures near $1400 \mathrm{C}$ in bending creep studies. Above $1700 \mathrm{C}, \mathrm{Day}$ and Stokes [67] concluded that sufficient independent slip systems were operating concurrently for continuity to be maintained between adjacent grains. The additional ability of the material to polygonize and recrystallize permitted a continuous relaxation of internal stresses and contributed to both the overall plasticity and change of shape under tension [67]. Deformation was found to proceed in fully dense specimens without intergranular separation and fracture occurred in a ductile manner [67]. In the presence of porosity, however, intergranular rupture was observed at temperatures as high as $2100 \mathrm{C}$, although plastic deformation occurred at temperatures below $1900 \mathrm{C}$ [67].

\section{b. Alumina}

It has been considered for some time that polycrystalline alumina creeps by a diffusioncontrolled mechanism rather than by dislocation motion, as a consequence of the limited number of independent slip systems available. It now appears that at temperature above $1400 \mathrm{C}$, the creep deformation of alumina is caused by a composite mechanism consisting partly of stressdirected diffusion, grain-boundary sliding and dislocation motion [1, 57]. As long as basal slip predominates (cf. table 3), there are only two independent slip systems in alumina, and even taking into account the additional flexibility afforded by dislocation climb, polycrystalline alumina should not be able to deform by slip without the generation of intergranular constraints [57]. Several experimental results, however, show that extensive inelastic nonrecoverable deformation is possible with dense, fine-gain material. 
Plastic deformation, with associated strain hardening, has possibly been observed by Passmore, et al. [57], in the range of $1300 \mathrm{C}$ to $1400 \mathrm{C}$. Furthermore, Vasilos and coworkers have been able to extensively deform polycrystalline alumina at temperatures above $1800 \mathrm{C}$ by press forging with a heavy resulting crystallographic texture. These observations, coupled with the recent discovery of $[1 \overline{0} 11]$ dislocations, suggest that limited to extensive ductility is possible in alumina at very high temperatures. Contributions due to diffusional creep, grain-boundary sliding, and dislocation motion all appear likely, with the relative contribution of each changing with changing experimental conditions.

\section{c. Steady-State Creep by Dislocation Motion}

In addition to the foregoing qualitative discussions concerning the role of dislocation motion in the creep of polycrystalline ceramics, a number of theories have been advanced to describe the steady-state creep of crystals by dislocation motion. As opposed to diffusional creep and grain boundary sliding, it should be noted that these dislocation motion theories do not apply specifically to polycrystalline materials and do not contain explicit grain size terms. Limited use of these theories has been made, however, as described below, to explain certain creep behaviors of polycrystalline material.

The most familiar of the so-called "microdynamical" dislocation motion theories of plasticity is that due to Weertman $[68,69]$, and relates to dislocation climb. Dislocation climb occurs when vacant lattice sites condense on or escape from edge dislocations, causing them to be displaced vertically in a direction perpendicular to the slip plane [34]. The climb process permits unlike dislocations to annihilate and also permits dislocations to detour around obstacles in the slip plane. Thus, dislocations trapped in a pileup against obstacles such as subgrain or grain boundaries can escape by climb and reariange themselves; the relaxation of the back stress enables the dislocation source to resume operation and deformation continues [34]. When the rate of dispersion of dislocations is approximately equal to the rate at which they are pumped into the pileup, a steady-state creep rate is established. The rate-controlling step in this process is vacancy diffusion, a fact which, according to Stokes [34], has been well established for all metals for which accurate data are available [60].

In a detailed analysis of the dislocation climb mechanism, Weertman $[68,69]$ has shown that the creep rate $(\dot{\epsilon})$ varies with the applied stress $(\sigma)$ and temperature $(T)$ according to the following relationship [34]:

$$
\dot{\epsilon} \propto \sigma^{9 / 2} \frac{\exp (-U / K T)}{T}
$$

where $U$ is generally the activation energy for self diffusion. In celamics, the presence of two ion species having different self-diffusion activation energies, and the possibility that diffusion may occur in pairs, makes the interpretation of $U$ more difficult [34].

It is seen in eq (7) that the creep rate depends on a high power of the stress (4.5) in contrast to the first powder dependence of diffusional creep (eqs (3), (4), (5), and (6)),

A theory similar to Weertman's, but due to McLean, also involving dislocation climb, has been applied to $\mathrm{TiO}_{2}$ (rutile) [70] and gave a creep rate dependence on stress to the 1.7 power $[1]$.

Chang [71] reviewed creep data for single crystals and large grain size polycrystals of UC, TiC, $\mathrm{Al}_{2} \mathrm{O}_{3}$, and $\mathrm{BeO}$, all of which give creep rates proportional to the stress raised to a powder from 4 to 6 ; Chang proposed a theory in which the creep rate is controlled by the dissolution by diffusion of debris left behind by moving dislocation [1]. Chang's theory gives a dependence of creep rate according to the fourth or fifth power of stress, depending upon which mechanism of dissolution is assumed. Weertman [72] has also derived creep rate equations for the case where the motion of individual dislocations is rate controlling. Other recent work on creep equations derived from dislocation dynamics models is also cited by Wachtman [1].

Stokes [34] outlined the work of Rogers et al., Kronberg, and Chang on the deformation of single-crystal alumina at elevated temperatures which has been related to dislocation motion. Briefly, in the temperature range of 900 to $1700 \mathrm{C}$, basal slip by simple slip on the basal plane, with an activation energy of $85 \mathrm{kcal} /$ mole, appears to be the rate-controlling mechanism, whereas dislocation climb, with an activation energy of $180 \mathrm{kcal} / \mathrm{mole}$ and a stress dependence of 4.5 , appears to be rate controlling at $1800 \mathrm{C}$ for single crystals of alumina.

\subsection{Creep by Grain Boundary Sliding}

Grain boundary sliding has been inferred from internal friction measurements and dynamic elastic modulus measurements at high temperatures [1]. Very little direct evidence exists, however, of the quantitative nature of grain boundary sliding in ceramics. It has usually been thought of as a separate process, due possibly to either the presence of an impure glassy phase or controlled by vacancy diffusion or even dislocation motion through the disordered boundary region [34]. Stokes [34] cites that the creep rate due to this process should be roughly proportional to the applied stress and inversely proportional to the grain size, i.e.,

$$
\dot{\epsilon} \propto \sigma / G S .
$$


On the other hand, Dorn [60] has shown that grain-boundary creep in metals is not a distinct mechanism but should follow the same stress and temperature relationships as intragranular creep. The recent efforts of Gifkins [39] to describe a stress-directed diffusional creep mechanism for grain boundary sliding have also been described in an earlier section (3.1).

Prior to Gifkins' work [39], it had generally been considered that grain boundary sliding could not contribute to deformation of a continuous nature without auxiliary processes, since intergranular deformation should occur coincidentally in order to maintain geometrical continuity between the grains [57]. Under such conditions, it has been argued that pores and eventually cracks would form at triple points, leading to overall fracture. Clearly, considerable additional study of grain boundary sliding mechanisms in ceramics is required.

\subsection{Creep of Glasses}

As cited by Wachtman [1], oxide glasses at high temperatures deform viscously. In this case, there is no detailed atomic model comparable to matter transport by vacancy motion in crystals undergoing Nabarro-Herring creep. However, viscosity in glass is related to self-diffusion and the flow is enhanced by an increase in free volume in a manner analogous to an increase in the NabarroHerring creep rate by an increase of vacancy concentration [1]. A theory taking into account both free volume and thermal activation effects has been advanced by Macedo and Litovitz [73]. The resulting equation correctly describes viscosity as a function of temperature over many orders of magnitude in viscosity. An alternate approach for associated liquids has been given by Adams and Gibbs [74].

\subsection{Effect of Porosity on Creep}

Insofar as possible, the major influences of structural factors, such as grain size, have been covered in the preceding sections. The influence of porosity, however, has not been mentioned and is briefly considered here.

An increase in the creep rate with porosity has long been recognized. Wygant [75], for example, found that the creep rate of a 12 percent porosity slip cast magnesia specimen was 10 times faster than a 2 percent porosity magnesia specimen; both specimens were tested at $1300 \mathrm{C}$ and 1800 psi. Coble and Kingery [76] studied the effect of porosity on the creep rate of sintered alumina with up to nearly 50 percent porosity; their results revealed that the torsional creep rate increased from $1.5 \times 10^{-6} \mathrm{in} / \mathrm{in} / \mathrm{hr}$ to $7.0 \times 10^{-5} \mathrm{in} / \mathrm{in} / \mathrm{hr}$ for an increase from 8 to 50 percent porosity under a stress of 600 psi at $1275 \mathrm{C}$.

Several functional relationships for the relation between creep rate and porosity have recently been suggested, largely as an outgrowth of at- tempts to describe hot pressing as a diffusional creep process. For example, Spriggs and Vasilos [77] suggested a linear relation between creep rate and porosity $\left(1 /\left(1-P^{2 / 3}\right)\right)$ when applied to the data of Coble and Kingery [76]. However, when applied by Vasilos and Passmore [57] to magnesia with porosity from 1 to 17 percent, a nonlinear relationship was observed. It is concluded that further study is required before specific comments can be made about observed differences and suggested relationships between creep rate and porosity.

\subsection{Combined Deformation Processes}

As suggested earlier, the observed deformation is frequently the result of more than one process and interactions also frequently occur. Deformation of a polycrystalline ceramic at high temperature is thus a complex process with several of the deformation processes previously discussed probably operating simultaneously.

At small grain sizes, low stresses, and high temperatures, Nabarro-Herring creep and grain boundary sliding probably take place together in most ceramics of commercial purity [1]. At larger grain sizes and higher stresses, a combination of grain boundary sliding and dislocation deformation may occur [1]. The transition with increasing grain size in alumina from creep controlled by the Nabarro-Herring mechanism to that controlled by dislocation glide has been suggested. [46]. Creep of polycrystalline thoria has been interpreted as grain boundary sliding plus dislocation motion [78]. Other examples have also been given.

It has generally been considered that [1], when creep occurs by more than one mechanism operating simultaneously, the rate will be controlled by the slower process if the faster process is unable to provide all the degrees of freedom needed to maintain contact between adjacent grains; too great a disparity between the rates will generally lead to the development of high tensile stresses of some grain boundaries and will favor the development of pores with attendant increase of creep rate and loss of strength [1].

An alternate approach to compound deformation has recently been suggested by Backofen et al. [79], in an effort to devise a comprehensive model to explain the phenomenon of superplasticity. Basically, a three-element mechanical model has been proposed with two elements in parallel (one of which is Newtonian in nature) and a third in series with that pair. A strongly developed Bingham-type behavior at low strain rates has been interpreted as a result of the parallel operation of diffusional creep and grain-boundary shear. The third element operates at high stresses or high strain rates and may be associated with plastic slip creep, e.g., dislocation climb. The three mechanisms in competition would thus produce the full strain-rate hardening behavior of a superplastic material and be capable of describing 
the three-branch log stress-log strain rate curve frequently observed for such materials.

\section{Summary}

The state-of-the-art of understanding the inelastic deformation of oxide ceramics has been briefly reviewed. Special emphasis was given to the permanent deformation caused by phenomena such as stress-enhanced diffusional creep, dislocation motion, and grain-boundary sliding. Brief reference was also made to recoverable inelastic deformation, especially anelasticity caused by point defect motion, and recoverable inelastiv deforination caused by both dislocations and grain boundary sliding. The information given dealt largely with polycrystalline oxide ceramics.

The primary mechanism by which most polycrystalline oxide ceramics appear to deform at high temperatures, relatively low stresses, and fine grain sizes was cited as Nabarro-Herring diffusional creep. Under most experimental conditions, however, it was recognized that the observed deformation is usually the result of more than one process, with complicating interaction frequently occurring, e.g., grain boundary sliding and Nabarro-Herring creep. At larger grain sizes and higher stresses, combinations such as grain boundary sliding and dislocation deformation may occur, but considerably less data are available to substantiate this.

A host of structural and compositional factors, as well as stress and temperature, influence the responsible mechanisms. Information concerning such influences exists for only a few ceramics, e.g., $\mathrm{Al}_{2} \mathrm{O}_{3}, \mathrm{MgO}, \mathrm{BeO}, \mathrm{SiC}$, and in no case is the information complete and unambiguous. The need for additional studies in essentially every area considered is clearly indicated.

\section{References}

[1] Wachtman, J. B., Jr., Mechanical properties of ceramics: An introductory survey, Am. Ceram. Soc. Bull. 46 (8) 756-774 (1967).

[2] Wachtman, J. B., Jr., Elastic deformation of crystalline ceramics and other refractory materials, this Syposium.

[3] Hollox, G. E., Inelastic deformation of non-oxide ceramics, this Symposium.

[4] Southgate, P. D., Mechanical relaxation of a point defect in magnesium oxide, J. Appl. Phys. 36, 2966 (1965).

[5] Southgate, P. D., Mendelson, K. S., and DePerro, P. L., Kilocycle-range dislocation damping in magnesium oxide, J. Appl. Phys. 37, 206 (1966).

[6] Chang, R., The elastic and anelastic properties of refractory materials for high-temperature applications, Chapter 14 in Mechanical Properties of Engineering Ceramics, Interscience, 1961.

[7] Wachtman, J. B., Jr., and Corwin, W. C., Internal friction in $\mathrm{ZrO}_{2}$ containing $\mathrm{CaO}$, J. Res. NंBS 69A, 457 (1965).

[8] Wachtman, J. B., Jr., Mechanical and electrical relaxation in $\mathrm{ThO}_{2}$ containing $\mathrm{CaO}$, Phys. Rev. 131, 517 (1963).
[9] Carnahan, R. D., and Brittain, J. O., Point-defect relaxation in rutile single crystals, J. Appl. Phys. 34, 3095 (1963).

[10] Wachtman, J. B., Jr., and Doyle, L. R., Internal friction in rutile containing point defects, Phys. Rev. 135, A276 (1964).

[11] Wachtman, J. B., Jr., Spinner, S., Brower, W. S., Fridinger, T., and Dickson, R. W., Internal friction in rutile containing $\mathrm{Ni}$ or $\mathrm{Cr}$, Ibid. 148, 811 (1966).

[12] Johnson, H. B., Tolar, N. J., Miller, G. R., and Cutler, I. B., Dipole relaxation in $\mathrm{CaF}_{2}$ doped with $\mathrm{NaF}$, J. Am. Ceram. Soc. 49 (8) 458 (1966).

[13] Cook, R. K., and Breckenridge, R. G., Anelasticity of quartz, Phys. Rev. 92, 1419 (1953).

[14] Van Houten, S., Mechanical losses in Li-doped $\mathrm{NiO}$ semiconductors, Phys. Chem. Solids 23, 1045 (1962).

[15] Astbury, N. F., and Davis, W. R., Internal friction in ceramics, Trans. Brit. Ceram. Soc. 63, 1 (1964).

[16] Kirby, P. L., XIX-Internal friction in glass. Part II, flexural and torsional vibrations, J. Soc. Glass Technol. 38, 383 (1954).

[17] Blum, S. L., Some physical factors affecting the internal dumping of glass, J. Am. Ceram. Soc. 38 (6) $205-10(1955)$.

[18] Kerper, M. J., and Scuderi, T. G., Mechanical properties of chemically strengthened glasses at elevated temperatures, Ibid. 49 (11) 613-18 (1966).

[19] Macedo, P. B., and Litovitz, T. A., Ultrasonic viscous relaxation in molten boron trioxide, Phys. Chem. Glasses 6, 69 (1965).

[20] Pratt, P. L., The role of point defects in mechanical properties, Proc. Brit. Ceram. Soc. 1, 177 (1964).

[21] Niblett, D. H., and Wilks, J., Dislocation damping in metals, Advan. Phys. 9, 1 (1960).

[22] Granato, A. V., Internal friction studies of dislocations; paper in Internal Friction, Damping and Cyclic Plastic, ASTM S.T.P. No. 378, 1965.

[23] Seeger, A., and Schiller, P., Kinks in dislocation lines and their effect on the internal friction in crystals; p. 361 in Physical Acoustics, Vol. IIIA, Academic Press, 1966.

[24] Chang, R., Dislocation relaxation phenomena in oxide crystals, J. Appl. Phys. 32, 1127 (1961).

[25] Carnahan, R. D., and Brittain, J. O., Dislocation damping in rutile single crystals, J.'Am. Ceram. Soc. 48 (1) $15-19$ (1966).

[26] Southgate, P. D., Mendelson, K. S., and DePerro, P. L., Kilocycle-Range Dislocation Damping in Magnesium Oxide, J. Appl. Phys. 37, 206 (1966).

[27] Fiore, N. F., A test of viscous dislocation damping theory, J. Appl. Phys. 3\%, 878 (1966).

[28] Wachtman, J. B., Jr., and Lam, D. G., Jr., Young's modulus of various refractory materials as a function of temperature, J. Am. Ceram. Soc. 42 (5) 254-60 (1959).

[29] Crandall, W. B., Chung, D. H., and Grey, T. J., Mechanical properties of ultra-fine hot-pressed alumina, Chapter 22 in Mechanical Properties of Engineering Ceramics, Interscience, 1961.

[30] Turnbaugh, J. E., Effect of additions on the hightemperature grain-boundary relaxation of alumina; Sc.D. Thesis, MIT, 1962.

[31] Stuart, J., Internal friction and creep in polycrystalline alumina; Task 5 in Studies of the Brittle Behavior of Ceramic Materials, Report ASD-TR-61-628, Part III, June 1964.

[32] Wachtman, J. B., Jr., and Maxwell, L. H., Factors controlling resistance to deformation and mechanical failure in polycrystalline (glass-free) ceramics, WADC Tech. Rept. No. 57-526, ASTIA Document No. AD 142245, 1957.

[33] Beauchamp, E. K., Baker, G. S., and Gibbs, P., Impurity dependence of creep aluminum oxide, Tech. Documentary Rept. No. ASD TR 61-481, University of Utah, April 1962.

[34] Stokes, R. J., Correlation of mechanical properties with microstructure, Chapter 4 in Microstructure 
of Ceramic Materials, NB5 Miscl. Publ. 257, April 1964.

[35] Nabarro, F. R. N., p. 75 in Report of a Conference on the Strength of Solids. Physical Society, London, 1948.

[36] Herring, C., Diffusional viscosity of a polycrystalline solid, J. Appl. Phys. 21, 437 (1950).

[37] Farnsworth, P. L., and Coble, R. L., Deformation behavior of dense polycrystalline SiC, J. Am. Ceram. Soc. 49 (5) 264-68 (1966).

[38] Coble, R. L., Model for boundary diffusion controlled creep in polycrystalline materials, J. Appl. Phys. 34, 1679 (1963).

[39] Gifkins, R. C., Diffusional creep mechanisms, J. Am. Ceram. Soc. 51 (2) 69-72 (1968).

[40] Gifkins, R. C., and Snowden, K. U., Mechanism for "viscous" grain-boundary sliding, Nature $\mathbf{2 1 2}$ (5065) 916-17 (1966).

[41] Ryan, H. J., and Suiter, J. W., Grain boundary topography of tungsten, Phil. Mag. 10, 727-29 (Oct. 1964).

[42] Gifkins, R. C., and Snowden, K. U., Stress sensitivity of creep of lead at low stresses, Trans. AIME 239 (6) 910-15 (1967).

[43] Gifkins, R. C., Superplasticity during creep, J. Inst. Metals 95 (12) 373-77 (1967).

[44] Hensler, J. H., and Cullen, G. V., Grain shape change during creep in magnesium oxide, J. Am. Ceram. Soc. 50, No. 11, 584-85 (1967).

[45] Folweiler, R. C., Creep behavior of pore-free polycrystalline aluminum oxide, J. Appl. Phys. 32, 773 (1961)

[46] Warshaw, S. J., and Norton, F. H., Deformation behavior of polycrystalline aluminum oxide, J. Am. Ceram. Soc. 45, No. 10, 479-86 (1962).

[47] Coble, R. L., and Guerard, Y. H., Creep of polycrystalline aluminum oxide, Ibid. 46, No. 7, 353-54 (1963).

[48] Coble, R. L., and Ellis, J. S., Hot pressing aluminamechanisms of material transport, Ibid. 46, No. 9. 438-41.

[49] Passmore, E. M., and Vasilos, T., Creep of dense, pure, fine-grained aluminum oxide, Ibid. 49, No. 3, 166-68 (1966).

[50] Vasilos, T., Mitchell, J. B., and Spriggs, R. M., Creep of polycrystalline magnesia, Ibid. 4\%, No.4, 203-204 (1964).

[51] Chang, R., High temperature creep and anelastic phenomena in polycrystalline refractory oxides, J. Nucl. Mater. 1, 174 (1959).

[52] Vandervoort, R. R., and Barmore, W. L., Compressive creep of polycrystalline beryllium oxide, J. Am. Ceram. Soc. 46, No. 4, 180-84 (1963).

[53] Fryxell, R. E., and Chandler, B. A., Creep, strength, expansion, and elastic moduli of sintered $\mathrm{BeO}$ as a function of grain size, porosity, and grain orientation, Ibid. $4 \%$, No. 6, 283-91 (1964).

[54] Vandervoort, R. R., and Barmore, W. L., Compressive creep of beryllium oxide-uranium dioxide mixtures, Ibid. 48, No. 11, 587-90 (1965).

[55] Kingery, W. D., and Montrone, E. D. diffusional creep in polycrystalline $\mathrm{NaCl}$, J. Appl. Phys. 86, 2412 (1965).

[56] Paladino, A. E., and Coble, R. L., Effect of grain boundaries on diffusion controlled processes in aluminum oxide, J. Am. Ceram. Soc. 46, No. 3, 133-36 (1963).

[57] Vasilos, T., and Passmore, E. M., Effect of microstructure on creep mechanisms in ceramics, Chap- ter 6 in Strengthening Mechanisms, Metals and Ceramics, Syracuse Univ. Press, 1966.

[58] Chang, R., High temperature creep and anelastic phenomena in polycrystalline materials, J. Nucl. Matls. 1, 174 (1959).

[59] Spriggs, R. M., Passmore, E. M., Brissette, L. A., and Vasilos, T., Microstructure studies in polycrystalline refractory oxides, Progress Report, Bureau of Naval Weapons, Contract NOw 64-0217-d (1964).

[60] Dorn, J. E., Creep and Recovery, Am. Soc. Metals, Cleveland (1957).

[61] Passmore, E. M., Duff, R. H., and Vasilos, T., Creep of dense, polycrystalline magnesium oxide, J. Am. Ceram. Soc. 49 (11) 594-600 (1966).

[62] Oishi, Y., and Kingery, W. D., Oxygen diffusion in periclase crystals, J. Chem. Phys. 33, 905-906 (1960).

[63] Lindner, R., and Parfitt, G. D., Diffusion of radioactive magnesium in magnesium oxide crystals, Ibid. 26, 182 (1957).

[64] Taylor, G. I., Plastic Strain in Metals, J. Inst. Metals 62, 307 (1938).

[65] Groves, G. W., and Kelly, A., Independent slip systems in crystals, Phil. Mag. 8, 877 (1963).

[66] Copley, S. M., and Pask, J. A., Plastic deformation of MgO single crystals up to $1600^{\circ} \mathrm{C}$, J. Am. Ceram. Soc. 48 (3) 139-145 (1965).

[67] Day, R. B., and Stokes, R. J., The mechanical behavior of magnesium oxide at high temperatures, Ibid. 47, 493 (1964).

[68] Weertman, J., Theory of steady-state creep based on dislocation climb, J. Appl. Phys. 26, 1213 (1955).

[69] Weertman, J., Steady-state creep through dislocation climb, Ibid. 28, 362 (1957).

[70] Hirthe, W. M., and Brittain, J. O. High-temperature steady-state creep in rutile, J. Am. Ceram. Soc. 46 (9) 411-17 (1963).

[71] Chang, R., Dislocation theories of the high-temperature creep of crystalline solids; paper in The Physics and Chemistry of Ceramics, Gordon and Breach (1963).

[72] Weertman, J., Steady-State creep of crystals, J. Appl. Phys. 28, 1185 (1957).

[73] Macedo, P. B., and Litovitz, T. A., On the relative roles of free volume and activation energy in the viscosity of liquids, J. Chem. Phys. 42, 245 (1965).

[74] Adam, G., and Gibbs, J. H., On the temperature dependence of cooperative relaxation properties in glass-forming liquids, Ibid. 43, No. 1, 139-46 (1965):

[75] Wygant, J. F., Elastic and flow properties of dense, Pure oxide refractories, J. Am. Ceram. Soc. 34, 374 (1951).

[76] Coble, R. L., and Kingery, W. D., Effect of porosity on physical properties of sintered alumina, Ibid. 39, 377 (1956).

[77] Spriggs, R. M. and Vasilos, T., Functional relaxation between creep rate and porosity for polycrystalline ceramics, Ibid. 47, No. 1, 47-48 (1964).

[78] Poteat, L. E., and Yust, C. S., Creep of polycrystalline thorium dioxide, Ibid. 49 (8) 410-14 (1966).

[79] Backofen, W. A., Azzarto, F. J., Murty, G. S., and Zehr, S. W., Deformation processing of anisotropic metals-superplasticity, Final Report on Contract N000 19-67-C-0089, Sept. 1967. 


\title{
Plastic Deformation of Carbides
}

\author{
Graham E. Hollox* \\ Research Institute for Advanced Studies (RIAS), \\ Martin Marietta Corporation, 1450 South Rolling Road, Baltimore, Maryland 21227
}

\begin{abstract}
A significant research effort is being directed towards obtaining technologically useful materials for high-temperature structural applications. The refractory metal carbides are of particular interest since (a) they include the materials having the highest melting point, (b) they are extremely strong, and (c) they deform plastically in a manner similar to the face centered cubic metals. This paper reviews the current understanding of the mechanical behavior of the carbides. It concentrates on evaluating the more meaningful information obtained from studies of single crystals rather than that from sintered polycrystalline materials, where inadequate characterization may have led to confusion in the literature. The present knowledge of the behavior of these materials suggests that they may be of technological importance in the future.
\end{abstract}

Key Words: Carbides; high-temperature; mechanical behavior; microstructure; plastic deformation.

\section{Introduction}

The need for improved materials for hightemperature structural applications has stimulated research into the mechanical behavior of high melting-point ceramics-carbides, borides, nitrides, aluminides, beryllides, silicides, etc. The properties of these materials have been discussed by, for example, Schwartzkopf and Kieffer [1], ${ }^{1}$ and Kendall [2]. Additionally, several tabulations of properties are available [3, 4, 5]. Material characteristics such as microstructural details have recently been clearly recognized as variables importantin determining the properties of ceramics [6]. Many of the materials used in past work on the carbides have lacked sufficient characterization and the results therefore need careful evaluation if an understanding of their properties is to be reached. It is evident that more scientifically meaningful information has been obtained from studies of single crystals of the carbides than has been obtained by some 50 years research on sintered, polycrystalline material. Although the value of this latter work in producing technologically useful material is not to be underrated or denied, the prospects for significant future developments of structural carbides will be considerably enhanced by an understanding of the relationship between microstructure and mechanical behavior.

The study of plastic deformation in ceramics is comparatively new, but some cubic materials,

\footnotetext{
*Present address: Brown Boveri Research Center, Baden, Switzerland.

Present address: Brown Boveri Research Center, Baden, Switzerland.
1 igures in brackets indicate the literature references at the end of this paper.
}

for example, $\mathrm{MgO}, \mathrm{NaCl}$, and $\mathrm{LiF}$ have been studied in recent years $[7,8,9]$. Much of this work is applicable to the even newer field of plastic deformation in the carbides. With the exception of the hexagonal carbide, WC $[10,11,12]$, plastic deformation studies appear to have been restricted to single crystals of cubic monocarbides. No equivalent effort on similarly characterized material has been directed towards understanding the other non-oxide ceramics, although single crystals of several diborides have been obtained and their structure examined $[13,14]$. Consequently, in this review the relationship between structure and properties of the carbides will be emphasized. The occurrence of these materials has been well documented $[1,15]$, and it is summarized in figure 1 . The titanium-carbon equilibrium diagram, figure 2, which is representative of the equilibrium between Group IV metals and carbon, illustrates the typical wide range in composition observed in these materials [15].

\section{Mechanical Behavior of Carbide Single Crystals}

\subsection{Titanium Carbide}

Single crystals of $\mathrm{TiC}$ deformed in compression or bending at temperatures below about $800^{\circ} \mathrm{C}$ are completely brittle [16]. No macroscopic plastic flow has been detected by etch-pit or slip line observations. As expected, the observed fracture strength is very dependent upon surface condition, but values as high as 800,000 psi ( 0.4 of the shear 


\begin{tabular}{|c|c|c|c|c|}
\hline $\mathrm{ScC} f \mathrm{fc}$ & $\mathrm{TiC} f \mathrm{fcc}$ & $\begin{array}{l}\text { VC fcc } \\
V_{2} C \begin{array}{l}\text { ortho } \\
\text {-hex }\end{array}\end{array}$ & $\begin{array}{l}\mathrm{Cr}_{23} \mathrm{C}_{6} \\
\mathrm{Cr}_{7} \mathrm{C}_{3} \\
\mathrm{Cr}_{3} \mathrm{C}_{2}\end{array}$ & $\begin{array}{l}\text { cubic } \\
\text { hex } \\
\text { ortho }\end{array}$ \\
\hline $\begin{array}{l}Y_{3} C \text { cubic } \\
Y C- \\
Y_{2} C_{3}- \\
Y C_{2} \text { tetrog }\end{array}$ & $\mathrm{ZrC}$ fcc & $\begin{array}{r}\mathrm{Nb}_{2} \mathrm{C} \text { ortho } \\
\text {-hex }\end{array}$ & $\mathrm{MO}_{2} \mathrm{C}$ & $\begin{array}{l}\text { ortho } \\
\text {-hex }\end{array}$ \\
\hline & $\mathrm{HfC}$ fcc & $\begin{array}{l}\text { TaC fcc } \\
\mathrm{To}_{2} \mathrm{C} \text { ortho } \\
\text { - hex }\end{array}$ & $\begin{array}{l}w_{2} C \\
\alpha-w C \\
\beta-w C\end{array}$ & $\begin{array}{l}\text { hex } \\
\text { hex } \\
\text { fcc }\end{array}$ \\
\hline $\begin{array}{l}\text { LANTHANIDES } \\
\text { ACTINIDES }\end{array}$ & \multicolumn{2}{|c|}{ 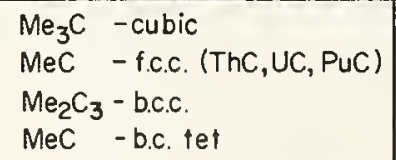 } & \multicolumn{2}{|c|}{$\begin{array}{r}\text { OTHERS... } \mathrm{Fe}_{3} \mathrm{C} \text {-cubic } \\
\mathrm{B}_{4} \mathrm{C}-\text {-hex } \\
\alpha-\mathrm{SiC}-\text { hex } \\
\beta-\text { SiC-f.c.C. }\end{array}$} \\
\hline
\end{tabular}

FIGURE 1. The occurrence of refractory carbides.

This review will be primarily concerned with the mechanical behavior of the cubic refractory carbides and these are indicated in heavier print.

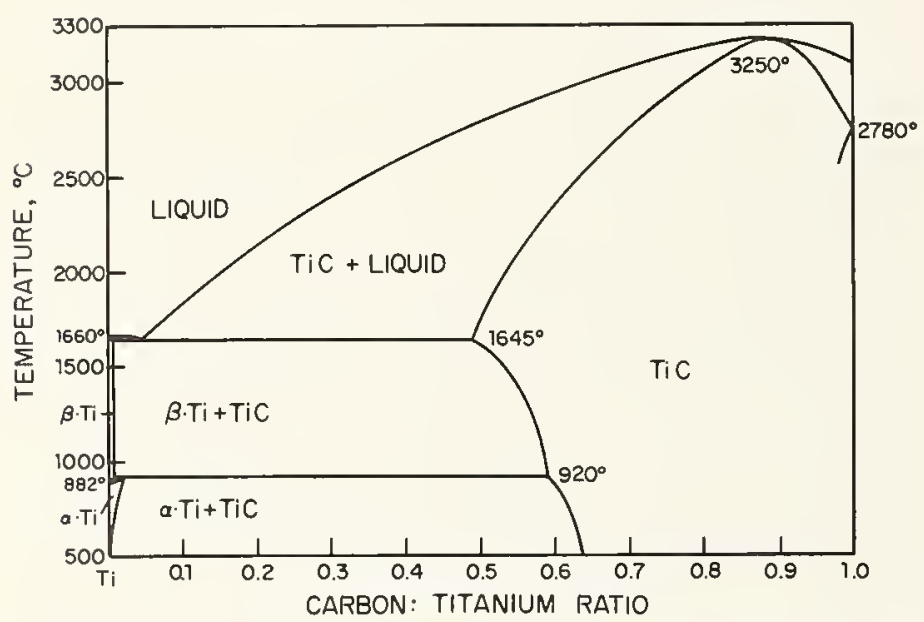

FIgURE 2. The titanium-carbon equilibrium diagram.

The TiC phase exists over a range in composition by incorporating vacancies in the carbon sublattice (after Storms [15]).

modulus) have been measured on carefully electropolished samples. Failure occurs by cleavage on $\{100\}$ planes initiated at surface or internal defects, although the possibility that microcracks are produced by dislocation interactions cannot be completely eliminated. Evidence for some dislocation motion at room temperature has been obtained from the surface markings close to Knoop microhardness indentations [17] or close to friction tracks [18], and these are consistent with slip on $\{111\}$ planes. Also, Williams [17] has shown that the microhardness number is dependent on the orientation of the indenter with respect to the active slip planes in $\mathrm{TiC}$, suggesting that dislocations participate in room temperature deformation.
At testing temperatures above 800 to $900^{\circ} \mathrm{C}$, plastic deformation takes place on the $\{111\}$ $\langle\overline{1} 0\rangle$ system $[16,19]$. The critical resolved shear stress for slip is very dependent upon carbon content, figure 3 . For example, at $900^{\circ} \mathrm{C}$ it increases linearly from about $12 \mathrm{~kg} / \mathrm{mm}^{2}$ for $\mathrm{TiC}_{0.79}$, to about $22 \mathrm{~kg} / \mathrm{mm}^{2}$ for $\mathrm{TiC}_{0.97}$ [20].

Vacancies have been commonly recognized as a cause of hardening in crystal lattices. For example, the yield strength of quenched aluminum is greater than annealed aluminum as a result of dislocationvacancy interactions [21]. Also, nonstoichiometric $\mathrm{TiO}_{2}$ is stronger than the stoichiometric composition because of the interactions between dislocations and vacancies [22, 23] or clustered vacancies [24]. In $\mathrm{TiC}$, however, the decrease in strength as the concentration of vacancies increases is attributed to a decrease in the contribution made by carbon atoms to cohesion in TiC. The nature of the electronic interactions between constituent atoms in the lattice has been deduced from studies of the band structure of this material. Lye [25] has shown that the predominant contribution to the bonding is from covalent metal-metal bonds, the strength of which increase with carbon content because (i) carbon atoms donate electrons to crystal states derived from metal atom wave functions and increase the number of $3 d$-electrons available for metal-metal bonding, and (ii) the presence of carbon atoms in overlap regions of neighboring metal atom $3 d$ -

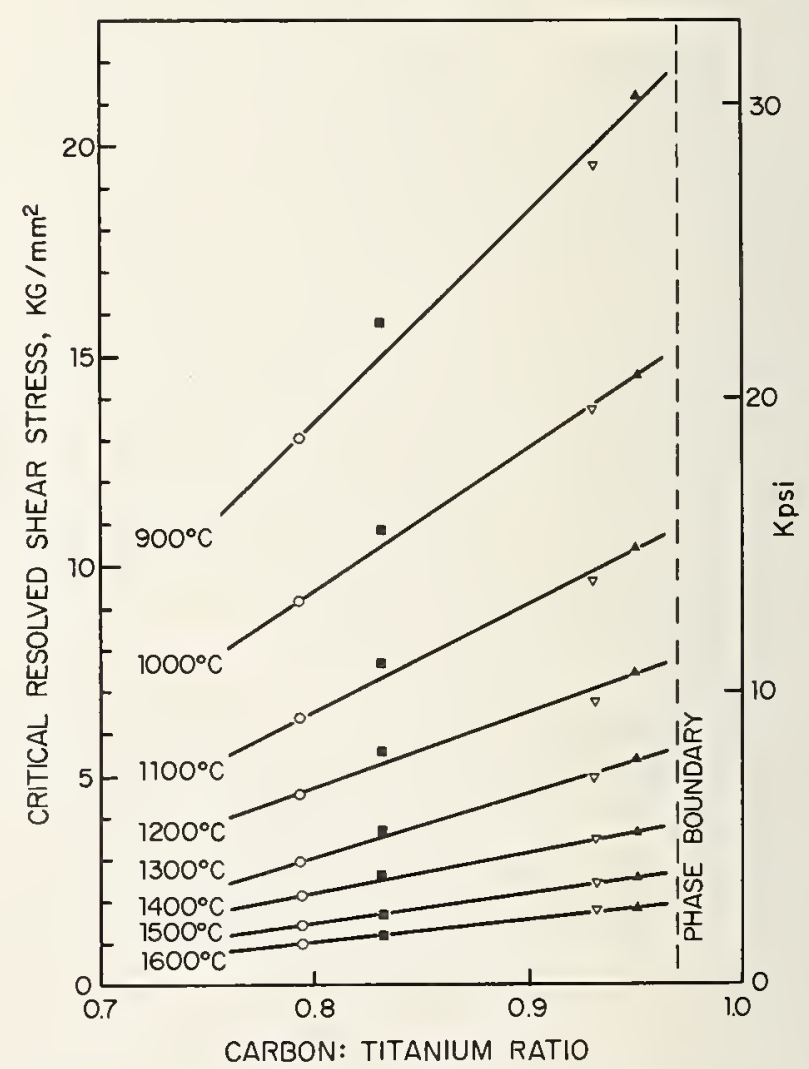

FIGURE 3. The critical resolved shear stress for slip in $\mathrm{TiC}$ as a function of carbon content.

(After Williams [20].) 


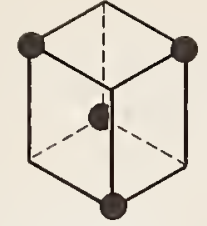

HYPOTHETICAL f.c.c. TITANIUM

Titonium

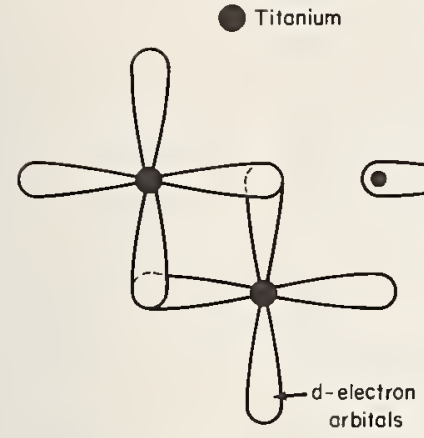

Flgure 4. Schematic representation of the bonding between $\pi$-oriented 3-d functions in $\mathrm{TiC}$ compared with that of hypothetical f.c.c. titanium.

This illustrates the position of carbon atoms in the overlap region between orbitals on neighboring atoms (after Lye [26]).

orbitals introduces a potential that increases the strength of the metal-metal interactions, figure 4. The observations of a $\{111\}<1 \overline{1} 0>$ slip system is consistent with the view that the structure should be considered as that of a f.c.c. metal rather than a rocksalt ionic compound.

The onset of bulk plastic flow in TiC is a gradual transition which involves an increasing strain at failure as the testing temperature is increased rather than the sharp brittle-to-ductile transition normally associated with bcc metals at low temperatures. It has been suggested that this gradual transition indicates that diffusion may play a role in determining the mobility of dislocations in $\mathrm{TiC}$ [27]. Some evidence to support this concept may be obtained by considering both the structure of the slip plane and the temperature dependence of the yield stress of TiC.

Figure 5 shows a plan of the $\mathrm{Ti}$ and $\mathrm{C}$ atoms on the $\{111\}$ planes of the TiC [28]. Following consideration of the synchro-shear process suggested by Kronberg [29], Rowcliffe [27] points out that for a unit of slip from $B_{1}$ to $B_{3}$, the titanium atom at $B_{1}$ must pass very close to that at $A_{1}$. This requires a large lattice expansion normal to the slip plane. Alternatively, if the carbon atom at $C_{1}$ can move in a $\langle 112\rangle$ direction to $B_{2}$ or $B_{3}$ at the same time as the titanium atom moves to $C_{1}$, then the unit displacement $B_{1}$ to $B_{3}$ can be accomplished by movements of partial dislocations by slip from $B_{1}$ to $C_{1}$ and $C_{1}$ to $B_{3}$. The motion of the carbon atom cannot be described by a single shear vector and will require the diffusion of carbon atoms to allow deformation to take place.

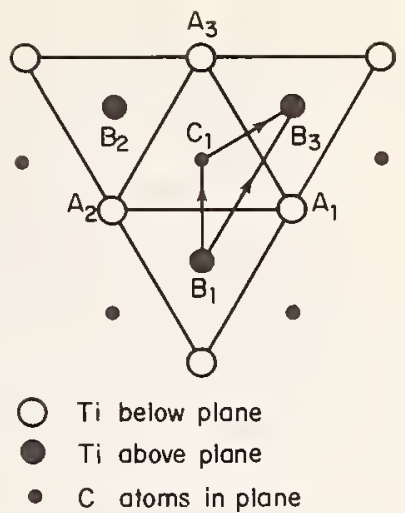

Figure 5. The slip plane
of TiC.
(After Kelly and Rowcliffe [28].)

The temperature dependence of the yield strength of $\mathrm{TiC}[20]$ is shown in figure 6 . In $\mathrm{TiC}_{0.97}$. the critical resolved shear stress $\sigma_{r}$ for slip on $\{111\}\langle 1 \overline{1} 0\rangle$ systems decreases from about 22 $\mathrm{kg} / \mathrm{mm}^{2}$ at $900^{\circ} \mathrm{C}$ to $2 \mathrm{~kg} / \mathrm{mm}^{2}$ at $1600^{\circ} \mathrm{C}$. Over limited temperature ranges, the relationship:

$$
\sigma_{\tau} \propto \exp \frac{B}{T}
$$

is applicable (fig. 8). This suggests that $B$ may be represented by $U / k$ where $U$ is the activation energy for plastic flow, and $k$ is the Boltzmann constant. Significantly, a characteristic change in the slope of this function occurs at a critical temperature [20] which is dependent on the carbon content of the TiC [19] i.e., it is approximately

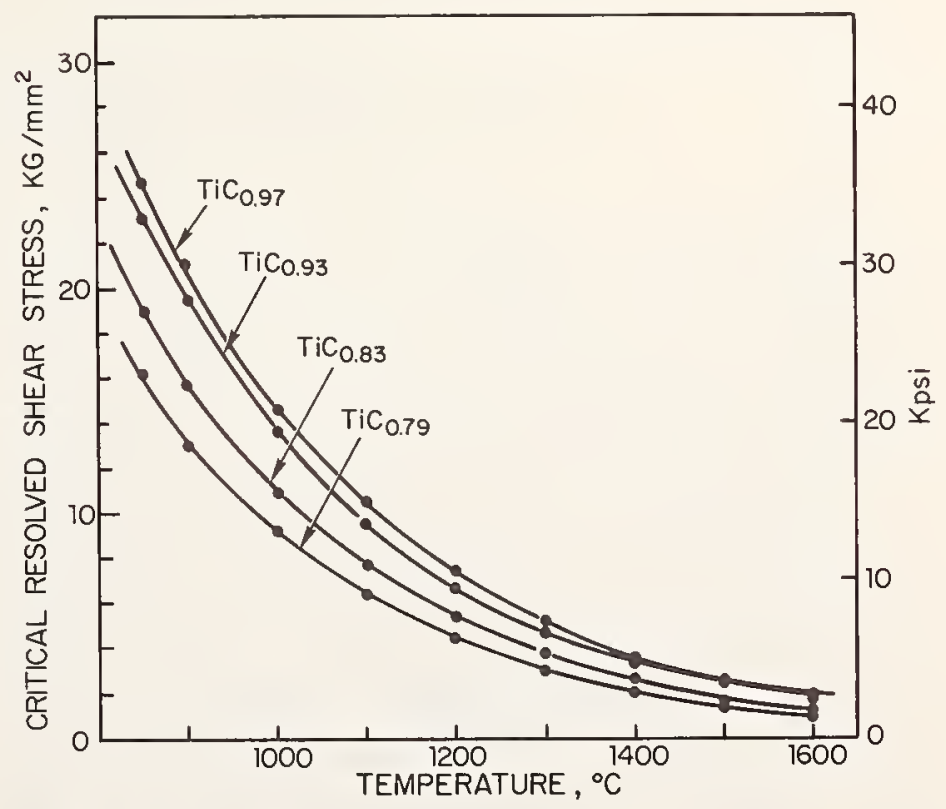

FIGURE 6. Temperature dependence of the critical resolved shear stress of $\mathrm{TiC}$ as a function of carbon content.

(After Williams [20].) 
$1150^{\circ} \mathrm{C}$ for $\mathrm{TiC}_{0.83}, 1305^{\circ} \mathrm{C}$ for $\mathrm{TiC}_{0.95}$, etc. Williams [17] has indicated that:

$$
\sigma_{r}{ }^{m} \propto \exp \frac{U}{k T}
$$

(where $m$ is the exponent in the relationship between dislocation velocity, $v$, and stress, $\sigma$; $v=\sigma^{m}[8]$ ) describes the variation in yield stress of $\mathrm{TiC}$ with temperature. Since the slope of the line in figure 7 must be multiplied by $m$, he suggests that the activation energy for plastic flow above about $1180^{\circ} \mathrm{C}$ in $\mathrm{TiC}$ is about $3.0 \mathrm{eV}$ [17]. Below the critical temperature the slope is almost halved, this being consistent with an activation energy for flow of about $1.5 \mathrm{eV}$.

Values of about $2.7 \mathrm{eV}$ and $5.1 \mathrm{eV}$ have been measured for the activation energy of carbon and titanium diffusion respectively in $\mathrm{TiC}[30,31]$, carbon having the lower activation energy consistent with its smaller size, interstitial position, and the presence of a large number of vacancies in its sublattice. Above about $0.5 T_{m}$, creep deformation appears to be controlled by titanium diffusion [33] and so it may be expected that the high-temperature deformation would exhibit an activation energy for flow of about 5.0 to $6.0 \mathrm{eV}$. This, however, is not observed, and as suggested by the mechanism proposed for dislocation motion [27], diffusion of the atomic species in the dislocation cores may have to be considered. In this context, Williams [17] has correlated the activation energy for flow $\sim 3.0 \mathrm{eV}$ with that for pipediffusion [32] of titanium atoms in TiC. Any correlations with the bulk activation energy for

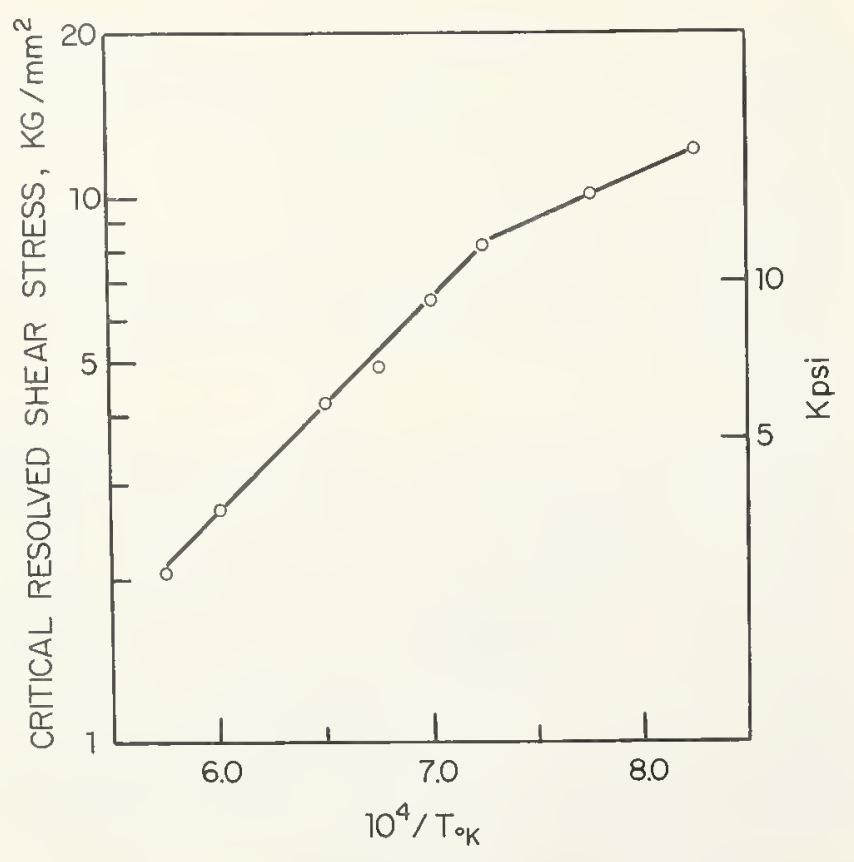

FIGURE 7. The temperature dependence of the yield stress of $\mathrm{TiC}_{0.83}$.

(After Williams [20].) The change in slope-close to $0.475 T_{m}-$ corresponds to a change in the mechanism controlling the deformation. diffusion of $\mathrm{C}$ and $\mathrm{Ti}$ in $\mathrm{TiC}$ must be regarded as tentative until more accurate measurements of these energies are available, particularly as a function of carbon content and in single crystal material.

Annealing of plastically deformed $\mathrm{TiC}$ is accompanied by the coalescence of vacancy dislocation loops, figure 8 [32]. The initial stages appear to be associated with the formation of trails of small loops, figure 8(b), produced from dislocation dipoles. The dislocation loop density as a function of isochronal annealing temperature for $\mathrm{TiC}_{0.97}$ and $\mathrm{TiC}_{0.88}$ is shown in figure 9. The "self-diffusion temperature," $T_{D}$, defined as the temperature at which loops disappear completely in a short time $(15 \mathrm{~min})$ anneal, is $1400^{\circ} \mathrm{C}$ for $\mathrm{TiC}_{0.97}$, and $1270^{\circ} \mathrm{C}$ for $\mathrm{TiC}_{0.88}$. The relationship $T_{D}=0.475 T_{m}$ [32] is obeyed in these alloys as it is in f.c.c. metals, and the activation energy for the annealing process involving the diffusion of vacancies is $5.25 \mathrm{eV}$ for $\mathrm{TiC}_{0.97}$ and $4.88 \mathrm{eV}$ for $\mathrm{TiC}_{0.88}$. The only reported value of the activation energy for the diffusion of titanium a toms in $\mathrm{TiC}, 5.1 \mathrm{eV}$, is in close agreement with these values [31].

It is significant to note that the "self-diffusion temperatures," $0.475 T_{m}$, are close to the values of the critical temperatures observed for the change in strength controlling mechanism. Above this temperature, therefore, it appears that some vacancy assisted process, involving dislocation climb, is controlling the deformation behavior of the material [19].

Measurements of the creep rate of large grained, polycrystalline $\mathrm{TiC}_{0.97}$ over the temperature range 1600 to $1700^{\circ} \mathrm{C}$ have indicated an activation energy of between 5 and $7 \mathrm{eV}$ [33]. Steady-state creep deformation is thought to be controlled by the climb of dislocations away from obstacles, a process governed by the activation energy for the diffusion of vacancies. This value is in reasonable agreement with the energy determined from loop annealing, and is consistent with titanium self-diffusion in TiC.

Williams [17] has applied Haasen's analysis [34] to the creep rate of $\mathrm{TiC}_{0.93}$, and deduces an activation energy of $3.3 \mathrm{eV}$ in the temperature range 1400 to $1700^{\circ} \mathrm{C}$. It would seem more reasonable to expect that the creep rate be governed by an activation energy of about $5 \mathrm{eV}$ in this temperature range, as titanium diffusion appears to control the strength in this temperature range, and consequently these results are not fully understood.

It has been mentioned already that the deformation characteristics of $\mathrm{TiC}$ are similar to those of a f.c.c. metal. In addition, this material has a high stacking-fault energy, since fringe contrast or dissociation of dislocations into partials has not been observed [19] (although this may occur within the width of the dislocation image, $\sim 100 \AA$ ). Moreover, the elongated dislocation loops 

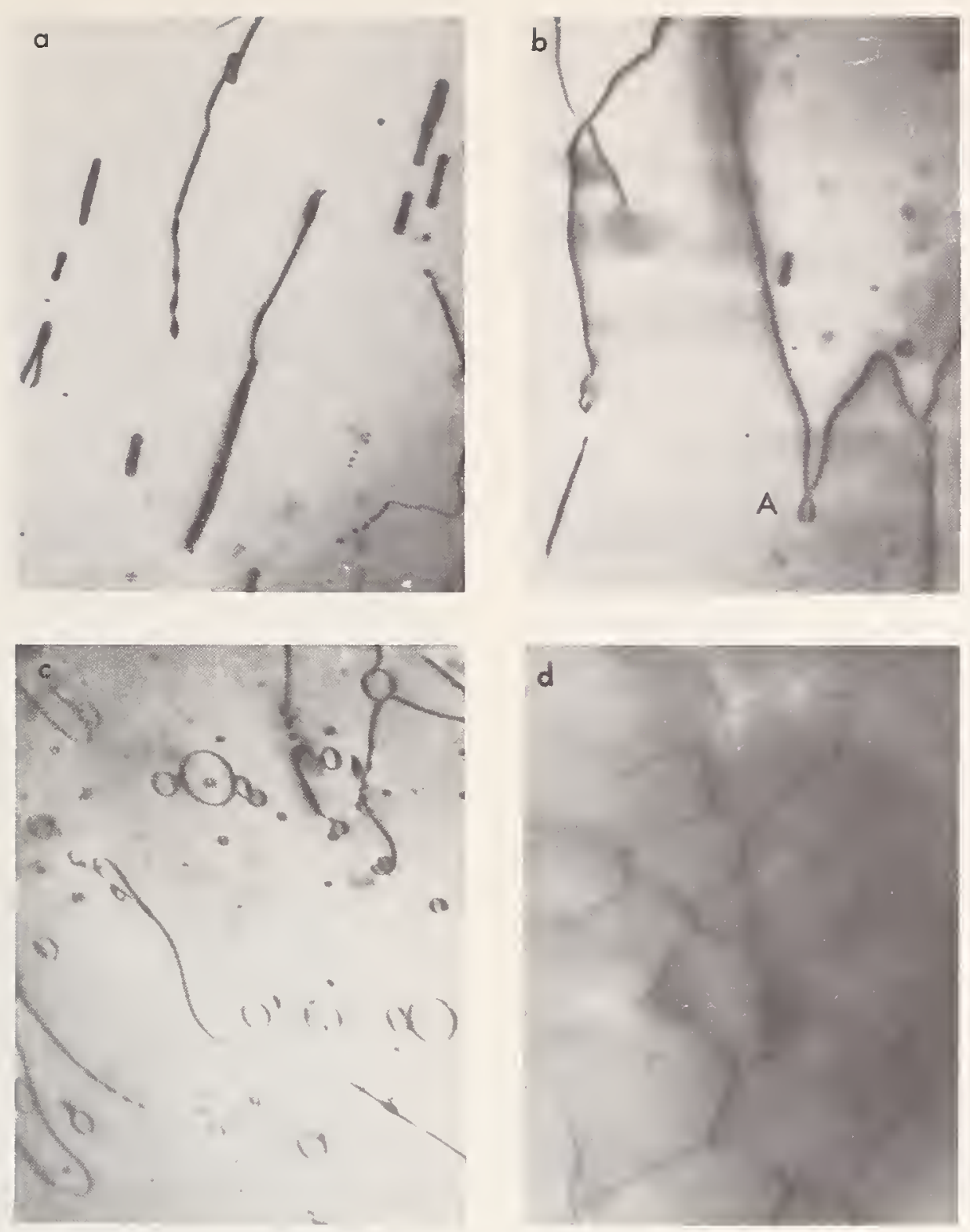

Figure 8. Dislocation structures in TiC.

(a) Early stage of deformation showing elongated dislocation loops, (b) annealing of an elongated loop, A, by pipe-diffusion along dislocation cores, (c) formation of vacancy dislocation loops on annealing at $1300^{\circ} \mathrm{C}$, (d) final annealed structure-a hexagonal dislocation network as observed in f.c.c. metals (after Hollox and Smallman [19, 23]).

observed in the early stages of deformation, figure $8(a)$, and also cell structures in more heavily deformed samples, are thought to result from a relative ease of cross slip in the material. The strong directional bonding in $\mathrm{TiC}$ probably accounts for the high stacking-fault energy of TiC, since the hexagonal symmetry in stacking faults in the f.c.c. structure requires a change in the bond directions. An alternative explanation relies on the observation that metals with a filled $d$-band have a lower stacking-fault energy than those with partially filled bands $[35,36]$. The latter case is applicable to $\mathrm{TiC}$, but no quantitative estimate of the stacking-fault energy has yet been made.

\subsection{Vanadium Carbide}

Although it is far from complete, the most detailed behavior of a carbide other than $\mathrm{TiC}$ has recently been established for VC [37, 38, 39].
When VC, having a carbon-to-metal ratio of 0.84 , is deformed in compression, the slip plane is $\{111\}$, exactly as is observed for TiC. Slip lines show a wavy appearance characteristic of a high stacking-fault energy material, and this also suggests that dislocation structures and slip mechanisms of $\mathrm{VC}_{0.84}$ are similar to those observed in TiC. On the basis of these observations, it was thought that the behavior of $\mathrm{TiC}$ might be representative of the behavior of all the cubic transition metal carbides. However, a generalization of this nature is invalid! Three significant differences in the behavior of this material and TiC of equivalent stoichiometry have been found (fig. 10): (i) the brittle-to-ductile transition temperature occurs between 1200 and $1250^{\circ} \mathrm{C}$, this being about 300 to $400{ }^{\circ} \mathrm{C}$ higher than that reported in $\mathrm{TiC}$; (ii) in the temperature range 1200 to $1500{ }^{\circ} \mathrm{C}$, the critical resolved shear stress for slip in $\mathrm{VC}_{0.84}$ is greater than that of $\mathrm{TiC}$; and (iii) the decrease 


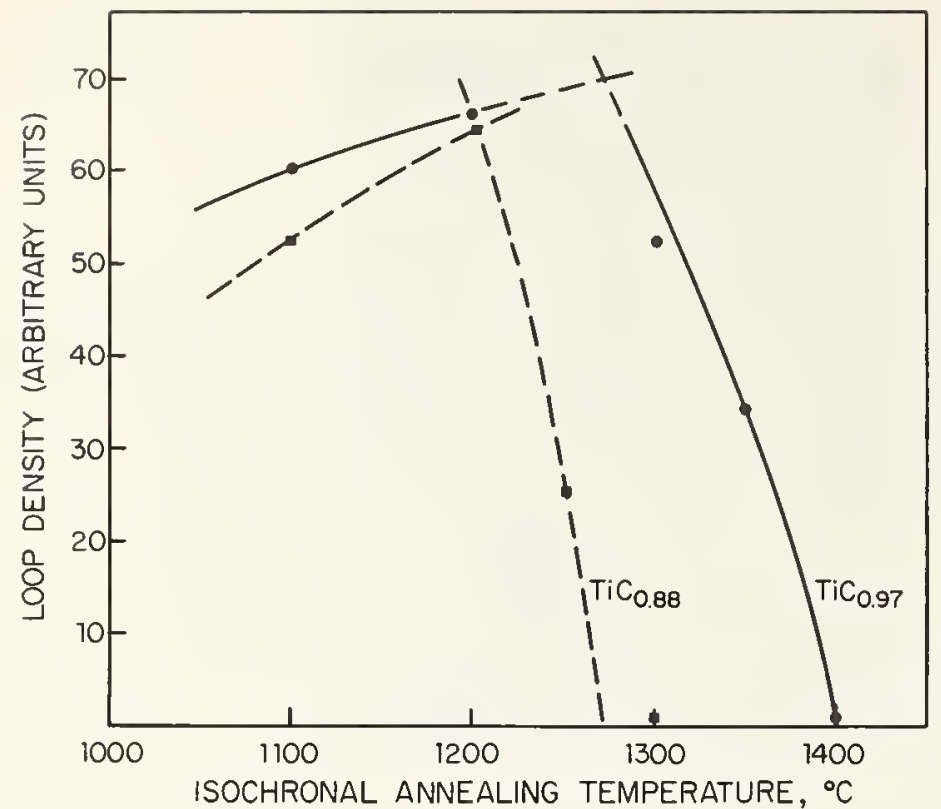

FIGURE 9. The variation in dislocation loop density as a function of isochronal annealing temperature in $\mathrm{TiC}$.

(After Hollox and Smallman [32].)

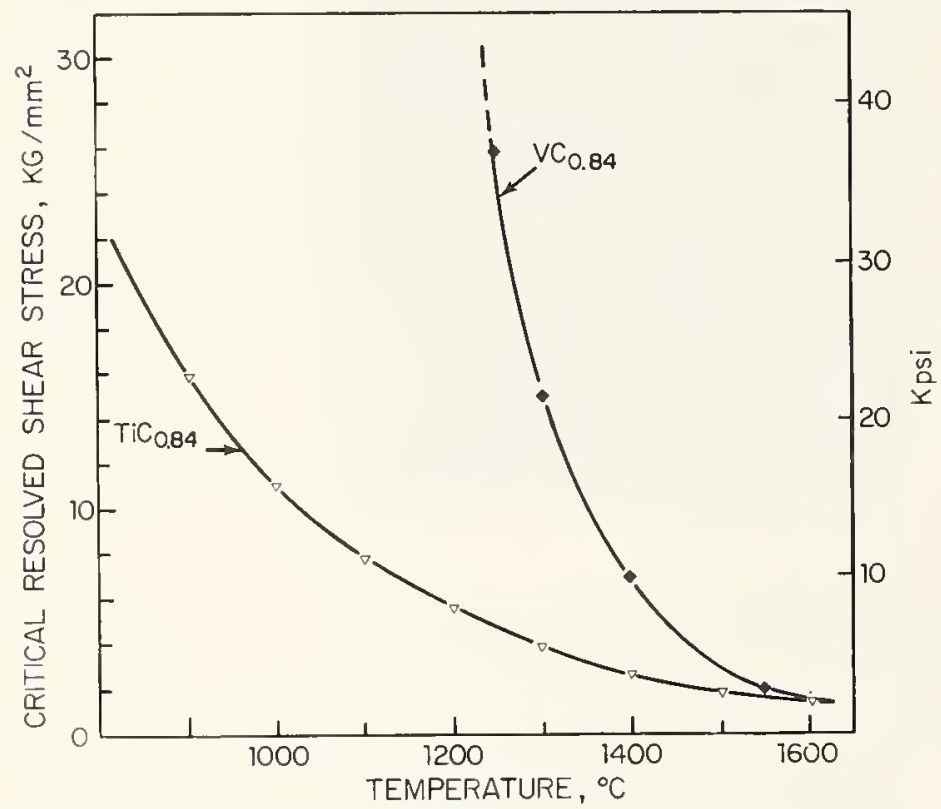

FIGURE 10. The critical resolved shear stress for slip in $\mathrm{VC}_{0.84}$ and $\mathrm{TiC}_{0.84}$ as a function of temperature.

(After Hollox and Venables [38].)

in critical resolved shear stress with increasing temperature is much more rapid in $\mathrm{VC}_{0.84}$ than that observed for other carbides, falling from about $25 \mathrm{~kg} / \mathrm{mm}^{2}(26,000 \mathrm{psi})$ at $1250{ }^{\circ} \mathrm{C}$ to about $3 \mathrm{~kg} /$ $\mathrm{mm}^{2}(4,200 \mathrm{psi})$ at $1500^{\circ} \mathrm{C}$.

The higher strength and increased brittle-toductile transition temperature of $\mathrm{VC}_{0.84}$ are surprising in view of the relative melting points of these carbides, since that of $\mathrm{VC}_{0.84}\left(2650^{\circ} \mathrm{C}\right)$ is some $600 \mathrm{C}$ below that of $\mathrm{TiC}\left(3250{ }^{\circ} \mathrm{C}\right)$. Lye [37] has suggested that the strength of the metalmetal $d$ - $d$ interactions in $\mathrm{VC}_{0.84}$ is greater than in $\mathrm{TiC}$ and this may account for the higher strength of this material. However, Venables et al. [39] have shown an important difference between TiC and $\mathrm{VC}_{0.84}$ is that carbon atoms in $\mathrm{VC}_{0.84}$ are ordered in a hexagonal superlattice. When the crystals are viewed in polarized light, a colored domain structure can be observed. This corresponds to the several possible orientations of the anisotropic superlattice within the metal lattice.

Metallographic observations, figure 11, suggest that disordering of the carbon superlattice occurs at some temperature between 1250 and $1300{ }^{\circ} \mathrm{C}$ [38]. Although the precise role of this superlattice is not fully understood, it significantly affects mechanical behavior, since the order-disorder transformation temperature corresponds closely with the brittle-to-ductile transition temperature.

Using nuclear magnetic resonance techniques, Froidevaux and Rossier [40] have obtained evidence for the ordering of carbon vacancies throughout the complete composition range of VC. de Novion et al. [41] have shown that a consequence of carbon vacancy ordering in $\mathrm{VC}_{0.89}$ is the formation of a superlattice of cubic symmetry. Venables et al. [39] have suggested that the symmetry of the superlattice cell depends on carbon concentration, and, as such, single-
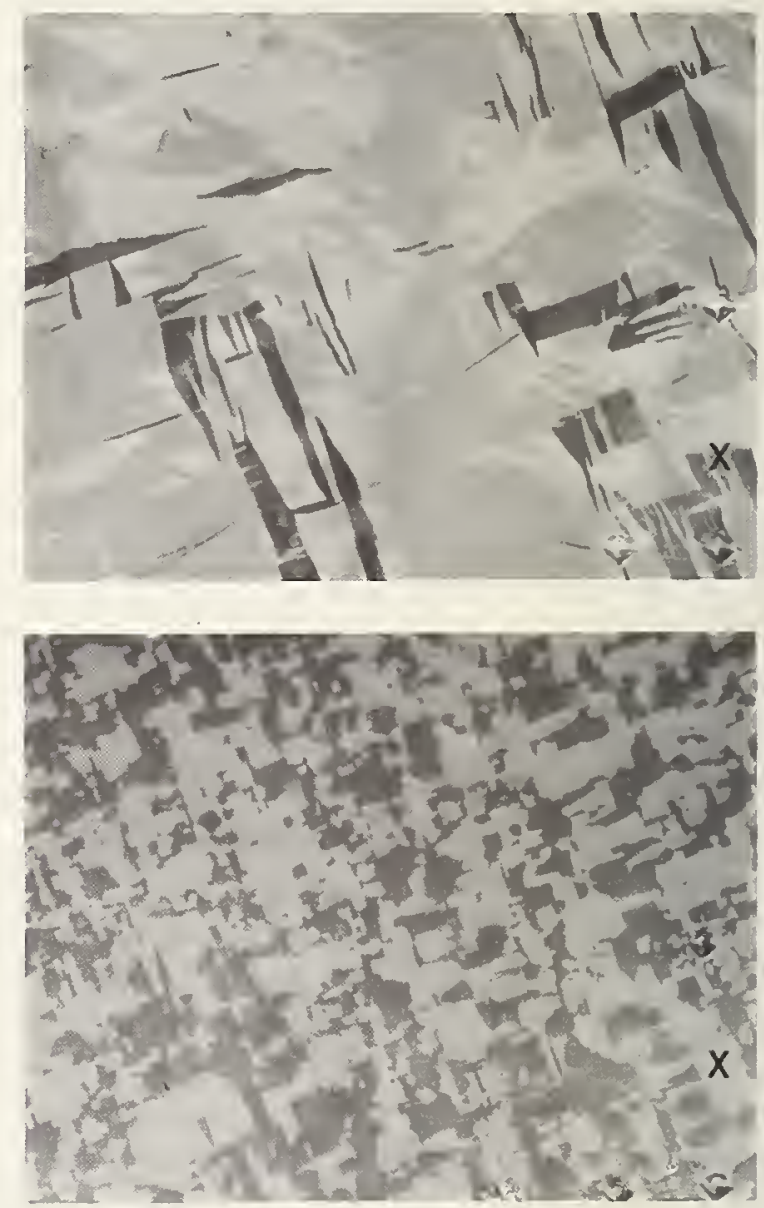

FIGURE 11. The change in domain structure of $\mathrm{VC}_{0.84}$ after an anneal at $1900^{\circ} \mathrm{C}$.

The upper micrograph shows the structure before annealing, and the lower one the final structure. No change is observed in a similaranneal at $1250^{\circ} \mathrm{C}$. These observation s are consistent with disordering of the carbon superlattice between 1250 and $1300^{\circ} \mathrm{C}$ (after Hollox and Venables [38]). 
phase VC might be more correctly described as a series of ordered compounds $\mathrm{V}_{8} \mathrm{C}_{7} \quad\left(\mathrm{VC}_{0.88}\right)$, $\mathrm{V}_{6} \mathrm{C}_{5}\left(\mathrm{VC}_{0.84}\right)$, etc. The occurrence of ordered structures at different compositions would be expected to have different effects on the strength of VC. Consequently, in comparing the properties of $\mathrm{VC}$ with TiC, composition is a very important variable. In this context, two other compositions of $\mathrm{VC}$, close to $\mathrm{VC}_{0.75}$ and $\mathrm{VC}_{0.89}$, both appear to be weaker than $V_{0.84}$ [42]. This suggests that the strength of $\mathrm{VC}$ passes through a maximum as carbon content is increased.

\subsection{Other Cubic Carbides}

Very little data is available for other carbides. Williams [20] has compared the properties of $\mathrm{ZrC}_{0.88}$ and $\mathrm{NbC}_{0.76}$ with $\mathrm{TiC}$ of varying carbon content. He observed that $\mathrm{ZrC}_{0.88}$ compressed in $\langle 001\rangle$ directions exhibited greater strength than $\mathrm{TiC}_{0.95}$, while both these were weaker than $\mathrm{NbC}_{0.76}$. The greater strengths of $\mathrm{ZrC}$ and $\mathrm{NbC}$ were attributed to an increased number of electrons in the bonding scheme. The variation in strength with carbon content of these other carbides has not been reported.

Lee and Haggerty [43] have measured the strength of $\mathrm{ZrC}_{0.9}$ as a function of crystal orientation. They observed slip on $\{111\}\langle 1 \overline{1} 0\rangle,\{110\}$ $\langle 1 \overline{1} 0\rangle$, and $\{001\}\langle 1 \overline{1} 0\rangle$ systems when the crystal orientation was chosen such that the Schmidt factor favored slip on these systems. These results confirm that $\mathrm{ZrC}_{0.9}$ is stronger than $\mathrm{TiC}_{0.9}$. In addition, the resolved shear stress for $\{110\}\langle 1 \overline{1} 0\rangle$ slip is slightly lower than for $\{111\}$ $\langle 1 \overline{10}\rangle$ slip. This cannot be explained at present. These workers have also investigated the creep behavior of single crystal $\mathrm{ZrC}$ and they obtain an activation energy of about $4.8 \mathrm{eV}$ for the steadystate creep. This value is slightly lower than that expected for the diffusion of zirconium in $\mathrm{ZrC}$ $(5.7 \mathrm{eV})$ [31] but is in fair agreement with the behavior of TiC.

\subsection{Binary Carbide Alloys}

Pseudo-binary solid solutions between carbides, nitrides, and oxides have been reported for many years. Schwartzkopf and Kieffer [1] state that complete solid solution occurs between these compounds except in cases where the lattice parameters differ by more than about 15 percent (e.g. $\mathrm{VC}-\mathrm{ZrC}$ ), reminiscent of the empirical relationships deduced by Hume-Rotherey for metallic solid solutions [44]. In addition, extensive solid solubility, for example, up to $70 \mathrm{w} / \mathrm{o}$ (weight percent) WC in $\mathrm{TiC}$, has been reported for noncubic carbides in the cubic carbides [1].

Single crystals of binary carbides have been prepared only recently [45] and their mechanical behavior studied [42]. The preliminary results, figure 12, however, are particularly interesting, for three reasons: (i) the alloys show considerably higher strength than the parent carbides; (ii) the

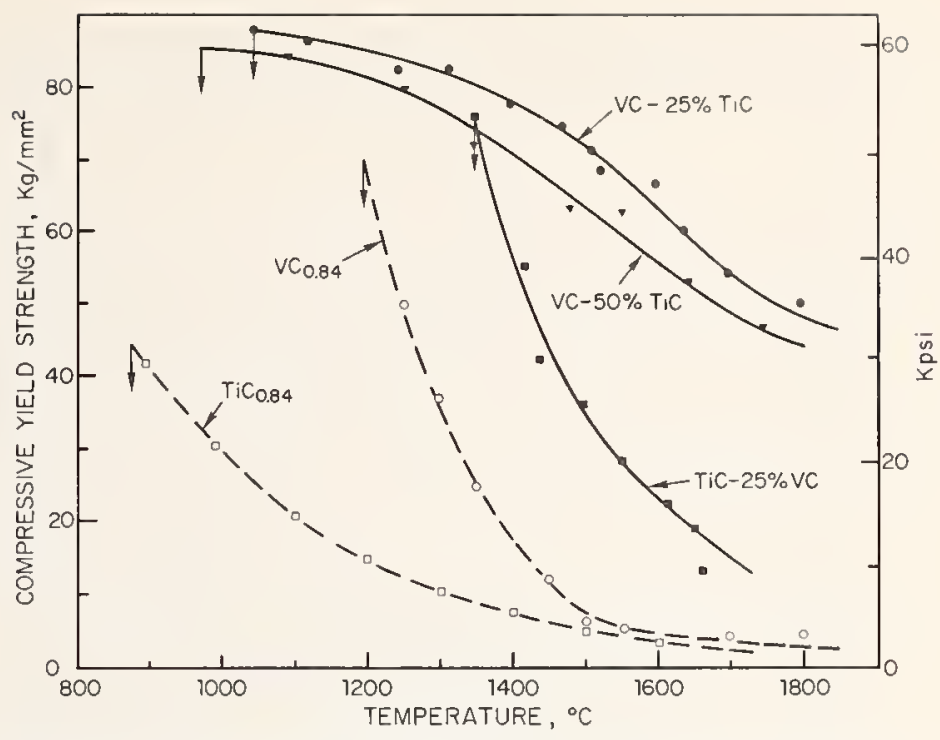

FIGURE 12. The yield strength ( $\langle 001\rangle$ compression) as a function of temperature for some $\mathrm{TiC}-\mathrm{VC}$ alloys, compared with that of $\mathrm{TiC}_{0.84}$ and $\mathrm{VC}_{0.84}$.

temperature dependence of the yield strength of the VC-25 a/o (atomic percent) $\mathrm{TiC}$ and $\mathrm{VC}-50$ a/o TiC compounds appears to have a form different from that of TiC-25 a/o VC and the pure carbides; (iii) there is evidence for a small amount of ductility $(\sim 1$ percent $)$ at about $1000^{\circ} \mathrm{C}$ in $\mathrm{VC}-25 \mathrm{a} / \mathrm{o} \mathrm{TiC}$ and $\mathrm{VC}-50 \mathrm{a} / \mathrm{o} \mathrm{TiC}$.

It is likely that these effects are dependent on stoichiometry and they have yet to be interpreted, but Venables [46] has shown that the VC-25 a/o TiC alloys are two-phase, one of which is ordered. The details of this phase separation is not fully understood, but the fine scale precipitation of a hard ordered phase in a "ductile" matrix may explain the mechanical behavior of this alloy [46].

\subsection{Effect of Boron on the Structure and Properties of TiC and VC}

Boron has been shown to significantly increase the strength of TiC and VC. For example, Williams [47] observed a tenfold increase in strength at $1600^{\circ} \mathrm{C}$ in $\mathrm{TiC}$ crystals, figure 13 , after they have been heated in contact with boron powder at $2000^{\circ}$ C. Similarly, the critical resolved shear stress of boron-doped $\mathrm{VC}_{0.84}$ is about $19 \mathrm{~kg} / \mathrm{mm}^{2}$ $(27,000 \mathrm{psi})$ at $1500^{\circ} \mathrm{C}$, compared with $3 \mathrm{~kg} / \mathrm{mm}^{2}$ $(4,200 \mathrm{psi})$ in the undoped carbide of the same composition, figure 13 [38]. These increased strengths have been correlated with the formation of boride precipitates in the carbides.

In TiC, precipitates form on $\{111\}$ planes, figure 14(a), and Williams [47] has suggested that they are $\mathrm{TiB}_{2}$. Using transmission electron microscopy, Venables [48] has confirmed this and shown that the precipitates are nucleated heterogeneously at dislocation nodes, figure 15 . Of particular importance is the fact that the precipitates form at boron concentrations of the order $100 \mathrm{ppm}$. An autoradiographic technique was used to show that the boron is almost totally 


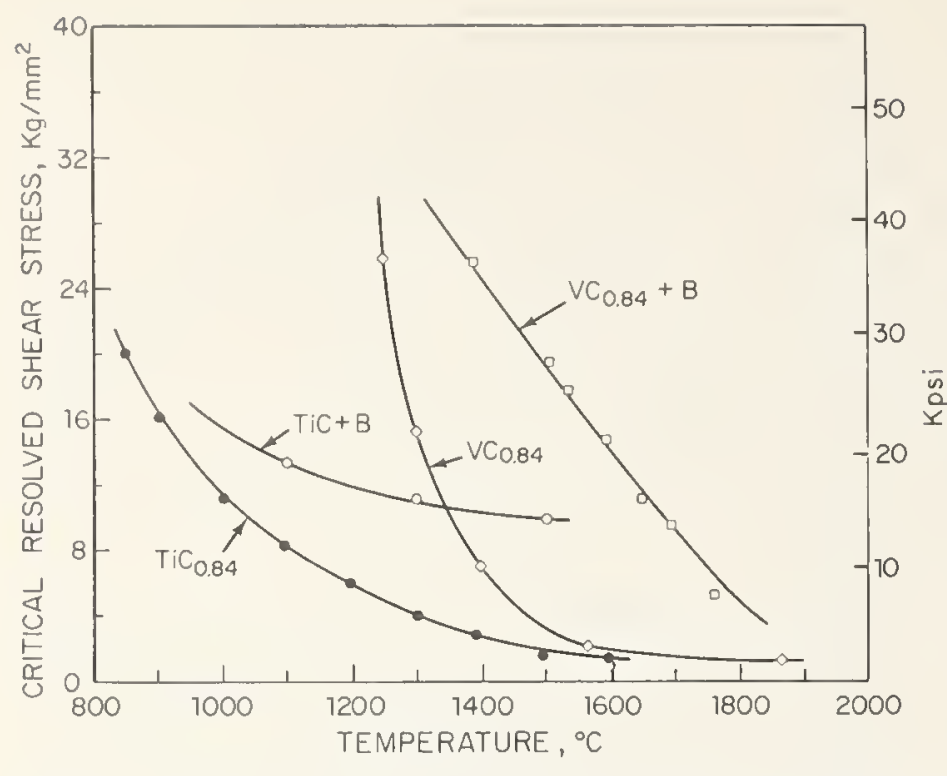

FIGURE 13. The critical resolved shear stress for slip as a function of temperature in boron-doped $\mathrm{TiC}$ (after Williams [49] and $\mathrm{VC}_{0.84}$ (after Hollox and Venables [38]).

associated with the precipitates, and little or none remains in solid solution in the lattice [49]. This technique relies on the observation by Fleischer et al. [50] and Armijo et al. [51] that the damage produced in thin films of cellulose nitrate by $\alpha$-particles resulting from the $\mathrm{B}^{10}(n, \alpha)$ reaction can be detected by etching with $6 N \mathrm{NaOH}$. A metallographic map, figure 14(a), and a boron map ("alphagraph") of the same area are shown for comparison in figure 14(b).

When $\mathrm{VC}_{0.84}$ is doped with boron by diffusion at $1800^{\circ} \mathrm{C}$ for $1 \mathrm{hr}$, precipitates form on $\{111\}$ planes in the surface layers where a high concentration of boron would be expected, but the plane of precipitation changes to $\{100\}$ with increasing distance from the surface, figure 16 [38]. The composition of the precipitates has not been con- clusively established, although the presence of boron has been demonstrated in both types by means of the alpharadiographic technique. It might be expected that the precipitates which form on $\{111$ \} planes would be similar to those which have been observed in boron-doped TiC. The identical structures and similar atomic mismatch in the vanadium compounds therefore suggests that the precipitates on $\{111\}$ planes of $\mathrm{VC}$ are probably of $\mathrm{VB}_{2}$.

It is possible that the precipitates on $\{100\}$ planes would be one of the lower borides of vanadium, since they appear in the more boron deficient regions of the sample. In order of increasing boron content, these borides are: (i) $V_{3} B_{2}$ (tetragonal, $\mathrm{U}_{3} \mathrm{Si}_{2}$-type structure), (ii) VB (orthorhombic, CrB-type structure, ) and (iii) $V_{3} B_{4}$ (orthorhombic $\mathrm{Mn}_{3} \mathrm{~B}_{4}$-type structure) [52]. Consequently a different plane of precipitation may be the result of a change in composition and structure of the precipitate.

\section{Mechanical Behavior of Polycrystal- line Carbides}

\subsection{Characterization}

Before examining the wealth of information in the published literature concerning the behavior of polycrystalline carbides, it is worthwhile to consider which variables may be critical in affecting the properties of such materials. From the behavior of single crystals, it is clear that to compare their properties, at least three variables are important:

\section{a. Stoichiometry}

The observed dependence of the strength of $\mathrm{TiC}$ and VC on carbon content has particular significance when properties of carbides are compared. Before precise conclusions about their
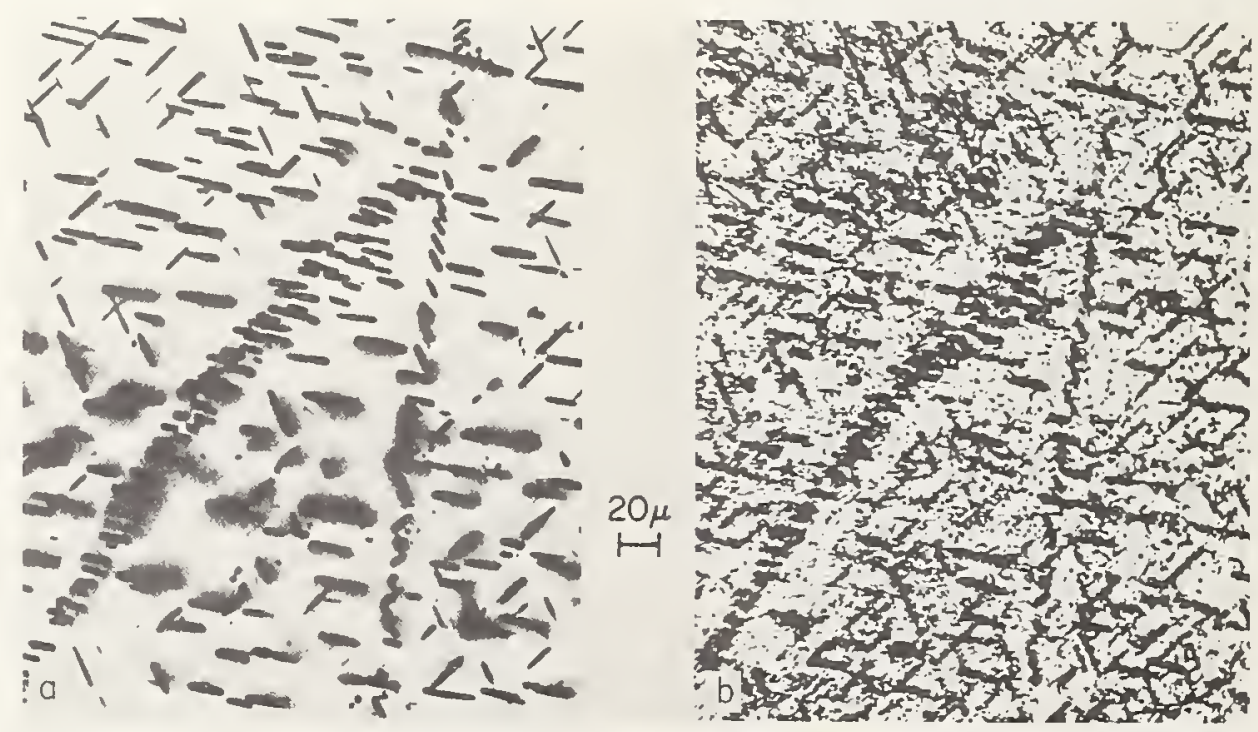

Figure 14. Precipitates on $\{111\}$ planes of TiC after boron doping at $2000{ }^{\circ} \mathrm{C}$.

A direct comparison between a metallographic map and an alphagraph (see text) indicates that most of the boron in the lattice is associated with these precipitates (after Venables [49]). 


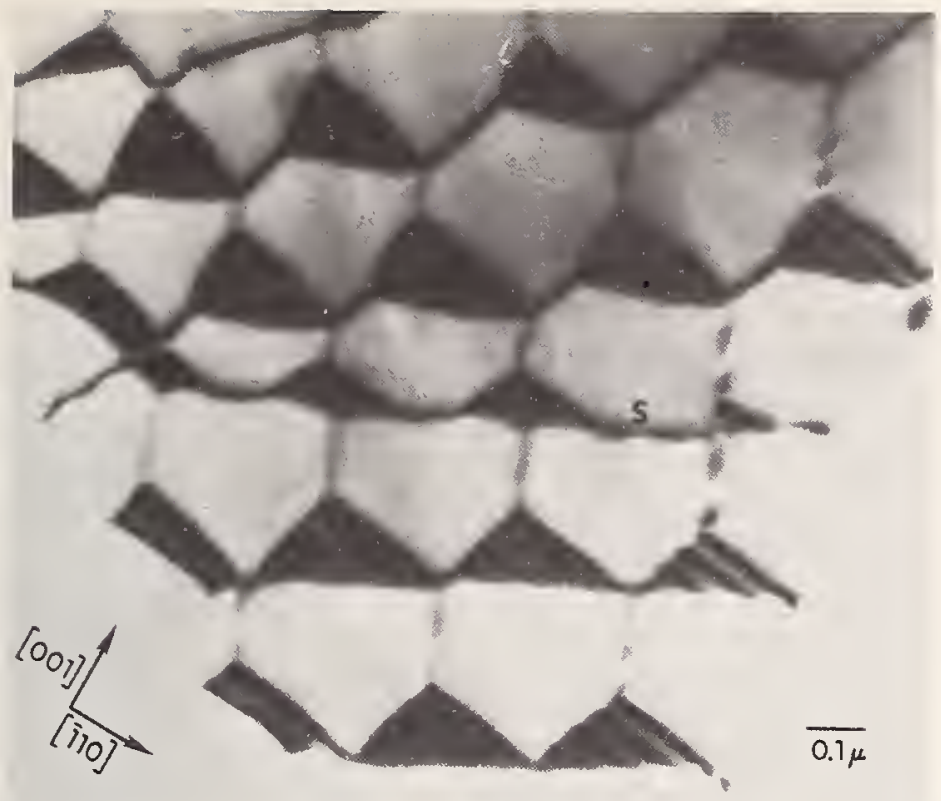

FiguRe 15. $\mathrm{TiB}_{2}$ precipitates nucleated at dislocation nodes. Their diffraction contrast resembles that of extrinsic stacking faults (after Venables [48]).

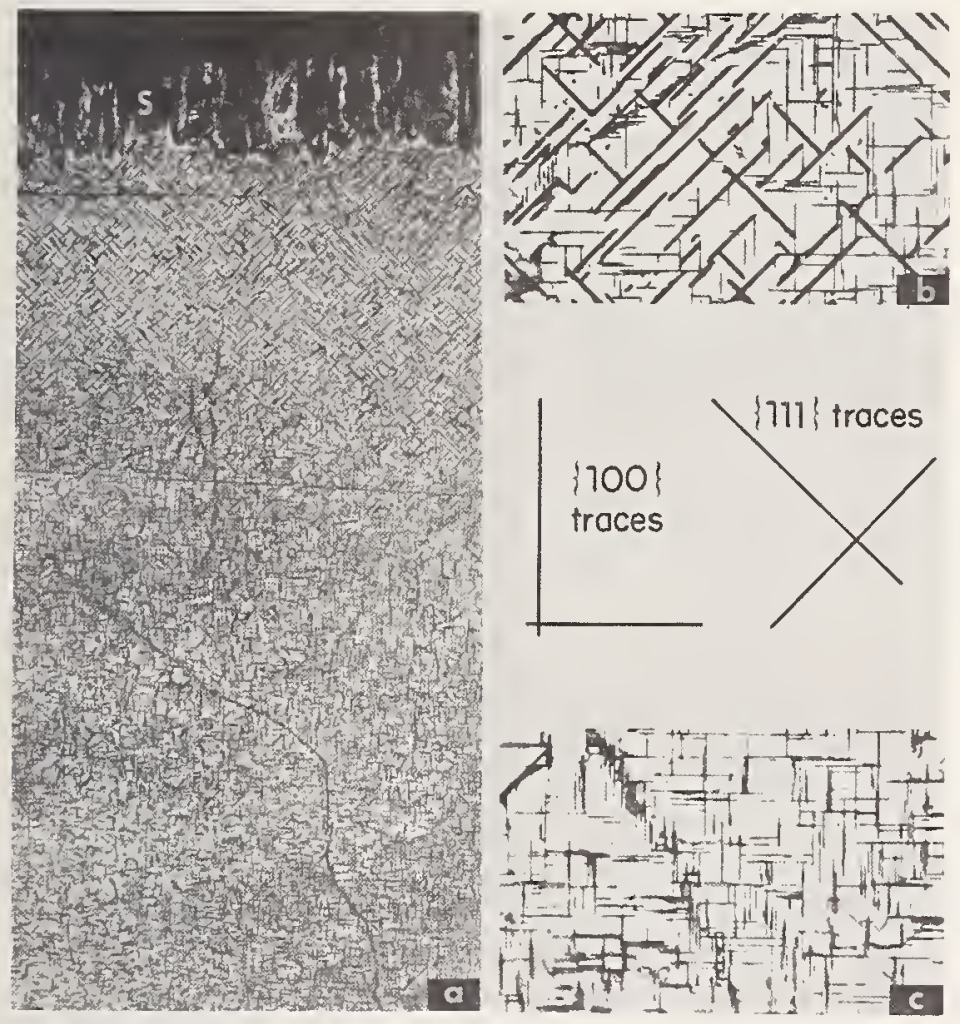

FIGURE 16. A $\{100\}$ section through a sample of boron-doped $\mathrm{VC}_{0.84}$.

In the surface layers where a high concentration of boron is expected, precipitates form on $\{111\}$ planes (b), but the plane of precipitation changes to $\{100\}$ with increasing distance from the surface (c). (After Hollox and Venables [38].)

relative behavior may be drawn, a full survey of mechanical properties as a function of carbon content is necessary. A comparison at equivalent carbon-to-metal ratios may be inadequate since ordering may occur in one carbide and not in the other, as in comparisons between the properties of $\mathrm{TiC}$ and $\mathrm{VC}$.

\section{b. Purity}

The dramatic increase in strength produced by $\sim 100$ ppm of boron in $\mathrm{TiC}$ single crystals illustrates an important influence of chemical composition on the properties of the carbides. In this case, the impurity forms a second phase having a different crystal structure. No mechanism for significantly changing the properties of the carbides can be visualized at present when impurities are in small quantities as long as they form solid solutions. This may be applicable to solutions of, for example, $\mathrm{TiN}$ and $\mathrm{TiO}$ in $\mathrm{TiC}$, although considerable differences in properties must be expected for appreciable quantities of these isomorphs in solid solution. In hardness tests, Cadoff et al. [53] have shown that polycrystalline $\mathrm{TiC}$ containing $45 \mathrm{a} / \mathrm{o}$ carbon and 5 a/o oxygen is more brittle than pure TiC. The impurity effects may be additionally important in polycrystalline samples and in sintered materials where equilibrium conditions may not be reached or where grain boundary segregation may be important.

\section{c. Temperature}

The observations on the high-temperature strength of $\mathrm{VC}$ and $\mathrm{TiC}$ are contrary to the room temperature microhardness of these compounds. At all loads, the room-temperature hardness of $\mathrm{VC}_{0.84}$ appears to be below that of $\mathrm{TiC}$ of equivalent stoichiometry [42]. Gilman [54] has shown a dependence of hardness on the elastic constant $\mathrm{C}_{44}$ in a group of materials of similar structure. The simple concept that the stiffer the material at room temperature the stronger it will be at high temperatures cannot be applied. Chang and Graham [55] have shown that $\mathrm{C}_{44}$ is lower for $\mathrm{ZrC}_{0.94}$ than for $\mathrm{TiC}_{0.91}$, yet the former material is stronger at high temperatures. In this case, the rate of decrease of the elastic constants of $\mathrm{TiC}$ with temperature is more rapid than that of $\mathrm{ZrC}$ [55]. Over the temperature range of 0 to $300{ }^{\circ} \mathrm{C}$, UC, which is isomorphous with these carbides [56], has a positive temperature coefficient for its elastic constants [55] and its hardness should therefore increase with temperature if Gilman's [54] correlations are valid, although these data are not available. The temperature dependence of the elastic constants in these materials would be a significant contribution to understanding their behavior.

Three additional variables which have to be considered in polycrystalline materials are:

\section{d. Crystal Structure}

The presence of grain boundaries in a crystal imposes a barrier to the propagation of slip, and 
unless each grain conforms to the deformation of its neighbors, grain boundary separation will occur. Taylor [57] and von Mises [58] have shown that for a polycrystalline aggregate to deform, five independent slip systems are necessary. As Groves and Kelly [59] have pointed out, most ceramic materials do not meet these requirements. In the cubic oxide ceramics, for example $\mathrm{MgO}$, slip takes place on $\{110\}\langle 1 \overline{1} 0\rangle$ systems. The number of independent slip systems is two, and ductility is not shown in polycrystalline material until slip is activated on additional systems [60]. Since the cubic carbides have the five independent slip systems, they are expected to be ductile in polycrystalline form at temperatures not far above the brittle-to-ductile transition temperature found in single crystals, although the grain boundaries could be weak due to the atomic mismatch in these directionally bonded materials. The hexagonal carbides and other similar ceramics are likely to possess more limited ductility.

\section{e. Porosity}

The yield strength of ceramics has been shown to decrease exponentially with increasing porosity $[61,62]$. Most of the published results compare the strengths of given carbides at different porosity levels, and this is very difficult to interpret on a fundamental basis. At low temperatures, porosity gives rise to increased brittleness since the pores act as crack nuclei, while at high temperatures the pores may increase the brittleness by restricting grain boundary sliding [63].

\section{f. Grain Size}

It is well known that the yield strength and fracture strength of metals and ceramics increases as the grain size decreases. The behavior of TiC is likely to follow this pattern. The room-temperature modulus of rupture of $\mathrm{TiC}$ has been shown to increase from about $31 \mathrm{~kg} / \mathrm{mm}^{2}(44,000 \mathrm{psi})$ at a grain size of $12 \mu \mathrm{m}$ to $51 \mathrm{~kg} / \mathrm{mm}^{2}(73,000 \mathrm{psi})$ when the grain size is $4 \mu$ [64]. These data cannot be accurately described because of the variation in porosity of intermediate grain sizes. For metals, an Orowan-Petch [65] type of relationship, i.e., $\sigma_{y} \propto d^{-1 / 2}$, is observed, while in some ceramics, for example $\mathrm{BeO} \cdot[66]$, the variation is more accurately described by $\sigma_{y} \alpha d^{-1}$.

\subsection{Discussion of Properties}

Very little use can be made of data which compare the properties of the carbides unless some attention is paid to the variables discussed in section 3.1. Unfortunately, nearly all the data in the literature has been obtained on inadequately characterized material, and although technologically useful, it does not readily lend itself to contribute to an understanding of the properties of the carbides.
The most recently available results on the hightemperature mechanical properties of polycrystalline carbides are those of Kelly and Rowcliffe [67], who have shown that hot-pressed $\mathrm{TiC}_{0.75}$, $\mathrm{VC}_{0.81}, \mathrm{NbC}_{0.88}, \mathrm{NbC}_{0.85}, \mathrm{TaC}$, and $\mathrm{WC}$, all behave in a ductile manner in bending tests performed at above $0.5-0.6 T_{m}$. Their results are represented in figure 17. At similar porosity levels, $\mathrm{NbC}_{0.85}$ exhibited higher strength than $\mathrm{NbC}_{0.88}$, indicating an increase in strength with carbon content in this range of composition and at these temperaatures. The strength of $\mathrm{TiC}_{0.75}$ was also shown to be greater than that of $\mathrm{NbC}_{0.95}$ over the above temperature range, which is in direct contrast to the results obtained by Williams [20], who found $\mathrm{NbC}_{0.75}$ single crystals to be stronger than those of $\mathrm{TiC}_{0.97}$. However, in this work, the porosity level was 97.5 percent for $\mathrm{TiC}_{0.75}$ and 92.5 percent for $\mathrm{NbC}_{0.85}$. The weakest carbide of the group was shown to be $\mathrm{VC}_{0.61}$. However, incipient melting of the sample was observed above $2000^{\circ} \mathrm{C}$, suggesting that the carbide was probably in the two-phase $\left(\mathrm{V}_{2} \mathrm{C}+\mathrm{VC}\right)$ region, the former carbide melting at about $2165{ }^{\circ} \mathrm{C}[15]$. Hence the properties of this lower melting, two-phase mixture probably cannot be compared directly with $\mathrm{TiC}$ and the other carbides.

Several attempts to determine the hot hardness (a measure of the yield strength) of carbides have been made. Westbrook [68] presents the microhardness of a number of carbides as a function of temperature (fig. 18) but no details of composition are given and it is difficult to evaluate the results. These results do suggest that the temperature dependence of the elastic constants is an important property, as suggested earlier in this section.

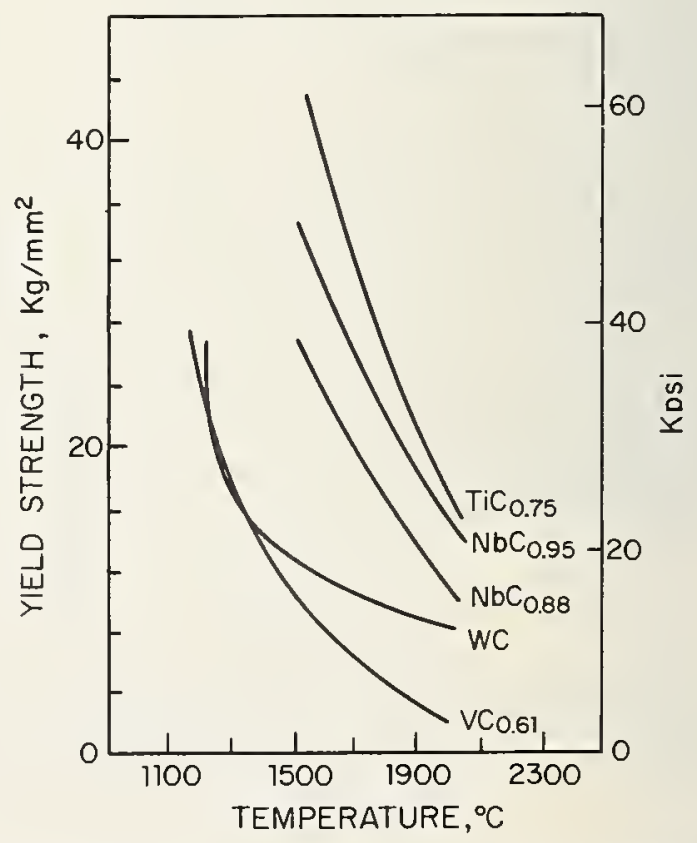

FIgURe 17. Yield strength of refractory carbides as a function of temperature.

(After Kelly and Rowcliffe [67].) 


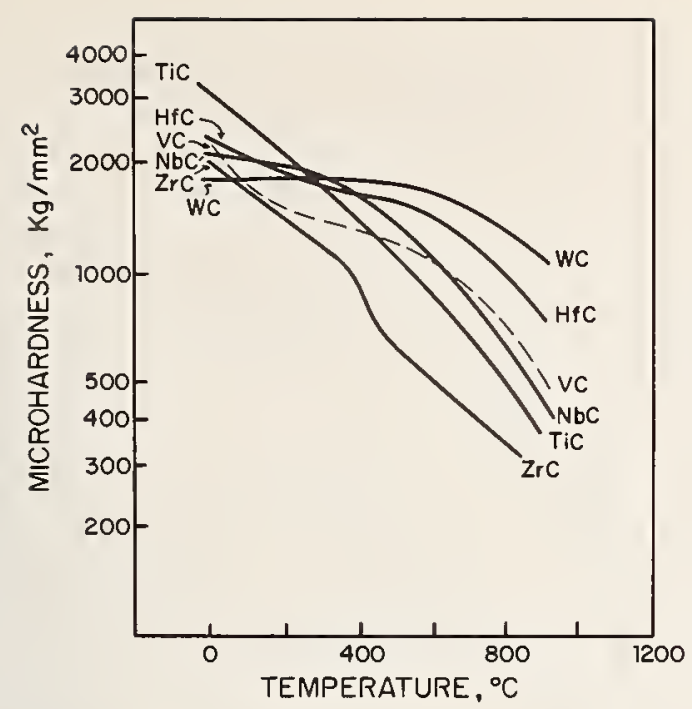

FIgURE 18. Microhardness of carbides as a function of temperature.

(After Westbrook [68].)

Atkins and Tabor [69, 70] have overcome the problems of softening and graphitization of the diamond indenter at high temperature by using a mutual indentation hardness technique. Their technique provides a measure of the plastic yield stress of the material, and measurements for single crystal $\mathrm{TiC}$ are in close agreement with those obtained in conventional compression testing. The hardness variation of a number of carbides- TiC, $\mathrm{VC}, \mathrm{ZrC}, \mathrm{NbC}, \mathrm{WC}$ - as a function of temperature was also reported, but again a constructive comparison is denied by variable porosity and composition. This mutual indentation technique has also been used to determine the activation energy for creep, which is again consistent with self-diffusion of the metal species in the carbide. This type of behavior seems to be established for the cubic carbides [71] including uranium carbide [72].

A hot-microhardness device for use up to $2000^{\circ} \mathrm{C}$ has been used by Koester and Moak [73] with various oxides and carbides. Using a $\mathrm{B}_{4} \mathrm{C}$ indenter, a linear dependence between log hardness and temperature was shown for $\mathrm{TiC}$ and $\mathrm{NbC}$. $\mathrm{NbC}$ is the stronger carbide at all temperatures above room temperature, but no composition specifications were given. This method has the inherent disadvantage of possible specimen contamination by boron at elevated temperatures, this impurity significantly affecting the strength of the carbides.

A particular example where specimen characterization may have played an important part in contributing to confusion in the literature is suggested by considering the properties reported for TaC, figure 19.

Santoro [74] has shown a maximum in the hardness and a minimum in the room-temperature strength at the same composition. He correlated these changes with several other physical changes

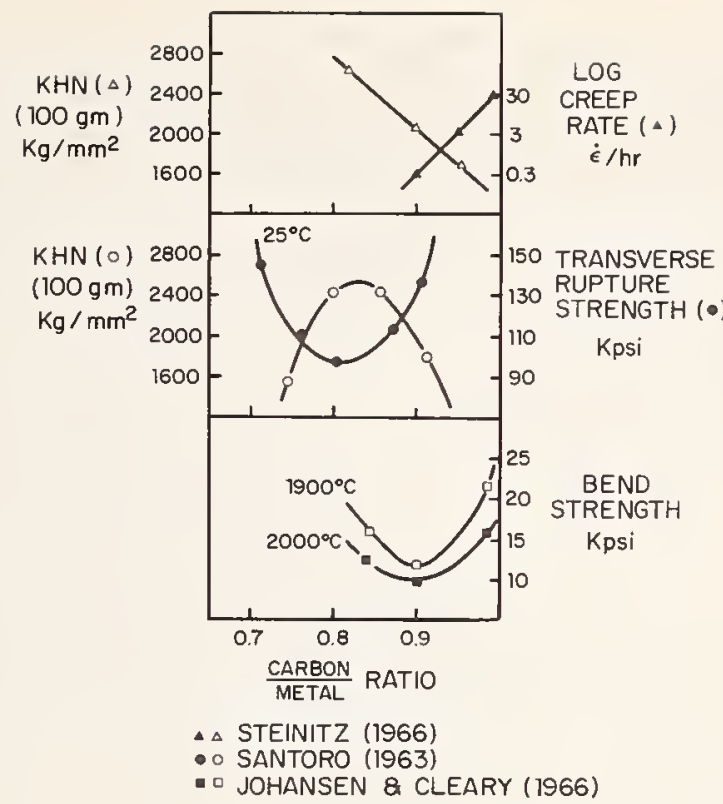

FIGURE 19. Mechanical behavior of tantalum carbides as a function of carbon content.

in the material, notably a change in color, and a maximum in the melting point. There is no simple explanation for such conflicting mechanical properties, although it may be suggested that measurements of tensile strengths in brittle materials at room temperature give results which are open to question because of stress concentrating effects which lead to premature failures. Qualitatively, the harder the material, the more susceptible it is to cracking, and this may be of some significance. In addition, a microhardness measurement implies measurement of the internal material properties, whereas the buk tensile properties are more likely to reflect the properties of the grain boundaries in the carbide.

Measurements of the bending strength of $\mathrm{TaC}$ at high temperatures by Johansen and Cleary [75], however, support the trend shown by the room temperature hardness measurements [74]. The material has a minimum strength at about $\mathrm{TaC}_{0.9}$. In addition, the brittle-to-ductile transition temperature increases linearly with increasing carbon content from compositions within the $\mathrm{TaC}+\mathrm{Ta}_{2} \mathrm{C}$ two-phase field across the $\mathrm{TaC}$ region to the two-phase $\mathrm{TaC}+\mathrm{C}$ field. However, if compositions with the $\mathrm{TaC}$ single-phase region are considered, which would appear more justifiable, then their data indicate a minimum in the transition temperature corresponding exactly with that of the bending strength.

Steinitz [71], in the most recent data, indicates that the room temperature hardness decreases and similarly the creep rate at high temperatures increases with carbon content over the composition range $\mathrm{TaC}_{0.8}$ to $\mathrm{TaC}_{10}$. He suggests that this decrease in strength with increasing carbon content is due to dislocation-vacancy interactions. This may not necessarily be true! The increase in strength of $\mathrm{TiC}$ with increasing carbon content 
has been interpreted as being due in part to an increase in the occupancy of the electron states which are responsible for cohesion in the lattice. It is possible that in a different carbide, the carbon atoms may contribute electrons to anti-bonding states in a given compound as a function of composition, and this change in electronic structure would give rise to a decreased strength of the material [26].

All these investigations have utilized $\mathrm{TaC}$ produced either by hot pressing with 4 percent $\mathrm{Co}_{0}$ as a binder $[71,75]$ or by carburization of $\mathrm{Ta}$ wires [74]. Although fully dense, and apparently of constant radial composition, the latter showed a large variation in grain size. It would seem that with these specifications, the material lacked metallurgical integrity and that these most confusing data must be evaluated by careful investigation of the properties of TaC single crystals. The possibility of ordering to give irregular variations in mechanical strength, as in VC, must also not be ignored. Ordering has not been detected in other carbides except in $\mathrm{TiC}$ [76] at low carbon-to-metal ratio ${ }^{2}(0.5-0.6)$. It is likely that further application of neutron diffraction, electron diffraction, and nuclear magnetic resonance will compensate for the lack of sensitivity of the conventional x-ray techniques in detecting ordering in these compounds.

It will therefore be appreciated that the present degree of understanding of the mechanical behavior of the carbides is very incomplete. TiC appears to deform in a manner similar to f.c.c. metals rather than rock-salt structure ceramics, and the strength arises from the complex electronic interactions between constituent atoms in the lattice. Diffusion of the atom species is important in determining the plastic behavior of this material. Very little is known about other Group IV carbides, although $\mathrm{ZrC}$ is stronger than ' $\mathrm{TiC}$, and there is some indication that the strength of HfC increases with carbon content [77] similar to that observed in TiC. The behavior of Group V carbides is even less clear. Ordering in the carbon sublattice has been shown to significantly affect the behavior of $\mathrm{VC}$, and initial results suggest that the strength of this carbide passes through a maximum as carbon content is increased. Some of the results presented for polycrystalline $\mathrm{TaC}$ suggest a similar trend [71], although this is uncertain.

The author feels that it is very difficult to base a fundamental understanding of the behavior of the carbides on the presently available data, much which has been accumulated on poor, inadequately characterized material. The situation would be clarified by experiments performed as single crystals produced from the melt $[45,78,79,80$, $81]$. In this way, undesirable specimen characteristics such as porosity could be eliminated.

${ }^{2}$ In the $\mathrm{Ti}+\mathrm{TiC}$ region according to some phase diagrams [15].
In particular, it would appear that measurements of such aspects of the mechanical behavior as activation energies and volumes involved in the flow process, in conjunction with diffusion experiments as a function of carbon content, could lead to a more complete understanding of the Peierls stress in these materials. With this knowledge, it would then be appropriate to discuss the effect of controlled grain size, strain-rate, temperature, and composition, etc., on the mechanical behavior. However, technological developments are not likely to wait for these data. Our present knowledge of these materials, although elementary, does enable an evaluation of their properties for high temperature structural applications to be made, and this will be discussed in section 4 .

The carbides appear to be a particularly appropriate field of study in which to relate electronic structure to mechanical behavior. Lye [25] has suggested that alloying may be expected to change the relative occupancy of the electron states which are responsible for cohesion in the carbides. By appropriate alloying, therefore, it may be possible to adjust the relative occupancy of bonding and anti-bonding electronic states such that differences in strength, ductility and brittleto-ductile transition temperature may be produced. Such a phenomenon may also account for the observed variations in properties of $\mathrm{TiC}$ and $\mathrm{TaC}$ as a function of carbon content, where carbon vacancies may be considered as the alloying constituents. The electronic structure of the carbides may be controlled by alloying with solid solutions of other carbides, nitrides, and oxides. Although ordering may introduce complications to these studies, a concurrent investigation of both electronic and mechanical properties should provide valuable information on the fundamental properties of these materials.

The present understanding of carbide base alloys is even less than that for the component materials. The mutual solubility between carbides was summarized by Schwartzkopf and Kieffer [1], but recent work suggests that many of these observations are incorrect [82]. One characteristic which has been reported in many of these binary systems is that of a melting-point maximum [1]. Examples of the maximum stability compositions include $\mathrm{TaC}-20$ percent $\mathrm{HfC}, \mathrm{TaC}-20$ percent $\mathrm{ZrC}$ and $\mathrm{TiC}-50$ percent $\mathrm{TiN}$. The $\mathrm{TaC}-20$ percent HfC has the highest reported melting temperature for any material, $4000{ }^{\circ} \mathrm{C}$ [83] and this has been associated with a hardness maximum [84]. These maxima suggest that ordering may be present in the solid state but this has yet to be detected. The application of more sensitive experimental techniques to determine phase equilibria and to generally characterize these materials is necessary. The scope for study of the properties of these materials also clearly is vast. 


\section{Carbides as High-Temperature Structural Materials}

The carbides are of particular interest for hightemperature structural applications for three reasons [85]: (i) they include the materials exhibiting the highest melting points; (ii) they are extremely strong; and (iii) at high temperatures, they deform in a manner similar to the ductile f.c.c. metals.

Because of their high brittle-to-ductile transition temperature, fabrication of the carbides currently presents problems. If a relatively ductile carbide could be developed by alloying to control electronic structure it would be of great technological importance. A thermomechanical treatment involving prestrain of a ductile carbide solid solution (perhaps in some forming process) followed by controlled high-temperature doping should produce an extremely strong structure.

Venables [48] has suggested that precipitate density and size, and hence the mechanical strength, may be controlled by varying the dislocation node density prior to or during doping. The notable effect of boron in increasing the hightemperature strength of $\mathrm{TiC}$ and $\mathrm{VC}$ must be considered as "casual" observations, since no control of precipitate size to optimize these properties has yet been attempted. However, the strength-to-density ratio of doped single crystals of $\mathrm{VC}_{0.84}$ is $280,000 \mathrm{in}$ at $1500^{\circ} \mathrm{C}$ (fig. 20). $\mathrm{TiC}-$ $\mathrm{VC}$ alloys show values in excess of this. In particular, that of the VC-50 a/o percent TiC alloy appears to exceed 600,000 in at $1000{ }^{\circ} \mathrm{C}$. It is possible that these values are greater than those of any conventional materials, and are of particu-

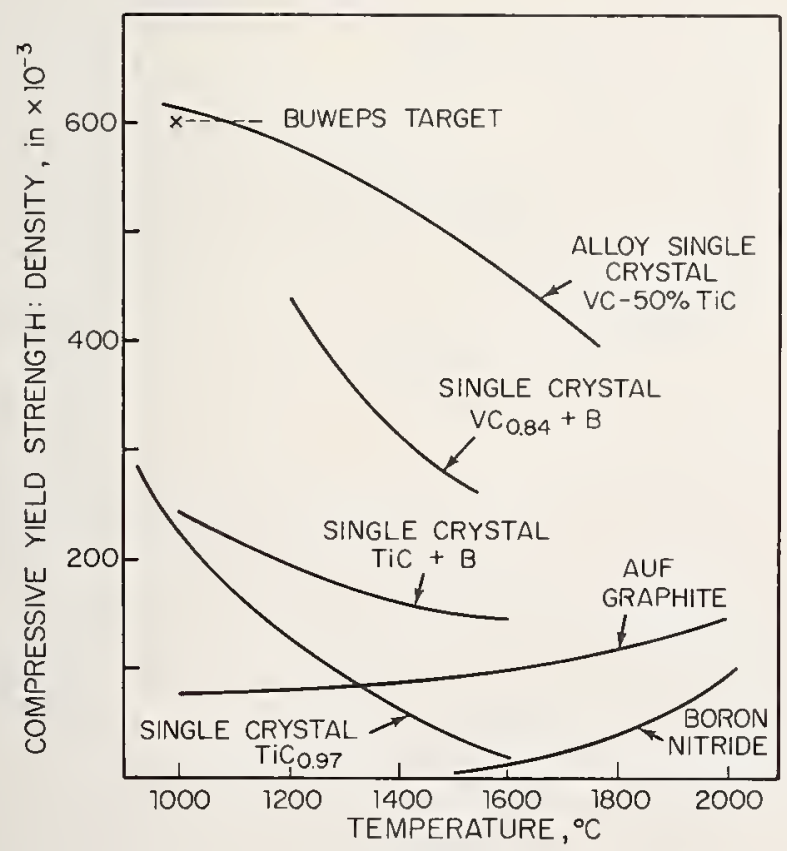

FIGURE 20. The strength-to-density ratio of $\mathrm{VC}-50$ a/o $\mathrm{TiC}$ and boron-doped $\mathrm{VC}_{0.84}$ as a function of temperature, compared with some other hightemperature materials. lar interest because they exceed the target set by the U.S. Bureau of Naval Weapons in 1964 [86]. Additionally, considerably higher values may be obtained when these alloy carbides are hardened by boron-induced precipitation. It is significant to note that if the strength-to-density ratio is a criterion for utilization, then it may not be the carbides of highest melting point $(\mathrm{NbC}$, $\mathrm{TaC}$, $\mathrm{HfC}$ ) which will prove of greatest usefulness, since these carbides are of considerably greater density than TiC or VC, and would therefore be required to be very much stronger.

If a relatively ductile alloy-carbide cannot be developed by controlling the electronic structure, then cladding with a ductile metal may be required to facilitate forming operations. Alternatively, cast shapes can be produced, as has already been demonstrated [87]. In recent years, one of the major advances in turbine blade technology has been the development of directionally solidified "monocrystaloys" of nickel-base alloys [88]. The elimination of grain boundaries in these materials has produced marked improvements in creep rupture properties and thermal shock resistance in comparison with equiaxed or columnar grained structures. It would appear that similar techniques could be applied with advantage to alloyed carbides.

Ductility has been observed in polycrystalline TiC at temperatures above the brittle-to-ductile transition in single crystals. For example, at $1500{ }^{\circ} \mathrm{C}$, in coarse-grained $\mathrm{TiC}$ of about $2 \mathrm{~mm}$ grain size, about 30 percent ductility has been observed, the yield strength being about 10 times that of single crystals of the same composition at the same temperature [42]. Other polycrystalline cubic carbides may behave similarly, and additional strength may result from grain refinement. Conversely, porosity reduces strength and increases brittleness. It appears, therefore, that fine-grain, fully-dense, alloyed, polycrystalline carbides could provide useful mechanical strength and ductility at high temperatures, and may therefore be of significant technological importance in the future.

The author is indebted to R. G. Lye, C. M. Preece, J. D. Venables, and A. R. C. Westwood for their helpful discussions and constructive criticism of this manuscript. Special thanks are due also to W. Precht for developing a crystal growth technique and to D. L. Novak and R. D. Huntington for their assistance in the experimental programs on which some of this work was based. 'The financial support of the Research Division of the National Aeronautics and Space Administration under Contract NASw1290 and the Army Research Office (Durham) under Contract DA-31-124-ARO-D-467 is gratefully acknowledged. 


\section{References}

[1] Schwartzkopf, P., and Kieffer, R., Refractory Hard Metals, Macmillan, New York (1955).

[2] Kendall, E. G., in Ceramics for Advanced Technologies, John Wiley \& Sons, New York, p. 143 $(1965)$.

[3] Shaffer, P. T. B., Materials Index, Plenum Press (1964).

[4] Samsonov, G. V., Properties Index, Plenum Press (1964).

[5] Lynch, J. F., Duckworth, W. H., and Ruderer, C. G., Engineering Properties of Selected Ceramic $\mathrm{Ma}-$ terials, Am. Ceram. Soc. (1966).

[6] Report of the Materials Advisory Board Committee on Ceramic Processing, to be published (1968).

[7] Stokes, R. J., in Strengthening Mechanisms of Ceramics, Syracuse Univ. Press (1966).

[8] Gilman, J. J., Prog. Ceram. Sci. 1, 146 (1961).

[9] Gilman, J. J., and Johnston, W. G., Solid State Phys. 13, 147 (1962)

[10] Takahasi, T., and Freise, E. J., Phil. Mag. 12, 1 (1965).

[11] Corteville, J., Monier, J. C., and Pons, L., C.R. Acad. Sci Paris, 6, 260, 2773 (1965).

[12] French, D. N., and Thomas, D. A., Trans. AIME 233, 950 (1965)

[13] Vahldiek, F. W., Mersol, S. A., and Lynch, C. T., AFML Tech. Rept. No. AFML-TR-67-321, July 1967.

[14] Haggerty, J. S., and Lee, D. W., Arthur D. Little Corp., Cambridge, Mass., private communication.

[15] Storms, E. K., The Refractory Carbides, Academic Press (1967).

[16] Williams, W. S., and Schaal, R. D., J. Appl. Phys. 33, 955 (1962).

[17] Williams, W. S., presented at Intern. Conf. SemiMetallic Compounds, Orsay, France, September 1965 , to be published.

[18] Brookes, C. A., Special Ceramics, Academic Press, London (1962).

[19] Hollox, G. E., and Smallman, R. E., J. Appl. Phys. 37, 818 (1966).

[20] Williams, W. S., J. Appl. Phys, 35, 1329 (1964).

[21] Maddin, R., and Cottrell, A. H., Phil. Mag. 46, 735 (1966).

[22] Ashbee, K. H. G., and Smallman, R. E., Proc. Roy. Soc. A274, 195 (1963).

[23] Hollox, G. E., and Smallman, R. E., Proc. Brit. Ceram. Soc. 6, 317 (1966).

[24] Eikum, A., and Smallman, R. E., University of Birmingham, private communication.

[25] Lye, R. G., Atomic and Electronic Structure of Metals, A.S.M., Cleveland (1967).

[26] Lye, R. G., RIAS, private communication.

[27] Rowcliffe, D. J., Ph.D. dissertation, The Mechanical Properties of Transition-Metal Carbides, University of Cambridge (1965).

[28] Kelly, A., and Rowcliffe, D. J., Phys. Stat. Sol. 14, K29 (1966).

[29] Kronberg, M. L., Acta Met. 5, 507 (1951).

[30] Tobin, J. M., Adeslberg, L. M. Cadoff, L. H., and Brizes, W. F-, in Nuclear Applications of Nonfissionable Ceramics, Am. Nuclear Soc., Illinois (1966).

[31] Harrod, D. L., and Fleischer, L. R., Proc. Intern. Symp. Anisotropy on Single-Crystal Refractory Compounds, Dayton, June 1967, to be published.

[32] Hollox, G. E., and Smallman, R. E., Proc. Brit. Ceram. Soc. 1, 211 (1964).

[33] Keihn, F., and Kebler, R., J. Less Common Metals 6, 484 (1964).

[34] Haasen, P., in Proc. Conf. Relation between Structure and Strength, H.M.S.O., London, 587 (1963).

[35] Seegar, A., in Dislocations and Mechanical Properties of Crystals, John Wiley \& Sons, New York (1957).

[36] Henderson, B., J. Inst. Metals 92, 55 (1963).

[37] Lye, R. G., Hollox, G. E., and Venables, J. D., In Anisotropy in Single Crystal Refractory Com- pounds, Ed. F. W. Vahldiek and S. A. Mersol, Plenum Press, New York, p. 445, (1965).

[38] Hollox, G. E., and Venables, J. D., Proc. Intern. Conf. Strength of Metals and Alloys, Tokyo, Supp. Japan Inst. Metals 9, 295 (1968).

[39] Venables, J. D., Kahn, D., and Lye, R. G., Phil. Mag. 18, 177 (1968).

[40] Froidevaux, C., and Rossier, D., J. Phys. Chem. Solids 28, 1197 (1967)

[41] de Novion, C. H., Lorenzelli, R., and Costa, P., C.R. Acad. Sci. Paris 263, 775 (1966).

[42] Hollox, G. E., Mater. Sci. Eng. 3, 121 (1968).

[43] Lee, D. W., and Haggerty, J. S., Arthur D. Little Corp., Cambridge, Mass., private communication.

[44] Hume-Rotherey, W., Atomic Theory for Students of Metallurgy, Inst. Metals, London (1960).

[45] Precht, W., and Hollox, G. E., Proc. Inter. Conf. Crystal Growth, Birmingham, to be published in J. Crystal Growth, 1968.

[46] Venables, J. D., RIÁS, private communication.

[47] Williams, W. S., Trans. A.I.M.E. 236, 211 (1966).

[48] Venables, J. D., Phil. Mag. 16, 143 (1967).

[49] Venables, J. D., and Westwood, A. R. C., unpublished work. Also, Venables, J. D., RIAS Tech. Rept. No. 67-2, March 1967.

[50] Fleischer, R. L., Price, P. B., and Walker, R. M., Phys. Rev. A133, A1443 (1964).

[51] Armijo, J. S., and Rosenbaum, H. S., J. Appl. Phys. 38, 2064 (1967).

[52] Rudy, E., and Winisch, S., Aerojet General Corp., Tech. Rept. No. AFML-TR-65-2, Part 1, Vol. $\mathbf{X}$, May 1966 .

[53] Cadoff, I., Nielsen, J. P., and Miller, E., Plansee Procs. 2, 50 (1955.)

[54] Gilman, J. J., NBS Mono. 59, p. 79 (1963).

[55] Chang, R., and Graham, L. J., J. Appl. Phys. 37, 3778 (1966).

[56] Frost, B. R. T., J. Nucl. Mats. 10, 265 (1963).

[57] Taylor, G. I., Proc. Roy. Soc. A145, 362 (1934).

[58] von Mises, R., Z. anger. Math. und Mech. 8, 611 (1928).

[59] Groves, G. W., and Kelly, A., Phil. Mag. 8, 877 (1963).

[60] Stokes, R. W., in Materials Science Research, Vol. 1, Plenum Press, New York (1963).

[61] Glaser, F. W., and Ivanick, W., J. Metals 4, 387 (1952).

[62] Coble, R. C., in Ceramic Fabrication Processes, M.I.T. Press (1963).

[63] Day, R. B., and Stokes, R. J., J. Am. Ceram. Soc. 49, 345 (1966).

[64] Harrison, W. B., Honeywell Research Centre, Tech. Rept. AD 612253, Feb. 1965.

[65] Orowan, F., Repts. Prog. Phys. 12, 186 (1949).

[66] Hyde, C., Battelle Mem. Inst., in ref. [62].

[67] Kelly, A., and Rowcliffe, D. J., J. Am. Ceram. Soc. 50, 253 (1967).

[68] Westbrook. J. H., and Stover, E. R., GE Rept. No. 60-RL-2656M (1960).

[69] Atkins, A. G., and Tabor, D., Brit. J. Aupl. Phys. 16, 1015 (1965).

[70] Atkins, A. G., and Tabor, D., Proc. Roy. Soc. A292, 441 (1966).

[71] Steinitz, R., in Nuclear Applications of Non-fissionable Ceramics, Am. Nuclear Soc., Illinois (1966).

[72] Chang, R., J. Appl. Phys., 33, 858 (1962).

[73] Koesler, R. D., and Moak, D. P., J. Am. Ceram. Soc. 50, 290 (1967).

[74] Santoro, G., Trans. A.I.M.E. 227, 1361 (1963).

[75] Johansen, H. A., and Cleary, J. G., J. Electrochem. Soc. 113, 378 (1966).

[76] Goretzki, H., Phys. Stat. Sol. 20, K141 (1967).

[77] Adams, R. P., and Beall, R. A., U.S. Bureau of Mines Report of Investigations No. 6304 (1963).

[78] Kieffer, A. D., Linde Co., private communication in ref. [16].

[79] Haggerty, J. S., Wencus, J. F., and Lee, D. W., Proc. $3 \mathrm{~d}$ Intern. Symp. High Temperature Technology, Asilomar, Calif., Sept. 1967, to be published. 
[80] Bartlett, R. W., and Halden, F. A., Stanford Research Institute, Final Report on Contract NASr-49(19), June 1967.

[81] Tobin, J. M., and Fleischer, L. R., Proc. 3d Intern. Sympo. High Temperature Technology, Asilomar, Calif., Sept. 1967, to be published.

[82] Brukl, C. E., and Harrison, D. P., Aerojet General Corp., Tech. Rept. No. AFML-TR-65-2, Part II, Vol. IV, Feb. 1966.

[83] Agte, C., and Alterthum, H., Z. Tech. Physik 11, 182 (1930).
[84] Deadmore, D. L., and Zaplatynsky, I., NASA TN D-2768, April 1965.

[85] Hollox, G. E., Proc. 3d Intern. Symp. High Temperature Technology, Asilomar, Calif., Sept. 1967, to be published.

[86] U.S. Bureau of Naval Weapons Target, 1964.

[87] Leeds, D., Kendall, E., and Ward, J. F., Aerospace Corp., El Segundo, Calif., Tech. Rept. No. 55DTDR-63-216 (1963).

[88] Piearcey, B. J., and VerSnyder, P. L., Pratt and Whitney Aircraft Co., Report No. 66-007, February 1966. 



\title{
Fracture of Ceramics
}

\author{
S. M. Wiederhorn \\ Institute for Materials Research, \\ National Bureau of Standards, Washington, D.C. 20234
}

\begin{abstract}
In this paper the fracture of ceramics is considered primarily from a fracture mechanics point of view. The maximum theoretical strength of ceramics is discussed. The ideas of a stress intensity factor and a fracture surface energy are developed. Finally, examples are presented of the use of these parameters to describe strength, thermal shock resistance and stress corrosion of ceramic materials.
\end{abstract}

Key Words: Ceramics; delayed failure; fracture; fracture mechanics; fracture surface energy; static fatigue; strength; stress corrosion; stress intensity factor; thermal shock.

\section{GONTENTS}

Page

1. Introduction _..

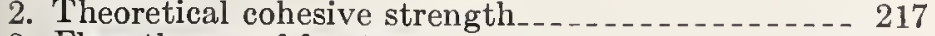

3. Flaw theory of fracture.

4. Continuum approach to fracture....... 220

4.1. Stress intensity viewpoint

4.2. Surface energy viewpoint

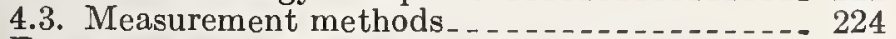

5. Experimental fracture surface energy values..... 225

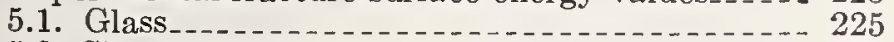

5.2. Single crystals

5.3. Bicrystal fracture energy measurements _. _- 226

5.4. Polyerystalline ceramics_._.

6. Applications of fracture mechanics to ceramics _-_ 227

6.1. Strength of glass_._._................ 228

6.2. Strength of single crystals $\ldots \ldots \ldots \ldots \ldots \ldots \ldots 28$

6.3. Strength of polycrystalline ceramics....... 228

6.4. Thermal fracture

6.5. Environmental effects

7. Conclusions

\section{Introduction}

Mechanical failure of ceramic materials is almost completely controlled by brittle fracture, which means that the failure occurs with negligible plastic deformation and without prior warning. This brittle behavior, combined with the presence of surface flaws leads to low strengths in ceramics and, consequently, severely limits the mechanical use of these materials. Investigations over the past decade on the deformation and fracture properties of ceramic materials have indicated that brittleness is an inherent property of ceramics; consequently, any improvement of their mechanical properties will necessitate designing around this brittle behavior by understanding the causes and effects of brittle behavior and by developing the necessary criteria for failure.

The primary purpose of this paper is to survey some aspects of brittle fracture that may be of use in the design of ceramic structures. Particular emphasis will be placed on the flaw theory of fracture and its generalization to the continuum approach of fracture mechanics. The ideas of a surface energy and a stress intensity factor in fracture will be developed and specific examples of their uses will be given. The effect of plastic deformation and environment on fracture will also be discussed.

\section{Theoretical Cohesive Strength}

The theoretical cohesive strength of a material depends on the physical and chemical forces holding it together and, as such, gives a meaningful measure of the stresses that must be overcome during fracture. All other things being equal, the material with the higher cohesive strength will be stronger. Thus the cohesive strength provides a means of comparing the potential strengths of different materials and gives the maximum strength to be expected from a given material.

This section will deal with a calculation of the theoretical strength of two ceramic materials, sapphire and glass, and will compare the calculated with the measured strengths of these materials. The theoretical cohesive strength will be shown to be approximately 100 times the normal engineering strength of these materials. The method of calculation is that described by Gilman [1]. ${ }^{1}$ Other discussions of the theoretical cohesive strengths of materials may be found in references [2-4].

Fracture is the process of separating a solid into two parts, each of which becomes bounded by a new surtace called the fracture surface. During the separation process attractive forces act across the incipient fracture surfaces, first increasing, then

\footnotetext{
1 Figures in brackets indicate the literature references at the end of this paper.
} 
decreasing with distance of separation. 'The maxiinum force corresponds to the theoretical cohesive strength of the solid. This behavior may be approximated by a potential energy function that is characteristic of the material under study and which relates potential energy per unit area of fracture surface, to distance of separation. The shape of a typical potential energy function and its first derivative is given in figure 1. Two typical potential energy functions are the Morse potential and the Born potential, eqs (1) and (2).

$$
\begin{aligned}
& U=U_{0}\left[e^{-2 a\left(x-x_{0}\right)}-2 e^{-a\left(x-x_{0}\right)}\right] \\
& U=-\frac{A}{x}+\frac{B}{x^{n}},
\end{aligned}
$$

where $U$ represents the potential energy per unit area of the fracture surfaces separated by a distance $x$. The stress on these two surfaces is equal to $\partial U / \partial x$ and the cohesive strength is calculated from the fact that the stress is a maximum when $\partial^{2} U / \partial x^{2}=0$.

The constants in eqs (1) and (2) determine the shape and position of the potential function. They are evaluated from measurable material properties such as the surface energy, $\gamma$, which determines the depth of the potential minimum; the zero stress separation distance, $x_{0}$, which determines the position of the potential minimum; and Young's modulus, $E$, which determines the curvature at the potential minimum. The general shape of the potential function at points away from the

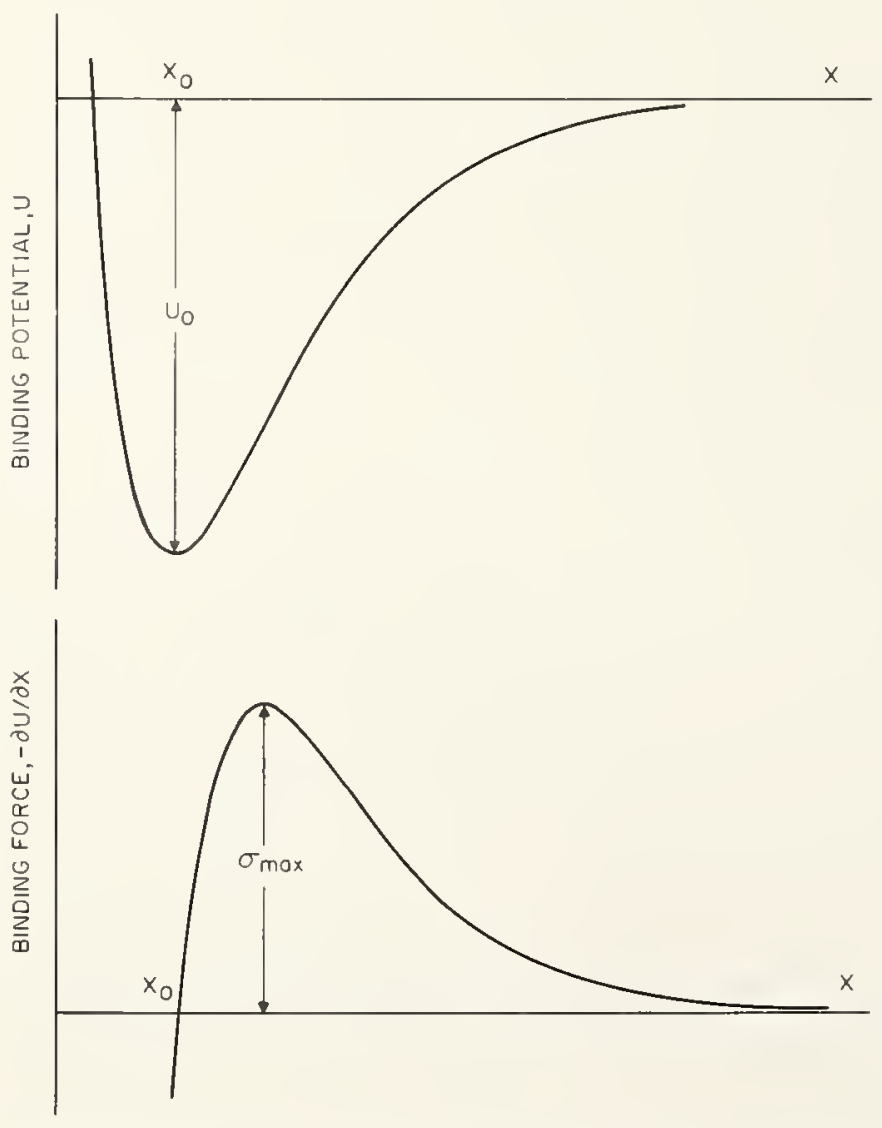

Figure 1. Binding potential function and first derivative. potential minimum is largely determined by the type of function selected to represent the potential. Consequently, to calculate the maximum stress, which occurs at the point of inflection of the potential energy curve, a potential function characteristic of the material under study should be selected. In this regard, a Born-type potential would be selected for ionic crystals, because it contains the long-range coulomb energy term characteristic of these materials, while a Morse-type potential would be selected for covalent solids which exhibit only short-range forces.

The use of the Morse and Born potential functions to calculate cohesive strength will now be illustrated for silica glass and sapphire respectively. The constants of eqs (1) and (2) must first be evaluated. At equilibrium displacement, $x=x_{0}$, the stress between the planes is zero. Consequently, $(\partial U / \partial x)_{x=x_{0}}=0$. By definition, the surface energy of a solid is equal to work necessary to form a unit area of surface. Therefore,

$$
\left.2 \gamma \equiv \int_{x_{0}}^{\infty} \sigma(x) d x=\int_{x_{0}}^{\infty}(\partial U / \partial x) d x=U\right]_{x_{0}}^{\infty}=-U\left(x_{0}\right)
$$

where $U\left(x_{0}\right)$ is the value of the potential minimum. At equilibrium displacement, $x_{0}$, the change in stress is directly proportional to the change in strain, Hooke's law, $d \sigma=E d x / x_{0}$, from which $E=x_{0}\left(\partial^{2} U / \partial x^{2}\right)_{x=x_{0}}$. Finally, the maximum stress occurs when $\partial^{2} U / \partial x^{2}=0$. Using these four conditions, it is easily shown that the maximum stress for the Morse potential is

$$
\sigma_{\max }=\sqrt{\gamma E / 4 x_{0}} .
$$

An equationn of this type was first derived by Orowan [3] using a linear approximation for the potential. The maximum stress calculated from the Born potential is

$$
\sigma_{\max }=\frac{E}{2}\left[\frac{2}{n+1}\right]^{n+1 / n-1},
$$

where $n=x_{0} E / 2 \gamma$.

The theoretical cohesive strength of silica glass, calculated from eq (3) using experimental values of $E, \gamma$, and $x_{0}$, table 1 , is $2.2 \times 10^{10} \mathrm{~N} / \mathrm{m}^{2}\left(3.2 \times 10^{6}\right.$ psi) while that of sapphire, calculated from eq (4), is $3.1 \times 10^{10} \mathrm{~N} / \mathrm{m}^{2}\left(4.5 \times 10^{6} \mathrm{psi}\right)$. While these values compare well with the highest strengths measured on these materials, it may be concluded that they are about 100 times larger than engineering strength values normally accepted for these materials, table 2. This conclusion leads naturally to questions of why ceramics are weak, how they can be made stronger, and how their strength can be characterized for design purposes. These questions will be discussed in succeeding sections of this paper. 
TABLE 1. Theoretical strength of silica glass and sapphire, (1010) plane

\begin{tabular}{|c|c|c|c|c|}
\hline Parameter & Fused silica & $\begin{array}{l}\text { Refer- } \\
\text { ence }\end{array}$ & Sapphire & $\begin{array}{l}\text { Refer- } \\
\text { ence }\end{array}$ \\
\hline $\begin{array}{l}\gamma \\
x_{0} \\
E \\
\sigma_{\max }\end{array}$ & $\begin{array}{l}4.56 \mathrm{~J} / \mathrm{m}^{2} \\
1.62 \times 10^{-10} \mathrm{~m} \\
7.2 \times 10^{10} \mathrm{~N} / \mathrm{m}^{2} \\
2.2 \times 10^{10} \mathrm{~N} / \mathrm{m}^{2}\end{array}$ & $\begin{array}{l}\text { Table } 3 \\
{[7]} \\
\text { eq (3) }\end{array}$ & $\begin{array}{l}7.3 \mathrm{~J} / \mathrm{m}^{2} \\
2.74 \times 10^{-10} \mathrm{~m} \\
42.5 \times 10^{10} \mathrm{~N} / \mathrm{m}^{2} \\
3.1 \times 10^{10} \mathrm{~N} / \mathrm{m}^{2}\end{array}$ & $\begin{array}{l}\text { Table } 4 \\
{[6]} \\
{[5]} \\
\text { eq (4) }\end{array}$ \\
\hline
\end{tabular}

In fused silica, the oxygen-silicon distance was taken as $x_{0}$. In sapphire, the oxygen-oxygen distance was taken as $x_{0}$. If half the oxygen-oxygen distance is taken as $x_{0}$ in sapphire, then $\sigma_{\max }=4.6 \times 10^{10} \mathrm{~N} / \mathrm{m}^{2}$.

TABLE 2. Measured strengths of silica glass and sapphire

\begin{tabular}{|c|c|c|}
\hline Material & Strength & Reference \\
\hline $\begin{array}{l}\text { Flame-polished silica, } \\
-196^{5} \mathrm{C} \text {. }\end{array}$ & $1.35 \times 10^{10} \mathrm{~N} / \mathrm{m}^{2}\left(1.96 \times 10^{8} \mathrm{psi}\right)$ & {$[8,9]$} \\
\hline Silica glass fibers, $-269 \mathrm{C}$. & $1.47 \times 10^{10} \mathrm{~N} / \mathrm{m}^{2}\left(2.1 \times 10^{6} \mathrm{psi}\right)$ & {$[10]$} \\
\hline Bulk silica glass............ & $1 \times 10^{3} \mathrm{~N} / \mathrm{m}^{2}\left(15 \times 10^{3} \mathrm{psi}\right)$ & [14] \\
\hline Flame-polished sapphire.... & $7 \times 10^{9} \mathrm{~N} / \mathrm{m}^{2}\left(1 \times 10^{6} \mathrm{psi}\right)$ & {$[13]$} \\
\hline Sapphire whiskers.......... & $1.1 \times 10^{10} \mathrm{~N} / \mathrm{m}^{2}\left(1.6 \times 10^{6} \mathrm{psi}\right)$ & [11] \\
\hline $\begin{array}{l}\text { Sapphire whiskers } \\
\text { Sapphire, single crystals...- }\end{array}$ & $\begin{array}{l}1 \times 10^{10} \mathrm{~N} / \mathrm{m}^{2}\left(1.5 \times 10^{8} \mathrm{psi}\right) \\
3.5-10 \times 10^{9} \mathrm{~N} / \mathrm{m}^{2}\left(50-150 \times 10^{3}\right.\end{array}$ & {$\left[\begin{array}{l}12 \\
{[14}\end{array}\right]$} \\
\hline & psi). & \\
\hline
\end{tabular}

\section{Flaw Theory of Fracture}

The low strength and brittle behavior of ceramics are almost entirely due to limitations on the amount and type of plastic deformation of these materials. Ceramics either do not deform plastically or possess an insufficient number of independent active slip systems to undergo generalized plastic deformation. An important consequence of this behavior is the fact that stress concentrations at crack tips or flaws cannot be relieved by generalized plastic flow. As a result any flaw contained within the body acts as a stress concentrator and is a potential nucleation site for fracture.

This section will deal with the flaw theory of fracture as developed by Griffith [15-17], who assumed that small cracks in the body of brittle materials were causing their low strength. Griffith was able to prove this by investigating the elastic stress distribution about an elliptically shaped crack in a uniform tensile stress field, figure 2. Using the stress analysis of Ingles [18], he was able to show that the tensile stress at the tip of such a crack was

$$
\sigma_{y y}=2 S \sqrt{L / \rho},
$$

where $L$ is half the length of the crack, $\rho$ is the radius of curvature at the crack tip, $S$ is the applied stress, and $L / \rho \gg 1$. If one assumes the flaw length to be of the order of $10^{-6} \mathrm{~m}$ and the radius of curvature of the crack tip to be $10^{-10} \mathrm{~m}$, then the stress concentration is of the order of 100 . Therefore, materials that ordinarily rupture at a stress of $10^{4}$ psi would have crack-tip stresses of the order of $10^{6} \mathrm{psi}$, which is approximately equal to the theoretical cohesive strength found pre-

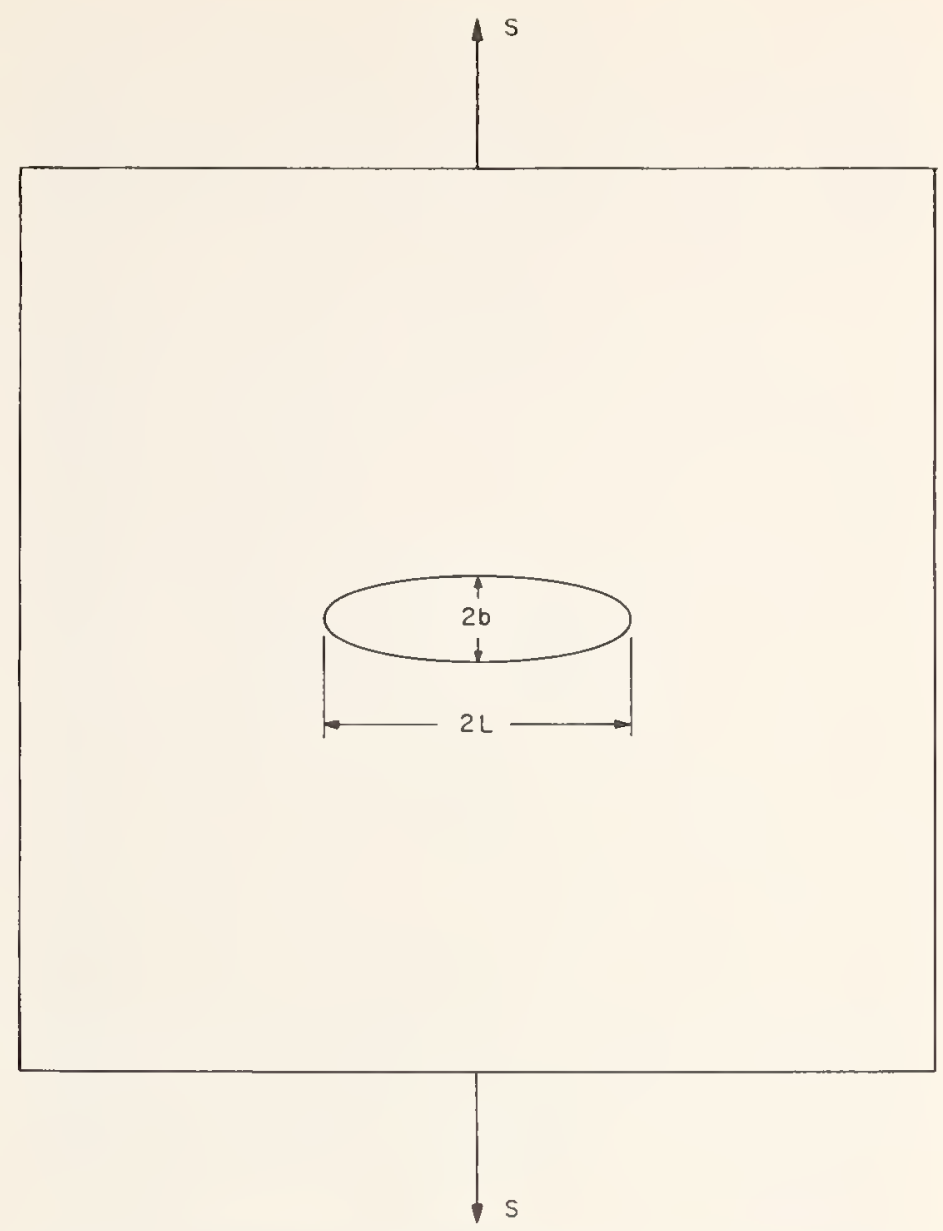

F1GURE 2. Elliptically shaped crack in an infinite twodimensional plane.

viously. Thus, the flaw theory seems to adequately account for the weakness of ceramic materials.

A second approach to the strength of materials was also conceived by Griffith $[15,16]$ by using a mechanical energy balance to determine the condition for material fracture. At incipient failure, the external work, $d W$, equals the change in energy stored in the material, $d V$, plus the energy necessary to form two new surfaces, $d \mathcal{S}=4 \gamma d L$, where $\gamma$ is the surface energy.

$$
d W=d V+d S
$$

From the elastic solution of the crack problem,

$$
\begin{aligned}
d(W-V)=[\partial(W-V) / \partial L] d L & \\
& =\left(2 \pi L S^{2} / E\right) d L=4 \gamma d L
\end{aligned}
$$

or

$$
S=\sqrt{2 E \gamma / \pi L},
$$

where $S$ is now the critical applied stress for failure. Equation 8 is the famous Griffith criterion for failure. Note the similarity between eqs (8) and (3). If one were to assume the crack length in the material to be of the order of the interatomic spacing, the rupture stress calculated by eq (8) would be of the order of the theoretical strength. In addition, the strength calculated for a crack of 
length $10^{-6} \mathrm{~m}$ is approximately one-hundredth of the theoretical strength, as found previously. Therefore, the energy balance approach for calculating weakening effects of cracks in completely brittle solids is consistent with the stress concentration approach in that they both predict the same degree of material weakening.

At first glance, the Griffith criterion for fracture might seem limited because it deals only with two-dimensional cracks and ignores the cohesive forces that must be acting between the fracture surfaces at the crack tip. In fact, the approach is applicable to a great variety of fracture problems. Three-dimensional crack problems that have been solved yield equations not greatly different from eq (8) $[19,20]$. Griffith recognized the fact that forces act across the crack tip, but avoided discussing them by assuming a finite radius of curvature at the crack tip. Crack problems with cohesive forces at the crack tip have been treated by Barenblatt [21], Elliot [22], and others [23, $24]$, with the conclusion that stresses are everywhere finite and the crack tip is cusp-shaped. 'These treatments are compatible with the Griffith assumption of a finite radius of curvature provided that radius is assumed to be of atomic dimensions. Therefore, Griffith's equation is valid provided an appropriate radius of curvature is assumed.

Several authors $[25,26]$ have discussed the relative merits of the stress concentration and energy balance methods as criteria for fracture, eqs (5) and (8) respectively. The stress concentration method assumes that a inaximum stress must be overcome for fracture, eq (5), while the energy balance method assumes fracture results after the creation of maximum amount of stored energy. The two approaches are equivalent for crack radii of the order of the interatomic spacing; however, for a large radius of curvature, the stress concentration approach, eq (5), is more fundamental since it always presents the criterion for fracture in terms of the maximum cohesive strength, which means that fracture always results when $\sigma_{y y}>\sigma_{\text {max }}$. Unfortunately, this more fundamental approach is not too useful since the crack root radius cannot be measured in most cases. In contrast, the energy approach can be used to establish fracture criteria provided it is recognized that the surface energy term reflects the chemical and mechanical processes occurring at the crack tip during fracture. The development of the Griffith ideas into useful fracture criteria will be discussed in the next section.

\section{Continuum Approach to Fracture}

\subsection{Stress Intensity Viewpoint}

The basic reason for the weakness of brittle materials is that cracks tend to redistribute internal stress fields, causing high stress concentrations at crack tips. Fracture occurs when the stress fields near the crack tip reach a critical value. This local criterion for fracture depends entirely on the stress fields at the crack tip. To determine the maximum load at fracture it is necessary to relate the crack tip stress field to the crack shape and applied load. 'Therefore, some understanding of the stress distribution around a crack tip is essential.

The most general form for the stress distribution near a crack tip was given by Irwin [27] and Williams [28], who noted that the stress field at any point near a crack tip, figure 3 , is related to the distance, $r$, from the crack tip, the angle of inclination, $\theta$, and a constant, $K$, called the stress intensity factor. The radial dependence is always inversely proportional to the square root of the distance from the crack tip, $r^{-1 / 2}$, regardless of the type of loading, crack geometry, or magnitude of the load. The angular dependence is independent of the magnitude of the load and flaw geometry, but does vary with stress component and type of loading. The stress intensity factor, $K$, is always proportional to the magnitude of the applied load and is related to the crack geometry and type of load, but is independent of inclination angle $\theta$. Before giving examples of stress field distributions, three general types of loading will be discussed.

Stress fields near a crack tip can be related to three basic modes of deformation, figure 4 . Mode $\mathrm{I}$, the opening mode, is associated with displacements perpendicular to the fracture surface.

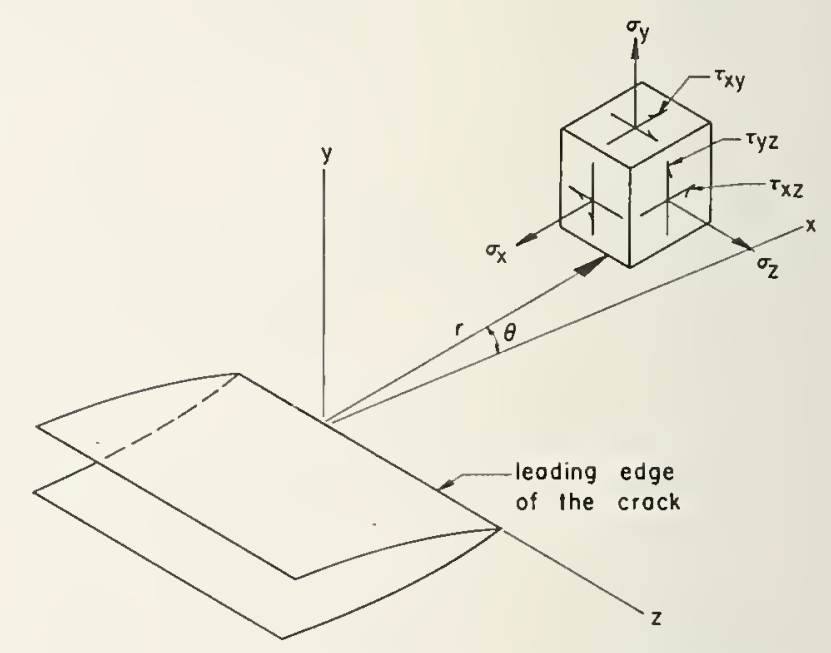

Figure 3. Coordinate relationship near the crack tip After P. C. Paris and G. C. Sih [29].

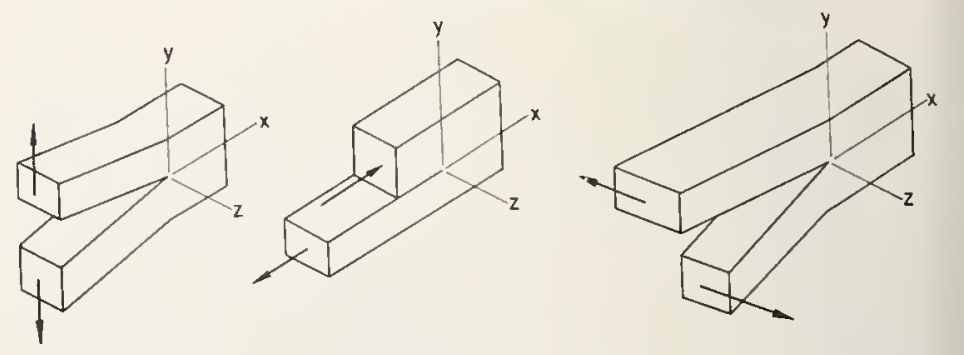

FIGURE 4. Three modes of loading After P. C. Paris and G. C. Sih [29]. 
Modes II and III are associated with displacements parallel to the fracture surface. Mode II is called the edge sliding mode and is characterized by shear displacements perpendicular to the crack tip. The tearing mode, mode III, is characterized by shearing displacements parallel to the crack edge. The superposition of these three modes is sufficient to describe the most general type of crack tip deformation in a general stress field. It is necessary to distinguish among these three modes for a particular application or test because the stress intensity factor and angular distribution depend on the mode of load application. Because ceramic materials fail in tension, mode $I$ is the most important type of loading. Consequently, most fracture tests in ceramics are designed for mode I failure. Torsion tests on ductile metals are an example of mode III loading.

The stress and displacement fields for mode I loading are listed below for the case of plane strain deformation [29]. The notation used is given in figure 3 .

$$
\begin{aligned}
\sigma_{x x} & =\left[K_{\mathrm{I}} /(2 \pi r)^{1 / 2}\right] \cos \theta / 2[1-\sin \theta / 2 \sin 3 \theta / 2] \\
\sigma_{y y} & =\left[K_{\mathrm{I}} /(2 \pi r)^{1 / 2}\right] \cos \theta / 2[1+\sin \theta / 2 \sin 3 \theta / 2] \\
\sigma_{x y} & =\left[K_{\mathrm{I}} /(2 \pi r)^{1 / 2}\right] \sin \theta / 2 \cos \theta / 2 \cos 3 \theta / 2 \\
\sigma_{z z} & =\nu\left(\sigma_{x x}+\sigma_{y y}\right), \sigma_{x z}=\sigma_{y z}=0 \\
u & =\left(K_{\mathrm{I}} / G\right)(r / 2 \pi)^{1 / 2} \cos \theta / 2\left[1-2 \nu+\sin ^{2} \theta / 2\right] \\
v & =\left(K_{\mathrm{I}} / G\right)(r / 2 \pi)^{1 / 2} \sin \theta / 2\left[2-2 \nu-\cos ^{2} \theta / 2\right] \\
w & =0
\end{aligned}
$$

$K_{\mathrm{I}}$ is the stress intensity factor for mode I loading, $u, v$, and $w$ are displacements in the $x, y$, and $z$ directions, and $G$ and $\nu$ are the shear modulus and Poisson's ratio, respectively. Equations for mode II and mode III deformation may be found in reference [29]. Stress intensity factors for these types of loading are denoted by $K_{\mathrm{II}}$ and $K_{\text {III. }}$.

The most significant feature of eqs (9) through (15) is the separation of the stresses and displacements into radial, angular, and stress intensity dependent portions. The angular and radial distribution of stress about the crack tip are identical for a given mode of loading. The level of stress, or stress intensity, depends entirely on the stress intensity factor which, in turn, is a function of the crack geometry and method of loading. In fact, the stress intensity factor is given by $K_{\mathrm{I}}=\alpha_{\mathrm{I}} S$, where $S$ is the applied load or applied stress and $\alpha_{I}$ is a geometric factor, in this case for opening mode failure. The geometric dependence of the stress intensity factor can be obtained by solving the appropriate elasticity equations, see reference [29]. Figures 5 through 7 give important examples of test specimen geometries for which the stress intensity factor has been solved [30-32]. In all cases, $K_{\mathrm{I}}$

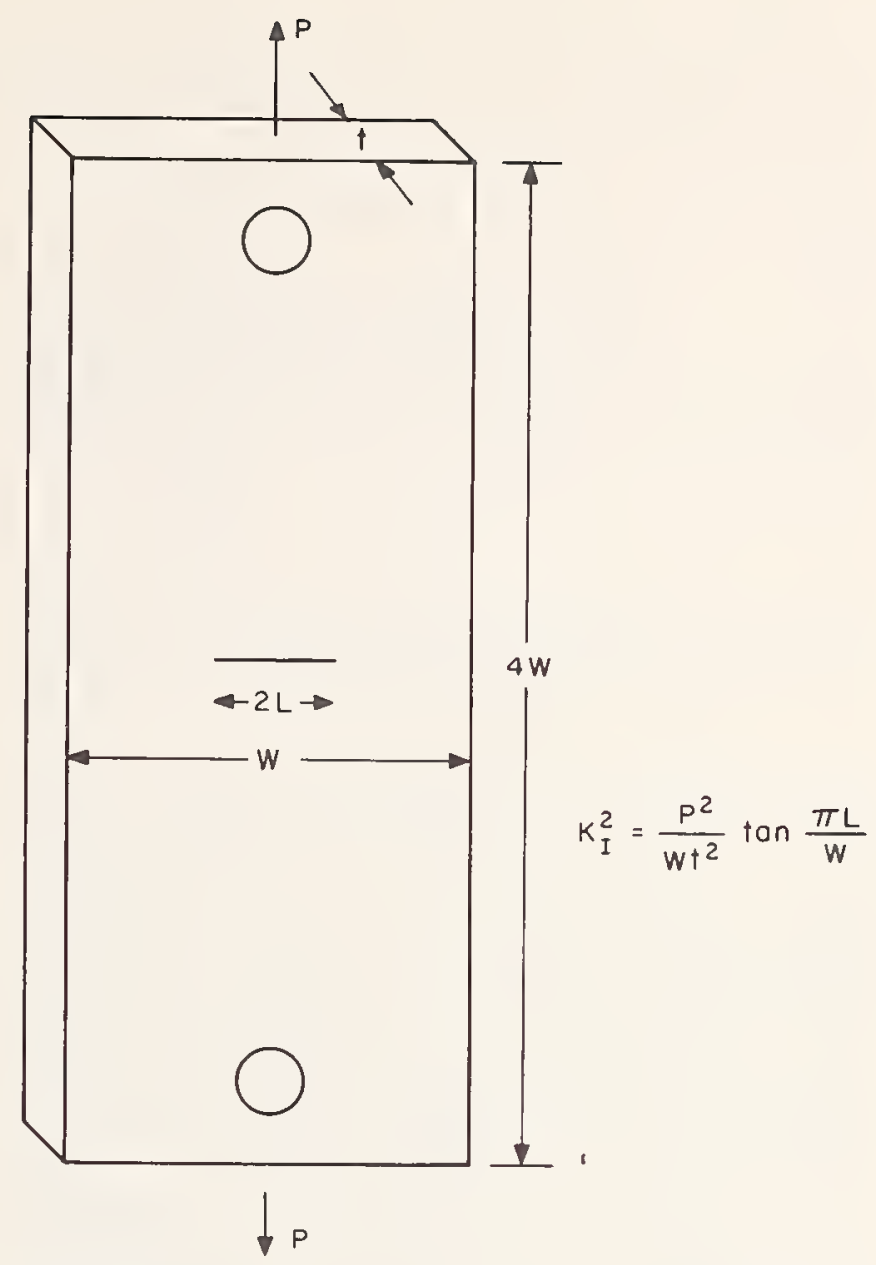

Figure 5. Center-notched plate tension specimen containing a crack of length $2 L$.

After J. E. Srawley and W. F. Brown [30].

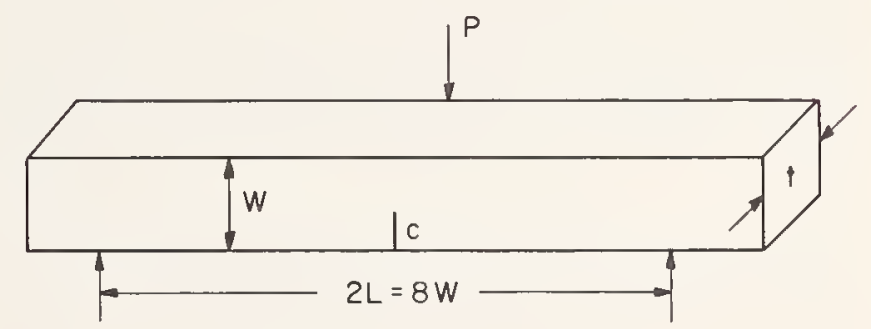

$K_{I}^{2}=\left(\frac{P}{\dagger}\right)^{2} \frac{L^{2}}{W^{2}}\left[31.7 c / W-64.8(c / W)^{2}+211(c / W)^{3}\right]$

FIGURE 6. Edge-cracked bend specimen containing a crack of length $c$.

For four-point loading see ref. [30]. After J. E. Srawley and W. F. Brown [30].

is dependent on the applied load $S$, the crack length and the specimen dimensions. A wide variety of other geometries is given in reference [29].

The radial dependence of the stresses in eqs (9) through (11) leads to the conclusion that the stresses are infinite at the crack tip, $r=0$. This conclusion is, of course, erroneous and arises from the assumption of a true continuum in deriving 


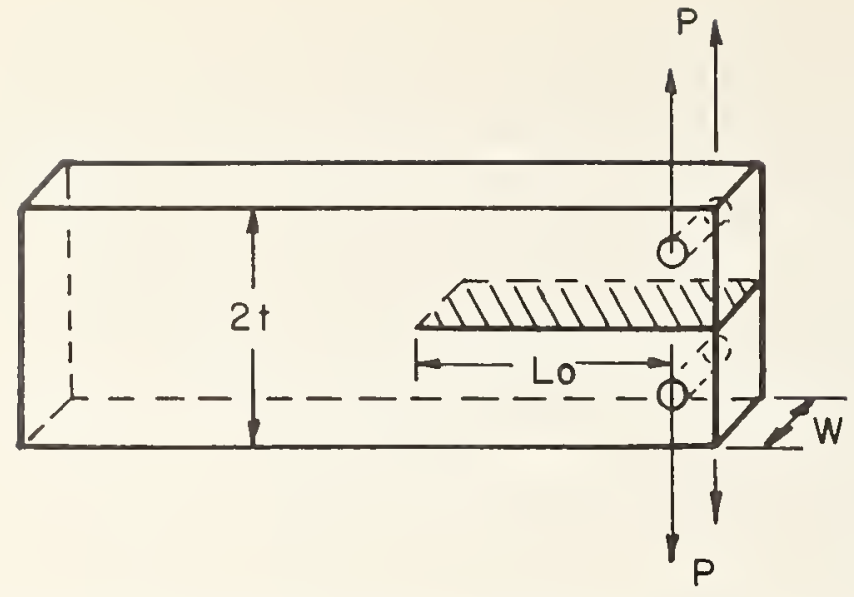

FIGURE 7. Double-cantilever specimen, $\mathrm{K}_{\mathrm{I}}=\left(\mathrm{PL} / \mathrm{Wt} \mathrm{t}^{1.5}\right)$ $(3.467+2.31, \mathrm{t} / \mathrm{L})$.

eqs (9) through (11). Since matter is not continuous, but discrete, deviations from eqs (9) through (14) will occur in brittle materials at distances from the crack tip of the order of several atomic dimensions, say $10^{-9} \mathrm{~m}$. In ductile materials crack blunting occurs and deviations from these equations result over even greater distances, depending on the amount of plastic flow. If the discrete nature of matter or size of the plastic zone is regarded as a disturbance in the continuum, then it can be concluded from St. Venant's principle that the effect of the disturbance is negligible at large distances with respect to the size disturbance and that the real stress fields approach those given by eqs (9) through (11) for sufficiently large distances. It is usually assumed in the continuum approach to fracture that the disturbance is small and is contained within the limits of applicability of eqs (9) through (11). This assumption is quite adequate for ceramics which exhibit little plastic relaxation at crack tips.

Because the stress fields near the crack tip of a real material approach those given by eqs. (9) through (11), a one-to-one relationship will exist between the maximum tensile stress at a crack tip and the value of the stress intensity factor at fracture. The strength of a material can therefore be characterized by a critical value of the stress intensity factor, usually denoted $K_{\text {IC }}$ for openingmode failure. $K_{\text {IC }}$ is a material constant dependent on physical processes occurring at the crack tip during fracture, but independent of crack geometry, specimen shape, or test procedure. It characterizes the inherent difficulty of propagating a crack in a material and provides information that is independent of the flaw distribution in the material. For a given load condition and crack geometry, the maximum load at fracture is obtained by equating $K_{\mathrm{I}}$ to $K_{\mathrm{IC}}$.

A valuable use of $K_{\text {IC }}$ is to predict the size of a plastic zone at a crack tip. The estimation of the plastic zone size presented below is based on the Dugdale $[33,34]$ model of plastic flow at a crack tip. The plastic zone is assumed to extend a distance $R$ in front of the crack, figure 8 . The length of the zone is

$$
R=(\pi / 8)\left(K_{\mathrm{IC}} / \sigma_{\nu}\right)^{2},
$$

where $K_{\mathrm{IC}}$ is the critical stress intensity factor and $\sigma_{\nu}$ is the yield stress of the material. When the plastic zone extends from the crack tip a distance $R$, the sides of the crack become displaced a distance $2 V(C)$ at the crack tip.

$$
V(C)=4 \sigma_{\nu} R / \pi E .
$$

Equations (16) and (17) are valid provided the breaking stress is much less than the general yield stress of the material, a condition that holds for most ceramics. Equations similar to (16) and (17) have been derived for other plastic flow models [35]. Equations (16) and (17) are valuable because they relate the extent of plastic flow at the crack tip to macroscopic measurements of yield stress and critical stress intensity factor at fracture.

\subsection{Surface Energy Vlewpoint}

Another method of treating fracture problems is the surface energy method, which is based on the fact that work is associated with the creation of new surfaces. As envisioned by Griffith, this energy was considered to be the thermodynamic free energy for the surface. In most cases, however, the energy associated with a fracture surface is not the surface free energy, but is much greater than the free energy of the surface. Irwin [17] and Orowan [3, 26] first noted that the surface energy in eq (8) should be written $\gamma=\gamma_{S}+\gamma_{P}$ where $\gamma_{S}$ is the surface free energy and $\gamma_{P}$ represents the amount of plastic work going into the formation of the surface. A number of other surface energy terms may enter into eq (8). For example, if crack propagation were accompanied by a chemical reaction a term $\gamma_{C}$, representing the energy of reaction per unit area of surface formed, would have to be subtracted from $\gamma_{S}$. Similar surface energy terms would be added for other processes and the total fracture surface energy $\gamma$ could be larger or smaller than the surface free energy $\gamma_{S}$. In metals, for example, $\gamma_{P}$ is typically $10^{4} \mathrm{~J} / \mathrm{m}^{2}$ while $\gamma_{S}$ is $1 \mathrm{~J} / \mathrm{m}^{2}$. Consequently, $\gamma \simeq \gamma_{P}$.

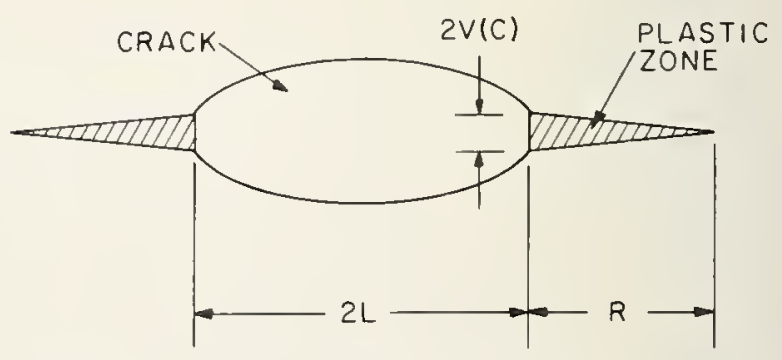

Figure 8. Dugdale model of plastic flow near a crack tip.

After G. R. Hahn and A. R. Rosenfield [34]. 
A general relationship between the fracture surface energy and experimental parameters can be derived using a mechanical energy balance $[17,37]$. Consider a crack in a solid, subjected to a constant load $P$, figure 9 . As the crack extends the work, $d W$, performed on the solid by the external load must equal the change of internal strain energy, $d V$, I lus the change of surface energy, $d \mathcal{S}, d W=d V+d \mathcal{S}$. The work by the external load is $P d u$, where $d u$ is the differential displacement of the $I$ ad $P$ as the crack extends a small amount. Thi total elastic strain energy of the body is $V=\int_{0}^{-} P d u$. For a linear elastic body, the displacement is proportional to the applied load, $u=\lambda P$, where $\lambda$ is called the compliance of the system. It therefore follows that the total elastic energy is $V=u^{2} / 2 \lambda=\mathrm{P} u / 2$, and at constant load $d V=1 / 2 P d u=1 / 2 d W$. The change in surface energy is $2 \gamma d A$ where $2 d A$ is the amount of surface formed as the crack propagates. As mentioned above, the surface energy per unit area of surface formed, $\gamma$, includes contributions from chemical and mechanical processes associated with crack motion. Substituting expressions for $d W, d V$, and $d \mathcal{S}$ into the energy balance, the following equation is obtained

$$
\gamma=(P / 4)(\partial u / \partial A)_{P}=\left(P^{2} / 4\right)(\partial \lambda / \partial A)_{P}
$$

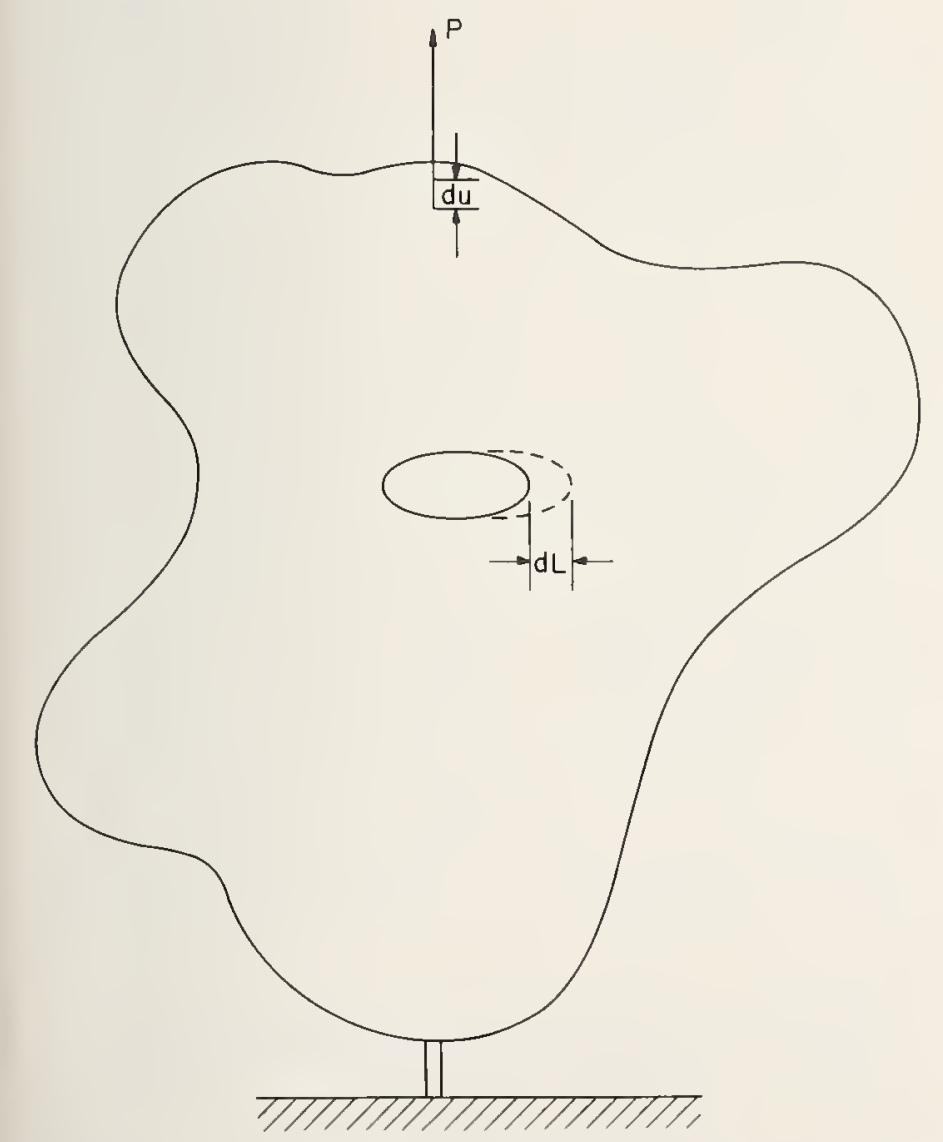

Figure 9. A body containing a crack that is extended a distance, $\mathrm{dL}$, by a force $\mathrm{P}$.

Displacement of the point of load application is $d u$. After P. C. Paris and G. C. Sih [29].
For specimens of constant thickness, $t, d A=t d L$ where $d L$ is the increase in crack length. Equation (18) then becomes

$$
\gamma=\left(P^{2} / 4 t\right)(\partial \lambda / \partial L)_{P}
$$

As discussed below, eq (18a) provides a general experimental means of measuring $\gamma$.

Irwin originally expressed many of his results in terms of a strain energy release rate, $\mathcal{G}$, which is just equal to twice the fracture surface energy, $\gamma$. In parallel to the stress intensity factor definitions, $\mathscr{G}_{\text {IC }}, \mathscr{G}_{\text {IIC }}$, and $\mathscr{C}_{\text {IIIC }}$ represent the critical strain energy release rates for crack motion, modes I, II, and III respectively. They are material constants dependent on the physical processes occurring at the crack tip during fracture, but independent of crack geometry, specimen shape or test procedure. Finally, it should be noted that eq (18) is valid for fixed grip as well as fixed load conditions.

The relationship between the surface energy and stress intensity approaches to fracture can be easily proven $[17,27,37]$. The calculation will be given for opening mode failure and then the general relationship between stress intensity factors and strain energy release rate values will be presented. As a result of these relationships, the two fracture viewpoints may be used interchangeably.

Consider the crack shown in figure 10 and assume that the crack faces are gradually loaded in some reversible manner to cause the crack to close a distance $\alpha$. Prior to closing the crack, the surfaces are stress free and the contour of the crack surfaces is described by eqs (13) through (15). After closing the surfaces, the stresses are described by eqs (9) through (12) and the crack surfaces are coplanar with the surface $y=0$. The reversible work to close the fracture surfaces is

$$
\mathscr{C}_{\mathrm{IC}}=(2 / \alpha) \int_{0}^{\infty}\left(\frac{1}{2} \sigma_{v y} v\right) d x
$$

Other possible energy terms containing $\left(\sigma_{x x} u\right) / 2$ or $\left(\sigma_{x y} v\right) / 2$ all equal zero. The stress $\sigma_{y y}$ is given by eq (10) with $r=x$ and $\theta=0$. The displacement $v$ is given by eq (14) with $r=\alpha-x$ and $\theta=\pi$. Substituting eqs (10) and (14) into eq (19) and integrating, one finds,

$$
G_{1 C}=\frac{(1-\nu) K_{\mathrm{IC}}{ }^{2}}{2 G}=\frac{\left(1-\nu^{2}\right) K_{\mathrm{IC}}{ }^{2}}{E}
$$

Values for other modes of displacement are

$$
\begin{aligned}
& \mathscr{C}_{\mathrm{IIC}}=\frac{\left(1-\nu^{2}\right)}{E} K_{\mathrm{IIC}}{ }^{2} \\
& \mathcal{G}_{\mathrm{IIIC}}=\frac{(1+\nu)}{E} K_{\mathrm{IIIC}}{ }^{2} .
\end{aligned}
$$




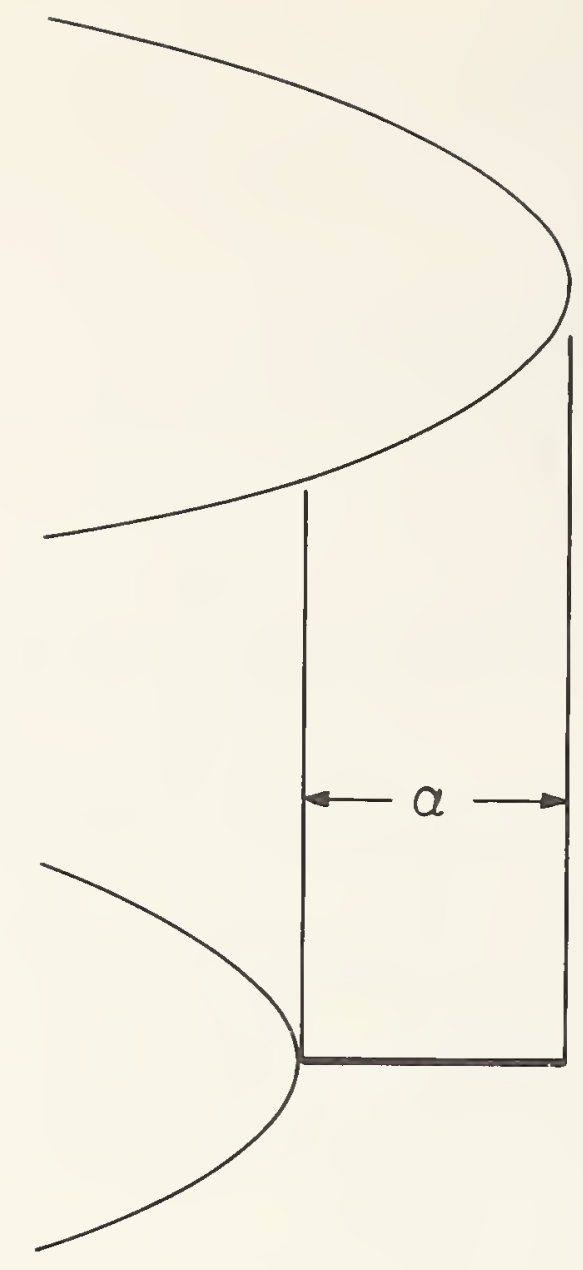

Figure 10. Closure of a crack a distance $\alpha$ by applying the stresses of equations (9)-(11) to the free surface of the crack.

After P. C. Paris and G. T. Sih [29].

For combined modefailure $\mathscr{C}_{\mathcal{L}}=2 \gamma=\mathscr{G}_{\mathrm{I}}+\mathscr{G}_{\mathrm{II}}+\mathscr{G}_{\text {III }}$, which constitutes a generalized Griffith condition for crack propagation. Equations (20) and (21) are for plane strain. Equations for plane stress are obtained by dropping $\nu^{2}$ from eqs (20) and (21). Equations (20) through (22) are given for isotropic media. Other types of media are discussed in the literature [29] and relationships between the stress intensity factors and strain energy release rates values are available.

\subsection{Measurement Methods}

There are three experimental methods for determining the critical fracture parameters, $K_{\text {IC }}$ and $\mathscr{C}_{\text {IC }}$. In each of these methods $K_{\text {IC }}$ and $\mathscr{G}_{\text {IC }}$ are related through eq (20). One method requires a solution of the crack problem for the specimen geometry under consideration so that $K_{\mathrm{I}}$ can be expressed as a function of applied load, crack size, and sample dimensions. $K_{\text {IC }}$ is obtained from a strength measurement on the material being studied. At failure $K_{\mathrm{IC}}=K_{\mathrm{I}}$. Therefore, $K_{\mathrm{IC}}$ is calculated from the specimen dimensions, crack size and critical load at failure. For a given set of physical circumstances, $K_{\mathrm{IC}}$ should be independent of test method.

Several practical test geometries are shown in figures 5 through 7 . The center notched plate tension specimen (fig. 5) and the edge-cracked bend specimen (fig. 6) have been used extensively in the testing of metals and the dimensions suggested are for metallic materials. The double cantilever specimen (fig. 7) also known as the infinite crack-line loaded edge-crack or Majoine specimen, has been used in various forms for both metals and ceramic materials.

The second method is called the compliance method and requires the use of eq (18a) to determine $\mathcal{G}_{\text {IC }}$. A series of specimens is prepared containing a range of crack sizes. As the specimen is loaded, the relative displacements of the loading points, $u$, must be measured as a function of $P$ for each crack length. Since $\lambda=u / P, \lambda$ can be plotted as a function of $L$ and $\partial \lambda / \partial L$ can be determined from the slope of the plot at any crack length. Because the derivative of $\lambda$ is important in the determination of $\mathscr{L}_{\text {IC }}$, displacements and loads must be measured to a high degree of accuracy. The main limitation of this method is the displacement measurements. The method has the great advantage of being applicable to any specimen shape and can be used to check elastic solutions of crack problems.

In the third method of measurement, the work to propagate a crack over a complete cross section is determined [38, 39]. The test is conducted in three point bending and edge cracked bend specimens are used (fig. 6). This type of test requires a testing machine with a large spring constant. Specimens are slowly deformed at a continuous, constant rate throughout the test, and the test is continued until specimens have been completely fractured. The method depends on the fact that as the crack propagates, the compliance, $\lambda$, decreases, and crack propagation occurs continuously and stably with gradually decreasing load. The force-displacement curve during the test is as depicted in figure 11, and the area under the curve is equal to the total work performed during the experiment. There is no elastic energy stored in the testing machine at the completion of the test, since the machine is load free once the crack has passed through the specimen. Consequently, all of the work performed during the experiment has gone into the fracture process and the strain energy release rate, $\mathscr{G}_{\mathrm{IC}}$, can be determined by dividing the total work by half the total fracture area.

The three methods of measurement just described are based on three different criteria for fracture. Method 1, the stress intensity method, is based on the assumption that crack motion occurs when the stress intensity in the vicinity of the crack tip has reached a certain level. The critical stress intensity will depend on the physical 


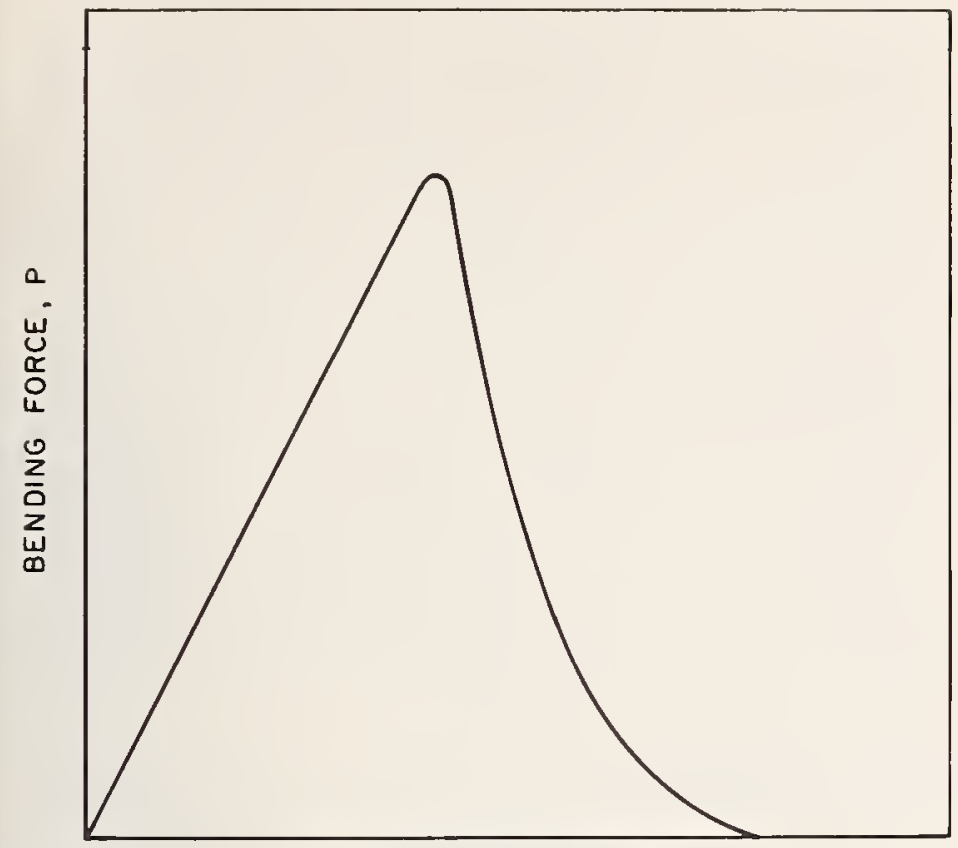

DISPLACEMENT, U

Figure 11. Force displacement curve for the work of fracture method of determining the fracture surface energy.

processes occurring at the crack tip, but not on crack size or shape.

In the second method of measurement, the compliance method, fracture occurs when the net mechanical energy input into the body equals the surface energy required for the production of new fracture surfaces. The method is differential in that energy values are expressed in the limit as the amount of new fracture surface area approaches zero. Thus, the second method, as the first, is only concerned with initial crack motion. The equivalence of the two methods is proven by eqs (20) through (22).

The third method, the work of fracture method, is also based on an energy criterion for fracture. It differs from the second method in that the fracture surface energy is determined by an integral technique. In other words, the surface energy is determined by an averaging procedure over the entire fracture cross section. Fracture surface energy values obtained by the third method are related more to crack propagation than crack initiation. This difference has been emphasized recently by Davidge and Tappin [40], who classify fracture surface energy measurements with reference to crack initiation, $\gamma_{I}$, or crack propagation, $\gamma_{F}$. Their experiments on graphite, alumina, polymethylmethacrolate and glass show that $\gamma_{I}$ and $\gamma_{F}$ are not necessarily equal, but depend on physical processes occurring during fracture.

\section{Experimental Fracture Surface Energy Values}

The available fracture surface energy data will be reviewed in this section, first for glass, then for single crystals and bicrystals, and finally for polycrystalline ceramics.

\subsection{Glass}

Glass provides an excellent material for testing the various ideas presented in previous sections. It is elastically isotropic so that linear elastic theory is applicable. It can be made homogeneous so there is no concern with the interaction of the crack tip with internal flaws or grain boundaries. Finally, glass is one of the most brittle materials known, so there is little concern with plastic flow at crack tips. The one limitation of glass is its extreme susceptibility to stress corrosion. The data presented will be that for which there was little or no stress corrosion.

Table 3 presents a summary of data for various glasses tested in various environments. Most of the data were obtained by the double cantilever method; however, two sets were obtained by other means. Agreement among the various test methods is good. Variations in measured fracture energy due to differences in composition and temperature were as high as 50 percent, suggesting a maximum difference of about 25 percent in the inherent cohesive strength of silica based glasses. The surface energy values of table 3 can be substituted into eq (3), yielding maximum tensile strengths of approximately $2 \times 10^{10} \mathrm{~N} / \mathrm{m}^{2}$ $\left(3 \times 10^{6} \mathrm{psi}\right)$, which agree within a factor of 2 with data available in the literature, table 2 .

\section{TABLE 3. Surface energies of glass, $J / \mathrm{m}^{2}$}

Double cantilever specimens, method I

\begin{tabular}{|c|c|c|c|}
\hline Glass & $N_{2}(g)$ & $N_{2}(l)$ & Toluene- $\mathrm{CO}_{2}$ \\
\hline $\begin{array}{l}\text { Fused silica } \\
\text { 96 percent silica } \\
\text { Soda lime-_ime } \\
\text { Borosilicate- } \\
\text { Aluminosilicate... } \\
\text { High lead. }\end{array}$ & $\begin{array}{r}25 C \\
4.32 \\
4.00 \\
3.82 \\
4.75 \\
4.65 \\
3.50\end{array}$ & $\begin{array}{r}-196 C \\
4.56 \\
4.17 \\
4.53 \\
4.80 \\
5.21 \\
4.11\end{array}$ & $\begin{array}{r}-60 C \\
4.86 \\
4.60 \\
4.38\end{array}$ \\
\hline
\end{tabular}

Plate glass 3.4-5.2, $25 \mathrm{C}$, ref. [38], modified edge crack bend specimen, method III.

Soda-lime glass 4.06, Vacuum, ref. [41], center notch plate tension specimen, method I.

It is of some interest to use eqs (16) and (17) to estimate the size of the plastic zone at the crack tip. The yield stresses for soda-lime glass and fused silica at liquid nitrogen temperature are $1 \times 10^{10} \mathrm{~N} / \mathrm{m}^{2}$ and $1.95 \times 10^{10} \mathrm{~N} / \mathrm{m}^{2}$ respectively [42]. Data for the critical values of the stress intensity factor are given in table 3. The lengths, of $R$, the plastic zones for soda-lime glass and silica glass are $2.6 \times 10^{-9} \mathrm{~m}$ and $6.4 \times 10^{-10} \mathrm{~m}$ respectively. The displacements, $V(C)$, for the same glasses are $4.5 \times 10^{-10} \mathrm{~m}$ and $2.2 \times 10^{-10} \mathrm{~m}$ respectively. The very small value of the calculated plastic zones satisfies all of the assumptions of fracture mechanics and, in addition, dramatizes the reason for the very brittle nature of glass. In the absence of large amounts of plastic flow there is no mechanism for absorbing energy during crack motion. As a result, a crack once started tends to propagate catastrophically. 


\subsection{Single Grystals}

The fracture of single crystals depends on the crystallographic orientation of the fracture plane and occurs more easily along those planes having the lowest fracture surface energy [4]. In extreme, but common, cases, this behavior leads to cleavage fracture, where fracture occurs only on a single crystallographic plane characterized by a fracture surface energy considerably lower than any other plane in the crystal. In less extreme cases, an orientation dependence of the fracture strength is observed, as in the case of sapphire [43]. Thus, a complete characterization of the fracture behavior of single crystals requires the determination of $\gamma, \mathscr{G}_{\mathrm{I}}$, or $K_{\mathrm{IC}}$ as a function of the orientation of the fracture plane.

Most fracture surface energy determinations on single crystals have been made on crystals that cleave. Thus, the energy values published are characteristic only of the cleavage planes in the crystal. Since many crystals that cleave exhibit fracture on no other crystal planes, these fracture surface energy values completely characterize the fracture in these materials.

A summary of some of the fracture surface energy data on crystals that cleave is given in table 4 . 'The data obtained by the double cantilever technique have been recalculated by the author according to the equation in figure 7 , which was not available at the time of the original publications. The data presented in table 4 were obtained on specimens tested in dry environments or in vacuum. The test conditions were such as to eliminate environmental effects and to reduce plastic flow at crack tips. Since the results presented in table 4 were close to those expected theoretically, plasticity and environmental effects may have been eliminated.

TABI 4 . Surface energies of single crystals

\begin{tabular}{|c|c|c|}
\hline Crystal & Energy $J / m^{2}$ & Reference \\
\hline $\begin{array}{l}\text { Mica, vacuum, } 25 \mathrm{C} \\
\text { Mica, vacuum, } 25 \mathrm{C} \\
\mathrm{LiF}, \mathrm{N}_{2}(1), 196 \mathrm{C}, \\
\mathrm{MgO}, \mathrm{N}_{2}(1),-196 \mathrm{C} \\
\mathrm{CaF}_{2}, \mathrm{~N}_{2}(1),-196 \mathrm{C}\end{array}$ & $\begin{array}{l}\text { 4. } 5 \\
4.57 \\
0.40 \\
1.5 \\
0.55\end{array}$ & $\begin{array}{l}{[44]} \\
{[45]} \\
{[46]} \\
{[46]} \\
{[46]}\end{array}$ \\
\hline 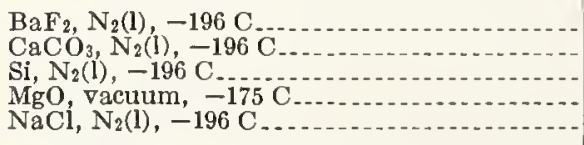 & $\begin{array}{l}0.31 \\
0.35 \\
1.8 \\
1.6 \\
0.28\end{array}$ & $\begin{array}{l}{[46]} \\
{[46]} \\
{[46]} \\
{[47]} \\
{[47]}\end{array}$ \\
\hline 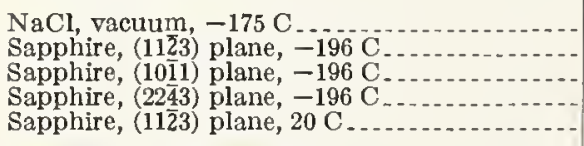 & $\begin{array}{l}0.24 \\
32.2 \\
24 \\
16.4 \\
24.4\end{array}$ & $\begin{array}{l}{[47]} \\
{[48]} \\
{[48]} \\
{[48]} \\
{[48]}\end{array}$ \\
\hline $\begin{array}{l}\text { Sapphire, (101̄1) plane, } 25 \text { C } \ldots \ldots \\
\text { Sapphire, }(10 \overline{10}) \text { plane, } 25 \text { C } \ldots \ldots\end{array}$ & $\begin{array}{l}6.0 \\
7.3\end{array}$ & {$\left[\begin{array}{l}122] \\
{[122]}\end{array}\right]$} \\
\hline
\end{tabular}

Data from references [46] and [47] were recalculated using the equation in figure 7 . The values given represent averages of the three smallest surface energy values in each group. Data from reference [48] were obtained on were obtained on double cantilever specimens. Morphological indices are used for sapphire, $c / a=1.365$.
Fracture surface energy measurements on noncleavable single crystals have been obtained only on sapphire, $\alpha-\mathrm{Al}_{2} \mathrm{O}_{3}$. The rather meager available data are presented in table 4. Energy values obtained by Petch et al. [48] are about 10 times that expected theoretically. The authors explain the high values as due either to energy absorption by plastic deformation or to the possible fact that the drilled hole did not offer a suitably sharp crack from which fracture could initiate. Considering the low fracture energy values obtained by Wiederhorn [122], it is improbable that plastic deformation plays a role in increasing the surface energy for fracture. Mechanical twinning induced by fracture has been observed by several investigators and may be playing a role in the (10 $\overline{1} 1$ ) fracture energy measurements [49-52].

\subsection{Bicrystal Fracture Energy Measurements}

Bicrystals occupy an intermediate place between single crystals and polycrystalline solids. They contain a single grain boundary, which is characterized by the relative orientation of the crystals on each side of the boundary. Because polycrystalline ceramics fracture primarily along grain boundaries, a measurement of grain boundary fracture energies is important. In theory, the grain boundary fracture energy can be obtained on specimen geometries such as those given in figures 5 through 7, provided the boundary is coplanar with the crack. Class and Machlin [53] have performed this type of experiment on twist boundaries of $\mathrm{KCl}$ using the double cantilever method for their study. Their results, figure 12, indicate that grain boundaries are weaker than single crystals. For large angle grain boundaries, the fracture surface energy is less than 50 percent of that for normal cleavage. These first experiments suggest the necessity for additional work on bicrystals.

\subsection{Polycrystalline Ceramics}

Fracture surface energy values obtained on polycrystalline ceramics, table 5 , are an order of magnitude greater than those obtained from single crystals. In addition, for small grain sizes the fracture surface energies of polycrystalline $\mathrm{MgO}$ and $\mathrm{Al}_{2} \mathrm{O}_{3}$ increase with increasing grain size, table 5. Since the strength of a material is expected to be proportional to the square root of the fracture surface energy, eq (8), polycrystalline ceramics should be stronger than single-crystal ceramics and strength should increase with increasing grain size. From the best available strength data on polycrystalline ceramics [56-58], these conclusions are erroneous. Single crystals are stronger than polycrystals and strengths increase with decreasing grain size. Consequently, it appears there is no correlation between strength and fracture energy measurements for polycrystalline ceramics. As will be discussed in a later section 


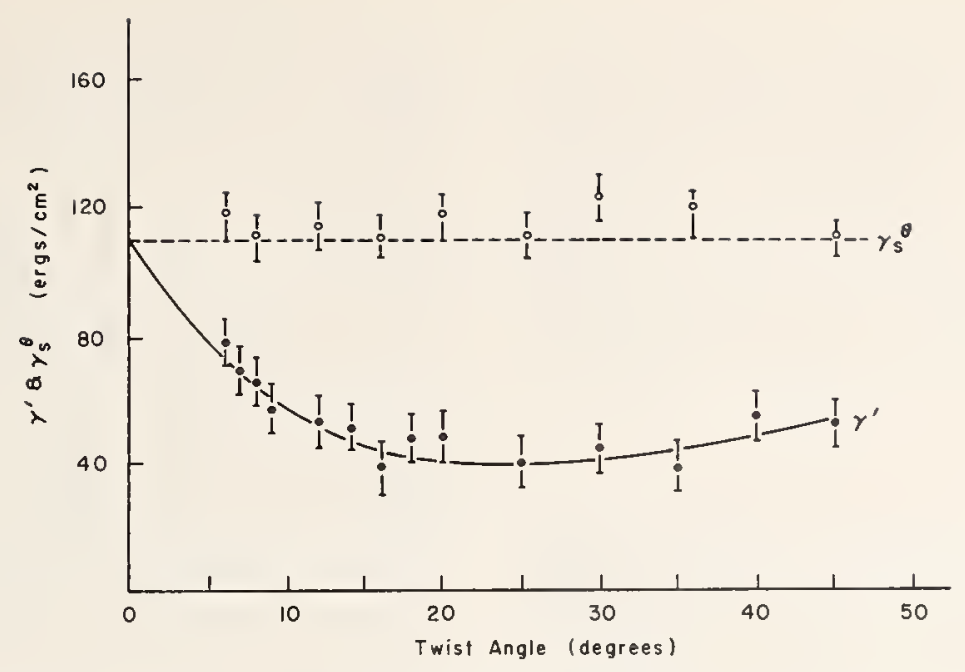

FIGURE 12. Fracture surface energy of $\mathrm{KCl}$ bicrystal twist boundaries.

The upper curve gives the fracture surface energy of the (001) surface and the twist angle refers to the direction of crack propagation on the (001) surface. The lower curve gives the fracture surface energy of the twist boundary. The angle of twist is given by the ordinate. After W. H. Class and E. S. Machlin [53].

of the paper, this lack of correlation suggests that the strength of polycrystalline materials depends more on single crystal and grain boundary fracture surface energies than on polycrystalline values of fracture surface energy. The polycrystalline values of surface energy are valuable for some types of mechanical and thermal shock.

The high fracture energy of polycrystalline ceramics is due to the tortuous nature of the crack path in these materials [54, 59], figure 13. Crack perimeters must extend through and around many grains and can be pinned at many points along the boundary by high-energy obstacles consisting of poorly alined grains and grain boundaries. As a result, the crack must overcome all of these obstacles to propagate, requiring an amount of work greater than is necessary to propagate in single crystals. Some energy absorbing processes for fracture have been discussed recently by Clarke et al. [54, 59] and include secondary crack formation, plastic flow, area increase due to roughness of the fracture surface, and step formation which leads to high-energy tear-

TABLE 5. Surface energies of polycrystalline ceramics

\begin{tabular}{|c|c|c|c|c|}
\hline Material & Density & Grain size & Energy & Reference \\
\hline Firebrick, Douglas X.... & $\mathrm{kg} / \mathrm{m}^{3} \times 10^{3}$ & meters $\times 10^{-6}$ & $\begin{array}{c}J / m^{2} \\
30\end{array}$ & [54] \\
\hline $\begin{array}{l}\text { MgO } \\
\text { MgO } \\
\text { MgO }\end{array}$ & $\begin{array}{l}3.48 \\
3.51 \\
3.56\end{array}$ & $\begin{array}{r}10 \\
50 \\
100\end{array}$ & $\begin{array}{l}16 \\
19.5 \\
35\end{array}$ & $\begin{array}{l}{[54]} \\
{[54]} \\
{[54]}\end{array}$ \\
\hline $\begin{array}{l}\text { MgO, theoretical density- } \\
\text { MgO, theoretical density- } \\
\text { MgO, theoretical density- } \\
\text { MgO, theoretical density-- } \\
\text { MgO, theoretical density- } \\
\text { MgO, theoretical density- }\end{array}$ & $\begin{array}{l}\text { 3. } 58 \\
\text { 3. } 58 \\
3.58 \\
\text { 3. } 58 \\
\text { 3. } 58 \\
\text { 3. } 58\end{array}$ & $\begin{array}{r}7 \\
13 \\
23 \\
38 \\
130 \\
150\end{array}$ & $\begin{array}{r}4.2 \\
8.9 \\
16 \\
17 \\
14 \\
7.9\end{array}$ & $\begin{array}{l}{[54]} \\
{[54]} \\
{[54]} \\
{[54]} \\
{[54]} \\
{[54]}\end{array}$ \\
\hline $\begin{array}{l}\mathrm{BeO} \\
\mathrm{Graphite} \\
\mathrm{SiN} \\
\mathrm{Al}_{2} \mathrm{O}_{3} \text {, Lucalox } \\
\mathrm{Al}_{2} \mathrm{O}_{3} \text {, Lucalox } \\
\mathrm{Al}_{2} \mathrm{O}_{3}, \text { Lucalox........ }\end{array}$ & \begin{tabular}{l}
2.79 \\
\hdashline 2.2 \\
3.97 \\
3.97 \\
3.97
\end{tabular} & $\begin{array}{l}10 \\
30 \\
45\end{array}$ & $\begin{array}{r}15 \\
100 \\
20 \\
18 \\
27 \\
46\end{array}$ & $\begin{array}{l}{[54]} \\
54] \\
{[54]} \\
{[55]} \\
{[55]} \\
{[55]}\end{array}$ \\
\hline
\end{tabular}

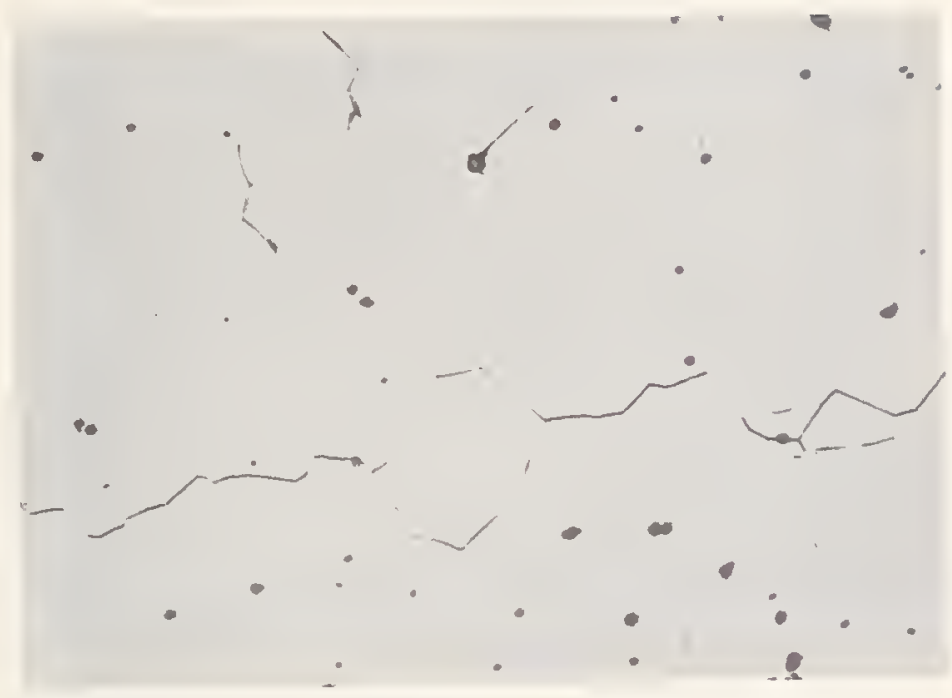

FIGURE 13. Crack propagating through polycrystalline $\mathrm{Al}_{2} \mathrm{O}_{3}$. Note the random nature of the crack path. After R. L. Coble, ref. [14]. p. 623 .

ing, mode III fracture. The exact role played by each of these processes has not yet been elucidated.

\section{Applications of Fracture Mechanics to Ceramics}

Discussions of previous sections were phenomenological in outlook. No attempt was made to relate the fracture process to fundamental phenomena such as microstructure, chemical composition, and ability to deform plastically. Instead, fracture was assumed to be controlled by three experimental parameters, fracture surface energy, Young's modulus, and flaw size. From an engineering point of view, this approach is valuable because it provides a convenient means of classifying materials. The fracture mechanics approach will be continued in this section where it will be 
applied to problems of strength, thermal shock, and stress corrosion. Where applicable, the phenomenological approach will be correlated with the microscopic approach. The effect of plastic deformation, grain size, chemical composition, and crystal structure on the fracture surface energy will be discussed. For an excellent coverage of the microscopic aspects of fracture, the reader is referred to the literature, references [60-62].

\subsection{Strength of Glass}

Materials that are homogeneous, isotropic, and completely brittle with absolutely no plastic flow, are the simplest to classify mechanically. Crack propagation in these materials is not influenced by the presence of grain boundaries, cleavage planes, plastic deformation, or stress inhomogeneities due to anisotropic thermal expansion. Fracture surface energies are isotropic, but may depend on environment. Many inorganic glasses fit this category and in the absence of environmental effects have surface energies given in table 3. Since these energies are nearly independent of composition, as is Young's modulus for these glasses, fracture is almost completely controlled by flaws contained in these glasses $[63,64]$. Experiments have shown that flaws are almost entirely limited to the external surfaces. It follows that glass can be strengthened either by eliminating surface flaws or by preventing them from propagating. The latter approach has been used in the recent development of surface toughened glasses [65]. The surfaces of these glasses are put into a state of compressive stress either by chemical or thermal treatment. Surface flaws and cracks then cannot result in fracture until the surface stress has been overcome by the applied load. In this manner, the strength of ordinary glass has been increased from 10,000 psi to $70,000 \mathrm{psi}$, an increase of sevenfold. The ideas of surface strenghtening have been extended to recrystallized glasses with even more impressive results [65].

\subsection{Strength of Single Crystals}

As noted by Stokes [62], the mechanical behavior of ceramics may be divided into three categories. Depending on the amount and type of plastic deformation that can occur, ceramic crystals are completely brittle, ductile, or semi-brittle.

Completely brittle crystalline ceramics cannot be deformed plastically as dislocations are immobile and all deformation is elastic. As a result, failure occurs by brittle fracture. Generally, covalent crystals at less than $0.5 T_{m}$ are in this category where $T_{m}$ is the melting temperature. Since inorganic glasses fit this category, the conclusions and statements concerning the strength of glass are equally valid for completely brittle single crystals. Fracture is governed by accidental surface flaws, the elastic constants and the surface energy, the latter two depending on crystallographic orientation of the fracture plane. In the absence of surface flaws, single crystal strengths exceed $10^{6}$ psi.

Ductile crystals can be deformed quite easily. Dislocations move readily with a great deal of flexibility in choice of slip plane. Ductile crystals can be deformed into arbitrary shapes by applied loads, and stress concentrations can always be relieved by plastic flow. As a result, stresses never exceed the cohesive strength of the solid and failure occurs by plastic instability, in which the solid necks to a point and then separates. Plastic behavior is demonstrated by the silver halides [66] at low temperature and by many ionically bonded materials at high temperatures [67,68], greater than $0.5 T_{m}$.

Semi-brittle crystals can be deformed plastically but, in contrast to ductile solids, cannot be deformed into arbitrary shapes by external loads. As a result, stress concentrations cannot be relieved by random shape changes and fracture occurs when local stresses exceed the cohesive strength of the material. This behavior results from the fact that the number of active slip systems in these materials is insufficient for ductile behavior. In addition, screw dislocations in semibrittle crystals are limited to specific slip planes and cannot maneuver from one plane to another by cross slip [62]. Semi-brittle crystals fail by fracture and are sensitive to the presence of surface cracks and notches $[66,69]$. Fracture is also caused by stress concentrations due to slip band interactions with grain boundaries [70-74] and other slip bands [75]. Semi-brittle crystals consist of ionic materials below $0.5 T_{m}$ and covalent crystals above $0.5 T_{m}$.

The type of failure of a solid may be qualitatively related to its plastic properties via the Von Mise criterion [76-78], which states that at least five independent slip systems must be active for ductile behavior. Any number less than this results in semi-brittle behavior and the complete absence of slip results in completely brittle behavior. The plastic behavior of several ceramic materials is given in table 6. Most ceramics are semi-brittle or completely brittle at room temperature. As the temperature is increased above 0.5 $T_{m}$, ceramics become semi-brittle or ductile.

For further discussion of the topics presented in this section, references $[61,62]$ are suggested.

\subsection{Strength of Polycrystalline Ceramics}

In this section, a discussion will be presented of some of the factors that effect the strength of polycrystalline ceramics. Crack nucleation due to grain boundary blocking of plactic deformation will be discussed first. Then a theory of crack nucleation due to anisotropic thermal expansion will be presented. Finally, the effect of grain boundaries on crack propagation will be discussed. 


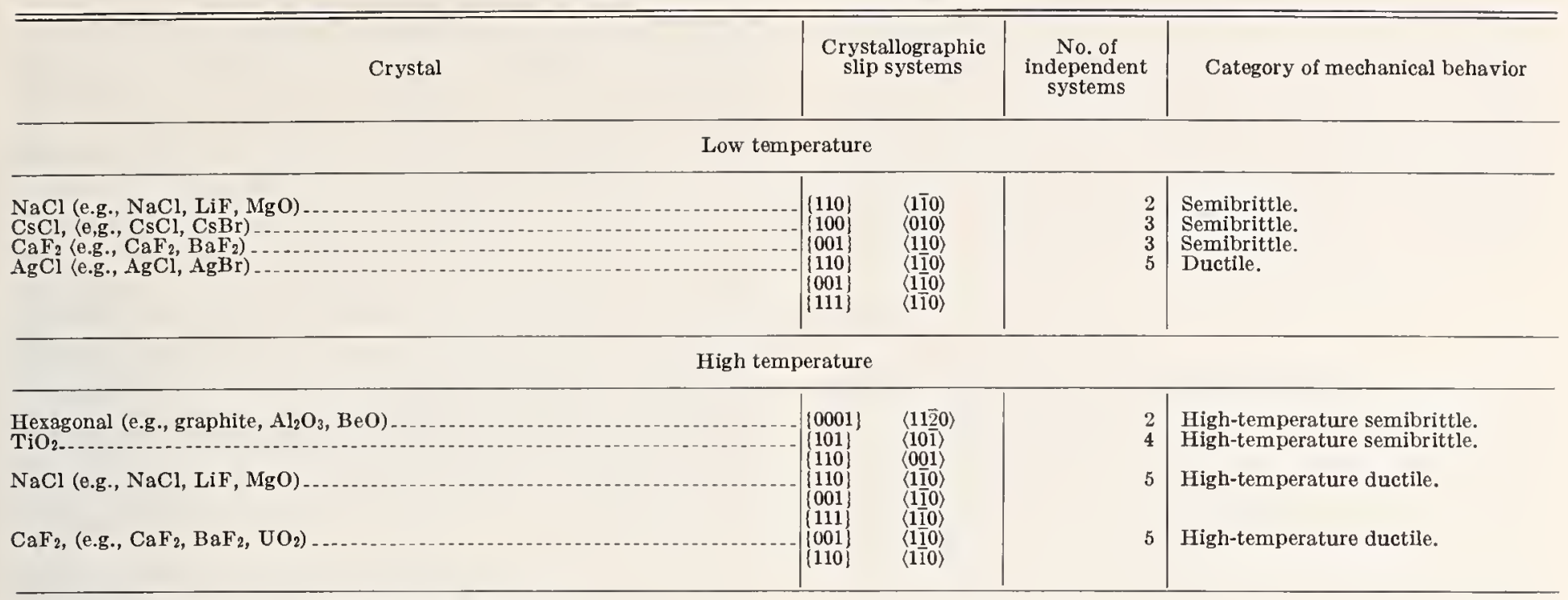

Taken from ref. [79]. Original sources, refs. [62, 78, 121].

Crack nucleation due to grain boundary blocking of plastic deformation was first suggested by Zener [80], developed by Stroh [81] and most recently discussed by Smith and Barnby [82]. Suppose a dislocation slip band has impinged upon a grain boundary as depicted in figure 14. Since the slip planes on either side of the boundary are not alined, the dislocations will be blocked at the boundary, setting up a stress field at the point of impingement. The stress field is described by an equation very similar to that obtained for a freely slipping crack under a shear stress [81], mode II deformation,

$$
\sigma=\left(\sigma_{s}-\sigma_{i}\right)(L / r)^{1 / 2} f(\theta)
$$

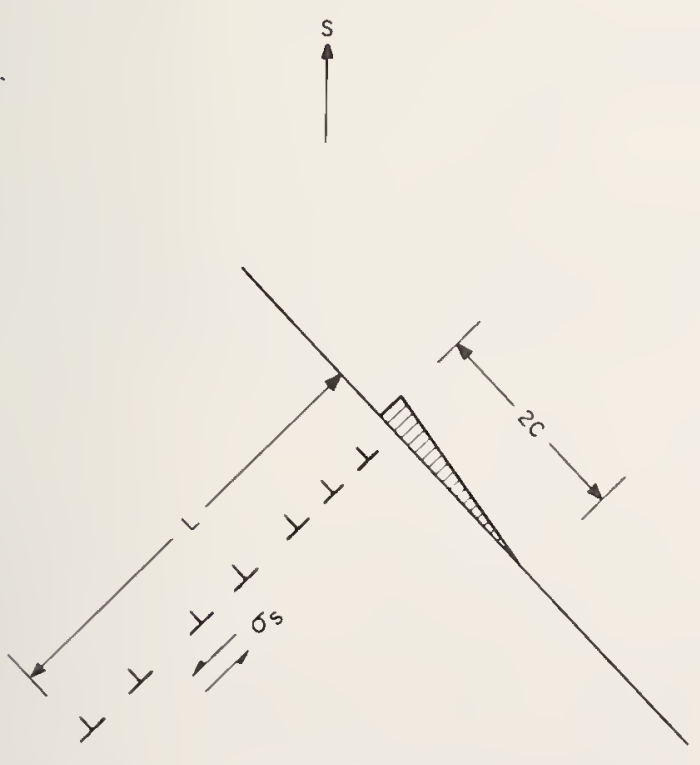

FIGURE 14. Slip band of length, L, impinging on a grain boundary to nucleate a crack of length $2 C$.

Applied tensile stress is $S$ and resolved shear stress along the slip plane is $\sigma S$ where $\sigma_{3}$ is the resolved shear stress along a slip band of length $L$ and $\sigma_{i}$ is the shear resistance of the material referred to as the friction stress. To a first approximation the friction stress is the minimum stress for dislocation motion.

The stress field at the crack tip will nucleate plastic deformation in the adjacent grain and the stress field will be relieved by plastic flow if the material is ductile. If the material is not ductile, but semi-brittle, stress will build up at the grain boundary and fracture will occur when the stresses at the boundary exceed the cohesive strength of the solid. For a Morse type potential the following equation is obtained as a condition for failure,

$$
\sqrt{\gamma E / 4 x_{0}}=\left(\sigma_{s}-\sigma_{i}\right)(L / r)^{1 / 2} f(\theta) .
$$

Assuming $L$ is proportional to the grain size, $D$, and $\sigma_{s}$ is equal to half the applied tensile stress, $\sigma_{s}=1 / 2 S$, eq (24) can be rearranged to give a Petch-type relationship [83],

$$
S=\sigma_{0}+k D^{-1 / 2}
$$

which relates the stress to nucleate fracture, $S$, to the grain size, $D$, and the minimum applied tensile stress, $\sigma_{0}$, for dislocation motion.

The constant, $k$, can be evaluated by obtaining a relationship between $L$ and the grain size $D$ and by evaluating $f(\theta)$. Eshelby et al. [84] give $L=D / 4$ while the maximum value of $f(\theta)$ is approximately 1 . Substituting these values into eq (24) and setting $r=x_{0}, k=\left(4 E_{\gamma}\right)^{1 / 2}$ is obtained. The exact value of $k$ depends on assumptions made in the derivation of eq (25). Using an energy balance and a more exact procedure Stroh [81] obtained $k=\left[3 \pi \gamma E /\left(1-\nu^{2}\right)\right]^{1 / 2}$, which is about 1.7 times that given above.

A second mechanism for crack nucleation was given by Clarke [85] and is based on the fact that internal stresses occur in polycrystalline ceramics 
due to anisotropic thermal contraction or radiation induced anisotropic expansion. Fracture is assumed to initiate at grain boundary pores and then to propagate along the grain boundary, figure 15, absorbing energy from both the external and internal strain fields. These fields arise, respectively, from the applied stresses and the expansion anisotropy. A Griffith type energy balance is used to derive the critical stress for fracture

$$
\sigma=\left\{E\left[2 \gamma_{b}-E \epsilon^{2}\left(l-c_{0}\right) / 12\left(1-\nu^{2}\right)\right] / \pi c_{0}\right\}^{1 / 2},
$$

where $\epsilon$ is the grain boundary strain; the grain size is $2 l ; \gamma_{b}$ is the grain boundary surface energy; and $c_{0}$ is the grain boundary pore diameter.

Equation (26) is essentially the Griffith equation with an additional term to account for the thermal expansion strain energy release per unit area of crack formed. The equation predicts spontaneous fracture when the term inside the square brackets equals zero,

$$
2 \gamma_{b}=E \epsilon^{2}\left(l-c_{0}\right) / 12\left(1-\nu^{2}\right)
$$

or for $c_{0} \ll l$,

$$
\epsilon=\left(24 \gamma_{b} / E l\right)^{1 / 2} .
$$

The validity of this last equation has been demonstrated recently by Davidge and Tappin [86] on polycrystalline $\mathrm{BeO}$.

Once a crack has been nucleated, it must be able to propagate for complete fracture to occur [87]. Ubstacles, such as grain boundaries, may

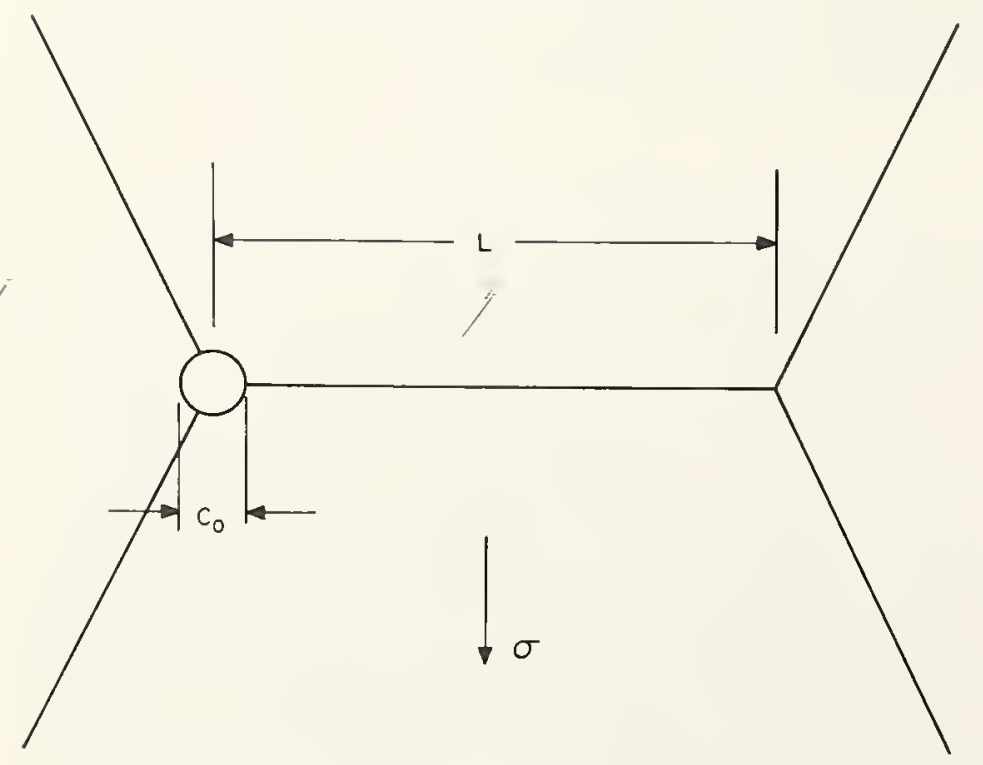

FIGURE 15. Model for crack initiation at a grain boundary pore due to thermal stresses.

After F. J. P. Clarke [85]. hinder crack growth and thus prevent complete fracture. The increase in stress required for a crack to abruptly change directions at a grain boundary has been recently calculated by Gell and Smith [88], and by Barnet and Tetelman [60]. The method of treatment is identical to that used for dislocation pileups at grain boundaries.

Consider a crack that has been momentarily arrested at a grain boundary, figure 16. Crack motion is assumed to reinitiate either at some tilt angle, $\theta$, to the original crack or at a twist angle, $\phi$, The stress distribution around the arrested crack, given by eqs (9) through (12) may be resolved along the projected plane of propagation by the appropriate tensor transformation. For the case of a two dimensional Griffith crack reinitiating along a tilt plane, $\theta$, these stresses are,

$$
\begin{aligned}
& \sigma_{12}=K_{S} /(2 \pi r)^{1 / 2} \\
& \sigma_{22}=K_{T} /(2 \pi r)^{1 / 2}
\end{aligned}
$$

where $K_{S}$ and $K_{T}$ are given by,

$$
\begin{gathered}
K_{S}=-S(\pi d)^{1 / 2} \sin \theta / 2 \cos ^{2} \theta / 2 \\
K_{T}=S(\pi d)^{1 / 2} \cos ^{3} \theta / 2
\end{gathered}
$$

$\sigma_{12}$ and $\sigma_{22}$ are respectively the shear and tensile stress on the projected plane of propagation. The stresses on these planes are identical to those obtained on the projection of the original crack plane provided combined mode I and mode II deformation is assumed with stress intensity actors $K_{\mathrm{I}}=K_{T}$ and $K_{\mathrm{II}}=K_{\mathrm{S}}$. Therefore, the condition for crack reinitiation for the case of plane strain is given by the sum of eqs (20) and (21),

$$
K_{S}{ }^{2}+K_{T}{ }^{2}=2 E \gamma /\left(1-\nu^{2}\right) .
$$

The criterion for reinitiation on the new plane therefore becomes

$$
\sigma=\left[2 E \gamma / \pi d\left(1-\nu^{2}\right)\right]^{1 / 2} \sec ^{2} \theta / 2 .
$$

Note that the condition for crack reinitiation along a new direction is the Griffith equation modified by the angular dependence $\sec ^{2} \theta / 2$. This conclusion is also true for other crack shapes.
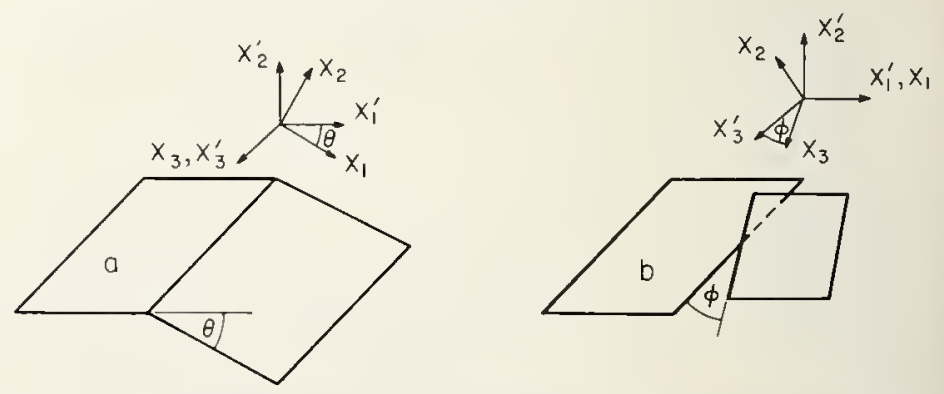

Figure 16. Change of crack plane orientation as crack traverses a grain boundary.

a) tilt propagation, b) twist propagation. After M. Gell and E. Smith [88]. 
The treatment by Gell and Smith [88] considers the nucleation of a new crack at the edge of a penny shaped crack of radius $d$. The equations for propagation on a plane of tilt $\theta$, or twist $\varphi$, figure 16 , can be obtained by methods identical to those given above. The reinitiation stress required for the tilt and twist case respectively are

$$
\begin{aligned}
& \sigma=\left[\pi \gamma E / 2 d\left(1-\nu^{2}\right)\right]^{1 / 2} \sec ^{2} \theta / 2 \\
& \sigma=\left[\pi \gamma E / 2 d\left(1-\nu^{2}\right)\right]^{1 / 2} \sec ^{2} \varphi .
\end{aligned}
$$

Again, the Griffith equation for crack motion is modified by trigonometric functions of $\theta$ and $\varphi$. Equations (34) through (36) give the condition for reinitiation of crack propagation and are rigorous only in this limit, since changes in the stress field due to the reinitiated crack are not considered.

The increase in stress for crack reinitiation depends on the angle of tilt or twist as would be expected. For fracture along grain boundaries, tilt propagation is most important, since all grain boundaries meet at tilt angles. If a polycrystalline aggregate is assumed to be a nested assembly of truncated octahedra (ref [14], page 410) the angle of tilt will always be $60^{\circ}$. In a real material, however, the angle of tilt scatters around this value and ranges from 0 to $90^{\circ}$. Since the larger angles will control the ease of fracture, it might be expected that tilt angles ranging from $60^{\circ}$ to $90^{\circ}$ will account for the more important obstacles to crack propagation. Tilt angles in this range are equivalent to surface energy increases of from 1.8 to 4 times the single crystal or grain boundary surface energy value. Similar increases in measured surface energies can occur for twist propagation, which plays an important role in limiting transgranular crack propagation [88].

In the above discussions of crack nucleation and propagation, a critical length and a surface energy parameter were always involved in establishing the criteria for fracture. In general, both of these parameters must be considered to decide which of the possible mechanisms controls fracture. In the dislocation pileup mechanism, eq (25) the length parameter is the grain diameter, while the surface energy parameter is the grain boundary or single crystal surface energy, table 4 . For the thermal expansion mechanism, eq (26), the parameters are the pore diameter and the grain boundary fracture energy. For the crack propagation mechanism, the critical surface energy depends on the size of the fracture nucleus. If the starting crack is several grain diameters in size, the critical surface energy will be the same as that measured on polycrystalline specimens, table 5 . If the starting crack is of the order of a grain diameter in size, then the critical surface energy will be the grain boundary or single crystal fracture surface energy, possibly modified by a trigonometric function as in eqs (34) through (36). These mechanisms will now be discussed with regard to experimental results.
Conclusive experimental evidence of the pileup mechanism in $\mathrm{MgO}$ at room temperature has been given by several people. $\mathrm{Ku}$ and Johnston [71] proved the validity of the Petch equation, eq (15), for bicrystals of $\mathrm{MgO}$. $D$ was shown to be the distance between the origin of the glide band and the grain boundary, $\sigma_{\circ}$ was shown to be the stress for dislocation multiplication at room temperature rather than the minimum stress to move dislocations, and $k$ gave a qualitative measure of the cohesive strength. The value of the friction stress, $\sigma_{0}$, was $5 \times 10^{7} \mathrm{~N} / \mathrm{m}^{2}\left(7.25 \times 10^{3} \mathrm{psi}\right)$.

Stokes and $\mathrm{Li}$ [70] investigated the effect of mobile dislocations on the strength of $\mathrm{MgO}$ and found that bicrystals possessed a tensile strength of over $7 \times 10^{8} \mathrm{~N} / \mathrm{m}^{2}(100,000 \mathrm{psi})$ in the absence of dislocation mobility, but only $7 \times 10^{7} \mathrm{~N} / \mathrm{m}^{2}$ ( $10,000 \mathrm{psi})$ in the presence of dislocation mobility. The reduction in strength resulted from an interaction of the dislocations with the bicrystal grain boundary. Stokes and Li also showed that dislocation mobility reduces the strength of 100 percent dense polycrystalline $\mathrm{MgO}$ from $20 \times 10^{7} \mathrm{~N} / \mathrm{m}^{2}$ (30,000 psi) to between 10 and $14 \times 10^{7} \mathrm{~N} / \mathrm{m}^{2}$ $\left(15-20 \times 10^{3} \mathrm{psi}\right)$.

Recently Rice presented evidence for the dislocation pileup mechanism in hot pressed and hot extruded MgO [89]. Fracture data on both types of material were found to give a straight line on a Petch type plot. The calculated friction stress was $4 \times 10^{7} \mathrm{~N} / \mathrm{m}^{2}(6,000 \mathrm{psi})$, which is close to the value obtained by $\mathrm{Ku}$ and Johnston. Fracture was transgranular so that the initial source of fracture could always be identified. Microscopic examination of the sources of fracture gave evidence of slip band pileups at the source of fracture. Thus there can be no doubt as to the importance of dislocation mobility to the strength of $\mathrm{MgO}$ at room temperature or of the importance of the pileup mechanism to crack nucleation in polycrystalline $\mathrm{MgO}$.

The expansion anisotropy mechanism of crack nucleation has been demonstrated on polycrystalline $\mathrm{BeO}$ and also may be important in $\mathrm{Al}_{2} \mathrm{O}_{3}$. The experimental evidence on $\mathrm{BeO}$ was obtained by Davidge and Tappin [86], who used neutron irradiation to develop expansion anisotropy strains in $\mathrm{BeO}$. Spontaneous cracking along grain boundaries was observed when eq (28) was satisfied, with $\gamma_{b}$ assumed to be about $1 \mathrm{~J} / \mathrm{m}^{2}\left(10^{3} \mathrm{ergs} / \mathrm{cm}^{2}\right)$. Further evidence for fracture induced by thermal expansion anisotropy was presented by Coble [90] who demonstrated spontaneous cracking in completely dense $\mathrm{Al}_{2} \mathrm{O}_{3}$, Lucalox, for grain sizes greater than $100 \mu \mathrm{m}$.

Crack propagation as a limiting mechanism failure has been suggested by Davidge and Tappin [86], who have shown that polycrystalline $\mathrm{BeO}$ does not exhibit significant weakening until cracks of the order of 1 to 3 grain diameters have appeared. Consequently, strength in $\mathrm{BeO}$ is believed to be limited by crack propagation from 
flaws 1 to 3 grain diameters in size with a surface energy of about $10 \mathrm{~J} / \mathrm{m}^{2}$.

An interesting correlation for fracture of $\mathrm{MgO}$, $\mathrm{BeO}$, and $\mathrm{Al}_{2} \mathrm{O}_{3}$ was presented by Carniglia $[91,92]$ who plotted published strength data of several authors $[56,93-95]$ as a function of $\mathrm{D}^{-1 / 2}$ and obtained bimodal curves such as the one shown in figure 17 for $\mathrm{MgO}$. Fracture data from the upper portion of this curve was attributed to plastic flow and could be represented by the Petch relationship, eq (25). The friction stress can be obtained by extrapolating the upper portion of the curve to infinite grain size and should be of the same order of magnitude as the yield strength of the material. Fracture behavior depicted by the steeply sloped portion of the curve was attributed to elastic crack propagation from internal cracks or flaws of the order of a grain size in dimensions and could be represented by a Griffith type relationship, eq (8) where $L$ is proportional to the grain size. At high temperatures the upper portion of the curves for $\mathrm{BeO}$ and $\mathrm{Al}_{2} \mathrm{O}_{3}$ had negative slopes that were attributed to grain boundary sliding. Thus, failure of polycrystalline $\mathrm{BeO}, \mathrm{Al}_{2} \mathrm{O}_{3}$, and $\mathrm{MgO}$ is believed to be limited by crack growth for large grain size, by crack nucleation due to plastic flow or grain boundary sliding for small grain size. Although Carniglia's explanations of fracture behavior of ceramics might seem reasonable, additional research is necessary to prove his views, since the shapes of the fracture curves are not sufficient evidence in themselves to prove his viewpoint.

One drawback to Carniglia's correlation is its failure to predict friction stresses for $\mathrm{MgO}, \mathrm{Al}_{2} \mathrm{O}_{3}$,

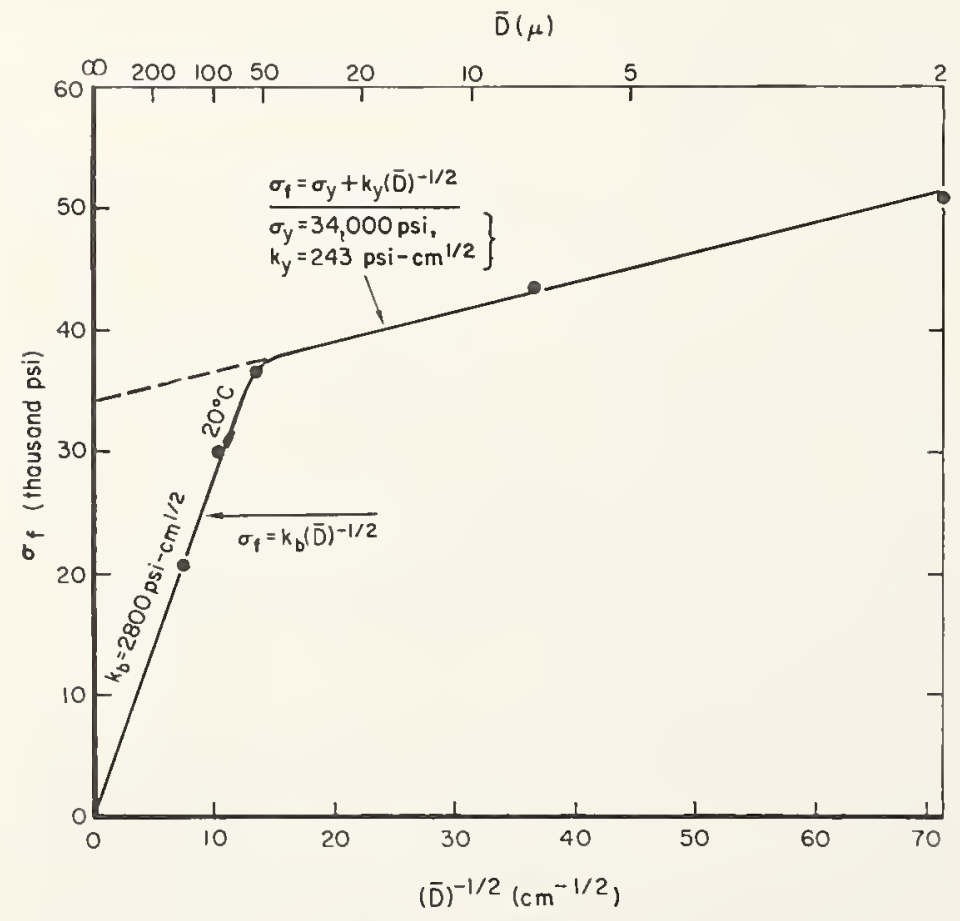

Figure 17. Petch plot of MgO bending data. After S. C. Carniglia [91, 92] and $\mathrm{BeO}$ that agree with other data in the literature. For example, the friction stress for $\mathrm{MgO}$ obtained from figure 17 is $2.35 \times 10^{8} \mathrm{~N} / \mathrm{m}^{2}(34,000$ $\mathrm{psi})$. This value is about five times the friction stress obtained by $\mathrm{Ku}$ and Johnston, $5 \times 10^{7} \mathrm{~N} / \mathrm{m}^{2}$ $\left(7.25 \times 10^{3} \mathrm{psi}\right)$ on bicrystals of $\mathrm{MgO}$; four times the stress required to initiate yielding in $\mathrm{MgO}$ single crystals [96], $5.5 \times 10^{7} \mathrm{~N} / \mathrm{m}^{2}\left(8 \times 10^{3} \mathrm{psi}\right)$; and six times the friction stress obtained by Rice on polycrystalline $\mathrm{MgO}, 4 \times 10^{7} \mathrm{~N} / \mathrm{m}^{2}\left(6 \times 10^{3} \mathrm{psi}\right)$. Similarly, friction stresses of $2.9 \times 10^{8} \mathrm{~N} / \mathrm{m}^{2}(42 \times$ $\left.10^{3} \mathrm{psi}\right)$ and $2.5 \times 10^{8} \mathrm{~N} / \mathrm{m}^{2}\left(37 \times 10^{3} \mathrm{psi}\right)$ are obtained for $\mathrm{Al}_{2} \mathrm{O}_{3}$ and $\mathrm{BeO}$ at room temperature despite the fact that single crystals of these materials are not known to deform plastically at room temperature $[43,123]$.

Some comment should be made on the relationship between the fracture energy measurements presented in tables 4 and 5 and the observed strength of $\mathrm{MgO}$ and $\mathrm{Al}_{2} \mathrm{O}_{3}$. It is possible to use the Griffith equation for a penny shaped crack [19], $\sigma=\sqrt{\pi E_{\gamma} / D\left(1-\nu^{2}\right)}$, to calculate the strength of fracture of polycrystalline $\mathrm{MgO}$ and $\mathrm{Al}_{2} \mathrm{O}_{3}$ from the data in table 5. Calculated strength results for 100 percent dense $\mathrm{MgO}$ and $\mathrm{Al}_{2} \mathrm{O}_{3}$ are in agreement with measured values provided crack nuclei are assumed to be 10 grain diameters for $\mathrm{MgO}$ and 10 to 20 grain diameters for $\mathrm{Al}_{2} \mathrm{O}_{3}$. Nucleation cracks of this magnitude have never been reported in these materials, therefore, it is reasonable to conclude that the fracture surface energy values listed in table 5 are not to be associated with the strength of these materials. In contrast, a similar calculation on the strength of $\mathrm{MgO}$ and $\mathrm{Al}_{2} \mathrm{O}_{3}$ using surface energies characteristic of the single crystal, table 4 , gives a predicted flaw size of approximately one to three grain diameters, which is in better agreement with that expected. It therefore appears that the strength of these materials is controlled either by crack nucleation or propagation from nuclei the order of a grain size in diameter, In either case, the surface energy is of the order of the single crystal value.

Discussions of the preceding sections have been limited to three materials, alumina, beryllia, and magnesia, and to mechanisms that are important at low temperatures. It is felt that the mechanisms presented have general applicability to many other types of ceramics at low temperatures. At high temperatures, where diffusion can occur, fracture is affected by other phenomena such as grain boundary sliding and Nabarro-Herring creep [97]. Figure 18 is illustrative of the type of failure that can occur at high temperatures during creep. This type of behavior has not yet been treated by methods of fracture mechanics, however, there may be important applications of fracture mechanics in this direction. 


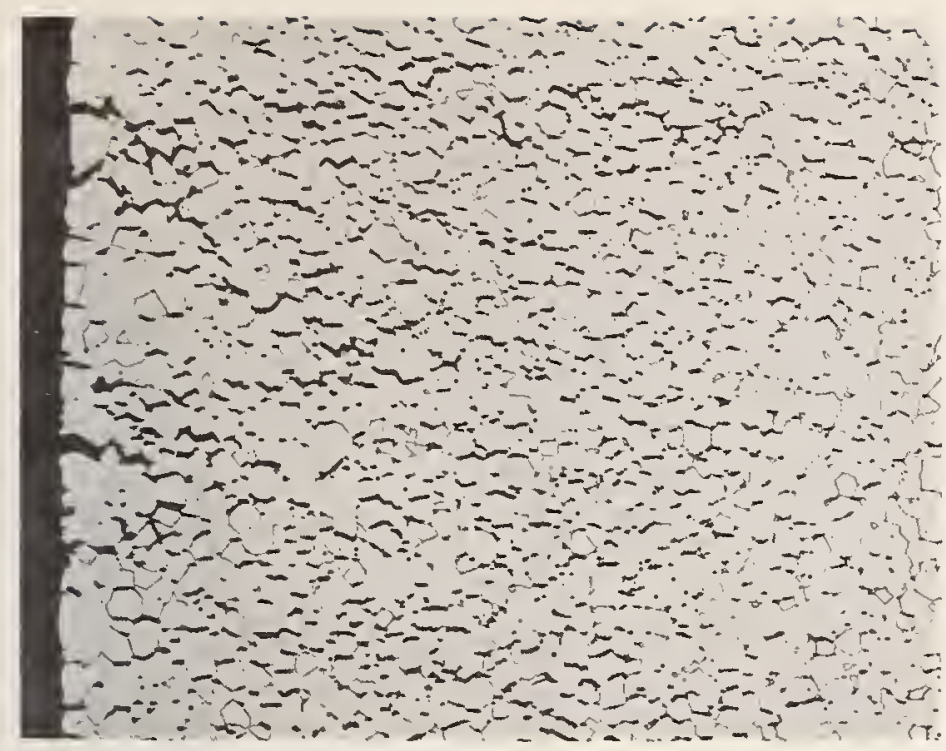

FIGURE 18. Creep fracture of initially dense $\mathrm{Al}_{2} \mathrm{O}_{3}$ which has been deformed at high temperatures in a bend test.

After R. L. Coble, reference 14, p. 619.

\subsection{Thermal Fracture}

Fracture is often observed to occur in ceramic materials after a rapid change in temperature known as thermal shock. The fracture results from stresses that arise from nonlinear temperature gradients or restraints to thermal expansion. The thermal stresses causing fracture depend on the rapidness of the temperature change and on the shape of the body being shocked. To determine the onset of fracture, it is necessary to solve the heat transfer equations and obtain the temperature distribution in the structure from which the stress distribution can be calculated. A review of the results of such analysis has been given by Kingery [98]. Failure is deemed to have occurred once the thermal tensile stresses exceed the tensile strength of the material, as in the case of static loading. Thus a maximum stress criterion is used to describe thermal failure of ceramic materials. (Table 6.)

Hasselman [99] and Clarke et al. [54] have suggested an exception to the maximum tensile strength criterion for thermal shock failure. Their criterion for failure is based on the availability of strain energy for crack propagation. During crack propagation, strain energy imparted to the structure by thermal shock is converted into fracture surface energy. The amount of strain energy available for fracture is limited by the severity of the shock. If the shock is not too severe, the conversion of strain energy into fracture surface energy may be complete before structural integrity is lost. Structural materials may crack in a limited way due to thermal shock, but still may be able to serve their original design function. Thermal failure therefore contrasts with constant load failure for which the available elastic strain energy is always sufficient for complete failure once a critical load is exceeded. Mechanical shock and failure under fixed grip conditions is similar to thermal shock in that the initial elastic strain energy is fixed so that crack propagation may cease before structural usefulness has been destroyed. In the following paragraphs the approach taken by Clarke et al. [54] will be used to illustrate thermal shock.

In engineering practice it is always useful to have a number that can be used to classify materials for a given end use. For the shock conditions described above, a useful parameter is the ratio of the fracture surface energy to the elastic strain energy available for fracture, $V$. Since this ratio may be large or small, the logarithm of the ratio will be more manageable for engineering purposes. Thus, the thermal shock resistance can be defined by a parameter $\tau$, where

$$
\tau=\log _{10}(\gamma / V) \text {. }
$$

This definition is essentially the same as the one suggested by Clarke et al., differing by an additive and multiplicative constant. Larger values of $\tau$ indicate greater resistance to thermal shock.

To evaluate $\tau$, the surface energy, $\gamma$, and the excess elastic strain energy must be determined. The fracture surface energies can be determined by methods described earlier in the paper. The excess strain energy can be estimated as follows. The strain imposed by thermal shock at a free surface of a solid is always of the form $\epsilon=A \alpha \Delta T$ [98], where $\alpha$ is the thermal coefficient of expansion, $\Delta T$ the temperature difference between the initial body temperature and that of the environment and $A$ is a constant that depends on the rate of heat transfer, thermal conductivity, and the dimensions of the structure. The value of $A$ is usually less than 1 . The stress at the free surface of the material is proportional to the thermal strain up to the fracture stress. At higher strains, cracks form so that the surface stress cannot exceed the fracture stress of the material. For purposes of approximation, it is assumed that crack formation releases strain energy in such a manner that the surface stresses remain constant as the material fractures. The stress-strain relationship of a region near the surface of the structure is assumed to be as depicted in figure 19. The excess strain energy for fracture is given by the shaded portion of figure 19. In quantitative terms,

$$
V=\int_{\sigma / E}^{A \alpha \Delta T} \sigma d \epsilon=(\sigma / E)(E A \alpha \Delta T-\sigma)
$$

where $\sigma$ is the strength of the material and $E$ is Young's modulus. From eq (38) it may be concluded that fracture does not initiate unless $\sigma=E A \alpha \Delta T$, which is Kingery's maximum stress criterion for thermal failure [98]. Therefore, the assumption used in the derivation of eq (38) are consistent with the treatment given by Kingery. 


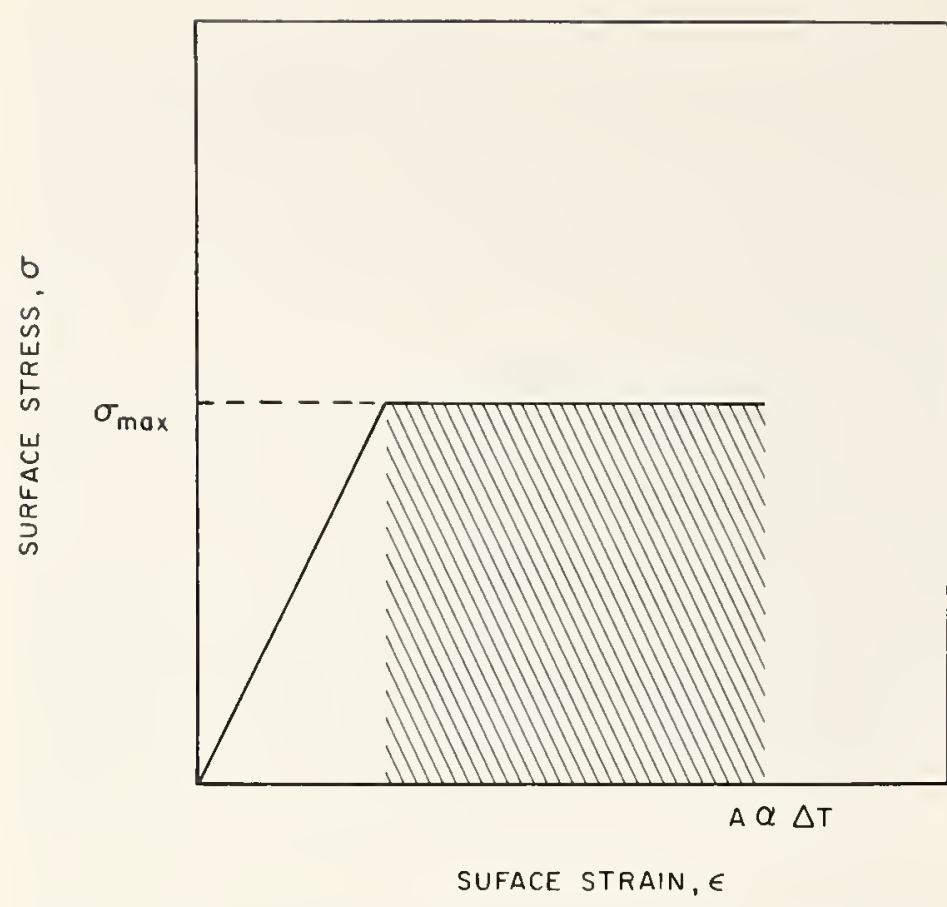

Figure 19. Assumed stress-strain curve in the surface layer of a thermally shocked ceramic.

Substituting eq (38) into eq (37), the following equation is obtained for thermal shock resistance,

$$
\tau=\log _{10}[\gamma E / \sigma(E A \alpha \Delta T-\sigma)] .
$$

For identical amounts of overstressing, $E A \alpha \Delta T$ $-\sigma=$ constant $>0$, the resistance to thermal :hock increases with increasing Young's modulus and surface energy, but decreases with increasing strength. The increased thermal shock resistance with decreasing strength has been noted previously [99].

Clark et al. have shown that a thermal shock Adugtion similar in form to eq (39) compares favorably with other methods of determining shock resistance. Data presented by Clarke et al. are shown in tables 7 and 8 . A comparison of $\tau$ with the British Standard Thermal Shock Test, table 7 , for commercial alumina ceramics shows that the thermal shock resistance of the various ceramics occupy similar positions on both scales. The fracture surface energy can also be used to measure the thermal shock resistance for these ceramics, table 7. For a broader range of materials, the fracture surface energy cannot be used as the only criterion for fracture, as can be seen from table 8 . The high thermal shock resistance of firebrick, table 8 , is due to its low strength.

\subsection{Environmental Effects}

Up to this point fracture has been assumed to occur in the absence of environment, controlled only by flaw geometry and inherent physical properties of materials. This assumption is very poor for ceramic materials, which despite their chemical inertness, are very sensitive to stresschemical effects in external environments. In this section, the effect of environment on strength will be discussed and the role of $K_{I}$ and $\gamma$ will be described.

Perhaps the first evidence for strength reduction by external environment was made by Grenet [100] who observed a time dependence in the strength of glass. Glass lathes loaded rapidly were stronger than those loaded slowly. In addition, a time delay to failure was observed in which glass lathes would support a given load for a period of time before failure. All of these observations were caused by water vapor in the atmosphere, although Grenet did not realize this at the time. The phenomenon first observed by Grenet has been termed static fatigue or delayed failure and is common to many ceramic solids.

TABLE 7. Thermal shock of commercial alumina

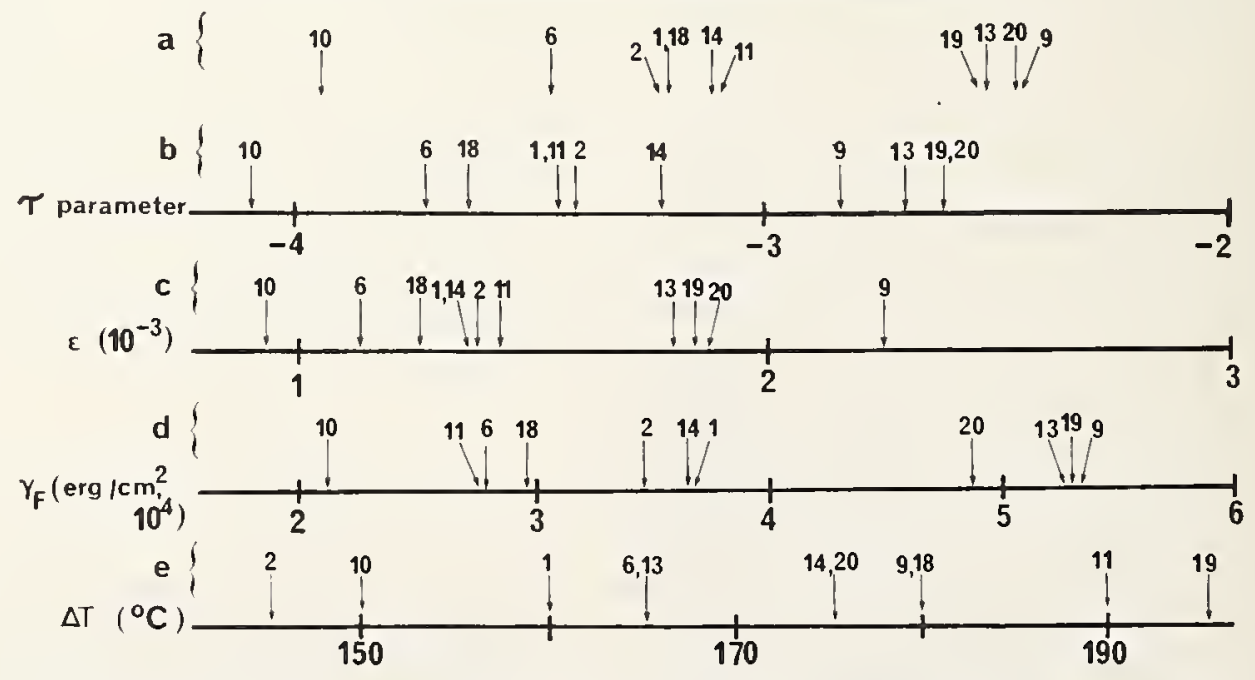

Comparison of a series of commercial aluminas. a) and b) are two different methods of calculating $\tau$. c) represents the strain that can be accommodated before a fresh amount of cracking can occur. d) is the fracture surface energy determined by method 3. e) is the British Standard thermal shock test. The numbers refer to the specimens in ref. [120]. After F. J. P. Clarke, H. G., Tattersall, and G. Tappin [54]. 
TABLE 8. Thermal shock of ceramics

\begin{tabular}{|c|c|c|c|c|c|}
\hline Material & $\begin{array}{c}Y F \\
\left(10^{4} \mathrm{ergs}\right. \\
\left.\mathrm{cm}^{-2}\right)\end{array}$ & $\mid \begin{array}{c}\sigma F \\
\left(10^{\circ} d y\right. \\
\left.\mathrm{cm}^{-2}\right)\end{array}$ & $\begin{array}{c}x \\
(10 \\
C\end{array}$ & $\begin{array}{l}\text { Youmgs } \\
\text { Modulus } \\
\text { (1012 dwn. } \\
\left(m^{-2}\right)\end{array}$ & $\tau$ \\
\hline Firebrick ${ }^{i}$ & 3 & 0.1 & 60 & 0.13 & +1 \\
\hline $\begin{array}{l}\text { Polycrystalline aluminaii } \\
\text { No. } 9 \\
\text { No. } 10\end{array}$ & $\begin{array}{l}5 \cdot 3 \\
2 \cdot 1\end{array}$ & $\begin{array}{l}\frac{2 \cdot 2}{3 \cdot 45} \\
\text { L }\end{array}$ & $\begin{array}{l}7.55 \\
7.8\end{array}$ & $\begin{array}{l}3.5 \\
3.9\end{array}$ & $\begin{array}{l}-2.9 \\
-4.5\end{array}$ \\
\hline Single-crystal alumina ${ }^{i i}$ & $1 \cdot 2$ & $3 \cdot 5$ & $7 \cdot 0$ & $3 \cdot 0$ & $-4 \cdot 8$ \\
\hline $\begin{array}{l}\text { Polycrystalline magnesiaiv } \\
3.48 \mathrm{g.cm}^{-3}, 10 \mu \mathrm{m} \\
3.51 \mathrm{g.cm}, 50 \mu \mathrm{m} \\
3.56 \mathrm{g.cm}, 100 \mu \mathrm{m} \\
\text { Theoretical density. } 7 \mu \mathrm{m}^{v}\end{array}$ & $\begin{array}{l}1 \cdot 6 \\
1.95 \\
3 \cdot 5 \\
0 \cdot 42\end{array}$ & $\begin{array}{l}0.4 \\
0.7 \\
1.0 \\
2.5\end{array}$ & $\begin{array}{l}12 \\
12 \\
12 \\
12\end{array}$ & $\begin{array}{l}3 \cdot 0 \\
3 \cdot 0 \\
3 \cdot 0 \\
3 \cdot 0\end{array}$ & $\begin{array}{l}-3 \cdot 17 \\
-3 \cdot 5 \\
-3 \cdot 2 \\
-6 \cdot 9\end{array}$ \\
\hline Single-crystal magnesia'i & $<0 \cdot 2$ & $1 \cdot 0$ & 12 & $3 \cdot 0$ & $-6 \cdot 8$ \\
\hline Polycrystalline beryllia'iii & $1 \cdot 5$ & $2 \cdot 0$ & $7 \cdot 0$ & $3 \cdot 4$ & $-4 \cdot 2$ \\
\hline
\end{tabular}

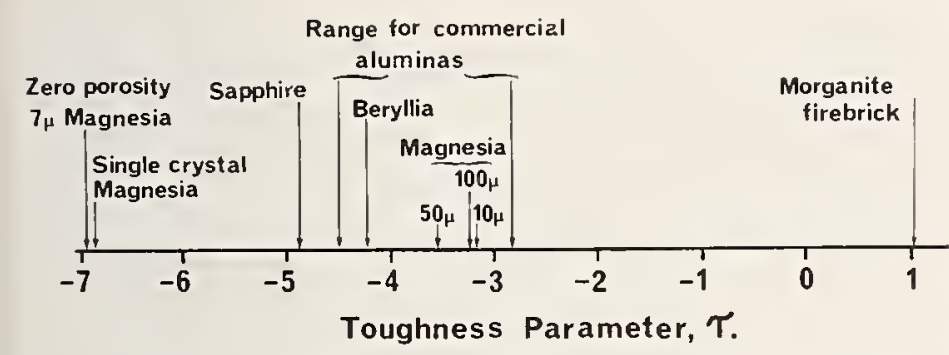

Comparison of some ceramics in respect to their resistance to thermal shock. Specimens are the same as those given in table 5. After F. J. P. Clarke, H. G., Tattersall, and G. Tappin [54].

More recent experimental results on glass clearly showed the effects observed by Grenet to be a form of stress corrosion caused by water vapor in the environment. Reviews of the subject may be found in references [63] and [100-102]. Most studies were made using rods or lathes in three of four point bending from which stresstime-to-failure curves were obtained as a function of temperature and environment, figure 20. From such studies it was concluded that delayed failure did not-occur in the absence of water vapor. A lower stress limit for failure was inferred from the long-time strength limit of the stress-time-tofailure curve. This limit, called the static fatigue limit, was approximately 20 to 30 percent of the short-time strength $[64,105]$. Charles was able to show that delayed failure was an activated process with an activation energy of $18.8 \mathrm{kcal} / \mathrm{mole}^{*}$ [106], about the same as that obtained for the corrosion of glass by water [107]. Finally Mould and Southwick [108] were able to reduce data from specimens of various surface treatments to a single curve, called the universal fatigue curve. Theories of delayed failure must satisfy the universal fatigue curve to be acceptable.

Recently Pearson [109] and Charles and Shaw $[58,110]$ were able to demonstrate a moisture induced, time-dependent weakening of single and

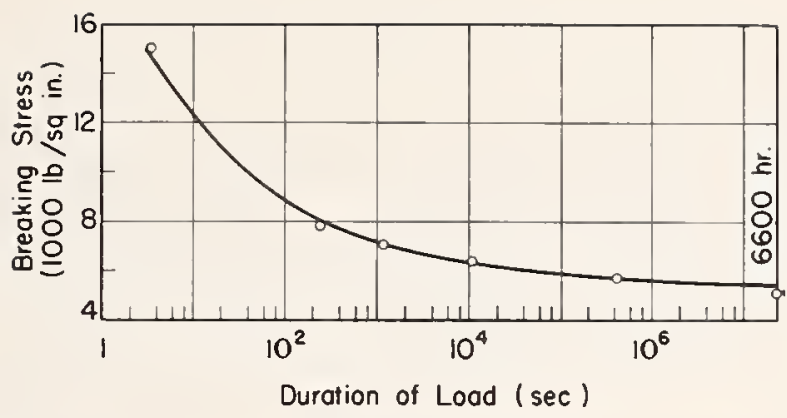

Figure 20. Stress time to failure curve for annealed soda-lime glass rods, $1 / 4$ in diam, tested in bending. After E. B. Shand [64].

polycrystalline alumina. The experimental observations were very similar to those on glass and it is reasonable to conclude that the static fatigue of sapphire is also caused by a stress corrosion process. The activation energy for the processes is $14 \mathrm{kcal} / \mathrm{mole}$ and the static fatigue limit is about 50 percent of the short-term strength for single crystals. Static fatigue on other ceramics has also been observed and is apparently quite common [111].

The theory that has been most successful in explaining static fatigue in ceramic materials is the one proposed by Charles and Hillig [112, 113]. They assume that static fatigue is caused by a stress enhanced chemical reaction that occurs most rapidly at crack tips where stresses are high. Cracks extend at subcritical stresses under the influence of the chemical reaction until the Griffith condition is satisfied, at which point rapid fracture ensues. Because of the general nature of this theory, it is worthwhile examining some of its underlying premises and conclusions.

Chemical reactions are well described as activated processes, in which reactants combine to form intermediate unstable chemical complexes that decompose to form the final reaction products [114]. The rates of reaction are controlled by the rate of formation of the activated complex. If $\Delta F_{T}^{*}$ is equal to the free energy of formation of the activated complex from the reactants and $\Delta F_{p}{ }^{*}$ is the free energy of formation of the activated complex from the reaction products then according to the absolute rate theory, the rate of reaction will be

$$
K=(k T / h)\left[\exp \left(-\Delta F_{r}{ }^{*} / R T\right)-\exp \left(-\Delta F_{p}{ }^{*} / R T\right)\right],
$$

where $k$ is Boltzmann's constant, $h$ is Planck's constant, $R$ is the ideal gas constant, and $T$ is the temperature. If the reaction is assumed to be a heterogeneous chemical reaction between a ceramic surface and a corrosive environment, then the rate or velocity of recession of the surface, $v$, will be proportional to the reaction rate $K$. Finally, if $\Delta F_{r}{ }^{*} \gg \Delta F_{p}{ }^{*}$, the velocity of recession is

$$
v=v_{0} \exp \left(-\Delta F_{r}^{*} / R T\right)
$$


which is the starting point for the Charles-Hillig theory.

The activation energy, $\Delta F_{r}{ }^{*}$, is the difference between the free energy of formation of the activated complex and that of the reactants. Since free energies of formation of materials are stress dependent [115], it follows that activation energies of chemical reactions are normally stress dependent. For simplicity it will be assumed that the stress dependent activation energy may be expanded into a Taylor's series as a function of stress,

$$
\Delta F_{r}^{*}(\sigma)=\Delta F_{r}^{*}(\sigma=0)+\left.\frac{\partial \Delta F_{r}^{*}(\sigma)}{\partial \sigma}\right|_{\sigma=0} \sigma+---
$$

where only the first two terns of the expansion have been retained. To simplify notation let

$$
\Delta F_{r}^{*}(\sigma=0) \text { be } E_{0}^{*} \text { and }\left.\frac{\partial \Delta F_{r}^{*}(\sigma)}{\partial \sigma}\right|_{\sigma=0} \text { be }-V^{*}
$$

$E_{0}^{*}$ is the activation energy of the chemical reaction in the absence of stress and $V^{*}$ has units of volume per mole and has been called the activation volume by Charles and Hillig.

In a stress corrosion process, the chemical reaction is most rapid at the roots of cracks, which act as stress concentrators. Since crack roots are curved, the effect of curvature on the activation energy must be considered. From thermodynamics, the free energy difference between a flat and curved surface is $\gamma V_{M} / \rho$ where $V_{M}$ is the molar volume of the solid and $\rho$ is the radius of curvature of the surface [116]. This free energy difference will tend to oppose the motion of the crack and is therefore subtracted from eq (42). With curvature taken into account, the velocity of recession of the crack tip under the combined effects of stress and chemical reaction is

$$
v=v_{0} \exp -\left[E_{0}^{*}-V^{*} \sigma+\gamma V_{M} / \rho\right] / R T .
$$

Equation (43) may be used directly to obtain an approximation for the static fatigue limit. A reasonable criterion for the static fatigue limit is that the velocity of the crack tip should be equal to the general rate of surface recession, a condition that is satisfied when

$$
V^{*} \sigma=\gamma V_{M} / \rho \text {. }
$$

If the crack tip is assumed to be elliptical in shape then the crack tip stress, $\sigma$, is related to the applied stress, $S$, by the Inglis relation [18], $\sigma=\sqrt{L / \rho} / S$, which when substituted into eq (44) gives $S^{*}=\left(\gamma V_{M}\right) /$ $\left(2 V^{*} \sqrt{L \rho}\right)$ for the static fatigue limit.

A more exact procedure followed by Charles and Hillig accounts for the dependence of crack geometry on stress corrosion process. Their criterion for the static fatigue limit is that the ratio of the crack tip stress to the applied load $\sigma / S$, should be time independent, which occurs when $d(L / \rho) / d t=0$. Charles and Hillig obtain the following equation for the static fatigue limit:

$$
S^{*}=\left(3 \gamma V_{M}\right) /\left(4 V^{*} \sqrt{L_{\rho}}\right),
$$

which differs from that given above by a numerical factor of $3 / 2$.

Equation (45) may be generalized for any crack geometry, mode I failure, by expressing it in terms of the stress intensity factor $K_{I}$, which for an elliptically shaped crack is $S \sqrt{\pi L}$. Therefore, eq (45) becomes

$$
\mathrm{K}_{I}^{*}=\left(3 \gamma V_{M} / 4 V^{*}\right) \sqrt{\pi / \rho}
$$

The critical stress intensity factor for the static fatigue limit is seen to depend inversely on the square root of the crack tip radius of curvature. Charles and Hillig [112] have shown that during stress corrosion, $d \rho / d L \approx-2 V^{*} \sigma / R T \approx-10$, which means that during stress corrosion a small change in crack length is accompanied by a decrease in flaw tip radius. The flaw tip radius cannot decrease indefinitely and will be limited by the atomic nature of the solid. Therefore, $\rho$ will be of the order of $10^{-9}$ to $10^{-10} \mathrm{~m}$.

A good stress corrosion theory should not only describe the stress dependence of material failure, but also the environmental dependence. The Charles-Hillig theory is particularly well suited for this purpose since the activation energy, $E_{0}^{*}$, can be related to the difference between the thermodynamic chemical potential of the various reactants [117]. For example, consider a gaseous reaction at the tip of a moving crack, where species $A$ is reacting with species $B$ in the glass to form an activated complex $B^{*}$. $B$ may be considered to be proportional to the number of bonds in the solid so that each time the reaction occurs, the crack tip advances a small distance. Therefore, the rate of crack advancement is proportional to the rate of reaction. Based on 1 mole of reacting $B$, the equation for the chemical reaction is

$$
n A+B \rightarrow B^{*},
$$

and the change of free energy is

$$
E_{0}^{*}=\mu_{B}^{*}-\mu_{B}-n \mu_{A},
$$

where $\mu_{B}^{*}$ is the chemical potential of the activated complex and $\mu_{B}$ and $\mu_{A}$ are the chemical potentials of species $B$ and $A$ respectively. At constant total pressure and temperature, $\mu_{B}$ and $\mu_{B}^{*}$ should be constant, since they depend only on the structure and state of the solid to 
which they are attached. $\mu_{A}$, by contrast, depends on environment. Assuming $A$ to be a perfect gas,

$$
\mu_{A}=R T\left[\Phi(T)+\ln p_{A}\right]
$$

where $\Phi(T)$ is some function of temperature, $T$, and $p_{A}$ is the partial pressure of species $A$ immediately adjacent to the crack tip. Substituting eqs (49) and (48) into (43), the following equation is obtained for the crack velocity dependence on environment and crack tip stress;

$$
v=A p_{A}^{n} \exp \left[V^{*} \sigma-V_{M} \gamma / \rho\right] / R T,
$$

where all of the constant terms have been collected into the constant $A$.

The important aspects of the Charles-Hillig theory have been verified by several experiments. The theory successfully predicts the universal fatigue curve developed by Mould and Southwick [108]. Using the data of Mould and Southwick, Hillig and Charles [113] calculated the static fatigue limit to be 0.17 of the critical stress intensity, $K_{I}^{*}=0.17 K_{I C}$. They also find that $\gamma=0.5 \mathrm{~J} / \mathrm{m}^{2}\left(500 \mathrm{ergs} / \mathrm{cm}^{2}\right)$ and $V^{*} \approx 1 \times 10^{-6} \mathrm{~m}^{3} /$ mole, which can be compared with an activation volume of $4.9 \times 10^{-6} \mathrm{~m}^{3} /$ mole for alkali diffusion in a typical soda lime glass.

A more direct confirmation of the CharlesHillig theory comes from the stress-corrosion experiments of Wiederhorn [117, 118]. Crack velocities were measured as a function of temperature, environment and $K_{I}$, using the double cantilever configuration. The pertinent experimental evidence is shown in figures 21 and 22 . In figure 21 , the logarithm of the crack velocity is plotted as a function of $K_{I}$ for environments of several different water vapor concentrations. Each water vapor concentration is represented by a tri-modal curve. In region I of these curves, crack velocity depends exponentially on $K_{I}$ and exhibits a water vapor pressure dependency. In region II, the plateau region, crack velocity is nearly independent of applied load, but depends on environment. In region III, crack velocity is again dependent on applied load, but is independent of environment. The data of figure 21, regions I and II, can be adequately explained using the theory of Charles and Hillig [117]. In region I the crack velocity is limited by the kinetics of the chemical reaction, while in region II the crack velocity is limited by rate of transport of water vapor from the surrounding environment to the crack tip.

Figure 22 presents crack velocity data obtained in water at four different temperatures [118]. The data were found to satisfy the following equation:

$$
v=1.2 \times 10^{2} \exp -\left(19,500-1.92 \times 10^{-2} K_{I}\right) / R T,
$$

which is similar in form to that proposed by Charles and Hillig. The activation energy, $E_{0}{ }^{*}=$

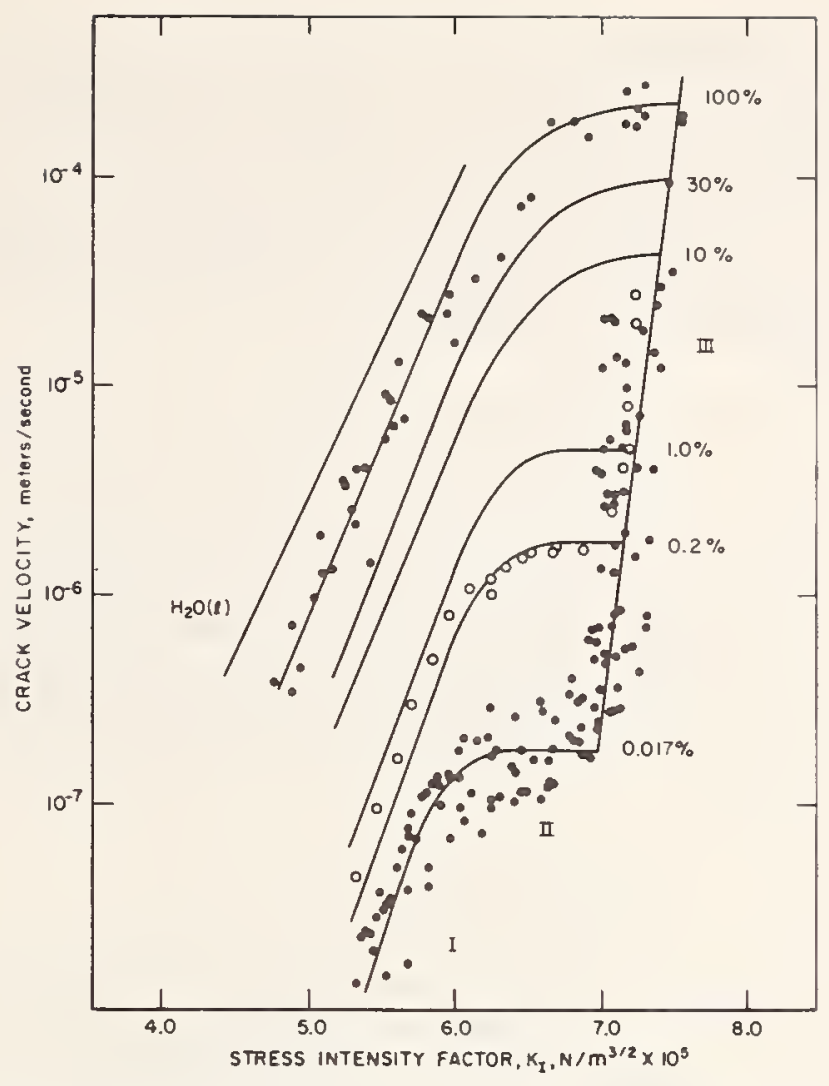

Figure 21. Dependence of crack velocity on stress intensity factor, $\mathrm{K}_{\mathrm{I}}$, in soda-lime-silica glass.

The percent relative humidity for each set of runs is given on the righthand side of the diagram. The Roman numerals identify the different regions of crack propagation.

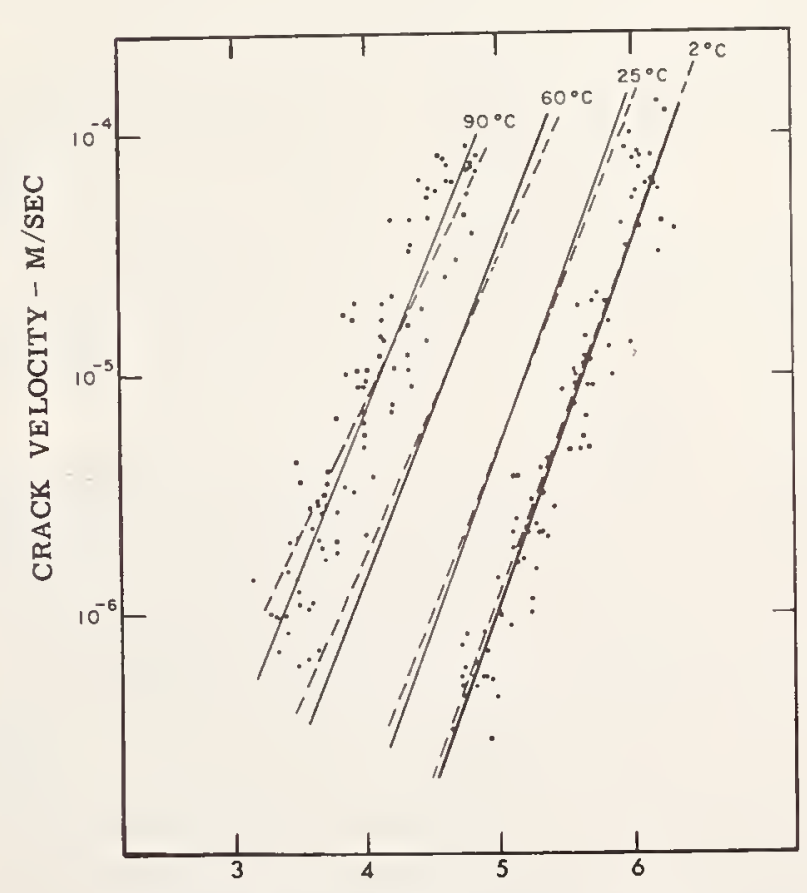
STRESS INTENSITY FACTOR $-\mathrm{N} / \mathrm{M}^{3 / 2} \times 10^{5}$

Figure 22. Dependence of crack velocity on stress intensity factor, $\mathrm{K}_{\mathrm{I}}$, and temperature in sodalime-silica glass. 
$19.5 \mathrm{kcal} / \mathrm{mole}$, compares favorably with values of $20 \mathrm{kcal} / \mathrm{mole}$ and $18.8 \mathrm{kcal} / \mathrm{mole}$ obtained by Charles respectively for fracture and corrosion of glass in saturated steam [106, 107].

An estimate for $V^{*}$ may be obtained by equating $V^{*} \sigma$ to the second term in the bracket of eq (51). Since $V^{*} \sigma=V^{*} 2 S \sqrt{L / \rho}=K_{I} 2 V^{*} / \sqrt{\pi \rho}$, the following equation is obtained for $V^{*}$ :

$$
V^{*}=4.02 \times 10^{-2} \sqrt{\pi \rho},
$$

where a conversion factor of 0.239 has been used to convert from calories to joules. Assuming a reasonable radius of curvature of $5 \times 10^{-10} \mathrm{~m}$, $V^{*}=1.6 \times 10^{-6} \mathrm{~m}^{3}\left(1.6 \mathrm{~cm}^{3}\right)$, which compares well with the value obtained by Hillig and Charles.

Equation (46) may now be simplified with the aid of eq (52) and from the fact that $K^{2} / 2 E=\gamma$, obtaining

$$
K_{I}^{*}=32 E_{\rho} \times 10^{-2} / 3 V_{M}
$$

For the soda lime glass used in the above experiments, $V_{M}=24.2 \times 10^{-6} \mathrm{~m}^{3}\left(24.2 \mathrm{~cm}^{3}\right)$ and $E=$ $7.35 \times 10^{10} \mathrm{~N} / \mathrm{m}^{2}$. With a crack tip radius of $5 \times 10^{-10} \mathrm{~m}$, a value of $2.2 \times 10^{5} \mathrm{~N} / \mathrm{m}^{3 / 2}$ is obtained for $K_{I}{ }^{*}$, which is 28 percent of $K_{I C}$ in liquid nitrogen and agrees quite well with experiment.

One of the most significant aspects of the equations presented in this section is their utility in predicting the relative resistance of glass to static fatigue. The crack velocity data necessary for the estimate require less time to obtain than comparable stress-time to failure data. Consequently, fracture mechanics techniques have a clear advantage over the usual strength techniques for this purpose.

Data on the stress corrosion of sapphire are not as plentiful as those on glass. Nevertheless, from the data available, the processes in sapphire seem to be very similar to those occurring in glass. Static fatigue curves [58, 110] and crack velocity data, figure 23, bear a strong resemblance to those obtained on glass. Consequently, it is reasonable to conclude that delayed failure in sapphire is also caused by a water-induced stress corrosion process.

Data on other ceramic materials are sparse; nevertheless, there is good evidence that delayed failure is a general property of ceramic materials and that the strength of these materials is limited by stress corrosion [111]. The effect of water vapor on the strength of several ceramic materials is given in table 9 , where it is evident that even a complex material such as granite can exhibit static fatigue. To establish the maximum usable strength of each of these materials, it will be necessary to determine their static fatigue limit either through strength time to failure studies or by fracture mechanics techniques. Considering the superiority of the latter, fracture mechanics techniques should be used where possible.

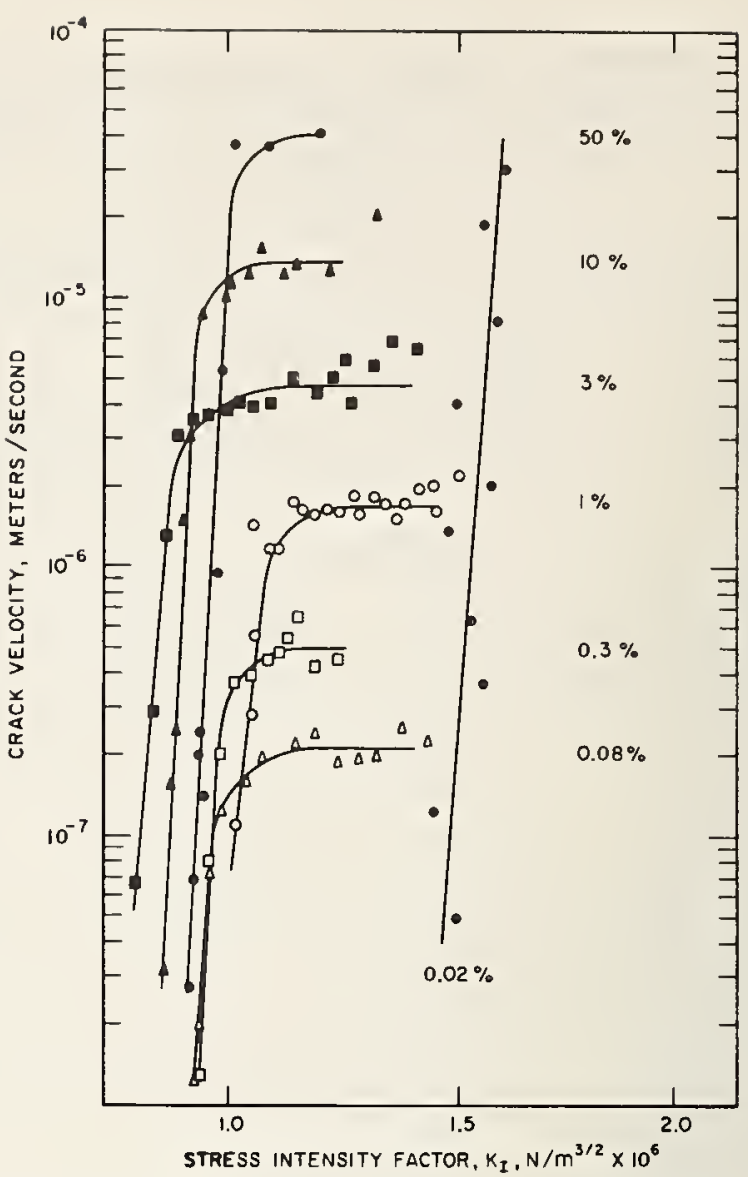

FIgURE 23. Dependence of crack velocity on stress intensity factor, $\mathrm{K}_{\mathrm{I}}$, in sapphire.

The percent relative humidity for each set of runs is given on the right hand side of the diagram. Crack propagation occurred on the (10̄11) rhombohedral plane.

TABLE 9. Effect of water vapor on the strength of ceramic materials

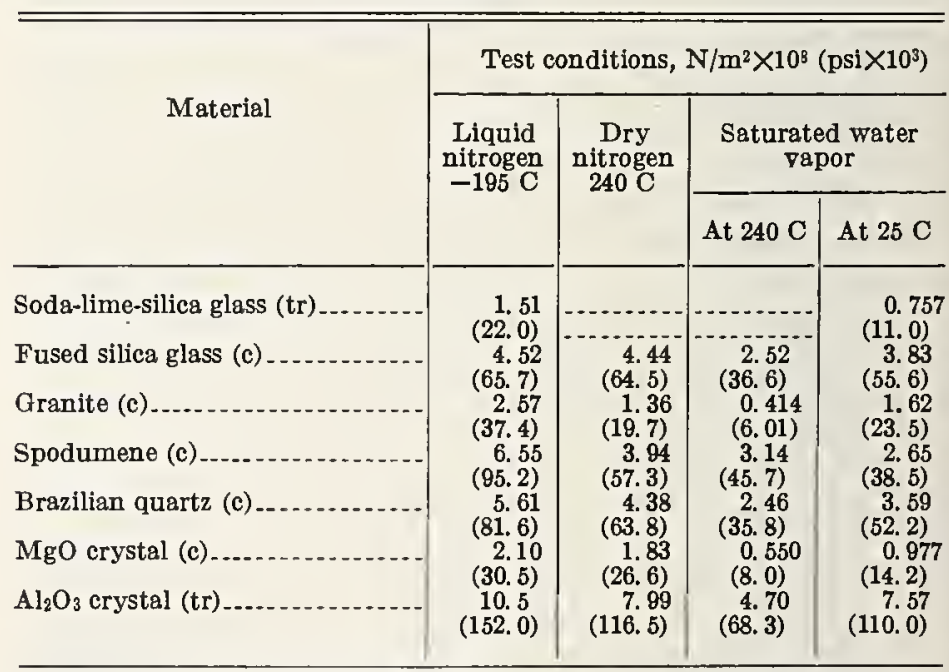

Compressive (c) or transverse (tr) strengths under conditions indicated with machine-loading rate of 0.005 in $1 \mathrm{~min}$. Table taken from ref. [14], page 617. Original data from ref. [111].

\section{Conclusions}

The main purpose of this paper has been to discuss some aspects of brittle fracture that may be of use in the design and manufacture of ceramic materials. Strong emphasis has been placed on the 
flaw theory of failure and its generalization into a continuum approach to fracture. The stress intensity factor and fracture surface energy have been described and the relationship between the two has been given. One of the main reasons for using these parameters to describe fracture is their independence of flaw size. They are material properties dependent on microstructure and environment, but independent of structure shape or flaw distribution. Examples have been given of the use of these parameters in describing strength, thermal shock resistance, and environmental effects on strength. It may be concluded that the continuum approach to fracture should provide a valuable adjunct to the usual strength measurements. Readers interested in a more intensive survey of this subject are referred to the recent book by Tetelman and McEvily [60], which should appeal to both the materials scientist and the structural engineer. More theoretical aspects of the subject will be found in references [17, 29], while engineering aspects of the subject are discussed in references $[30,119]$.

Support for this work by the U. S. Army Research Office-Durham, N.C. and the Advanced Research Projects Agency is gratefully acknowledged.

\section{References}

[1] Gilman, J. J., The strength of ceramic crystals, in The Physics and Chemistry of Ceramics, C. Klingsberg (ed.), Gordon and Breach, New York (1963), p. 240.

[2] Theoretical Strength of Materials, Materials Advisory Board, National Academy of Sciences, Publication MAB-221-M, Aug. 1966.

[3] Orowan, F., Fracture and strength of solids, Repts. Prog. Phys. 12, 185-232 (1948).

[4] Gilman, J. J., Cleavage, ductility and tenacity in crystals, in Fracture, Averbach, B. L., Felbeck, D. K. Hahn, G. T., and Thomas D. A., (eds.), John Wiley \& Sons, New York (1959), pp. 193-222.

[5] Wachtman, J. B., Jr., Tefft, W. E., Lam, D. G., Jr., and Stinchfield, R. P., Elastic constants of synthetic single crystal corundum at room temperature, J. Research NBS 64A, 213-228 (1960).

[6] Kronberg, M. L., Plastic deformation of single crystals of sapphire: Basal slip and twinning, Acta Met. 5, 507-524 (1957).

[7] Warren, B. E., and Biscoe, J., Fourier analysis of x-ray patterns of soda-silica glass, J. Am. Ceram. Soc. 21, 259-265 (1938).

[8] Hillig, W. B., The factors affecting the ultimate strength of bulk fused silica, in Symposium on Mechanical Strength of Glass and Ways of Improving It, Florence, Italy, Sept. 25-29, 1961, Union Scientific Continentale du Verre, Charleroi, Belgium, pp. 206-236 (1962).

[9] Hillig, W. B., Strength of bulk fused quartz, J. Appl. Phys. 32, 741 (1961).

[10] Proctor, B. A., Whitney, I., and Johnston, J. W., The strength of fused silica, Proc. Roy. Soc. A297, 534-557 (1967).

[11] Mehan, R. L., and Feingold, F., Strength and structure of very fine sapphire whiskers, pp. 355-406 in Strength Mechanisms, Burke, J. J., Reed, N. L., and Weiss, V. (eds.), Syracuse Univ. Press, Syracuse, N.Y. (1966).
[12] Brenner, S. S., Mechanical behavior of sapphire whiskers at elevated temperatures, J. Appl. Phys. 33, 33-39 (1962).

[13] Mallinder, F. P., and Proctor, B. A., The strengths of flame-polished sapphire crystals, Phil. Mag. 197-208 (1966).

[14] Kingery, W. D., Introduction to Ceramics, p. 610, John Wiley \& Sons, New York (1960).

[15] Griffith, A. A., Phenomena of rupture and flow in solids, Phil. Trans. Roy. Soc. (London) 221A, 163-198 (1920).

[16] Griffith, A. A., Theory of rupture, Proc. First Intern. Congr. Appl. Mechanics, Delft, pp. 55-63 (1924).

[17] Irwin, G. R., Fracture, in Encyclopedia of Physics, Vol. VI, Springer, Heidelberg (1958).

[18] Inglis, C. E., Stresses in a plate due to the presence of cracks and sharp corners, Trans. Inst. Naval Architects (London) 55, 219 (1913).

[19] Sneddon, I. N., The distribution of stress in the neighborhood of a crack in an elastic solid, Proc. Roy. Soc. (London) A18\%, 229 (1946).

[20] Sadowski, M. A., and Sternberg, E. G., Stress concentration around a triaxial ellipsoidal cavity, J. Appl. Mech. 16, 149 (1949).

[21] Barenblatt, G. I., The mathematical theory of equilibrium cracks in brittle fracture, pp. 55-126 in Advances in Applied Mechanics, Vol. VII, $\mathrm{H}$. L. Dryden and T. von Karman (eds.), Academic Press, New York (1962).

[22] Elliot, H. A., An analysis of the conditions for rupture due to Griffith cracks, Proc. Phys. Soc. (London) 59, 208-223 (1947).

[23] Willis, J. R., A comparison of the fracture criteria of Griffith and Barenblatt, J. Mech. Phys. Solids 15, $151-162$ (1967).

[24] Cribb, J. L., and Tomkins, B., On the nature of the stress at the tip of a perfectly brittle crack, J. Mech. Phys. Solids 15, 135-140 (1967).

[25] Hillig, W. B., Sources of weakness and the ultimate strength of brittle amorphous solids, pp. 152-194 in Modern Aspects of the Vitreous State, Vol. 2, J. D. Mackenzie (ed.), Butterworths, London (1962).

[26] Orowan, E., Energy criteria of fracture, Weld. J., London 34, 157s-160s (1955).

[27] Irwin, G. R., Analysis of stresses and strains near the end of a crack traversing a plate, J. Appl. Mech. 24, 361 (1957).

[28] Williams, M. L., On the stress distribution at the base of a stationary crack, J. Appl. Mech. 24, 109-114 (1957).

[29] Paris, P. C., and Sih, G. C., Stress analysis of cracks, pp. $30-81$ in Fracture Toughness Testing and its Applications, ASTM Special Technical Publication No. 381 .

[30] Srawley, J. E., and Brown, W. F., Fracture toughness testing methods, pp. 133-196 in Fracture Toughness Testing and its Applications, ASTM Special Technical Publication No. 381.

[31] Srawley, J. E., and Gross, B., Stress intensity factors for crackline-loaded edge-crack specimens, Mat. Res. Stds. 7, 155-162 (1967).

[32] Wiederhorn, S. M., Shorb, A. M., and Moses, R. L., A critical analysis of the theory of the double cantilever method of measuring fracture surface energies, J. Appl. Phys. 39, 1569-1572 (1968).

[33] Dugdale, D. S., Yielding of steel sheets containing slits, J. Mech. Phys. Solids 8, 100 (1960).

[34] Hahn, G. T., and Rosenfield, A. R., Local yielding and extension of a crack under plane stress, Acta Met. 13, 293-306 (1965).

[35] McClintock, F. A., and Irwin, G. R., Plasticity aspects of fracture mechanics, pp. $84-113$ in Fracture Toughness Testing and its Applications, ASTM Special Tech. Pub. No. 381.

[36] Irwin, G. R., and Kies, J. A., Critical energy rate analysis of fracture strength, Welding Research Suppl. 33, 193s-198s (1954). 
[37] Irwin, G. R., Fracture mechanics, pp. 557-592 in Structural Mechanics, Goodier J. N., and Hoff, N. J. (eds.), Pergamon Press, New York (1960).

[38] Nakayama, J., Direct measurement of fracture energies of brittlc heterogeneous materials, J. Am. Ceram. Soc. 48, 583-587 (1965).

[39] Tattersall, H. G., and Tappin, G., The work of fracture and its measurement in metals, ceramics and other materials, J. Mat. Sci. 1, 296-301 (1966).

[40] Davidge, R. W., and Tappin, G., The effective surface energy of brittle materials, J. Mat. Sci. 3, 162-173 (1968).

[41] Berdennikov, W. P., Messung der Oberflachenspannung von Festen Korpern, Soviet Phys. Z.S. 4, 397-419 (1933); Zhur. Fiz. Khim. 5(2-3), 358 (1934). (A summary of these articles is given in Surface Energy of Solids by V. D. Kuznetsov, Her Majesty's Stationery Office, London (1957), pp. 224-234. Error in the original data corrected by S. M. Wiederhorn in Materials Science Research, Vol. 3, W. W. Kriegel and H. Palmour III (eds.), Plenum Press (1966), p. 514.)

[42] Marsh, D. M., Plastic flow in glass, Proc. Roy. Soc. A2\% 9, 420-435 (1964).

[43] Wachtman, J. B., Jr., and Maxwell, L. H., Plastic deformation of ceramic-oxide single crystals, J. Am. Ceram. Soc. 37, 291-299 (1954).

[44] Orowan, E., The tensile strength of mica and the problem of the technical strength, Z. Physik. 82, 235-266 (1933)

[45] Bryant, P. J., Taylor, L. H., and Gutshall, P. L., Cleavage studies of lamellar solids in various gas environments, pp. 21-26 in Transactions of the Tenth National Vacuum Symposium, American Vacuum Society (1963).

[46] Gilman, J. J., Direct measurements of the surface energies of crystals, J. Appl. Phys. 31, 2208-2218 (1960).

[47] Gutshall, P. L., and Gross, G. E., Cleavage surface energies of $\mathrm{NaCl}$ and $\mathrm{MgO}$ in vacuum, J. Appl. Phys. 36, 2459-2460 (1965).

[48] Petch, N. J., Congleton, J., Hardie, D., and Perkins, R. N., Effect of surface energy, Study of the Brittle Behavior of Ceramic Materials, Technical Documentary Report No. ASD-TR-61-628, Parts I, II, III.

[49] Judd, J. W., On the structure planes of corundum, J. Mineralogical Soc. 11, 49-55 (1895).

[50] Conrad, H., Mechanical behavior of sapphire, J. Am. Ceram. Soc. 48, 195-201 (1965).

[51] Stofel, E., and Conrad, H., Fracture and twinning in sapphire (alpha- $\mathrm{Al}_{2} \mathrm{O}_{3}$ crystals), Trans. AIME 22\%, 1053-1060 (1963).

[52] Heuer, A. H., Deformation twinning in corundum, Phil. Mag. 13, 379-393 (1966).

[53] Class, W. H., and Machlin, E. S., Crack propagation method for measuring grain boundary energies in brittle materials, J. Am. Ceram. Soc. 49, 306 (1966)

[54] Clarke, F. J. P., Tattersall, H. G., and Tappin, G., Toughness of ceramics and their work of fracture, Proc. Brit. Ceram. Soc. 6, 163-172 (1966).

[55] Gutshall, P. L., and Gross, G. E., Observations and mechanisms of fracture in polycrystalline alumina, to be published in the Proc. Nat. Symp. Fracture Mechanics, Lehigh University, June 19-21, 1967.

[56] Vasilos, T., Mitchell, J. B., and Spriggs, R. M., Mechanical properties of pure, dense magnesium oxide as a function of temperature and grain size, J. Am. Ceram. Soc. 47, 606-610 (1964).

[57] Passmore, E. M., Spriggs, R. M., and Vasilos, T., Strength-grain size-porosity relations in alumina, J. Am. Ceram. Soc. 48, 1-7 (1965).

[58] Charles, R. J., and Shaw, R. R., Delayed failure of polycrystalline and single-crystal alumina, General Electric Research Laboratory Report No. 62RL-3081 M, July 1962.
[59] Clarke, F. J. P., and Wilks, R. S., Effects of irradiation on mechanical properties of nonfissionable ceramics, pp. 57-74 in Proc. Conf. Nuclear Applications of Nonfissionable Cermaics, Boltan, A., and Handwerk, J. H., (eds.), sponsored by American Ceramic Society and American Nuclear Society, May 9-11, 1966.

[60] Tetelman, A. S., and McEvily, A. J., Jr., Fracture of Structural Materials, John Wiley \& Sons, New York (1967).

[61] Johnston, T. L., Fracture of polycrystals, pp. 1-22 in Fundamental Phenomena in the Material Sciences, Vol. 4: Fracture of Metals, Polymers, and Glasses, Bonis, L. J., Duga, J. J., and Gilman, J. J. (eds.), Plenum Press, New York (1967).

[62] Stokes, R. J., Fracture of ceramics, pp. 151-175 in Fundamental Phenomena in the Material Sciences, Vol. 4: Fracture of Metals, Polymers, and Glasses, Bonis, L. J., Duga, J. J., and Gilman, J. J. (eds.), Plenum Press, New York (1967).

[63] Mould, R. E., The strength of inorganic glasses, pp. 119-149, in Fundamental Phenomena in the Material Sciences, Vol. 4: Fracture of Metals, Polymers, and Glasses, Bonis, L. J., Duga, J. J., and Gilman, J. J. (eds.), Plenum Press, New York (1967)

[64] Shand, E. B., Experimental study of fracture of glass: I, The fracture process, J. Am. Ceram. Soc. 37, 52-60 (1954).

[65] Stookey, S. D., Strengthening glass and glassceramics by built-in surface compression, pp. 669-680 in High-Strength Materials, Zackay, V. F. (ed.), John Wiley Sons, New York (1965)

[66] Johnston, T. L., Li, C. H., and Stokes, R. J., The strength of icnic solids, pp. 341-374 in Strengthening Mechanisms in Solids, American Society of Metals, Cleveland (1962).

[67] Stokes, R. J., Mechanical properties of polycrystalline sodium chloride, Proc. Brit. Ceram. Soc. 6, 189-207 (1966).

[68] Day, R. B., and Stokes, R. J., Grain boundaries and the mechanical behavior of magnesium oxide, pp. 355-386 in Material Science Research, Vol. 3, Kriegel, W. W., and Palmour, H., III (eds.), Plenum Press, New York (1966).

[69] Stokes, R. J., Johnston, T. L., and Li, C. H., Environmental effects on the mechanical properties of ionic solids with particular reference to the Joffe effect, Trans. AIME 218, 655-662 (1960).

[70] Stokes, R. J., and Li, C. H., Dislocations and the tensile strength of magnesium oxide, J. Am. Ceram. Soc. 46, 423-434 (1963).

[71] Ku, R. C., and Johnston, T. L., Fracture strength of MgO bicrystals, Phil. Mag. 9, 231-247 (1964).

[72] Westwood, A. R. C., On the fracture behaviour of magnesium oxide bi-crystals, Phil. Mag. 6, 195-200 (1961)

[73] Clarke, F. J. P., Sambell, R. A. J., and Tattersall, H. G., Cracking at grain boundaries due to dislocation pile-up, Trans. Brit. Ceram. Soc. 61, 61-66 (1962).

[74] Johnston, T. L., Stokes, R. J., and Li, C. H., Crack nucleation in magnesium oxide bicrystals under compression, Phil. Mag. 7, 23-34 (1962).

[75] Stokes, R. J., Johnston, T. L., and Li, C. H., The relationship between plastic flow and the fracture mechanism in magnesium oxide single crystals, Phil. Mag. 4, 20-32 (1959).

[76] von Mises, R., Mechanik der plastichen formanderung von kristallen, Zeit. Angew. Math. und Mech. 8, 161 (1938).

[77] Taylor, G. I., Plastic strain in metals, J. Inst. Metals 62, 307-324 (1938).

[78] Groves, G. W., and Kelly, A., Independent slip systems in crystals, Phil. Mag. 8, 877-887 (1963).

[79] Wachtman, J. B., Jr., Mechanical properties of ceramics: An introductory survey, Bull. Am. Ceram. Soc. 46, 756-772 (1967). 
[80] Zener, C., Micro-mechanism of fracture, pp. 3-31 in Fracturing of Metals, Am. Soc. Metals, Novelty, Ohio (1948).

[81] Stroh, A. N., A theory of the fracture of metals, Advances in Physics 6, 418-465 (1957).

[82] Smith, E., and Barnby, J. T., Crack nucleation in crystalline solids, Metal Sci. J. 1, 56-64 (1967).

[83] Petch, N. J., The cleavage strength of polycrystals, J. Iron Steel Inst. 174, 25-28 (1953).

[84] Eshelby, J. D., Frank, F. C., and Nabarro, F. R. N., The equilibrium of linear arrays of dislocations, Phil. Mag. 42, 351 (1951).

[85] Clarke, F. J. P., Residual strain and the fracture stress-grain size relationship in brittle solids, Acta Met. 12, 139-143 (1964).

[86] Davidge, R. W., and Tappin, G., Internal strain and the strength of brittle materials, J. Mat. Sci. 3, 297-301, (1968).

[87] Cottrell, A. H. Theory of brittle fracture in steel and similar metals, Trans AIME 212, 192-203 (1958).

[88] Gell, M., and Smith, E., The propagation of cracks through grain boundaries in polycrystalline $3 \%$ silicon-iron, Acta Met. 15, 253-258 (1967).

[89] Rice, R. W., Strength and fracture of dense $\mathrm{MgO}$, presented at Third Intern. Materials Symp., Ceramic Microstructures-Their Analysis, Significance and Production, Univ. of California, Berkeley, Calif., June 13-16, 1966.

[90] Coble, R. L., Personal communication.

[91] Carniglia, S. C., Grain boundary and surface influence on mechanical behavior of refractory oxides-experimental and deductive evidence, pp. 425-471 in Materials Science Research, Vol. 3, W. W. Kriegel and H. Palmour III (eds.), Plenum Press, New York (1966).

[92] Carniglia, S. C., Petch relation in single-phase oxide ceramics, J. Am. Ceram. Soc. 48, 580-583 (1965).

[93] Spriggs, R. M., Mitchell, J. B., and Vasilos, T., Mechanical properties of pure, dense aluminum oxide as a function of temperature and grain size, J. Am. Ceram. Soc. 4\%, 323-327 (1964).

[94] Fryxell, R. E., and Chandler, B. A., Creep, strength, expansion and elastic moduli of sintered $\mathrm{BeO}$ as a function of grain size, porosity and grain orientation, J. Am. Ceram. Soc. 47, 283-291 (1964).

[95] Bentle, G. G. and Kniefel, R. M., Brittle and plastic behavior of hot-pressed BeO, J. Am. Ceram. Soc. 48, 570-577 (1965).

[96] Stokes, R. J., Johnston, T. L., and Li, C. H., Effect of surface condition on the initiation of plastic flow in magnesium oxide, Trans. AIME 215, 437-444 (1959).

[97] Kingery, W. D., Introduction to Ceramics, p. 581, John Wiley \& Sons, New York (1960).

[98] Kingery, W. D., Factors affecting thermal stress resistance of ceramic materials, J. Am. Ceram. Soc. 38, 3-15 (1955).

[99] Hasselman, D. P. H., Elastic energy at fracture and surface energy as design criteria for thermal shock, J. Am. Ceram. Soc. 46, 535-540 (1963).

[100] Grenet, L., Mechanical strength of glass, Bull. Soc. Enc. Industr. Nat. Paris (ser. 5), 4, 838-848 (1899). Partial translations of this article may be found in Glass Ind. 15, 277 (1934) and J. Am. Ceram. Soc. 18, 219 (1935).

[101] Charles, R. J., A review of glass strength, pp. 1-38 in Progress in Ceramic Science, Vol. 1, J. E. Burke (ed.), Pergamon Press, New York (1961).

[102] Hillig, W. B., Sources of weakness and the ultimate strength of brittle amorphous solids, pp. 152-194 in Modern Aspects of the Vitreous State, Vol. 2,
J. D. Mackenzie (ed.), Butterworth and Co. (London) (1962) .

[103] Phillips, C. J., Strength and weakness of brittle materials, Am. Scientist 53, 20-51 (1965).

[104] Phillips, C. J., Mechanical strength of glass, report Research Laboratory, Corning Glass Works, 1937 (see also ref. [64] above).

[105] Holland, A. J. and Turner, W. E. S., The effect of sustained loading on the breaking strength of sheet glass, J. Soc. Glass Technol. 24, 46T (1940).

[106] Charles, R. J., Static fatigue of glass II, J. Appl. Phys. 29, 1554-1560 (1958).

[107] Charles, R. J., Static fatigue of glass I, J. Appl. Phys. 29, 1549-1553 (1958).

[108] Mould, R. E., and Southwick, R. D., Strength and static fatigue of abraded glass under controlled ambient conditions: II Effect of various abrasions and the universal fatigue curve, J. Am. Ceram. Soc. 42, 582-592 (1959).

[109] Pearson, S., Delayed fracture of sintered alumina, Proc. Phys. Soc. (Lond on) 69B, 1293-1296 (1956).

[110] Charles, R. J., Static fatigue: delayed fracture, pp. 469 in Studies of the Brittle Behavior of Ceramic Materials, N. A. Weil (ed.), Technical Report ASD-TR-61-628, Part II (April 1963); see also refs. [113] and [58].

[111] Charles, R. J., The strength of silicate glasses and some crystalline oxides, pp. 225-249 in Fracture, Averbach, B. L., Felbeck, D. K., Hahn, G. T., and Thomas, D. A. (eds.), John Wiley \& Sons, New York (1959).

[112] Charles, R. J., and Hillig, W. B., The kinetics of glass failure by stress corrosion, pp. 511-527 in Symp. Mechanical Strength and Ways of Improving It. Florence, Italy, Sept. 25-29, 1961, Union Scientifique Continentale du Verre, Charleroi, Belgium (1962).

[113] Hillig, W. B., and Charles, R. J., Surfaces, stressdependent surface reactions and strength, pp. 682-705 in High-Strength Materials, V. F. Zackey (ed.), John Wiley \& Sons, Inc., New York (1965).

[114] Glasstone, S., Laidler, K. J., and Eyring, H., Theory of Rate Processes, Mc Graw-Hill Book Co., New York (1941).

[115] Callen, H. B., Thermodynamics, pp. 213-237, John Wiley \& Sons, New York (1960).

[116] Adamson, A. W., Physicai Chemistry of Surfaces, p. 56, Interscience Publishers (1960).

[117] Wiederhorn, S. M., Influence of water vapor on crack propagation in soda-lime glass, J. Am. Ceram. Soc. 50, 407-414 (1967).

[118] Wiederhorn, S. M., Effects of environment on the fracture of glass, pp. 293-317 in EnvironmentSensitive Mechanical Behavior of Materials, A. R. C. Westwood and N. S. Stoloff (eds.), Gordon and Breach, New York (1966).

[119] Brown, W. F., Jr. and Srawley, J. E., Plane strain crack toughness testing of high strength metallic materials, ASTM Spec. Tech. Publ. 410, Am. Soc. Testing Mats. (1967).

[120] Binns, D. B., and Popper, P., Mechanical properties of some commercial alumina ceramics, Proc. Brit. Ceram. Soc. 6, 71-82 (1966)

[121] Stokes, R. J., "Mechanical behavior of polycrystalline ceramics", Third Intern. Materials Symp., Ceramic Microstructures-Their Analysis, Significance and Production, Univ, of California, Berkeley, Calif., June 13-16, 1966.

[122] Wiederhorn, S. M., To be published.

[123] Bentle, G. G., and Miller, K. T., Dislocations, slip, and fracture in BeO, J. Appl. Phys. 38, 4248-57 (1967). 



\title{
Strength Testing Of Ceramics-A Survey
}

\author{
Leonard Mordfin and Matthew J. Kerper \\ Office of Aerospace Research, 1400 Wilson Boulevard, Arlington, Virginia 22209
}

\begin{abstract}
Mechanical test methods for brittle ceramics are reviewed, and the state-of-the-art is presented for tensile and compressive strength determinations. Included are various indirect tension, flexure, and torsion test methods and the relatively new stress wave technique. The emphasis is on technological factors, particularly on improvements achieved since 1963, but the importance of the human element is also stressed. References are provided for more detailed study and to guide the selection of an appropriate method for a given set of circumstances.
\end{abstract}

Key Words: Brittleness; ceramics; mechanical testing; strength.

\section{Introduction}

The unique combinations of properties offered by ceramics make them attractive for many specialized structural applications where metals are inadequate. On the other hand, the inherent brittleness of most ceramics makes the design and development of reliable ceramic structural members exceedingly difficult. This problem has been discussed perceptively by Harmsworth [1]. ${ }^{1}$ One of the difficulties is the determination of reliable strength data. Such determinations have frequently been characterized by excessive variability, or scatter, due to testing errors and material variability. The reduction in testing errors is the subject of this paper. We assume some understanding of this problem on the part of the reader, particularly as it applies to brittle materials, but where this is not the case we recommend Duckworth's clear exposition [2] as preliminary reading.

In 1963 Shook [3] prepared an extremely comprehensive survey of mechanical property test methods for brittle materials. It is our intention here to update his work insofar as strength-test methods are concerned. In both Shook's work and our own, the emphasis is on technological improvements in testing techniques. Nevertheless, it may be appropriate to say a few words on the human element involved. It cannot be denied that much of the unreliable strength data on ceramics have been generated as a result of improper testing practices at the hands of inexperienced or incompetent personnel. All of the improvements in testing apparatus are for nought if not utilized by experienced, capable personnel. Obtaining strength measurements on a new brittle material, or an old one under a new

\footnotetext{
1 Figures in brackets indicate the literature references at the end of this
} paper. set of conditions, is anything but a routine operation. It should be treated as a research experiment.

Our point is that mechanical testing is not a degrading occupation. Good people and good equipment are both needed to obtain reliable, useful data on brittle materials as well as to develop good test methods.

Although many of the strength test methods for ceramics were originally developed for concrete, most of the recent advances in these methods have been accomplished by the Air Force; principally the Air Force Materials Laboratory and its contractors, the IIT Research Institute, the Stanford Research Institute, the Southern Research Institute, and the Battelle Memorial Institute, to name a few. This is not surprising. Most of the major innovations in structures technology, for several decades now, were born from aerospace requirements. Unfortunately, much of this work has not found its way into the open literature, for a variety of reasons. We have, therefore, found it necessary to reference many Air Force reports in preparing this paper. In many cases we have included the AD number, by which these reports are cataloged by the Defense Documentation Center, Cameron Station, Alexandria, Virginia 22314. Copies of these are readily available to qualified requestors. Many of the reports are similarly available to the general public from the Clearinghouse for Federal Scientific and Technical Information, 5285 Port Royal Road, Springfield, Virginia 22151.

\section{Direct Tension Test}

In order to evaluate the fracture strength of a material, it is necessary to have a means of relating the load on a specimen, at the instant of fracture, to the stress at the fracture location. For certain 
loading and specimen configurations, the loadstress relationship is directly obtainable from equilibrium considerations. The most obvious example is the ideal tension test, wherein a prismatic rod is subjected to uniformly distributed, axial end loads. The stress is then everywhere equal to the force intensity, or the load divided by the cross sectional area. The simplicity of this concept has made the tension test the principal tool for evaluating the strengths of materials in general.

In practice, it is virtually impossible to apply tensile load uniformly over the end of a rod. Instead, the various gripping methods available, such as threads, button heads, tapered shoulders, collets, etc., apply loads chiefly through a shear mechanism. ${ }^{2}$ However, recourse to Saint-Venant's Principle still enables our objective to be fulfilled. That is, if the resultant of the applied load is pure tension, and if the specimen is long enough, then the central portion of the specimen will still be in the required state of tension.

Unfortunately, even this approach is not realistic in practice. Friction, imperfect machining, inhomogeneity, and other factors make the attainment of a purely tensile resultant force on the end of a rod specimen impossible. Instead, the resultant consists of a tensile force plus a moment, however small. The effect of the moment is to introduce bending stresses into the central portion of the specimen in addition to the desired tensile stresses.

If, through careful testing technique, the bending moment is kept reasonably small, its effect on strength determinations can be ignored when testing most metals, plastics, and other ductile materials. The deformation capabilities of ductile materials enable specimens to respond to small, superposed bending moments in such a way that the bending stresses in the central portion are relieved, and a nearly pure state of tension is achieved prior to fracture.

In most ceramics and other brittle materials, on the other hand, this deformation capability does not exist, and fracture is initiated at a point in the specimen where the sum of the tensile and bending stresses is greater than the tensile stress alone.

The above discussion is not intended to create the impression that satisfactory tension testing of brittle materials is impossible, but rather that it can be achieved only with special care and techniques that reduce the maximum bending stress to less than, say, 2 percent of the tensile stress. Above all, we are trying to show why tensile testing of brittle materials is not a routine procedure.

To illustrate the point, Barnett et al. [5] subjected an instrumented tensile specimen to 67 consecutive cycles of loading and unloading, and recorded the ratio of maximum bending stress to average tensile stress each time. Between cycles the specimen was removed from the grips, cleaned, reinserted in the grips and carefully alined. The

${ }^{2}$ An exception applicable to large, low-strength specimens uses thick steel plates bonded with epoxies to the ends of concrete specimens [4]. data obtained are shown in figure 1. It may be seen that the bending stresses amounted to as much as 80 percent of the tensile stresses. Furthermore, the nearly linear nature of the cumulative distribution indicates that high bending stresses are just as likely to occur as lower stress values. It is not surprising, therefore, that relatively large amounts of scatter are obtained in results from tests of this kind.

In another study, which involved even more careful alinement of specimens in grips and the use of crushable shims, Bortz [6] still obtained bending strains as high as 10 percent of the total strain.

The only solution is to instrument all test specimens and to reject or re-start all tests which produce excessive bending. The intent of the recommendations and descriptions which follow is merely to reduce the difficulties encountered in initiating a successful test. We may also reiterate, at this point, that there is no substitute for experience and diligence on the part of the testing personnel.

The objective is to make the applied force coincident with the centerline of the specimen. This involves all elements of the load train, which includes the specimen, the grips, and any extension rods. All of these elements must be accurately machined for concentricity and precisely alined. Specimens should preferably be round, for easier machining and elimination of corner effects, and have a reduced, uniform diameter over the gage length. Bortz and Wade [7] recommend a gage length of at least four times the gage diameter to reduce the effects of fillets. The collet type of grip is widely consider ed the best for repeatedly and reliably holding the necessary alinement.

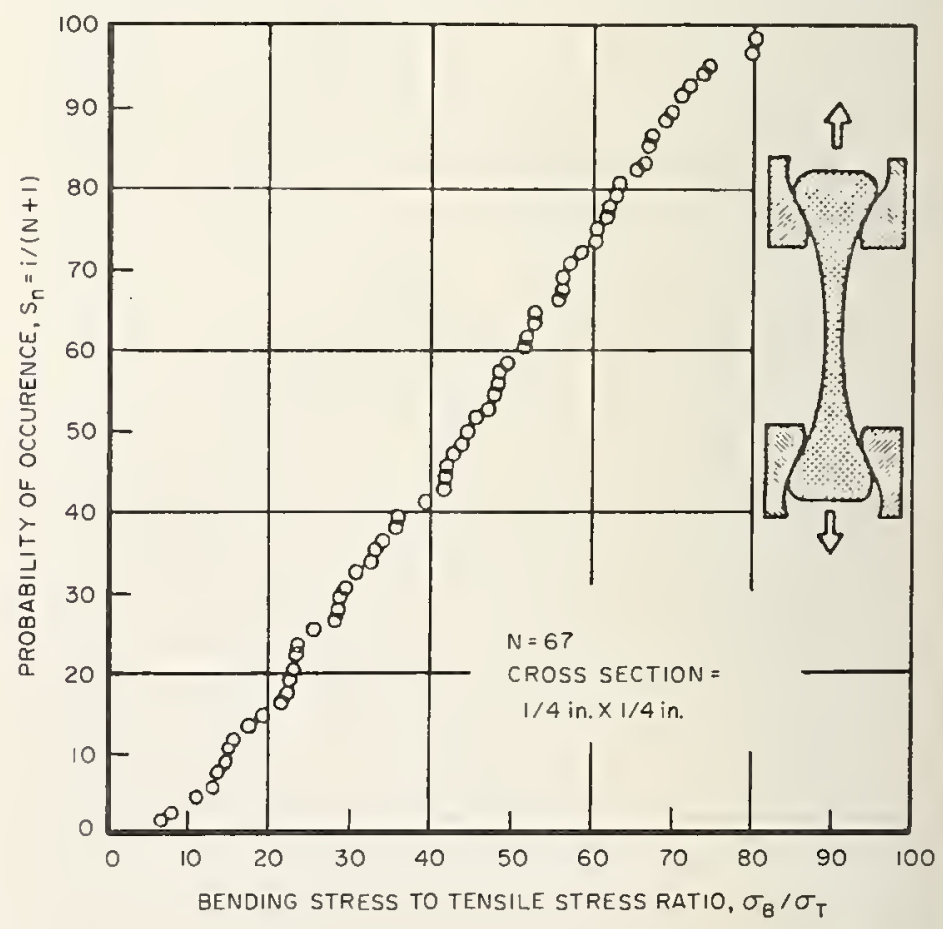

Figure 1. Occurrence of errors due to eccentric loading in tension tests. 
Carefully machined, threaded grips have also proved satisfactory where the specimen material permits it.

Digesu and Pears [8,9] developed a tapered collet grip much like those used in precision lathes (see fig. 2). The grooves at each end of the specimen that receive the grips are machined concentrically about the axis of the gage section. In fact, the specimen configuration was designed to allow machining of all surfaces to a common centerline without intermittent removal of the specimen from the lathe, thus permitting concentricity to within 0.0005 in. All of the other elements of the load train were similarly designed and fabricated to the same tolerance. To eliminate other sources of bending, the assembled load train was statically balanced and instrumentation leads were routed through the center of the extension rods.

In using this collet grip, the three-piece split ring is pressed into the groove by advancing the compression nut. This provides the required gripping force, and also forces the ground end faces of the specimen and the pull rod together, to provide axial alinement.

Having achieved satisfactory alinement in the load train, the next problem requiring consideration is the attachment of the load train to the testing machine crossheads. If the movable

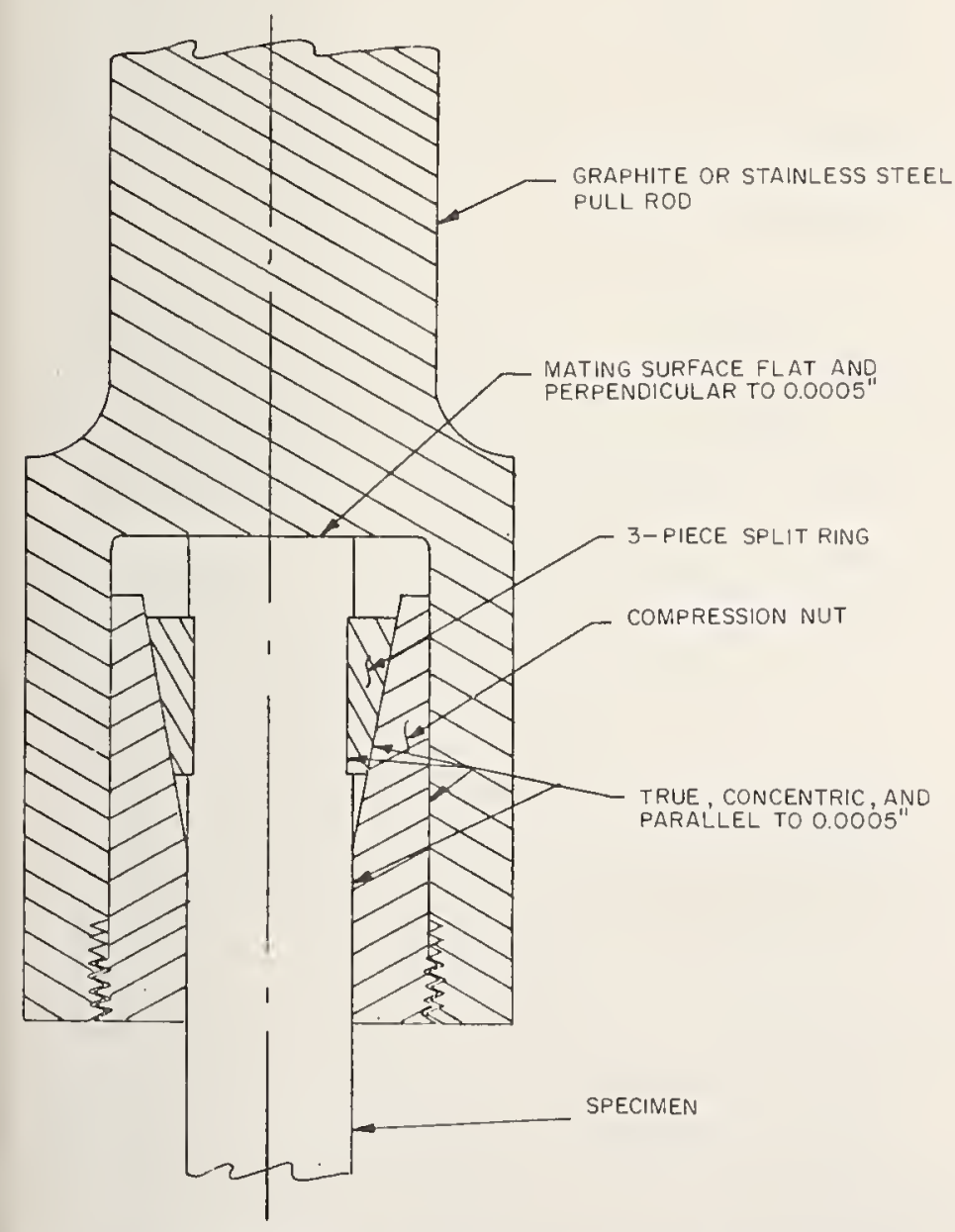

Figure 2. Precision collet grip for tensile specimens. crosshead is constrained to move precisely parallel to the load train centerline, then the attachments may be rigid. Unfortunately, conventional testing machines are often inadequate in this respect, so the attainment of uniaxial loading requires a frictionless universal bearing. Mechanical universal joints are hardly frictionless under load. Chains are better. The best attachment available at this time is the gas-bearing universal (fig. 3) developed by Digesu and Pears [8,9]. This apparatus provides $\mathrm{a}$ 0.0005-in-thick gas film between the extension rods and the machine crossheads at each end of the load train. With 9-in diam bearings and a gas pressure of 300 psi in the contact area, the universals transmit less than 1 in-lb of torque and essentially no bending moment to the specimen, at an applied load of $15,000 \mathrm{lb}$.

Since the elimination of bending stresses permits greater tensile stresses to be applied to a specimen before fracture, improvements in tension testing techniques manifest themselves in higher strength determinations as well as in reduced scatter. Through the use of the above innovations, Pears obtained tensile strength data which were at least 30 percent higher than those previously reported for the same materials. His values agree more closely with reported flexural strengths.

An entirely different approach to the alinement problem was proposed by Todd [10]. He compensated for the effects of eccentric loading by applying an external moment over the middle third of the tensile specimen. While good results were apparently obtained, this technique is rather slow and tedious and requires very strict control.

Electric resistance strain gages appear to be a convenient means of monitoring alinement during

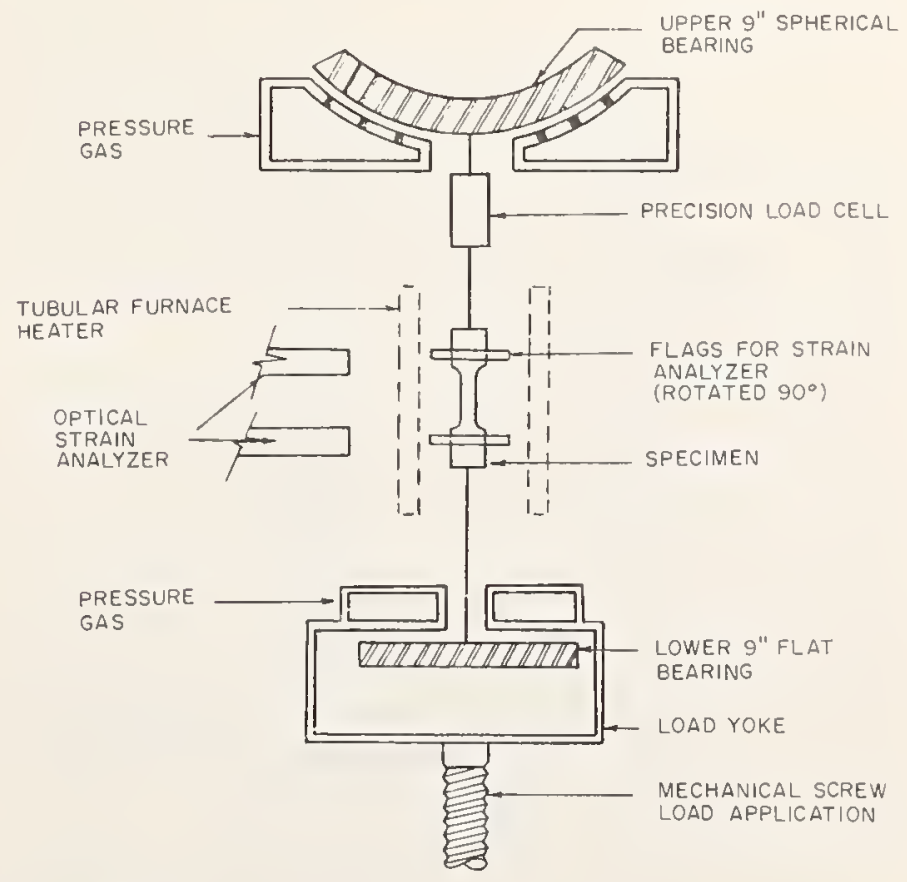

FIGURE 3. Schematic of gas bearings for attachment of load train to testing machine crossheads. 
tests, and also provide the strain data needed for the determination of stress-strain characteristics. At elevated temperatures or in special environments, for which the direct tension test is well suited, other strain-measuring instrumentation may be more desirable. Examples are the optical strain analyzer used by Digesu and Pears [8,9], and the photographic strain-measuring technique [11]. Mechanical extensometers should, perhaps, be avoided. Bortz and Wade [7] found that they caused a 13 percent reduction in strength. This has been attributed to surface damage produced by the extensometer, which magnifies the load eccentricity, however small it may be initially.

In the determination of elastic moduli from tensile stress-strain measurements, abnormally large scatter is obtained with certain materials even when proper testing techniques have been observed. This may be explained by the possibility that the deformation is not truly elastic but includes the creation and propagation of numerous microcracks.

Recognition of the problems associated with proper tension testing has caused this method to achieve less acceptance for brittle materials than it has enjoyed for ductile materials. Instead, numerous other tests have been used or proposed for evaluating tensile strengths. These are called indirect tension tests, and will be discussed in following sections.

(We have omitted any discussion of tension tests for ceramic fibers. This area of endeavor is still suffering growing pains and, while the literature on it is rapidly increasing, a comparative evaluation of available methods may be premature at this time.)

\section{Pressurized Ring Test}

This test was developed by Sedlacek and Halden in 1962 [12]. The specimens are carefully machined in the form of short, thin-walled tubes, and the loading is applied by means of an internal pressurized bag. Like the direct tension test, this test permits the stresses to be evaluated solely from equilibrium considerations, providing the specimen wall thickness does not exceed onetenth of the radius.

Numerous improvements in the testing technique have been made since this method was introduced. Cornish and Broutman [13] used an apparatus involving two hardened tool steel platens with dowel pins which facilitate the alinement of one platen above the other. A neoprene bulb, which is formed to the internal dimensions of the test specimen, serves as the pressure diaphragm. The inlet to the bulb passes through a tapered plug centrally located in the upper platen. The bulb is approximately 0.010 -in thick.

The cylindrical test specimen is positioned in the apparatus and the platens are mounted between the bed and crosshead of a testing machine, which prevent separation of the platens due to the axial force exerted by the pressure bulb. Spacers maintain a gap of approximately 0.001-in between the specimen and the top platen to permit radial expansion of the specimen.

Sedlacek [14] has provided details of suitable equipment for pressure generation, sensing, monitoring, calibrating, and recording.

This type of test has many advantages for the determination of tensile strengths. It is relatively simple to perform, the loading is self-alining, and the extraneous stresses caused by gripping in direct tensile tests are eliminated. 'The uncomplicated stress distribution is highly reproducible and failure cannot occur outside of the gage section.

Babel [15] examined the bending stresses that are introduced by possible variations in the wall thickness of the specimen, and by imperfect contact between the specimen and the pressure bulb at all points. His analysis showed that for ceramics these stresses are negligible. Differences between measured and calculated hoop strains were found to be less than 2 percent. Babel also concluded that frictional effects, if any are present, do not significantly affect the fracture strength of a specimen.

These advantages, relative to direct tension testing, have resulted in the measurement of tensile strengths which exceed previously reported values and approach flexural values. Most important is that a low scatter of results is observed. On materials such as alumina, individual groups of specimens have yielded coefficients of variation as low as 1 or 2 percent. This compares favorably with the value of $\mathbf{5}$ or more that is frequently associated with flexural strength data.

It is entirely feasible that, by proper positioning of strain gages, determinations of stress-strain characteristics for a wide range of stress rates can be made during strength tests. As mentioned earlier, however, wide scatter in results will obtain when investigating materials that are prone to microcrack development. On Boride $\mathrm{Z}$, for example, which is a multi-phase material that is believed to develop microcracks on cooling after the sintering process, strength tests reportedly gave a coefficient of variation of 35 percent [16].

The principal disadvantage of the pressurized ring test is the temperature limitation of bulb materials, which is presently about $600 \mathrm{~F}$. In an attempt to overcome this limitation, a modified test technique, called the floated ring test (fig. 4), has been proposed. In this approach, the specimen ring is floated between two conical end pieces that permit a controlled gas leakage through the gaps between the specimen and the end pieces. The specimen ends are unrestrained, and the temperature limitation introduced by a pressure bulb is removed. A disadvantage is that large power inputs are required to preheat the gas in order to achieve high-temperature operation. 


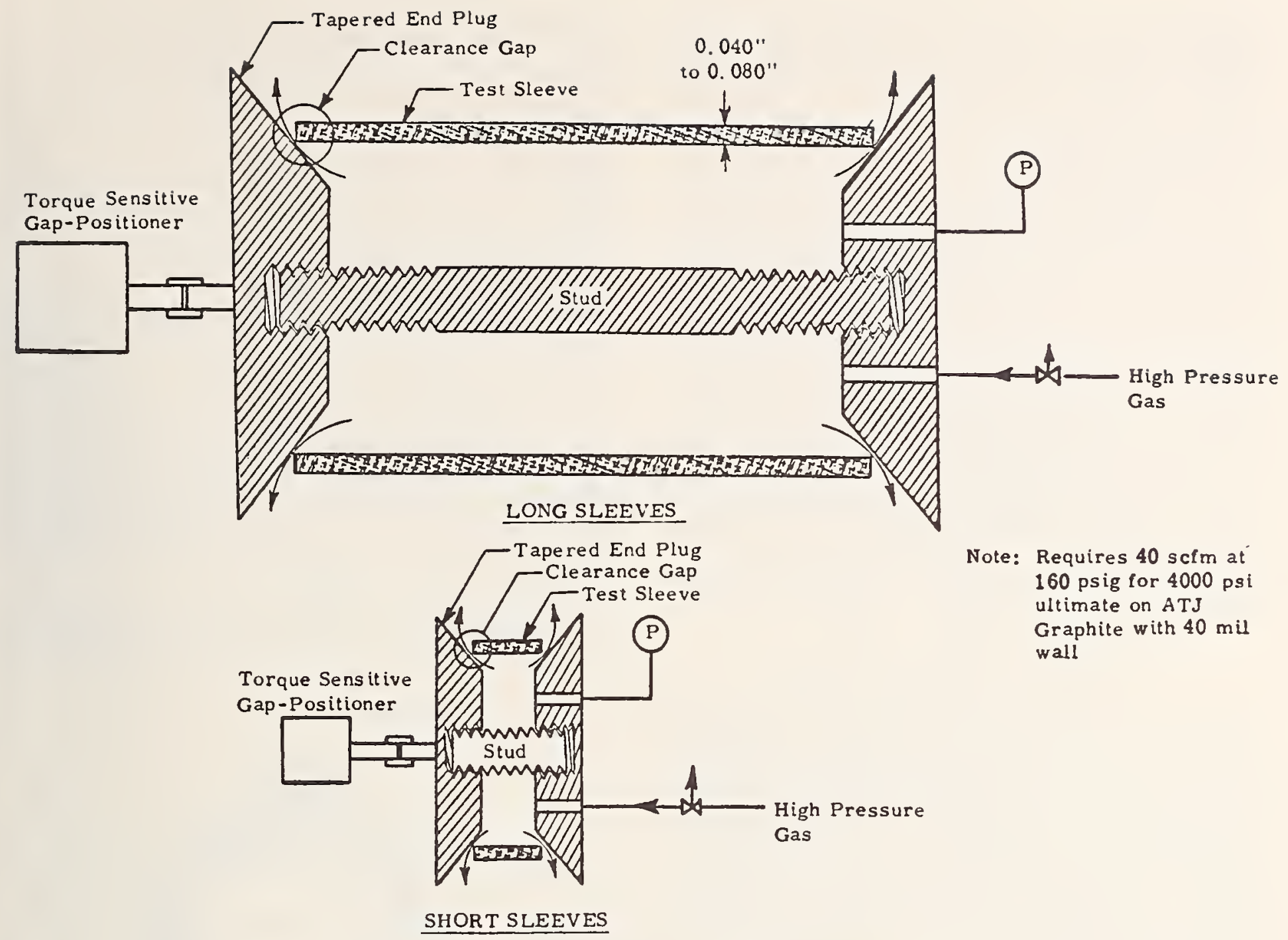

FIGURE 4. Schematic of gas-floated ring test setup.

Several other interesting variations of the pressurized ring method have been proposed. In one of these [17], which is shown in figure 5, a universal testing machine is used to apply a compressive force to a floating piston. This force is transferred to the silicone rubber plug which, reportedly, acts as an incompressible fluid and applies a hydrostatic force to the ring specimen. A tough rubber bladder protects the soft rubber plug. The pressure developed within the plug as a function of applied load was measured by means of a small liquid reservoir within it, which was tapped with a pressure gage. No significant error was observed, but it still remains to verify that the plug acts isotropically. If the bladder, which gets chewed up, is replaced after every tenth test, coefficients of variation of about 4 percent are apparently attainable with alumina specimens. This apparatus is less expensive than gas-pressured arrangements, and has been recommended for field or routine production control uses.

Still another approach to pressurized ring testing was introduced by Malhotra et al. [18, 19]. The modification here is the use of thick-walled cylindrical specimens instead of thin-walled ones. The

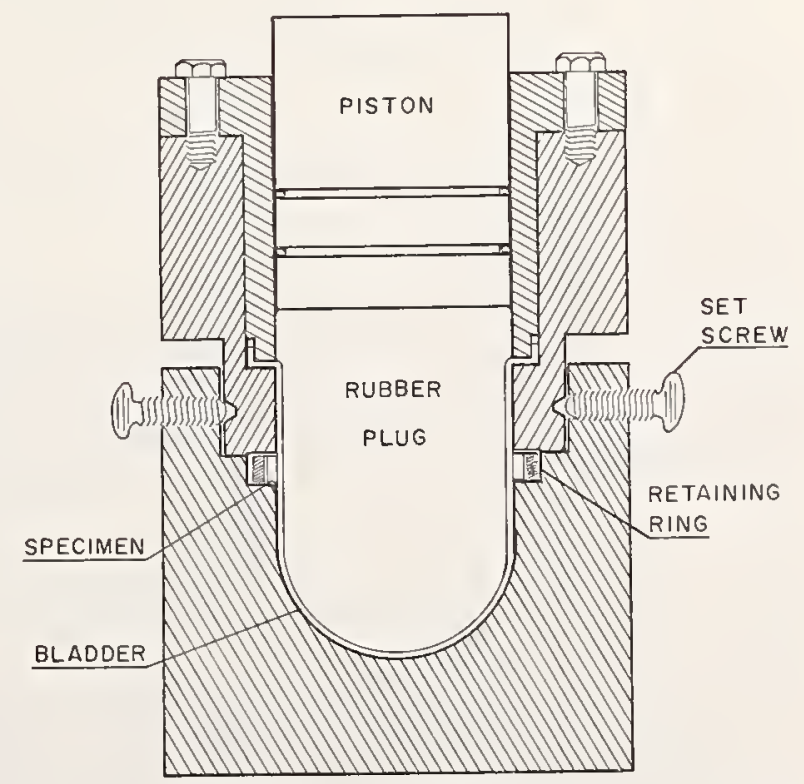

FIGURE 5. Modified Sedlacek-Halden pressurized ring test setup. 
application is to concrete, where the size of the aggregate may not permit the fabrication of thinwalled member's. 'Tests yielded strength values which exceed those obtained by conventional tensile tests but which are lower than those from flexure tests. Coefficients of variation were reported to be about 5 percent.

The principal disadvantage of this method is that the load-stress relation is no longer statically determinate. 'The maximum tensile stress is developed on the innel periphery of the ring and it was calculated by assuming linear stress-strain behavior of the specimen material to fracture. Since concrete normally exhibits some inelastic behavior, the fracture strengths determined from the test results are probably higher than the actual stresses at fracture and may not exceed the values obtained by conventional tension tests.

In the tests, 6 -in ring specimens were placed in a jig consisting of two 11-in-diam mild steel plates held together by five tie bolts. Pressure was applied through a specially molded bladder made of $1 / 8$-in-thick nitrile rubber.

\section{Theta Specimen Test}

The direct tension test and the thin-walled pressurized ring test, described above, have the advantage of providing nominally uniform tensile stresses that may be calculated from equilibrium considerations, irrespective of the nature of the stress-strain characteristic of the specimen material. In the remaining indirect tensile tests which will be discussed the tensile stresses are not statically determinate. As is the case with the thick-walled pressurized ring test, the determination of the load-stress relationship in these cases requires an assumption regarding the nature of the stress-strain characteristic. Since we are concerned with brittle materials it is most convenient to assume a linear stress-strain characteristic all the way to fracture. This assumption includes the condition that the elastic moduli in tension and compression are equal. If the specimen material does not, in fact, behave in this manner the fracture strengths obtained from these tests are accordingly in error.

One test that falls in this category was mentioned by Daniels and Weil [20]. It consists of a diametrally loaded disk containing two holes separated by a narrow strip of material (fig. 6). Photoelastic studies reportedly show [3] that a uniform tensile stress area exists in the strip under the diametral compressive load. This test has not received wide acceptance but it apparently led to the theta specimen, which has been investigated in several studies.

The theta specimen was introduced by Durelli, Morse, and Parks [21] and Daniels and Weil [20] as a means for determining tensile strengths at elevated temperatures in an oven, where gripping and alinement problems are more difficult to contend with. The specimen (fig. 6) is intended to be loaded between two parallel platens with the central bar parallel to the platens. Outward motion of the ring on the horizontal axis subjects the central bar to tension. The recommended design (fig. 7) is the final choice of over 60 shapes based on the same principle. Most of the other designs were rejected because they failed in the ring rather than in the bar. 'The recommended design generally fails only in the bar.

Photoelastic studies showed that (1) the maximum tensile stress occurs in the bar; (2) the stress in the bar is uniform; and (3) the fillets at the ends of the bar do not produce unacceptable stress concentrations.

The principal advantage of this specimen is that it does not require excessive precautions to achieve alinement. Alinement by eye is said to be adequate to produce the desired symmetrical stress distribution. A major disadvantage is obviously the considerable precision machining that the specimen requires.

Other factors to be considered in evaluating this test method are apparently not so well understood.
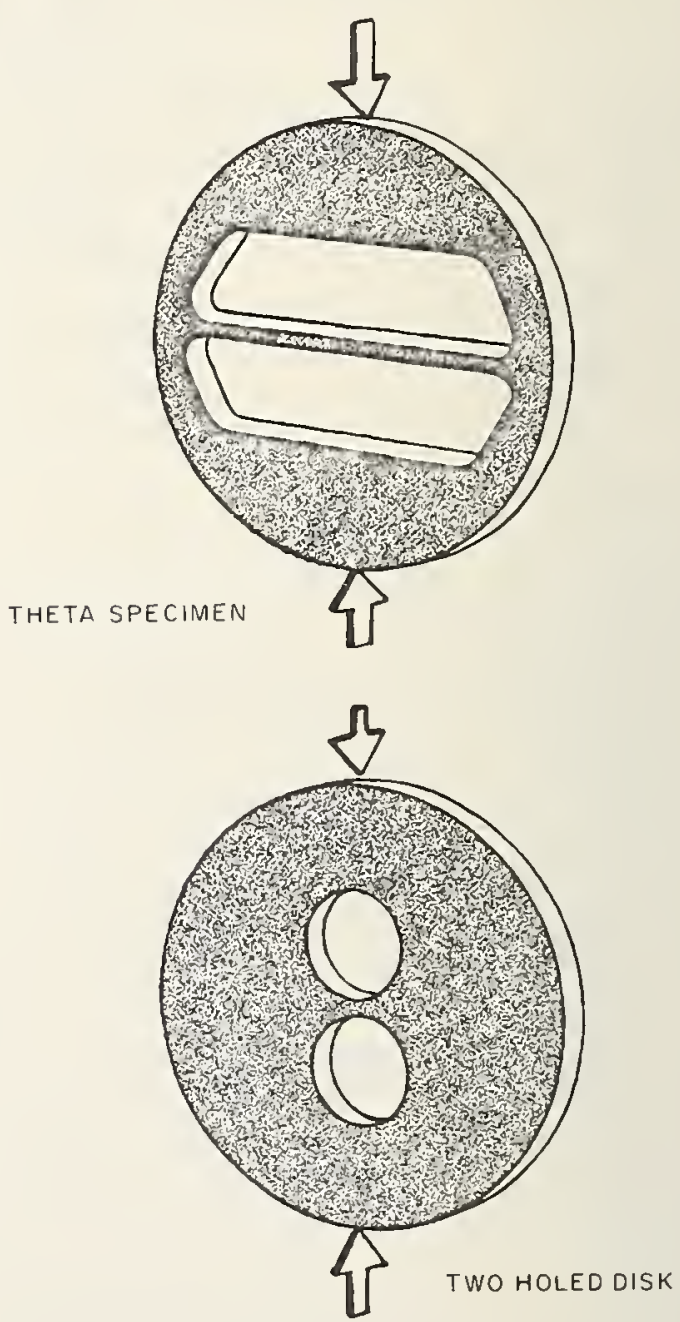

Figure 6. Loading configurations for theta specimen (top) and two-holed disk specimen (bottom). 


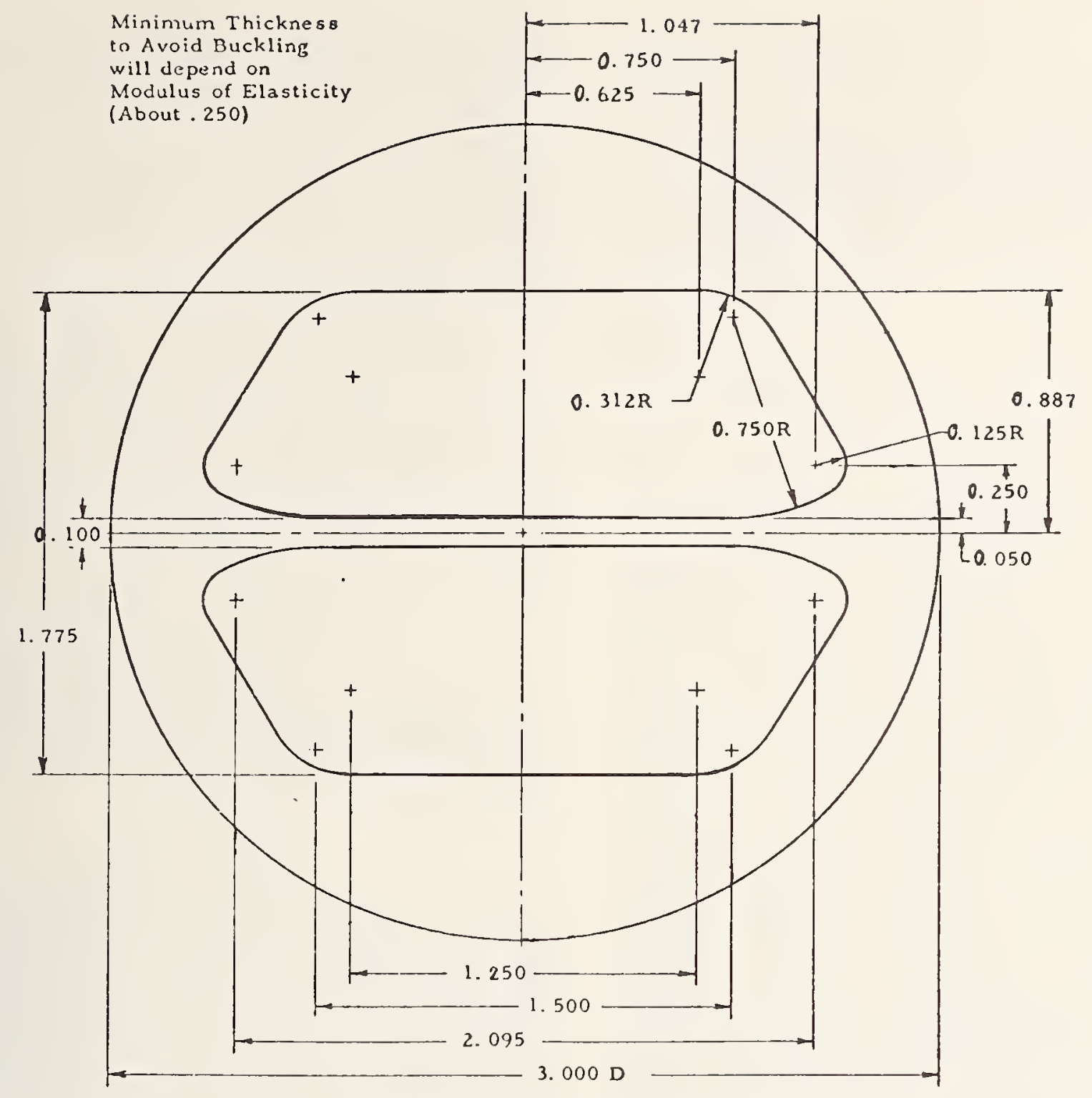

FIGURE 7. Theta specimen geometry.

The photoelastic studies indicated the following load-stress relationship:

where

$$
\sigma=\frac{k P}{D t}
$$

$$
\begin{aligned}
\sigma & =\text { stress in bar } \\
P & =\text { applied load } \\
D & =\text { outer diameter } \\
t & =\text { thickness of specimen }
\end{aligned}
$$

and $k$ is a constant equal to 13.8 (according to Durelli et al.) or 16.4 (according to $\mathrm{D}$ aniels and Weil). If the deformations of the specimen are small, and if the material is elastic, isotropic, and homogeneous, this relationship should hold for any model with plane dimensions proportional to those in the figure. (The thickness need not be proportional.) Dimensional accuracy is apparently not critical [21] except, perhaps, in the width of bar where significant deviations produce correspondingly significant changes in the stress [20]. Further study of this test method is apparently required before it can warrant wide acceptance.

\section{Truss Beam Tensile Test}

This test, proposed by Bortz in 1963 [22], uses a bending mechanism to achieve a uniform, uniaxial, tensile stress. The specimen, figure 8, consists of a heavy upper beam and a comparatively thin lower beam. Flexural loading of the specimen results in bending of the upper beam and a nominally uniform tensile stress throughout most of the lower portion, which acts like a tension tie rod.

The advantage of this test method is that it provides a uniform tensile stress over a relatively large gage section, which makes it attractive for the evaluation of stress-strain characteristics. Measured strains in the lower beam of the specimen agreed with theoretical predictions within 1.4 percent [23]. However, Bortz [7] recently concluded that the specimen is, in fact, subject to stress conditions other than those predicted, and recommended further study of the method. 


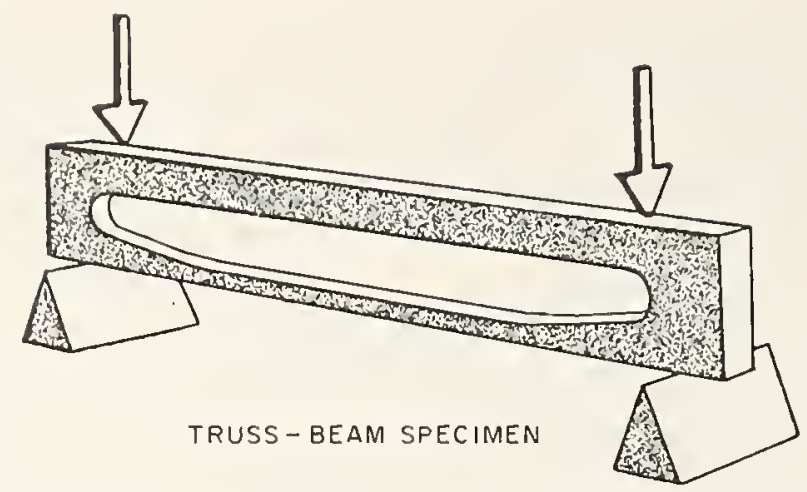

Figure 8. Loading configuration for truss beam tensile specimen.

\section{Stress Wave Technique}

An entirely new approach to the tensile strength testing of brittle materials has been developed by Cornish and Abbott [24, 25]. The method produces a dynamic state of pure tension, free of most of the difficulties characteristic of direct tension testing. It is applicable to many brittle materials such as alumina which does not experience a change in tensile strength with changes in strain rate at room temperature.

It is impractical for us to describe the technique in detail here, and the reader is referred to one or the other of the referenced descriptions, which are written in a clear, interesting fashion. Briefly, however, consider a long, slender, freely supported bar. Applying an impulse to one end of the bar causes an unchanging longitudinal compressive wave to travel down the bar. At the other end this wave is reflected as a mirror-image tensile wave which propagates back up the bar toward the first end. This is illustrated schematically in figure 9 . Since the tensile strength of a ceramic is small relative to the compression strength, a ceramic specimen can be made to fail in tension under the action of the reflected wave.

In a test, a strain gage is mounted longitudinally near the loaded end of the bar in order to determine its "weakest link" tensile strength. On the basis of previous information, or from a trial and error process, a compressive impulse is applied whose reflected value exceeds the tensile strength of the specimen by a small amount. The reflected wave travels back up the bar until it reaches a point where the tensile strength of the bar is less than the peak value of the wave. At this point fracture occurs. That portion of the tensile wave which is behind the fracture section is lost, but the amount of strain which caused fracture continues to travel up the bar and is measured by the strain gage.

In their experiments Cornish and Abbott used 1/4-in square alumina bars supported in a rubbercoated chemical clamp and loaded with an electrically ignited charge of lead azide. Their results are noteworthy. The average tensile strength they obtained differed by less than 2 percent from average values obtained by the pressurized ring test and by the gas-bearing tensile test. The coefficient of variation was essentially the same for both the stress wave technique and the pressurized ring test, about 8 percent. (The coefficient for the direct tension test was not available.)

The stress wave technique appears to be applicable to elevated temperature use although the strain rate sensitivity of the specimen materials will first have to be evaluated.

\section{Flexure Test}

Because of the problems associated with direct tension testing, the ceramics industry promoted the well-known flexure test as a means for evaluating tensile strengths. This test, which is also referred to as the bend test or the transverse test, avoids the need for placing the entire cross section of a specimen in uniform tension, and places only a portion of the cross section in tension. The maximum tensile stresses occur only in the extreme outer fibers on one side of the specimen, with the tensile stresses decreasing to zero at the neutral plane and being balanced by compressive stresses in the specimen on the other side of the neutral plane. Thus, while the test is intended to provide a tensile strength value, it is classified as a bending test because it involves a non-uniform tensile

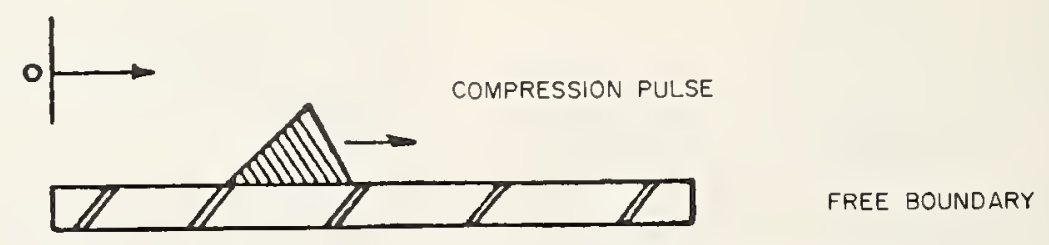

IMPULSIVELY LOADED SURFACE

FREELY SUPPORTED BAR

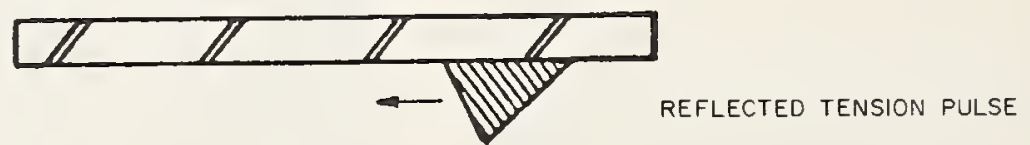

FiguRe 9. Schematic of stress wave reflection at a free boundary. 
stress field instead of the uniform field considered in our discussion of tension test methods.

The flexure test at first appears to be simplicity itself. As shown in figure 10, a specimen is supported close to each end and on the side opposite the supports a uniformly increasing load is applied at a single point centrally located between the supports (also called mid-point, center point, or three-point loading) or at two points (four-point loading) equidistant from the supports. The load is increased until the specimen fractures. At present, ASTM recommends three-point loading for the strength testing of brittle materials [26].

The maximum stress at fracture is defined as the flexural strength, and is frequently referred to as the modulus of rupture, or MOR. The maximum stress is obtained by using the following equation:

$$
\sigma=\frac{M C}{I}
$$

where $\sigma=$ extreme fiber stress

$\mathrm{M}=$ bending moment

$\mathrm{C}=$ distance from the neutral plane of the specimen to the extreme tension fiber

$\mathrm{I}=$ moment of inertia of the cross section of the specimen about the neutral axis.

Some assumptions have to be made for the equation to be valid. These are:

(1) The material obeys Hooke's Law.

(2) The stress is distributed linearly across the bent beam and is directly proportional to the distance from the neutral plane.

(3) Bending occurs in a plane of symmetry of the beam.

(4) The original transverse plane remains a plane after bending occurs and is perpendicular to all longitudinal fibers after bending.

(5) There are no shear stresses present in the area where the maximum fiber stress is to be measured.

In order to justify the assumptions, the flexure test loses its simplicity and becomes more complicated. Some of the more important factors

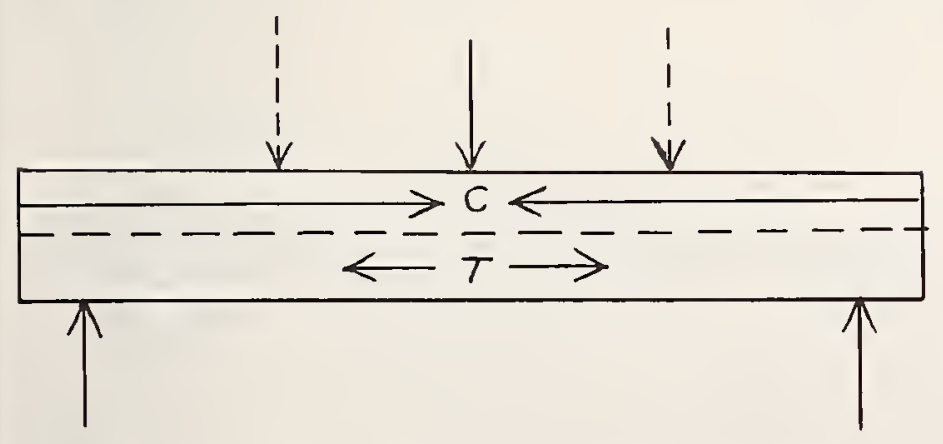

FIgURE 10. Diagram of a bar flexure specimen.

The specimen is supported near the ends and loaded centrally (solid arrow at top) or in four-point bending (dashed arrows at top). The dashed horizontal line is the neutral axis. During a test the specimen is in tension below this line and in compression above it. involved in designing a flexure test to give valid, reliable results will be discussed.

The specimen should be of a size that is large enough to be representative of the material for which it is serving as a sample. It should also be of a size convenient to handle but not too large or expensive to fabricate so as to reduce the number of available specimens for test to a point where meaningful results cannot be developed. It is felt that the specimen shape should be kept as simple as possible and, where necessary, the test assembly made complicated in order to meet the test requirements.

The most commonly used shapes for specimens are bars, cylinders, and enlarged-end specimens, and a specialized enlarged-end specimen called the dog-bone specimen. These are shown in figure 11. The bar and cylindrical specimens have the advantage of economy, ease of preparation, and generally being representative of the material, particularly the surfaces and edges, as found in use. Care must be taken not to damage the surface and edges of the bar specimen that will be in tension when tested. The cylindrical specimen has the disadvantage of having only a small part of the specimen under maximum stress. In fourpoint loading enlarged-end and dog-bone specimens have the advantage of eliminating concentrated loads and shear stresses in the reduced gage length, while the dog-bone specimen, which is loaded and supported along the neutral plane, has the further advantage of minimizing errors introduced by frictional forces.

To minimize torsional stresses arising from warped specimens or nonplanar test beds, rockers are generally used on the bottoms of the supports (which are commonly knife edges) and loading is often done through spheres.

Frictional forces should be reduced to a minimum. Since these forces act by grabbing and slipping they produce an extremely erratic effect on the results. These forces are generally reduced

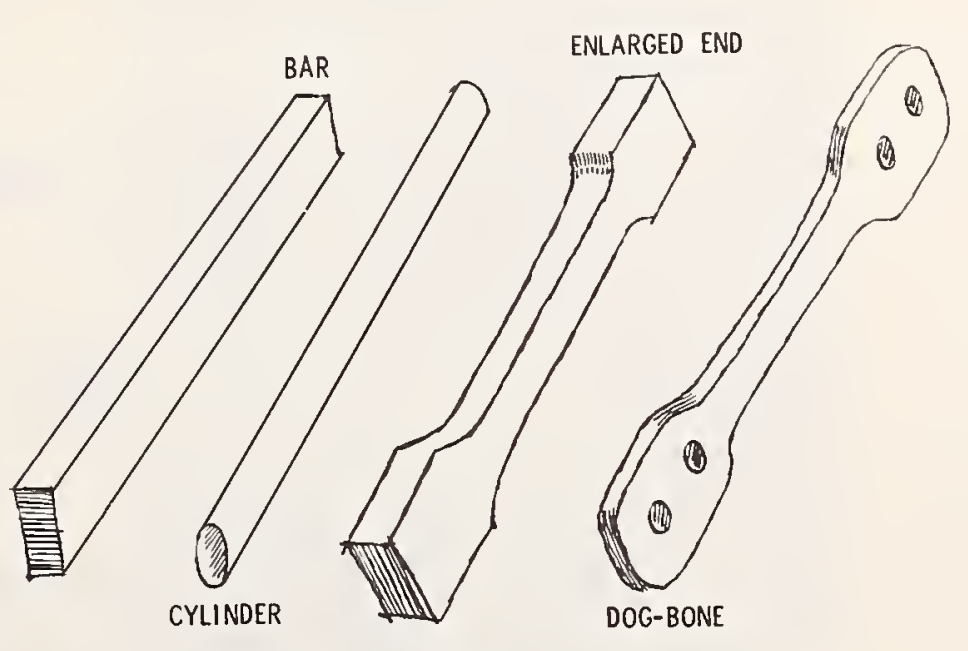

Figure 11. Common flexure specimens.

The bar and cylinder specimens are probably the most common types while the enlarged-end and dog-bone specimens can provide an area of uniform stress in their reduced sections. 
by using rounded or rolling knife edges and lubricants with the bar, cylindrical, and enlargedend specimens, and by loading along the neutral plane in the dog-boned specimen. A picture of a flexure test assembly is shown in figure 12. The long loading arms allowed the specimens to be tested in various atmospheres.

Some materials that are classed as brittle nevertheless show some ductility. Using the regular beam equation will give results higher than they should be. A correction by Nadai [27] will compensate for this departure from Hookean behavior before fracture.

Three-point (or center-point) loading is not the preferred flexure test method. There may be a wedging action due to the concentrated load which could lead to erroneous results. In addition, with four-point loading a much larger area of the specimen is under maximum stress, giving a more representative value of the usable strength of the material. Figure 13 [28] shows that center-point loading tends to "force" the glass to fail near the loading point which is the point of maximum stress. In four-point loading it can be seen that as the loading knife edges are moved farther apart the failure origins fall between them in a fairly uniform manner. The strengths indicated along the sides of the specimen representations in the figure show that as the area under uniform maximum stress increases the strength decreases. This is representative of the size effect. Figure 14 shows photoelastically the large area placed under uniform stress when four-point loading is used.

\section{Large Area Bending Tests}

One of the problems in the flexure test is that even a modestly large specimen may not be

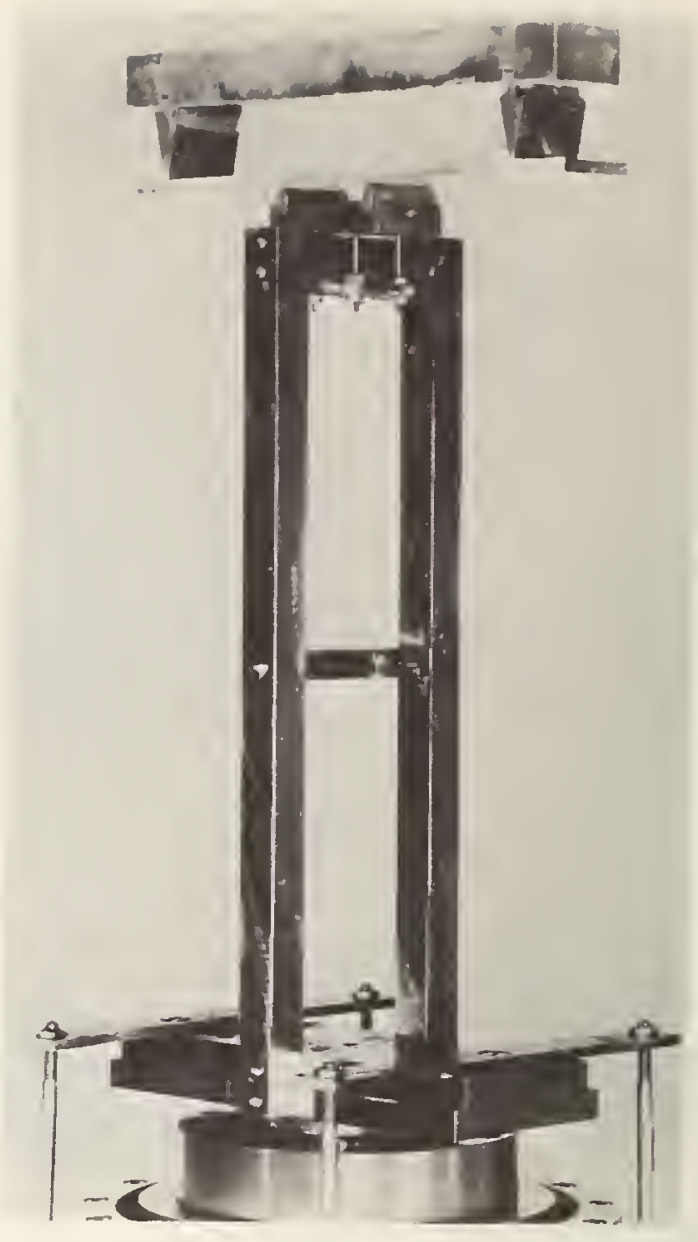

Figure 12. Four-point bending test of a glass specimen.

One support knife-edge fixes the plane of the specimen while the other has a rocker bottom to compensate for twist in the specimen. The loading knifeedges have rocker tops and are mounted in a fixture which is loaded through a fifth knife-edge. These precautions minimize the possibilities of nonvertical load application. The long arms on this apparatus permit testing of specimens in furnaces and cold boxes.
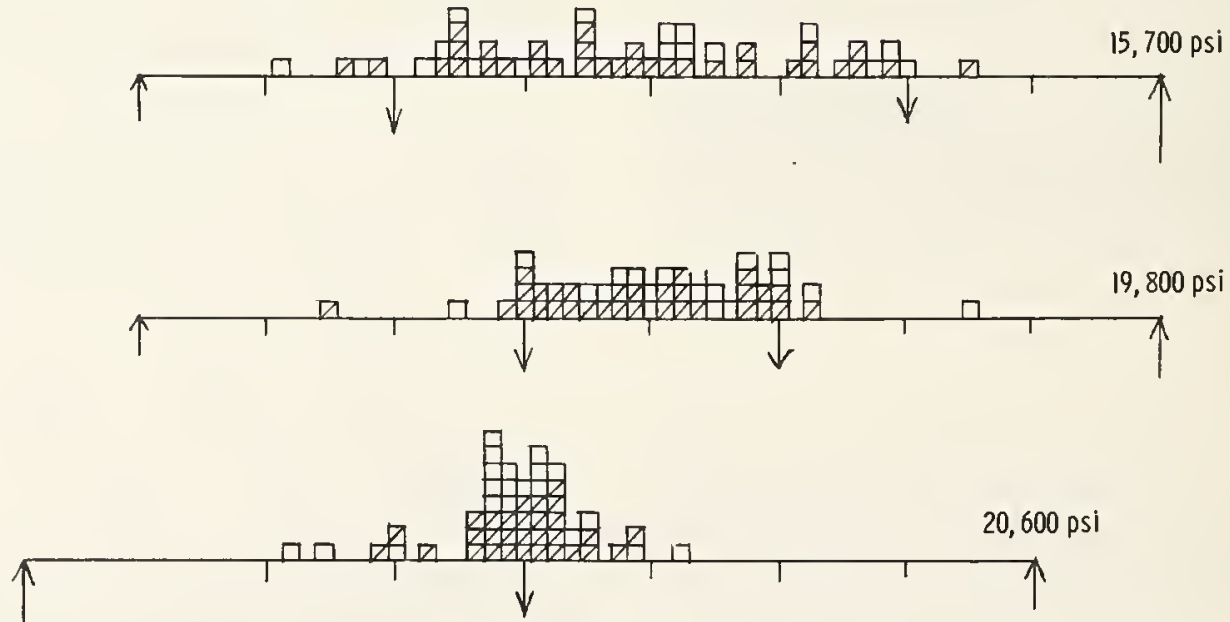

Figure 13. Locations of fracture origins and strengths for $10 \times 1 \frac{1}{2} \times 1 / 4-$ in annealed soda-lime-silica glass specimens.

Slashed squares and hollow squares indicate fractures originating on the surface and at the edge, respectively. Specimens were supported on an 8-in span and tested in three-point bending (bottom diagram), four-point bending with loads 2 in apart (middle diagram), and four-point bending with loads 4 in apart (top diagram). The fracture origins cluster around the point of maximum stress for three-point bending, and are uniformly dispersed throughout the maximum stress regions in four-point bending. As the area under maximum stress increases, the strength decreases. 


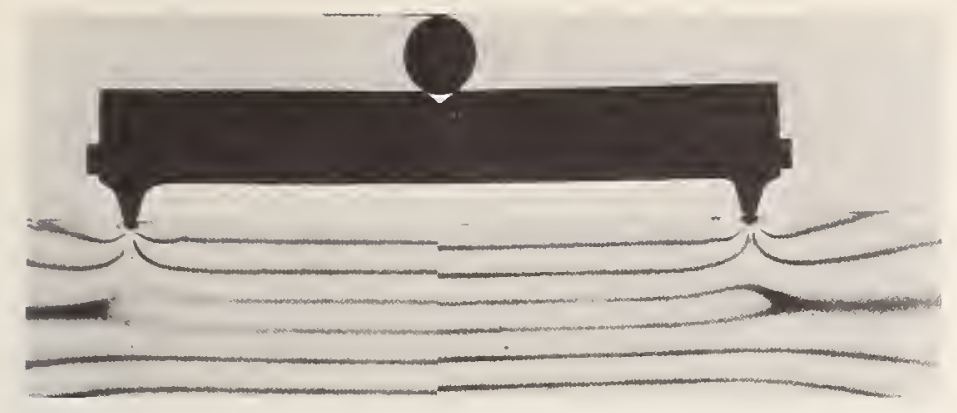

FIgURE 14. This composite photoelastic print shows the central portion of a flexure specimen in four-point bending.

The dark lines are lines of uniform stress which indicate that the tension face of the specimen experiences uniform stress over a length slightly longer than the distance between loading points.

representative of large applications such as glass windows. Pressure tests have been used with some success to minimize this problem. In this test the specimen, generally a flat plate, is held near the edges and a uniform load is applied over the entire surface of the specimen. There are no recognized standards and this test is generally designed to meet specific conditions. This test method has the advantage of being able to place large areas of a material under load and under conditions similar to those found in practice (e.g., glass windows under wind load or water pressure). It has the disadvantage of placing only a small part of the specimen under maximum stress.

A test method presently being considered by several laboratories to place large areas of a material under uniform stress is the concentric ring test. In this test a flat specimen is supported on a ring and loaded through a concentric ring of appropriately smaller diameter than the support ring. There is much that needs to be learned before this method can be used with confidence, such as the effects of specimen overhang, specimen deflection, and the ratio of support ring diameter to loading ring diameter. Advantages of this test method are its ability to place large areas under uniform stress and to test only the surface of the specimen and thus eliminate the influence of edges on the results.

\section{Diametral Compression Test}

In this test method a cylindrical specimen is compressed diametrically between two flat platens. Under proper conditions fracture occurs along the loaded diameter ( $A B$ in fig. 15) as a result of tensile stresses which develop across this diameter. Like the flexure test, this test is used for the determination of tensile strength although it does not develop a uniform uniaxial state of tensile stress.

The method is also known as the splitting tension test, or the Brazilian test, and was originally developed in Brazil and Japan around 1943

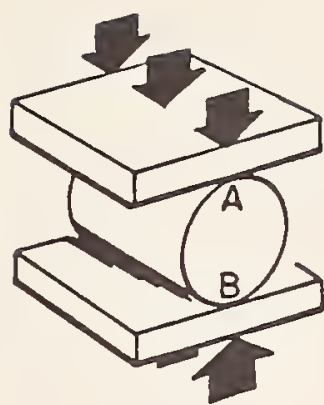

(a) LOADING

FIGURE 15. Diametral compression of a solid cylindrical specimen.

for concrete [19]. Figure 16 shows a diametral compression test on an asphalt specimen [29].

This test has gained considerable favor because of its relative simplicity. The specimen alines itself between the platens and can be easily tested without elaborate fixtures.

If the specimen is not long in comparison with its diameter, the diametral loading sets up a biaxial stress distribution in the cylinder. Frocht [30] evaluated the stress distribution using elasticity theory and T. S. Love [31] verified this solution photoelastically. The maximum tensile

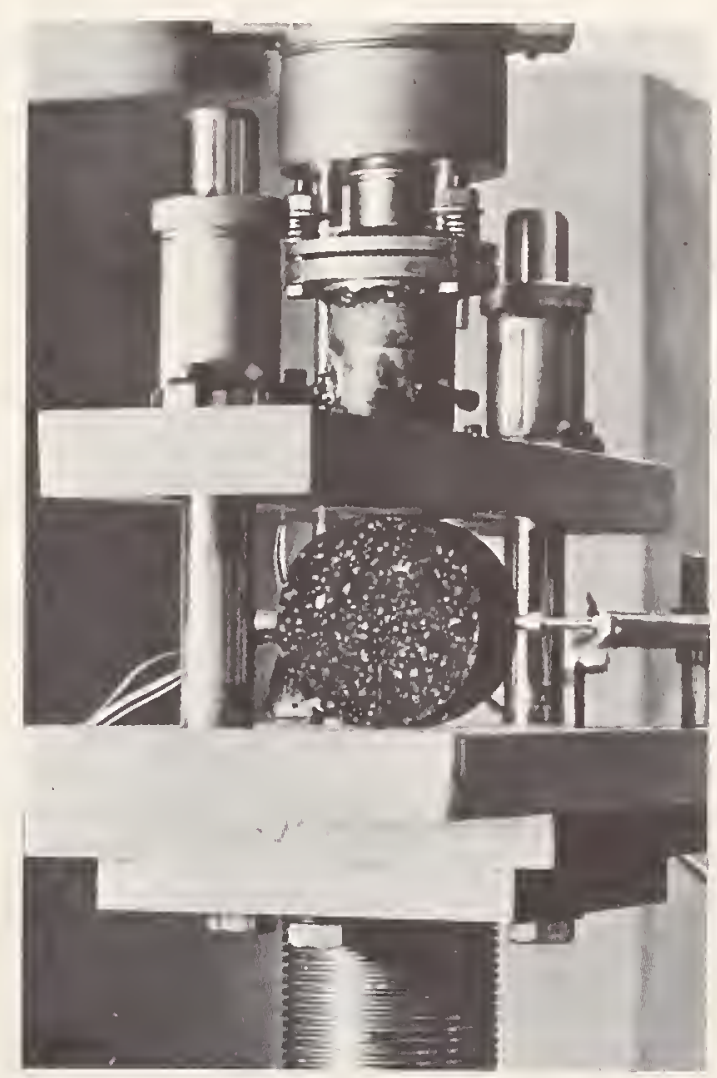

Figure 16. Diametral compression test of an asphalt specimen at the University of Texas. 
stresses which act uniformly across the loaded diameter are given by:

$$
\sigma=\frac{2 P}{D t \pi}
$$

where $P=$ applied load

$D=$ specimen diameter

$t=$ specimen thickness.

This analysis assumes that the specimen is isotropic and homogeneous as well as elastic, and that the compression loads are restricted to lines of contact between the cylinder and the platens. In a real situation, local deformation of the cylinder and platens distributes the compression load over an area, but Rudnick, Hunter, and Holden [32] have shown that this does not significantly alter the stress distribution near the center of the specimen. In fact, it is this feature of the problem that makes the test feasible. It is considered good practice to place narrow bearing pads of suitable material between the specimen and the platens to compensate for surface irregularities and to reduce the infinite compressive stresses immediately under the applied load. Figure 17 shows the elastic stress distribution along the loaded diameter in a specimen having a length equal to onetenth of the diameter, and assuming uniform load distribution over a contact width of one-tenth of the diameter. $\sigma_{1}$ is the maximum tensile stress (which acts normal to the loaded diameter), $\sigma_{2}$ is the maximum compressive stress (which acts along this diameter), and $\tau$ is the maximum shear stress (which is inclined to the diameter). It may be seen that very high compressive stresses act at the loaded surfaces and that high shear stresses exist immediately below these surfaces. Thus, in order to achieve the desired tensile failure the compressive and shear strengths of the specimen must be several times higher than its tensile strength, and the applied load must be distributed over an adequate area.

The selection of the proper bearing pad material is the key to a successful test. As Rudnick, Hunter, and Holden have pointed out, if the pad is too hard the load application will approach line contact and fracture may initiate in compression or shear rather than in tension. If the pad is too soft and the load is thereby distributed over too great an area the tensile stresses in the central portion of the specimen are reduced and the apparent tensile strength is increased. The selection of the proper pad material is done experimentally. One criterion is that it produce tensile, rather than compressive or shear, failures. (Compressive failures appear as local crushing, shear failures start at an angle to the loaded diameter, and tensile failures break the specimen along the loaded diameter.) Another factor involved is friction between the
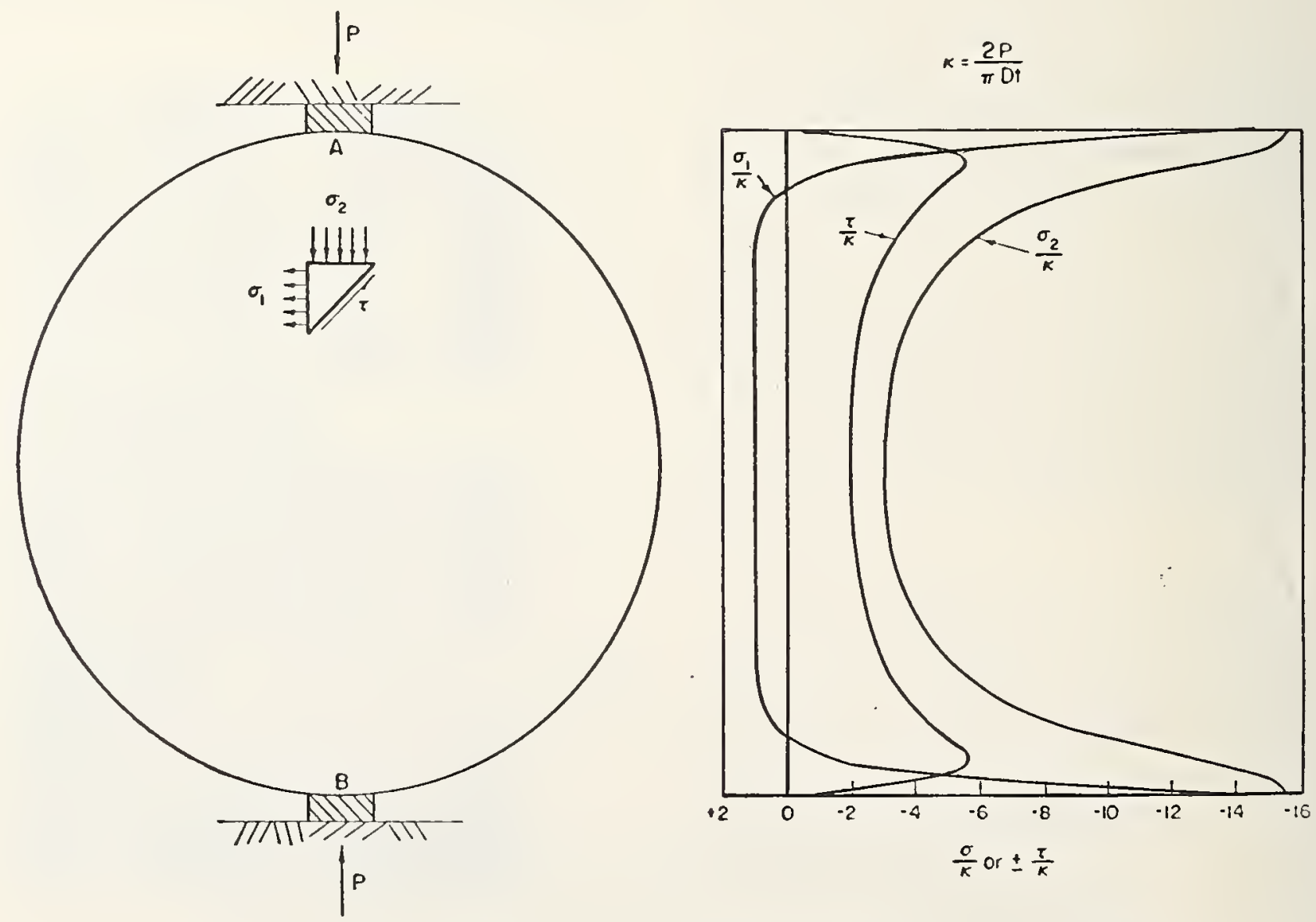

FIGURE 17. Stress distribution along loaded diameter for a diametrally compressed cylinder, assuming uniform contact pressure over the contact area. 
specimen and the pads. This tends to increase the apparent tensile strength [33]. Blotting paper and punch cards are two pad materials currently used for tests on ceramics, while plywood is standard for concrete [34].

The most critical alinement factor in the diametral compression test is the parallelism of the platens in the direction of the axis of the cylinder. In order to maintain this as loading progresses, it is important that the specimen be centered with respect to the loading heads of the testing machine. Ivey and Buth [35] found that a longitudinal displacement of the specimen by 8 percent of its length caused an apparent strength reduction of 12 percent. On the other hand, parallelism of the platens in the direction normal to the cylinder axis was not found to be critical. An angularity of 6 degrees reduced the apparent strength by only 4 percent. If the bearing pads are narrow and are not centered with respect to the loaded diameter, strength reductions of the order of 8 percent may be expected. Any kind of misalinement increases the coefficient of variation of the test results beyond the 5 percent value obtainable with homogeneous materials and good testing technique.

Another possible source of error in the diametral compression test was described by Bortz and Wade [7]. If the tensile strength of the specimen material is high, and its elastic modulus is low, considerable flattening can develop at the loading areas before failure is attained. This distortion invalidates the theory used to develop the loadstress relation and results in lowered values of apparent tensile strength.

Finally, it should be mentioned that the diametral compression test is not amenable to the direct determination of stress-strain characteristics.

On the other hand, the test has numerous advantages besides simplicity. It is suitable for elevated temperature testing and may be used for very small specimens. It is less affected by surface conditions of the specimen than any of the other test methods. Only at the ends of the cylinder do maximum tensile stresses appear at a surface. Shook [3] has described a means of eliminating even this surface interaction. If the ends of the specimen are reduced in diameter by a gradual tapering, the applied load and resulting tensile stresses will be smaller in this region. However, the effects of this non-uniform load distribution on the stress distribution near the mid-length of the specimen are not immediately obvious.

\section{Brittle Ring Test}

The brittle ring test is a variation of the diametral compression test and employs a thickwalled, hollow, cylindrical specimen instead of a solid cylinder. Loading is accomplished by compressing the specimen diametrally between flat platens. A steep stress gradient (fig. 18) is produced in the ring similar to that obtained in the flexure

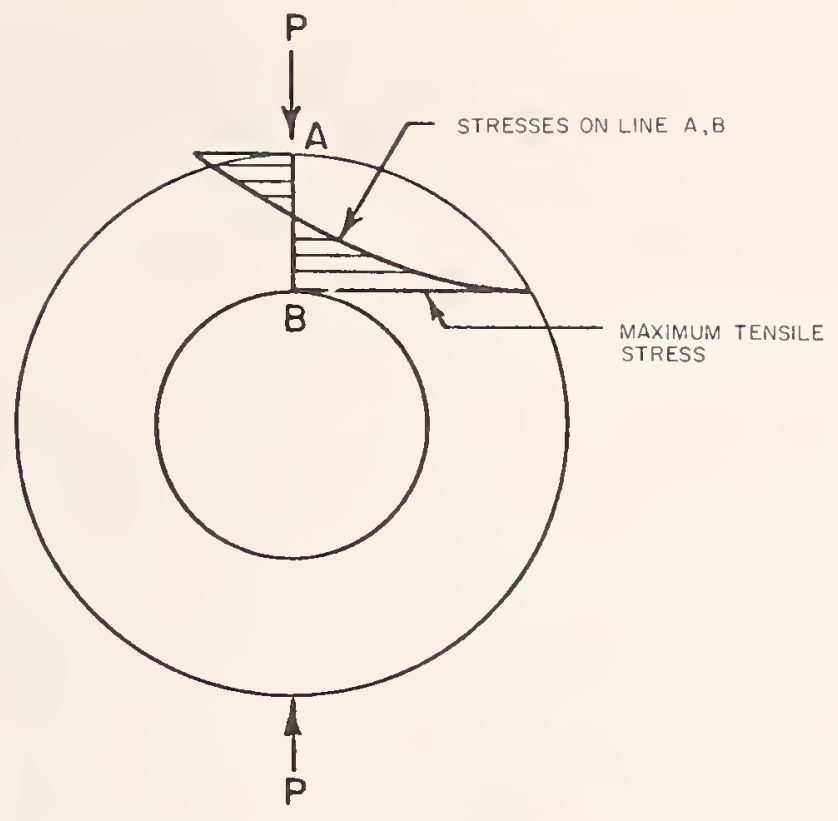

Figure 18. Stresses developed in a ring subjected to diametral compression.

test. The maximum tensile stress appears on the inner surface of the ring, at the loaded diameter, in the tangential direction. Fracture always initiates here [23].

This test method has many of the advantages of the diametral compression test method. The specimen alines itself between the parallel platens without any special gripping apparatus. Furthermore, the test is easily adaptable for elevated temperature use.

The principal deficiency of the method is the determination of the load-stress relationship. This problem has been studied theoretically by Timoshenko [36], photoelastically by Frocht [37], with brittle coatings by Durelli et al. [38], and with strain gages by Bortz and Lund [39]. From a comparison of available data, the latter investigators concluded that the relationship between the maximum tensile stress and the applied load depends only on the inner and outer radii of the specimen, independent of the elastic constants. In his review of this work Shook [3] expressed a less optimistic viewpoint. It appears that more work is required to evaluate the suitability of this test method insofar as numerical strength determinations are concerned. The statistical variation of test results with this method, however, is reportedly better than that obtained with standard tensile specimens [39].

Because of the steep stress gradient, only a small volume of the specimen experiences the maximum tensile stress. Rudnick and Duckworth [40] have pointed out that with large-grained materials the highly stressed region may be confined to a single grain, in which case transgranular fracture would be encouraged. With small-grained materials fracture at the grain boundary would be encouraged. Properly interpreted, the test can be valuable in both cases. 
Bortz and Lund [39] have observed that with this test method there is no strength variation with volume variation such as occurs with other test methods. This, too, is probably due to the maximum tensile stress being confined to a small volume of material.

\section{Torsion Test}

In most of the test methods discussed thus far, the state of stress at the fracture location is uniaxial. The exceptions are the pressurized ring test, the large area bending tests, and the diametral compression test. Classical theory holds that fracture is dependent only on the maximum tensile stress, i.e., that a transverse stress component does not affect longitudinal strength. While there are insufficient data available to categorically verify this principle for brittle ceramics, it is equally true that evidence to contradict it is likewise lacking.

In a torsion test of a circular cylindrical specimen the stresses are zero at the cylinder axis and increase linearly with the radius. The maximum tensile stresses occur on the surface at a 45-degree angle to the axis. Numerically equal compressive stresses act perpendicular to these tensile stresses. The maximum shear stresses, which are also numerically equal to the maximum tensile and compressive stresses, act parallel and perpendicular to the axis of the specimen. In a brittle material, which has lower strength in tension than in shear, fracture occurs normal to the maximum tensile stresses, i.e., at 45-degrees to the specimen axis. An interesting example of such a fracture is shown in figure 19, which shows a torsion failure of a human leg bone, similar to those experienced in skiing accidents [41].

The torsion test has not been used much for determining tensile strengths in brittle materials. An often quoted objection to it $[2,42]$ relates to the consequences of improper gripping. If the specimen is not straight, or if the torque axis is not coincident with the specimen axis, undesirable bending stresses are introduced. However, this alinement problem is not as severe as the one in the tension test, and if specimens having large slenderness ratios (i.e., length/diameter) are used, visual alinement should be satisfactory. In any case, the presence of bending stresses can be checked with strain gages.

Another objection to the torsion test is similar to that raised in connection with the flexure test. If the material is not, in fact, linearly elastic to failure then the magnitude of the fracture stress is somewhat obscured. The possibility of some plastic deformation prior to failure is greater in the torsion test than it is in the tension or flexure tests because the shear stress is higher here. But this deficiency can be overcome by using a thin-walled tubular specimen instead of a solid cylinder. In this case the load-stress relation is statically determinate, regardless of the shape of the stress-strain curve. Furthermore,

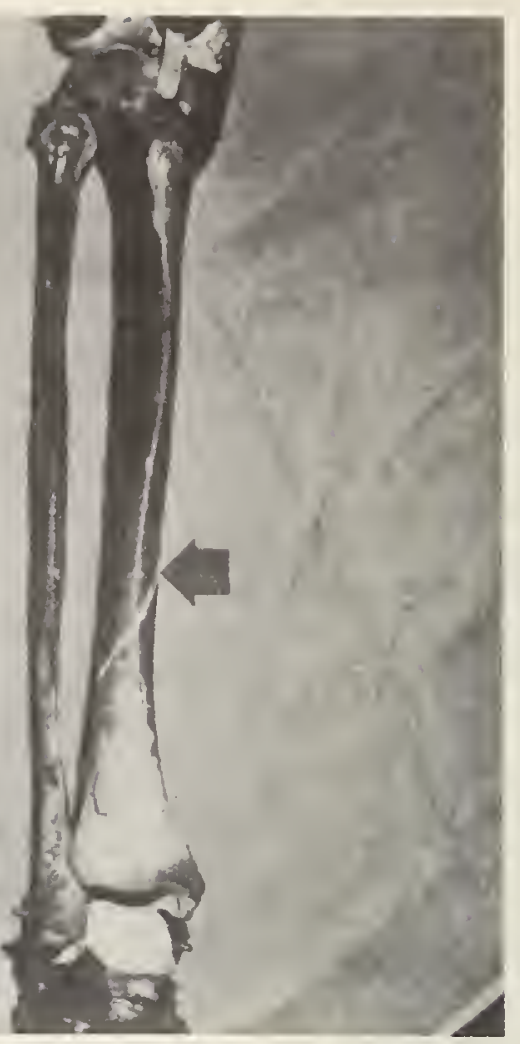

FIGURE 19. Spiral fracture of a tibia resulting from a torsion test.

with the thin-walled tube the stresses through the thickness may be considered uniform; more so, in fact, than in the pressurized ring specimen [43].

The torsion test is readily adaptable to elevated temperature use.

\section{Compression Test}

All of the test methods discussed thus far are used, to some extent at least, to determine the tensile strengths of brittle materials. The compression test, on the other hand, is used to evaluate "compression strengths." However, it is realized that the values obtained are not truly representative of the compressive strength of the materials since, even in this case, failure occurs as a result of induced tensile stresses [44].

"In the strength testing of ceramics analyses indicate that with proper and known precautions specific to each test method, the use of bending, torsion, direct tension, and diametral compression can allow the stress that causes failure to be determined without serious doubt. However, with direct compression, the actual failure stress is a subject of doubt ..." [2].

The major problem, in compression testing, is related to the tendency of the specimen to expand laterally under load. Frictional forces are created between the specimen ends and the load plates due to differences in geometry and Poisson's ratio. The forces can be reduced but not eliminated by lubrication, and the nominally uniaxial compressive stress distribution is thereby distorted in the end portions of the specimen. 
Another problem in compression testing involves alinement. Specimen ends should be ground perfectly flat and parallel, and normal to the axis of the specimen. These ends should be concentrically mated with the flat and parallel loading plates in the testing machine. Deviations from ideality contribute to eccentric loading which, like the friction forces, superpose undesirable bending stresses on the uniaxial compressive stress distribution.

In order to reduce the effects of end friction, Bortz and Wade [7] recommend the use of enlarged end specimens in which the length-to-diameter ratio of the reduced section is at least 2 . For uniform specimens this ratio should be increased to 2.5. This exceeds standard requirements [45]. Of course, too large a ratio must also be avoided since this may cause buckling. Specimens with round cross sections are favored because their transverse expansion under load tends to be radial and uniform. However, friction forces cannot be relied upon to behave uniformly. The use of a lubricating pad (e.g., Teflon) is usually recommended, but the high compressive strengths of some ceramics require that end bearing plates be made of very hard material such as tungsten carbide [13]. In this case specimen ends may be lubricated with graphite and oil.

Lynch, Ruderer, and Duckworth [42] recommend using three specimens in series to eliminate the effects of end restraints on the middle one. This appears to be a remedy for the case where specimen lengths are limited for some reason; however, it will have to be demonstrated that failure initiates in the middle specimen.

The use of a universal, or a spherically seated, loading block in the testing machine is sometimes recommended as a means of minimizing load eccentricity [46]. Such devices are never entirely friction-free and are, therefore, only a poor substitute for precision machining and accurate alinement.

An interesting approach to good compression testing technique was recently described by $\mathrm{B}$ abel $[15,47]$. This approach employs hollow cylinders for the specimen and end cylinders (see figs. 20 and 21). The test cylinder has a curved exterior surface which provides a reduced thickness in the gage area and thus precludes edge-initiated failures. The specimen and the end cylinders have conical ends which are lapped together to minimize stress discontinuities at their interfaces. (A better, although more expensive, design would include the end cylinders as integral parts of the specimen.) This design limits bending stresses in the specimen to less than 5 percent and is not susceptible to buckling. Nevertheless, it yields greater scatter in compression test results than does the pressurized ring test using the same specimen. It is recommended that strain gages be used in every test to monitor the uniformity of stress. It is interesting to compare a typical compression failure obtained with this specimen design (fig. 22) with that obtained with a solid cylinder (fig. 23) [48].

The compression test is adaptable to elevated temperature use although, like the direct tension test, limiting accessibility to the specimen usually is conducive to alinement problems. Evans [49] has described a high-temperature compression testing apparatus capable of use to $2,200 \mathrm{C}$ (see fig. 24). Testing is carried out in a helium atmosphere using a tantalum heating element. To avoid damaging the ends of the rams, flat disks were placed between the specimens and the rams. These disks were removed after each test, ground to
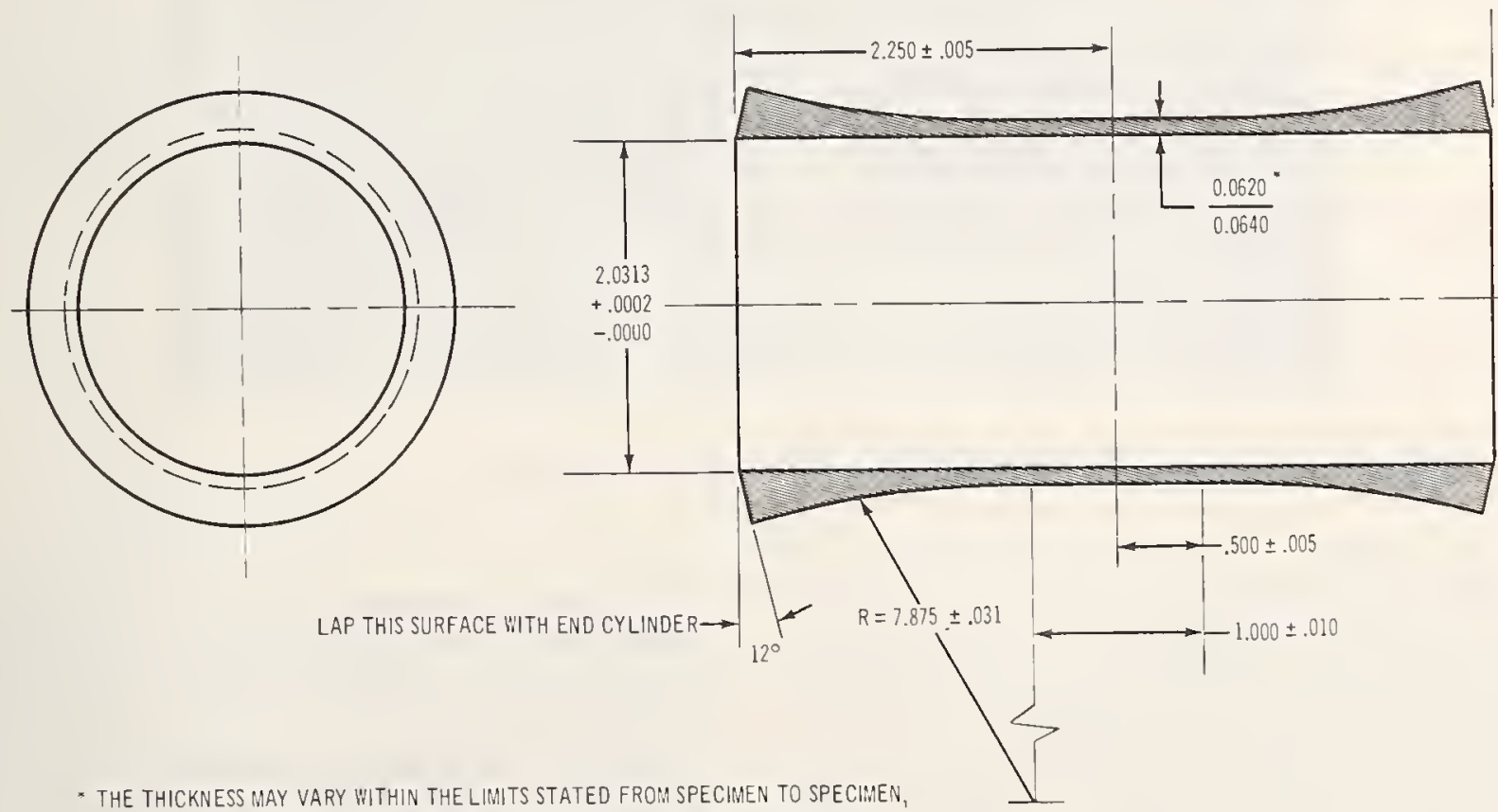

* THE THICKNESS MAY VARY WITHIN THE LIMITS STATED FROM SPECIMEN TO SPECIMEN BUT DN ANY ONE SPECIMEN THE THICKNESS MUST NOT VARY MORE THAN \pm 0.0002

Figure 20. Hollow compression specimen. 

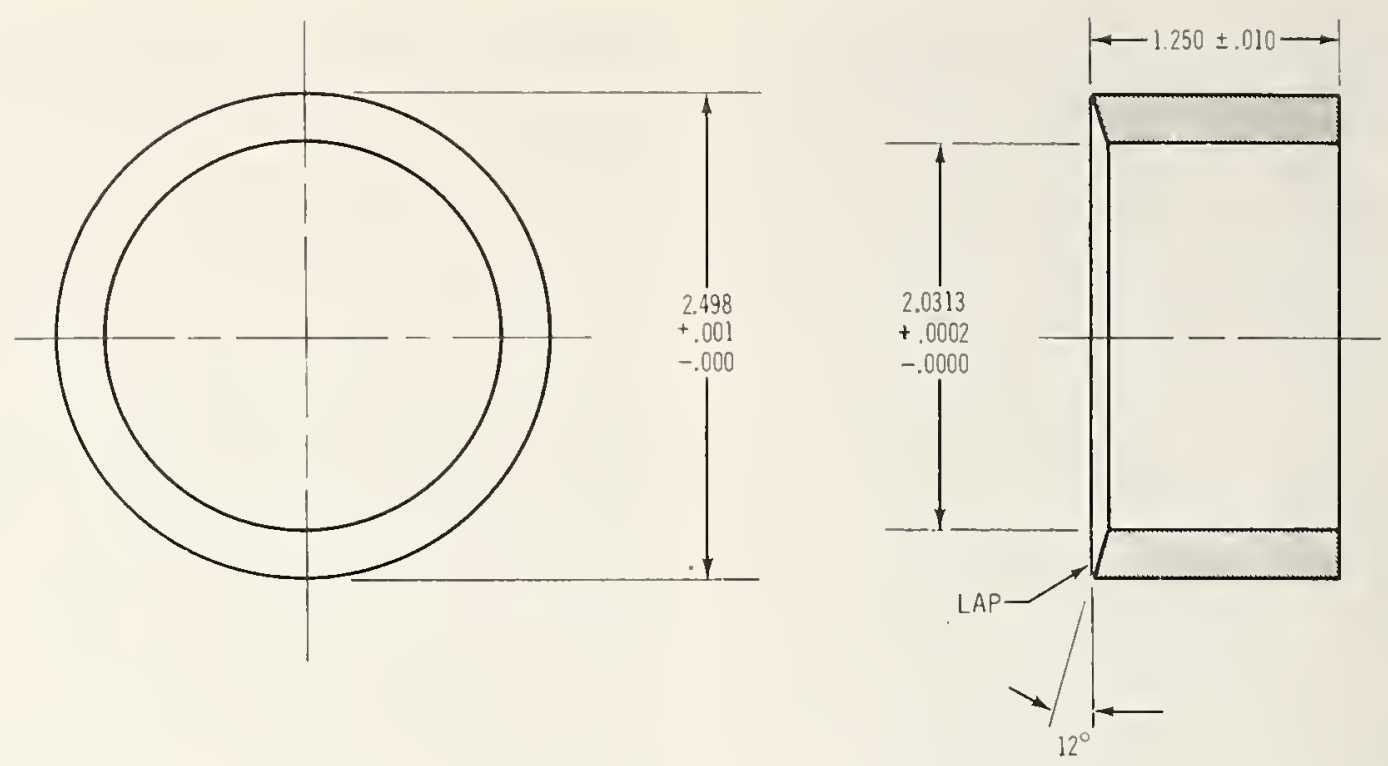

Figure 21. End cylinder for use with the hollow compression specimen.

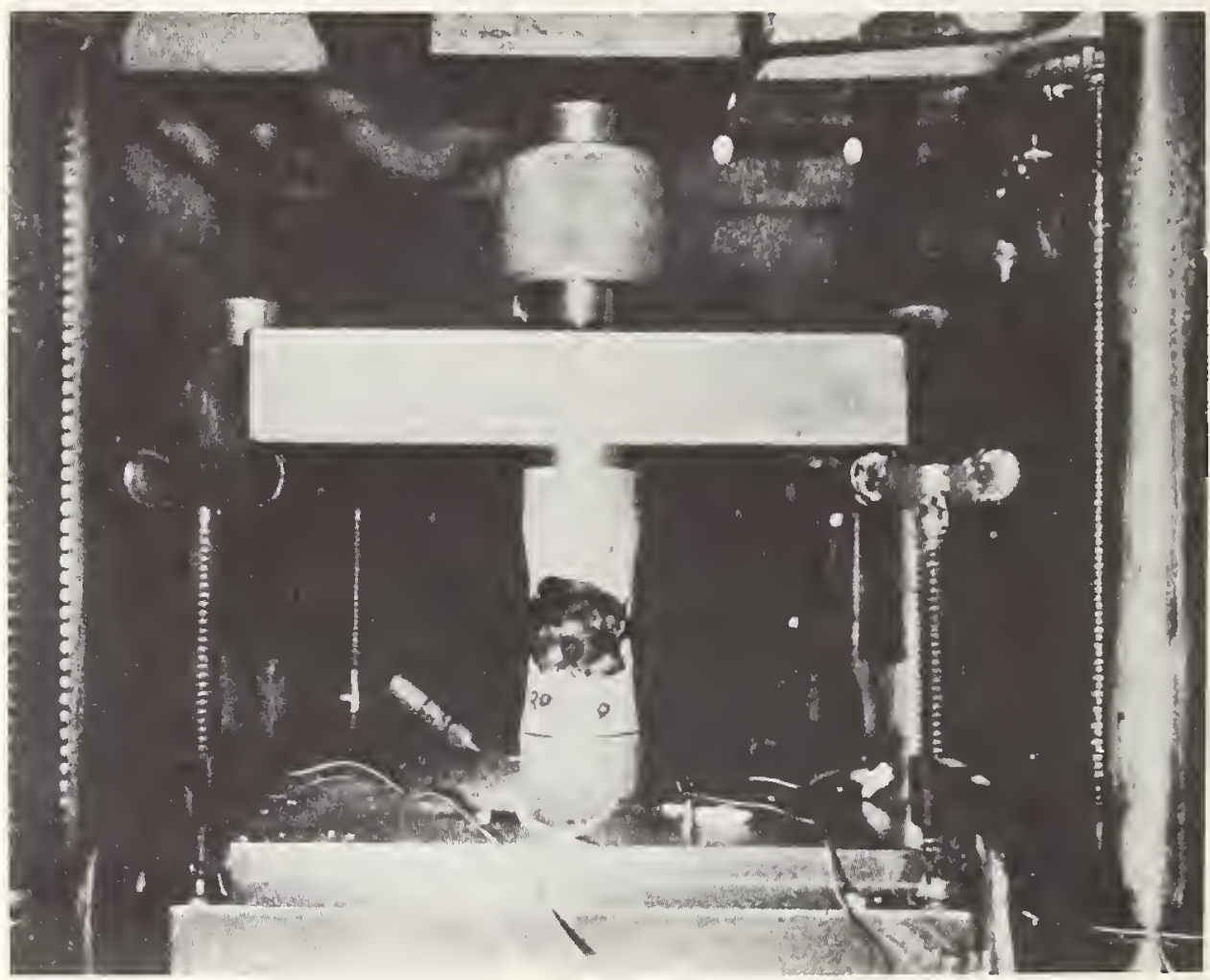

Figure 22. Typical failure of hollow zirconia specimen in compression.

yield a flat surface, and re-used. In tests of magnesia and alumina, tungsten rams and disks were found to be adequate to $1600 \mathrm{C}$, but for higher temperatures alumina was required.

The Griffith criterion for fracture of brittle materials specifies a compressive strength eight times the tensile strength. This is based on the assumption of infinitely sharp cracks in the specimen, distributed with random orientations. Babel and Sines [50] extended the Griffith analysis to cracks of finite sharpness and arrived at compressive strengths from three to eight times the tensile strength, depending on the sharpness of the cracks. Measured compressive strength data for ceramics are limited. They are of ten higher than reported tensile strengths by a factor of 6 to 10, although factors of 4 to 5 are reported for some amorphous materials [44]. 


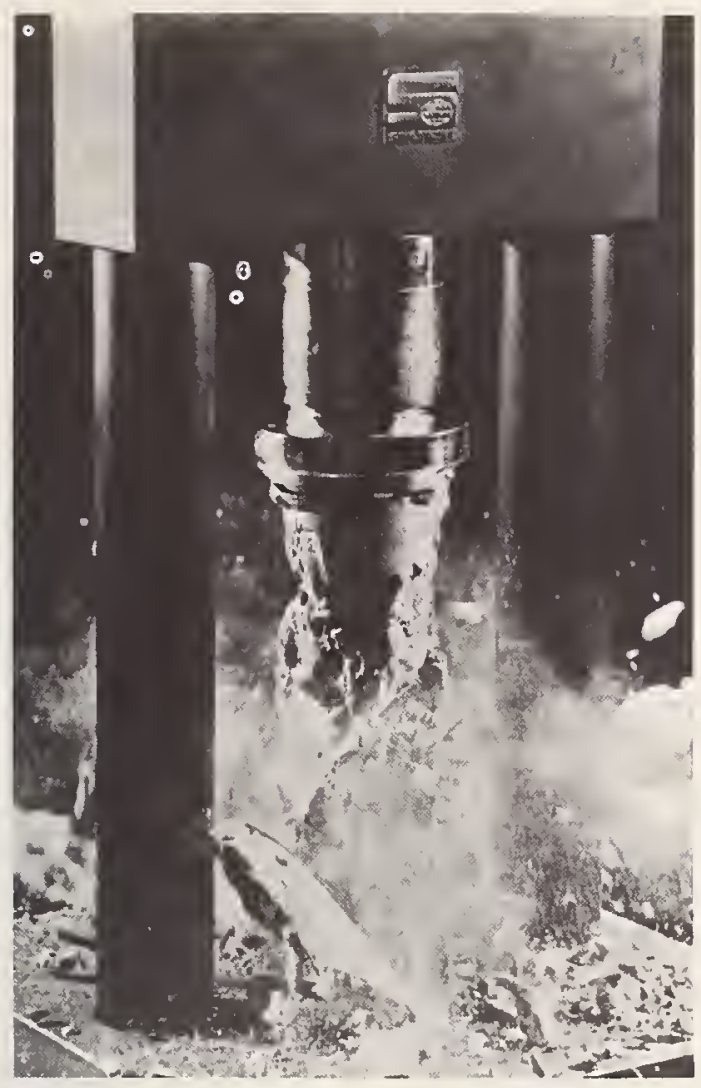

Figure 23. Explosive failure of standard concrete cylinder specimen in compression test by Soiltest, Inc.

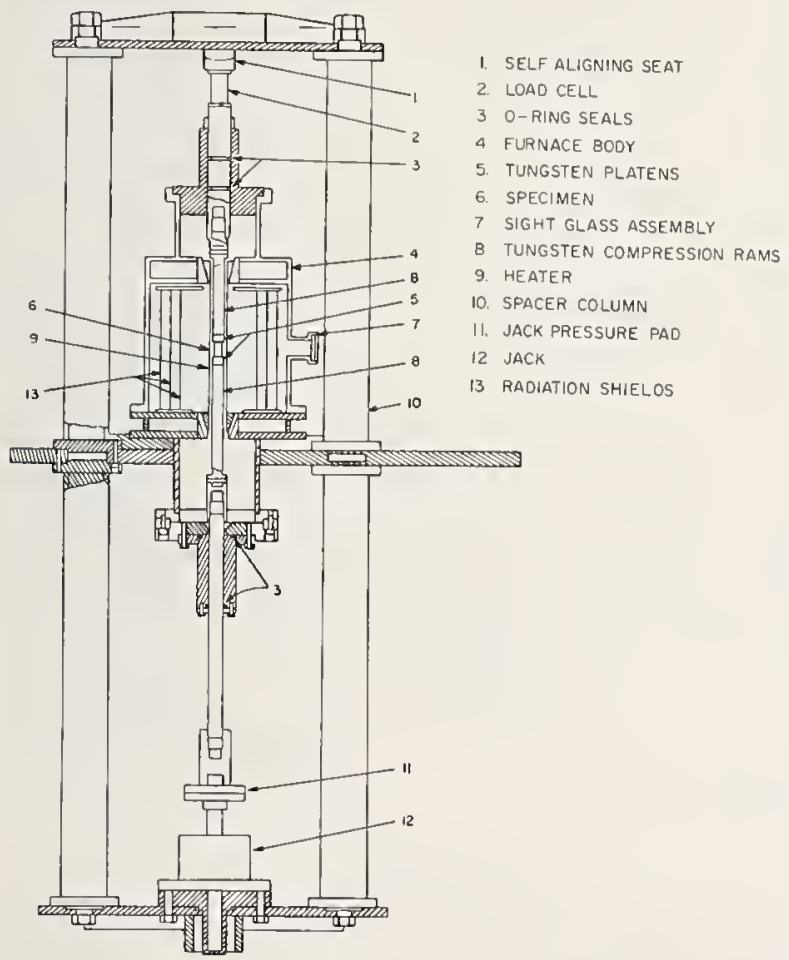

FIGURE 24. High-temperature compression testing apparatus.

\section{General Considerations}

There are a great many factors that can influence the results of a strength test. A good testing program is designed so that only one property is measured at a time. In other words, a strength test program may be initiated to evaluate strengths for a particular set of conditions, or it may be intended to evaluate the influence of some other single factor on strength. In either case, the possible effects of other factors must be considered. Some brittle materials are particularly sensitive to ambient conditions, surface and edge conditions, strain rate, or size effects. With a new material, precautions should be taken until it is understood. Only when the sensitivity of the material to these factors has been studied can the required degrees of control over them be established.

An example of the importance of not treating a new material in a routine manner was shown when the chemically strengthened glasses were first tested in flexure. Peculiarities were noted in some of the strain gage readings. Further investigation showed these glasses had considerable delayed elastic effects. This is shown best in figure 25 [51] in which dead weights were used. As can be seen, the chemically strengthened glasses showed considerable deflection and recovery long after the load was applied and removed-a rather unusual behavior for a brittle material like glass. Clearly, in tests of this material it is important that loads be applied in a controlled manner and rate and that these conditions should be well documented.

This precaution is as applicable to quasi-static and dynamic strength tests (e.g., fatigue, stress rupture) as it is to static strength testing. (We might mention, at this point, that our omission of these types of tests from this survey is intentional. The requirements for these tests that are peculiar to brittle materials are the same as they are for the static tests, e.g., alinement. On the other hand, the equipment for generating cyclic loads or for maintaining constant loads is essentially the same regardless of whether the specimen material is brittle or ductile.)

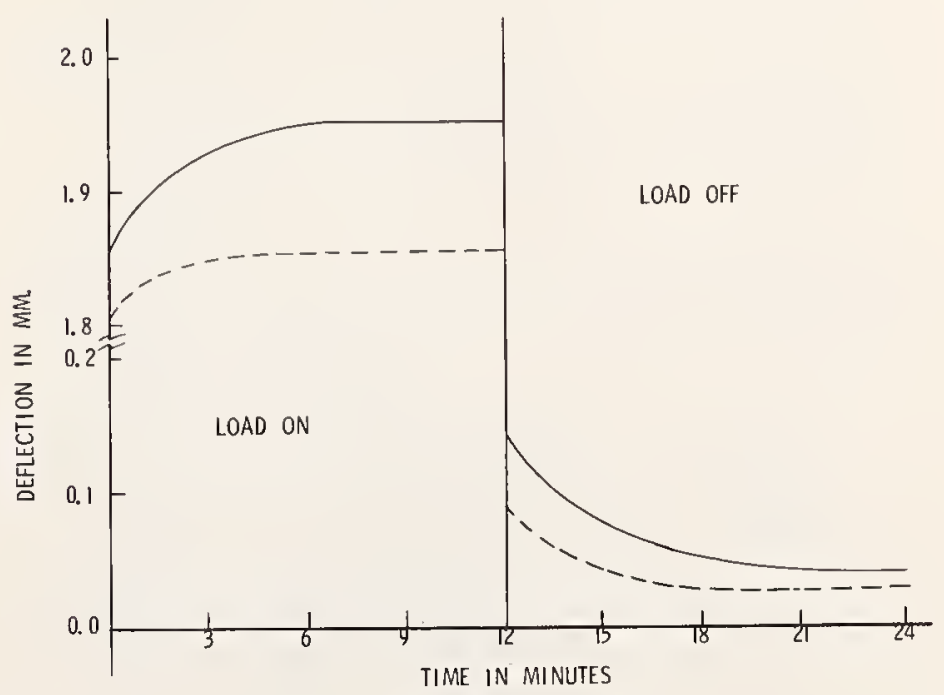

FIGURE 25. Deflection and recovery of two chemically strengthened glasses, as a function of time. 
When evaluating the effects of some environmental condition on strength, a different specimen geometry is often used under the special condition than is used under normal conditons. A comparison should be made of different types or sizes of specimens under identical conditions before comparisons of results under different conditions are attempted. An example of the effect of size on the strength of glass rods is shown in figure 26 [52], and it can be seen that there is a considerable variation in strength with size.

In this connection it is instructive to consider Rudnick's [32] view. "The relationship between tensile strengths of brittle materials determined by any two test techniques is not a universal constant. It depends on the stress distributions developed by each technique, the specimen sizes used, and, particularly, the nature of the material itself. According to the statistical concepts of the strength of brittle materials, it is theoretically possible to achieve any desired value of strength, within limits, from any test procedure simply by varying the specimen size. Thus, while the bend strength is higher than the uniaxial tensile strength if specimens of equal size are used, this order can be reversed by employing very small tensile specimens and very large bend specimens."

An example of the effect of surfaces and edges on the strength is shown in table 1 [28]. This table presents strength test results for annealed, ground, and polished soda-lime-silica glass from two different manufacturers. The table gives the number of fractures that occurred at the edge or on the surface of the specimen and the average modulus of rupture. The data indicate that glass $B$ is stronger than glass $A$ and that glass $B$ has less severe flaws in the surface than glass $A$. Also shown, for both glasses, is that the average

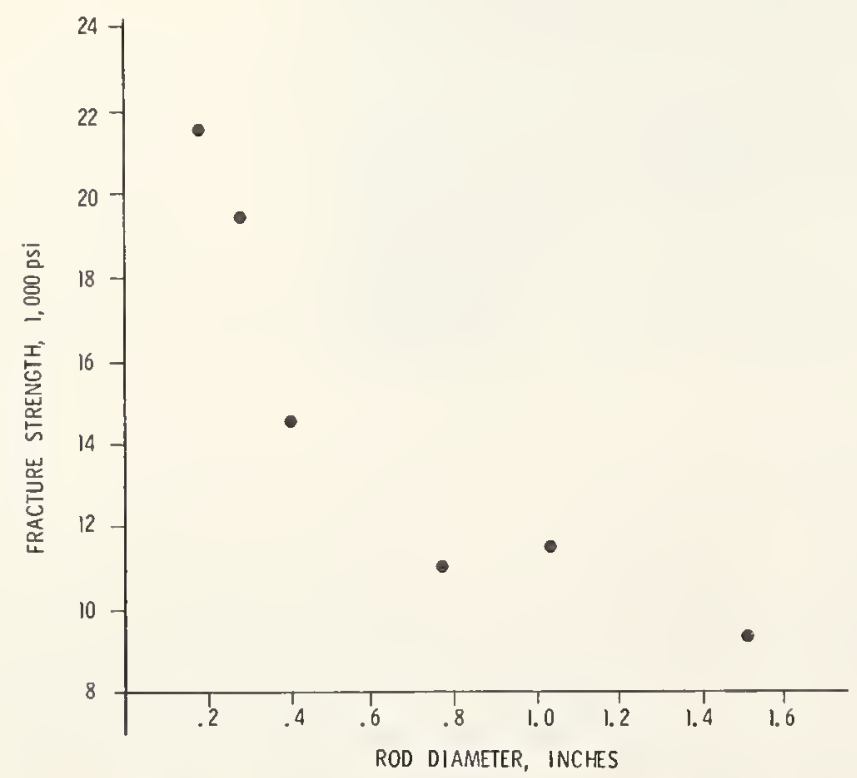

Figure 26. Average fracture strength versus rod diameter for annealed Pyrex glass, showing how strength decreases as rod diameter increases. strength of those specimens that fracture from the edge is consistently lower than that of those fracturing from the surface. The table also shows another example of the size effect, for as the area under uniform stress increases the strength decreases.

TABLE 1. Effects of surfaces and edges on flexural strength

\begin{tabular}{|c|c|c|c|c|c|c|}
\hline \multirow{2}{*}{$\begin{array}{l}\text { Area } \\
\text { under } \\
\text { max. } \\
\text { stress }\end{array}$} & \multicolumn{3}{|c|}{ Glass A } & \multicolumn{3}{|c|}{ Glass B } \\
\hline & $\begin{array}{l}\text { Fracture } \\
\text { origin }^{1}\end{array}$ & $\begin{array}{l}\text { Number of } \\
\text { fractures }\end{array}$ & $\begin{array}{l}\text { Mod. of } \\
\text { rupture }\end{array}$ & $\begin{array}{l}\text { Fracture } \\
\text { origin } 1\end{array}$ & $\begin{array}{l}\text { Number of } \\
\text { fractures }\end{array}$ & $\begin{array}{l}\text { Mod. of } \\
\text { rupture }\end{array}$ \\
\hline$\stackrel{i n^{2}}{0}$ & $\stackrel{\mathrm{S}}{\mathrm{E}}$ & $\begin{array}{l}37 \\
13\end{array}$ & $\begin{array}{c}p s i \\
19308 \\
16550\end{array}$ & $\stackrel{\mathrm{S}}{\mathrm{E}}$ & $\begin{array}{l}30 \\
20\end{array}$ & \begin{tabular}{cc}
\multicolumn{2}{c}{$p s i$} \\
21 & 572 \\
19 & 091
\end{tabular} \\
\hline 3 & $\stackrel{\mathrm{S}}{\mathrm{E}}$ & $\begin{array}{l}34 \\
16\end{array}$ & $\begin{array}{l}17546 \\
15016\end{array}$ & $\stackrel{S}{E}$ & $\begin{array}{l}33 \\
17\end{array}$ & $\begin{array}{l}20712 \\
17994\end{array}$ \\
\hline 6 & $\stackrel{\mathrm{S}}{\mathrm{E}}$ & $\begin{array}{l}33 \\
17\end{array}$ & $\begin{array}{l}15260 \\
13341\end{array}$ & $\stackrel{\mathrm{S}}{\mathrm{E}}$ & $\begin{array}{l}32 \\
18\end{array}$ & $\begin{array}{l}19215 \\
14919\end{array}$ \\
\hline 12 & $\stackrel{\mathrm{S}}{\mathrm{E}}$ & $\begin{array}{l}19 \\
31\end{array}$ & $\begin{array}{l}13365 \\
11942\end{array}$ & $\stackrel{\mathrm{S}}{\mathrm{E}}$ & $\begin{array}{l}21 \\
28\end{array}$ & $\begin{array}{l}16968 \\
14788\end{array}$ \\
\hline
\end{tabular}

$\mathrm{S}=$ surface

$\mathrm{E}=$ edge.

\section{Selecting a Test Method}

The criteria involved in selecting a test method are more complex than immediately meets the eye, and are beyond the scope of this paper. The reader faced with this problem is referred to Rudnick and Duckworth [40], who have described the principles guiding the selection of a test method exceptionally well. The principles are based upon the intended use of the test results. If it is intended to determine, as closely as possible, the "true" tensile strength of a brittle material, the work of Bortz and Wade [7] merits careful study. On the other hand, the designer concerned with a particular piece of hardware would be wise to follow Boland and Walton's [44] suggestion, i.e., he should see that the materials evaluated for his program are tested in such a manner that stress fields are similar to those encountered in service. To use data obtained under near-ideal conditions for an application in which the material will be used in a very non-ideal situation would almost guarantee failure.

To this we add that no test method is better than the experience and competence with which it is used.

\section{References}

[1] Harmsworth, C. L., Design criteria and test techniques, Air Force Materials Symposium/1965Technical Papers, AF Materials Lab Technical Report No. AFML-TR-65-29, 831 (1965).

[2] Duckworth, W., Strength testing of ceramics, Presented to Electronics Div, Am. Ceram. Soc., Chicago (Sept. 1966).

[3] Shook, W. B., Critical survey of mechanical property test-methods for brittle materials, AF Materials Lab Technical Documentary Report No. ASDTDR-63-491 (July 1963). 
[4] Hughes, B. P., and Chapman, G. P., Direct tensile test for concrete using modern adhesives, RILEM Bull. 26, 77 (Mar. 1965).

[5] Barnett, R. L., Costello, J. F., Hermann, P. C., and Hofer, K. E., Jr., The behavior and design of brittle structures, AF Flight Dynamics Lab Technical Report No. AFFDL-TR-65-165 (Sept. 1965) AD 475040.

[6] Bortz, S. A., Effect of structural size-the "zero strength," Studics of the Brittle Behavior of Ceramic Materials, AF Directorate of Materials and Processes Technical Documentary Report No. ASD-TR-61-628, 36 (April 1962) AD 277659.

[7] Bortz, S. A., and Wade, T. B., Analysis and review of mechanical testing procedures for brittle materials, Presented at Army seminar on Analysis of Mechanical Testing Procedures for Brittle Materials, Chicago (Mar. 1967).

[8] Pears, C. D., Evaluation of tensile data for brittle materials obtained with gas bearing concentricity, AF Directorate of Materials and Processes Technical Documentary Report No. ASD-TDR-63-245 (May 1963) AD 410770.

[9] Digesu, F. J., and Pears, C. D., Some parameters of the mechanical behavior of graphites, Presented to Am. Ceram. Soc., Los Angeles (Oct. 1963).

[10] Todd, J. D., The determination of tensile stress/strain curves for concrete, Proc. Inst. Civil Engrs. (London) 4, Part 1, 201 (1955).

[11] Mordfin, L., and Robusto, T., Jr., Photographic strain measuring technique for use above $3000 \mathrm{~F}$, Proc. Instrument Soc. Am. 1\%, Part 1, 3.4.62-1 (1962)

[12] Sedlacek, R., and Halden, F. C., Method for tensile testing of brittle materials, Rev. Sci. Instr. 33, 298 (1962).

[13] Cornish, R. H., and Broutman, L. J., Effects of polyaxial stresses, Studies of the Brittle Behavior of Ceramic Materials, AF Materials Lab Technical Documentary Report No. ASD-TR-61-628, Part 3, 289 (June 1964) AD 444829.

[14] Sedlacek, R., Tensile strength of brittle materials, AF Materials Lab Technical Report No. AFMLTR-65-129 (Aug. 1965).

[15] Babel, H. W., Biaxial-fracture strength of brittle materials, AF Materials Lab Technical Report No. AFML-TR-66-51 (Mar. 1966).

[16] Dally, J. W., Design data for materials employed in thermal protective systems on advanced aerospace vehicles, AF Materials Lab Technical Documentary Report No. ML-TDR-64-204, Vol. 3 (Aug. 1965) AD 476964.

[17] Woldokoff, V. E., Briggs, D. D., and Moss, J. R., Modified Sedlacek-Halden ring test, Presented to Am. Ceram. Soc., New York (May 1967).

[18] Malhotra, V. M., Zoldners, N. G., and Woodrooffe, H. M., Ring test for tensile strength of concrete, Materials Research \& Standards 6, 2 (1966).

[19] Malhotra, V. M., and Zoldners, N. G., Comparison of ring-tensile strength of concrete with compressive, flexural, and splitting-tensile strengths, J. Materials 2, 160 (1967).

[20] Daniels, I. M., and Weil, N. A., Effect of non-uniform stress fields, Studies of the Brittle Behavior of Ceramic Materials, AF Directorate of Materials and Processes Technical Documentary Report No. ASD-TR-61-628, 119 (April 1962) AD 277659.

[21] Durelli, A. J., Morse, S., and Parks, V., The theta specimen for determining tensile strength of brittle materials, Materials Research \& Standards 2, 114 (1962).

[22] Bortz, S. A., A means for obtaining axial tension from a bending test, Presented to Am. Ceram. Soc., Pittsburgh (April 1963).

[23] Barnett, R. L., Utilization of refractory nonmetallic materials in future aerospace vehicles, AF Flight Dynamics Lab Technical Documentary Report No. FDL-TDR-64-123, Part 1, Review of struc- tural design techniques for brittle components under static loads (Sept. 1964) AD 607977.

[24] Cornish, R. H., and Abbott, B. W., Strain rate effects, Studies of the Brittle Behavior of Ceramic Materials, AF Materials Lab Technical Documentary Report No. ASD-TR-61-628, Part 3, 77 (June 1964) AD 444829.

[25] Abbott, B. W., and Cornish, R. H., A stress-wave technique for determining the tensile strength of brittle materials, Experimental Mechanics 5, 148 (1965).

[26] Standard method of flexure testing of glass, ASTM Designation C158-43 (1965), 1966 Book of ASTM Standards, Part 13, 113 (Am. Soc. Testing Materials, Philadelphia, 1966).

[27] Nadai, A., Plasticity, 128 (McGraw-Hill Book Co., New York, 1931)

[28] Kerper, M. J., and Scuderi, T. G., Comparison of single-point and two-point loading for determining the strength of flat glass, Proc. Am. Soc. Testing Materials 64, 1037 (1964).

[29] Anon., Versatility is the watchword, Closed Loop 1, No. 5, 11 (1966).

[30] Frocht, M. M., Photoelasticity, Vol. 2 (John Wiley \& Sons, New York, 1948).

[31] Love, T. S.; Structural analyses using photoelastic techniques, General Dynamics/Convair Applied Research Report No. 11, 131 (1967).

[32] Rudnick, A., Hunter, A. R., and Holden, F. C., An analysis of the diametral-compression test, Materials Research \& Standards 3, 283 (1963).

[33] Pincus, G., and Gesund, H., Evaluating the tensile strength of concrete, Materials Research \& Standards 5, 454 (1965).

[34] Standard method of test for splitting tensile strength of molded concrete cylinders, ASTM Designation C496-66, 1967 Book of ASTM Standards, Part 10, 356 (Am. Soc. Testing Materials, Philadelphia, 1967).

[35] Ivey, D. L., and Buth, E., Splitting tension test of structural lightweight concrete, J. Materials $\mathbf{1}, 859$ (1966).

[36] Timoshenko, S., and Goodier, J. N., Theory of elasticity, 2d cd., 121-123 (McGraw-Hill Book Co., New York, 1951).

[37] Frocht, M. M., Strength of materials, 175-177 (Ronald Press Co., New York, 1951).

[38] Durelli, A. J., Phillips, F. A., and Tsao, C. H., Introduction to the theorctical and experimental analysis of stress and strain, 441-455 (Mc Graw-Hill Book Co., New York, 1958).

[39] Bortz, S. A., and Lund, H. H., The brittle ring test, Mechanical Properties of Engineering Ceramics (Kriegel and Palmour, eds.), 383 (Interscience Publishers, New York, 1961).

[40] Rudnick, A., and Duckworth, W. H., Considerations in testing brittle materials, Presented at Army seminar on Analysis of Mechanical Testing Procedures for Brittle Materials, Chicago (Mar. 1967).

[41] Outwater, J. O., and Woodard, M. S., Skiing forces and fractures, Mech. Eng. 89, No. 12, 26 (Dec. 1967).

[42] Lynch, J. F., Ruderer, C. G., and Duckworth, W. H., Engineering properties of ceramics, AF Materials Lab Technical Report No. AFML-TR-66-52 (June 1966) AD 803765.

[43] Mordfin, L., Torsion creep of circular and noncircular tubes, J. Research NBS $\mathbf{7 1 C ,} 209$ (1967).

[44] Boland, P., and Walton, J. D., Jr., Aerospace ceramics-characteristics and design principles, AF Materials Lab Technical Report No. AFML-TR65-171 (June 1965) AD 469154.

[45] Standard method of test for compressive (crushing) strength of fired whiteware materials, ASTM Designation C407-58, 1966 Book of ASTM Standards, Part 13, 349 (Am. Soc. Testing Materials, Philadelphia, 1966). 
[46] Standard method of test for compressive strength of molded concrete cylinders, ASTM Designation C3966, 1967 Book of ASTMI Standards, Part 10, 27 (Am. Soc. Testing Materials, Philadelphia, 1967).

[47] Babel, H. W., and Sines, G., An improved method for uniaxial and biaxial testing of brittle materials, Douglas Aireraft Co. Paper No. 4244 (Mar. 1967) submitted to Am. Soc. Testing Materials.

[48] Test Engineering 18, No. 10, 38 (Oct. 1967).

[49] Evans, P. R. V., Effect of microstructure, Studies of the Brittle Behavior of Ceramic Materials, AF Directorate of Materials and Processes Technical
Documentary Report No. ASD-TR-61-628, Part 2, 164 (April 1963) AD 408925.

[50] Babel, H.W., and Sines, G., A biaxial fracture criterion for porous brittle materials, Douglas Aircraft Co. Paper No. 4221 (Feb. 1967), submitted to J. Appl. Mechanics.

[51] Kerper, M. J., and Scuderi, T. G., Mechanical properties of chemically strengthened glasses at high temperatures, J. Am. Ceram. Soc. 49, 613 (1966).

[52] Kerper, M. J., and Scuderi, T. G., Relation of the fracture stress to the fracture pattern for glass rods of various diameters, Bull. Am. Ceram. Soc. 45. 1065 (1966). 
A

Absorptance, 74, 126

Absorption coefficient, ultrasonic, 74, 174, 178

Alloying, effect on thermal conductivity, 85

Alumina, 2, 3, 15, 69, 111

crack nucleation, 232

creep, 192, 196

elastic constants, 146, 147, 153

emittance, 136

fracture, 232

fracture surface energy, 226

strength, 2, 3, 232, 246, 247, 250, 258

American Ceramic Society, 1, 5, 260-262

Anelasticity, 159, 189, 190, 259

Anisotropy, 146, 83, 90

effect on elastic moduli, 146, 154

effect on elastic waves, 151

effect on resonance, 152

Anisotropic grains, 15, 41, 42, 45

Annealing, 177, 180, 204

effect on emittance, 132

effect on thermal conductivity, 95

Annealing point, 180

Applications of ceramics, 1, 3, 13, 16, 2 213,260

ASTM, 1, 5, 9, 101, 102, 251, 261

B

Bend testing (see Flexure testing), 15, 210, 211

Beryllia, 110

crack nucleation, 232

creep, 193

fracture, 232

strength, 210, 232

thermal conductivity, 110

Binary systems, 3, 24, 87-92, 207, 212

Blackbodies, 38,126

Bonding, 202, 211, 212

Borides, 201, 208, 246

Brillouin zone, 66

Brittleness, 13, 14, 243 effect on design, 13, 14, 243, 260

Bulk modulus (see Compressibility), 142, $147,149,155$
Carbides, 201, 257

Ceramic industry, 1, 2, 15, 250

Ceramics, 1, 2, 3

Ceramic society, 1

Ceramics, IV applications, 13

ASTM activities, 9

relation to materials technology, 1

Character, IV, 14-16, 41

Characterization, 3, 14-16, 112, 208

with respect to elastic moduli, 161

Chemical composition, 3, 14-16, 41, 83

Clapeyron equation, 22

Coble creep, 191

Cohesive strength, 217

Compliance method, 223

Component, 19

Composites, 154

elastic moduli, 154

thermal conductivity, 87-92

thermal expansion, 55

Composition, 2, 3, 16

effect on thermal conductivity, $3,14,83$

effect on thermal expansion, 55

effect on elastic constants, 161

Compressibility (see Bulk modulus), 142, $147,149,155$

Compression testing, 247, 248, 250, 253, 255,256

Compromises in standards, 5

Conductivity

$$
\text { electrical, 70-73 }
$$

thermal, 3, 63-123

Congruent melting, 212

Consensus, 10

Continuing education, 1,2

Crack nucleation, 229, 231, 246

Crack propagation, 230, 235, 246

Crack tip, 220

nature of, 220

plastic deformation at, 222, 225

Creep, 189, 191-192, 195, 197-198, 204, 211

Critical point, 177

Crystal structure, 42, 83-84, 209 effect on reflectance, 132

Crystallinity, effect on thermal conductivity, 85 


\section{D}

Deformation, 189, 198, 201, 243

Deformation point, 177

Degree of freedom, 19, 77

Delayed failure, 234

Density, 16, 41, 95

measurement, 172

Design techniques, 14-15

Dielectric constant, 156

Dielectric strength, 3

Diffusion, 64, 78, 191, 204

Dislocations, 2, 70, 202, 203, 204

motion, 195, 197, 204

\section{$\mathrm{E}$}

Edge and surface effects, 15, 159, 246, $251-253,255,259,260$

on thermal radiation properties, 127

Education (see Continuing education), 1, 2 Elastic constants, 14, 139, 209, 246, 248,

255,256

glasses and melts, 177

isotropic body, second order, 147

isotropic body, third order, 149

measurement, 160

porosity dependence, 153

pressure dependence, 149

single crystal, second order, 145

single crystal, third order, 148

temperature dependence, 157, 209

Electrical conductivity, 70-73

Electrical properties, 2, 3

Electrical resistance, 3

Electromagnetic fields, effect on thermal conductivity, 81

Electromigration, 78

Electron microscopy, 204, 205, 207, 212

Electronic conduction, 70-72

Electronic structure, 70, 83, 202, 212

Electrons, 70-72

free, effect on expansivity, 45

Emittance, 37, 73-76, 126

Engineering, 2, 3

Exciton, 78

Extinction coefficient, 74, 132

F

Fermi function, 70

Firing temperature, effect on thermal conductivity, 95
Firing time, effect on thermal conductivity, 95

Flaw theory, 14, 219

Flexure testing, 248-253, 255, 256, 259

Fourier's law, 64

Fracture, 14, 217, 243

continuum approach, 220

grain boundaries, 226, 231

surface energy approach, 222

Fracture mechanics, 14, 217

Fracture surface energy, 217

bicrystals, 226

glass, 225

measurement methods, 224

polycrystalline ceramics, 226

single crystals, 226

Friction effects in testing, 244-246, 251, $254,256,257$

Fulcher equation, 183

Fused silica, 80, 85, 111

Fusion curve, 22, 23, 25

G

Gas conduction, 77, 81

Gifkins creep, 191

Glass, 1, 2, 14, 53, 85-86, 111, 177, 190, $198,217,225,228,234,252,253,259$, 260

Glass-crystal system, 54, 55, 111

Glass transformation, 177

Glass transition, 177, 180, 182

Grain boundaries, 2, 3, 14, 189, 210, 226,

255

effect on thermal conductivity, 93-95

Grain boundary sliding, 190, 197

Grain size (or growth), 2, 14, 15, 16, 210

effect on expansivity, 41, 55, 210

Graphite, elastic constants, 160

Griffith theory, 219

Griffith flaws, 14, 219

$\mathrm{H}$

Hard metals, 201

Heat capacity, 64

Heat conduction, 63-123

Heat transfer, 125, 63-123

Heat treatment, effect on thermal

conductivity, 95

Heterogeneous materials, 87

emittance of, 131

thermal conductivity of, 87-95 
Holes, 72

I

Imperfections, effect on thermal conductivity, 86-87

Impurities, 16

effect on expansivity, 41

effect on thermal conductivity, 84-85

Index of refraction, 73, 75-76, 156

Inelastic deformation (see Plastic deformation), 189, 190, 191, 201, 228, 231, 248,252

Insulators, spark plug, 1, 3

Interferometer, 48

\section{$\mathbf{L}$}

Lattice conduction, 65-70

Lattice dynamics, 44, 65-67, 156

Lorenz function, 71-72

\section{M}

Magnesia, III

crack nucleation, 232

creep, 194, 196

elastic constants, 146, 147, 149, 150,

153

emittance, 136

fracture, 232

plastic deformation, 201, 231

strength, 231, 232, 258

Materials, 5, 14

Mechanical behavior, 1, 2, 3, 14, 189,

201,243

elasticity, 139

fracture, 217,243

plastic deformation, 189, 201

viscoelasticity, 169

testing, 15, 243

Mechanical properties (see Mechanical

behavior), 1, 2, 3, 14, 189, 201

Mechanical testing (see Methods of testing), $15,47,96-108,133,243$

Melting point, 19

methods, 33

Metastability, 20, 23

Metastable equilibria, 23
Methods of testing, 15, 47, 96-108, 133, 243

brittle ring, 255

compression, 256

diametral compression, 253

flexure, 250

large area bending, 252

pressurized ring, 246

stress wave, 250

tension, 243

theta specimen, 248

torsion, 256

truss beam, 249

Mic roc racks, 189, 202, 246, 258

effect on expansivity, 55

effect on the rmal conductivity, 94

Microcrystalline glass, 111

Microhardness, 3, 209, 210, 211, 212

Microstructure (see Character), IV, 1, 2,

$3,15,210,255$

effect on thermal conductivity, 9394

effect on viscoelasticity, 182

Modulus of rupture, 210, 251, 260

Moduli of glasses and liquids, 178

Multi-component system, 28, 55, 87-93

\section{$\mathrm{N}$}

Nabarro-Herring creep, 191

National Bureau of Standards, II, III, IV, 1,111

National Research Council, IV, 2, 112

Neutron irradiation, effect on thermal conductivity, 86-87

O

One component system, 20

Optical absorption, 156

Optical comparator, 49

Optical thickness, 75

Ordered compounds, 206, 212

$\mathbf{P}$

Petch relationship, 210, 229

Phase diagrams, 2, 201

Phase rule, 19 
Phonons, 65-69

Photoelasticity, 248, 249, 252, 253, 255

Photons, 73-77

Planck's law, 36, 73, 128

Plastic deformation (see Inelastic deformation), 189, 190, 191, 201, 228, 231, 256 at crack tip, 222 crack nucleation due to, 228,229 in crystals, 189,228 in glass, 225

Point defects, 70, 159, 189

Polycrystalline ceramics, 2, 3, 208 crack nucleation, 228, 229

creep, 189

elastic moduli, 147, 153

fracture surface energies, 226

plastic deformation, 228

strength, 3, 228

Porosity, 2, 3, 14-16, 210

effect on creep, 198

effect on elastic moduli, 153

effect on expansivity, 41,53

effect on thermal conductivity, 90-93

Predictability, 14, 16

Pressure

effect on elastic moduli, 142, 149, 155

effect on thermal conductivity, 81-82

Pressure testing, 246-250, 253, 257

Processing, IV, 14-16

Properties, 2, 3

Pyrometry, 36

Q

Quartz, 44, 54, 80, 85, 111

R

Radiation "conduction", 73-77

Raoult-Van't Hoff equation, 26

Reference standards

glass, 172

thermal conductivity, 108-112

thermal expansion, 53

Reflectance, 126

Relaxation time, 178

average, 180

Reliability, 13, 15, 95-96

Resonance, 152
Sapphire, 69

fracture surface energy, 226

strength, 217

Scattering coefficient, 74,131

Semiconductor, 72-73

Silica, 44, 54, 80, 85, 111

Silicon, thermal conductivity, 110

Silicon carbide, creep, 195

Simon equation, 22

Single crystals, 201

elastic constants, 145

fracture surface energy, 226

plastic deformation, 197, 201

strength, 228

Sintering, 2, 85

Size effects, 252, 256, 259, 260

Slip systems, 209

Solid solution, effect on elastic constants, 157

Soret Effect, 78

Sound velocity, 150, 156

Spark plug insulators, 1, 3

Specific heat, 64

relation to elastic constants, 156

Spectrum of relaxation times, 178,179

Stable equilibria, 20

Standards, 5, 9, 37, 38, 53, 108-112, 253, $255,257,261$

glasses, 172

thermal conductivity, 108-112

thermal expansion, 53

Static fatigue, limit, 236

Stoichiometry, 202, 208

effect on expansivity, 41

effect on thermal conductivity, 85

Strain, 144, 202, 244-250, 255, 256

Strain energy release rate, measurement methods, 223

Strain gages, 245, 246, 250, 255-259

Strain point, 182

Strain rate effects, 204, 250, 259

Strength, 2, 3, 13-15, 201, 243

alumina, 2, 3, 232, 246, 247, 250, 258

cohesive, 217

effect on environment on, 14-15, 234

glass, 225, 228, 252, 253, 259, 260

magnesia, 231, 232, 258

polycrystalline ceramics, 3,228 
Strength-Continued

single crystals, 228

theoretical, 13, 217

Stress, 13-16, 81-82, 143, 243

effect on expansivity, 56

effect on thermal conductivity, 81-82

Stress corrosion, 14-15, 217, 225, 234, 235

activation energy for, $236,237,238$

activation volume for, 236,238

in alumina, 235, 238

in glass, $14,235,237,238$

Stress intensity factor, 217,220

measurement methods, 224

Structral applications, 1, 13, 16, 201 213,243

Structural ceramics, 1, 213, 243

Structural relaxation, 178

Superplasticity, 198

Surface, 14

Surface effects (see Edge and surface effects), 14, 15

Surface energy (see Fracture surface energy), 217

Surface flaws, 201

Surface topography, 16

$\mathrm{T}$

Technology, 1, 5

Temperature, 36

effect on elastic constants, 157

effect on expansivity, 44

effect on thermal conductivity, 78-81

mechanical testing at elevated, 202,

$$
246,248,250,255-259
$$

Temperature coefficient of emittance, 127, 128

Temperature scales, 36

Tension testing, 244, 246, 248-253, 255257

Tentative standards, 9

Ternary systems, 28,32

Test data (see also Methods of testing), 1415,243

Test procedures (see also Methods of testing), 14-15, 133, 243

Thermal activation, 203

Thermal conductivity, $3,63-123$ measurement methods, 96-108
Thermal diffusivity, 64

Thermal expansion, 41,94

Thermal expansion anisotropy, stresses due to, 94,230

Thermal history, effect on thermal conductivity, 94-95

Thermal properties, IV, $1,2,3,13-14$, 41,63

melting points, 19

thermal conductivity, 63

thermal expansion, 41

thermal radiation properties, 7677,125

thermal shock, 217, 230, 233

Thermal radiation, $73-77,125$

Thermal radiation properties, 76-77, 1\%5

Thermal shock, 3, 217, 230, 233

crack nucleation, $217,230,233$

Thoria, 112, 136, 190

Torsion testing, 256

$\mathrm{U}$

Ultrasonic propagation

electronic apparatus, 175

measurement technique, 176

Umklapp process, 67-68

\section{V}

Variability, 13-15, 243-248, 250, 255-257

Viscoelasticity, 169,177

Viscosity, 169, 191, 198

definition of shear and volume viscosity, 179

measurement of longitudinal and shear viscosity by ultrasonic propagation, 174

measurement of shear viscosity

by rotating cylinder, 169

by fiber elongation, 171

by beam bending, 171

non-equilibrium viscosity, 182

non-linear viscosity, 182

temperature dependence of, 183

theories of, 185

Viscous relaxation, 178

Vitreous-silica dilatometer, 47

Voluntary standardization process, 5, 9

Voluntary standards, 5, 9 
Wave propagation, 65-67, 150, 174, 250

Wave vector, 66-67

Weight efficiency, 13

$\mathrm{X}$

$\mathrm{X}$-ray diffractometer, 50

$\mathrm{Y}$

Yield strength, 202

Z

Zirconia, 93, 136, 190, 258

emittance, 136 
PERIODICALS

JOURNAL OF RESEARCH reports National Bureau of Standards research and development in physics, mathematics, chemistry, and engineering. Comprehensive scientific papers give complete details of the work, including laboratory data, experimental procedures, and theoretical and mathematical analyses. Illustrated with photographs, drawings, and charts.

\section{Published in three sections, available separately:}

\section{- Physics and Chemistry}

Papers of interest primarily to scientists working in these fields. This section covers a broad range of physical and chemical research, with major emphasis on standards of physical measurement, fundamental constants, and properties of matter. Issued six times a year. Annual subscription: Domestic, $\$ 6.00$; foreign, $\$ 7.25 *$.

\section{- Mathematical Sciences}

Studies and compilations designed mainly for the mathematician and theoretical physicist. Topics in mathematical statistics, theory of experiment design, numerical analysis, theoretical physics and chemistry, logical design and programming of computers and computer systems. Short numerical tables. Issued quarterly. Annual subscription: Domestic, $\$ 2.25$; foreign, $\$ 2.75^{*}$.

\section{- Engineering and Instrumentation}

Reporting results of interest chiefly to the engineer and the applied scientist. This section includes many of the new developments in instrumentation resulting from the Bureau's work in physical measurement, data processing, and development of test methods. It will also cover some of the work in acoustics, applied mechanics, building research, and cryogenic engineering. Issued quarterly. Annual subscription: Domestic, $\$ 2.75$; foreign, $\$ 3.50 *$.

\section{TECHNICAL NEWS BULLETIN}

The best single source of information concerning the Bureau's research, developmental, cooperative and publication activities, this monthly publication is designed for the industry-oriented individual whose daily work involves intimate contact with science and technology-for engineers, chemists, physicists, research managers, product-development managers, and company executives. Annual subscription: Domestic, $\$ 3.00$; foreign, $\$ 4.00^{*}$.

* Difference in price is due to extra cost of foreign mailing.

Order NBS publications from:

Superintendent of Documents

Government Printing Office
Washington, D.C. 20402

Government Printing Office
Washington, D.C. 20402
NONPERIODICALS

Applied Mathematics Series. tables, manuals, and studies.

Building Science Series. Research results, test methods, and performance criteria of building materials, components, systems, and structures.

Handbooks. Recommended codes of engineering and industrial practice (including safety codes) developed in cooperation with interested industries, professional organizations, and regulatory bodies.

Special Publications. Proceedings of NBS conferences, bibliographies, annual reports, wall charts, pamphlets, etc.

Monographs. Major contributions to the technical literature on various subjects related to the Bureau's scientific and technical activities.

National Standard Reference Data Series. NSRDS provides quantitative data on the physical and chemical properties of materials, compiled from the world's literature and critically evaluated.

Product Standards. Provide requirements for sizes, types, quality and methods for testing various industrial products. These standards are developed cooperatively with interested Government and industry groups and provide the basis for common understanding of product characteristics for both buyers and sellers. Their use is voluntary.

Technical Notes. This series consists of communications and reports (covering both other agency and NBS-sponsored work) of limited or transitory interest.

Federal Information Processing Standards Publications. This series is the official publication within the Federal Government for information on standards adopted and promulgated under the Public Law 89-306, and Bureau of the Budget Circular A-86 entitled, Standardization of Data Elements and Codes in Data Systems.

\section{CLEARINGHOUSE}

The Clearinghouse for Federal Scientific and Technical Information, operated by NBS, supplies unclassified information related to Governmentgenerated science and technology in defense, space, atomic energy, and other national programs. For further information on Clearinghouse services, write:

Clearinghouse

U.S. Department of Commerce

Springfield, Virginia 22151 






